\title{
Fluidized Bed Asbestos Sampler Design and Testing
}

Karen E. Wright

Barry H. O'Brien

December 2007

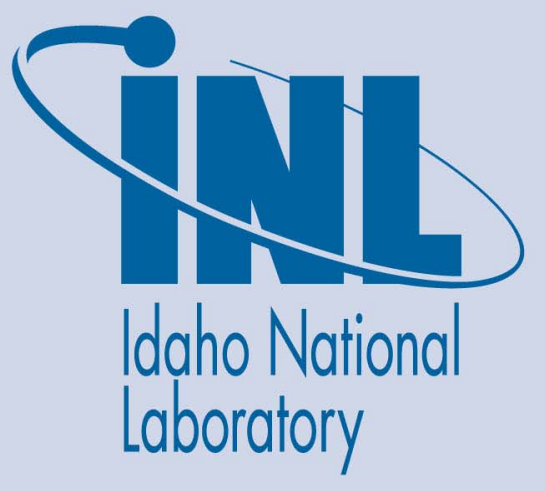

The INL is a U.S. Department of Energy National Laboratory operated by Battelle Energy Alliance 


\title{
Fluidized Bed Asbestos Sampler Design and Testing
}

\author{
Karen E. Wright
}

Barry H. O'Brien

December 2007

\section{Idaho National Laboratory \\ Idaho Falls, Idaho 83415}

\author{
Prepared for \\ Office of Research and Development \\ National Exposure Research Laboratory \\ Environmental Sciences Division \\ U.S. Environmental Protection Agency \\ Las Vegas NV 89193-3478 \\ Task Order Manager: Dr. Brian Schumacher \\ and the \\ U.S. Department of Energy \\ Under DOE Idaho Operations Office \\ Contract DE-AC07-05ID14517
}





\section{Fluidized Bed Asbestos Sampler Design and Testing}

INL/EXT-07-13122

Approved by

K. E. Wright, Principal Investigator

B. H. O’Brien,

, Reviewer
Date

Date

Date 



\section{ABSTRACT}

A large number of samples are required to characterize a site contaminated with asbestos from previous mine or other industrial operations. Current methods, such as EPA Region 10's glovebox method, or the Berman Elutriator method are time consuming and costly primarily because the equipment is difficult to decontaminate between samples. EPA desires a shorter and less costly method for characterizing soil samples for asbestos. The objective of this was to design and test a qualitative asbestos sampler that operates as a fluidized bed. The proposed sampler employs a conical spouted bed to vigorously mix the soil and separate fine particulate including asbestos fibers on filters. The filters are then analyzed using transmission electron microscopy for presence of asbestos.

During initial testing of a glass prototype using ASTM 20/30 sand and clay fines as asbestos surrogates, fine particulate adhered to the sides of the glass vessel and the tubing to the collection filter - presumably due to static charge on the fine particulate. This limited the fines recovery to $\sim 5 \%$ of the amount added to the sand surrogate. A second prototype was constructed of stainless steel, which improved fines recovery to about $10 \%$. Fines recovery was increased to $15 \%$ by either humidifying the inlet air or introducing a voltage probe in the air space above the sample. Since this was not a substantial improvement, testing using the steel prototype proceeded without using these techniques.

Final testing of the second prototype using asbestos suggests that the fluidized bed is considerably more sensitive than the Berman elutriator method. Using a sand/tremolite mixture with $0.005 \%$ tremolite, the Berman elutriator did not segregate any asbestos structures while the fluidized bed segregated an average of 11.7. The fluidized bed was also able to segregate structures in samples containing asbestos at a $0.0001 \%$ concentration, while the Berman elutriator method did not detect any fibers at this concentration.

Opportunities for improvement with the fluidized bed include improving reproducibility among replicates, increasing mass recovery, improving the lid gasket seal. 



\section{CONTENTS}

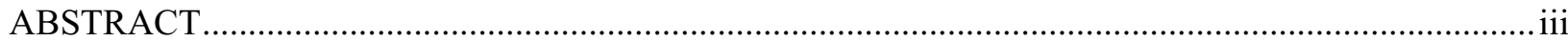

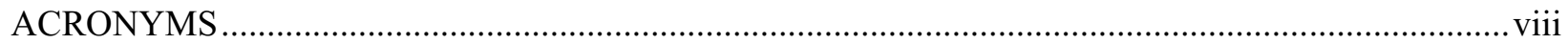

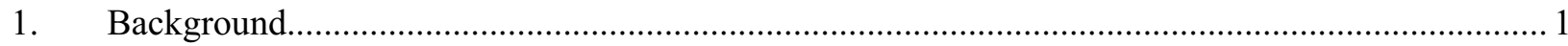

1.1 Theory of Fluidized Bed Operation.......................................................................... 1

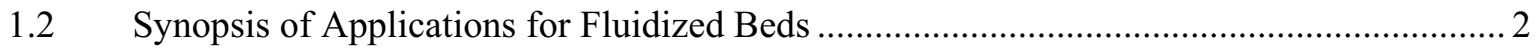

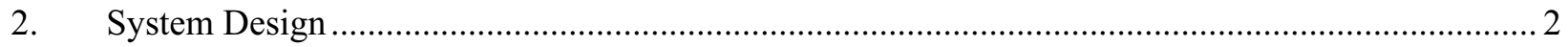

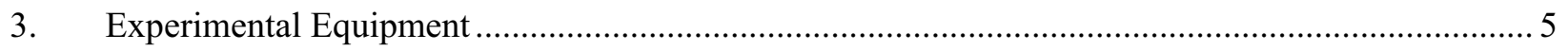

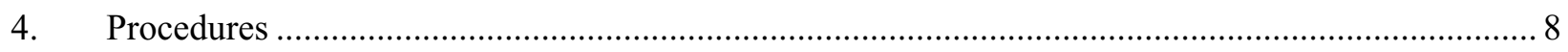

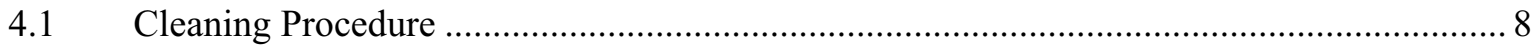

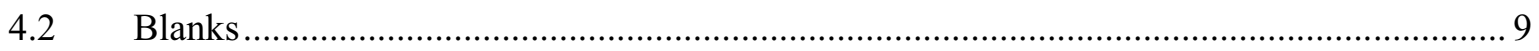

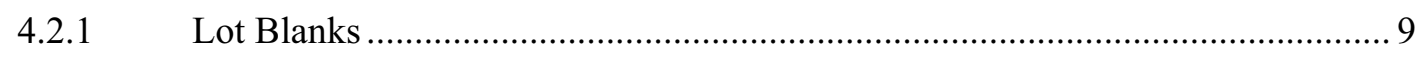

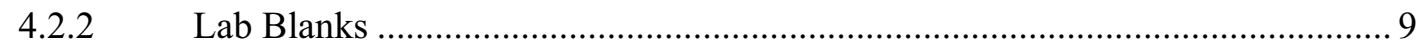

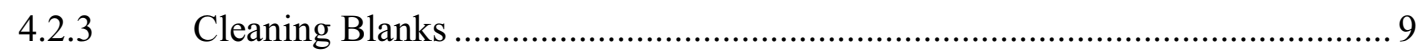

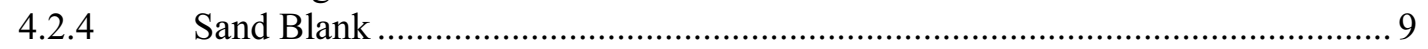

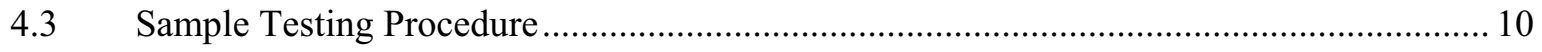

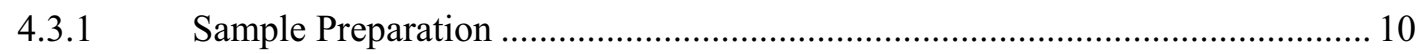

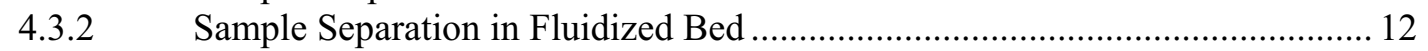

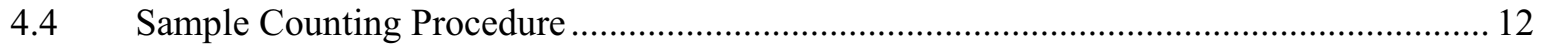

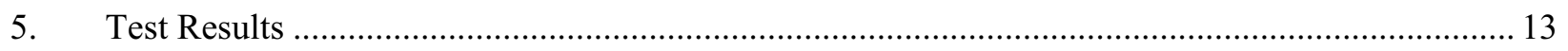

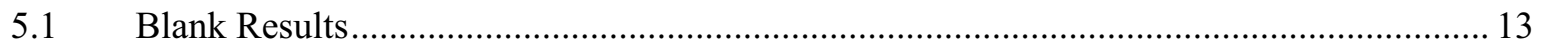

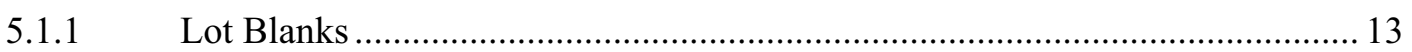

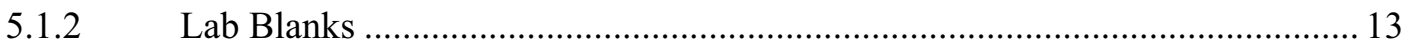

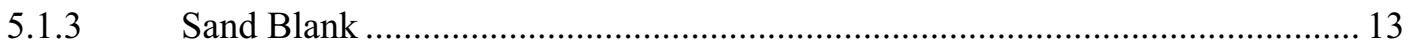

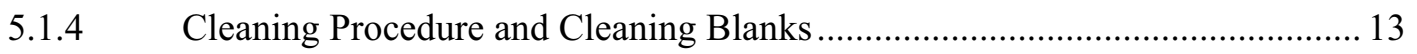

$5.2 \quad$ Fluidized Bed Sample Test Results ............................................................................... 14

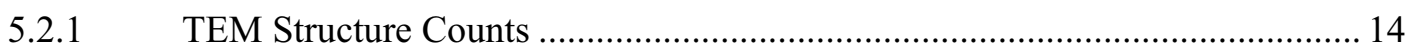

5.2.2 Comparison of TEM Data with Sample Concentrations ..................................... 16

5.2.3 Size Distributions for Asbestos Structures .................................................... 16

$5.3 \quad$ Berman Elutriator Test Results .................................................................................. 18

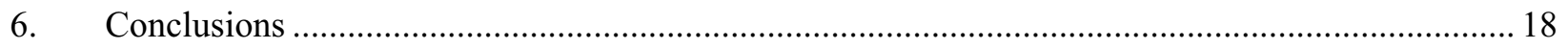

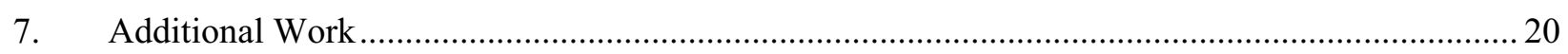




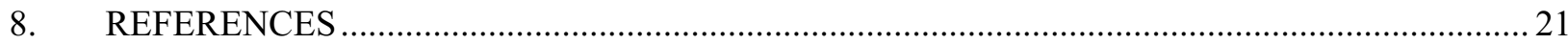

Appendix A Design Calculations for the Conical Spouted Bed Elutriator ............................................ 22

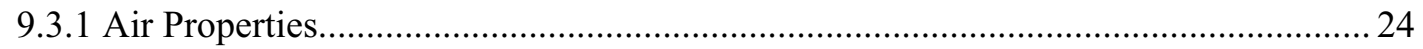

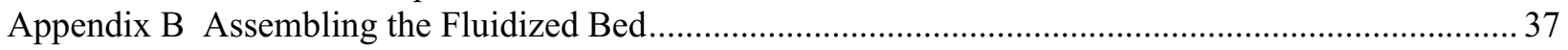

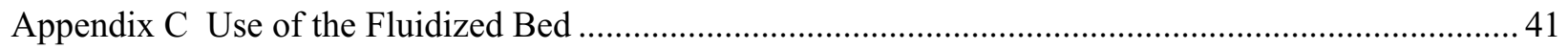

Appendix D Mathematical Foundation for the Release of Asbestos into a Confined Volume................. 44

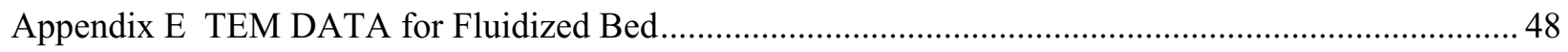

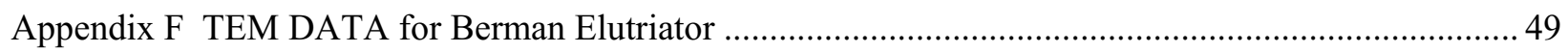

\section{FIGURES}

Figure 1. Solids mixing pattern for a conical spouted bed................................................................ 2

Figure 2. Conical spouted bed test unit (dimensions in $\mathrm{cm}$ ) ........................................................... 4

Figure 3. Calculated particle sizes elutriated versus air flow for the prototype conical spouted bed. ....... 5

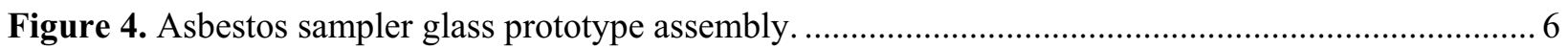

Figure 5 (a-b). Asbestos sampler stainless steel prototype test assembly. a. schematic of working prototype (this prototype uses three $25 \mathrm{~mm}$ collection filters); b. photograph of actual steel prototype.

Figure 6. Number of structures versus sample concentration. ............................................................. 16

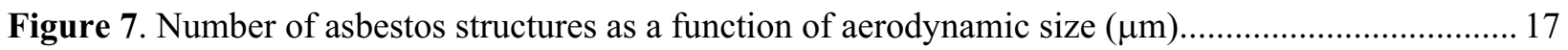

\section{TABLES}

Table 1. Test Matrix (wt $\%$ tremolite in an ASTM 20/30 quartz sand matrix)...................................... 10

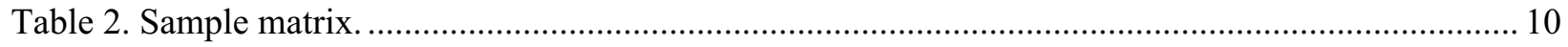

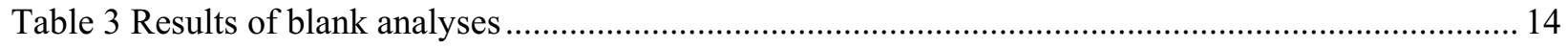

Table 4. Results of samples segregated by fluidized bed and counted using TEM............................... 15

Table 5. Results of samples segregated by the Berman Elutriator and counted using TEM .................... 18

Table 6. Comparison of 0.005 wt.\% tremolite run in Berman Elutriator versus Fluidized Bed..........27 


\section{ACRONYMS}

$\begin{array}{ll}\text { alpm } & \text { actual liters per minute } \\ \text { ASTM } & \text { American Society for Testing Materials } \\ \text { FB } & \text { Fluidized Bed } \\ \text { CAS } & \text { Chemical Abstract System } \\ \text { cfm } & \text { cubic feet per minute } \\ \text { DC } & \text { Direct Current } \\ \text { INL } & \text { Idaho National Laboratory } \\ \text { NIST } & \text { National Institute for Standards and Testing } \\ \text { lpm } & \text { liters per minute } \\ \text { ppmwt } & \text { Parts per million by weight } \\ \text { ppmv } & \text { Parts per million by volume } \\ \text { psia } & \text { pounds per square inch absolute } \\ \text { slpm } & \text { Standard liters per minute } \\ \text { TEM } & \text { Transmission Electron Microscopy } \\ \text { USEPA } & \text { United States Environmental Protection Agency } \\ \text { USGS } & \text { United States Geological Survey } \\ & \\ \text { IN } & \end{array}$




\section{Background}

Asbestos is a generic term for two mineral groups that include six naturally occurring, fibrous silicates (Occupational Safety and Health Administration, 1992). Asbestos has been widely used in commercial products because of its useful properties, including resistance to heat and fire. However, it is hazardous if inhaled. Asbestos in soil and soil-like material at multiple locations such as those in and around Libby, Montana endangers public health. In addition, there are numerous locations around the US where asbestos-containing material will need to be evaluated for health risk and cost-effective methods for measuring the potential release of asbestos.

The hazard posed by asbestos-bearing materials depends on the amount of asbestos that can be suspended from the material when it is disturbed. A qualitative procedure was developed (Januch and McDermott, SOP EPA Region 10-IEU-001, 2005) to evaluate the potential for asbestos to become airborne when a soil or other similar asbestos-containing matrix is disturbed. This procedure involves disturbing a quantity of soil or soil-like material in a glovebox, while sampling the air in the glovebox and capturing released particles on a filter. This procedure was effective at indicating the potential for suspension of asbestos from soil at the site, but was relatively time-consuming. A mathematical foundation for release of asbestos into such a box is provided in Appendix D of the current report. Another method was developed by Berman (Modified Elutriator Method for the Determination of Asbestos in Soils and Bulk Material, Revision 1 May 23, 2000). This method involves putting soil or soil-like material into a cylindrical, rotating tumbler while pulling air through the cylinder, upwards through tubes. The method separates the PM10 fraction of the material and pulls it up through the tubes to capture it on filters. Drawbacks of this method include its expense and low sample throughput.

Processing material to suspend asbestos for subsequent characterization is one of several timeconsuming steps in current methods. Increased sample throughput and decreased cost per sample could be achieved by developing alternative methods for sample suspension and particle collection for subsequent microscopy analysis. The use of a fluidized bed (FB) for sample suspension could potentially address these issues. Fluidization of the soil samples with air will effectively mix the entire sample, contact it with the air and entrain the fine particulates from the soil where they can be collected on a filter for later analysis.

\subsection{Theory of Fluidized Bed Operation}

Solids fluidization involves flowing gas or liquid upward through a bed of particulate solids. When the flow is increased to where the pressure drop through the bed equals the weight of the bed, the solids begin to circulate and act like a fluid. The flow rate at which the bed particles become fluidized is the minimum fluidization velocity or minimum spouting velocity. The fluidized bed velocities for fluidized bed design and operation are usually reported in terms of the superficial velocity, which is the velocity of the gas in the empty vessel. The minimum velocity to fluidize solids is dependent on the shape, density, and average size of the particles, and on the velocity of the gas at the bottom of the vessel.

The most common type of fluidized bed consists of a cylindrical vessel with a porous gas distributor plate at the bottom. Cylindrical fluidized-beds are generally limited to a narrow particle size range that can be completely fluidized under moderate velocities. Larger solids in the mixture require higher gas flow rates to keep them from settling at the bottom of the bed, but this increases elutriation of smaller particles from the bed.

Another type of fluidizing vessel is the spouted-bed, in which gas flows up through a small orifice into a conical vessel containing the solids. This type of fluidizing system will fluidize a much wider range of particle sizes than a cylindrical bed because the higher velocity in the lower part of the cone keeps 
large particles flowing while the large diameter in the upper part of the cone reduces the gas velocity, thus limiting the size of particles that are elutriated out of the vessel.

One version of the conical fluidized bed is the 'Pachuca Tank' (Perry 1973 pg 19-12), which uses introduction of gas or liquid through a tank consisting of a conical bottom attached to an upper cylindrical section (Figure 1). Pachuca tanks have been used to mix and fluidize large, dense solids such as gravel.

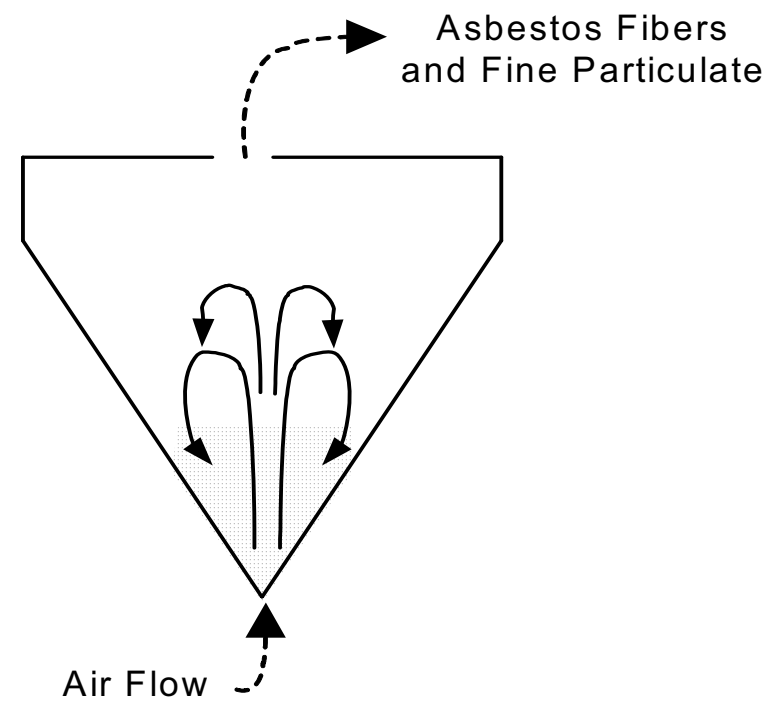

Figure 1. Solids mixing pattern for a conical spouted bed.

\subsection{Synopsis of Applications for Fluidized Beds}

Desirable features of fluidized beds for solids processing are their high mass transfer, excellent mixing and high heat transfer. Fluidization of particulate solids has many industrial and commercial applications, such as coal combustion, fluid-catalytic cracking of heavy oils into gasoline, liquid radioactive waste solidification, spray drying aqueous solutions, drying of solids such as cement and limestone, purification of silicon by decomposition of silane gas into silicon and hydrogen gas, and separation of fine particulates from solids.

Dust control and containment is a primary concern when processing and handling dusty solids. One common method to contain toxic, dusty, or radioactive materials is to operate the solids processing system under a vacuum. With the process piping and fluidized bed operated under vacuum, any leaks will be inward thus containing the contaminants inside the processing system and preventing contamination of the external surfaces of the equipment and personnel exposure.

\section{System Design}

Correlations for minimum fluidization and spouting velocities are available in literature, allowing for design of a system based on the expected operating conditions and desired operating characteristics. 
Settling velocity correlations are also available, allowing calculation of the size of particles expected to be elutriated out of the bed and onto the sample filter. Detailed design calculations were made for the conical spouted vessel design and are attached as Appendix A.

Several assumptions had to be made for the design calculations. These assumptions do not necessarily represent what was desirable for final performance of the device. They represent parameters that were used in the initial calculations to design the device. It is important to recognize that actual performance of the device may be highly sensitive to some parameters, but relatively insensitive to others. Empirical testing of the device is necessary to achieve the desired performance characteristics. Design assumptions include:

- Average soil particle diameter of $1 \mathrm{~mm}$ (about that for no. 20 US standard mesh) and maximum size of $0.25 \mathrm{in}$, assuming the samples will be sieved through a $1 / 4 \mathrm{in}$. sieve.

- Bulk properties of the soil (void fraction, sphericity, and particle density) are similar to those for round sand.

- Asbestos particle density is that of amphibole, at $3.0 \mathrm{~g} / \mathrm{cc}$

- The asbestos fibers have a diameter of 0.5 to $5 \mu \mathrm{m}$ and have a length to diameter ratio of 10 to 1 .

- Asbestos content in the soil is up to $1 \mathrm{wt} \%$ and the fine particulate in the soil is $1 \mathrm{wt} \%$ for a total fine particulate content of $2 \mathrm{wt} \%$ in the sample.

- $\quad$ Maximum desired filter loading is $0.4 \mathrm{~g}$.

- The fluidizing air is at $20^{\circ} \mathrm{C}$ and the local pressure at the INL altitude is 645 torr (12.47 psia).

- Inlet hole size of $3 \mathrm{~mm}$.

A conical vessel angle with sides $30^{\circ}$ from the vertical $\left(60^{\circ}\right.$ included cone angle) was selected for the sampler to give a reasonable bed height for the sample size and a reasonable vessel height, which reduces the velocity in the top of the cone to de-entrain larger particles. The soil sample size and height in the conical vessel, based on the weight percent of fine particulates and maximum filter loading were calculated to be (see Appendix A):

$$
\mathrm{m}_{\text {sample }}=20 \mathrm{~g} \quad \mathrm{H}_{\text {bed_ } 30 \mathrm{deg}}=3.32 \mathrm{~cm}
$$

The minimum spouting air flow for $1 \mathrm{~mm}$ average sand and for $1 / 4$ " average rocks/soil were calculated to be:

$$
\mathrm{Q}_{\mathrm{ms} \_30 \mathrm{deg}}=17.68 \frac{\mathrm{L}}{\mathrm{min}} \quad \mathrm{Q}_{\mathrm{ms} \_30 \operatorname{deg}_{-} \max }=44.56 \frac{\mathrm{L}}{\mathrm{min}}
$$

The equivalent spherical diameter for fibers with a length to diameter ratio of 10 , based on equivalent volumes, is 2.466 times the fiber diameter. For a maximum fiber diameter of $5 \mu \mathrm{m}$ and length of $50 \mu \mathrm{m}$, the equivalent spherical diameter and settling velocity were calculated to be: 


$$
\begin{aligned}
& \mathrm{D}_{\text {sph_asbestosMax }}=2.4662 D_{\text {asbestosMax }} \\
& \mathrm{u}_{\text {mintopofcone }}=2.19 \frac{\mathrm{cm}}{\mathrm{sec}}
\end{aligned}
$$$$
\mathrm{D}_{\text {sph_asbestosMax }}=12.08 \mu \mathrm{m}
$$

Based on the minimum air flow needed for spouting for samples having maximum expected particle size, the dimensions of the conical vessel to achieve the minimum velocity at the top of the cone were calculated to be:

$$
\mathrm{D}_{\text {topofcone_30deg }}=20.79 \mathrm{~cm} \quad \mathrm{H}_{\text {cone_30deg }}=18 \mathrm{~cm}
$$

A smaller cone than this will entrain and elutriate larger particles than the maximum assumed asbestos size. A larger cone than this would result in: 1) decreased velocity at the top; and 2) more surface area on which to deposit particles.

Initially, the first prototype vessel was fabricated of glass. Although the glass allowed visual observation of the spouted bed, the initial tests with sand and fine clay particulate resulted in poor recovery. About $5 \%$ of the fines were recovered on the filter. Fine particulate was adhering to the sides of the glass and tubing due to static charge, so a stainless steel funnel of similar in dimensions was procured and an inlet plug with inlet hole was installed in it. The dimensions for the stainless steel test unit are shown in Figure 2. Note that this funnel was obtained commercially thus deviated somewhat from the theoretical geometry indicated by our calculations. Desired air flows were recalculated for the commercially available funnel, and these flows were used for testing of the unit. A mounting stand and removable flat lid with rubber gasket seal were also fabricated for the second prototype.

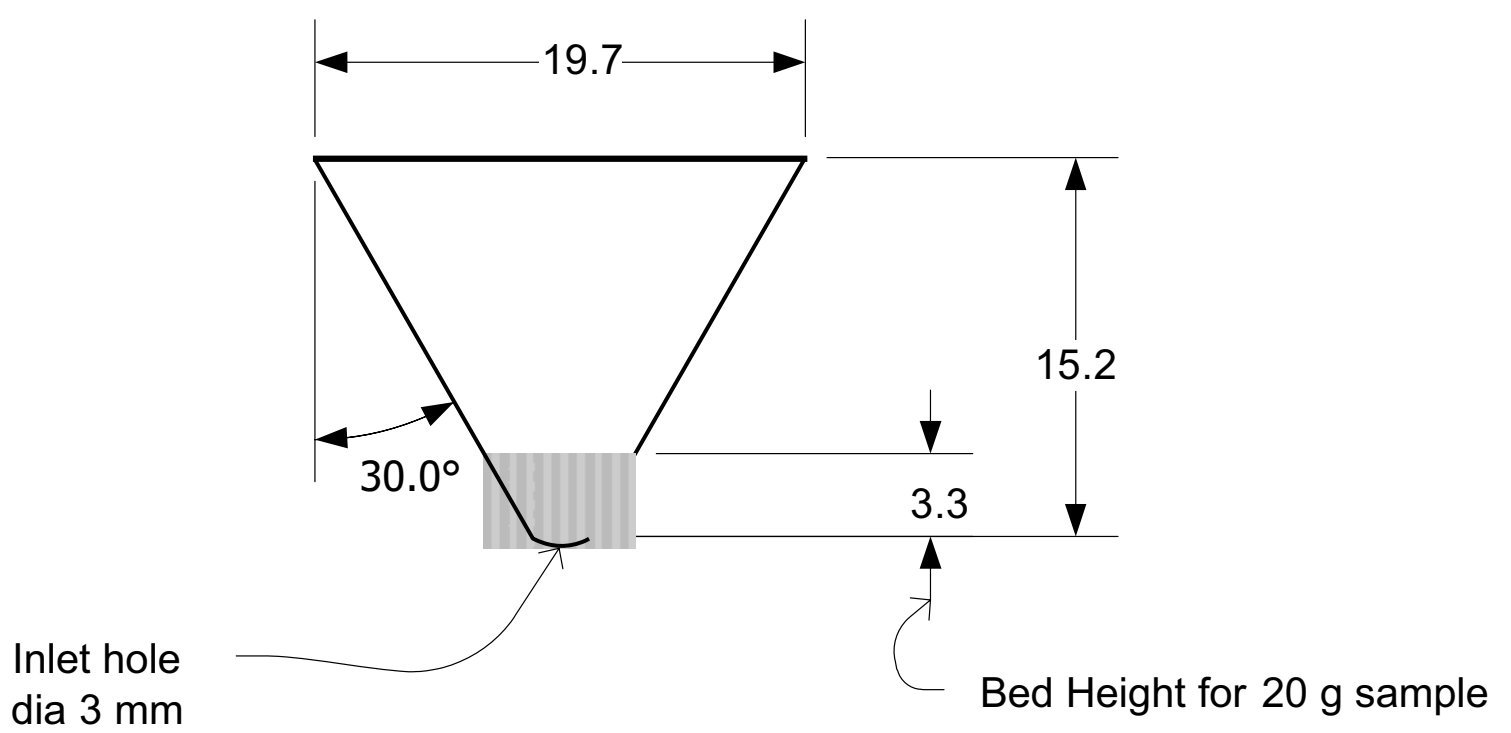

Figure 2. Conical spouted bed test unit (dimensions in $\mathrm{cm}$ ). 
The equivalent spherical particle sizes elutriated out the vessel were calculated for varying flow rates for the conical spouted bed test unit, along with the equivalent asbestos particle diameter for fibers with a length to diameter ratio of 8.6. The results of these calculations are provided in Figure 3.

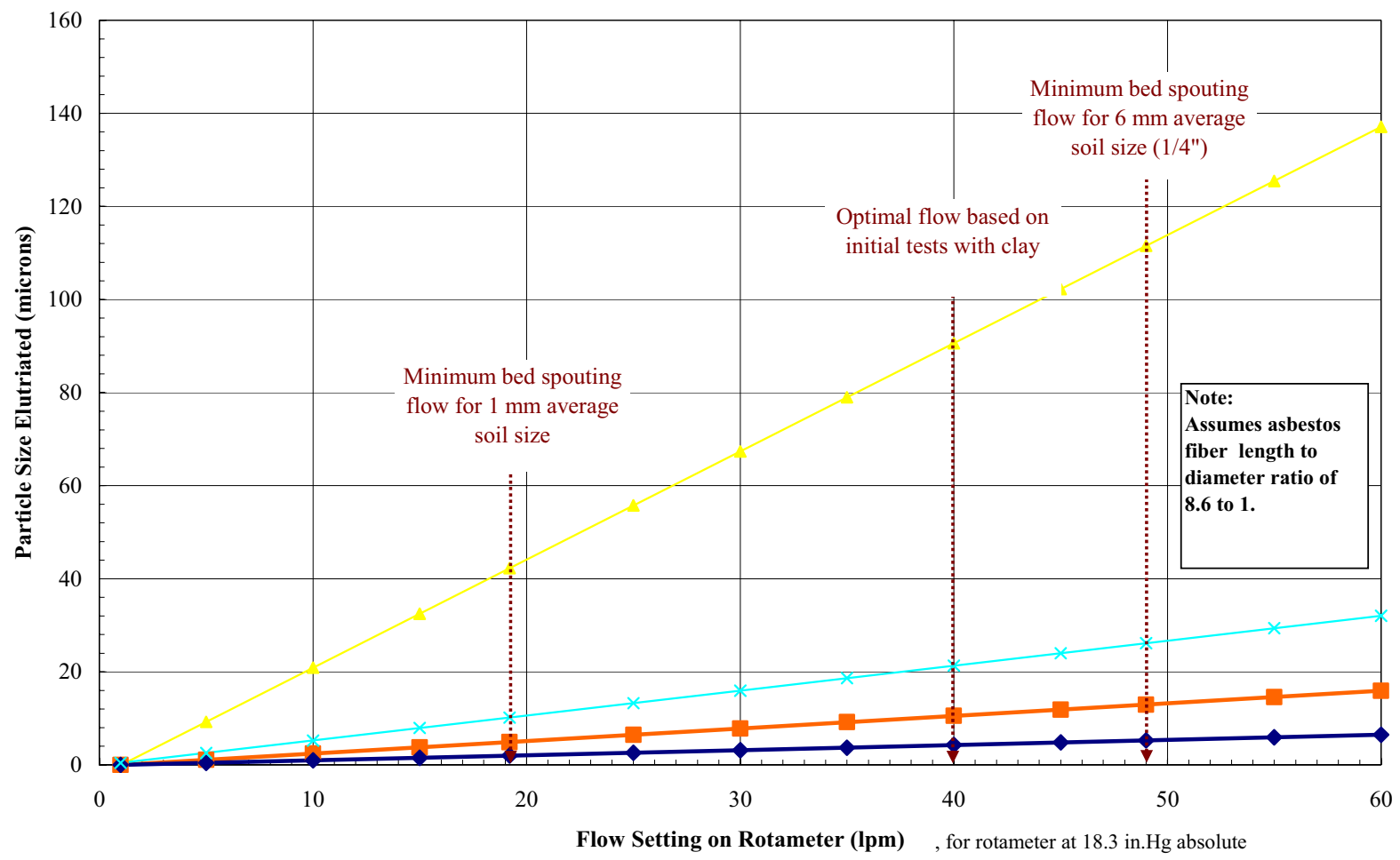

Equivalent Density Spherical Particle Elutriated (um) —Diameter of Asbestos Fibers Elutriated (um) Length of Asbestos Fibers Elutriated (um) $\rightarrow$ Equivalent Aerodynamic Size Elutriated (um)

Figure 3. Calculated particle sizes elutriated versus air flow for the prototype conical spouted bed.

Also shown on the figure are the minimum air flows needed to cause the bed to fluidize for a typical soil size $(1 \mathrm{~mm})$ and the maximum expected soil size. A flow somewhere between these two flows will be needed for the sample to adequately mix the soil and elutriate fine particulate. For an air flow of $40 \mathrm{slpm}$, particles with an equivalent spherical diameter of up to $13 \mu \mathrm{m}$ and asbestos fibers up to about $5 \mu \mathrm{m}$ diameter will be elutriated from the soil sample and collected on the filter.

\section{Experimental Equipment}

To test the engineering design, a glass prototype (Figure 4) was constructed at the INL glass shop. The glass funnel was $20.3 \mathrm{~cm}$ across the top and tapered to $2 \mathrm{~cm}$ at the bottom. The height was $22.9 \mathrm{~cm}$, 
with inlet and outlet ports $1 \mathrm{~cm}$ each in diameter. The glass prototype was advantageous because the degree of fluidization could be readily observed. In application, glass is impractical because it is difficult to clean and breaks easily.

Initial testing suggested that the $1 \mathrm{~cm}$. inlet orifice was too large to allow fluidization to occur even at high flow rates $(>60 \mathrm{lpm})$, thus a $3 \mathrm{~mm}$ diameter restrictor was inserted into the orifice, which allowed fluidization at substantially lower flow rates.

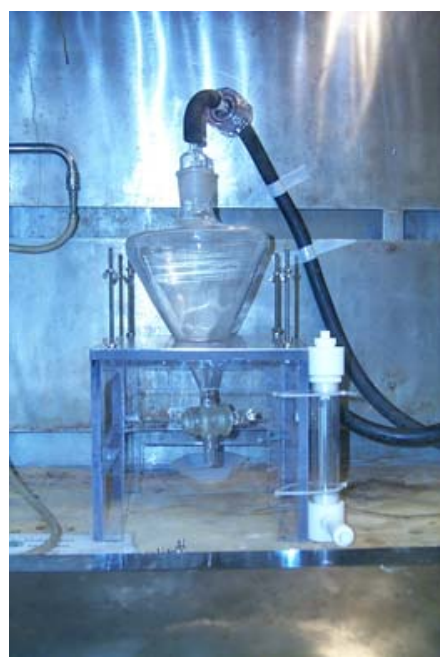

Figure 4. Asbestos sampler glass prototype assembly.

Based on the initial experiments with the glass prototype, a second prototype was constructed, this time out of stainless steel (304). A stainless steel funnel was purchased from Cole-Parmer (part number K-07268-00). This was modified by the INL machine shop so that the orifice tapered to a $3 \mathrm{~mm}$. inlet, which was fitted with a threaded fitting for a valve. A square stainless steel lid was constructed for the funnel and was drilled to accommodate one $47 \mathrm{~mm} 0.8 \mu \mathrm{m}$ pore collection filter. The filter pore size was chosen to provide greater air flow through the cowl than would be permitted by a $0.45 \mu \mathrm{m}$ pore collection filter.

Subsequent conversations with EPA personnel suggested that although the $47 \mathrm{~mm}$ filter provided adequate flow, EPA traditionally used $25 \mathrm{~mm}$ filter cowls for sampling. Thus, we incorporated the use of a $25 \mathrm{~mm} 0.8 \mu \mathrm{m}$ pore filter cowl into our design. However, one cowl did not provide adequate flow, thus three were put in manifold across the lid of the funnel (Figure 5, a and b) 
a.

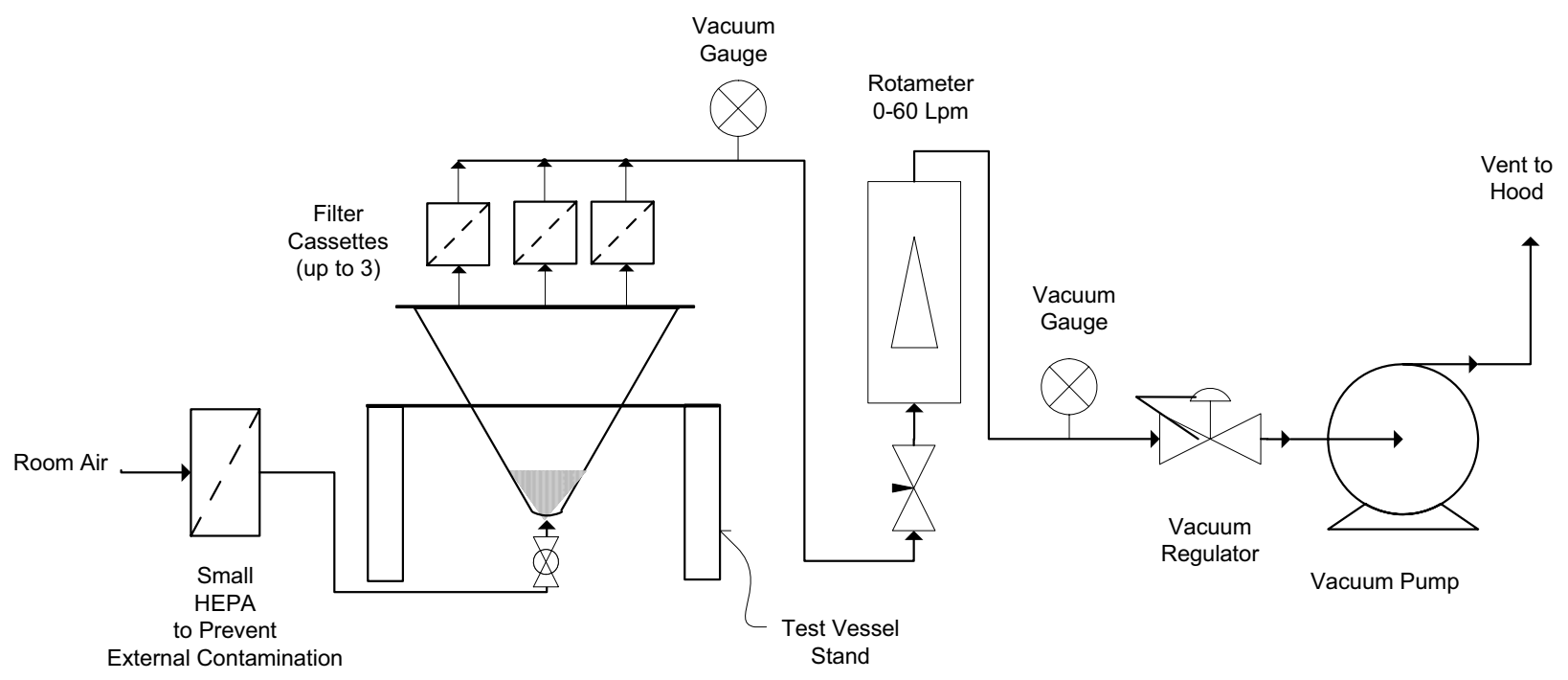

b.

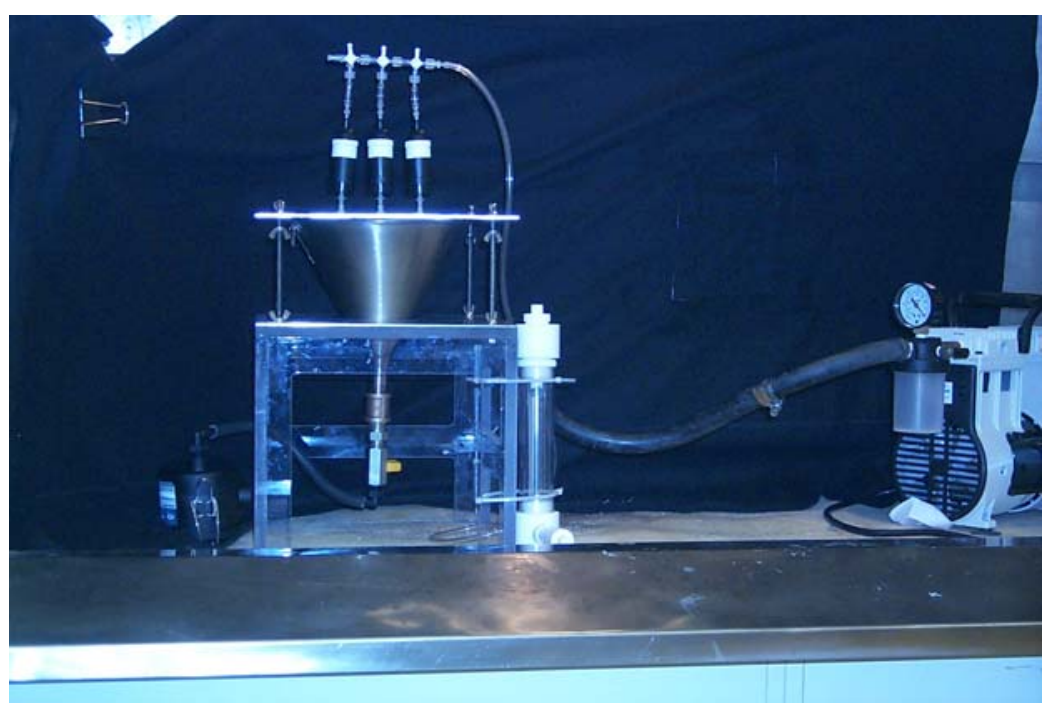

Figure 5 (a-b). Asbestos sampler stainless steel prototype test assembly. a. schematic of working prototype (this prototype uses three $25 \mathrm{~mm}$ collection filters); b. photograph of actual steel prototype.

Initial experiments with the steel fluidized bed used calcium silicate (CAS 1344-95-2) as a substitute for asbestos, in a matrix of ASTM 20/30 quartz sand. The goals for initial testing included the following: 
1. Determine the optimum flow rate (the highest flow rate that can be used without collecting significant amounts of sand onto the collection filter)

2. Determine the percent recovery of the calcium silicate

3. Qualitative determination of filter loading

Initial flow rate tests showed that at flow rates exceeding $50 \mathrm{lpm}$, significant quantities of sand were collected on the filters $(\mathrm{n}=1)$, but at flow rates less than $30 \mathrm{lpm}$, very little calcium silicate was collected $(n=1) .40 \mathrm{lpm}$ was selected as the flow rate to be used for the testing process. In order to achieve this rate, it was necessary to use three collection filters rather than one.

Calcium silicate recovery experiments were conducted using a $20 \mathrm{~g}$ sample (19.8g quartz sand and $0.2 \mathrm{~g}$ calcium silicate). Filters were weighed before and after fluidization to determine the amount of particulate captured. Each of the three filters was weighed to determine whether particle recovery was similar between the three filters. Weighing (and later microscopic analysis of the filters) showed that recovery was similar with each of the filters regardless of their position on the lid $( \pm 17 \%(1 \sigma)$ based on three replicates). Recovery for each of the filters was added together to arrive at a total recovery. At a 40 $1 \mathrm{pm}$ flow rate, approximately $10 \%$ of the calcium silicate was recovered. The majority of the sample was retained via static charge along the surfaces of the filter cowls and the interior surface of the fluidized bed.

Several techniques were explored to reduce static charge. These included grounding the fluidized bed, running a DC current through an electrode placed in the fluidized bed, and running influent air through water to humidify it. Grounding the bed had no impact on recovery, but the application of DC current and humidifying the air stream both increased recovery to about $15 \%$. Because this is not a substantial improvement, none of these techniques is currently being used with the fluidized bed.

Filter loading was studied by running a $0.1 \%$ sample of USGS tremolite through the fluidized bed and examining the captured material under a phase contrast microscope. Particles were captured on a $0.8 \mu \mathrm{M}$ mixed cellulose ester filter, which was then placed onto a glass slide. Acetone was dripped onto the filter until it was rendered transparent. Using this procedure, it was determined that the minimum filter loading was about $50 \%$, and that the bulk of the captured material was tremolite, not sand. Further testing and development work will be needed to address the filter loading problem.

\section{Procedures}

\subsection{Cleaning Procedure}

Two cleaning procedures were tested. The first involved taking apart the fluidized bed, rinsing it in water and ethanol, reassembling the fluidized bed, and running it without a sample 5 minutes. The second involved only wiping down the internal surfaces of the fluidized bed, reassembling the fluidized bed, and running it without a sample for 5 minutes. Samples from each test were collected on clean filters and the filters were sent to a contract laboratory (Lab/Cor, Seattle, WA) for analysis. After the results were received by INL, testing of the bed continued using various concentrations tremolite in sand, and using the cleaning procedure that yielded the fewest extraneous fibers. However, due to the fact that each procedure was tested only once, conclusions cannot be made concerning the absolute performance and variability of each cleaning technique. 


\subsection{Blanks}

\subsubsection{Lot Blanks}

Two unused filters representative of the lot of filters used to collect air samples for this project were analyzed by ISO 10312. The analysis was performed in such a manner as to achieve equivalent analytical sensitivity (number of grid openings counted) as to be comparable to those of the sample set. The result of analysis of the lot blanks were used to determine whether there is any asbestos contamination of the lot. The sample lot is rejected if the number of asbestos structures or fibers exceed 10 structures $/ \mathrm{mm}^{2}$ or if the mean fiber count for asbestos fibers and bundles longer than $5 \mu \mathrm{m}$ is more than 0.1 fibers $/ \mathrm{mm}^{2}$.

\subsubsection{Lab Blanks}

Two lab blanks were prepared and analyzed by ISO 10312. The lab blank cassettes were uncapped exposing the filters during preparation of the sample set. Wedges of the blank filters were prepared along side the wedges of the sample set filters. The purpose was to show that no asbestos contamination occurs in the laboratory during sample preparation. The sample lot is rejected if the number of asbestos structures or fibers exceed 10 structures $/ \mathrm{mm}^{2}$ or if the mean fiber count for asbestos fibers and bundles longer than $5 \mu \mathrm{m}$ is more than 0.1 fibers $/ \mathrm{mm}^{2}$.

\subsubsection{Cleaning Blanks}

To ensure that the fluidized bed equipment had been adequately cleaned between samples, a blank was prepared between samples of different asbestos concentrations. This was accomplished by loading a clean collection filter onto the bed and running air through the sample for 5 minutes, the specified collection time for an actual sample. The fluidized bed equipment will be considered contaminated if the asbestos on the cleaning blank filter is $>\left(\bar{x}_{\min }-\mathbf{2} s_{\min }\right)$ where $\bar{x}_{\min }$ and $s_{\min }$ denote the sample mean and standard deviation of replicates for the number of asbestos particles on the filter for the minimum soil concentration $(0.0001 \mathrm{wt} \%$ tremolite) for which the concentration is significantly $>0$ at the 0.05 probability level as determined by Student's t-test.

\subsubsection{Sand Blank}

One sand blank using clean, 20 grams of the ASTM 20/30 sand was tested in the fluidized bed. This was accomplished by loading a clean collection filter onto the bed and running air through the sample for 5 minutes, the specified collection time for an actual sample. The purpose was to show that no asbestos contamination was present in the sand used for sample preparation. Sand would be considered contaminated if the number of asbestos structures or fibers exceed 10 structures $/ \mathrm{mm}^{2}$ or if the mean fiber count for asbestos fibers and bundles longer than $5 \mu \mathrm{m}$ is more than 0.1 fibers $/ \mathrm{mm}^{2}$. 


\subsection{Sample Testing Procedure}

\subsubsection{Sample Preparation}

Test samples containing $0.0001 \%$ to $0.1 \%$ tremolite using ASTM $20 / 30$ sand as the matrix (Table 1 and 2) were prepared. The ASTM 20/30 sand had a mean particle diameter of $714 \mu \mathrm{m}$ and a particle density of $2.4 \mathrm{~g} / \mathrm{cm}^{3}$. The appropriate amount of tremolite was weighed out and added to sand, which had been submerged in deionized water. The sample was shaken and rotated for 5 minutes to allow for complete mixing. It was then put in an oven to dry at $105^{\circ} \mathrm{C}$ to constant weight. Samples were then split using a riffle splitter to obtain splits of homogeneous $20 \mathrm{~g}$ samples used in the testing procedure for the fluidized bed, and splits of $100 \mathrm{~g}$ for the Berman Elutriator procedure.

Table 1. Test Matrix (wt $\%$ tremolite in an ASTM 20/30 quartz sand matrix). All Berman Elutriator samples were diluted 1:1 with uncontaminated soil to increase particulate releasability. Concentrations shown below indicate the ACTUAL asbestos concentration tested by the indicated method.

\begin{tabular}{|l|l|l|l|l|l|l|l|l|}
\hline TechniquelWt\%Asbestos & $0.05 \%$ & $0.01 \%$ & $0.005 \%$ & $0.001 \%$ & $0.0005 \%$ & $0.0001 \%$ & $0.00005 \%$ & Blank \\
\hline Fluidized Bed & 0 & 7 & 7 & 7 & 0 & 7 & 0 & 8 \\
\hline Berman Elutriator & 1 & 0 & 1 & 0 & 1 & 0 & 1 & 1 \\
\hline
\end{tabular}

The Berman Elutriator procedure was executed by a contracted lab (LabCor, Seattle WA). LabCor used $40 \mathrm{~g}$ aliquots, which were then mixed with $40 \mathrm{~g}$ uncontaminated soil. This was done to increase the release of fine particulate as the Berman method relies on collecting a particular mass on the filter to determine the conclusion of the experiment.

Samples with $0.1 \%$ tremolite would likely overload the filter, thus they were diluted 1:20 with ASTM 20/30 sand prior to running them through the fluidized bed. The $0.1 \%$ tremolite split used for the Berman Elutirator procedure was not diluted, except by the addition of soil as previously described.

Table 2. Sample matrix.

\begin{tabular}{|l|l|l|}
\hline INL Sample Name & LabCor sample number & Description \\
\hline FB-4-R1 & S-12 & $0.0001 \%$ tremolite with ASTM 20/30 sand \\
\hline FB-4-R2 & S-13 & $0.0001 \%$ tremolite with ASTM 20/30 sand \\
\hline FB-4-R3 & S-14 & $0.0001 \%$ tremolite with ASTM 20/30 sand \\
\hline FB-4-R4 & S-15 & $0.0001 \%$ tremolite with ASTM 20/30 sand \\
\hline FB-4-R5 & S-16 & $0.0001 \%$ tremolite with ASTM 20/30 sand \\
\hline FB-4-R6 & S-17 & $0.0001 \%$ tremolite with ASTM 20/30 sand \\
\hline FB-4-R7 & S-18 & $0.0001 \%$ tremolite with ASTM 20/30 sand \\
\hline FB-3-R1 & S-19 & $0.001 \%$ tremolite with ASTM 20/30 sand \\
\hline FB-3-R2 & S-20 & $0.001 \%$ tremolite with ASTM 20/30 sand \\
\hline FB-3-R3 & S-21 & $0.001 \%$ tremolite with ASTM 20/30 sand \\
\hline FB-3-R4 & S-22 & $0.001 \%$ tremolite with ASTM 20/30 sand \\
\hline FB-3-R5 & S-23 & $0.001 \%$ tremolite with ASTM 20/30 sand \\
\hline
\end{tabular}




\begin{tabular}{|c|c|c|}
\hline FB-3-R6 & S-24 & $0.001 \%$ tremolite with ASTM $20 / 30$ sand \\
\hline FB-3-R7 & S-25 & $0.001 \%$ tremolite with ASTM 20/30 sand \\
\hline FB-2-R1 & S-26 & $0.01 \%$ tremolite with ASTM $20 / 30$ sand \\
\hline FB-2-R2 & S-27 & $0.01 \%$ tremolite with ASTM $20 / 30$ sand \\
\hline FB-2-R3 & S-28 & $0.01 \%$ tremolite with ASTM $20 / 30$ sand \\
\hline FB-2-R4 & S-29 & $0.01 \%$ tremolite with ASTM $20 / 30$ sand \\
\hline FB-2-R5 & $\mathrm{S}-30$ & $0.01 \%$ tremolite with ASTM $20 / 30$ sand \\
\hline FB-2-R6 & S-31 & $0.01 \%$ tremolite with ASTM $20 / 30$ sand \\
\hline FB-2-R7 & $\mathrm{S}-32$ & $0.01 \%$ tremolite with ASTM $20 / 30$ sand \\
\hline FB-1-R1 & S-33 & $0.005 \%$ tremolite with ASTM 20/30 sand \\
\hline FB-1-R2 & S-34 & $0.005 \%$ tremolite with ASTM 20/30 sand \\
\hline FB-1-R3 & S-35 & $0.005 \%$ tremolite with ASTM 20/30 sand \\
\hline FB-1-R4 & S-36 & $0.005 \%$ tremolite with ASTM 20/30 sand \\
\hline FB-1-R5 & S-37 & $0.005 \%$ tremolite with ASTM 20/30 sand \\
\hline FB-1-R6 & S-38 & $0.005 \%$ tremolite with ASTM 20/30 sand \\
\hline FB-1-R7 & S-39 & $0.005 \%$ tremolite with ASTM 20/30 sand \\
\hline BR-0-R1 & S-1 & Sand blank for Berman elutriator \\
\hline BR-1-R1 & $\mathrm{S}-2$ & $\begin{array}{l}0.1 \% \text { tremolite with ASTM } 20 / 30 \text { sand for Berman } \\
\text { elutriator diluted to } 0.05 \%\end{array}$ \\
\hline BR-2-R1 & S-3 & $\begin{array}{l}0.01 \% \text { tremolite with ASTM } 20 / 30 \text { sand for Berman } \\
\text { elutriator diluted to } 0.005 \%\end{array}$ \\
\hline BR-3-R1 & S-4 & $\begin{array}{l}0.001 \% \text { tremolite with ASTM } 20 / 30 \text { sand for Berman } \\
\text { elutriator diluted to } 0.0005 \%\end{array}$ \\
\hline BR-4-R1 & S-5 & $\begin{array}{l}0.0001 \% \text { tremolite with ASTM } 20 / 30 \text { sand for Berman } \\
\text { elutriator diluted to } 0.00005 \%\end{array}$ \\
\hline Cleaning blank \#1 & $\mathrm{S}-1$ & $\begin{array}{l}\text { Air pulled through empty fluidized bed before running } \\
\text { sample FB-4-R1 }\end{array}$ \\
\hline Cleaning blank \#2 & $\mathrm{S}-2$ & $\begin{array}{l}\text { Air pulled through empty fluidized bed before running } \\
\text { sample FB-3-R1 }\end{array}$ \\
\hline Cleaning blank \#3 & $\mathrm{S}-3$ & $\begin{array}{l}\text { Air pulled through empty fluidized bed before running } \\
\text { sample FB-2-R1 }\end{array}$ \\
\hline Cleaning blank \#4 & S-4 & $\begin{array}{l}\text { Air pulled through empty fluidized bed before running } \\
\text { sample FB-1-R1 }\end{array}$ \\
\hline Lab blank \#1 & S-5 & Unused collection filter cowl for LabCor \\
\hline Lab blank \#2 & S-6 & Unused collection filter cowl for LabCor \\
\hline Lot blank \#1 & S-7 & $\begin{array}{l}\text { Unused collection filter cowl to test to see if filter is } \\
\text { asbestos free }\end{array}$ \\
\hline Lot blank \#2 & $\mathrm{S}-8$ & $\begin{array}{l}\text { Unused collection filter cowl to test to see if filter is } \\
\text { asbestos free }\end{array}$ \\
\hline
\end{tabular}




\begin{tabular}{|l|l|l|}
\hline Cleaning protocol 1 & S-9 & $\begin{array}{l}\text { Dismantle Fluid Bed, rinse with water and ethanol, } \\
\text { and replace inlet and outlet fittings }\end{array}$ \\
\hline Cleaning protocol 2 & S-10 & $\begin{array}{l}\text { Dismantle Fluid Bed, wipe inside, and replace inlet } \\
\text { and outlet fittings }\end{array}$ \\
\hline Sand blank & S-11 & Washed ASTM 20/30 sand \\
\hline
\end{tabular}

\subsubsection{Sample Separation in Fluidized Bed}

To minimize cross-contamination, samples were tested in order from lowest to highest concentration. For each sample test, the fluidized bed was cleaned using the first protocol (section 4.1). The inlet valve and outlet fittings and filters were then replaced with new items, the $20 \mathrm{~g}$ sample was placed in the fluidized bed, the lid and gasket were clamped to the top, inlet and outlet hoses reconnected, and the vacuum pump turned on. The flow was adjusted to $40 \mathrm{lpm}$ setting on the rotameter $(31 \mathrm{slpm}, 37$ actual lpm in the fluidized bed after correcting the rotameter's reading for vacuum of $7 \mathrm{in}$. $\mathrm{Hg}$ and local pressure of $25.35 \mathrm{in}$. Hg absolute), and the vacuum pump shut off after 5 minutes. Flow on the rotameter measures the total air flow from all three filter cowls manifolded into one air stream. Individual flow rates through each cowl were not measured.

After testing, the middle filter of the three filters on the fluidized bed outlet was removed and labeled for analysis. The other two filters were not replaced between replicates, but were replaced and the used filters disposed between sample sets.

\subsection{Sample Counting Procedure}

For asbestos samples segregated by the fluidized bed, one of three collection filters (the one in the center of the cone, Figure 5 (a-b)) was sent to a contract laboratory (Lab/Cor, Seattle, WA) for ISO 10312 TEM analysis. In this case, we used the counting protocol from the ISO procedure, but did not extrapolate to a fiber per volume of air concentration. Since the fluidized bed samples soil, not air, it would be misleading to report data in the form of fibers per volume of air. However, the counting rules used in the ISO 10312 process were used to count the structures impacted on filters by the fluidized bed.

For samples containing $0.0001,0.001$, and $0.01 \%$ tremolite, 35 grid openings were counted. For samples containing $0.1 \%$ tremolite (which were diluted $1 / 20$ to achieve a $0.005 \%$ tremolite concentration), 10 grid openings were counted. Due to the large number of structures found during the counting of the $0.01 \%$ samples, samples FB-2-R6 and FB-2-R7 had 10 grid openings counted. Percent recovery was based on the mass of fibers counted on the TEM grids (calculated by multiplying length $\mathrm{X}$ width $X$ width $X$ amphibole density of each fiber), extrapolated to the total area of one $25 \mathrm{~mm}$ collection filter, multiplied by three to account for the three collection filters on the top of the funnel.

For asbestos samples segregated by the Berman elutriator, counting methods follow from Berman's protocols (Modified Elutriator Method for the Determination of Asbestos in Soils and Bulk Material, Revision 1 May 23, 2000). 


\section{Test Results}

\subsection{Blank Results}

The results of the TEM analysis of the filters from cassettes collected for blanks and cleaning protocol testing are provided in Table 3. A small number of asbestos structures were detected on two of the eleven blanks. These were 6.3 Structures $/ \mathrm{mm}^{2}$ counted on the cleaning protocol 2 filter, and 3.2 Structures $/ \mathrm{mm}^{2}$ structure counted on cleaning blank (using protocol 1) \#3 taken between testing sample sets of $0.001 \%$ and $0.01 \%$ tremolite.

\subsubsection{Lot Blanks}

No asbestos structures were detected by the lab on the filters from unused filter cassettes from the two lots used for the testing. This showed that no asbestos contamination was present in the filter cassettes from the two lots.

\subsubsection{Lab Blanks}

No asbestos structures were detected by the lab on the lab blank filters. This showed that the filters in the cassettes were not contaminated with asbestos during preparation of the sample set for TEM analysis.

\subsubsection{Sand Blank}

No asbestos structures were detected by the lab on the filter from testing the sand blank. This showed that no asbestos contamination was present in the sand used for sample preparation.

\subsubsection{Cleaning Procedure and Cleaning Blanks}

Two fluidized bed equipment cleaning protocols were tested (see Section 4.1). For both, the fluidized bed was loaded with 20/30 ASTM silica sand containing $0.1 \mathrm{wt} \%$ tremolite and run for 5 minutes prior to each cleaning. No asbestos structures were detected by the lab on the filter after using Cleaning Protocol 1; however, asbestos structures were detected on the filter generated after using Cleaning Protocol 2. Cleaning protocol 1 was selected for use during the test procedure.

None of the cleaning blanks taken between sample sets showed asbestos structures except for cleaning blank \#3 taken between testing of the $0.001 \%$ and $0.01 \%$ sets. This result suggests that in general, Cleaning Protocol 1 was sufficient.

If we compare the number of primary asbestos fibers recovered from Cleaning Protocol 1 sample and the four cleaning blank samples to the seven replicates run of the $0.0001 \%$ tremolite samples, we can make several observations. Firstly, using a two-sample t-test assuming unequal variances, the probability that the means of these two populations are the same is $0.5 \%$. Using a Kolmogorov-Smirnov test, we can show that the results of the $0.0001 \%$ analyses follow a normal distribution $(\mathrm{p}>0.2)$. The probability that 1 (one fiber found in one cleaning blank) is part of the distribution of data encompassed by the $0.0001 \%$ sample results is $7.28 \%$. This suggests that it is unlikely that this one fiber compromises the data set. 
Table 3 Results of blank analyses

\begin{tabular}{|c|c|c|c|c|}
\hline $\begin{array}{l}\text { INL Sample } \\
\text { Name }\end{array}$ & $\begin{array}{l}\text { Lab/Cor } \\
\text { sample } \\
\text { number }\end{array}$ & $\begin{array}{l}\text { Primary } \\
\text { Asbestos } \\
\text { Structures }\end{array}$ & $\begin{array}{l}\text { Filter Density } \\
\left(\text { Structures } / \mathrm{mm}^{2}\right)\end{array}$ & Description \\
\hline $\begin{array}{l}\text { Cleaning protocol } \\
1\end{array}$ & S-9 & 0 & 0 & $\begin{array}{l}\text { Dismantle, rinse with filtered and de- } \\
\text { ionized water and replace fittings. }\end{array}$ \\
\hline $\begin{array}{l}\text { Cleaning protocol } \\
2\end{array}$ & $\mathrm{~S}-10$ & 2 & 6.3 & $\begin{array}{l}\text { Dismantle, wipe down inside of } \\
\text { fluidized bed and replace fittings. }\end{array}$ \\
\hline Lot blank \#1 & S-7 & 0 & 0 & $\begin{array}{l}\text { Unused collection filter cowl to test to } \\
\text { see if filter is asbestos free }\end{array}$ \\
\hline Lot blank \#2 & S-8 & 0 & 0 & $\begin{array}{l}\text { Unused collection filter cowl to test to } \\
\text { see if filter is asbestos free }\end{array}$ \\
\hline Lab blank \#1 & S-5 & 0 & 0 & $\begin{array}{l}\text { Unused collection filter cowl for } \\
\text { LabCor }\end{array}$ \\
\hline Lab blank \#2 & S-6 & 0 & 0 & $\begin{array}{l}\text { Unused collection filter cowl for } \\
\text { LabCor }\end{array}$ \\
\hline Sand blank & S-11 & 0 & 0 & Washed ASTM 20/30 sand \\
\hline Cleaning blank \#1 & $\mathrm{S}-1$ & 0 & 0 & $\begin{array}{l}\text { Air pulled through empty fluidized bed } \\
\text { before running sample FB-4-R1 }\end{array}$ \\
\hline Cleaning blank \#2 & $\mathrm{S}-2$ & 0 & 0 & $\begin{array}{l}\text { Air pulled through empty fluidized bed } \\
\text { before running sample FB-3-R1 }\end{array}$ \\
\hline Cleaning blank \#3 & $\mathrm{S}-3$ & 1 & 3.2 & $\begin{array}{l}\text { Air pulled through empty fluidized bed } \\
\text { before running sample FB-2-R1 }\end{array}$ \\
\hline Cleaning blank \#4 & S-4 & 0 & 0 & $\begin{array}{l}\text { Air pulled through empty fluidized bed } \\
\text { before running sample FB-1-R1 }\end{array}$ \\
\hline
\end{tabular}

\subsection{Fluidized Bed Sample Test Results}

\subsubsection{TEM Structure Counts}

The total number of TEM structure counts for each replicate sample segregated using the fluidized bed and the corresponding percent of asbestos mass recovered are shown in Table 4. Several observations can be made concerning the data.

1. The percentage by weight of asbestos recovered in each group of replicates varies greatly. This is likely because the weight recovered is dominated by the small number of large structures elutriated and collected on the sample filters. Structures with an aerodynamic diameter greater than $10 \mu \mathrm{m}$ by number comprised only $12 \%$ of the number of structures but were $94 \%$ of the weight of the structures.

2. In the analyses of the $0.001 \%$ samples, there was no recovery of fibers in four of the seven replicates. This was likely due to poor seating of the gasket between the lid and the funnel. 
3. There was significant variability in the number of structures counted for the replicates. Possible causes/contributors could have been: 1) uneven flow distribution in the top of the cone near the outlets to the filters, 2) poor lid sealing, 3) uneven loading on the filters leading to uneven numbers of fibers in the grid areas counted by TEM. Deposition of fibers should be heaviest in the center of the filter and should taper off radially toward the edges of the filter. The ideal way to sample and count such a filter would be to sample a "pie-shaped" wedge extracted from the center of the filter, extending to the edges. Fiber counts on the wedge would be integrated over the entire area of the filter. Because TEM grids are circular and of fixed diameter, such a counting method is precluded. The uneven distribution of fibers on the filter can be accentuated by examining a relatively small portion of the filter. This can result in abnormally low or high apparent recoveries.

4. At very low asbestos concentrations (i.e. $0.0001 \%$ ) a minimum of three fibers were detected, suggesting the method may be highly sensitive to low concentrations.

5. Two of the samples contained some chrysotile fibers $(0.0001 \mathrm{wt} . \%$, Replicate 1 and 6$)$. The reason for this is not known. However, it is not likely to have come from the sand as the sand blank showed no asbestos detected. In additional, the same sand was used for all 28 samples, so if chrysotile was in the sand, we would expect it to have been detected much more frequently.

Table 4. Results of samples segregated by fluidized bed and counted using TEM.

\begin{tabular}{|c|c|c|c|c|c|c|c|c|c|c|c|c|}
\hline \multirow[b]{2}{*}{ Replicate } & \multicolumn{3}{|c|}{ Concentration 0.0001 wt $\%$} & \multicolumn{3}{|c|}{ Concentration 0.001 wt\% } & \multicolumn{3}{|c|}{ Concentration $0.005 \mathrm{wt} \%$} & \multicolumn{3}{|c|}{ Concentration 0.01 wt\% } \\
\hline & $\begin{array}{c}\text { Struc- } \\
\text { tures }\end{array}$ & $\begin{array}{c}\text { Filter } \\
\text { Loading } \\
(\mathbf{S} / \\
\left.\mathbf{m m}^{\mathbf{2}}\right) \\
\end{array}$ & $\begin{array}{c}\text { Recov- } \\
\text { ery } \\
(\mathbf{w t} \%)\end{array}$ & $\begin{array}{c}\text { Struc- } \\
\text { tures }\end{array}$ & $\begin{array}{c}\text { Filter } \\
\text { Loading } \\
(\mathbf{S} / \\
\left.\mathbf{m m}^{2}\right)\end{array}$ & $\begin{array}{c}\text { Recov- } \\
\text { ery } \\
(w t \%)\end{array}$ & $\begin{array}{c}\text { Struc } \\
- \\
\text { tures }^{\mathrm{a}}\end{array}$ & $\begin{array}{c}\text { Filter } \\
\text { Loading } \\
(\mathbf{S} / \\
\left.\mathbf{m m}^{\mathbf{2}}\right)\end{array}$ & $\begin{array}{c}\text { Recov- } \\
\text { ery } \\
(w t \%)\end{array}$ & $\begin{array}{c}\text { Struc- } \\
\text { tures }\end{array}$ & $\begin{array}{c}\text { Filter } \\
\text { Loading } \\
(\mathbf{S} / \\
\left.\mathbf{m m}^{\mathbf{2}}\right) \\
\end{array}$ & $\begin{array}{c}\text { Recov- } \\
\text { ery } \\
(w t \%)\end{array}$ \\
\hline 1 & $13^{\mathrm{c}}$ & 85.7 & 18.6 & 0 & 0.0 & 0.0 & 36 & 400 & 8.4 & 262 & 832 & 21.3 \\
\hline 2 & 8 & 25.4 & 5.0 & 65 & 206.3 & 183.4 & $0^{\mathbf{b}}$ & $0^{\mathbf{b}}$ & $0.0^{\mathrm{b}}$ & 190 & 603 & 53.4 \\
\hline 3 & 14 & 44.4 & 130.4 & $0^{\mathbf{b}}$ & $0.0^{\mathrm{b}}$ & $0.0^{\mathrm{b}}$ & 34 & 378 & 3.9 & 230 & 730 & 6.6 \\
\hline 4 & 5 & 15.9 & 5.6 & 0 & 0.0 & 0.0 & 36 & 400 & 15.2 & 220 & 698 & 8.9 \\
\hline 5 & 7 & 22.2 & 16.4 & 29 & 92.1 & 13.2 & 29 & 322 & 19.5 & 173 & 549 & 1.7 \\
\hline 6 & $11^{\mathrm{c}}$ & 38.1 & 9.2 & 0 & 0.0 & 0.0 & 27 & 300 & 1.5 & 50 & 556 & 2.4 \\
\hline 7 & 4 & 12.7 & 1.8 & 25 & 79.4 & 41.5 & 31 & 344 & 17.1 & 74 & 822 & 14.8 \\
\hline Std Dev & 3.89 & 25.1 & 46.1 & 24.7 & 78.5 & 67.6 & 12.6 & 140 & 7.9 & 80.2 & 119 & 18.0 \\
\hline $\begin{array}{r}\text { Total } \\
\text { Struct- } \\
\text { ures } \\
\end{array}$ & 63 & & & 119 & & & 193 & & & 1199 & & \\
\hline Average & 8.86 & 34.9 & 26.7 & 17.0 & 54.0 & 34.0 & 27.6 & 306 & 9.4 & 171.3 & 684 & 15.6 \\
\hline $\begin{array}{r}\text { Total } \\
\text { Struc- } \\
\text { tures (All } \\
\text { Samples) } \\
\end{array}$ & 1574 & & & & & & & & & & & \\
\hline $\begin{array}{l}\mathbf{a} \text { Ten grids } \\
\text { b The lid ga } \\
{ }^{\mathbf{c}}\end{array}$ & was fo & $\begin{array}{l}\text { or each } 0 \text {. } \\
\text { to not be }\end{array}$ & $\begin{array}{l}5 \mathrm{wt} \% \text { re } \\
\text { operly se }\end{array}$ & $\begin{array}{l}\text { cate. Th } \\
\text { d after }\end{array}$ & $\begin{array}{l}\text { y-five grids } \\
\text { sample wa }\end{array}$ & $\begin{array}{l}\text { ere cour } \\
\text { In in th }\end{array}$ & for a & $\begin{array}{l}\text { hers. } \\
\text { d. Theref }\end{array}$ & air in-1 & ge like & iluted the & aple. \\
\hline
\end{tabular}




\subsubsection{Comparison of TEM Data with Sample Concentrations}

The mean Total Asbestos structures and PCM Equivalent structures per gram of sample for the each set of replicates are plotted versus the sample asbestos concentration in Figure 6 . The $\mathrm{R}^{2}$ values shown in the figure are for linear correlations of the means. The number of asbestos structures elutriated from the simulated soil sample by the fluidized bed device and collected on the filters was roughly proportional to the concentration in the sample; however, the repeatability was poor for the sample replicates as shown in the figure by the error bars for the $95 \%$ confidence limits for each set of replicates.

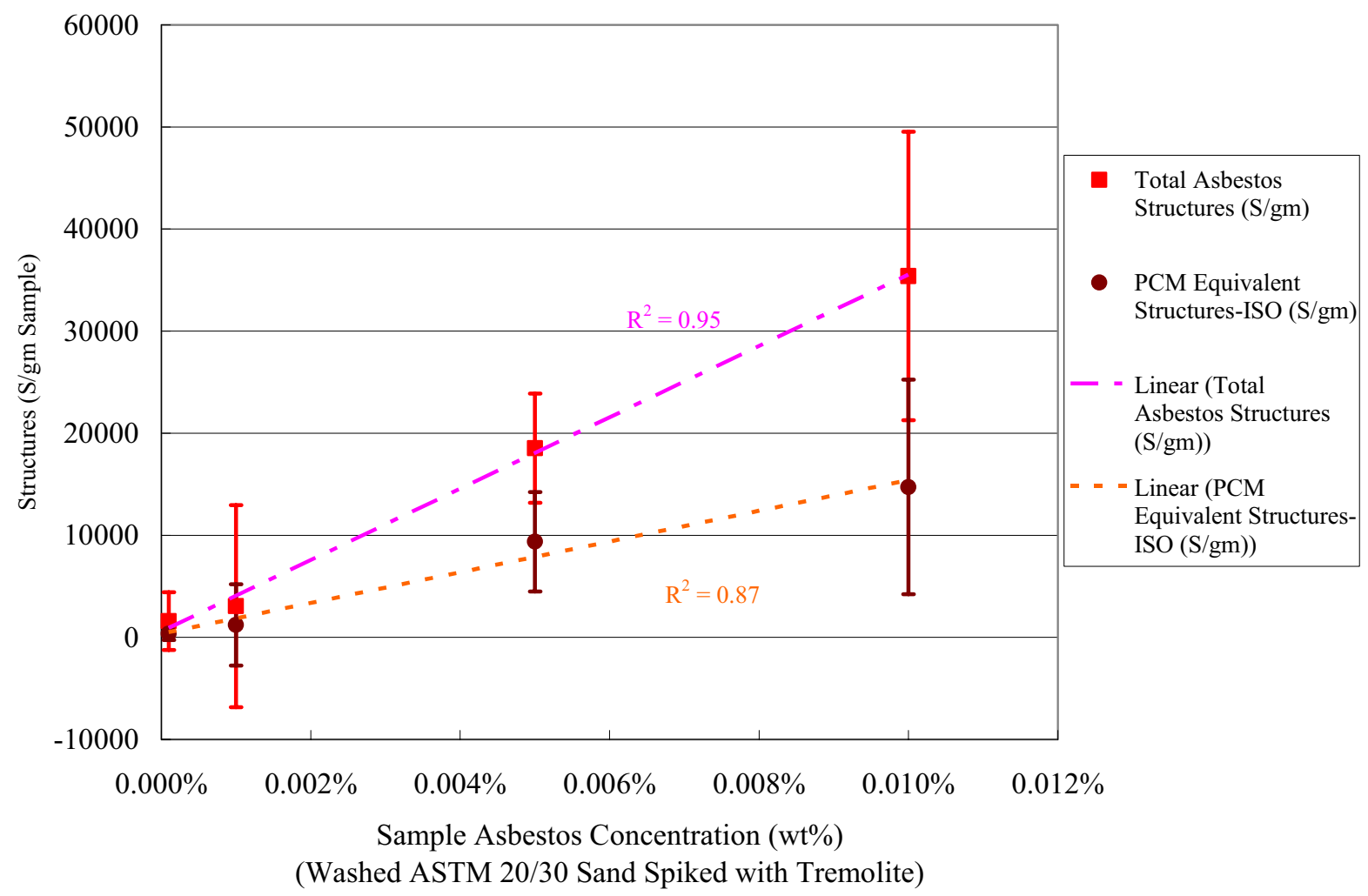

Figure 6. Number of structures versus sample concentration.

\subsubsection{Size Distributions for Asbestos Structures}

The average aspect ratio for all the asbestos structures counted on the filters was 8.6 with a standard deviation of 6.5. The average aerodynamic diameter (diameter of water droplet having same settling velocity as the fiber/structure) was $5.7 \mu \mathrm{m}$ with a standard deviation of $6.3 \mu \mathrm{m}$. The median aerodynamic diameter was $3.0 \mu \mathrm{m}$.

If all the structures collected from all samples are grouped together and plotted as a function of each structure's aerodynamic diameter, several features become apparent (Figure ). On the figure, the median size for each sample concentration is denoted by a diamond, and the mean size is denoted by a box. 
1. The distribution of aerodynamic diameters present in each concentration is similar, suggesting the size range of particles elutriated from the sample by the fluidized bed is the same regardless of the asbestos concentration. The mean and median aerodynamic diameter for each concentration are also very close, with the means varying from 5.2 to 7.2 microns, and the medians varying from 2.7 to 3.4 microns.

2. Ninety percent of the particles have an aerodynamic diameter less than $10 \mu \mathrm{m}$, and ninety-seven percent of the particles are less than $20 \mu \mathrm{m}$. Seven structures were identified that have aerodynamic diameters greater than $40 \mu \mathrm{m}$, with one of these measuring $90 \mu \mathrm{m}$. The longest structure identified had an actual length of $70 \mu \mathrm{m}$ and a diameter of $1 \mu \mathrm{m}$. Calculations suggest that when using the test flow of $31 \mathrm{slpm}$ (37 alpm in the fluidized bed when corrected for ambient pressure), the fluidized bed is capable of elutriating fibers as large as $91 \mu \mathrm{m}$ with a $4.3 \mu \mathrm{m}$ diameter (aerodynamic diameter of 21 $\mu \mathrm{m})$.

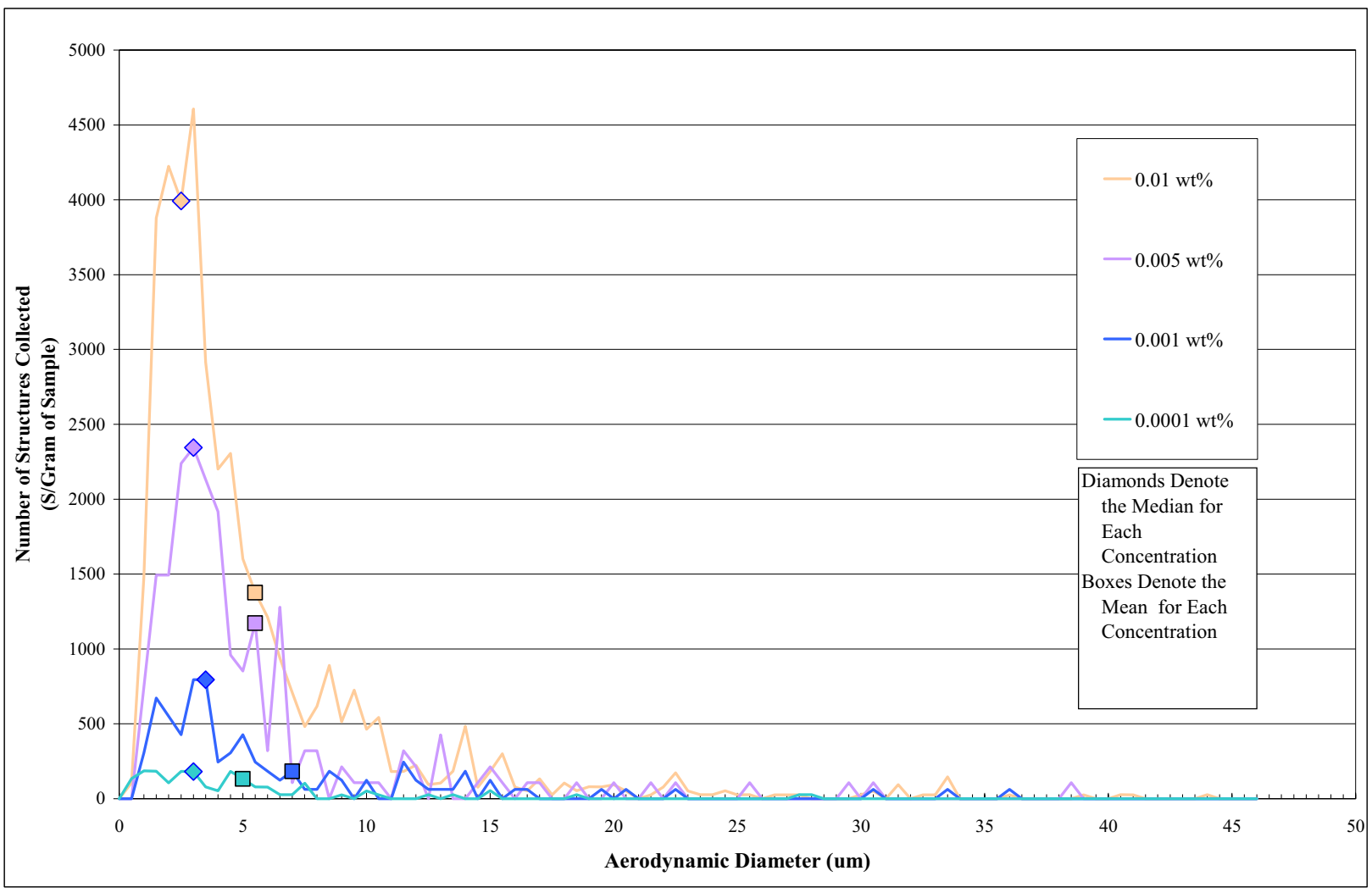

Figure 7. Number of asbestos structures as a function of aerodynamic size $(\mu \mathrm{m})$ 


\subsection{Berman Elutriator Test Results}

Table 5 shows the results of $40 \mathrm{~g}$ splits of the four asbestos concentrations tested using the Berman Elutriator method. Although twice as many grids were counted for the Berman method compared to the fluidized bed method, and the samples were two times larger than those used in the fluidized bed, no structures were found in the $0.0001 \%, 0.001 \%$ and $0.01 \%$ (which had been diluted to $0.05,0.005,0.0005$, and $0.00005 \mathrm{wt} \%$ for Elutriator testing) asbestos samples using the Berman method. In contrast, using the fluidized bed, structures were found in the $0.0001 \%, 0.001 \%, 0.01 \%$, and $0.1 \%$ (which had been diluted to $0.005 \%$ to prevent overloading the filter).

Making a direct comparison between the two methods was problematic for this study. Budgetary constraints made performing replicates using the Berman Elutriator impossible. In addition, because the elutriator samples had to be diluted with soil to enhance particle release, the weight percentage of tremolite in the elutriator samples was half that of the samples in the fluidized bed. However, $0.005 \mathrm{wt} \%$ tremolite was analyzed using both methods. Results for this are shown in Table 6.

Table 5. Results of samples segregated by the Berman Elutriator and counted using TEM

\begin{tabular}{|l|l|l|}
\hline Tremolite wt.\% & Total Asbestos Structures & Grids Counted \\
\hline 0.05 & 6 & 74 \\
\hline 0.005 & 0 & 70 \\
\hline 0.0005 & 0 & 71 \\
\hline 0.00005 & 0 & 74 \\
\hline
\end{tabular}

Table 6. Comparison of 0.005 wt.\% tremolite run in Berman Elutriator versus Fluidized Bed

\begin{tabular}{|l|l|l|}
\hline & Berman Elutriator & Fluidized Bed \\
\hline Wt. \% tremolite in samples & 0.005 & 0.005 \\
\hline Quantity of sample in device $(\mathrm{g})$ & $40 \mathrm{~g}$ & $20 \mathrm{~g}$ \\
\hline Replicate analyses & 1 & 7 \\
\hline Grid openings counted & 74 & 10 \\
\hline $\begin{array}{l}\text { Number of asbestos structures } \\
\text { recovered (using Berman criteria } \\
\text { for counting) }\end{array}$ & 0 & $0-21$ (average of 11.7) \\
\hline
\end{tabular}

\section{Conclusions}

- Advantages of fluidized be separator/sampler and the test method include the following:

- $\quad$ The time to clean the equipment was minimal since the system was operated under vacuum to preclude contamination from escaping. 
- $\quad$ Cleaning Protocol 1 (dismantle fluid bed, rinse with water and ethanol, and replace inlet and outlet fittings) was superior to Cleaning Protocol 2 (dismantle fluid bed, wipe inside, and replace inlet and outlet fittings). The phase II fluid bed separator will use relatively inexpensive disposable fluid bed units and fittings to preclude the need for cleaning.

- $\quad$ The distribution of aerodynamic diameters present in each concentration is similar; suggesting the size range of particles elutriated from sample by the fluidized bed is the same regardless of the asbestos concentration. The mean and median aerodynamic diameter for each concentration are also very close, with the means varying from 5.2 to $7.2 \mu \mathrm{m}$, and the medians varying from 2.7 to $3.4 \mu \mathrm{m}$.

- $\quad$ The number of asbestos structures elutriated from the simulated soil sample by the fluidized bed separator and collected on the filters was roughly proportional to the concentration in the sample.

- $\quad$ At very low asbestos concentrations (i.e. $0.0001 \%$ ) fibers are detected, suggesting the method will be highly sensitive to low concentrations.

- $\quad$ Although twice as many grids were counted for the Berman method compared to the fluidized bed method, and the samples were two times larger than those used in the fluidized bed, $0.005 \mathrm{wt} \%$ asbestos samples using the Berman method. However from 0 to 21 (mean of 11.7) structures were found using the fluidized bed.

Limitations of sampler and test method are as follows:

- $\quad$ The repeatability of the sample method was poor, which was likely because the weight recovered is dominated by the small number of large structures elutriated and collected on the sample filters.

- $\quad$ Some larger fibers were entrained in the outlet. This was either due to the short height of the cylindrical de-entrainment section above fluidized bed or to entrainment of particles thrown upward from the spouted bed.

- $\quad$ The bed flow used for the tests was calculated to elutriate asbestos fibers with an equivalentdensity size of $10 \mu \mathrm{m}$ rather than an aerodynamic size. The bed flow for future tests should be set at that for elutriation of particles with an aerodynamic diameter of $10 \mu \mathrm{m}$ and smaller, which is the size for respirable particles (PM10) that are the primary toxicological concern. A lower flow will also reduce loading on the filter.

- $\quad$ Tremolite was the only small particulate added to the sand samples. No small particulate was added as would be present in normal soil samples and that would occlude the filter/reduce effectiveness of identification and counting of asbestos structures. The phase II design will incorporate a method(s) to limit collection on the filters (by splitting flow, or increasing the number of filters in parallel on the outlet, depending on the amount of dilution required).

- $\quad$ The lid gasket on the fluidized bed did not seal well, which likely have resulted in poor collection on the filters during some of the tests. The phase II fluid bed separator design does not rely on alignment of a top gasket for sealing the lid but instead uses a non-removal lid that is glued to the cone. 
Use of the fluidized bed should be a substantial improvement over EPA's glovebox method for the qualitative determination of asbestos in soil or soil-like materials primarily because the fluidized bed is easier to clean, will increase sample throughput, and is field-deployable. Insufficient data exists at this time to determine whether the fluidized bed will be a substantial improvement over the Berman Elutriator. More extensive testing is required.

\section{Additional Work}

Before the fluidized bed can be used predictably and defensively as an asbestos screening tool, the following work should be completed:

1. Actual soil samples spiked with asbestos should be run through the fluidized bed to check for filter loading. Sample sizes may have to be adjusted to compensate for filter loading.

2. Asbestos-spiked samples should be thoroughly characterized as to the size population and distribution prior to running them in the fluidized bed, and after the run is complete so that potential damage to the fibers, or segregation within the bed could be observed.

3. The gasket should be glued to the lid so that it cannot unseat itself when the lid is applied.

4. Many replicates of Cleaning Procedure 1 should be run (following contamination of the bed) to determine more accurately how well this procedure performs. 


\section{REFERENCES}

Berman, D.W., and Kolk, A., 2000, Modified Elutriator Method for the Determination of Asbestos in Soils and Bulk Material, Revision 1 May 23, 2000.

H. T. Bi, A. Macchi, J. Chaouki and R. Legros, "Minimum Spouting Velocity of Conical Spouted Beds,"Canadian Journal of Chemical Engineering, Vol. 75, April 1997, pp 460-465.

ISO 10312:1995(E), Ambient air-determination of asbestos fibres-direct-transfer transmission electron microscopy method

Occupational Safety and Health Administration (1992) 29 CFR Parts 1910 and 1926 (Docket No. H-033d): occupational exposure to asbestos, tremolite, anthophyllite and actinolite. Rederal Register 57(110) (Monday, June 8, 1992), p 24310-24331.

Perry, R. H, and C. H. Chilton, Chemical Engineer's Handbook, 5th ed., New York: McGraw-Hill, 1973.

Wright, K. E., Idaho National Laboratory, "Quality Assurance Project Plan for Evaluation of Field Device for Separation of Asbestos from Soil," Version 1, March 6, 2007.

US EPA, 2005, Standard Operating Procedure for Sampling Airborne Asbestos Fibers in a Laboratory Enclosure-A Qualitative Procedure, SOP\# EPA Region 10-IEU-001.

US Agency for Toxic Substances and Disease Registry (ATSDR), 2003, Superfund Site Assessment Branch, "Public Health Assessment, Libby Asbestos Site, Libby, Lincoln County, Montana, EPA Facility ID: M500090983840,” May 15, 2003. 


\section{Appendix A}

\section{Design Calculations for the Conical Spouted Bed Elutriator}




\section{Design Calculations for the RARE Asbestos Sampler Test Unit}

\section{Purpose:}

A simplified method is desired to separate and quantify asbestos in soil samples.

Scope:

Calculate design parameters for operation of a conical spouted bed separator for sampling of asbestos in soil.

\section{Safety Category}

This design calculation is for a research and development laboratory system with a safety category of CG Commercial grade.

Natural Hazards Phenomena (NPH) Performance Category (PC)

$\mathrm{NPH}$ performance category does not apply to these calculations.

\section{Subject-Specific Data}

NA

\section{Assumptions:}

Operation is at atmospheric conditions of $20 \mathrm{C}$ and local pressure at Idaho Falls of 12.47 psia.

Desired maximum loading on filter is $2 \mathrm{wt} \%$ of the total soil sample

Fine particulate and asbestos in the soil are each $1 \mathrm{wt} \%$ of the soil sample.

\section{Acceptance Criteria}

NA

\section{Software Validation}

MathCad version 13.0 was used for the calculations; however, the calculations are simple enough that they can be checked by hand calculations and, as such, software validation is not required.

\section{Calculations}

\subsection{Method:}

- Obtain properties of gases and materials at the temperatures and pressures in unit.

- Estimate desired sample volume based on maximum cutoff for asbestos weight \% in the sample.

- Calculate minimum spouting velocity for several cone angles and for small and large bed particulate.

- Calculate minimum velocity in freeboard to entrain the asbestos.

- Calculate diameter at the top of the cone required to reduce the velocity to only elutriate respirable sized

- particles.

- Calculate minimum spouting velocities for typical and maximum particle size along with corresponding

- volumetric flow rates.

- Calculate expected pressure drop for the fluidized bed based on initial and final bed weights.

- Compare flow with that desired for the collection filter and adjust sample size until the required flow is

- near the filter design flow.

- Calculate size of outlet tubing to accommodate flow

- Summarize the calculations

- Draw conclusions and make recommendations. 


\subsection{Define additional units for Mathcad}

$$
\begin{aligned}
& \text { lbmole }:=\operatorname{mole} \frac{\mathrm{lb}}{\mathrm{gm}} \quad \mathrm{cp}:=10^{-2} \text {.poise } \\
& \operatorname{deg} \mathrm{C}:=1 \mathrm{~K} \quad \operatorname{degF}:=1 \cdot \mathrm{R} \quad \text { in_wc }:=\frac{1}{27.673} \cdot \mathrm{psi}
\end{aligned}
$$

\subsection{Properties of Fluidizing Gas and Solids}

\subsubsection{Air Properties}

Air thermodynamic properties at $20 \mathrm{C}$ (Ref. $3 \mathrm{pg} 520$ ) and $\mu$ is proportional to $\mathrm{T}$ :

$$
\begin{aligned}
& \mathrm{T}_{\text {air20 }} \equiv(20+273.15) \mathrm{K} \quad \mathrm{P}_{\mathrm{INL}} \equiv 12.47 . \mathrm{psi} \quad \mathrm{P}_{\mathrm{INL}}=644.9 \text { torr } \\
& \rho_{\text {air20 }}:=1.164 \frac{\mathrm{kg}}{\mathrm{m}^{3}} \quad \rho_{2}\left(\mathrm{~T}_{2}, \mathrm{P}_{2}\right):=\rho_{\text {air20 }} \cdot \frac{\mathrm{P}_{2}}{1 \cdot \mathrm{atm}} \cdot \frac{(20+273.15) \cdot \mathrm{K}}{\mathrm{T}_{2}} \\
& \rho_{\text {air20INL }}:=\rho_{2}\left(T_{\text {air20 }}, P_{I N L}\right) \quad \rho_{\text {air20INL }}=1 \frac{\mathrm{kg}}{\mathrm{m}^{3}} \\
& \mu_{\text {air20 }}:=18.2410^{-6} \cdot \frac{\mathrm{N} \cdot \mathrm{s}}{\mathrm{m}^{2}} \quad \mu_{2}\left(\mathrm{~T}_{2}\right):=\mu_{\text {air20 }} \cdot \frac{\mathrm{T}_{2}}{(20+273.15) \cdot \mathrm{K}} \\
& \mu_{\text {air20 }}=0 \mathrm{cp}
\end{aligned}
$$

Molecular Weight of Air per the CRC Handbook of Chemistry and Physics 62nd Ed (Ref. 4).:

$$
\mathrm{MW}_{\text {air }}:=28.96 \frac{\mathrm{gm}}{\mathrm{mole}}
$$

\subsubsection{Asbestos properties:}

Amphibole asbestos sizes and densities to assume for the design were provided by Karen (Ref. 6):

$$
\begin{aligned}
& \mathrm{D}_{\text {asbestos }} \equiv 0.5 \cdot \text { micron } \quad \mathrm{D}_{\text {asbestosMax }} \equiv 4.9 \cdot \text { micron } \quad \text { FiberLtoD } \equiv 8.6 \\
& \mathrm{~L}_{\text {asbestosMin }}:=\text { FiberLtoD } \cdot \mathrm{D}_{\text {asbestos }} \quad \mathrm{L}_{\text {asbestosMin }}=4.3 \text { micron } \\
& \mathrm{L}_{\text {asbestosMax }} \equiv \text { FiberLtoD } \cdot \mathrm{D}_{\text {asbestosMax }} \mathrm{L}_{\text {asbestosMax }}=42.1 \text { micron } \\
& \rho_{\text {chrysotile }} \equiv 2.5 \cdot \frac{\mathrm{gm}}{\mathrm{mL}} \quad \rho_{\text {amphibole }} \equiv 3.0 \cdot \frac{\mathrm{gm}}{\mathrm{mL}}
\end{aligned}
$$

The asbestos particles can be approximated as long cylinders. Kunii and Levenspiel (Ref. 1 pg 62) give the sphericity of cylinders with a height to diameter ratio of 8 as:

$$
\phi_{\text {asbestos }}:=\frac{0.58-0.70}{10-5} \cdot(8-5)+0.7 \mathrm{C} \quad \phi_{\text {asbestos }}=0.63
$$

\subsubsection{Soil Properties:}

Estimate soil size and properties assuming it is approximated by round sand. The harmonic mean particle diameter is used rather than the mass mean partilce size for fluidizing and elutriation calculations because it gives a diameter that has the same total surface area for the same bed volume: 


$$
\mathrm{D}_{\text {SoilHMPD }}=\frac{1}{\sum_{\mathrm{n}=1}^{\mathrm{i}} \frac{\mathrm{x}_{\mathrm{i}}}{\mathrm{D}_{\mathrm{i}}}}
$$

For the calculations, assume a soil harmonic mean particle diameter of $1 \mathrm{~mm}$ (about that for U.S. Standard Sieve no. 20) and that the soil has been sieved through a 1/4 in screen:

$$
\mathrm{D}_{\text {soilHMPD }} \equiv 1 \cdot \mathrm{mr} \quad \mathrm{D}_{\text {soilMax }} \equiv 0.25 \text {. in }
$$

Sphericity and void fraction at minimum fluidization from Kunii and Levenspiel (Ref.1) pg 69 for round sand:

$$
\begin{aligned}
& \varepsilon_{\text {soil }}:=0.4 \mathrm{C} \\
& \phi_{\text {soil }}:=0.8 \mathrm{C}
\end{aligned}
$$

Estimated soil particle density and corresponding bulk density are:

$$
\begin{aligned}
& \rho_{\text {soil } \_p} \equiv 2.6 \cdot \frac{\mathrm{gm}}{\mathrm{mL}} \\
& \rho_{\text {soil_bulk }}:=\rho_{\text {soil_p}} \cdot\left(1-\varepsilon_{\text {soil }}\right) \quad \quad \rho_{\text {soil_bulk }}=1.6 \frac{\mathrm{gm}}{\mathrm{mL}}
\end{aligned}
$$

\subsubsection{Sample Collection Filter Properties}

Perry (Ref. 8) notes that the NIOSH 7400 method is the most prevalent method for asbestos sampling from air for analysis by phase control microscopy (PCM). Filter pore size of $0.45 \mu \mathrm{m}$ is used for air sampling and $0.8 \mu \mathrm{m}$ for personal monitoring.

For the calculation, we'll assume that a standard $25 \mathrm{~mm}$ mixed cellulose ester (MCE) filter with $0.45 \mu \mathrm{m}$ pore size. Millipore gives the maximum desired flow rate for this filter as

$$
\begin{aligned}
& D_{\text {filter }}:=25 \cdot \mathrm{mr} \\
& \mathrm{Q}_{\text {filter_per_area }}:=4 \frac{\mathrm{L}}{\min \cdot \mathrm{cm}^{2}} \quad \mathrm{Q}_{25 \mathrm{~mm} \_ \text {filter }}:=\mathrm{Q}_{\text {filter_per_area }} \frac{\pi}{4} \cdot \mathrm{D}_{\text {filter }}{ }^{2} \quad \mathrm{Q}_{25 \mathrm{~mm} \_ \text {filter }}=19.6 \frac{\mathrm{L}}{\mathrm{min}}
\end{aligned}
$$

\subsection{Estimate Soil Sample Size Desired and Bed Height in the Cone}

Assume a weight fraction of asbestos in soil of:

$$
\mathrm{X}_{\text {asbestos }} \equiv 1 \%
$$

Assume a weight fraction of fines in soil equal to or smaller than the asbestos of:

$$
X_{\text {fines }} \equiv 1 \% \quad X_{\text {fines }}+X_{\text {asbestos }}=2 \%
$$

Assume a desired maximum filter loading;

$$
\mathrm{m}_{\text {filter_max }} \equiv 0.4 \cdot \mathrm{gm}
$$


Soil sample size can then be calculated based on the weight fraction of fines and asbestos and the maximum filter loading:

$$
\begin{array}{ll}
\mathrm{m}_{\text {sample }}:=\frac{\mathrm{m}_{\text {filter_max }}}{\mathrm{X}_{\text {asbestos }}+\mathrm{X}_{\text {fines }}} & \mathrm{m}_{\text {sample }}=20 \mathrm{gm} \\
\mathrm{V}_{\text {sample }}:=\frac{\mathrm{m}_{\text {sample }}}{\rho_{\text {soil_bulk }}} & \mathrm{V}_{\text {sample }}=12.8 \mathrm{~mL}
\end{array}
$$

The height of the soil sample in the conical bed is that of a right circular cone with a volume equal to the sample volume:

$$
\begin{array}{ll}
\mathrm{V}_{\text {cone }}=\frac{\pi}{3} \cdot \mathrm{r}^{2} \cdot \mathrm{h} & \mathrm{r}=\mathrm{h} \cdot \tan (\gamma) \\
\mathrm{V}_{\text {cone }}=\frac{1}{3} \cdot \pi \cdot \mathrm{h}^{3} \cdot \tan (\gamma)^{2} &
\end{array}
$$

Solving for bed height gives:

$$
\mathrm{h}=\left(3 \cdot \frac{\mathrm{V}_{\text {cone }}}{\pi \cdot \tan (\gamma)^{2}}\right)^{\frac{1}{3}}
$$

For a cone angle of $30 \mathrm{deg}$ from the vertical, the height and top radius of the bed are:

$$
\begin{aligned}
& \gamma:=30 \cdot \operatorname{deg} \\
& \mathrm{H}_{\text {bed_30deg }}:=\left(3 \cdot \frac{\mathrm{V}_{\text {sample }}}{\pi \cdot \tan (\gamma)^{2}}\right)^{\frac{1}{3}} \quad \mathrm{H}_{\text {bed_30deg }}=3.3 \mathrm{~cm} \quad \mathrm{H}_{\text {bed_30deg }}=1.3 \mathrm{in} \\
& \mathrm{r}_{\text {bed_30deg }}:=\mathrm{H}_{\text {bed_30deg }} \tan (\gamma) \quad \mathrm{r}_{\text {bed_30deg }}=1.9 \mathrm{~cm} \quad \mathrm{r}_{\text {bed_30deg }} \cdot 2=1.5 \mathrm{in}
\end{aligned}
$$

\subsection{Particle Elutriation Size and Elutriation Velocity}

Particles with a terminal velocity less than the velocity in the freeboard above the spouted bed will be elutriated into the gas exit line while large particles will settle back into the conical vessel or the spouted bed. The terminal velocity can be approximated for particles with sphericity of between 0.5 and 1 by an equation in Kunii and Levenspiel (Ref. 1 pg 80):

$$
\mathrm{u}_{\mathrm{t} \_ \text {star }}=\left(\frac{18}{\mathrm{~d}_{\mathrm{p}_{\mathrm{s}} \mathrm{star}}}+\frac{2.335-1.744 \phi_{\mathrm{s}}}{\sqrt{\mathrm{d}_{\mathrm{p}_{\mathrm{s}} \mathrm{star}}}}\right)^{-1}
$$

Where $u_{t_{\_} \text {star }}$ and $d_{p_{-} \_ \text {stal }}$ are dimensionless velocity and particle size given by:

$$
d_{p_{-} \text {star }}=d_{p} \cdot\left[\frac{\rho_{\mathrm{g}} \cdot\left(\rho_{\mathrm{s}}-\rho_{\mathrm{g}}\right) \cdot \mathrm{g}}{\mu^{2}}\right]^{\frac{1}{3}} \quad \mathrm{u}_{\mathrm{t}_{-} \text {tar }}=\mathrm{u}_{\mathrm{t}} \cdot\left[\frac{\rho_{\mathrm{g}}{ }^{2}}{\mu \cdot\left(\rho_{\mathrm{s}}-\rho_{\mathrm{g}}\right) \cdot \mathrm{g}}\right]^{\frac{1}{3}}
$$


Substituting these terms and rearranging for u.t gives the following relation as a function of the other parameters:

$$
\left.\mathrm{u}_{\mathrm{t}}\left(\mathrm{d}_{\mathrm{p}}, \mu, \rho_{\mathrm{g}}, \rho_{\mathrm{s}}, \phi_{\mathrm{s}}\right):=\left[\frac{\rho_{\mathrm{g}}^{2}}{\mu \cdot\left(\rho_{\mathrm{s}}-\rho_{\mathrm{g}}\right) \cdot \mathrm{g}}\right]^{\frac{1}{3}}\right]^{-1} \cdot\left[\frac{18}{\mathrm{~d}_{\mathrm{p}} \cdot\left[\frac{\rho_{\mathrm{g}} \cdot\left(\rho_{\mathrm{s}}-\rho_{\mathrm{g}}\right) \cdot \mathrm{g}}{\mu^{2}}\right]^{\frac{1}{3}}}+\frac{2.335-1.744 \phi_{\mathrm{s}}}{\left.\sqrt{\mathrm{d}_{\mathrm{p}} \cdot\left[\frac{\rho_{\mathrm{g}} \cdot\left(\rho_{\mathrm{s}}-\rho_{\mathrm{g}}\right) \cdot \mathrm{g}}{\mu^{2}}\right]^{\frac{1}{3}}}\right]^{-1}}\right.
$$

Find maximum diameter of particles elutriated from sampler based on actual velocity. The equivalent spherical diameter is used for the correlations and is defined as the diameter of a sphere having the same volume as the particle. For the maximum size of asbestos fibers and the fiber length to diameter ratio from the assumptions, the equivalent spherical diameter for the asbestos fibers is:

$$
\begin{aligned}
& \frac{\pi}{6} \cdot \mathrm{D}_{\text {equivSphere }}{ }^{3}=\left[\frac{\pi}{4} \cdot \mathrm{D}_{\text {asbestos }}{ }^{2} \cdot\left(\text { FiberLtoD } \cdot \mathrm{D}_{\text {asbestos }}\right)\right] \\
& \mathrm{D}_{\text {equivSphere }}=\left(\frac{3}{2} \cdot \text { FiberLtoD }\right)^{3} \cdot \mathrm{D}_{\text {Asbestos }} \\
& \left(\left(\frac{3}{2} \cdot 10\right)^{\frac{1}{3}}\right)=\text { । } \\
& \left(\left(\frac{3}{2} \cdot 8 .\right)^{\frac{1}{3}}\right)=2.2894 \\
& \left(\left(\frac{3}{2} \cdot 8.6\right)^{\frac{1}{3}}\right)=2.3453
\end{aligned}
$$

Solving for the equivalent spherical diameter gives:

$$
\left(\frac{D_{\text {equivSphere }}}{D_{\text {asbestos }}}=2.4662\right) \quad \text { for } \mathrm{L} / \mathrm{D} \text { of } 10 \text {, and } \quad\left(\frac{D_{\text {equivSphere }}}{D_{\text {asbestos }}}=2.29\right) \quad \text { for } \mathrm{L} / \mathrm{D} \text { of } 8
$$

To ensure that all asbestos particles are elutriated, set the freeboard velocity to that required to elutriate the largest asbestos particles.

$$
\begin{aligned}
& \mathrm{D}_{\text {sph_asbestosMax }}:=\left(\frac{3}{2} \cdot \text { FiberLtoD }\right)^{\frac{1}{3}} \cdot \mathrm{D}_{\text {asbestosMax }} \quad \mathrm{D}_{\text {sph_asbestosMax }}=11.5 \text { micron } \\
& \mathrm{u}_{\text {mintopofcone }}:=\mathrm{u}_{\mathrm{t}}\left(\mathrm{D}_{\text {sph_asbestosMax }}, \mu_{\text {air20 }}, \rho_{\text {air20 }}, \rho_{\text {amphibole }}, \phi_{\text {asbestos }}\right) \\
& \mathrm{u}_{\text {mintopofcone }}=2.1 \frac{\mathrm{cm}}{\mathrm{sec}}
\end{aligned}
$$

\subsubsection{Determine Maximum Size Spheres And Asbestos Fibers Elutriated From Sampler For A Given Air Flow Rate}

The velocity in the sampler decreases as the diameter increases towards the top of the vessel. Fibers and particles with a settling velocity less than the velocity in the freeboard at the top of the sampler will be elutriated out of the bed while larger particles will settle out of the gas stream and remain in the sampler. Some particles may also be thrown out of the fluidized bed to the top of the vessel and be captured in the outlet, but this will depend on how vigorously the bect is fluidized. 
Determine size elutriated vs velocity for a range of equivalent spherical particle sizes, and flowrate for the diameter at the top of the conical vessel:
$\mathrm{D}_{\text {elut }}:=.5 \cdot$ micron, $2.5 \cdot$ micron.. 30 micron
$\mathrm{D}_{\text {testunit }}:=19.7 \cdot \mathrm{cm}$
$\mathrm{D}_{\text {testunit }}=7.8 \mathrm{in}$

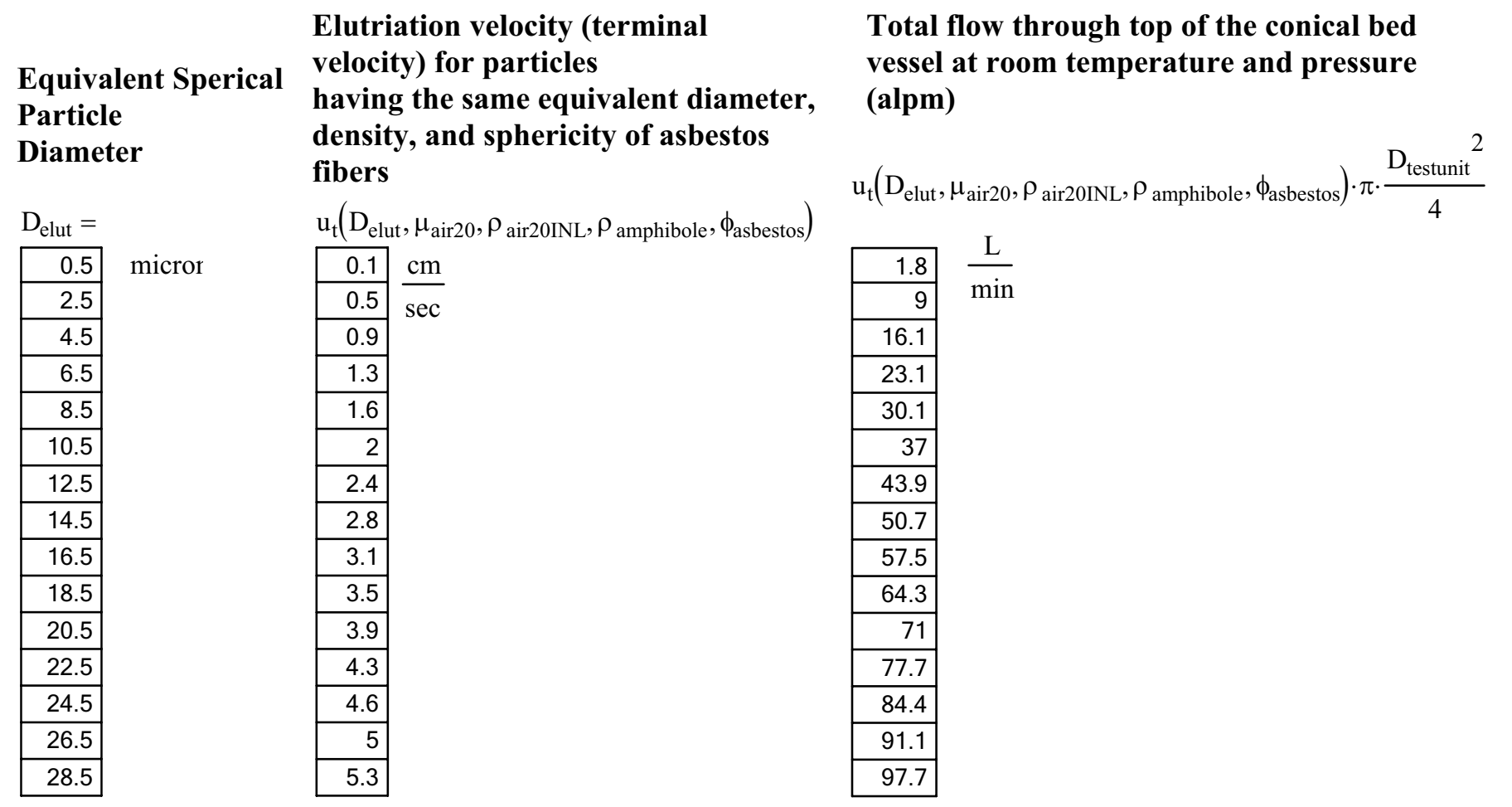

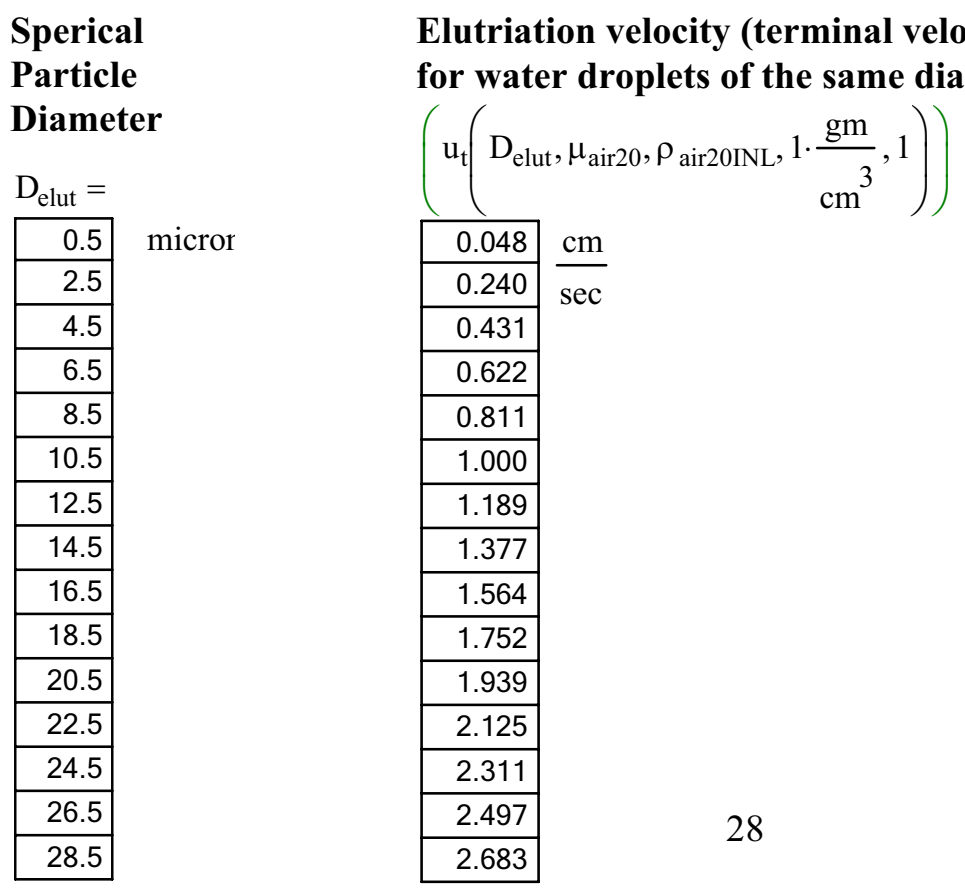


The optimal flow into the sampler based on initial tests with sand, combined with the test sampler conical bed size, gives a velocity at the top of the sampler of:

$$
\begin{array}{ll}
\mathrm{Q}_{\text {testflow }}:=37 \cdot \frac{\mathrm{L}}{\mathrm{min}} \text { alpm } & \mathrm{D}_{\text {testunit }}=19.7 \mathrm{~cm} \\
\mathrm{H}_{\text {testunit }}:=15.24 \mathrm{~cm} & \mathrm{H}_{\text {testunit }}=6 \mathrm{in}
\end{array}
$$

For the actual height of the test bed unit and typical test flow rate, the velocity at the top of the sampler is

$$
\mathrm{u}_{\text {testflow }}:=\frac{\mathrm{Q}_{\text {testflow }}}{\frac{\pi}{4} \cdot \mathrm{D}_{\text {testunit }}{ }^{2}} \quad \mathrm{u}_{\text {testflow }}=2 \frac{\mathrm{cm}}{\mathrm{sec}}
$$

The maximum asbestos size elutriated from the conical sampler vessel can then be iteratively determined to find the size that has the same terminal velocity as the actual velocity at the top of the vessel.

$$
\begin{array}{cc}
D_{\text {asbestosMaxElut }}:=4.9 m i c r o n & \mathrm{~L}_{\text {asbestosMaxElut }}:=\mathrm{L}_{\text {asbestosMax }} \cdot \mathrm{D}_{\text {asbestosMaxElut }} \\
\mathrm{D}_{\text {equiv_Max_asb }}:=(2.29) \cdot \mathrm{D}_{\text {asbestosMaxElut }} & \mathrm{D}_{\text {equiv_Max_asb }}=11.2 \mathrm{micron} \\
\mathrm{u}_{\text {equiv_Dp_Max_asb_elut }}:=\mathrm{u}_{\mathrm{t}}\left(\mathrm{D}_{\text {equiv_Max_asb }}, \mu_{\text {air20 }}, \rho_{\text {air20INL }}, \rho_{\text {amphibole }}, \phi_{\text {asbestos }}\right) \\
\mathrm{u}_{\text {equiv_Dp_Max_asb_elut }}=2.2 \frac{\mathrm{cm}}{\mathrm{sec}}
\end{array}
$$

\subsection{Calculation of Minimum Spouting Velocity for the Conical Bed}

Bi et. al (Ref. 5) reviewed several correlations for minimum spouting velocity and derived a semi-empirical equation from the Ergun equation for the minimum spouting velocity based on the inlet diameter:

$\operatorname{Re}_{i_{-} m s}=\operatorname{if}\left[\frac{D_{b}}{D_{i}}<1.66,0.202 \cdot \sqrt{\left.\operatorname{Ar} \cdot \frac{D_{b}}{D_{i}} \cdot\left(\frac{D_{b}}{D_{i}}\right)^{2}+\frac{D_{b}}{D_{i}}+1\right] \cdot \frac{1}{3}},\left[0.30-0.27 \cdot\left(\frac{D_{i}}{D_{b}}\right)^{2}\right] \cdot \sqrt{\left.\left.\operatorname{Ar} \cdot \frac{D_{b}}{D_{i}} \cdot\left(\frac{D_{b}}{D_{i}}\right)^{2}+\frac{D_{b}}{D_{i}}+1\right] \cdot \frac{1}{3}\right]}\right.$

Inserting the equations for the Reynold's number Re and the Arrhenius number Ar and solving for velocity gives:

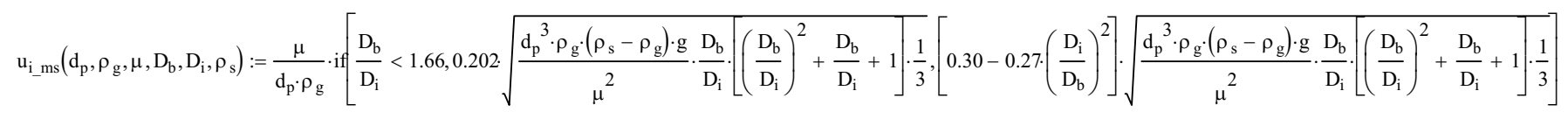




\subsubsection{Calculate Flows for Selected Bed Distributor Open Area/Diameter}

Selecting and inlet opening diameter of $3 \mathrm{~mm}$, then for a $30 \mathrm{deg}$ bed

$$
\mathrm{D}_{\mathrm{i}}:=3 \cdot \mathrm{mm} \quad \mathrm{D}_{\mathrm{i}}=0.1 \mathrm{in}
$$

The ratio of top bed diameter to inlet diameter is:

$$
\mathrm{r}_{\text {bed_30deg }}=1.9 \mathrm{~cm} \quad \frac{2 \cdot \mathrm{r}_{\text {bed_30deg }}}{\mathrm{D}_{\mathrm{i}}}=12.8
$$

The minimum spouting velocity for the average soil size based on the inlet opening diameter is:

$$
\begin{aligned}
& \mathrm{u}_{\mathrm{ms} \_30 \mathrm{deg}}:=\mathrm{u}_{\mathrm{i}_{\_} \mathrm{ms}}\left(\mathrm{D}_{\text {soilHMPD }}, \rho_{\text {air20INL }}, \mu_{\text {air20 }}, 2 \cdot \mathrm{r}_{\text {bed_3 } 30 \mathrm{deg}}, \mathrm{D}_{\mathrm{i}}, \rho_{\text {soil_p }}\right) \\
& \mathrm{u}_{\mathrm{ms} \_30 \mathrm{deg}}=4.2 \times 10^{3} \frac{\mathrm{cm}}{\mathrm{sec}}
\end{aligned}
$$

The minimum air flow for the calculated minimum spouting velocity is:

$$
\begin{aligned}
& \mathrm{Q}_{\mathrm{ms} \_30 \mathrm{deg}}:=\mathrm{u}_{\mathrm{ms} \_30 \mathrm{deg}} \cdot \mathrm{D}_{\mathrm{i}}^{2} \cdot \frac{\pi}{4} \\
& \mathrm{Q}_{\mathrm{ms} \_30 \mathrm{deg}}=17.7 \frac{\mathrm{L}}{\mathrm{min}} \quad \mathrm{Q}_{\mathrm{ms} \_30 \mathrm{deg}}=0.6 \frac{\mathrm{ft}^{3}}{\mathrm{~min}}
\end{aligned}
$$

The superficial velocity at the top of the sample bed (not the top of the vessel) at the minimum spouting flow is:

$$
\mathrm{u}_{\text {bms_30deg }}:=\mathrm{u}_{\mathrm{ms} \_30 \mathrm{deg}} \cdot \frac{\mathrm{D}_{\mathrm{i}}^{2}}{\left(2 \cdot \mathrm{r}_{\text {bed_30deg }}\right)^{2}} \quad \mathrm{u}_{\text {bms_30deg }}=25.5 \frac{\mathrm{cm}}{\mathrm{sec}}
$$

Minimum spouting velocity and air flow for a bed composed entirely of $1 / 4$ " rocks with the same density as the soil in a standard cylindrical fluidized bed is:

$$
\begin{aligned}
& \mathrm{u}_{\mathrm{ms} \_30 \text { deg_max }}:=\mathrm{u}_{\mathrm{i} \_\mathrm{ms}}\left(\mathrm{D}_{\text {soilMax }}, \rho_{\text {air20INL }}, \mu_{\text {air20 }}, 2 \cdot \mathrm{r}_{\text {bed_30deg }}, \mathrm{D}_{\mathrm{i}}, \rho_{\text {soil__p}}\right) \\
& \mathrm{u}_{\mathrm{ms} \_30 \text { deg_max }}=1.1 \times 10 \frac{\mathrm{cm}}{\mathrm{sec}} \quad \quad \mathrm{u}_{\mathrm{ms} \_30 \text { deg_max }}=105.1 \frac{\mathrm{m}}{\mathrm{s}}
\end{aligned}
$$

Velocity at the top of the bed (not the top of the vessel) and air flow for the minimum spouting velocity for a bed of all $1 / 4$ " rocks are then:

$$
\mathrm{u}_{\text {bms_30deg_max }}:=\mathrm{u}_{\text {ms_30deg_max }} \cdot \frac{\mathrm{D}_{\mathrm{i}}^{2}}{\left(2 \cdot \mathrm{r}_{\text {bed_3 deg }}\right)^{2}} \quad \mathrm{u}_{\text {bms_30deg_max }}=64.2 \frac{\mathrm{cm}}{\mathrm{sec}}
$$




$$
\begin{aligned}
& \mathrm{Q}_{\mathrm{ms} \_30 \text { deg_max }}:=\mathrm{u}_{\mathrm{ms} \_30 \text { deg_max }} \cdot \mathrm{D}_{\mathrm{i}}^{2} \cdot \frac{\pi}{4} \\
& \mathrm{Q}_{\mathrm{ms} \text { 30deg_max }}=44.6 \frac{\mathrm{L}}{\mathrm{min}} \quad \mathrm{Q}_{\mathrm{ms} \_30 \text { deg_max }}=1.6 \frac{\mathrm{ft}^{3}}{\min }
\end{aligned}
$$

The superficial velocity is reduced as the diameter of the cone increases with the cone height.

The minimum velocity is at the top of the cone. If this velocity is set at the elutriation velocity for the maximum size asbestos fibers, then the height of the cone calculated for the minimum spouting velocity for the maximum size bed particles is then:

$$
\begin{aligned}
& D_{\text {topofcone_30deg }}:=\sqrt{\frac{4 \cdot Q_{\mathrm{ms}_{\_} 30 \mathrm{deg} \_ \text {max }}}{\pi \cdot \mathrm{u}_{\text {mintopofcone }}}} \\
& D_{\text {topofcone_30deg }}=21.3 \mathrm{~cm} \quad D_{\text {topofcone_30deg }}=8.4 \mathrm{in}
\end{aligned}
$$

Cone height needed when fluidizing $1 / 4$ " rocks is then:

$$
\begin{aligned}
& \mathrm{H}_{\text {cone_30deg }}:=\frac{\mathrm{D}_{\text {topofcone_30deg }}}{2} \cdot \frac{1}{\tan (\gamma)} \\
& \mathrm{H}_{\text {cone_30deg }}=18.4 \mathrm{~cm} \quad \mathrm{H}_{\text {bed_30deg }}=3.3 \mathrm{~cm} \quad \text { D }_{\text {topofcone_30deg }}=21.3 \mathrm{~cm}
\end{aligned}
$$

In reality, the particulates in the soil sample will not all be $1 / 4$ ", so the flow needed to cause the bed to spout will usually be less than this and the diameter at the top of the cone and corresponding height will not need to be this large. 


\subsection{Bed and Distributor Design Pressure Drops}

For cylindrical beds, fluidization occurs at the point when the pressure drop through the packed bed is equal to the hydraulic pressure in the bottom of the bed. This is because after the bed becomes fluidized, it behaves as a fluid, and as such has a pressure due to the height of the 'liquid' column.

For spouted beds, the bed pressure drop starts out less than that for a fluidized bed and increases as the velocity increases up until the entire bed is fluidized rather than spouting.

The pressure drops for fluidized beds of the same height as the conical beds, and therefore the maximum pressure drop for the conical bed is:

$$
\begin{aligned}
& \mathrm{H}_{\text {bed_30deg }}=3.3 \mathrm{~cm} \\
& \mathrm{dP}_{\mathrm{fb} \_30 \mathrm{deg}}:=\mathrm{H}_{\text {bed_30deg }} \mathrm{g} \cdot \rho_{\text {soil_bulk }} \\
& \rho_{\text {soil_bulk }}=1.6 \frac{\mathrm{gm}}{\mathrm{cm}^{3}} \quad \mathrm{~g}=980.7 \frac{\mathrm{cm}}{\mathrm{sec}^{2}} \\
& \mathrm{dP}_{\mathrm{fb} \_} 30 \mathrm{deg}=2 \text { in_wc }
\end{aligned}
$$

These are fairly small and thus the absolute pressure in the freeboard above the bed and the corresponding gas density will not be significantly different than that in the inlet air, provided that the pressure drop across the distributor at the inlet to the cone does not also have a significant pressure drop.

Distributor pressure drop.

\subsection{Freeboard Height Above the Bed}

The spout erupts to a significant height above the surface of the spouting bed. Therefore, either the height of the vessel must be greater than the disengaging height or a baffle plate is needed above the bed to ensure that particles thrown above the bed are not entrained in the outlet. Kunii and Levenspiel (Ref. 1) give the following relationship for the Transport Disengaging Height based on a Froude number of 10e-3.

$$
\begin{aligned}
& \mathrm{TDH}=\frac{\mathrm{u}_{\mathrm{o}}{ }^{2}}{\mathrm{~g} \cdot 10^{-3}} \\
& \mathrm{H}_{\text {freeboard_30deg }}:=\frac{\mathrm{u}_{\text {mintopofcone }}}{\mathrm{g} \cdot 10^{-3}} \\
& \mathrm{H}_{\text {freeboard_30deg }}=4.5 \mathrm{~cm} \quad \mathrm{H}_{\text {freeboard_30deg }}=1.8 \text { in }
\end{aligned}
$$

The height of the portion of the cone above the bed is much greater than the calculated TDH, so no additional height is needed at the top of the cone for disengagement of bed particles.

\subsection{Time to Entrain Particulate}

The maximum time needed to elutriate all the fine particulates from the bed can be estimated by assuming that the vessel is perfectly mixed. This is conservative, since flow in the top of the vessel will be something between plug flow and perfectly mixed flow. From Branan (Ref. 8), the purging cycle time to obtain a ratio of initial to final concentration of 1000 to 1 in a perfectly mixed vessel (Ref. 8 pg 272) is: 


$$
\begin{aligned}
& \mathrm{R}_{\mathrm{i}_{\_} \mathrm{t} \_\mathrm{f}}:=100 \mathrm{C} \\
& \mathrm{V}_{\text {fluidbed }}:=\frac{\pi}{3} \cdot \frac{\mathrm{D}_{\text {topofcone_30deg }}}{4} \cdot \mathrm{H}_{\text {cone_30de }} \\
& \mathrm{V}_{\text {fluidbed }}=2.2 \mathrm{~L} \\
& \mathrm{Q}_{\mathrm{ms} \_30 \mathrm{deg}}=17.7 \frac{\mathrm{L}}{\mathrm{min}}
\end{aligned}
$$

Number of bed volumes required for purging is

$$
\mathrm{N}_{\text {volumes }}:=\frac{\log \left(\mathrm{R}_{\mathrm{i} \_ \text {to_f }}\right)}{0.4227} \quad \mathrm{~N}_{\text {volumes }}=7.1
$$

Maximum purge time at the minimum spouting velocity for $1 \mathrm{~mm}$ bed particles is

$$
\mathrm{T}_{\text {purgingMin }}:=\frac{\mathrm{N}_{\text {volumes }} \cdot \mathrm{V}_{\text {fluidbed }}}{\mathrm{Q}_{\mathrm{ms} \_30 \text { deg }}} \quad \mathrm{T}_{\text {purgingMin }}=52.5 \mathrm{sec}
$$

Purge time at the optimal velocity based on the initial tests is

$$
\mathrm{T}_{\text {purging }}:=\frac{\mathrm{N}_{\text {volumes }} \cdot \mathrm{V}_{\text {fluidbed }}}{\mathrm{Q}_{\text {testflow }}} \quad \mathrm{T}_{\text {purging }}=25.1 \mathrm{sec}
$$

Therefore, a purge time of 1 minute will be more than adequate to elutriate the asbestos.

\subsection{Tubing Size}

Size tubing inside diameter based on typical design velocities for 0-30 psig air (Ref. 8)

$$
\begin{aligned}
& \mathrm{u}_{\text {airdesign }}:=4000 \frac{\mathrm{ft}}{\mathrm{min}} \\
& \mathrm{u}_{\text {airdesign }}=66.7 \frac{\mathrm{ft}}{\mathrm{s}} \\
& \mathrm{u}_{\text {airdesign }}=2 \times 10^{3} \frac{\mathrm{cm}}{\mathrm{sec}} \\
& D_{\text {tubing }}:=\sqrt{\frac{4 \cdot \mathrm{Q}_{\mathrm{ms} \_30 \operatorname{deg} \_\max }}{\mathrm{u}_{\text {airdesign }} \cdot \pi}} \\
& \mathrm{D}_{\text {tubing }}=0.7 \mathrm{~cm}
\end{aligned}
$$

Velocity for $1 / 4$ " ID tubing

$$
\mathrm{u}_{0.25 \text { in_tubing }}:=\frac{4 \mathrm{Q}_{\mathrm{ms} \_30 \mathrm{deg} \_\max }}{\pi \cdot(0.25 \mathrm{in})^{2}} \quad \mathrm{u}_{0.25 \text { in_tubing }}=4.6 \times 10^{3} \frac{\mathrm{ft}}{\mathrm{min}}
$$

Velocity for 3/8" ID tubing

$$
\mathrm{u}_{0.375 \text { in_tubing }}:=\frac{4 \mathrm{Q}_{\mathrm{ms} \_30 \mathrm{deg} \_ \text {max }}}{\pi \cdot(0.375 \mathrm{in})^{2}} \quad \mathrm{u}_{0.375 \text { in_tubing }}=2.1 \times 10^{3} \frac{\mathrm{ft}}{\mathrm{min}}
$$




\section{Summary and Recommended Spouted Conical Bed Design}

For soil and asbestos properties of

$$
\begin{aligned}
& \mathrm{D}_{\text {soilHMPD }}=1 \mathrm{mr} \\
& \mathrm{D}_{\text {soilMax }}=0.3 \text { in } \\
& \rho_{\text {soil } \_p}=2.6 \frac{\mathrm{gm}}{\mathrm{cm}^{3}} \\
& \mathrm{D}_{\text {asbestos }}=0.5 \mathrm{micron} \\
& \mathrm{D}_{\text {asbestosMax }}=4.9 \text { micron } \\
& \frac{\mathrm{L}_{\text {asbestosMin }}}{\mathrm{D}_{\text {asbestos }}}=8.6 \\
& \rho_{\text {amphibole }}=3 \frac{\mathrm{gm}}{\mathrm{cm}^{3}} \\
& \mathrm{X}_{\text {fines }}+\mathrm{X}_{\text {asbestos }}=2 \%
\end{aligned}
$$

For an estimated maximum desired filter loading, the sample size is calculated to be

$$
\begin{aligned}
& \mathrm{m}_{\text {filter_max }}=0.4 \mathrm{gm} \\
& \mathrm{m}_{\text {sample }}=20 \mathrm{gm}
\end{aligned}
$$

$$
\mathrm{V}_{\text {sample }}=12.8 \mathrm{~mL}
$$

$\mathrm{H}_{\text {bed_30deg }}=3.3 \mathrm{~cm}$

Per Millipore literature, the maximum flowrate for their $0.45 \mu \mathrm{m}$ pore size $25 \mathrm{~mm}$ dia MCE filters is

$$
\mathrm{Q}_{25 \mathrm{~mm} \_ \text {filter }}=19.6 \frac{\mathrm{L}}{\mathrm{min}}
$$

Based upon the desire to elutriate the maximum asbestos particle size and smaller, the velocity at the top of the vessel should be greater than:

$$
\mathrm{u}_{\text {mintopofcone }}=2.1 \frac{\mathrm{cm}}{\mathrm{sec}}
$$

The conical spouted bed for fluidizing from $1 \mathrm{~mm}$ to $1 / 4$ in. $(6.35 \mathrm{~mm})$ particles should have a 30 deg angle from the vertical and have inlet air flow rates of:

$$
\begin{aligned}
& \gamma=30 \mathrm{deg} \\
& \mathrm{Q}_{\mathrm{ms} \_30 \mathrm{deg}}=17.7 \frac{\mathrm{L}}{\mathrm{min}} \quad \mathrm{Q}_{\mathrm{ms} \_30 \text { deg_ }_{-} \max }=44.6 \frac{\mathrm{L}}{\mathrm{min}} \\
& \mathrm{Q}_{\mathrm{ms} \_30 \mathrm{deg}}=0.6 \frac{\mathrm{ft}^{3}}{\mathrm{~min}} \quad \mathrm{Q}_{\mathrm{ms} \_30 \mathrm{deg} \_\max }=1.6 \frac{\mathrm{ft}^{3}}{\min }
\end{aligned}
$$

The maximum time to purge and elutriate the fine particulates from the conical sample vessel at the minimum spouting velocity for $1 \mathrm{~mm}$ particles is

$$
\mathrm{T}_{\text {purgingMin }}=52.5 \mathrm{sec}
$$

This flow is about $50 \%$ higher than the desired filter flowrate, but should still give reasonably low pressure drop across the filter.

The bed height, cone height, freeboard diameter and freeboard height are: 


$$
\begin{array}{ll}
\mathrm{H}_{\text {bed_30deg }}=3.3 \mathrm{~cm} & \\
\mathrm{H}_{\text {cone_30deg }}=18.4 \mathrm{~cm} & \mathrm{H}_{\text {cone_30deg }}=7.3 \mathrm{in} \\
\mathrm{D}_{\text {topofcone_30deg }}=21.3 \mathrm{~cm} & \mathrm{D}_{\text {topofcone_30deg }}=8.4 \mathrm{in}
\end{array}
$$

Maximum bed pressure drop is:

$$
\mathrm{dP}_{\mathrm{fb} \_30 \mathrm{deg}}=2 \text { in_wc }
$$

The conical bed sampler dimensions for the test unit are shown in Figure 1. The sample separator is mostly wasted space due to the large diameter needed to reduce the velocity at the top of the vessel to that needed to allow particles larger than the asbestos fibers to settle out. A cyclone or similar device could be added to the outlet so that the velocity in the freeboard can be increased (diameter decreased) and the overall dimensions reduced.

Alternatively, if it is decided that entrainment of particulate larger than asbestos up to a certain size is acceptable for the filter collection and subsequent analysis, then the bed diameter and height can be decreased without the need for a cyclone.

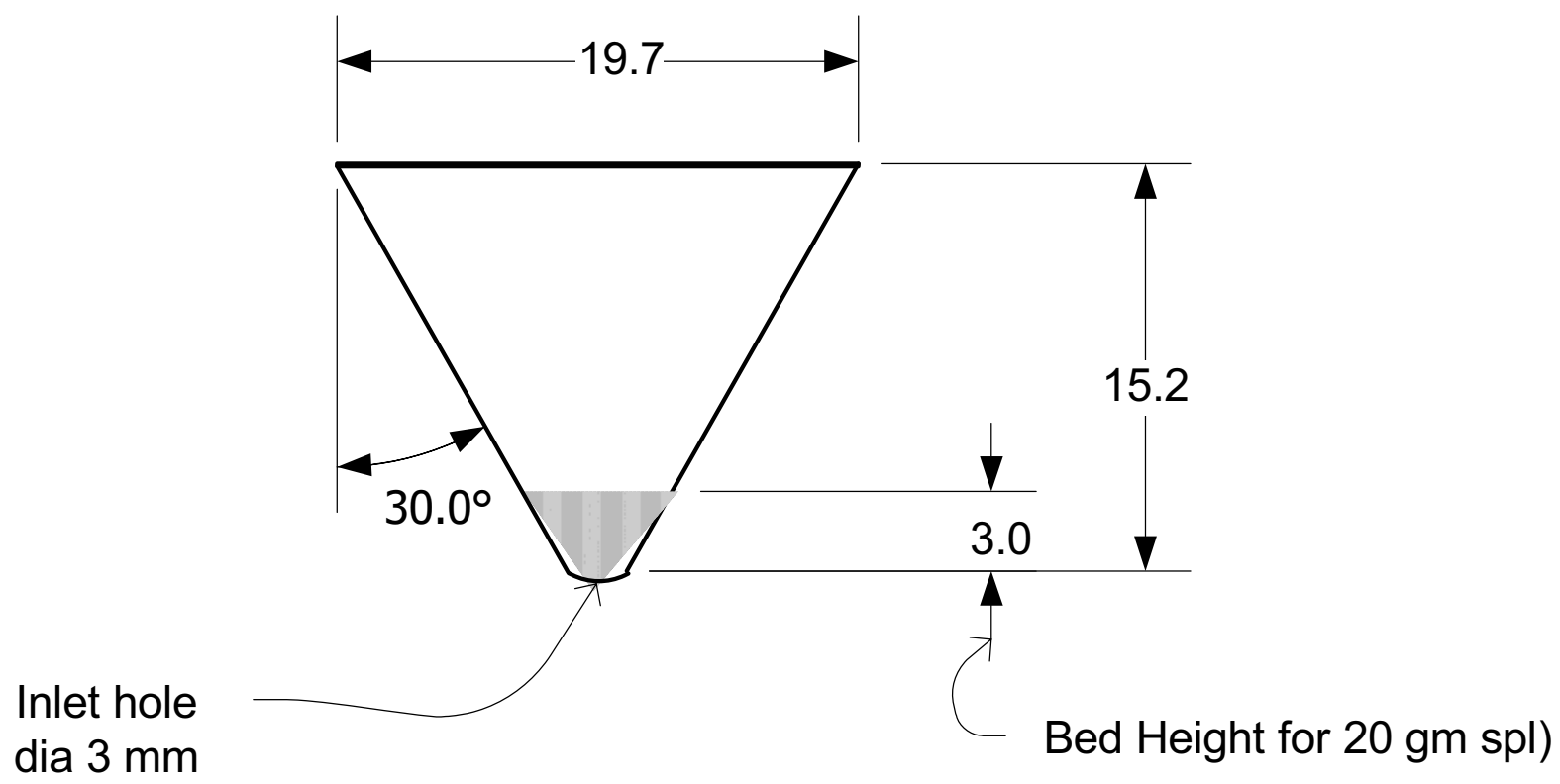

Figure 1: Conical Spouted Bed Test Unit Dimensions (Dimensions in Centimeters) 


\section{References:}

D. Kunii, and O. Levenspiel, Fluidization Engineering, Second Edition, Butterworth-Heinemann: Newton, MA, 1991.

R. H. Perry,and C. H. Chilton, Chemical Engineer's Handbook, 5th ed., New York: McGraw-Hill, 1987.

F. Krieth and W. Z. Black, Basic Heat Transfer, New York: Harper and Row, 1980.

CRC Handbook of Chemistry and Physics, 62nd ed., Chemical Rubber Co., 1981.

H. T. Bi, A. Macchi, J. Chaouki and R. Legros, "Minimum Spouting Velocity of Conical Spouted Beds,"Canadian Journal of Chemical Engineering, Vol. 75, April 1997, pp 460-465.

K. E. Wright, INL, E-mail to B. H. O'Brien, "Sizes to segregate," January 16, 2006.

A. Perry, "A Discussion of Asbestos Dection Techniques for Air and Soil," prepared for the U.S. EPA Office of Solid Waste and Emergency Reponse, August 2004.

C. Branan, Rules of Thumb for Chemical Engineers, Second Edition, Houston: Gulf Publishing, 1998. 
Appendix B

Assembling the Fluidized Bed 


\section{Materials Required}

- Fluidized bed apparatus (steel funnel with $3 \mathrm{~mm}$ orifice and $1 / 2$ " NPT male thread on inlet)

- Steel lid, drilled to accommodate luer fittings (fittings listed by size and part number below)

- Lexan support stand with 4 wing nuts

- Inlet HEPA filter (such as part number 5169K72 and 9179K14 from McMaster Carr)

- Neoprene rubber gasket (such as part number 97725K42 from McMaster Carr)

- Rotameter (0-60 lpm)

- Filter Cassettes (such as part number 225-231 from SKC)

- Ball valve (1/2" NPT female, $1 / 2$ " NPT female)

- Vacuum tubing, $1 / 2$ " ID x $1 / 4$ " wall

- Tygon tubing, $1 / 8$ " ID

- Tubing, 1/4" ID

- Vacuum pump (such as Welch dry vacuum \#2581)

- Luer Fittings (Part numbers listed for fittings correspond to Cole-Parmer)

1- Male luer plug (K-45505-58)

3- Male luer x male luer (K-45505-72)

3- Stopcocks (K-30600-02)

3- Female luer x $1 / 4-28$ UNF (K-45502-66)

1 - Female luer x $1 / 8$ " hose barb (K-45502-04)

3- Male luer lock x 1/8" hose barb (K-45505-04)

3- Male luer slip x $1 / 8$ " hose barb (K-45505-26)

- 1 Multi barb elbow x male $1 / 8$ " ID x $1 / 2$ " NPT

- 1 Multi barb straight x male $1 / 8$ " ID x $1 / 2$ " NPT

- Teflon tape $(<1 / 4$ " wide)

- Zip ties ( 6-8" long) 


\section{PRELIMINARY ASSEMBLY}

\section{HEPA FILTER ASSEMBLY}

1. Replace the nylon filter that comes in the inlet filter cartridge with the HEPA filter.

2. Put a multibarb elbow $\left(1 / 8^{\prime \prime} \times 1 / 2 " N P T\right)$ on the filter cartridge outlet to fluidized bed.

3. Attach about 12-15" 1/4"ID tubing to multibarb elbow, and connect it to the inlet of the fluidized bed.

\section{FUNNEL SET-UP}

1. Attach the ball valve to the NPT inlet of the funnel.

2. Attach a straight barb elbow $\left(1 / 8 " x \frac{1}{2} " \mathrm{NPT}\right)$ to the inlet to the ball valve.

3. Put the funnel in the hole in the steel base of the lexan stand.

4. Attach the tubing on the outlet of the HEPA filter to the inlet barb of the funnel.

5. Using zip ties, attach the rotameter to one leg of the lexan stand.

\section{GASKET ALTERATION}

1. Trim the gasket such that the outer diameter remains the same, but the width of the gasket is about $1 / 2$ " (thus the inner diameter will be about 1 " larger).

\section{MANIFOLD ASSEMBLY}

1. Attach the three stopcocks together:

a. orient one stopcock so that it looks like a "T".

b. connect all three stopcocks in series by attaching the male luer lock of the right side of the " $T$ " to the female receiver side on the left side of the " $T$ " of another stopcock.

c. repeat with all three stopcocks so that the tops of the "T's" are all attached and the base of the "T's" all point down. 
2. Hold the stopcocks so that the base of the "T's" point down and the valves face you. Put the male plug into the left side of the " $T$ " in the stopcock on the far left. Put the female luer-hose barb into the luer lock of the right side of the " $\mathrm{T}$ " in the stopcock on the far right.

3. Attach one male luer lock x $1 / 8$ " barb to each of the intake ports on the 3 stopcocks.

4. Cut three 1-inch sections of $1 / 8$ " ID tygon tubing and attach each section to the barb end of the male luer lock x $1 / 8$ " barb on the stopcocks.

5. Into each free end of tubing, insert the barbed end of a male luer slip x $1 / 8$ " barb. This assembly constitutes the manifold.

\section{LID ASSEMBLY}

1. Obtain the three female luer $\mathrm{x} 1 / 4 "-28$ UNF fittings. Wrap each with Teflon tape and screw into the three holes in the lid.

2. Put a male luer $\mathrm{x}$ male luer into each of the female luer $\mathrm{x} 1 / 4$ " -28 UNF fittings.

\section{FINAL ASSEMBLY}

1. Put the gasket on the lip of the funnel so that it covers the whole lip.

2. Put the lid on the funnel such that the fittings extend upward and right-to-left (rather than frontto- back). Make sure the gasket is not moved out of place.

3. Put one wing nut on each all-thread and tighten each iteratively until nut is finger tight.

4. Put one cassette on each male luer extending out of the lid such that the inlet of the cassette faces the lid and the outlet of the cowl extends upward.

5. Attach the manifold so the male leur fitting of each inlet goes into the outlet of each cassette.

6. Affix one end of tygon tubing to the outlet of the manifold and the other end to the inlet of the rotameter.

7. Attach one end of the vacuum tubing to the outlet of the rotameter and the other end to the inlet of the vacuum pump. 


\section{Appendix C}

\section{Use of the Fluidized Bed}




\section{USE OF THE FLUIDIZED BED FOR SEPARATION OF ASBESTOS FROM NON- ASBESTOS MATRIX AND QUALITATIVE ANALYSIS}

1. Assemble the fluidized bed as described in Appendix B.

2. Turn on the vacuum pump and open the ball valve on the bottom of the funnel.

3. Adjust the rotameter so that flow is $40 \mathrm{lpm}$. If the vacuum pump has adjustment, this may require opening or closing down the adjustment valve to provide the required $40 \mathrm{lpm}$. Note: The specific amount of vacuum provided is not particularly important as long as adequate flow is maintained.

4. Turn off the vacuum and close the ball valve on the bottom of the funnel.

5. Using a riffle splitter, split the sample to $20 \mathrm{~g} \pm 2 \mathrm{~g}$. Record the weight of the sample used.

6. Remove the 4 wing nuts holding the lid down, remove the lid, and put the sample in the funnel.

7. Being careful not to dislodge the gasket, replace the lid and the 4 wing nuts.

8. Turn on the vacuum and open the ball valve on the bottom of the funnel. Start a stopwatch.

9. At the end of 5 minutes, close the ball valve and turn off the funnel. Note: Complete fluidization should occur within the first 30 seconds, so running the fluidized bed 10-15 seconds more or less than 5 minutes should pose no problem.

10. Remove one of the filter cowls to the right or left of the center cowl. Using established methods (e.g. NIOSH 7400), prepare the filter for viewing under phase contrast microscopy.

11. Examine the filter using phase contrast microscopy to determine whether it is $<50 \%$ loaded. If it is $<50 \%$ loaded, take the CENTER filter cowl off the fluidized bed and perform ISO 10312 analysis on it. Proceed to step 13.

12. If in step 11 , the filter $>50 \%$ loaded, use the riffle splitter to obtain a smaller sample weight (note the weight). Proceed to step 13.

13. Clean the fluidized bed

\section{Cleaning the Fluidized Bed}

1. Remove the collection cowls and the fittings that connect the cowls to the lid. Dispose of the outer two cowls and save the middle cowl for analysis.

2. Remove the ball valve and dispose of it.

3. Dispose of excess sample in the funnel.

4. Wipe the interior of the funnel, the lid, and both sides of the gasket with a damp paper towel. 
5. Run deionized water through the funnel for about a minute. Make sure to run water in both directions through the orifice at the bottom.

6. Run deionized water across both sides of the lid and both sides of the gasket.

7. Rinse all equipment with ethanol (this allows it to dry quicker).

8. Reassemble when dry using a new ball valve and fittings between the lid and cowls. 


\section{Appendix D}

Mathematical Foundation for the Release of Asbestos into a Confined Volume 


\section{Release of Asbestos into a Confined Volume of Air \\ Carl D. Palmer and Karen Wright \\ Idaho National Laboratory}

Consider a confined volume with dimensions

$\Delta \mathrm{x}_{\mathrm{c}} \times \Delta y_{c} \times \Delta z_{c}$ containing a volume of soil with dimensions $\Delta \mathrm{x}_{\text {soil }} \times \Delta y_{\text {soil }} \times \Delta z_{\text {soil }}$ (Figure 6). The areas of the confined volume and the soil normal to the $\mathrm{z}$-direction are $\mathrm{A}_{\mathrm{c}}=\left(\Delta \mathrm{x}_{\mathrm{c}}\right.$ )$\left(\Delta \mathrm{y}_{\mathrm{c}}\right)$ and $\mathrm{A}_{\text {soil }}=\left(\Delta \mathrm{x}_{\text {soil }}\right)\left(\Delta \mathrm{y}_{\text {soil }}\right)$, respectively. The mass of soil within the box, $\mathrm{M}_{\text {soil }}$, is

$$
M_{\text {soil }}=\rho_{b, \text { soil }} A_{\text {soil }} \Delta z_{\text {soil }}
$$

If the concentration of asbestos in the soil, $\mathrm{C}_{\mathrm{asb}, \text { soil }}$, is given in fibers per gram of soil, the number of releasable fibers, $\mathrm{N}_{\text {fiber }}$, can be written as

$$
N_{\text {fiber }}=C_{a s b, \text { soil }} M_{\text {soil }}=C_{\text {asb }, \text { soil }} \rho_{b, \text { soil }} A_{\text {soil }} \Delta z_{\text {soil }}
$$

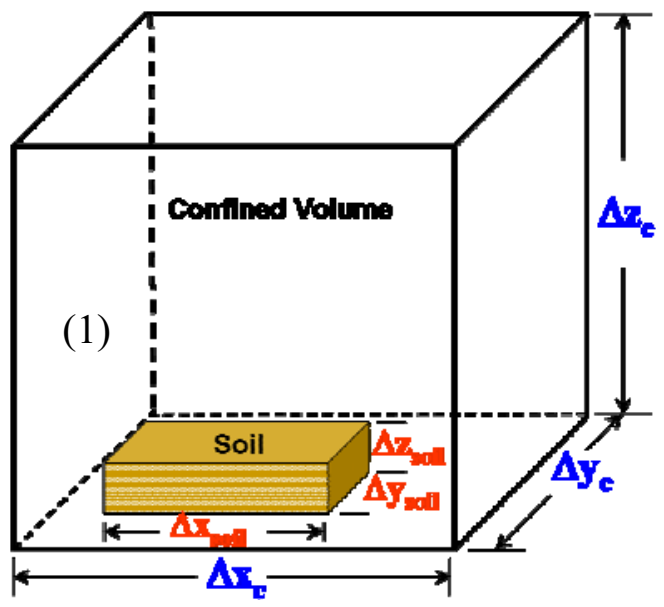

The concentration of asbestos fibers in air, $\mathrm{C}_{\mathrm{asb}, \mathrm{air}}$, can be estimated by

Figure 6. Volume of soil in a confined volume.

$$
C_{\text {asb }, \text { air }}=\frac{N_{\text {fibers }} E_{r}}{V_{\text {air }}}=\frac{N_{\text {fibers }} E_{r}}{A_{c} \Delta z_{c}-A_{\text {soil }} \Delta z_{\text {soil }}\left(\mathbf{1}-\theta_{s}\right)}
$$

where $E_{r}$ is the efficiency of particle release (i.e., the fraction of the total number of fibers in the soil released to the air) and $\theta_{\mathrm{s}}$ is the saturated water content (porosity) of the soil. Substituting Eq. (2) into Eq. (3) we obtain

$$
C_{a s b, a i r}=\frac{E_{r} C_{a b s, \text { soil }} \rho_{b, \text { soil }} A_{\text {soil }} \Delta z_{\text {soil }}}{A_{c} \Delta z_{c}-A_{\text {soil }} \Delta z_{\text {soil }}\left(\mathbf{1}-\theta_{s}\right)}
$$

or after rearranging Eq. (4)

$$
C_{a s b, a i r}=\frac{E_{r} C_{a b s, s o i l} \rho_{b, s o i l}}{\left(\frac{A_{c}}{A_{\text {soil }}}\right)\left(\frac{\Delta z_{c}}{\Delta z_{\text {soil }}}\right)-\left(\mathbf{1}-\theta_{s}\right)}
$$

The $\rho_{\mathrm{b} \text {,soil }}$ and $\theta_{\mathrm{s}}$ are material properties of the soil that can either be measured or reasonably estimated. The dimensionless ratios $\left(A_{c} / A_{\text {soil }}\right),\left(\Delta z_{c} / \Delta z_{\text {soil }}\right)$ and $\mathrm{E}_{\mathrm{r}}$ are activity-based parameters that need to be developed from careful, detailed field measurements.

In this derivation, we have assumed that the airflow is sufficiently large relative to the confined air volume that essentially all of the particles have been removed and collected on the filter over the time period of interest. In an alternative approach, the air flow rate may be relatively small and not all of the particles are removed over the sampling interval. Assume there is complete mixing in the confined volume as air is added. Further, assume that there is no loss of particles except by the airflow out of the 
confined volume (i.e., particles are not adhering to the sides of the container or settling in the chamber). The change in the number of particles per unit time is

$$
\frac{d N_{a s b, a i r}}{d t}=-\left(\frac{Q}{V_{c}}\right) N_{a s b, a i r}
$$

where $\mathrm{N}_{\text {asb,air }}$ are the number of asbestos fibers in the air in the confined space, $\mathrm{Q}$ is the air flow in liters per unit time and $\mathrm{V}_{\mathrm{c}}$ is the volume of air in the confined space which is

$$
V_{c}=A_{c} \Delta z_{c}-A_{\text {soil }} \Delta z_{\text {soil }}\left(\mathbf{1}-\theta_{s}\right)
$$

At $\mathrm{t}=0$, the number of fibers in the air is $\mathrm{N}_{0, \text { air }}$. Equation (6) can be rearranged and integrated to yield

$$
\int_{N_{\mathbf{0}, a i r}}^{N_{a b s, a i r}} \frac{d N_{a b s, a i r}}{N_{a b s, a i r}}=-\int_{0}^{t} \frac{Q}{V_{c}} d t
$$

If $\mathrm{Q}$ is constant over the sampling interval, the number of fibers in the air within the confined space at time $t$ is

$$
N_{a b s, \text { air }}(t)=-N_{\mathbf{0}, \text { air }} e^{-\left(Q / V_{c}\right) t}=-E_{r} N_{\text {fibers }} e^{-\left(Q / V_{c}\right) t}
$$

If all of the fibers that leave the confined volume are captured by the filter, the number of fibers on the filter at time $\mathrm{t}, \mathrm{N}_{\text {filter }}(\mathrm{t})$ is

$$
N_{\text {filter }}(t)=N_{\mathbf{0}, \text { air }}-N_{\mathbf{0}, \text { air }} e^{-\left(Q / V_{c}\right) t}=N_{\mathbf{0}, \text { air }}\left(\mathbf{1}-e^{-\left(Q / V_{c}\right) t}\right)=E_{r} N_{\text {fibers }}\left(\mathbf{1}-e^{-\left(Q / V_{c}\right) t}\right)
$$

Eq. (10) can be solved for $\mathrm{N}_{0, \text { air }}$ :

$$
N_{\mathbf{0}, \text { air }}=\frac{N_{\text {filter }}}{\left(\mathbf{1}-e^{-\left(Q / V_{c}\right) t}\right)}
$$

Dividing both sides of Eq. (11) by $V_{c}$, we obtain the concentration of fibers at time $t=0$ :

$$
\frac{N_{\mathbf{0}, \text { air }}}{V_{c}}=C_{\mathbf{0}, \text { air }}=\frac{N_{\text {filter }}}{V_{c}\left(\mathbf{1}-e^{-\left(Q / V_{c}\right) t}\right)}
$$

Substituting Eqs. (1) and (7) into Eq. (12) and taking the limit as $t \rightarrow \infty$, one obtains Eq. (5). Similarly, by dividing Eq. (11) through by the mass of soil, $M_{\text {soil }}$, we can obtain the concentration of releasable fibers in the soil $\left(C_{a b s, r, s o i l}\right)$ or the total number of fibers in the soil $\left(C_{a b s, s o i l}\right)$ :

$$
\frac{N_{\mathbf{0}, \text { air }}}{M_{\text {soil }}}=C_{a b s, r, \text { soil }}=E_{r} C_{a b s, \text { soil }}=\frac{N_{\text {filter }}}{M_{\text {soil }}\left(\mathbf{1}-e^{-\left(Q / V_{c}\right) t}\right)}
$$

Substituting Eq. (1) into Eq. (13) and taking the limit as $t \rightarrow \infty$, one obtains an expression for $\mathrm{C}_{\text {asb,soil }}$ that can also be obtained from Eq.(2). Based on these equations, the expectation is that the asbestos concentrations in the air within the confined volume should decrease exponentially with time (Figure 7). 


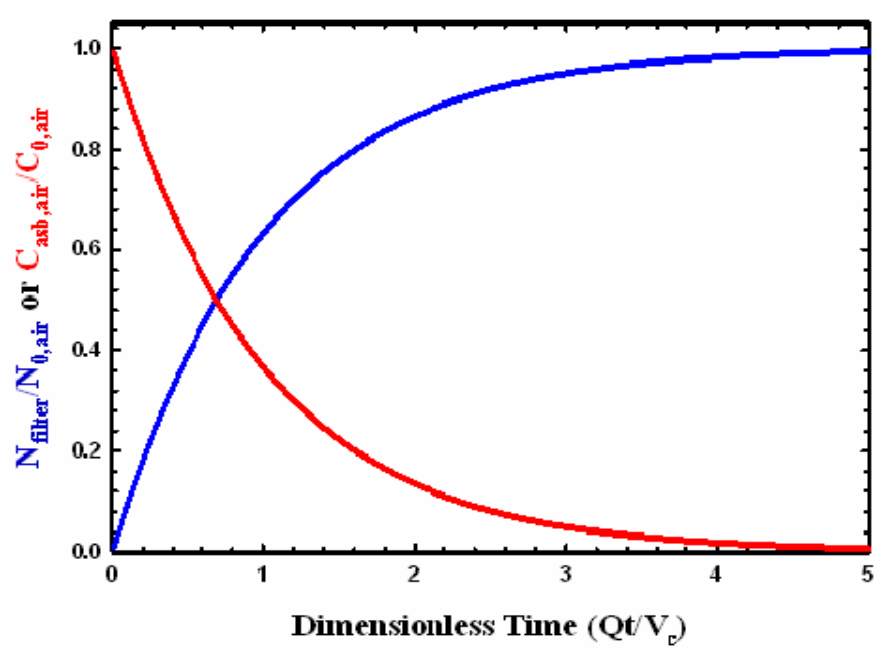

Figure 7. Relative concentration of fibers in the air in the confined volume (red line) and the relative number of fibers captured by the filters as a function of dimensionless time.

The cumulative number of asbestos fibers on the filters should increase in an exponential function of dimensionless time.

If a filter is placed in the system for a specific time interval, $\Delta t$, the number of fibers collected on that filter $\left(\mathrm{N}_{\text {filter }}(\mathrm{t}+\Delta \mathrm{t})-\mathrm{N}_{\text {filter }}(\mathrm{t})\right)$ can be obtained by application of Eq. (11). It follows that

$$
\begin{aligned}
N_{\text {filter }}(t+\Delta t)-N_{\text {filter }}(t) & =\left[N_{\mathbf{0} \text {.air }}-N_{\mathbf{0} \text {. air }} e^{-\left(Q / V_{c}\right)(t+\Delta t)}\right]-\left[N_{\mathbf{0} \text {.air }}-N_{\mathbf{0} \text {.air }} e^{-\left(Q / V_{c}\right) t}\right] \\
& =N_{\mathbf{0} \text {. } \text { air }} e^{-\left(Q / V_{c}\right) t}\left(\mathbf{1}-e^{-\left(Q / V_{c}\right) \Delta t}\right)
\end{aligned}
$$

Thus, for the same sample sampling interval, the number of fibers collected should decrease with time Figure 8.

It should be pointed out that the air flow rate, $\mathrm{Q}$, does not have to be constant. If the functional form of $\mathrm{Q}(\mathrm{t})$ is known, it can be substituted into (8) and integrated. One of the more common situations that will be encountered will be when Q is varied in a stepwise fashion. The integration can then be obtained in the same stepwise fashion to yield a series of recursive equations:

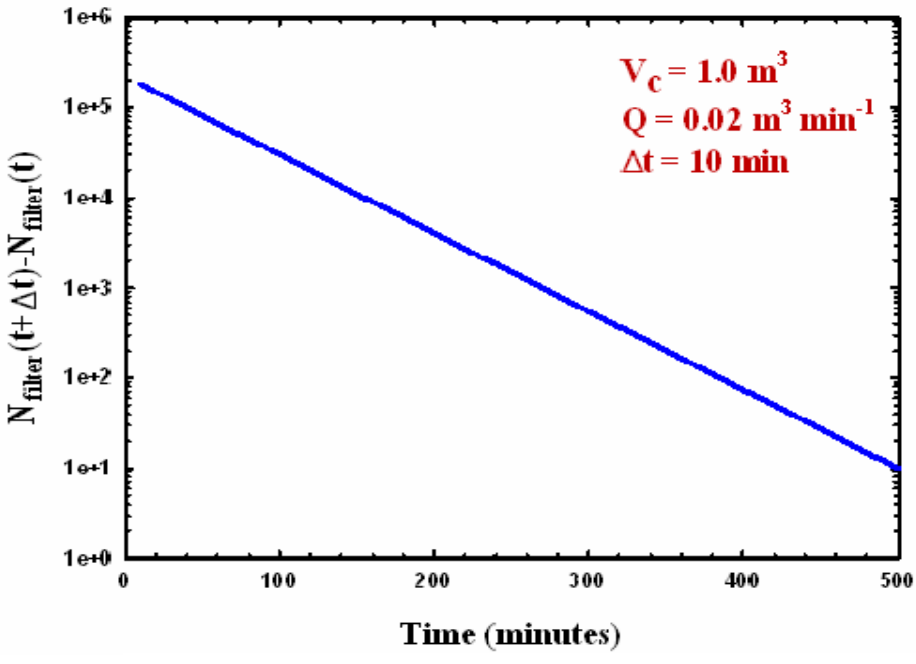

Figure 8 . Number of fibers captured by a filter over the time interval from $t$ to $t+\Delta t$ for $\Delta t=10$ minutes and for $t=0$ to 500 minutes 
Appendix E

TEM DATA for Fluidized Bed 
L Lab/Cor, Inc.

7619 6th Ave Nw Seattle, WA 98117
Analysis Report Cover Final Report
Phone: (206) 781-0155

Fax: (206) 789-8424

http://www.labcor.net
Job Number: 070434

Client: Idaho National Laboratory

Address: PO Box 1625 MS 2107

Idaho Falls, ID 83415-2107

Project Name: RARE

Project Num:

PO Number:

Sub Project:
Report Number: 070434R06

Report Date: 6/7/2007

Enclosed please find results for samples submitted to our laboratory. A list of samples and analyses follows:

\begin{tabular}{|c|c|c|c|}
\hline $\begin{array}{l}\text { Lab/Cor Sample \# } \\
070434 \text { - S1 }\end{array}$ & $\begin{array}{l}\text { Client Sample \# and Description } \\
\text { cleaning blank \#1 - }\end{array}$ & $\begin{array}{ll}\text { Analysis } & \text { Analysis Notes } \\
\text { ISO 10312, Direct } & \end{array}$ & $\begin{array}{l}\text { Date Received: } \\
4 / 23 / 2007\end{array}$ \\
\hline 070434 - S2 & cleaning blank \#2 - & ISO 10312, Direct & $4 / 23 / 2007$ \\
\hline $070434-\mathrm{S} 3$ & cleaning blank \#3 - & ISO 10312, Direct & $4 / 23 / 2007$ \\
\hline 070434 - S4 & cleaning blank \#4 - & ISO 10312, Direct & $4 / 23 / 2007$ \\
\hline 070434 - S5 & Lab blank \#1 - & ISO 10312, Direct & $4 / 23 / 2007$ \\
\hline 070434 - S6 & Lab blank \#2 - & ISO 10312, Direct & $4 / 23 / 2007$ \\
\hline 070434 - S7 & Lot blank \#1 - & ISO 10312, Direct & $4 / 23 / 2007$ \\
\hline 070434 - S8 & Lot blank \#2 - & ISO 10312, Direct & $4 / 23 / 2007$ \\
\hline 070434 - S9 & cleaning protocol 1 - & ISO 10312, Direct & $4 / 23 / 2007$ \\
\hline $070434-\mathrm{S} 10$ & cleaning protocol 2 - & ISO 10312, Direct & $4 / 23 / 2007$ \\
\hline $070434-\mathrm{S} 11$ & Sand blank - & ISO 10312, Direct & $4 / 23 / 2007$ \\
\hline $070434-\mathrm{S} 12$ & FB-4-R1 - & ISO 10312, Direct & $4 / 23 / 2007$ \\
\hline $070434-\mathrm{S} 13$ & FB-4-R2 - & ISO 10312, Direct & $4 / 23 / 2007$ \\
\hline $070434-\mathrm{S} 14$ & FB-4-R3 - & ISO 10312, Direct & $4 / 23 / 2007$ \\
\hline $070434-\mathrm{S} 15$ & FB-4-R4 - & ISO 10312, Direct & $4 / 23 / 2007$ \\
\hline $070434-\mathrm{S} 16$ & FB-4-R5 - & ISO 10312, Direct & $4 / 23 / 2007$ \\
\hline $070434-\mathrm{S} 17$ & FB-4-R6 - & ISO 10312, Direct & $4 / 23 / 2007$ \\
\hline 070434 - S18 & FB-4-R7 - & ISO 10312, Direct & $4 / 23 / 2007$ \\
\hline $070434-$ S19 & FB-3-R1 - & ISO 10312, Direct & $4 / 23 / 2007$ \\
\hline 070434 - S20 & FB-3-R2 - & ISO 10312, Direct & $4 / 23 / 2007$ \\
\hline $070434-\mathrm{S} 21$ & FB-3-R3 - & ISO 10312, Direct & $4 / 23 / 2007$ \\
\hline 070434 - S22 & FB-3-R4 - & ISO 10312, Direct & $4 / 23 / 2007$ \\
\hline 070434 - S23 & FB-3-R5 - & ISO 10312, Direct & $4 / 23 / 2007$ \\
\hline 070434 - S24 & FB-3-R6 - & ISO 10312, Direct & $4 / 23 / 2007$ \\
\hline 070434 - S25 & FB-3-R7 - & ISO 10312, Direct & 4/23/2007 \\
\hline $070434-$ S26 & FB-2-R1 - & ISO 10312, Direct & $4 / 23 / 2007$ \\
\hline 070434 - S27 & FB-2-R2 - & ISO 10312, Direct & $4 / 23 / 2007$ \\
\hline $070434-\mathrm{S} 28$ & FB-2-R3 - & ISO 10312, Direct & $4 / 23 / 2007$ \\
\hline 070434 - S29 & FB-2-R4 - & ISO 10312, Direct & $4 / 23 / 2007$ \\
\hline $070434-$ S30 & FB-2-R5 - & ISO 10312, Direct & $4 / 23 / 2007$ \\
\hline 070434 - S31 & FB-2-R6 - & ISO 10312, Direct & $4 / 23 / 2007$ \\
\hline $070434-$ S32 & FB-2-R7 - & ISO 10312, Direct & $4 / 23 / 2007$ \\
\hline 070434 - S33 & FB-1-R1 - & ISO 10312, Direct & $4 / 23 / 2007$ \\
\hline 070434 - S34 & FB-1-R2 - & ISO 10312, Direct & $4 / 23 / 2007$ \\
\hline 070434 - S35 & FB-1-R3 - & ISO 10312, Direct & $4 / 23 / 2007$ \\
\hline $070434-\mathrm{S} 36$ & FB-1-R4 - & ISO 10312, Direct & $4 / 23 / 2007$ \\
\hline 070434 - S37 & FB-1-R5 - & ISO 10312, Direct & $4 / 23 / 2007$ \\
\hline
\end{tabular}


Project Name: RARE

ISO 10312, Preparation and analysis of the above samples was conducted in accordance with the ISO method 10312 (Direct) for the

Direct - identification of asbestos. Briefly, the samples were collapsed with acetone, then etched in a low temperature plasma etcher to remove the top surface of the filter and other organics. The samples were carbon coated at high vacuum with a thin layer of carbon, placed on 200 mesh copper grids and allowed to dissolve in acetone until cleared of filter debris.

TEM analysis was performed using a transmission electron microscope equipped with an EDS $X$ ray analyzer. The air samples were analyzed at various approximate screen magnifications of 5,000x for PCM equivalent structures, 10,000x for asbestos structures greater than 5.0 micrometer lengths, and 20,000x for asbestos structures greater than 0.5 micrometer lengths. An accelerating voltage of $100 \mathrm{KV}$ was applied. The sizing of grid openings was performed on the microscope at a magnification of approximately 550X.

Disclaimer The results reported relate only to the samples tested or analyzed. Interpretation of these results is the sole responsibility of the client.

If further clarification of these results is needed, please call us. Thank you for allowing the staff at Lab/Cor, Inc. the opportunity to provide you with the analytical services.

Sincerely

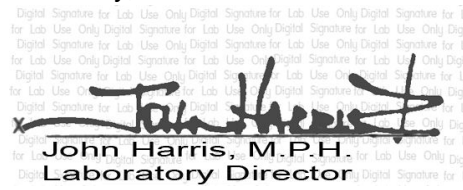


Job Number: 070434

\section{SEA}

\section{ISO 10312, Direct Summary Data}

Client: Idaho National Laboratory

Report Number: 070434R06

Date Received: 4/23/2007

Project Name: RARE

Lab/Cor Sample No.: S1

Client Sample No.: cleaning blank \#1

Description:

$\begin{array}{cc}\text { Analyst(s) } & \text { Analysis Date } \\ \text { KM } & 4 / 30 / 2007\end{array}$
Volume (L) : 0

Lab Filter Area (mm2) : 385

Grid Openings Analyzed : 35

Average Grid Opening Area : 0.009

Area Analyzed (mm2) : 0.315

Analytical Sens. (struc/cc) : NA

Dectection Limit. (struc/cc) : NA

\begin{tabular}{|c|r|r|r|c|}
\hline $\begin{array}{c}\text { Structure } \\
\text { Type }\end{array}$ & $\begin{array}{c}\text { Filter } \\
\text { Density } \\
(\mathbf{s} / \mathbf{m m} \text { 2) }\end{array}$ & $\begin{array}{c}\text { Concen- } \\
\text { tration* } \\
\text { (struc/cc) }\end{array}$ & $\begin{array}{c}\text { Structure } \\
\text { Count } \\
\text { Prim/Total }\end{array}$ \\
\hline Primary Asbestos Structures & 0 & Not Applicable & $\begin{array}{c}\text { 95\% Confidence } \\
\text { Interval } \\
\text { (struc/cc) }\end{array}$ \\
\hline Total Asbestos Structures & 0 & Not Applicable & Not Applicable & Not Applicable \\
\hline PCM Equivalent Fibers-ISO & 0 & Not Applicable & Not Applicable & Not Applicable \\
\hline PCM Equivalent Fibers-NIOSH & 0 & Not Applicable & Not Applicable & 0 \\
\hline PCM Equivalent Structures-ISO & 0 & Not Applicable & Not Applicable \\
\hline PCM Equivalent Structures-NIOSH & 0 & Not Applicable & Not Applicable & 0 \\
\hline Asbestos Structures >5um and 3:1 & 0 & Not Applicable & Not Applicable & 0 \\
\hline Asbestos Fibers and Bundles > 5um and 3:1 & 0 & Not Applicable & 0 & 0 \\
\hline
\end{tabular}

1 Concentration and $95 \%$ Confidence Level are calculated based upon the number showing under the Structure Count header.

Lab/Cor Sample No.: S2

Client Sample No.: cleaning blank \#2 Description:
Analyst(s)
Analysis Date
KM $4 / 30 / 2007$

Volume (L) : 0

Lab Filter Area (mm2) : 385

Grid Openings Analyzed : 35

Average Grid Opening Area : 0.009

Area Analyzed (mm2) : 0.315

Analytical Sens. (struc/cc) : NA

Dectection Limit. (struc/cc) : NA

\begin{tabular}{|c|c|c|c|c|c|}
\hline $\begin{array}{c}\text { Structure } \\
\text { Type }\end{array}$ & $\begin{array}{c}\text { Filter } \\
\text { Density } \\
(\mathbf{s} / \mathrm{mm} 2)\end{array}$ & $\begin{array}{l}\text { Concen- } \\
\text { tration* } \\
\text { (struc/cc) }\end{array}$ & $\begin{array}{l}95 \% \text { Confidence } \\
\text { Interval } \\
\text { (struc/cc) }\end{array}$ & \multicolumn{2}{|c|}{$\begin{array}{l}\text { Structure } \\
\text { Count }^{1} \\
\text { Prim/Total }\end{array}$} \\
\hline Primary Asbestos Structures & 0 & Not Applicable & Not Applicable & 0 & \\
\hline Total Asbestos Structures & 0 & Not Applicable & Not Applicable & & 0 \\
\hline PCM Equivalent Fibers-ISO & 0 & Not Applicable & Not Applicable & & 0 \\
\hline PCM Equivalent Fibers-NIOSH & 0 & Not Applicable & Not Applicable & & 0 \\
\hline PCM Equivalent Structures-ISO & 0 & Not Applicable & Not Applicable & 0 & \\
\hline PCM Equivalent Structures-NIOSH & 0 & Not Applicable & Not Applicable & 0 & \\
\hline Asbestos Structures >5um and 3:1 & 0 & Not Applicable & Not Applicable & 0 & \\
\hline Asbestos Fibers and Bundles > 5um and 3:1 & 0 & Not Applicable & Not Applicable & & 0 \\
\hline
\end{tabular}

\footnotetext{
${ }_{1}$ Concentration and $95 \%$ Confidence Level are calculated based upon the number showing under the Structure Count header.
} 
Job Number: 070434

\section{SEA}

\section{ISO 10312, Direct Summary Data}

Client: Idaho National Laboratory

Report Number: 070434R06

Date Received: 4/23/2007

Project Name: RARE

Lab/Cor Sample No.: S3

Client Sample No.: cleaning blank \#3

Description:

$\begin{array}{cc}\text { Analyst(s) } & \text { Analysis Date } \\ \text { KM } & 5 / 1 / 2007\end{array}$
Volume (L) : 0

Lab Filter Area (mm2) : 385

Grid Openings Analyzed : 35

Average Grid Opening Area : 0.009

Area Analyzed (mm2) : 0.315

Analytical Sens. (struc/cc) : NA

Dectection Limit. (struc/cc) : NA

\begin{tabular}{|c|r|r|r|c|}
\hline $\begin{array}{c}\text { Structure } \\
\text { Type }\end{array}$ & $\begin{array}{c}\text { Filter } \\
\text { Density } \\
\text { (s/mm2) }\end{array}$ & $\begin{array}{c}\text { Concen- } \\
\text { tration* } \\
\text { (struc/cc) }\end{array}$ & $\begin{array}{c}\text { Structure } \\
\text { Count } \\
\text { Prim/Total }\end{array}$ \\
\hline Primary Asbestos Structures & 3.2 & Not Applicable & $\begin{array}{c}\text { 95\% Confidence } \\
\text { Interval } \\
\text { (struc/cc) }\end{array}$ & Not Applicable \\
\hline Total Asbestos Structures & 3.2 & Not Applicable & Not Applicable & Not Applicable \\
\hline PCM Equivalent Fibers-ISO & 3.2 & Not Applicable & Not Applicable & 1 \\
\hline PCM Equivalent Fibers-NIOSH & 3.2 & Not Applicable & Not Applicable & Not Applicable \\
\hline PCM Equivalent Structures-ISO & 3.2 & Not Applicable & Not Applicable & 1 \\
\hline PCM Equivalent Structures-NIOSH & 3.2 & Not Applicable & Not Applicable & 1 \\
\hline Asbestos Structures >5um and 3:1 & 3.2 & Not Applicable & Not Applicable & 1 \\
\hline
\end{tabular}

${ }_{1}$ Concentration and $95 \%$ Confidence Level are calculated based upon the number showing under the Structure Count header.

Lab/Cor Sample No.: S4

Client Sample No.: cleaning blank \#4 Description:
Volume (L) : 0

Lab Filter Area (mm2) : 385

Grid Openings Analyzed : 35

Average Grid Opening Area : 0.009

Area Analyzed (mm2) : 0.315

Analytical Sens. (struc/cc) : NA

Dectection Limit. (struc/cc) : NA

\begin{tabular}{|c|c|c|c|c|c|}
\hline $\begin{array}{c}\text { Structure } \\
\text { Type }\end{array}$ & $\begin{array}{c}\text { Filter } \\
\text { Density } \\
(\mathbf{s} / \mathbf{m m 2})\end{array}$ & $\begin{array}{c}\text { Concen- } \\
\text { tration }^{\star} \\
(\text { struc/cc) }\end{array}$ & $\begin{array}{c}95 \% \text { Confidence } \\
\text { Interval } \\
\text { (struc/cc) }\end{array}$ & \multicolumn{2}{|c|}{$\begin{array}{l}\text { Structure } \\
\text { Count }^{1} \\
\text { Prim/Total }\end{array}$} \\
\hline Primary Asbestos Structures & 0 & Not Applicable & Not Applicable & 0 & \\
\hline Total Asbestos Structures & 0 & Not Applicable & Not Applicable & & 0 \\
\hline PCM Equivalent Fibers-ISO & 0 & Not Applicable & Not Applicable & & 0 \\
\hline PCM Equivalent Fibers-NIOSH & 0 & Not Applicable & Not Applicable & & 0 \\
\hline PCM Equivalent Structures-ISO & 0 & Not Applicable & Not Applicable & 0 & \\
\hline PCM Equivalent Structures-NIOSH & 0 & Not Applicable & Not Applicable & 0 & \\
\hline Asbestos Structures >5um and 3:1 & 0 & Not Applicable & Not Applicable & 0 & \\
\hline Asbestos Fibers and Bundles > 5um and 3:1 & 0 & Not Applicable & Not Applicable & & 0 \\
\hline
\end{tabular}

\footnotetext{
${ }_{1}$ Concentration and $95 \%$ Confidence Level are calculated based upon the number showing under the Structure Count header.
} 
Job Number: 070434

\section{SEA}

ISO 10312, Direct Summary Data

Client: Idaho National Laboratory

Report Number: 070434R06

Date Received: 4/23/2007

Project Name: RARE

Lab/Cor Sample No.: S5

Client Sample No.: Lab blank \#1

Description:

$\begin{array}{cc}\text { Analyst(s) } & \text { Analysis Date } \\ \text { KM } & 5 / 1 / 2007\end{array}$
Volume (L) : 0

Lab Filter Area (mm2) : 385

Grid Openings Analyzed : 35

Average Grid Opening Area : 0.009

Area Analyzed (mm2) : 0.315

Analytical Sens. (struc/cc) : NA

Dectection Limit. (struc/cc) : NA

\begin{tabular}{|c|r|r|r|c|}
\hline $\begin{array}{c}\text { Structure } \\
\text { Type }\end{array}$ & $\begin{array}{c}\text { Filter } \\
\text { Density } \\
(\mathbf{s} / \mathbf{m m} \text { 2) }\end{array}$ & $\begin{array}{c}\text { Concen- } \\
\text { tration* } \\
\text { (struc/cc) }\end{array}$ & $\begin{array}{c}\text { Structure } \\
\text { Count } \\
\text { Prim/Total }\end{array}$ \\
\hline Primary Asbestos Structures & 0 & Not Applicable & $\begin{array}{c}\text { 95\% Confidence } \\
\text { Interval } \\
\text { (struc/cc) }\end{array}$ \\
\hline Total Asbestos Structures & 0 & Not Applicable & Not Applicable & Not Applicable \\
\hline PCM Equivalent Fibers-ISO & 0 & Not Applicable & Not Applicable & Not Applicable \\
\hline PCM Equivalent Fibers-NIOSH & 0 & Not Applicable & Not Applicable & 0 \\
\hline PCM Equivalent Structures-ISO & 0 & Not Applicable & Not Applicable & 0 \\
\hline PCM Equivalent Structures-NIOSH & 0 & Not Applicable & Not Applicable & 0 \\
\hline Asbestos Structures >5um and 3:1 & 0 & Not Applicable & Not Applicable & 0 \\
\hline Asbestos Fibers and Bundles > 5um and 3:1 & 0 & Not Applicable & 0 \\
\hline
\end{tabular}

1 Concentration and $95 \%$ Confidence Level are calculated based upon the number showing under the Structure Count header.

Lab/Cor Sample No.: S6

Client Sample No.: Lab blank \#2 Description:
Volume (L) : 0

Lab Filter Area (mm2) : 385

Grid Openings Analyzed : 35

Average Grid Opening Area : 0.009

Area Analyzed (mm2) : 0.315

Analytical Sens. (struc/cc) : NA

Dectection Limit. (struc/cc) : NA

\begin{tabular}{|c|c|c|c|c|c|}
\hline $\begin{array}{c}\text { Structure } \\
\text { Type }\end{array}$ & $\begin{array}{c}\text { Filter } \\
\text { Density } \\
(\mathbf{s} / \mathbf{m m 2})\end{array}$ & $\begin{array}{c}\text { Concen- } \\
\text { tration }^{\star} \\
(\text { struc/cc) }\end{array}$ & $\begin{array}{c}95 \% \text { Confidence } \\
\text { Interval } \\
\text { (struc/cc) }\end{array}$ & \multicolumn{2}{|c|}{$\begin{array}{l}\text { Structure } \\
\text { Count }^{1} \\
\text { Prim/Total }\end{array}$} \\
\hline Primary Asbestos Structures & 0 & Not Applicable & Not Applicable & 0 & \\
\hline Total Asbestos Structures & 0 & Not Applicable & Not Applicable & & 0 \\
\hline PCM Equivalent Fibers-ISO & 0 & Not Applicable & Not Applicable & & 0 \\
\hline PCM Equivalent Fibers-NIOSH & 0 & Not Applicable & Not Applicable & & 0 \\
\hline PCM Equivalent Structures-ISO & 0 & Not Applicable & Not Applicable & 0 & \\
\hline PCM Equivalent Structures-NIOSH & 0 & Not Applicable & Not Applicable & 0 & \\
\hline Asbestos Structures >5um and 3:1 & 0 & Not Applicable & Not Applicable & 0 & \\
\hline Asbestos Fibers and Bundles > 5um and 3:1 & 0 & Not Applicable & Not Applicable & & 0 \\
\hline
\end{tabular}

\footnotetext{
${ }_{1}$ Concentration and $95 \%$ Confidence Level are calculated based upon the number showing under the Structure Count header.
} 
Job Number: 070434

\section{SEA}

ISO 10312, Direct Summary Data

Client: Idaho National Laboratory

Report Number: 070434R06

Date Received: 4/23/2007

Project Name: RARE

Lab/Cor Sample No.: S7

Client Sample No.: Lot blank \#1

Description:

$\begin{array}{cc}\text { Analyst(s) } & \text { Analysis Date } \\ \text { KM } & 5 / 1 / 2007\end{array}$
Volume (L) : 0

Lab Filter Area (mm2) : 385

Grid Openings Analyzed : 35

Average Grid Opening Area : 0.009

Area Analyzed (mm2) : 0.315

Analytical Sens. (struc/cc) : NA

Dectection Limit. (struc/cc) : NA

\begin{tabular}{|c|r|r|r|c|}
\hline $\begin{array}{c}\text { Structure } \\
\text { Type }\end{array}$ & $\begin{array}{c}\text { Filter } \\
\text { Density } \\
(\mathbf{s} / \mathbf{m m} \text { 2) }\end{array}$ & $\begin{array}{c}\text { Concen- } \\
\text { tration* } \\
\text { (struc/cc) }\end{array}$ & $\begin{array}{c}\text { Structure } \\
\text { Count } \\
\text { Prim/Total }\end{array}$ \\
\hline Primary Asbestos Structures & 0 & Not Applicable & $\begin{array}{c}\text { 95\% Confidence } \\
\text { Interval } \\
\text { (struc/cc) }\end{array}$ \\
\hline Total Asbestos Structures & 0 & Not Applicable & Not Applicable & Not Applicable \\
\hline PCM Equivalent Fibers-ISO & 0 & Not Applicable & Not Applicable & Not Applicable \\
\hline PCM Equivalent Fibers-NIOSH & 0 & Not Applicable & Not Applicable & 0 \\
\hline PCM Equivalent Structures-ISO & 0 & Not Applicable & Not Applicable & 0 \\
\hline PCM Equivalent Structures-NIOSH & 0 & Not Applicable & Not Applicable & 0 \\
\hline Asbestos Structures >5um and 3:1 & 0 & Not Applicable & Not Applicable & 0 \\
\hline Asbestos Fibers and Bundles > 5um and 3:1 & 0 & Not Applicable & 0 \\
\hline
\end{tabular}

1 Concentration and $95 \%$ Confidence Level are calculated based upon the number showing under the Structure Count header.

Lab/Cor Sample No.: S8

Client Sample No.: Lot blank \#2 Description:

Analyst(s) Analysis Date

KM 5/1/2007

Volume (L) : 0

Lab Filter Area (mm2) : 385

Grid Openings Analyzed : 35

Average Grid Opening Area : 0.009

Area Analyzed (mm2) : 0.315

Analytical Sens. (struc/cc) : NA

Dectection Limit. (struc/cc) : NA

\begin{tabular}{|c|c|c|c|c|c|}
\hline $\begin{array}{l}\text { Structure } \\
\text { Type }\end{array}$ & $\begin{array}{c}\text { Filter } \\
\text { Density } \\
(\mathbf{s} / \mathrm{mm} 2)\end{array}$ & $\begin{array}{l}\text { Concen- } \\
\text { tration* } \\
\text { (struc/cc) }\end{array}$ & $\begin{array}{c}\text { 95\% Confidence } \\
\text { Interval } \\
\text { (struc/cc) }\end{array}$ & \multicolumn{2}{|c|}{$\begin{array}{l}\text { Structure } \\
\text { Count }^{1} \\
\text { Prim/Total }\end{array}$} \\
\hline Primary Asbestos Structures & 0 & Not Applicable & Not Applicable & 0 & \\
\hline Total Asbestos Structures & 0 & Not Applicable & Not Applicable & & 0 \\
\hline PCM Equivalent Fibers-ISO & 0 & Not Applicable & Not Applicable & & 0 \\
\hline PCM Equivalent Fibers-NIOSH & 0 & Not Applicable & Not Applicable & & 0 \\
\hline PCM Equivalent Structures-ISO & 0 & Not Applicable & Not Applicable & 0 & \\
\hline PCM Equivalent Structures-NIOSH & 0 & Not Applicable & Not Applicable & 0 & \\
\hline Asbestos Structures >5um and 3:1 & 0 & Not Applicable & Not Applicable & 0 & \\
\hline Asbestos Fibers and Bundles > 5um and 3:1 & 0 & Not Applicable & Not Applicable & & 0 \\
\hline
\end{tabular}

\footnotetext{
1 Concentration and 95\% Confidence Level are calculated based upon the number showing under the Structure Count header.
} 
Job Number: 070434

\section{SEA}

\section{ISO 10312, Direct Summary Data}

Client: Idaho National Laboratory

Report Number: 070434R06

Date Received: 4/23/2007

Project Name: RARE

Lab/Cor Sample No.: S9

Client Sample No.: cleaning protocol 1

Description:

$\begin{array}{cc}\text { Analyst(s) } & \text { Analysis Date } \\ \text { KM } & 5 / 1 / 2007\end{array}$
Volume (L) : 0

Lab Filter Area (mm2) : 385

Grid Openings Analyzed : 35

Average Grid Opening Area : 0.009

Area Analyzed (mm2) : 0.315

Analytical Sens. (struc/cc) : NA

Dectection Limit. (struc/cc) : NA

\begin{tabular}{|c|r|r|r|c|}
\hline $\begin{array}{c}\text { Structure } \\
\text { Type }\end{array}$ & $\begin{array}{c}\text { Filter } \\
\text { Density } \\
(\mathbf{s} / \mathbf{m m} \text { 2) }\end{array}$ & $\begin{array}{c}\text { Concen- } \\
\text { tration* } \\
\text { (struc/cc) }\end{array}$ & $\begin{array}{c}\text { Structure } \\
\text { Count } \\
\text { Prim/Total }\end{array}$ \\
\hline Primary Asbestos Structures & 0 & Not Applicable & $\begin{array}{c}\text { 95\% Confidence } \\
\text { Interval } \\
\text { (struc/cc) }\end{array}$ \\
\hline Total Asbestos Structures & 0 & Not Applicable & Not Applicable & Not Applicable \\
\hline PCM Equivalent Fibers-ISO & 0 & Not Applicable & Not Applicable & Not Applicable \\
\hline PCM Equivalent Fibers-NIOSH & 0 & Not Applicable & Not Applicable & 0 \\
\hline PCM Equivalent Structures-ISO & 0 & Not Applicable & Not Applicable & 0 \\
\hline PCM Equivalent Structures-NIOSH & 0 & Not Applicable & Not Applicable & 0 \\
\hline Asbestos Structures >5um and 3:1 & 0 & Not Applicable & Not Applicable & 0 \\
\hline Asbestos Fibers and Bundles > 5um and 3:1 & 0 & Not Applicable & 0 \\
\hline
\end{tabular}

1 Concentration and $95 \%$ Confidence Level are calculated based upon the number showing under the Structure Count header.

Lab/Cor Sample No.: S10

Client Sample No.: cleaning protocol 2 Description:
Analyst(s)
Analysis Date

KM $5 / 1 / 2007$
Volume (L) : 0

Lab Filter Area (mm2) : 385

Grid Openings Analyzed : 35

Average Grid Opening Area : 0.009

Area Analyzed (mm2) : 0.315

Analytical Sens. (struc/cc) : NA

Dectection Limit. (struc/cc) : NA

\begin{tabular}{|c|c|c|c|c|c|}
\hline $\begin{array}{c}\text { Structure } \\
\text { Type }\end{array}$ & $\begin{array}{c}\text { Filter } \\
\text { Density } \\
(\mathbf{s} / \mathbf{m m 2})\end{array}$ & $\begin{array}{c}\text { Concen- } \\
\text { tration }^{\star} \\
(\text { struc/cc) }\end{array}$ & $\begin{array}{c}95 \% \text { Confidence } \\
\text { Interval } \\
\text { (struc/cc) }\end{array}$ & \multicolumn{2}{|c|}{$\begin{array}{c}\text { Structure } \\
\text { Count }{ }^{1} \\
\text { Prim/Total }\end{array}$} \\
\hline Primary Asbestos Structures & 6.3 & Not Applicable & Not Applicable & 2 & \\
\hline Total Asbestos Structures & 6.3 & Not Applicable & Not Applicable & & 2 \\
\hline PCM Equivalent Fibers-ISO & 6.3 & Not Applicable & Not Applicable & & 2 \\
\hline PCM Equivalent Fibers-NIOSH & 6.3 & Not Applicable & Not Applicable & & 2 \\
\hline PCM Equivalent Structures-ISO & 3.2 & Not Applicable & Not Applicable & 1 & \\
\hline PCM Equivalent Structures-NIOSH & 3.2 & Not Applicable & Not Applicable & 1 & \\
\hline Asbestos Structures >5um and 3:1 & 6.3 & Not Applicable & Not Applicable & 2 & \\
\hline Asbestos Fibers and Bundles > 5um and 3:1 & 6.3 & Not Applicable & Not Applicable & & 2 \\
\hline
\end{tabular}

1 Concentration and 95\% Confidence Level are calculated based upon the number showing under the Structure Count header.

* One-sided upper 95\% Poisson confidence limits may be used to calculate sample concentrations ([Struc count] *

[Analytical Sensitivity]) when the structure count is below 4 . The limits are: $0 \mathrm{str}-2.99,1 \mathrm{str}-4.74,2 \mathrm{str}-6.3,3 \mathrm{str}$ - 
Job Number: 070434

\section{SEA}

ISO 10312, Direct Summary Data

Client: Idaho National Laboratory

Report Number: 070434R06

Date Received: 4/23/2007

Project Name: RARE

Lab/Cor Sample No.: S11

Client Sample No.: Sand blank

Description:

$\begin{array}{cc}\text { Analyst(s) } & \text { Analysis Date } \\ \text { KM } & 5 / 1 / 2007\end{array}$
Volume (L) : 0

Lab Filter Area (mm2) : 385

Grid Openings Analyzed : 35

Average Grid Opening Area : 0.009

Area Analyzed (mm2) : 0.315

Analytical Sens. (struc/cc) : NA

Dectection Limit. (struc/cc) : NA

\begin{tabular}{|c|r|r|r|c|}
\hline $\begin{array}{c}\text { Structure } \\
\text { Type }\end{array}$ & $\begin{array}{c}\text { Filter } \\
\text { Density } \\
(\mathbf{s} / \mathbf{m m} \text { 2) }\end{array}$ & $\begin{array}{c}\text { Concen- } \\
\text { tration* } \\
\text { (struc/cc) }\end{array}$ & $\begin{array}{c}\text { Structure } \\
\text { Count } \\
\text { Prim/Total }\end{array}$ \\
\hline Primary Asbestos Structures & 0 & Not Applicable & $\begin{array}{c}\text { 95\% Confidence } \\
\text { Interval } \\
\text { (struc/cc) }\end{array}$ \\
\hline Total Asbestos Structures & 0 & Not Applicable & Not Applicable & Not Applicable \\
\hline PCM Equivalent Fibers-ISO & 0 & Not Applicable & Not Applicable & Not Applicable \\
\hline PCM Equivalent Fibers-NIOSH & 0 & Not Applicable & Not Applicable & 0 \\
\hline PCM Equivalent Structures-ISO & 0 & Not Applicable & Not Applicable & 0 \\
\hline PCM Equivalent Structures-NIOSH & 0 & Not Applicable & Not Applicable & 0 \\
\hline Asbestos Structures >5um and 3:1 & 0 & Not Applicable & Not Applicable & 0 \\
\hline Asbestos Fibers and Bundles > 5um and 3:1 & 0 & Not Applicable & 0 \\
\hline
\end{tabular}

1 Concentration and $95 \%$ Confidence Level are calculated based upon the number showing under the Structure Count header.

Lab/Cor Sample No.: S12

Client Sample No.: FB-4-R1 Description:

Analyst(s) Analysis Date

KM 5/7/2007

Volume (L) : 0

Lab Filter Area (mm2) : 385

Grid Openings Analyzed : 35

Average Grid Opening Area : 0.009

Area Analyzed (mm2) : 0.315

Analytical Sens. (struc/cc) : NA

Dectection Limit. (struc/cc) : NA

\begin{tabular}{|c|c|c|c|c|c|}
\hline $\begin{array}{c}\text { Structure } \\
\text { Type }\end{array}$ & $\begin{array}{l}\text { Filter } \\
\text { Density } \\
\text { (s/mm2) }\end{array}$ & $\begin{array}{l}\text { Concen- } \\
\text { tration }^{\star} \\
\text { (struc/cc) }\end{array}$ & $\begin{array}{l}95 \% \text { Confidence } \\
\text { Interval } \\
\text { (struc/cc) }\end{array}$ & \multicolumn{2}{|c|}{$\begin{array}{c}\text { Structure } \\
\text { Count } \\
\text { Prim/Total }\end{array}$} \\
\hline Primary Asbestos Structures & 54 & Not Applicable & Not Applicable & 17 & \\
\hline Total Asbestos Structures & 66.7 & Not Applicable & Not Applicable & & 21 \\
\hline PCM Equivalent Fibers-ISO & 15.9 & Not Applicable & Not Applicable & & 5 \\
\hline PCM Equivalent Fibers-NIOSH & 15.9 & Not Applicable & Not Applicable & & 5 \\
\hline PCM Equivalent Structures-ISO & 9.5 & Not Applicable & Not Applicable & 3 & \\
\hline PCM Equivalent Structures-NIOSH & 12.7 & Not Applicable & Not Applicable & 4 & \\
\hline Asbestos Structures >5um and 3:1 & 19 & Not Applicable & Not Applicable & 6 & \\
\hline Asbestos Fibers and Bundles > 5um and 3:1 & 19 & Not Applicable & Not Applicable & & 6 \\
\hline
\end{tabular}

\footnotetext{
1 Concentration and 95\% Confidence Level are calculated based upon the number showing under the Structure Count header.
} 
Job Number: 070434

\section{SEA}

ISO 10312, Direct Summary Data

Client: Idaho National Laboratory

Report Number: 070434R06

Date Received: 4/23/2007

Project Name: RARE

Lab/Cor Sample No.: S13

Client Sample No.: FB-4-R2

Description:

$\begin{array}{cc}\text { Analyst(s) } & \text { Analysis Date } \\ \text { KM } & 5 / 7 / 2007 \\ \text { KM } & 5 / 8 / 2007\end{array}$

Volume (L) : 0

Lab Filter Area (mm2) : 385

Grid Openings Analyzed : 35

Average Grid Opening Area : 0.009

Area Analyzed (mm2) : 0.315

Analytical Sens. (struc/cc) : NA

Dectection Limit. (struc/cc) : NA

\begin{tabular}{|c|c|c|c|c|c|}
\hline $\begin{array}{c}\text { Structure } \\
\text { Type }\end{array}$ & $\begin{array}{c}\text { Filter } \\
\text { Density } \\
(\mathrm{s} / \mathrm{mm} 2)\end{array}$ & $\begin{array}{c}\text { Concen- } \\
\text { tration* }^{*} \\
(\text { struc/cc) }\end{array}$ & $\begin{array}{c}\text { 95\% Confidence } \\
\text { Interval } \\
\text { (struc/cc) }\end{array}$ & \multicolumn{2}{|c|}{$\begin{array}{c}\text { Structure } \\
\text { Count }{ }^{1} \\
\text { Prim/Total }\end{array}$} \\
\hline Primary Asbestos Structures & 22.2 & Not Applicable & Not Applicable & 7 & \\
\hline Total Asbestos Structures & 22.2 & Not Applicable & Not Applicable & & 7 \\
\hline PCM Equivalent Fibers-ISO & 12.7 & Not Applicable & Not Applicable & & 4 \\
\hline PCM Equivalent Fibers-NIOSH & 12.7 & Not Applicable & Not Applicable & & $\overline{4}$ \\
\hline PCM Equivalent Structures-ISO & 12.7 & Not Applicable & Not Applicable & 4 & \\
\hline PCM Equivalent Structures-NIOSH & 12.7 & Not Applicable & Not Applicable & 4 & \\
\hline Asbestos Structures >5um and 3:1 & 9.5 & Not Applicable & Not Applicable & 3 & \\
\hline Asbestos Fibers and Bundles > 5um and 3:1 & 9.5 & Not Applicable & Not Applicable & & 3 \\
\hline
\end{tabular}

${ }_{1}$ Concentration and $95 \%$ Confidence Level are calculated based upon the number showing under the Structure Count header.

Lab/Cor Sample No.: S14

Client Sample No.: FB-4-R3 Description:

Analyst(s) Analysis Date KM 5/8/2007
Volume (L) : 0

Lab Filter Area (mm2) : 385

Grid Openings Analyzed : 35

Average Grid Opening Area : 0.009

Area Analyzed (mm2) : 0.315

Analytical Sens. (struc/cc) : NA

Dectection Limit. (struc/cc) : NA

\begin{tabular}{|c|c|c|c|c|c|}
\hline $\begin{array}{c}\text { Structure } \\
\text { Type }\end{array}$ & $\begin{array}{l}\text { Filter } \\
\text { Density } \\
\text { (s/mm2) }\end{array}$ & $\begin{array}{l}\text { Concen- } \\
\text { tration }^{\star} \\
\text { (struc/cc) }\end{array}$ & $\begin{array}{l}95 \% \text { Confidence } \\
\text { Interval } \\
\text { (struc/cc) }\end{array}$ & \multicolumn{2}{|c|}{$\begin{array}{c}\text { Structure } \\
\text { Count } \\
\text { Prim/Total }\end{array}$} \\
\hline Primary Asbestos Structures & 34.9 & Not Applicable & Not Applicable & 11 & \\
\hline Total Asbestos Structures & 34.9 & Not Applicable & Not Applicable & & 11 \\
\hline PCM Equivalent Fibers-ISO & 15.9 & Not Applicable & Not Applicable & & 5 \\
\hline PCM Equivalent Fibers-NIOSH & 15.9 & Not Applicable & Not Applicable & & 5 \\
\hline PCM Equivalent Structures-ISO & 9.5 & Not Applicable & Not Applicable & 3 & \\
\hline PCM Equivalent Structures-NIOSH & 9.5 & Not Applicable & Not Applicable & 3 & \\
\hline Asbestos Structures >5um and 3:1 & 15.9 & Not Applicable & Not Applicable & 5 & \\
\hline Asbestos Fibers and Bundles > 5um and 3:1 & 12.7 & Not Applicable & Not Applicable & & $\overline{4}$ \\
\hline
\end{tabular}

\footnotetext{
1 Concentration and 95\% Confidence Level are calculated based upon the number showing under the Structure Count header.
} 
Job Number: 070434

\section{SEA}

ISO 10312, Direct Summary Data

Client: Idaho National Laboratory

Report Number: 070434R06

Date Received: 4/23/2007

Project Name: RARE

Lab/Cor Sample No.: S15

Client Sample No.: FB-4-R4

Description:

$\begin{array}{cc}\text { Analyst(s) } & \text { Analysis Date } \\ \mathrm{KM} & 5 / 9 / 2007\end{array}$
Volume (L) : 0

Lab Filter Area (mm2) : 385

Grid Openings Analyzed : 35

Average Grid Opening Area : 0.009

Area Analyzed (mm2) : 0.315

Analytical Sens. (struc/cc) : NA

Dectection Limit. (struc/cc) : NA

\begin{tabular}{|c|r|r|r|r|}
\hline $\begin{array}{c}\text { Structure } \\
\text { Type }\end{array}$ & $\begin{array}{c}\text { Filter } \\
\text { Density } \\
\text { (s/mm2) }\end{array}$ & $\begin{array}{c}\text { Concen- } \\
\text { tration* } \\
\text { (struc/cc) }\end{array}$ & $\begin{array}{c}\text { Structure } \\
\text { Count } \\
\text { Prim/Total }\end{array}$ \\
\hline Primary Asbestos Structures & 12.7 & Not Applicable & $\begin{array}{c}\text { 95\% Confidence } \\
\text { Interval } \\
\text { (struc/cc) }\end{array}$ & Not Applicable \\
\hline Total Asbestos Structures & 12.7 & Not Applicable & Not Applicable & Not Applicable \\
\hline PCM Equivalent Fibers-ISO & 3.2 & Not Applicable & Not Applicable & Not Applicable \\
\hline PCM Equivalent Fibers-NIOSH & 3.2 & Not Applicable & Not Applicable & 1 \\
\hline PCM Equivalent Structures-ISO & 3.2 & Not Applicable & Not Applicable & 1 \\
\hline PCM Equivalent Structures-NIOSH & 3.2 & Not Applicable & Not Applicable & 1 \\
\hline Asbestos Structures >5um and 3:1 & 6.3 & Not Applicable & Not Applicable & 2 \\
\hline
\end{tabular}

${ }_{1}$ Concentration and $95 \%$ Confidence Level are calculated based upon the number showing under the Structure Count header.

Lab/Cor Sample No.: S16

Client Sample No.: FB-4-R5 Description:

Analyst(s) Analysis Date

KM 5/9/2007

Volume (L) : 0

Lab Filter Area (mm2) : 385

Grid Openings Analyzed : 35

Average Grid Opening Area : 0.009

Area Analyzed (mm2) : 0.315

Analytical Sens. (struc/cc) : NA

Dectection Limit. (struc/cc) : NA

\begin{tabular}{|c|c|c|c|c|c|}
\hline $\begin{array}{c}\text { Structure } \\
\text { Type }\end{array}$ & $\begin{array}{c}\text { Filter } \\
\text { Density } \\
(\mathbf{s} / \mathbf{m m 2})\end{array}$ & $\begin{array}{c}\text { Concen- } \\
\text { tration }^{\star} \\
(\text { struc/cc) }\end{array}$ & $\begin{array}{c}95 \% \text { Confidence } \\
\text { Interval } \\
\text { (struc/cc) }\end{array}$ & \multicolumn{2}{|c|}{$\begin{array}{c}\text { Structure } \\
\text { Count }^{1} \\
\text { Prim/Total }\end{array}$} \\
\hline Primary Asbestos Structures & 15.9 & Not Applicable & Not Applicable & 5 & \\
\hline Total Asbestos Structures & 15.9 & Not Applicable & Not Applicable & & 5 \\
\hline PCM Equivalent Fibers-ISO & 9.5 & Not Applicable & Not Applicable & & 3 \\
\hline PCM Equivalent Fibers-NIOSH & 9.5 & Not Applicable & Not Applicable & & 3 \\
\hline PCM Equivalent Structures-ISO & 6.3 & Not Applicable & Not Applicable & 2 & \\
\hline PCM Equivalent Structures-NIOSH & 6.3 & Not Applicable & Not Applicable & 2 & \\
\hline Asbestos Structures >5um and 3:1 & 12.7 & Not Applicable & Not Applicable & 4 & \\
\hline Asbestos Fibers and Bundles > 5um and 3:1 & 9.5 & Not Applicable & Not Applicable & & 3 \\
\hline
\end{tabular}

\footnotetext{
1 Concentration and $95 \%$ Confidence Level are calculated based upon the number showing under the Structure Count header.
} 
Job Number: 070434

\section{SEA}

ISO 10312, Direct Summary Data

Client: Idaho National Laboratory

Report Number: 070434R06

Date Received: 4/23/2007

Project Name: RARE

Lab/Cor Sample No.: S17

Client Sample No.: FB-4-R6

Description:

$\begin{array}{cc}\text { Analyst(s) } & \text { Analysis Date } \\ \text { KM } & 5 / 10 / 2007 \\ \text { KM } & 5 / 11 / 2007\end{array}$

Volume (L) : 0

Lab Filter Area (mm2) : 385

Grid Openings Analyzed : 35

Average Grid Opening Area : 0.009

Area Analyzed (mm2) : 0.315

Analytical Sens. (struc/cc) : NA

Dectection Limit. (struc/cc) : NA

\begin{tabular}{|c|c|c|c|c|c|}
\hline $\begin{array}{c}\text { Structure } \\
\text { Type }\end{array}$ & $\begin{array}{l}\text { Filter } \\
\text { Density } \\
\text { (s/mm2) }\end{array}$ & $\begin{array}{l}\text { Concen- } \\
\text { tration* } \\
\text { (struc/cc) }\end{array}$ & $\begin{array}{l}95 \% \text { Confidence } \\
\text { Interval } \\
\text { (struc/cc) }\end{array}$ & \multicolumn{2}{|c|}{$\begin{array}{c}\text { Structure } \\
\text { Count }^{1} \\
\text { Prim/Total }\end{array}$} \\
\hline Primary Asbestos Structures & 31.7 & Not Applicable & Not Applicable & 10 & \\
\hline Total Asbestos Structures & 31.7 & Not Applicable & Not Applicable & & 10 \\
\hline PCM Equivalent Fibers-ISO & 6.3 & Not Applicable & Not Applicable & & 2 \\
\hline PCM Equivalent Fibers-NIOSH & 6.3 & Not Applicable & Not Applicable & & 2 \\
\hline PCM Equivalent Structures-ISO & 3.2 & Not Applicable & Not Applicable & 1 & \\
\hline PCM Equivalent Structures-NIOSH & 3.2 & Not Applicable & Not Applicable & 1 & \\
\hline Asbestos Structures >5um and 3:1 & 9.5 & Not Applicable & Not Applicable & 3 & \\
\hline Asbestos Fibers and Bundles > 5um and 3:1 & 6.3 & Not Applicable & Not Applicable & & 2 \\
\hline
\end{tabular}

1 Concentration and $95 \%$ Confidence Level are calculated based upon the number showing under the Structure Count header.

Lab/Cor Sample No.: S18

Client Sample No.: FB-4-R7 Description:

Analyst(s) Analysis Date

KM 5/11/2007

Volume (L) : 0

Lab Filter Area (mm2) : 385

Grid Openings Analyzed : 35

Average Grid Opening Area : 0.009

Area Analyzed (mm2) : 0.315

Analytical Sens. (struc/cc) : NA

Dectection Limit. (struc/cc) : NA

\begin{tabular}{|c|c|c|c|c|c|}
\hline $\begin{array}{c}\text { Structure } \\
\text { Type }\end{array}$ & $\begin{array}{c}\text { Filter } \\
\text { Density } \\
(\mathrm{s} / \mathrm{mm} 2)\end{array}$ & $\begin{array}{l}\text { Concen- } \\
\text { tration }^{\star} \\
\text { (struc/cc) }\end{array}$ & $\begin{array}{l}95 \% \text { Confidence } \\
\text { Interval } \\
\text { (struc/cc) }\end{array}$ & \multicolumn{2}{|c|}{$\begin{array}{c}\text { Structure } \\
\text { Count }^{1} \\
\text { Prim/Total }\end{array}$} \\
\hline Primary Asbestos Structures & 9.5 & Not Applicable & Not Applicable & 3 & \\
\hline Total Asbestos Structures & 9.5 & Not Applicable & Not Applicable & & 3 \\
\hline PCM Equivalent Fibers-ISO & 0 & Not Applicable & Not Applicable & & 0 \\
\hline PCM Equivalent Fibers-NIOSH & 0 & Not Applicable & Not Applicable & & 0 \\
\hline PCM Equivalent Structures-ISO & 0 & Not Applicable & Not Applicable & 0 & \\
\hline PCM Equivalent Structures-NIOSH & 0 & Not Applicable & Not Applicable & 0 & \\
\hline Asbestos Structures >5um and 3:1 & 3.2 & Not Applicable & Not Applicable & 1 & \\
\hline Asbestos Fibers and Bundles $>5$ um and 3:1 & 0 & Not Applicable & Not Applicable & & 0 \\
\hline
\end{tabular}

\footnotetext{
1 Concentration and 95\% Confidence Level are calculated based upon the number showing under the Structure Count header.
} 
Job Number: 070434

\section{SEA}

ISO 10312, Direct Summary Data

Client: Idaho National Laboratory

Report Number: 070434R06

Date Received: 4/23/2007

Project Name: RARE

Lab/Cor Sample No.: S19

Client Sample No.: FB-3-R1

Description:

$\begin{array}{cc}\text { Analyst(s) } & \text { Analysis Date } \\ \text { KM } & 5 / 16 / 2007 \\ \text { KM } & 5 / 17 / 2007\end{array}$

Volume (L) : 0

Lab Filter Area (mm2) : 385

Grid Openings Analyzed : 35

Average Grid Opening Area : 0.009

Area Analyzed (mm2) : 0.315

Analytical Sens. (struc/cc) : NA

Dectection Limit. (struc/cc) : NA

\begin{tabular}{|c|c|c|c|c|c|}
\hline $\begin{array}{c}\text { Structure } \\
\text { Type }\end{array}$ & $\begin{array}{c}\text { Filter } \\
\text { Density } \\
(\mathrm{s} / \mathrm{mm} 2)\end{array}$ & $\begin{array}{c}\text { Concen- } \\
\text { tration* }^{*} \\
(\text { struc/cc) }\end{array}$ & $\begin{array}{c}\text { 95\% Confidence } \\
\text { Interval } \\
\text { (struc/cc) }\end{array}$ & \multicolumn{2}{|c|}{$\begin{array}{c}\text { Structure } \\
\text { Count }{ }^{1} \\
\text { Prim/Total }\end{array}$} \\
\hline Primary Asbestos Structures & 0 & Not Applicable & Not Applicable & 0 & \\
\hline Total Asbestos Structures & 0 & Not Applicable & Not Applicable & & 0 \\
\hline PCM Equivalent Fibers-ISO & 0 & Not Applicable & Not Applicable & & 0 \\
\hline PCM Equivalent Fibers-NIOSH & 0 & Not Applicable & Not Applicable & & 0 \\
\hline PCM Equivalent Structures-ISO & 0 & Not Applicable & Not Applicable & 0 & \\
\hline PCM Equivalent Structures-NIOSH & 0 & Not Applicable & Not Applicable & 0 & \\
\hline Asbestos Structures >5um and 3:1 & 0 & Not Applicable & Not Applicable & 0 & \\
\hline Asbestos Fibers and Bundles $>5 u m$ and $3: 1$ & 0 & Not Applicable & Not Applicable & & 0 \\
\hline
\end{tabular}

1 Concentration and $95 \%$ Confidence Level are calculated based upon the number showing under the Structure Count header.

Lab/Cor Sample No.: S20

Client Sample No.: FB-3-R2 Description:

Analyst(s) Analysis Date

KM 5/17/2007

Volume (L) : 0

Lab Filter Area (mm2) : 385

Grid Openings Analyzed : 35

Average Grid Opening Area : 0.009

Area Analyzed (mm2) : 0.315

Analytical Sens. (struc/cc) : NA

Dectection Limit. (struc/cc) : NA

\begin{tabular}{|c|c|c|c|c|c|}
\hline $\begin{array}{c}\text { Structure } \\
\text { Type }\end{array}$ & $\begin{array}{l}\text { Filter } \\
\text { Density } \\
\text { (s/mm2) }\end{array}$ & $\begin{array}{l}\text { Concen- } \\
\text { tration }^{\star} \\
\text { (struc/cc) }\end{array}$ & $\begin{array}{l}95 \% \text { Confidence } \\
\text { Interval } \\
\text { (struc/cc) }\end{array}$ & \multicolumn{2}{|c|}{$\begin{array}{c}\text { Structure } \\
\text { Count } \\
\text { Prim/Total }\end{array}$} \\
\hline Primary Asbestos Structures & 168.3 & Not Applicable & Not Applicable & 53 & \\
\hline Total Asbestos Structures & 177.8 & Not Applicable & Not Applicable & & 56 \\
\hline PCM Equivalent Fibers-ISO & 85.7 & Not Applicable & Not Applicable & & 27 \\
\hline PCM Equivalent Fibers-NIOSH & 85.7 & Not Applicable & Not Applicable & & 27 \\
\hline PCM Equivalent Structures-ISO & 66.7 & Not Applicable & Not Applicable & 21 & \\
\hline PCM Equivalent Structures-NIOSH & 66.7 & Not Applicable & Not Applicable & 21 & \\
\hline Asbestos Structures >5um and 3:1 & 76.2 & Not Applicable & Not Applicable & 24 & \\
\hline Asbestos Fibers and Bundles > 5um and 3:1 & 69.8 & Not Applicable & Not Applicable & & 22 \\
\hline
\end{tabular}

\footnotetext{
1 Concentration and $95 \%$ Confidence Level are calculated based upon the number showing under the Structure Count header.
} 
Job Number: 070434

\section{SEA}

ISO 10312, Direct Summary Data

Client: Idaho National Laboratory

Report Number: 070434R06

Date Received: 4/23/2007

Project Name: RARE

Lab/Cor Sample No.: S21

Client Sample No.: FB-3-R3

Description:

$\begin{array}{cc}\text { Analyst(s) } & \text { Analysis Date } \\ \mathrm{KM} & 5 / 21 / 2007\end{array}$
Volume (L) : 0

Lab Filter Area (mm2) : 385

Grid Openings Analyzed : 35

Average Grid Opening Area : 0.009

Area Analyzed (mm2) : 0.315

Analytical Sens. (struc/cc) : NA

Dectection Limit. (struc/cc) : NA

\begin{tabular}{|c|r|r|r|c|}
\hline $\begin{array}{c}\text { Structure } \\
\text { Type }\end{array}$ & $\begin{array}{c}\text { Filter } \\
\text { Density } \\
(\mathbf{s} / \mathbf{m m} \text { 2) }\end{array}$ & $\begin{array}{c}\text { Concen- } \\
\text { tration* } \\
\text { (struc/cc) }\end{array}$ & $\begin{array}{c}\text { Structure } \\
\text { Count } \\
\text { Prim/Total }\end{array}$ \\
\hline Primary Asbestos Structures & 0 & Not Applicable & $\begin{array}{c}\text { 95\% Confidence } \\
\text { Interval } \\
\text { (struc/cc) }\end{array}$ \\
\hline Total Asbestos Structures & 0 & Not Applicable & Not Applicable & Not Applicable \\
\hline PCM Equivalent Fibers-ISO & 0 & Not Applicable & Not Applicable & Not Applicable \\
\hline PCM Equivalent Fibers-NIOSH & 0 & Not Applicable & Not Applicable & 0 \\
\hline PCM Equivalent Structures-ISO & 0 & Not Applicable & Not Applicable & 0 \\
\hline PCM Equivalent Structures-NIOSH & 0 & Not Applicable & Not Applicable & 0 \\
\hline Asbestos Structures >5um and 3:1 & 0 & Not Applicable & Not Applicable & 0 \\
\hline Asbestos Fibers and Bundles > 5um and 3:1 & 0 & Not Applicable & 0 \\
\hline
\end{tabular}

1 Concentration and $95 \%$ Confidence Level are calculated based upon the number showing under the Structure Count header.

Lab/Cor Sample No.: S22

Client Sample No.: FB-3-R4

Description:

$\begin{array}{cc}\text { Analyst(s) } & \text { Analysis Date } \\ \text { KM } & 5 / 21 / 2007 \\ \text { KM } & 5 / 22 / 2007\end{array}$

Volume (L) : 0

Lab Filter Area (mm2) : 385

Grid Openings Analyzed : 35

Average Grid Opening Area : 0.009

Area Analyzed (mm2) : 0.315

Analytical Sens. (struc/cc) : NA

Dectection Limit. (struc/cc) : NA

\begin{tabular}{|c|c|c|c|c|c|}
\hline $\begin{array}{c}\text { Structure } \\
\text { Type }\end{array}$ & $\begin{array}{c}\text { Filter } \\
\text { Density } \\
(\mathbf{s} / \mathbf{m m 2})\end{array}$ & $\begin{array}{c}\text { Concen- } \\
\text { tration }^{\star} \\
(\text { struc/cc) }\end{array}$ & $\begin{array}{c}95 \% \text { Confidence } \\
\text { Interval } \\
\text { (struc/cc) }\end{array}$ & \multicolumn{2}{|c|}{$\begin{array}{l}\text { Structure } \\
\text { Count }^{1} \\
\text { Prim/Total }\end{array}$} \\
\hline Primary Asbestos Structures & 0 & Not Applicable & Not Applicable & 0 & \\
\hline Total Asbestos Structures & 0 & Not Applicable & Not Applicable & & 0 \\
\hline PCM Equivalent Fibers-ISO & 0 & Not Applicable & Not Applicable & & 0 \\
\hline PCM Equivalent Fibers-NIOSH & 0 & Not Applicable & Not Applicable & & 0 \\
\hline PCM Equivalent Structures-ISO & 0 & Not Applicable & Not Applicable & 0 & \\
\hline PCM Equivalent Structures-NIOSH & 0 & Not Applicable & Not Applicable & 0 & \\
\hline Asbestos Structures >5um and 3:1 & 0 & Not Applicable & Not Applicable & 0 & \\
\hline Asbestos Fibers and Bundles > 5um and 3:1 & 0 & Not Applicable & Not Applicable & & 0 \\
\hline
\end{tabular}

1 Concentration and 95\% Confidence Level are calculated based upon the number showing under the Structure Count header.

* One-sided upper 95\% Poisson confidence limits may be used to calculate sample concentrations ([Struc count] *

[Analytical Sensitivity]) when the structure count is below 4. The limits are: $0 \mathrm{str}-2.99,1 \mathrm{str}-4.74,2 \mathrm{str}-6.3,3 \mathrm{str}$ - 
Job Number: 070434

\section{SEA}

ISO 10312, Direct Summary Data

Client: Idaho National Laboratory

Report Number: 070434R06

Date Received: 4/23/2007

Project Name: RARE

Lab/Cor Sample No.: S23

Client Sample No.: FB-3-R5

Description:

$\begin{array}{cc}\text { Analyst(s) } & \text { Analysis Date } \\ \mathrm{KM} & 5 / 22 / 2007\end{array}$
Volume (L) : 0

Lab Filter Area (mm2) : 385

Grid Openings Analyzed : 35

Average Grid Opening Area : 0.009

Area Analyzed (mm2) : 0.315

Analytical Sens. (struc/cc) : NA

Dectection Limit. (struc/cc) : NA

\begin{tabular}{|c|c|c|c|c|c|}
\hline $\begin{array}{c}\text { Structure } \\
\text { Type }\end{array}$ & $\begin{array}{c}\text { Filter } \\
\text { Density } \\
(\mathrm{s} / \mathrm{mm} 2)\end{array}$ & $\begin{array}{l}\text { Concen- } \\
\text { tration* } \\
\text { (struc/cc) }\end{array}$ & $\begin{array}{c}95 \% \text { Confidence } \\
\text { Interval } \\
\text { (struc/cc) }\end{array}$ & \multicolumn{2}{|c|}{$\begin{array}{l}\text { Structure } \\
\text { Count }^{1} \\
\text { Prim/Total }\end{array}$} \\
\hline Primary Asbestos Structures & 76.2 & Not Applicable & Not Applicable & 24 & \\
\hline Total Asbestos Structures & 76.2 & Not Applicable & Not Applicable & & 24 \\
\hline PCM Equivalent Fibers-ISO & 50.8 & Not Applicable & Not Applicable & & 16 \\
\hline PCM Equivalent Fibers-NIOSH & 54 & Not Applicable & Not Applicable & & 17 \\
\hline PCM Equivalent Structures-ISO & 44.4 & Not Applicable & Not Applicable & 14 & \\
\hline PCM Equivalent Structures-NIOSH & 50.8 & Not Applicable & Not Applicable & 16 & \\
\hline Asbestos Structures >5um and 3:1 & 50.8 & Not Applicable & Not Applicable & 16 & \\
\hline Asbestos Fibers and Bundles $>5 u m$ and $3: 1$ & 44.4 & Not Applicable & Not Applicable & & 14 \\
\hline
\end{tabular}

1 Concentration and $95 \%$ Confidence Level are calculated based upon the number showing under the Structure Count header.

Lab/Cor Sample No.: S24

Client Sample No.: FB-3-R6 Description:

Analyst(s) Analysis Date KM 5/24/2007
Volume (L) : 0

Lab Filter Area (mm2) : 385

Grid Openings Analyzed : 35

Average Grid Opening Area : 0.009

Area Analyzed (mm2) : 0.315

Analytical Sens. (struc/cc) : NA

Dectection Limit. (struc/cc) : NA

\begin{tabular}{|c|c|c|c|c|c|}
\hline $\begin{array}{c}\text { Structure } \\
\text { Type }\end{array}$ & $\begin{array}{c}\text { Filter } \\
\text { Density } \\
(\mathbf{s} / \mathbf{m m 2})\end{array}$ & $\begin{array}{c}\text { Concen- } \\
\text { tration }^{\star} \\
(\text { struc/cc) }\end{array}$ & $\begin{array}{c}95 \% \text { Confidence } \\
\text { Interval } \\
\text { (struc/cc) }\end{array}$ & \multicolumn{2}{|c|}{$\begin{array}{l}\text { Structure } \\
\text { Count }^{1} \\
\text { Prim/Total }\end{array}$} \\
\hline Primary Asbestos Structures & 0 & Not Applicable & Not Applicable & 0 & \\
\hline Total Asbestos Structures & 0 & Not Applicable & Not Applicable & & 0 \\
\hline PCM Equivalent Fibers-ISO & 0 & Not Applicable & Not Applicable & & 0 \\
\hline PCM Equivalent Fibers-NIOSH & 0 & Not Applicable & Not Applicable & & 0 \\
\hline PCM Equivalent Structures-ISO & 0 & Not Applicable & Not Applicable & 0 & \\
\hline PCM Equivalent Structures-NIOSH & 0 & Not Applicable & Not Applicable & 0 & \\
\hline Asbestos Structures >5um and 3:1 & 0 & Not Applicable & Not Applicable & 0 & \\
\hline Asbestos Fibers and Bundles > 5um and 3:1 & 0 & Not Applicable & Not Applicable & & 0 \\
\hline
\end{tabular}

\footnotetext{
1 Concentration and 95\% Confidence Level are calculated based upon the number showing under the Structure Count header.
} 
Job Number: 070434

\section{SEA}

ISO 10312, Direct Summary Data

Client: Idaho National Laboratory

Report Number: 070434R06

Date Received: 4/23/2007

Project Name: RARE

Lab/Cor Sample No.: S25

Client Sample No.: FB-3-R7

Description:

$\begin{array}{cc}\text { Analyst(s) } & \text { Analysis Date } \\ \text { KM } & 5 / 24 / 2007 \\ \text { KM } & 5 / 25 / 2007\end{array}$

Volume (L) : 0

Lab Filter Area (mm2) : 385

Grid Openings Analyzed : 35

Average Grid Opening Area : 0.009

Area Analyzed (mm2) : 0.315

Analytical Sens. (struc/cc) : NA

Dectection Limit. (struc/cc) : NA

\begin{tabular}{|c|c|c|c|c|c|}
\hline $\begin{array}{c}\text { Structure } \\
\text { Type }\end{array}$ & $\begin{array}{l}\text { Filter } \\
\text { Density } \\
\text { (s/mm2) }\end{array}$ & $\begin{array}{l}\text { Concen- } \\
\text { tration* } \\
\text { (struc/cc) }\end{array}$ & $\begin{array}{l}95 \% \text { Confidence } \\
\text { Interval } \\
\text { (struc/cc) }\end{array}$ & \multicolumn{2}{|c|}{$\begin{array}{c}\text { Structure } \\
\text { Count }^{1} \\
\text { Prim/Total }\end{array}$} \\
\hline Primary Asbestos Structures & 60.3 & Not Applicable & Not Applicable & 19 & \\
\hline Total Asbestos Structures & 63.5 & Not Applicable & Not Applicable & & 20 \\
\hline PCM Equivalent Fibers-ISO & 31.7 & Not Applicable & Not Applicable & & 10 \\
\hline PCM Equivalent Fibers-NIOSH & 28.6 & Not Applicable & Not Applicable & & 9 \\
\hline PCM Equivalent Structures-ISO & 15.9 & Not Applicable & Not Applicable & 5 & \\
\hline PCM Equivalent Structures-NIOSH & 15.9 & Not Applicable & Not Applicable & 5 & \\
\hline Asbestos Structures >5um and 3:1 & 31.7 & Not Applicable & Not Applicable & 10 & \\
\hline Asbestos Fibers and Bundles > 5um and 3:1 & 31.7 & Not Applicable & Not Applicable & & 10 \\
\hline
\end{tabular}

1 Concentration and $95 \%$ Confidence Level are calculated based upon the number showing under the Structure Count header.

Lab/Cor Sample No.: S26

Client Sample No.: FB-2-R1 Description:

Analyst(s) Analysis Date

Volume (L) : 0

Lab Filter Area (mm2) : 385

Grid Openings Analyzed : 35

Average Grid Opening Area : 0.009

Area Analyzed (mm2) : 0.315

Analytical Sens. (struc/cc) : NA

Dectection Limit. (struc/cc) : NA

\begin{tabular}{|c|c|c|c|c|c|}
\hline $\begin{array}{c}\text { Structure } \\
\text { Type }\end{array}$ & $\begin{array}{c}\text { Filter } \\
\text { Density } \\
(\mathbf{s} / \mathbf{m m 2})\end{array}$ & $\begin{array}{c}\text { Concen- } \\
\text { tration }^{\star} \\
(\text { struc/cc) }\end{array}$ & $\begin{array}{c}95 \% \text { Confidence } \\
\text { Interval } \\
\text { (struc/cc) }\end{array}$ & \multicolumn{2}{|c|}{$\begin{array}{c}\text { Structure } \\
\text { Count }^{1} \\
\text { Prim/Total }\end{array}$} \\
\hline Primary Asbestos Structures & 634.9 & Not Applicable & Not Applicable & 200 & \\
\hline Total Asbestos Structures & 666.7 & Not Applicable & Not Applicable & & 210 \\
\hline PCM Equivalent Fibers-ISO & 342.9 & Not Applicable & Not Applicable & & 108 \\
\hline PCM Equivalent Fibers-NIOSH & 358.7 & Not Applicable & Not Applicable & & 113 \\
\hline PCM Equivalent Structures-ISO & 244.4 & Not Applicable & Not Applicable & 77 & \\
\hline PCM Equivalent Structures-NIOSH & 257.1 & Not Applicable & Not Applicable & 81 & \\
\hline Asbestos Structures >5um and 3:1 & 307.9 & Not Applicable & Not Applicable & 97 & \\
\hline Asbestos Fibers and Bundles > 5um and 3:1 & 276.2 & Not Applicable & Not Applicable & & 87 \\
\hline
\end{tabular}

\footnotetext{
1 Concentration and 95\% Confidence Level are calculated based upon the number showing under the Structure Count header.
} 
Job Number: 070434

\section{SEA}

ISO 10312, Direct Summary Data

Client: Idaho National Laboratory

Report Number: 070434R06

Date Received: 4/23/2007

Project Name: RARE

Lab/Cor Sample No.: S27

Client Sample No.: FB-2-R2

Description:

$\begin{array}{cc}\text { Analyst(s) } & \text { Analysis Date } \\ \text { KM } & 5 / 30 / 2007\end{array}$

Volume (L) : 0

Lab Filter Area (mm2) : 385

Grid Openings Analyzed : 35

Average Grid Opening Area : 0.009

Area Analyzed (mm2) : 0.315

Analytical Sens. (struc/cc) : NA

Dectection Limit. (struc/cc) : NA

\begin{tabular}{|c|c|c|c|c|c|}
\hline $\begin{array}{c}\text { Structure } \\
\text { Type }\end{array}$ & $\begin{array}{c}\text { Filter } \\
\text { Density } \\
(\mathrm{s} / \mathrm{mm} 2)\end{array}$ & $\begin{array}{l}\text { Concen- } \\
\text { tration*}^{\star} \\
\text { (struc/cc) }\end{array}$ & $\begin{array}{c}95 \% \text { Confidence } \\
\text { Interval } \\
\text { (struc/cc) }\end{array}$ & \multicolumn{2}{|c|}{$\begin{array}{l}\text { Structure } \\
\text { Count }^{1} \\
\text { Prim/Total }\end{array}$} \\
\hline Primary Asbestos Structures & 498.4 & Not Applicable & Not Applicable & 157 & \\
\hline Total Asbestos Structures & 527 & Not Applicable & Not Applicable & & 166 \\
\hline PCM Equivalent Fibers-ISO & 298.4 & Not Applicable & Not Applicable & & 94 \\
\hline PCM Equivalent Fibers-NIOSH & 320.6 & Not Applicable & Not Applicable & & 101 \\
\hline PCM Equivalent Structures-ISO & 238.1 & Not Applicable & Not Applicable & 75 & \\
\hline PCM Equivalent Structures-NIOSH & 269.8 & Not Applicable & Not Applicable & 85 & \\
\hline Asbestos Structures >5um and 3:1 & 273 & Not Applicable & Not Applicable & 86 & \\
\hline Asbestos Fibers and Bundles $>5 u m$ and $3: 1$ & 257.1 & Not Applicable & Not Applicable & & 81 \\
\hline
\end{tabular}

1 Concentration and $95 \%$ Confidence Level are calculated based upon the number showing under the Structure Count header.

Lab/Cor Sample No.: S28

Client Sample No.: FB-2-R3 Description:

$\begin{array}{cc}\text { Analyst(s) } & \text { Analysis Date } \\ \text { KM } & 6 / 1 / 2007 \\ \text { KM } & 6 / 4 / 2007\end{array}$

Volume (L) : 0

Lab Filter Area (mm2) : 385

Grid Openings Analyzed : 35

Average Grid Opening Area : 0.009

Area Analyzed (mm2) : 0.315

Analytical Sens. (struc/cc) : NA

Dectection Limit. (struc/cc) : NA

\begin{tabular}{|c|c|c|c|c|c|}
\hline $\begin{array}{c}\text { Structure } \\
\text { Type }\end{array}$ & $\begin{array}{c}\text { Filter } \\
\text { Density } \\
(\mathbf{s} / \mathbf{m m 2})\end{array}$ & $\begin{array}{c}\text { Concen- } \\
\text { tration }^{\star} \\
(\text { struc/cc) }\end{array}$ & $\begin{array}{c}95 \% \text { Confidence } \\
\text { Interval } \\
\text { (struc/cc) }\end{array}$ & \multicolumn{2}{|c|}{$\begin{array}{c}\text { Structure } \\
\text { Count }^{1} \\
\text { Prim/Total }\end{array}$} \\
\hline Primary Asbestos Structures & 660.3 & Not Applicable & Not Applicable & 208 & \\
\hline Total Asbestos Structures & 663.5 & Not Applicable & Not Applicable & & 209 \\
\hline PCM Equivalent Fibers-ISO & 257.1 & Not Applicable & Not Applicable & & 81 \\
\hline PCM Equivalent Fibers-NIOSH & 273 & Not Applicable & Not Applicable & & 86 \\
\hline PCM Equivalent Structures-ISO & 244.4 & Not Applicable & Not Applicable & 77 & \\
\hline PCM Equivalent Structures-NIOSH & 260.3 & Not Applicable & Not Applicable & 82 & \\
\hline Asbestos Structures >5um and 3:1 & 257.1 & Not Applicable & Not Applicable & 81 & \\
\hline Asbestos Fibers and Bundles > 5um and 3:1 & 231.7 & Not Applicable & Not Applicable & & 73 \\
\hline
\end{tabular}

\footnotetext{
${ }_{1}$ Concentration and $95 \%$ Confidence Level are calculated based upon the number showing under the Structure Count header.
} 
Job Number: 070434

\section{SEA}

ISO 10312, Direct Summary Data

Client: Idaho National Laboratory

Report Number: 070434R06

Date Received: 4/23/2007

Project Name: RARE

Lab/Cor Sample No.: S29

Client Sample No.: FB-2-R4

Description:

$\begin{array}{cc}\text { Analyst(s) } & \text { Analysis Date } \\ \text { KM } & 6 / 4 / 2007\end{array}$

Volume (L) : 0

Lab Filter Area (mm2) : 385

Grid Openings Analyzed : 35

Average Grid Opening Area : 0.009

Area Analyzed (mm2) : 0.315

Analytical Sens. (struc/cc) : NA

Dectection Limit. (struc/cc) : NA

\begin{tabular}{|c|r|r|r|c|}
\hline $\begin{array}{c}\text { Structure } \\
\text { Type }\end{array}$ & $\begin{array}{c}\text { Filter } \\
\text { Density } \\
\text { (s/mm2) }\end{array}$ & $\begin{array}{c}\text { Concen- } \\
\text { tration* } \\
\text { (struc/cc) }\end{array}$ & $\begin{array}{c}\text { Structure } \\
\text { Count } \\
\text { Prim/Total }\end{array}$ \\
\hline Primary Asbestos Structures & 619 & Not Applicable & $\begin{array}{c}\text { 95\% Confidence } \\
\text { Interval } \\
\text { (struc/cc) }\end{array}$ & Not Applicable \\
\hline Total Asbestos Structures & 647.6 & Not Applicable & Not Applicable & Not Applicable \\
\hline PCM Equivalent Fibers-ISO & 323.8 & Not Applicable & Not Applicable & Not Applicable \\
\hline PCM Equivalent Fibers-NIOSH & 342.9 & Not Applicable & 102 \\
\hline PCM Equivalent Structures-ISO & 288.9 & Not Applicable & Not Applicable & 108 \\
\hline PCM Equivalent Structures-NIOSH & 307.9 & Not Applicable & Not Applicable & 97 \\
\hline Asbestos Structures >5um and 3:1 & 292.1 & Not Applicable & Not Applicable & 92 \\
\hline Asbestos Fibers and Bundles > 5um and 3:1 & 285.7 & Not Applicable & 90 \\
\hline
\end{tabular}

${ }_{1}$ Concentration and $95 \%$ Confidence Level are calculated based upon the number showing under the Structure Count header.

Lab/Cor Sample No.: S30

Client Sample No.: FB-2-R5 Description:

Analyst(s) Analysis Date

KM 6/5/2007

Volume (L) : 0

Lab Filter Area (mm2) : 385

Grid Openings Analyzed : 35

Average Grid Opening Area : 0.009

Area Analyzed (mm2) : 0.315

Analytical Sens. (struc/cc) : NA

Dectection Limit. (struc/cc) : NA

\begin{tabular}{|c|c|c|c|c|c|}
\hline $\begin{array}{c}\text { Structure } \\
\text { Type }\end{array}$ & $\begin{array}{l}\text { Filter } \\
\text { Density } \\
\text { (s/mm2) }\end{array}$ & $\begin{array}{l}\text { Concen- } \\
\text { tration }^{\star} \\
\text { (struc/cc) }\end{array}$ & $\begin{array}{l}95 \% \text { Confidence } \\
\text { Interval } \\
\text { (struc/cc) }\end{array}$ & \multicolumn{2}{|c|}{$\begin{array}{c}\text { Structure } \\
\text { Count } \\
\text { Prim/Total }\end{array}$} \\
\hline Primary Asbestos Structures & 504.8 & Not Applicable & Not Applicable & 159 & \\
\hline Total Asbestos Structures & 511.1 & Not Applicable & Not Applicable & & 161 \\
\hline PCM Equivalent Fibers-ISO & 228.6 & Not Applicable & Not Applicable & & 72 \\
\hline PCM Equivalent Fibers-NIOSH & 225.4 & Not Applicable & Not Applicable & & 71 \\
\hline PCM Equivalent Structures-ISO & 215.9 & Not Applicable & Not Applicable & 68 & \\
\hline PCM Equivalent Structures-NIOSH & 212.7 & Not Applicable & Not Applicable & 67 & \\
\hline Asbestos Structures >5um and 3:1 & 209.5 & Not Applicable & Not Applicable & 66 & \\
\hline Asbestos Fibers and Bundles > 5um and 3:1 & 209.5 & Not Applicable & Not Applicable & & 66 \\
\hline
\end{tabular}

\footnotetext{
1 Concentration and 95\% Confidence Level are calculated based upon the number showing under the Structure Count header.
} 
Job Number: 070434

\section{SEA}

ISO 10312, Direct Summary Data

Client: Idaho National Laboratory

Report Number: 070434R06

Date Received: 4/23/2007

Project Name: RARE

Lab/Cor Sample No.: S31

Client Sample No.: FB-2-R6

Description:

$\begin{array}{cc}\text { Analyst(s) } & \text { Analysis Date } \\ \text { KM } & 6 / 5 / 2007 \\ \text { KM } & 6 / 6 / 2007\end{array}$

Volume (L) : 0

Lab Filter Area (mm2) : 385

Grid Openings Analyzed : 10

Average Grid Opening Area : 0.009

Area Analyzed (mm2) : 0.09

Analytical Sens. (struc/cc) : NA

Dectection Limit. (struc/cc) : NA

\begin{tabular}{|c|c|c|c|c|c|}
\hline $\begin{array}{c}\text { Structure } \\
\text { Type }\end{array}$ & $\begin{array}{c}\text { Filter } \\
\text { Density } \\
(\mathbf{s} / \mathbf{m m} 2)\end{array}$ & $\begin{array}{c}\text { Concen- } \\
\text { tration* } \\
\text { (struc/cc) }\end{array}$ & $\begin{array}{c}\text { 95\% Confidence } \\
\text { Interval } \\
\text { (struc/cc) }\end{array}$ & \multicolumn{2}{|c|}{$\begin{array}{l}\text { Structure } \\
\text { Count }^{1} \\
\text { Prim/Total }\end{array}$} \\
\hline Primary Asbestos Structures & 500 & Not Applicable & Not Applicable & 45 & \\
\hline Total Asbestos Structures & 500 & Not Applicable & Not Applicable & & 45 \\
\hline PCM Equivalent Fibers-ISO & 177.8 & Not Applicable & Not Applicable & & 16 \\
\hline PCM Equivalent Fibers-NIOSH & 177.8 & Not Applicable & Not Applicable & & $\overline{16}$ \\
\hline PCM Equivalent Structures-ISO & \begin{tabular}{l|l}
155.6 \\
\end{tabular} & Not Applicable & Not Applicable & 14 & \\
\hline PCM Equivalent Structures-NIOSH & 155.6 & Not Applicable & Not Applicable & 14 & \\
\hline Asbestos Structures >5um and 3:1 & 166.7 & Not Applicable & Not Applicable & 15 & \\
\hline Asbestos Fibers and Bundles $>5$ um and 3:1 & 155.6 & Not Applicable & Not Applicable & & 14 \\
\hline
\end{tabular}

${ }_{1}$ Concentration and $95 \%$ Confidence Level are calculated based upon the number showing under the Structure Count header.

Lab/Cor Sample No.: S32

Client Sample No.: FB-2-R7 Description:

Analyst(s) Analysis Date

KM 6/6/2007

Volume (L) : 0

Lab Filter Area (mm2) : 385

Grid Openings Analyzed : 10

Average Grid Opening Area : 0.009

Area Analyzed (mm2) : 0.09

Analytical Sens. (struc/cc) : NA

Dectection Limit. (struc/cc) : NA

\begin{tabular}{|c|c|c|c|c|c|}
\hline $\begin{array}{c}\text { Structure } \\
\text { Type }\end{array}$ & $\begin{array}{c}\text { Filter } \\
\text { Density } \\
\text { (s/mm2) }\end{array}$ & $\begin{array}{l}\text { Concen- } \\
\text { tration* } \\
\text { (struc/cc) }\end{array}$ & $\begin{array}{l}95 \% \text { Confidence } \\
\text { Interval } \\
\text { (struc/cc) }\end{array}$ & \multicolumn{2}{|c|}{$\begin{array}{l}\text { Structure } \\
\text { Count }^{1} \\
\text { Prim/Total }\end{array}$} \\
\hline Primary Asbestos Structures & 755.6 & Not Applicable & Not Applicable & 68 & \\
\hline Total Asbestos Structures & 755.6 & Not Applicable & Not Applicable & & 68 \\
\hline PCM Equivalent Fibers-ISO & 422.2 & Not Applicable & Not Applicable & & 38 \\
\hline PCM Equivalent Fibers-NIOSH & 422.2 & Not Applicable & Not Applicable & & 38 \\
\hline PCM Equivalent Structures-ISO & 388.9 & Not Applicable & Not Applicable & 35 & \\
\hline PCM Equivalent Structures-NIOSH & 388.9 & Not Applicable & Not Applicable & 35 & \\
\hline Asbestos Structures >5um and 3:1 & 433.3 & Not Applicable & Not Applicable & 39 & \\
\hline Asbestos Fibers and Bundles > 5um and 3:1 & 411.1 & Not Applicable & Not Applicable & & 37 \\
\hline
\end{tabular}

\footnotetext{
1 Concentration and 95\% Confidence Level are calculated based upon the number showing under the Structure Count header.
} 
Job Number: 070434

\section{SEA}

ISO 10312, Direct Summary Data

Client: Idaho National Laboratory

Report Number: 070434R06

Date Received: 4/23/2007

Project Name: RARE

Lab/Cor Sample No.: S33

Client Sample No.: FB-1-R1

Description:

$\begin{array}{cc}\text { Analyst(s) } & \text { Analysis Date } \\ \text { KM } & 6 / 6 / 2007\end{array}$

Volume (L) : 0

Lab Filter Area (mm2) : 385

Grid Openings Analyzed : 10

Average Grid Opening Area : 0.009

Area Analyzed (mm2) : 0.09

Analytical Sens. (struc/cc) : NA

Dectection Limit. (struc/cc) : NA

\begin{tabular}{|c|c|c|c|c|c|}
\hline $\begin{array}{c}\text { Structure } \\
\text { Type }\end{array}$ & $\begin{array}{l}\text { Filter } \\
\text { Density } \\
\text { (s/mm2) }\end{array}$ & $\begin{array}{l}\text { Concen- } \\
\text { tration* } \\
\text { (struc/cc) }\end{array}$ & $\begin{array}{l}95 \% \text { Confidence } \\
\text { Interval } \\
\text { (struc/cc) }\end{array}$ & \multicolumn{2}{|c|}{$\begin{array}{c}\text { Structure } \\
\text { Count }^{1} \\
\text { Prim/Total }\end{array}$} \\
\hline Primary Asbestos Structures & 311.1 & Not Applicable & Not Applicable & 28 & \\
\hline Total Asbestos Structures & 344.4 & Not Applicable & Not Applicable & & 31 \\
\hline PCM Equivalent Fibers-ISO & 222.2 & Not Applicable & Not Applicable & & 20 \\
\hline PCM Equivalent Fibers-NIOSH & 222.2 & Not Applicable & Not Applicable & & 20 \\
\hline PCM Equivalent Structures-ISO & 188.9 & Not Applicable & Not Applicable & 17 & \\
\hline PCM Equivalent Structures-NIOSH & 211.1 & Not Applicable & Not Applicable & 19 & \\
\hline Asbestos Structures >5um and 3:1 & 222.2 & Not Applicable & Not Applicable & 20 & \\
\hline Asbestos Fibers and Bundles > 5um and 3:1 & 211.1 & Not Applicable & Not Applicable & & 19 \\
\hline
\end{tabular}

1 Concentration and $95 \%$ Confidence Level are calculated based upon the number showing under the Structure Count header.

Lab/Cor Sample No.: S34

Client Sample No.: FB-1-R2 Description:

Analyst(s) Analysis Date

KM 6/6/2007

Volume (L) : 0

Lab Filter Area (mm2) : 385

Grid Openings Analyzed : 35

Average Grid Opening Area : 0.009

Area Analyzed (mm2) : 0.315

Analytical Sens. (struc/cc) : NA

Dectection Limit. (struc/cc) : NA

\begin{tabular}{|c|c|c|c|c|c|}
\hline $\begin{array}{c}\text { Structure } \\
\text { Type }\end{array}$ & $\begin{array}{c}\text { Filter } \\
\text { Density } \\
(\mathbf{s} / \mathbf{m m 2})\end{array}$ & $\begin{array}{c}\text { Concen- } \\
\text { tration }^{\star} \\
(\text { struc/cc) }\end{array}$ & $\begin{array}{c}95 \% \text { Confidence } \\
\text { Interval } \\
\text { (struc/cc) }\end{array}$ & \multicolumn{2}{|c|}{$\begin{array}{l}\text { Structure } \\
\text { Count }^{1} \\
\text { Prim/Total }\end{array}$} \\
\hline Primary Asbestos Structures & 0 & Not Applicable & Not Applicable & 0 & \\
\hline Total Asbestos Structures & 0 & Not Applicable & Not Applicable & & 0 \\
\hline PCM Equivalent Fibers-ISO & 0 & Not Applicable & Not Applicable & & 0 \\
\hline PCM Equivalent Fibers-NIOSH & 0 & Not Applicable & Not Applicable & & 0 \\
\hline PCM Equivalent Structures-ISO & 0 & Not Applicable & Not Applicable & 0 & \\
\hline PCM Equivalent Structures-NIOSH & 0 & Not Applicable & Not Applicable & 0 & \\
\hline Asbestos Structures >5um and 3:1 & 0 & Not Applicable & Not Applicable & 0 & \\
\hline Asbestos Fibers and Bundles > 5um and 3:1 & 0 & Not Applicable & Not Applicable & & 0 \\
\hline
\end{tabular}

\footnotetext{
1 Concentration and 95\% Confidence Level are calculated based upon the number showing under the Structure Count header.
} 
Job Number: 070434

\section{SEA}

ISO 10312, Direct Summary Data

Client: Idaho National Laboratory

Report Number: 070434R06

Date Received: 4/23/2007

Project Name: RARE

Lab/Cor Sample No.: S35

Client Sample No.: FB-1-R3

Description:

$\begin{array}{cc}\text { Analyst(s) } & \text { Analysis Date } \\ \text { KM } & 6 / 6 / 2007\end{array}$
Volume (L) : 0

Lab Filter Area (mm2) : 385

Grid Openings Analyzed : 10

Average Grid Opening Area : 0.009

Area Analyzed (mm2) : 0.09

Analytical Sens. (struc/cc) : NA

Dectection Limit. (struc/cc) : NA

\begin{tabular}{|c|c|c|c|c|c|}
\hline $\begin{array}{c}\text { Structure } \\
\text { Type }\end{array}$ & $\begin{array}{c}\text { Filter } \\
\text { Density } \\
(\mathrm{s} / \mathrm{mm} 2)\end{array}$ & $\begin{array}{l}\text { Concen- } \\
\text { tration* } \\
\text { (struc/cc) }\end{array}$ & $\begin{array}{c}95 \% \text { Confidence } \\
\text { Interval } \\
\text { (struc/cc) }\end{array}$ & \multicolumn{2}{|c|}{$\begin{array}{l}\text { Structure } \\
\text { Count }^{1} \\
\text { Prim/Total }\end{array}$} \\
\hline Primary Asbestos Structures & 355.6 & Not Applicable & Not Applicable & 32 & \\
\hline Total Asbestos Structures & 355.6 & Not Applicable & Not Applicable & & 32 \\
\hline PCM Equivalent Fibers-ISO & 211.1 & Not Applicable & Not Applicable & & 19 \\
\hline PCM Equivalent Fibers-NIOSH & 222.2 & Not Applicable & Not Applicable & & 20 \\
\hline PCM Equivalent Structures-ISO & 211.1 & Not Applicable & Not Applicable & 19 & \\
\hline PCM Equivalent Structures-NIOSH & 222.2 & Not Applicable & Not Applicable & 20 & \\
\hline Asbestos Structures >5um and 3:1 & 200 & Not Applicable & Not Applicable & 18 & \\
\hline Asbestos Fibers and Bundles $>5 u m$ and $3: 1$ & 200 & Not Applicable & Not Applicable & & 18 \\
\hline
\end{tabular}

1 Concentration and $95 \%$ Confidence Level are calculated based upon the number showing under the Structure Count header.

Lab/Cor Sample No.: S36

Client Sample No.: FB-1-R4 Description:

$\begin{array}{cc}\text { Analyst(s) } & \text { Analysis Date } \\ \text { KM } & 6 / 6 / 2007 \\ \text { KM } & 6 / 7 / 2007\end{array}$

Volume (L) : 0

Lab Filter Area (mm2) : 385

Grid Openings Analyzed : 10

Average Grid Opening Area : 0.009

Area Analyzed (mm2) : 0.09

Analytical Sens. (struc/cc) : NA

Dectection Limit. (struc/cc) : NA

\begin{tabular}{|c|c|c|c|c|c|}
\hline $\begin{array}{c}\text { Structure } \\
\text { Type }\end{array}$ & $\begin{array}{c}\text { Filter } \\
\text { Density } \\
(\mathbf{s} / \mathbf{m m 2})\end{array}$ & $\begin{array}{c}\text { Concen- } \\
\text { tration }^{\star} \\
(\text { struc/cc) }\end{array}$ & $\begin{array}{c}95 \% \text { Confidence } \\
\text { Interval } \\
\text { (struc/cc) }\end{array}$ & \multicolumn{2}{|c|}{$\begin{array}{c}\text { Structure } \\
\text { Count }^{1} \\
\text { Prim/Total }\end{array}$} \\
\hline Primary Asbestos Structures & 366.7 & Not Applicable & Not Applicable & 33 & \\
\hline Total Asbestos Structures & 366.7 & Not Applicable & Not Applicable & & 33 \\
\hline PCM Equivalent Fibers-ISO & 166.7 & Not Applicable & Not Applicable & & 15 \\
\hline PCM Equivalent Fibers-NIOSH & 188.9 & Not Applicable & Not Applicable & & 17 \\
\hline PCM Equivalent Structures-ISO & 133.3 & Not Applicable & Not Applicable & 12 & \\
\hline PCM Equivalent Structures-NIOSH & 166.7 & Not Applicable & Not Applicable & 15 & \\
\hline Asbestos Structures >5um and 3:1 & 166.7 & Not Applicable & Not Applicable & 15 & \\
\hline Asbestos Fibers and Bundles > 5um and 3:1 & 166.7 & Not Applicable & Not Applicable & & 15 \\
\hline
\end{tabular}

\footnotetext{
${ }_{1}$ Concentration and $95 \%$ Confidence Level are calculated based upon the number showing under the Structure Count header.
} 
Job Number: 070434

\section{SEA}

ISO 10312, Direct Summary Data

Client: Idaho National Laboratory

Report Number: 070434R06

Date Received: 4/23/2007

Project Name: RARE

Lab/Cor Sample No.: S37

Client Sample No.: FB-1-R5

Description:

$\begin{array}{cc}\text { Analyst(s) } & \text { Analysis Date } \\ \text { KM } & 6 / 7 / 2007\end{array}$

Volume (L) : 0

Lab Filter Area (mm2) : 385

Grid Openings Analyzed : 10

Average Grid Opening Area : 0.009

Area Analyzed (mm2) : 0.09

Analytical Sens. (struc/cc) : NA

Dectection Limit. (struc/cc) : NA

\begin{tabular}{|c|c|c|c|c|c|}
\hline $\begin{array}{c}\text { Structure } \\
\text { Type }\end{array}$ & $\begin{array}{c}\text { Filter } \\
\text { Density } \\
(\mathrm{s} / \mathrm{mm} 2)\end{array}$ & $\begin{array}{l}\text { Concen- } \\
\text { tration* } \\
\text { (struc/cc) }\end{array}$ & $\begin{array}{c}95 \% \text { Confidence } \\
\text { Interval } \\
\text { (struc/cc) }\end{array}$ & \multicolumn{2}{|c|}{$\begin{array}{l}\text { Structure } \\
\text { Count }^{1} \\
\text { Prim/Total }\end{array}$} \\
\hline Primary Asbestos Structures & 288.9 & Not Applicable & Not Applicable & 26 & \\
\hline Total Asbestos Structures & 288.9 & Not Applicable & Not Applicable & & 26 \\
\hline PCM Equivalent Fibers-ISO & 144.4 & Not Applicable & Not Applicable & & 13 \\
\hline PCM Equivalent Fibers-NIOSH & 144.4 & Not Applicable & Not Applicable & & 13 \\
\hline PCM Equivalent Structures-ISO & 122.2 & Not Applicable & Not Applicable & 11 & \\
\hline PCM Equivalent Structures-NIOSH & 133.3 & Not Applicable & Not Applicable & 12 & \\
\hline Asbestos Structures >5um and 3:1 & 144.4 & Not Applicable & Not Applicable & 13 & \\
\hline Asbestos Fibers and Bundles $>5 u m$ and $3: 1$ & 144.4 & Not Applicable & Not Applicable & & 13 \\
\hline
\end{tabular}

${ }_{1}$ Concentration and $95 \%$ Confidence Level are calculated based upon the number showing under the Structure Count header.

Lab/Cor Sample No.: S38

Client Sample No.: FB-1-R6 Description:

Analyst(s) Analysis Date

KM 6/7/2007

Volume (L) : 0

Lab Filter Area (mm2) : 385

Grid Openings Analyzed : 10

Average Grid Opening Area : 0.009

Area Analyzed (mm2) : 0.09

Analytical Sens. (struc/cc) : NA

Dectection Limit. (struc/cc) : NA

\begin{tabular}{|c|c|c|c|c|c|}
\hline $\begin{array}{c}\text { Structure } \\
\text { Type }\end{array}$ & $\begin{array}{c}\text { Filter } \\
\text { Density } \\
\text { (s/mm2) }\end{array}$ & $\begin{array}{l}\text { Concen- } \\
\text { tration* } \\
\text { (struc/cc) }\end{array}$ & $\begin{array}{l}95 \% \text { Confidence } \\
\text { Interval } \\
\text { (struc/cc) }\end{array}$ & \multicolumn{2}{|c|}{$\begin{array}{l}\text { Structure } \\
\text { Count }^{1} \\
\text { Prim/Total }\end{array}$} \\
\hline Primary Asbestos Structures & 277.8 & Not Applicable & Not Applicable & 25 & \\
\hline Total Asbestos Structures & 277.8 & Not Applicable & Not Applicable & & 25 \\
\hline PCM Equivalent Fibers-ISO & 166.7 & Not Applicable & Not Applicable & & 15 \\
\hline PCM Equivalent Fibers-NIOSH & 166.7 & Not Applicable & Not Applicable & & 15 \\
\hline PCM Equivalent Structures-ISO & 177.8 & Not Applicable & Not Applicable & 16 & \\
\hline PCM Equivalent Structures-NIOSH & 177.8 & Not Applicable & Not Applicable & 16 & \\
\hline Asbestos Structures >5um and 3:1 & 177.8 & Not Applicable & Not Applicable & 16 & \\
\hline Asbestos Fibers and Bundles > 5um and 3:1 & 155.6 & Not Applicable & Not Applicable & & 14 \\
\hline
\end{tabular}

\footnotetext{
1 Concentration and 95\% Confidence Level are calculated based upon the number showing under the Structure Count header.
} 
Job Number: 070434

SEA

Report Number: 070434R06

Client: Idaho National Laboratory

Date Received: 4/23/2007

Project Name: RARE

Lab/Cor Sample No.: S39

Client Sample No.: FB-1-R7

Description:

Analyst(s)

KM

Analysis Date

$6 / 7 / 2007$
Volume (L) : 0

Lab Filter Area (mm2) : 385

Grid Openings Analyzed : 10

Average Grid Opening Area : 0.009

Area Analyzed (mm2) : 0.09

Analytical Sens. (struc/cc) : NA

Dectection Limit. (struc/cc) : NA

\begin{tabular}{|c|c|c|c|c|c|}
\hline $\begin{array}{c}\text { Structure } \\
\text { Type }\end{array}$ & $\begin{array}{c}\text { Filter } \\
\text { Density } \\
(\mathrm{s} / \mathrm{mm} 2)\end{array}$ & $\begin{array}{l}\text { Concen- } \\
\text { tration* }^{*} \\
\text { (struc/cc) }\end{array}$ & $\begin{array}{c}\text { 95\% Confidence } \\
\text { Interval } \\
\text { (struc/cc) }\end{array}$ & \multicolumn{2}{|c|}{$\begin{array}{l}\text { Structure } \\
\text { Count }^{1} \\
\text { Prim/Total }\end{array}$} \\
\hline Primary Asbestos Structures & 288.9 & Not Applicable & Not Applicable & 26 & \\
\hline Total Asbestos Structures & 300 & Not Applicable & Not Applicable & & 27 \\
\hline PCM Equivalent Fibers-ISO & 166.7 & Not Applicable & Not Applicable & & 15 \\
\hline PCM Equivalent Fibers-NIOSH & 166.7 & Not Applicable & Not Applicable & & 15 \\
\hline PCM Equivalent Structures-ISO & 144.4 & Not Applicable & Not Applicable & 13 & \\
\hline PCM Equivalent Structures-NIOSH & 144.4 & Not Applicable & Not Applicable & 13 & \\
\hline Asbestos Structures >5um and 3:1 & 144.4 & Not Applicable & Not Applicable & 13 & \\
\hline Asbestos Fibers and Bundles > 5um and 3:1 & 133.3 & Not Applicable & Not Applicable & & 12 \\
\hline
\end{tabular}

${ }_{1}$ Concentration and 95\% Confidence Level are calculated based upon the number showing under the Structure Count header.

Reviewed by:

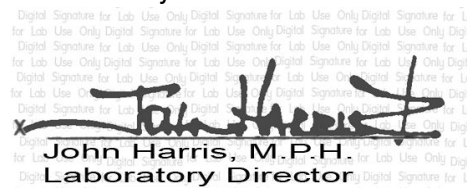

John Harris, M.P.H.

Laboratory Director

* One-sided upper 95\% Poisson confidence limits may be used to calculate sample concentrations ([Struc count] * 
Phone: (206) 781-0155

Fax: (206) 789-8424

7619 6th Ave Nw

ISO 10312, Direct Raw Data

Job Number: 070434

SEA

Report Number: 070434R06

Client: Idaho National Laboratory

Date Received: 4/23/2007

Project Name: RARE

Client Sample No: cleaning blank \#1

Lab/Cor Sample No: S1

Client Description:

Date Sampled:

$4 / 16 / 2007$

\begin{tabular}{|c|c|c|c|c|c|c|c|c|c|c|c|c|}
\hline Gr & No. & Loc. & ID & Prim Tot & Class & Len & Wid & Asp & Analyte & Elements & Comment & Count Categories \\
\hline G1 & 1 & A2 & & & NSD & & & & & & & \\
\hline G1 & 2 & $\mathrm{C} 2$ & & & NSD & & & & & & & \\
\hline G1 & 3 & E2 & & & NSD & & & & & & & \\
\hline G1 & 4 & G2 & & & NSD & & & & & & & \\
\hline G1 & 5 & 12 & & & NSD & & & & & & & \\
\hline G1 & 6 & J4 & & & NSD & & & & & & & \\
\hline G1 & 7 & $\mathrm{H} 4$ & & & NSD & & & & & & & \\
\hline G1 & 8 & $\mathrm{~F} 4$ & & & NSD & & & & & & & \\
\hline G1 & 9 & D4 & & & NSD & & & & & & & \\
\hline G1 & 10 & B4 & & & NSD & & & & & & & \\
\hline G1 & 11 & A7 & & & NSD & & & & & & & \\
\hline G1 & 12 & C7 & & & NSD & & & & & & & \\
\hline G1 & 13 & E7 & & & NSD & & & & & & & \\
\hline G1 & 14 & G7 & & & NSD & & & & & & & \\
\hline G1 & 15 & 17 & & & NSD & & & & & & & \\
\hline G1 & 16 & J9 & & & NSD & & & & & & & \\
\hline G1 & 17 & $\mathrm{H} 9$ & & & NSD & & & & & & & \\
\hline G1 & 18 & F9 & & & NSD & & & & & & & \\
\hline G1 & 19 & D9 & & & NSD & & & & & & & \\
\hline G1 & 20 & B9 & & & NSD & & & & & & & \\
\hline G2 & 21 & $\mathrm{~A} 2$ & & & NSD & & & & & & & \\
\hline G2 & 22 & C2 & & & NSD & & & & & & & \\
\hline G2 & 23 & E2 & & & NSD & & & & & & & \\
\hline G2 & 24 & G2 & & & NSD & & & & & & & \\
\hline G2 & 25 & 12 & & & NSD & & & & & & & \\
\hline G2 & 26 & J4 & & & NSD & & & & & & & \\
\hline G2 & 27 & $\mathrm{H} 4$ & & & NSD & & & & & & & \\
\hline G2 & 28 & F4 & & & NSD & & & & & & & \\
\hline G2 & 29 & D4 & & & NSD & & & & & & & \\
\hline G2 & 30 & B4 & & & NSD & & & & & & & \\
\hline G2 & 31 & A7 & & & NSD & & & & & & & \\
\hline G2 & 32 & C7 & & & NSD & & & & & & & \\
\hline G2 & 33 & E7 & & & NSD & & & & & & & \\
\hline G2 & 34 & G7 & & & NSD & & & & & & & \\
\hline
\end{tabular}

Count Categories

AFB $>5,3: 1$

PAS

PCMEF-US

PCMES-US
Asbestos Fibers and Bundles > 5um and 3:1

Primary Asbestos Structures

PCM Equivalent Fibers-NIOSH

PCM Equivalent Structures-NIOSH
AS $>5,3: 1$ PCMEF-ISO PCMES-ISO TAS
Asbestos Structures $>5$ um and 3:1 PCM Equivalent Fibers-ISO PCM Equivalent Structures-ISO Total Asbestos Structures 
Phone: (206) 781-0155

7619 6th Ave Nw

Job Number: 070434

SEA

ISO 10312, Direct Raw Data

Client: Idaho National Laboratory

Report Number: 070434R06

Name: RARE

Client Sample No: cleaning blank \#1

Lab/Cor Sample No: $\mathbf{S 1}$

Client Description:

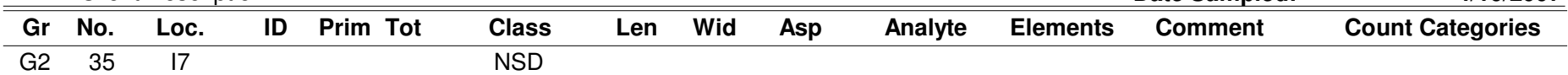

Count Categories

AFB $>5,3: 1$

PAS

PCMEF-US

PCMES-US
Asbestos Fibers and Bundles > 5um and 3:1

Primary Asbestos Structures

PCM Equivalent Fibers-NIOSH

PCM Equivalent Structures-NIOSH
AS>5, 3:1 PCMEF-ISO PCMES-ISO TAS
Fax: (206) 789-8424

http://www.labcor.net 
Phone: (206) 781-0155

Fax: (206) 789-8424

7619 6th Ave Nw

ISO 10312, Direct Raw Data

Job Number: 070434

SEA

Report Number: 070434R06

Client: Idaho National Laboratory

Date Received: 4/23/2007

Project Name: RARE

Client Sample No: cleaning blank \#2

Lab/Cor Sample No: S2

Client Description:

Date Sampled:

$4 / 16 / 2007$

\begin{tabular}{|c|c|c|c|c|c|c|c|c|c|c|c|c|}
\hline $\mathrm{Gr}$ & No. & Loc. & ID & Prim Tot & Class & Len & Wid & Asp & Analyte & Elements & Comment & Count Categories \\
\hline G1 & 1 & $\mathrm{~A} 2$ & & & NSD & & & & & & & \\
\hline G1 & 2 & $\mathrm{C} 2$ & & & NSD & & & & & & & \\
\hline G1 & 3 & E2 & & & NSD & & & & & & & \\
\hline G1 & 4 & G2 & & & NSD & & & & & & & \\
\hline G1 & 5 & 12 & & & NSD & & & & & & & \\
\hline G1 & 6 & J4 & & & NSD & & & & & & & \\
\hline G1 & 7 & $\mathrm{H} 4$ & & & NSD & & & & & & & \\
\hline G1 & 8 & $\mathrm{~F} 4$ & & & NSD & & & & & & & \\
\hline G1 & 9 & D4 & & & NSD & & & & & & & \\
\hline G1 & 10 & B4 & & & NSD & & & & & & & \\
\hline G1 & 11 & A7 & & & NSD & & & & & & & \\
\hline G1 & 12 & C7 & & & NSD & & & & & & & \\
\hline G1 & 13 & E7 & & & NSD & & & & & & & \\
\hline G1 & 14 & G7 & & & NSD & & & & & & & \\
\hline G1 & 15 & 17 & & & NSD & & & & & & & \\
\hline G1 & 16 & $\mathrm{H} 9$ & & & NSD & & & & & & & \\
\hline G1 & 17 & F9 & & & NSD & & & & & & & \\
\hline G1 & 18 & D9 & & & NSD & & & & & & & \\
\hline G1 & 19 & B9 & & & NSD & & & & & & & \\
\hline G2 & 20 & $\mathrm{~A} 2$ & & & NSD & & & & & & & \\
\hline G2 & 21 & $\mathrm{C} 2$ & & & NSD & & & & & & & \\
\hline G2 & 22 & E2 & & & NSD & & & & & & & \\
\hline G2 & 23 & G2 & & & NSD & & & & & & & \\
\hline G2 & 24 & 12 & & & NSD & & & & & & & \\
\hline G2 & 25 & J4 & & & NSD & & & & & & & \\
\hline G2 & 26 & $\mathrm{H} 4$ & & & NSD & & & & & & & \\
\hline G2 & 27 & F4 & & & NSD & & & & & & & \\
\hline G2 & 28 & D4 & & & NSD & & & & & & & \\
\hline G2 & 29 & B4 & & & NSD & & & & & & & \\
\hline G2 & 30 & A7 & & & NSD & & & & & & & \\
\hline G2 & 31 & C7 & & & NSD & & & & & & & \\
\hline G2 & 32 & E7 & & & NSD & & & & & & & \\
\hline G2 & 33 & G7 & & & NSD & & & & & & & \\
\hline G2 & 34 & 17 & & & NSD & & & & & & & \\
\hline
\end{tabular}

Count Categories

AFB $>5,3: 1$

PAS

PCMEF-US

PCMES-US
Asbestos Fibers and Bundles > 5um and 3:1

Primary Asbestos Structures

PCM Equivalent Fibers-NIOSH

PCM Equivalent Structures-NIOSH
AS>5, 3:1 PCMEF-ISO PCMES-ISO TAS
Asbestos Structures $>5$ um and 3:1 PCM Equivalent Fibers-ISO PCM Equivalent Structures-ISO Total Asbestos Structures 
Phone: (206) 781-0155

7619 6th Ave Nw

Job Number: 070434

SEA

ISO 10312, Direct Raw Data

Client: Idaho National Laboratory

Report Number: 070434R06

Name: RARE

Client Sample No: cleaning blank \#2

Lab/Cor Sample No: S2

Client Description:

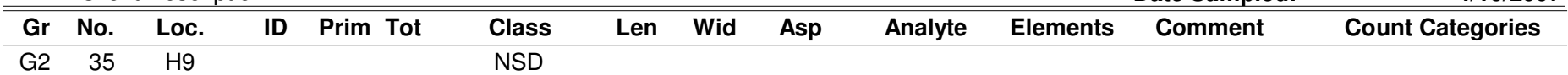

Count Categories

AFB $>5,3: 1$

PAS

PCMEF-US

PCMES-US
Asbestos Fibers and Bundles > 5um and 3:1

Primary Asbestos Structures

PCM Equivalent Fibers-NIOSH

PCM Equivalent Structures-NIOSH
AS $>5,3: 1$ PCMEF-ISO PCMES-ISO TAS
Fax: (206) 789-8424

http://www.labcor.net 
Phone: (206) 781-0155

7619 6th Ave Nw

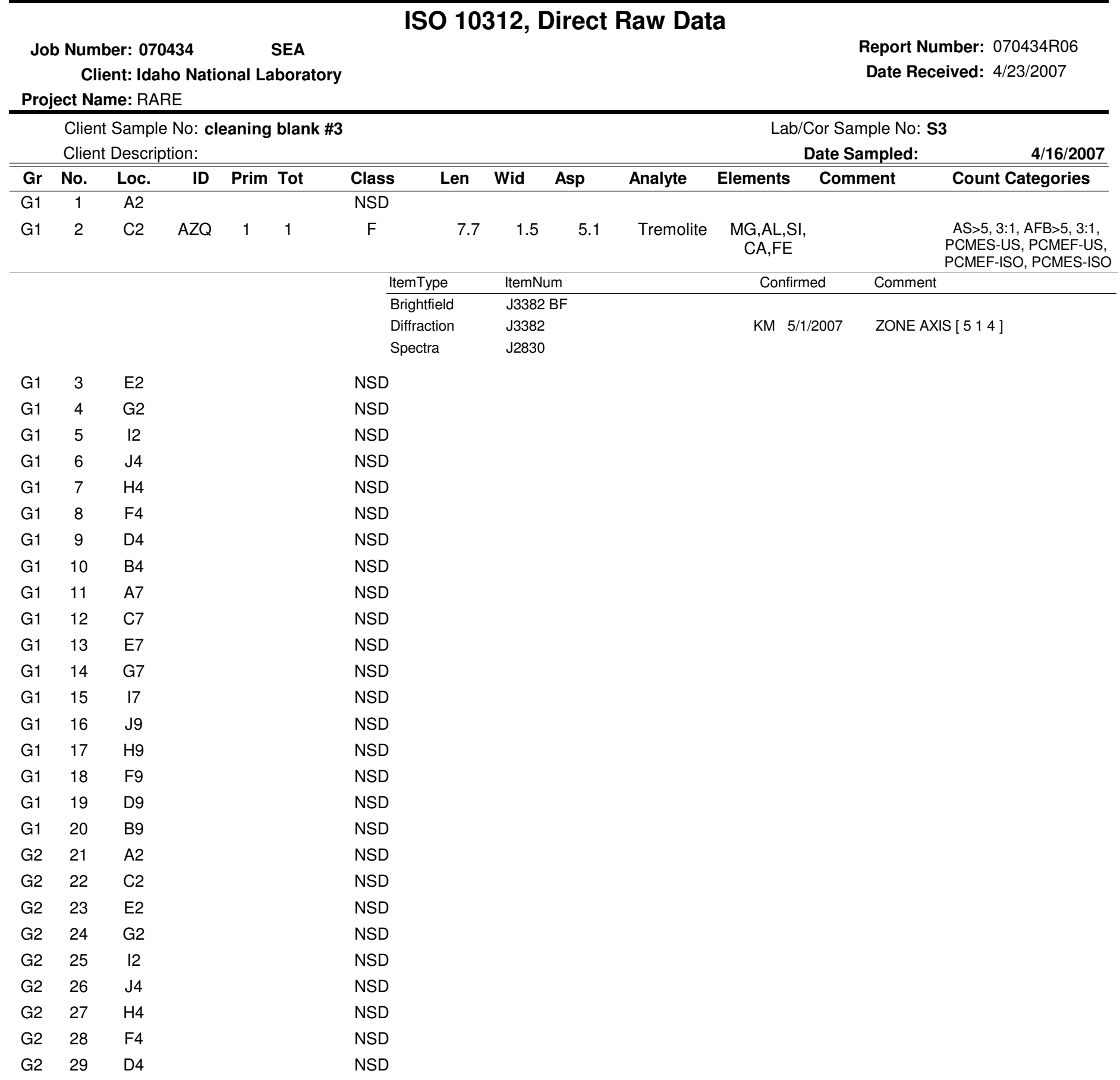

Count Categories AFB $>5,3: 1$

PAS

PCMEF-US

PCMES-US
Asbestos Fibers and Bundles $>$ 5um and 3:1

Primary Asbestos Structures

PCM Equivalent Fibers-NIOSH

PCM Equivalent Structures-NIOSH
AS>5, 3:1 PCMEF-ISO PCMES-ISO TAS
Asbestos Structures $>5$ um and 3:1 PCM Equivalent Fibers-ISO PCM Equivalent Structures-ISO Total Asbestos Structures 
Job Number: 070434

ISO 10312, Direct Raw Data

Client: Idaho National Laboratory

Report Number: 070434R06

Project Name: RARE

Date Received: 4/23/2007

Client Sample No: cleaning blank \#3

Lab/Cor Sample No: S3

Client Description:

Date Sampled:

$4 / 16 / 2007$

\begin{tabular}{|c|c|c|c|c|c|c|c|c|c|c|c|c|}
\hline $\mathrm{Gr}$ & No. & Loc. & ID & Prim Tot & Class & Len & Wid & Asp & Analyte & Elements & Comment & Count Categories \\
\hline $\mathrm{G} 2$ & 30 & B4 & & & NSD & & & & & & & \\
\hline $\mathrm{G} 2$ & 31 & A7 & & & NSD & & & & & & & \\
\hline G2 & 32 & $\mathrm{C} 7$ & & & NSD & & & & & & & \\
\hline $\mathrm{G} 2$ & 33 & E7 & & & NSD & & & & & & & \\
\hline G2 & 34 & G7 & & & NSD & & & & & & & \\
\hline G2 & 35 & 17 & & & NSD & & & & & & & \\
\hline
\end{tabular}

Asbestos Fibers and Bundles > 5um and 3:1

Primary Asbestos Structures

PCM Equivalent Fibers-NIOSH

PCMEF-US

PCM Equivalent Structures-NIOSH
AS>5, 3:1 PCMEF-ISO PCMES-ISO TAS
Asbestos Structures >5um and 3:1 PCM Equivalent Fibers-ISO PCM Equivalent Structures-ISO Total Asbestos Structures 
Phone: (206) 781-0155

Fax: (206) 789-8424

7619 6th Ave Nw

ISO 10312, Direct Raw Data

Job Number: 070434

SEA

Report Number: 070434R06

Client: Idaho National Laboratory

Date Received: 4/23/2007

Project Name: RARE

Client Sample No: cleaning blank \#4

Lab/Cor Sample No: S4

Client Description:

Date Sampled:

$4 / 16 / 2007$

\begin{tabular}{|c|c|c|c|c|c|c|c|c|c|c|c|c|}
\hline $\mathrm{Gr}$ & No. & Loc. & ID & Prim Tot & Class & Len & Wid & Asp & Analyte & Elements & Comment & Count Categories \\
\hline G1 & 1 & $\mathrm{~A} 2$ & & & NSD & & & & & & & \\
\hline G1 & 2 & $\mathrm{C} 2$ & & & NSD & & & & & & & \\
\hline G1 & 3 & E2 & & & NSD & & & & & & & \\
\hline G1 & 4 & G2 & & & NSD & & & & & & & \\
\hline G1 & 5 & 12 & & & NSD & & & & & & & \\
\hline G1 & 6 & J4 & & & NSD & & & & & & & \\
\hline G1 & 7 & $\mathrm{H} 4$ & & & NSD & & & & & & & \\
\hline G1 & 8 & $\mathrm{~F} 4$ & & & NSD & & & & & & & \\
\hline G1 & 9 & D4 & & & NSD & & & & & & & \\
\hline G1 & 10 & B4 & & & NSD & & & & & & & \\
\hline G1 & 11 & A7 & & & NSD & & & & & & & \\
\hline G1 & 12 & C7 & & & NSD & & & & & & & \\
\hline G1 & 13 & E7 & & & NSD & & & & & & & \\
\hline G1 & 14 & G7 & & & NSD & & & & & & & \\
\hline G1 & 15 & 17 & & & NSD & & & & & & & \\
\hline G1 & 16 & J9 & & & NSD & & & & & & & \\
\hline G1 & 17 & $\mathrm{H} 9$ & & & NSD & & & & & & & \\
\hline G1 & 18 & F9 & & & NSD & & & & & & & \\
\hline G1 & 19 & D9 & & & NSD & & & & & & & \\
\hline G1 & 20 & B9 & & & NSD & & & & & & & \\
\hline G2 & 21 & $\mathrm{~A} 2$ & & & NSD & & & & & & & \\
\hline G2 & 22 & $\mathrm{C} 2$ & & & NSD & & & & & & & \\
\hline G2 & 23 & E2 & & & NSD & & & & & & & \\
\hline G2 & 24 & G2 & & & NSD & & & & & & & \\
\hline G2 & 25 & 12 & & & NSD & & & & & & & \\
\hline G2 & 26 & J4 & & & NSD & & & & & & & \\
\hline G2 & 27 & $\mathrm{H} 4$ & & & NSD & & & & & & & \\
\hline G2 & 28 & F4 & & & NSD & & & & & & & \\
\hline G2 & 29 & D4 & & & NSD & & & & & & & \\
\hline G2 & 30 & B4 & & & NSD & & & & & & & \\
\hline G2 & 31 & A7 & & & NSD & & & & & & & \\
\hline G2 & 32 & $\mathrm{C} 7$ & & & NSD & & & & & & & \\
\hline G2 & 33 & E7 & & & NSD & & & & & & & \\
\hline G2 & 34 & G7 & & & NSD & & & & & & & \\
\hline
\end{tabular}

Count Categories

AFB $>5,3: 1$

PAS

PCMEF-US

Asbestos Fibers and Bundles > 5um and 3:1

PCMES-ISO

PCM Equivalent Fibers-NIOSH

$\mathrm{PCM}$ Equivalent Structures-NIOSH
AS>5, 3:1 PCMEF-ISO

TAS

Asbestos Structures $>5$ um and 3:1

PCM Equivalent Fibers-ISO

PCM Equivalent Structures-ISO

Total Asbestos Structures 
Phone: (206) 781-0155

7619 6th Ave Nw

Job Number: 070434

SEA

ISO 10312, Direct Raw Data

Client: Idaho National Laboratory

Report Number: 070434R06

Name: RARE

Client Sample No: cleaning blank \#4

Lab/Cor Sample No: S4

Client Description:

\begin{tabular}{|c|c|c|c|c|c|c|c|c|c|c|c|c|}
\hline $\mathrm{Gr}$ & No. & Loc. & ID & Prim Tot & Class & Len & Wid & Asp & Analyte & Elements & Comment & Count Categories \\
\hline G2 & 35 & 17 & & & NSD & & & & & & & \\
\hline
\end{tabular}

Count Categories

AFB $>5,3: 1$

PAS

PCMEF-US

PCMES-US
Asbestos Fibers and Bundles > 5um and 3:1

Primary Asbestos Structures

PCM Equivalent Fibers-NIOSH

PCM Equivalent Structures-NIOSH
AS>5, 3:1 PCMEF-ISO PCMES-ISO TAS
Fax: (206) 789-8424

http://www.labcor.net 
Phone: (206) 781-0155

Fax: (206) 789-8424

7619 6th Ave Nw

ISO 10312, Direct Raw Data

Job Number: 070434

SEA

Report Number: 070434R06

Client: Idaho National Laboratory

Date Received: 4/23/2007

Project Name: RARE

Client Sample No: Lab blank \#1

Lab/Cor Sample No: S5

Client Description:

Date Sampled:

$4 / 16 / 2007$

\begin{tabular}{|c|c|c|c|c|c|c|c|c|c|c|c|c|}
\hline $\mathrm{Gr}$ & No. & Loc. & ID & Prim Tot & Class & Len & Wid & Asp & Analyte & Elements & Comment & Count Categories \\
\hline G1 & 1 & A2 & & & NSD & & & & & & & \\
\hline G1 & 2 & $\mathrm{C} 2$ & & & NSD & & & & & & & \\
\hline G1 & 3 & E2 & & & NSD & & & & & & & \\
\hline G1 & 4 & G2 & & & NSD & & & & & & & \\
\hline G1 & 5 & 12 & & & NSD & & & & & & & \\
\hline G1 & 6 & J4 & & & NSD & & & & & & & \\
\hline G1 & 7 & $\mathrm{H} 4$ & & & NSD & & & & & & & \\
\hline G1 & 8 & $\mathrm{~F} 4$ & & & NSD & & & & & & & \\
\hline G1 & 9 & D4 & & & NSD & & & & & & & \\
\hline G1 & 10 & B4 & & & NSD & & & & & & & \\
\hline G1 & 11 & B7 & & & NSD & & & & & & & \\
\hline G1 & 12 & $\mathrm{C} 7$ & & & NSD & & & & & & & \\
\hline G1 & 13 & E7 & & & NSD & & & & & & & \\
\hline G1 & 14 & G7 & & & NSD & & & & & & & \\
\hline G1 & 15 & 17 & & & NSD & & & & & & & \\
\hline G1 & 16 & J8 & & & NSD & & & & & & & \\
\hline G1 & 17 & $\mathrm{H} 8$ & & & NSD & & & & & & & \\
\hline G1 & 18 & F8 & & & NSD & & & & & & & \\
\hline G1 & 19 & D8 & & & NSD & & & & & & & \\
\hline G1 & 20 & B8 & & & NSD & & & & & & & \\
\hline G2 & 21 & $\mathrm{~A} 2$ & & & NSD & & & & & & & \\
\hline G2 & 22 & $\mathrm{C} 2$ & & & NSD & & & & & & & \\
\hline G2 & 23 & E2 & & & NSD & & & & & & & \\
\hline G2 & 24 & G2 & & & NSD & & & & & & & \\
\hline G2 & 25 & 12 & & & NSD & & & & & & & \\
\hline G2 & 26 & J4 & & & NSD & & & & & & & \\
\hline G2 & 27 & $\mathrm{H} 4$ & & & NSD & & & & & & & \\
\hline G2 & 28 & $\mathrm{~F} 4$ & & & NSD & & & & & & & \\
\hline G2 & 29 & D4 & & & NSD & & & & & & & \\
\hline G2 & 30 & B4 & & & NSD & & & & & & & \\
\hline G2 & 31 & A7 & & & NSD & & & & & & & \\
\hline G2 & 32 & $\mathrm{C} 7$ & & & NSD & & & & & & & \\
\hline G2 & 33 & E7 & & & NSD & & & & & & & \\
\hline G2 & 34 & G7 & & & NSD & & & & & & & \\
\hline
\end{tabular}

Count Categories

AFB $>5,3: 1$

PAS

PCMEF-US

Asbestos Fibers and Bundles > 5um and 3:1

AS>5, 3:1 PCMEF-ISO

PCMES-ISO

PCM Equivalent Fibers-NIOSH

PCM Equivalent Structures-NIOSH
Asbestos Structures $>5$ um and 3:1

PCM Equivalent Fibers-ISO

PCM Equivalent Structures-ISO

Total Asbestos Structures 
Phone: (206) 781-0155

7619 6th Ave Nw

ISO 10312, Direct Raw Data

Job Number: 070434

SEA

Report Number: 070434R06

Client: Idaho National Laboratory

Date Received: 4/23/2007

Project Name: RARE

Client Sample No: Lab blank \#1

Lab/Cor Sample No: S5

Client Description:

Date Sampled:

$4 / 16 / 2007$

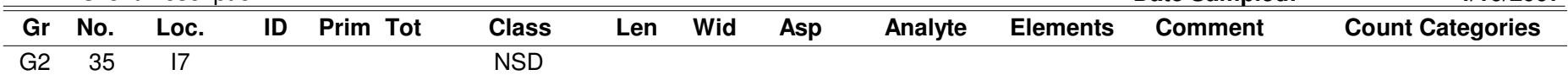

Count Categories

AFB $>5,3: 1$

PAS

PCMEF-US

PCMES-US
Asbestos Fibers and Bundles > 5um and 3:1

Primary Asbestos Structures

PCM Equivalent Fibers-NIOSH

PCM Equivalent Structures-NIOSH
AS>5, 3:1 PCMEF-ISO PCMES-ISO TAS
Asbestos Structures $>5$ um and 3:1 PCM Equivalent Fibers-ISO PCM Equivalent Structures-ISO Total Asbestos Structures 
Phone: (206) 781-0155

Fax: (206) 789-8424

7619 6th Ave Nw

ISO 10312, Direct Raw Data

Job Number: 070434

SEA

Report Number: 070434R06

Client: Idaho National Laboratory

Date Received: 4/23/2007

Project Name: RARE

Client Sample No: Lab blank \#2

Lab/Cor Sample No: S6

Client Description:

Date Sampled:

$4 / 16 / 2007$

\begin{tabular}{|c|c|c|c|c|c|c|c|c|c|c|c|c|}
\hline $\mathrm{Gr}$ & No. & Loc. & ID & Prim Tot & Class & Len & Wid & Asp & Analyte & Elements & Comment & Count Categories \\
\hline G1 & 1 & $\mathrm{~A} 2$ & & & NSD & & & & & & & \\
\hline G1 & 2 & $\mathrm{C} 2$ & & & NSD & & & & & & & \\
\hline G1 & 3 & E2 & & & NSD & & & & & & & \\
\hline G1 & 4 & G2 & & & NSD & & & & & & & \\
\hline G1 & 5 & 12 & & & NSD & & & & & & & \\
\hline G1 & 6 & J4 & & & NSD & & & & & & & \\
\hline G1 & 7 & $\mathrm{H} 4$ & & & NSD & & & & & & & \\
\hline G1 & 8 & $\mathrm{~F} 4$ & & & NSD & & & & & & & \\
\hline G1 & 9 & D4 & & & NSD & & & & & & & \\
\hline G1 & 10 & B4 & & & NSD & & & & & & & \\
\hline G1 & 11 & A7 & & & NSD & & & & & & & \\
\hline G1 & 12 & C7 & & & NSD & & & & & & & \\
\hline G1 & 13 & E7 & & & NSD & & & & & & & \\
\hline G1 & 14 & G7 & & & NSD & & & & & & & \\
\hline G1 & 15 & 17 & & & NSD & & & & & & & \\
\hline G1 & 16 & J9 & & & NSD & & & & & & & \\
\hline G1 & 17 & $\mathrm{H} 9$ & & & NSD & & & & & & & \\
\hline G1 & 18 & F9 & & & NSD & & & & & & & \\
\hline G1 & 19 & D9 & & & NSD & & & & & & & \\
\hline G1 & 20 & B9 & & & NSD & & & & & & & \\
\hline G2 & 21 & $\mathrm{~A} 2$ & & & NSD & & & & & & & \\
\hline G2 & 22 & $\mathrm{C} 2$ & & & NSD & & & & & & & \\
\hline G2 & 23 & E2 & & & NSD & & & & & & & \\
\hline G2 & 24 & G2 & & & NSD & & & & & & & \\
\hline G2 & 25 & 12 & & & NSD & & & & & & & \\
\hline G2 & 26 & J4 & & & NSD & & & & & & & \\
\hline G2 & 27 & $\mathrm{H} 4$ & & & NSD & & & & & & & \\
\hline G2 & 28 & F4 & & & NSD & & & & & & & \\
\hline G2 & 29 & D4 & & & NSD & & & & & & & \\
\hline G2 & 30 & B4 & & & NSD & & & & & & & \\
\hline G2 & 31 & A7 & & & NSD & & & & & & & \\
\hline G2 & 32 & $\mathrm{C} 7$ & & & NSD & & & & & & & \\
\hline G2 & 33 & E7 & & & NSD & & & & & & & \\
\hline G2 & 34 & G7 & & & NSD & & & & & & & \\
\hline
\end{tabular}

Count Categories

AFB $>5,3: 1$

PAS

PCMEF-US

Asbestos Fibers and Bundles > 5um and 3:1

AS>5, 3:1 PCMEF-ISO

PCMES-ISO

PCM Equivalent Fibers-NIOSH

PCM Equivalent Structures-NIOSH
TAS
Asbestos Structures $>5$ um and 3:1

PCM Equivalent Fibers-ISO

PCM Equivalent Structures-ISO

Total Asbestos Structures 
Phone: (206) 781-0155

7619 6th Ave Nw

ISO 10312, Direct Raw Data

Job Number: 070434

SEA

Report Number: 070434R06

Client: Idaho National Laboratory

Date Received: 4/23/2007

Project Name: RARE

Client Sample No: Lab blank \#2

Lab/Cor Sample No: S6

Client Description:

Date Sampled:

$4 / 16 / 2007$

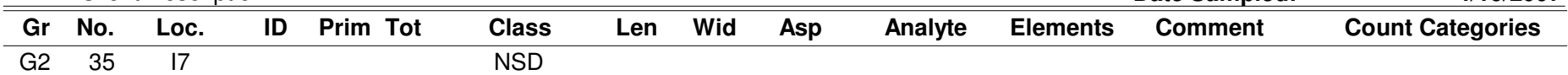

Count Categories

AFB $>5,3: 1$

PAS

PCMEF-US

PCMES-US
Asbestos Fibers and Bundles > 5um and 3:1

Primary Asbestos Structures

PCM Equivalent Fibers-NIOSH

PCM Equivalent Structures-NIOSH
AS>5, 3:1 PCMEF-ISO PCMES-ISO TAS
Asbestos Structures >5um and 3:1

PCM Equivalent Fibers-ISO

PCM Equivalent Structures-ISO

Total Asbestos Structures 
Phone: (206) 781-0155

Fax: (206) 789-8424

7619 6th Ave Nw

ISO 10312, Direct Raw Data

Job Number: 070434

SEA

Report Number: 070434R06

Client: Idaho National Laboratory

Date Received: 4/23/2007

Project Name: RARE

Client Sample No: Lot blank \#1

Lab/Cor Sample No: S7

Client Description:

Date Sampled:

$4 / 16 / 2007$

\begin{tabular}{|c|c|c|c|c|c|c|c|c|c|c|c|c|}
\hline $\mathrm{Gr}$ & No. & Loc. & ID & Prim Tot & Class & Len & Wid & Asp & Analyte & Elements & Comment & Count Categories \\
\hline G1 & 1 & $\mathrm{~A} 2$ & & & NSD & & & & & & & \\
\hline G1 & 2 & $\mathrm{C} 2$ & & & NSD & & & & & & & \\
\hline G1 & 3 & E2 & & & NSD & & & & & & & \\
\hline G1 & 4 & G2 & & & NSD & & & & & & & \\
\hline G1 & 5 & 12 & & & NSD & & & & & & & \\
\hline G1 & 6 & J4 & & & NSD & & & & & & & \\
\hline G1 & 7 & $\mathrm{H} 4$ & & & NSD & & & & & & & \\
\hline G1 & 8 & $\mathrm{~F} 4$ & & & NSD & & & & & & & \\
\hline G1 & 9 & D4 & & & NSD & & & & & & & \\
\hline G1 & 10 & B4 & & & NSD & & & & & & & \\
\hline G1 & 11 & A7 & & & NSD & & & & & & & \\
\hline G1 & 12 & C7 & & & NSD & & & & & & & \\
\hline G1 & 13 & E7 & & & NSD & & & & & & & \\
\hline G1 & 14 & G7 & & & NSD & & & & & & & \\
\hline G1 & 15 & 17 & & & NSD & & & & & & & \\
\hline G1 & 16 & $\mathrm{H} 9$ & & & NSD & & & & & & & \\
\hline G1 & 17 & F9 & & & NSD & & & & & & & \\
\hline G1 & 18 & D9 & & & NSD & & & & & & & \\
\hline G1 & 19 & B9 & & & NSD & & & & & & & \\
\hline G2 & 20 & $\mathrm{~A} 2$ & & & NSD & & & & & & & \\
\hline G2 & 21 & $\mathrm{C} 2$ & & & NSD & & & & & & & \\
\hline G2 & 22 & E2 & & & NSD & & & & & & & \\
\hline G2 & 23 & G2 & & & NSD & & & & & & & \\
\hline G2 & 24 & 12 & & & NSD & & & & & & & \\
\hline G2 & 25 & J4 & & & NSD & & & & & & & \\
\hline G2 & 26 & $\mathrm{H} 4$ & & & NSD & & & & & & & \\
\hline G2 & 27 & F4 & & & NSD & & & & & & & \\
\hline G2 & 28 & D4 & & & NSD & & & & & & & \\
\hline G2 & 29 & B4 & & & NSD & & & & & & & \\
\hline G2 & 30 & A7 & & & NSD & & & & & & & \\
\hline G2 & 31 & C7 & & & NSD & & & & & & & \\
\hline G2 & 32 & E7 & & & NSD & & & & & & & \\
\hline G2 & 33 & G7 & & & NSD & & & & & & & \\
\hline G2 & 34 & 17 & & & NSD & & & & & & & \\
\hline
\end{tabular}

Count Categories

AFB $>5,3: 1$

PAS

PCMEF-US

Asbestos Fibers and Bundles > 5um and 3:1

AS>5, 3:1 PCMEF-ISO

PCMES-ISO

PCM Equivalent Fibers-NIOSH

PCM Equivalent Structures-NIOSH
TAS
Asbestos Structures $>5$ um and 3:1

PCM Equivalent Fibers-ISO

PCM Equivalent Structures-ISO

Total Asbestos Structures 
Phone: (206) 781-0155

7619 6th Ave Nw

ISO 10312, Direct Raw Data

Job Number: 070434

SEA

Report Number: 070434R06

Client: Idaho National Laboratory

Date Received: 4/23/2007

Project Name: RARE

Client Sample No: Lot blank \#1

Lab/Cor Sample No: S7

Client Description:

Date Sampled:

$4 / 16 / 2007$

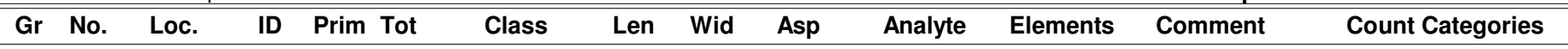

G2 $35 \quad \mathrm{H} 9$

Asbestos Fibers and Bundles > 5um and 3:1

Primary Asbestos Structures

PCM Equivalent Fibers-NIOSH

PCMEF-US

PCM Equivalent Structures-NIOSH
AS>5, 3:1 PCMEF-ISO PCMES-ISO TAS
Asbestos Structures $>5$ um and 3:1 PCM Equivalent Fibers-ISO PCM Equivalent Structures-ISO Total Asbestos Structures 
Phone: (206) 781-0155

Fax: (206) 789-8424

7619 6th Ave Nw

ISO 10312, Direct Raw Data

Job Number: 070434

SEA

Report Number: 070434R06

Client: Idaho National Laboratory

Date Received: 4/23/2007

Project Name: RARE

Client Sample No: Lot blank \#2

Lab/Cor Sample No: S8

Client Description:

Date Sampled:

$4 / 16 / 2007$

\begin{tabular}{|c|c|c|c|c|c|c|c|c|c|c|c|c|}
\hline $\mathrm{Gr}$ & No. & Loc. & ID & Prim Tot & Class & Len & Wid & Asp & Analyte & Elements & Comment & Count Categories \\
\hline G1 & 1 & $\mathrm{~A} 2$ & & & NSD & & & & & & & \\
\hline G1 & 2 & $\mathrm{C} 2$ & & & NSD & & & & & & & \\
\hline G1 & 3 & E2 & & & NSD & & & & & & & \\
\hline G1 & 4 & G2 & & & NSD & & & & & & & \\
\hline G1 & 5 & 12 & & & NSD & & & & & & & \\
\hline G1 & 6 & J4 & & & NSD & & & & & & & \\
\hline G1 & 7 & $\mathrm{H} 4$ & & & NSD & & & & & & & \\
\hline G1 & 8 & $\mathrm{~F} 4$ & & & NSD & & & & & & & \\
\hline G1 & 9 & D4 & & & NSD & & & & & & & \\
\hline G1 & 10 & B4 & & & NSD & & & & & & & \\
\hline G1 & 11 & A7 & & & NSD & & & & & & & \\
\hline G1 & 12 & C7 & & & NSD & & & & & & & \\
\hline G1 & 13 & E7 & & & NSD & & & & & & & \\
\hline G1 & 14 & G7 & & & NSD & & & & & & & \\
\hline G1 & 15 & 17 & & & NSD & & & & & & & \\
\hline G1 & 16 & J9 & & & NSD & & & & & & & \\
\hline G1 & 17 & $\mathrm{H} 9$ & & & NSD & & & & & & & \\
\hline G1 & 18 & F9 & & & NSD & & & & & & & \\
\hline G1 & 19 & D9 & & & NSD & & & & & & & \\
\hline G1 & 20 & B9 & & & NSD & & & & & & & \\
\hline G2 & 21 & $\mathrm{~A} 2$ & & & NSD & & & & & & & \\
\hline G2 & 22 & $\mathrm{C} 2$ & & & NSD & & & & & & & \\
\hline G2 & 23 & E2 & & & NSD & & & & & & & \\
\hline G2 & 24 & G2 & & & NSD & & & & & & & \\
\hline G2 & 25 & 12 & & & NSD & & & & & & & \\
\hline G2 & 26 & J4 & & & NSD & & & & & & & \\
\hline G2 & 27 & $\mathrm{H} 4$ & & & NSD & & & & & & & \\
\hline G2 & 28 & F4 & & & NSD & & & & & & & \\
\hline G2 & 29 & D4 & & & NSD & & & & & & & \\
\hline G2 & 30 & B4 & & & NSD & & & & & & & \\
\hline G2 & 31 & A7 & & & NSD & & & & & & & \\
\hline G2 & 32 & $\mathrm{C} 7$ & & & NSD & & & & & & & \\
\hline G2 & 33 & E7 & & & NSD & & & & & & & \\
\hline G2 & 34 & G7 & & & NSD & & & & & & & \\
\hline
\end{tabular}

Count Categories

AFB $>5,3: 1$

PAS

PCMEF-US

Asbestos Fibers and Bundles > 5um and 3:1

AS>5, 3:1 PCMEF-ISO

PCMES-ISO

PCM Equivalent Fibers-NIOSH

PCM Equivalent Structures-NIOSH
TAS
Asbestos Structures $>5$ um and 3:1

PCM Equivalent Fibers-ISO

PCM Equivalent Structures-ISO

Total Asbestos Structures 
Phone: (206) 781-0155

7619 6th Ave Nw

ISO 10312, Direct Raw Data

Job Number: 070434

SEA

Report Number: 070434R06

Client: Idaho National Laboratory

Date Received: 4/23/2007

Project Name: RARE

Client Sample No: Lot blank \#2

Lab/Cor Sample No: $\mathbf{S 8}$

Client Description:

Date Sampled

$4 / 16 / 2007$

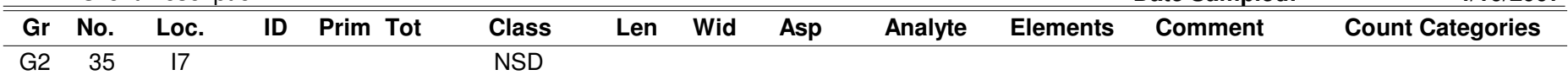

Count Categories

AFB $>5,3: 1$

PAS

PCMEF-US

PCMES-US
Asbestos Fibers and Bundles > 5um and 3:1

Primary Asbestos Structures

PCM Equivalent Fibers-NIOSH

PCM Equivalent Structures-NIOSH
AS>5, 3:1 PCMEF-ISO PCMES-ISO TAS
Asbestos Structures >5um and 3:1

PCM Equivalent Fibers-ISO

PCM Equivalent Structures-ISO

Total Asbestos Structures 
Phone: (206) 781-0155

Fax: (206) 789-8424

7619 6th Ave Nw

ISO 10312, Direct Raw Data

Job Number: 070434

SEA

Report Number: 070434R06

Client: Idaho National Laboratory

Date Received: 4/23/2007

Project Name: RARE

Client Sample No: cleaning protocol 1

Lab/Cor Sample No: $\mathbf{S 9}$

Client Description:

Date Sampled

$4 / 16 / 2007$

\begin{tabular}{|c|c|c|c|c|c|c|c|c|c|c|c|c|}
\hline $\mathrm{Gr}$ & No. & Loc. & ID & Prim Tot & Class & Len & Wid & Asp & Analyte & Elements & Comment & Count Categories \\
\hline G1 & 1 & $\mathrm{~A} 2$ & & & NSD & & & & & & & \\
\hline G1 & 2 & $\mathrm{C} 2$ & & & NSD & & & & & & & \\
\hline G1 & 3 & E2 & & & NSD & & & & & & & \\
\hline G1 & 4 & G2 & & & NSD & & & & & & & \\
\hline G1 & 5 & 12 & & & NSD & & & & & & & \\
\hline G1 & 6 & J4 & & & NSD & & & & & & & \\
\hline G1 & 7 & $\mathrm{H} 4$ & & & NSD & & & & & & & \\
\hline G1 & 8 & $\mathrm{~F} 4$ & & & NSD & & & & & & & \\
\hline G1 & 9 & D4 & & & NSD & & & & & & & \\
\hline G1 & 10 & B4 & & & NSD & & & & & & & \\
\hline G1 & 11 & A7 & & & NSD & & & & & & & \\
\hline G1 & 12 & C7 & & & NSD & & & & & & & \\
\hline G1 & 13 & E7 & & & NSD & & & & & & & \\
\hline G1 & 14 & G7 & & & NSD & & & & & & & \\
\hline G1 & 15 & 17 & & & NSD & & & & & & & \\
\hline G1 & 16 & J9 & & & NSD & & & & & & & \\
\hline G1 & 17 & $\mathrm{H} 9$ & & & NSD & & & & & & & \\
\hline G1 & 18 & F9 & & & NSD & & & & & & & \\
\hline G1 & 19 & D9 & & & NSD & & & & & & & \\
\hline G1 & 20 & B9 & & & NSD & & & & & & & \\
\hline G2 & 21 & $\mathrm{~A} 2$ & & & NSD & & & & & & & \\
\hline G2 & 22 & $\mathrm{C} 2$ & & & NSD & & & & & & & \\
\hline G2 & 23 & E2 & & & NSD & & & & & & & \\
\hline G2 & 24 & G2 & & & NSD & & & & & & & \\
\hline G2 & 25 & 12 & & & NSD & & & & & & & \\
\hline G2 & 26 & $\mathrm{H} 4$ & & & NSD & & & & & & & \\
\hline G2 & 27 & F4 & & & NSD & & & & & & & \\
\hline G2 & 28 & D4 & & & NSD & & & & & & & \\
\hline G2 & 29 & B4 & & & NSD & & & & & & & \\
\hline G2 & 30 & A7 & & & NSD & & & & & & & \\
\hline G2 & 31 & C7 & & & NSD & & & & & & & \\
\hline G2 & 32 & E7 & & & NSD & & & & & & & \\
\hline G2 & 33 & G7 & & & NSD & & & & & & & \\
\hline G2 & 34 & 17 & & & NSD & & & & & & & \\
\hline
\end{tabular}

Count Categories

AFB $>5,3: 1$

PAS

PCMEF-US

Asbestos Fibers and Bundles > 5um and 3:1

AS>5, 3:1 PCMEF-ISO

PCMES-ISO

PCM Equivalent Fibers-NIOSH

PCM Equivalent Structures-NIOSH
TAS
Asbestos Structures $>5$ um and 3:1

PCM Equivalent Fibers-ISO

PCM Equivalent Structures-ISO

Total Asbestos Structures 
Phone: (206) 781-0155

7619 6th Ave Nw

Job Number: 070434

SEA

ISO 10312, Direct Raw Data

Client: Idaho National Laboratory

Name: RARE

Client Sample No: cleaning protocol 1

Client Description:

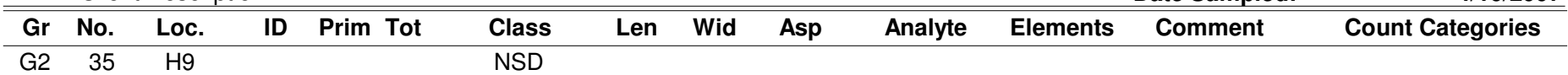

Count Categories

AFB $>5,3: 1$

PAS

PCMEF-US

PCMES-US
Asbestos Fibers and Bundles > 5um and 3:1

Primary Asbestos Structures

PCM Equivalent Fibers-NIOSH

PCM Equivalent Structures-NIOSH
AS>5, 3:1 PCMEF-ISO PCMES-ISO TAS
Report Number: 070434R06

Fax: (206) 789-8424

http://www.labcor.net

Date Received: 4/23/2007
Lab/Cor Sample No: S9 Date Sampled $4 / 16 / 2007$

Asbestos Structures $>5$ um and 3:1 PCM Equivalent Fibers-ISO PCM Equivalent Structures-ISO Total Asbestos Structures 
Phone: (206) 781-0155

7619 6th Ave Nw

ISO 10312, Direct Raw Data

Job Number: 070434

SEA

Report Number: 070434R06

Client: Idaho National Laboratory

Date Received: 4/23/2007

Project Name: RARE

Client Sample No: cleaning protocol 2

Lab/Cor Sample No: S10

Client Description:

Date Sampled:

$4 / 16 / 2007$

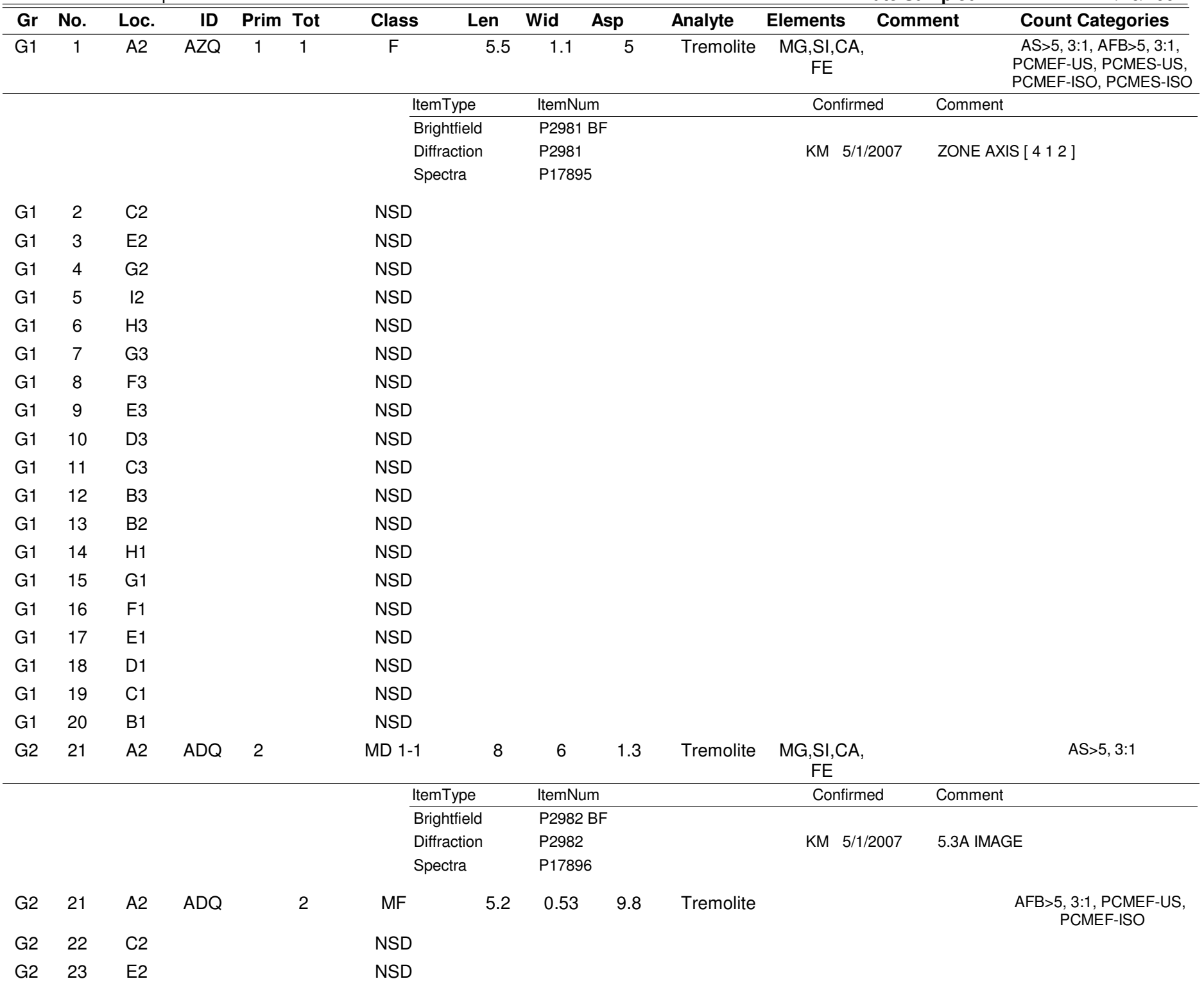

Count Categories

$A F B>5,3: 1$

Asbestos Fibers and Bundles > 5um and 3:1

PAS

PCMEF-US

Primary Asbestos Structures

PCM Equivalent Fibers-NIOSH

PCMES-US

PCM Equivalent Structures-NIOSH
AS $>5,3: 1$ PCMEF-ISO PCMES-ISO

TAS
Asbestos Structures $>5$ um and 3:1

PCM Equivalent Fibers-ISO

PCM Equivalent Structures-ISO

Total Asbestos Structures 
Job Number: 070434

SEA

ISO 10312, Direct Raw Data

Client: Idaho National Laboratory

Report Number: 070434R06

Project Name: RARE

Date Received: 4/23/2007

Client Sample No: cleaning protocol 2

Lab/Cor Sample No: S10

Client Description:

Date Sampled:

$4 / 16 / 2007$

\begin{tabular}{|c|c|c|c|c|c|c|c|c|c|c|c|c|}
\hline Gr & No. & Loc. & ID & Prim Tot & Class & Len & Wid & Asp & Analyte & Elements & Comment & Count Categories \\
\hline G2 & 24 & G2 & & & NSD & & & & & & & \\
\hline G2 & 25 & 12 & & & NSD & & & & & & & \\
\hline G2 & 26 & $\mathrm{~J} 4$ & & & NSD & & & & & & & \\
\hline G2 & 27 & $\mathrm{H} 4$ & & & NSD & & & & & & & \\
\hline G2 & 28 & $\mathrm{~F} 4$ & & & NSD & & & & & & & \\
\hline G2 & 29 & D4 & & & NSD & & & & & & & \\
\hline G2 & 30 & B4 & & & NSD & & & & & & & \\
\hline G2 & 31 & A7 & & & NSD & & & & & & & \\
\hline G2 & 32 & $\mathrm{C7}$ & & & NSD & & & & & & & \\
\hline G2 & 33 & E7 & & & NSD & & & & & & & \\
\hline G2 & 34 & G7 & & & NSD & & & & & & & \\
\hline G2 & 35 & 17 & & & NSD & & & & & & & \\
\hline
\end{tabular}

Asbestos Fibers and Bundles > 5um and 3:1

Primary Asbestos Structures

PCM Equivalent Fibers-NIOSH

PCMEF-US

PCM Equivalent Structures-NIOSH
AS $>5,3: 1$ PCMEF-ISO PCMES-ISO TAS
Asbestos Structures $>5$ um and 3:1 PCM Equivalent Fibers-ISO PCM Equivalent Structures-ISO Total Asbestos Structures 
Phone: (206) 781-0155

Fax: (206) 789-8424

7619 6th Ave Nw

ISO 10312, Direct Raw Data

Job Number: 070434

SEA

Report Number: 070434R06

Client: Idaho National Laboratory

Date Received: 4/23/2007

Project Name: RARE

Client Sample No: Sand blank

Lab/Cor Sample No: S11

Client Description:

Date Sampled

$4 / 16 / 2007$

\begin{tabular}{|c|c|c|c|c|c|c|c|c|c|c|c|c|}
\hline $\mathrm{Gr}$ & No. & Loc. & ID & Prim Tot & Class & Len & Wid & Asp & Analyte & Elements & Comment & Count Categories \\
\hline G1 & 1 & $\mathrm{C} 2$ & & & NSD & & & & & & & \\
\hline G1 & 2 & E2 & & & NSD & & & & & & & \\
\hline G1 & 3 & G2 & & & NSD & & & & & & & \\
\hline G1 & 4 & 12 & & & NSD & & & & & & & \\
\hline G1 & 5 & $\mathrm{~J} 4$ & & & NSD & & & & & & & \\
\hline G1 & 6 & $\mathrm{H} 4$ & & & NSD & & & & & & & \\
\hline G1 & 7 & $\mathrm{~F} 4$ & & & NSD & & & & & & & \\
\hline G1 & 8 & D4 & & & NSD & & & & & & & \\
\hline G1 & 9 & B4 & & & NSD & & & & & & & \\
\hline G1 & 10 & A7 & & & NSD & & & & & & & \\
\hline G1 & 11 & $\mathrm{C} 7$ & & & NSD & & & & & & & \\
\hline G1 & 12 & E7 & & & NSD & & & & & & & \\
\hline G1 & 13 & G7 & & & NSD & & & & & & & \\
\hline G1 & 14 & 17 & & & NSD & & & & & & & \\
\hline G1 & 15 & J9 & & & NSD & & & & & & & \\
\hline G1 & 16 & $\mathrm{H} 9$ & & & NSD & & & & & & & \\
\hline G1 & 17 & F9 & & & NSD & & & & & & & \\
\hline G1 & 18 & D9 & & & NSD & & & & & & & \\
\hline G1 & 19 & B9 & & & NSD & & & & & & & \\
\hline G2 & 20 & $\mathrm{~A} 2$ & & & NSD & & & & & & & \\
\hline G2 & 21 & $\mathrm{C} 2$ & & & NSD & & & & & & & \\
\hline G2 & 22 & E2 & & & NSD & & & & & & & \\
\hline G2 & 23 & G2 & & & NSD & & & & & & & \\
\hline G2 & 24 & 12 & & & NSD & & & & & & & \\
\hline G2 & 25 & J4 & & & NSD & & & & & & & \\
\hline G2 & 26 & $\mathrm{H} 4$ & & & NSD & & & & & & & \\
\hline G2 & 27 & F4 & & & NSD & & & & & & & \\
\hline G2 & 28 & D4 & & & NSD & & & & & & & \\
\hline G2 & 29 & B4 & & & NSD & & & & & & & \\
\hline G2 & 30 & A7 & & & NSD & & & & & & & \\
\hline G2 & 31 & C7 & & & NSD & & & & & & & \\
\hline G2 & 32 & E7 & & & NSD & & & & & & & \\
\hline G2 & 33 & G7 & & & NSD & & & & & & & \\
\hline G2 & 34 & 17 & & & NSD & & & & & & & \\
\hline
\end{tabular}

Count Categories

AFB $>5,3: 1$

PAS

PCMEF-US

Asbestos Fibers and Bundles > 5um and 3:1

AS>5, 3:1 PCMEF-ISO

PCMES-ISO

PCM Equivalent Fibers-NIOSH

PCM Equivalent Structures-NIOSH
TAS
Asbestos Structures $>5$ um and 3:1

PCM Equivalent Fibers-ISO

PCM Equivalent Structures-ISO

Total Asbestos Structures 
Phone: (206) 781-0155

7619 6th Ave Nw

Job Number: 070434

SEA

ISO 10312, Direct Raw Data

Client: Idaho National Laboratory

Report Number: 070434R06

Name: RARE

Client Sample No: Sand blank

Lab/Cor Sample No: S11

Client Description:

\begin{tabular}{|c|c|c|c|c|c|c|c|c|c|c|c|c|}
\hline Gr & No. & Loc. & ID & Prim Tot & Class & Len & Wid & Asp & Analyte & Elements & Comment & Count Categories \\
\hline G2 & 35 & H9 & & & NSD & & & & & & & \\
\hline
\end{tabular}

Count Categories

AFB $>5,3: 1$

PAS

PCMEF-US

PCMES-US
Asbestos Fibers and Bundles > 5um and 3:1

Primary Asbestos Structures

PCM Equivalent Fibers-NIOSH

PCM Equivalent Structures-NIOSH
AS $>5,3: 1$ PCMEF-ISO PCMES-ISO TAS
Fax: (206) 789-8424

http://www.labcor.net 
Job Number: 070434

SEA

ISO 10312, Direct Raw Data

Client: Idaho National Laboratory

Report Number: 070434R06

Name: RARE

Client Sample No: FB-4-R1

Lab/Cor Sample No: S12

Client Description:

Date Sampled:

$4 / 16 / 2007$

\begin{tabular}{|c|c|c|c|c|c|c|c|c|c|c|c|c|c|}
\hline \multirow{2}{*}{$\begin{array}{l}\mathrm{Gr} \\
\mathrm{G} 1\end{array}$} & \multirow{2}{*}{ No. } & \multirow{2}{*}{$\begin{array}{l}\text { Loc. } \\
\text { E4 }\end{array}$} & \multirow{2}{*}{$\begin{array}{r}\text { ID } \\
A Z Q\end{array}$} & \multirow{2}{*}{$\frac{\text { Prim }}{1}$} & \multirow[t]{2}{*}{ Tot } & Class & \multirow{2}{*}{$\frac{\text { Len }}{20.5}$} & \multirow{2}{*}{$\begin{array}{r}\text { Wid } \\
9\end{array}$} & Asp & \multirow{2}{*}{$\frac{\text { Analyte }}{\text { Tremolite }}$} & \multirow{2}{*}{$\begin{array}{c}\text { Elements } \\
\text { Mg, } \mathrm{Si}, \mathrm{Ca}, \\
\mathrm{Fe}\end{array}$} & lent & Count Categories \\
\hline & & & & & & MD 1-1 & & & 2.3 & & & & $\mathrm{AS}>5,3: 1$ \\
\hline & & & & & & \multicolumn{2}{|c|}{ ItemType } & \multicolumn{2}{|l|}{ ItemNum } & & Confirmed & \multicolumn{2}{|c|}{ Comment } \\
\hline & & & & & & \multicolumn{2}{|c|}{ Brightfield } & \multicolumn{2}{|c|}{ J3389 BF } & & \multirow{3}{*}{ KM 5/7/2007 } & \multirow{3}{*}{\multicolumn{2}{|c|}{ ZONE AXIS [ $\left.\begin{array}{lll}-2 & -1 & 1\end{array}\right]$}} \\
\hline & & & & & & & & J3389 & & & & & \\
\hline & & & & & & \multicolumn{2}{|c|}{ Spectra } & $\mathrm{J} 2849$ & & & & & \\
\hline G1 & 1 & E4 & $A Z Q$ & & 1 & MF & 20 & 0.75 & 26.7 & Tremolite & & & $\begin{array}{c}\text { AFB }>5,3: 1, \text { PCMEF-US, } \\
\text { PCMEF-ISO }\end{array}$ \\
\hline G1 & 2 & G4 & $A Z Q$ & 2 & 2 & B & 11.2 & 1.25 & 9 & Tremolite & & & $\begin{array}{l}\text { AFB }>5,3: 1, \text { AS }>5,3: 1, \\
\text { PCMES-US, PCMEF-US, } \\
\text { PCMES-ISO, PCMEF-ISO }\end{array}$ \\
\hline G1 & 3 & 14 & & & & NSD & & & & & & & \\
\hline G1 & 4 & A4 & & & & NSD & & & & & & & \\
\hline G1 & 5 & $\mathrm{C} 4$ & & & & NSD & & & & & & & \\
\hline G1 & 6 & $\mathrm{~A} 2$ & & & & NSD & & & & & & & \\
\hline G1 & 7 & $\mathrm{C} 2$ & & & & NSD & & & & & & & \\
\hline \multirow[t]{5}{*}{ G1 } & 8 & E2 & $\mathrm{CDQ}$ & 3 & & CD 3-1 & 1.15 & 1 & 1.1 & Chrysotile & $\mathrm{Mg}, \mathrm{Si}$ & & \\
\hline & & & & & & \multirow{2}{*}{\multicolumn{2}{|c|}{$\begin{array}{l}\text { ItemType } \\
\text { Brightfield }\end{array}$}} & \multicolumn{2}{|l|}{ ItemNum } & & Confirmed & \multicolumn{2}{|c|}{ Comment } \\
\hline & & & & & & & ield & J3391 B & & & & & \\
\hline & & & & & & & & J3391 & & & KM 5/7/2007 & & \\
\hline & & & & & & & & J2851 & & & & & \\
\hline G1 & 8 & E2 & $\mathrm{CDQ}$ & & 3 & $\mathrm{CF}$ & 1.1 & 0.1 & 11 & Chrysotile & & & \\
\hline G1 & 8 & E2 & $\mathrm{CDQ}$ & & 4 & $\mathrm{CF}$ & 1 & 0.12 & 8.3 & Chrysotile & & & \\
\hline G1 & 8 & E2 & $\mathrm{CDQ}$ & & 5 & $\mathrm{CF}$ & 8 & 0.1 & 80 & Chrysotile & & & $A F B>5,3: 1$ \\
\hline G1 & 8 & E2 & $C D$ & 4 & 6 & B & 1.2 & 0.2 & 6 & Chrysotile & & & \\
\hline G1 & 8 & E2 & $C D$ & 5 & 7 & B & 1.25 & 0.2 & 6.2 & Chrysotile & & & \\
\hline G1 & 9 & G2 & & & & NSD & & & & & & & \\
\hline G1 & 10 & 12 & $A D Q$ & 6 & 8 & $\mathrm{~F}$ & 2.7 & 0.51 & 5.3 & Tremolite & $\begin{array}{l}\mathrm{Mg}, \mathrm{Si}, \mathrm{Ca} \\
\mathrm{Fe}\end{array}$ & & \\
\hline & & & & & & & & ItemNum & & & Confirmed & Comr & \\
\hline & & & & & & & & J3392 B & & & & & \\
\hline & & & & & & & & J3392 & & & KM 5/7/2007 & $5.3 \mathrm{~A}$ & \\
\hline & & & & & & & & J2852 & & & & & \\
\hline G1 & 11 & J7 & $\mathrm{CMQ}$ & 7 & & CD 2-0 & 2.2 & 1.2 & 1.8 & Chrysotile & $\mathrm{Mg}, \mathrm{Si}$ & & \\
\hline G1 & 11 & J7 & $\mathrm{CMQ}$ & & 9 & CF & 2.2 & 0.05 & 44 & Chrysotile & & & \\
\hline G1 & 11 & J7 & $\mathrm{CMQ}$ & & 10 & CF & 1.2 & 0.05 & 24 & Chrysotile & & & \\
\hline
\end{tabular}

Count Categories

$\mathrm{AFB}>5,3: 1$

PAS

PCMEF-US

PCMES-US
Asbestos Fibers and Bundles $>$ 5um and 3:1

Primary Asbestos Structures

PCM Equivalent Fibers-NIOSH

PCM Equivalent Structures-NIOSH
AS $>5,3: 1$ PCMEF-ISO PCMES-ISO TAS
Asbestos Structures $>5$ um and 3:1

PCM Equivalent Fibers-ISO

PCM Equivalent Structures-ISO

Total Asbestos Structures 
ISO 10312, Direct Raw Data

Job Number: 070434

SEA

Report Number: 070434R06

Client: Idaho National Laboratory

Date Received: 4/23/2007

Project Name: RARE

Client Sample No: FB-4-R1

Lab/Cor Sample No: S12

Client Description:

Date Sampled:

4/16/2007

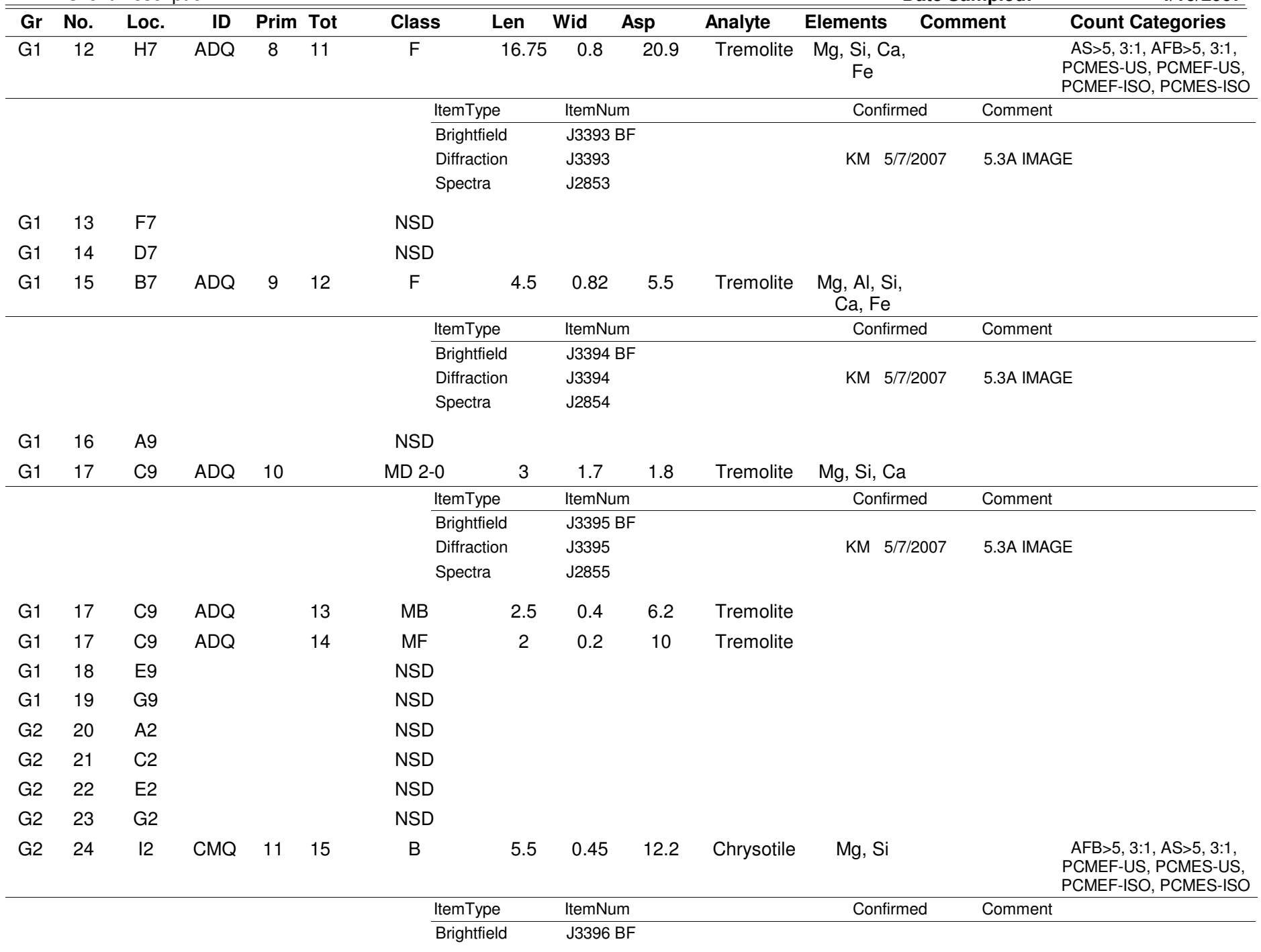

G2 $25 \quad J 4$

NSD

Count Categories

AFB $>5,3: 1$

PAS

PCMEF-US

PCMES-US
Asbestos Fibers and Bundles > 5um and 3:1

Primary Asbestos Structures

PCM Equivalent Fibers-NIOSH

PCM Equivalent Structures-NIOSH
AS $>5,3: 1$ PCMEF-ISO PCMES-ISO TAS
Asbestos Structures $>5$ um and 3:1

PCM Equivalent Fibers-ISO

PCM Equivalent Structures-ISO

Total Asbestos Structures 
Phone: (206) 781-0155

7619 6th Ave Nw

ISO 10312, Direct Raw Data

Job Number: 070434

SEA

Report Number: 070434R06

Client: Idaho National Laboratory

Date Received: 4/23/2007

Project Name: RARE

Client Sample No: FB-4-R1

Lab/Cor Sample No: S12

Client Description:

Date Sampled:

$4 / 16 / 2007$

\begin{tabular}{|c|c|c|c|c|c|c|c|c|c|c|c|c|c|}
\hline$\overline{\mathrm{Gr}}$ & No. & Loc. & IID & Prim & Tot & Class & Len & Wid & Asp & Analyte & Elements & Comment & Count Categories \\
\hline $\mathrm{G} 2$ & 26 & $\mathrm{H} 4$ & $A D Q$ & 12 & & MD 1-1 & 16.5 & 4 & 4.1 & Tremolite & $\begin{array}{l}\mathrm{Mg}, \mathrm{Si}, \mathrm{Ca} \\
\mathrm{Fe}\end{array}$ & & AS $>5,3: 1$, PCMES-US \\
\hline & & & & & & \multicolumn{2}{|c|}{ ItemType } & ItemNum & & & Confirme & Comment & \\
\hline & & & & & & \multicolumn{2}{|c|}{ Brightfield } & J3397 B & & & & & \\
\hline & & & & & & \multicolumn{2}{|c|}{ Spectra } & $\mathrm{J} 2856$ & & & & & \\
\hline G2 & 26 & $\mathrm{H} 4$ & $A D Q$ & & 16 & MF & 10 & 1.5 & 6.7 & Tremolite & & & $\begin{array}{c}\text { AFB }>5,3: 1, \text { PCMEF-US, } \\
\text { PCMEF-ISO }\end{array}$ \\
\hline $\mathrm{G} 2$ & 27 & $\mathrm{~F} 4$ & $\mathrm{AMQ}$ & 13 & 17 & $\mathrm{~F}$ & 4 & 0.3 & 13.3 & Tremolite & $\begin{array}{l}\mathrm{Mg}, \mathrm{Si}, \mathrm{Ca} \\
\mathrm{Fe}\end{array}$ & & \\
\hline $\mathrm{G} 2$ & 28 & A4 & $\mathrm{AMQ}$ & 14 & & MD 1-0 & 9 & 5.5 & 1.6 & Tremolite & $\begin{array}{l}\mathrm{Mg}, \mathrm{Si}, \mathrm{Ca} \\
\mathrm{Fe}\end{array}$ & & $A S>5,3: 1$ \\
\hline $\mathrm{G} 2$ & 28 & A4 & $\mathrm{AMQ}$ & & 18 & MB & 3 & 0.22 & 13.6 & Tremolite & & & \\
\hline G2 & 29 & D4 & & & & NSD & & & & & & & \\
\hline $\mathrm{G} 2$ & 30 & A7 & & & & NSD & & & & & & & \\
\hline $\mathrm{G} 2$ & 31 & $\mathrm{C} 7$ & & & & NSD & & & & & & & \\
\hline $\mathrm{G} 2$ & 32 & E7 & $\mathrm{AMQ}$ & 15 & 19 & $\mathrm{~F}$ & 2.2 & 0.35 & 6.3 & Tremolite & $\begin{array}{l}\mathrm{Mg}, \mathrm{Si}, \mathrm{Ca} \\
\mathrm{Fe}\end{array}$ & & \\
\hline G2 & 33 & G7 & $\mathrm{AMQ}$ & 16 & 20 & $\mathrm{~F}$ & 2.5 & 0.5 & 5 & Tremolite & $\begin{array}{l}\mathrm{Mg}, \mathrm{Si}, \mathrm{Ca} \\
\mathrm{Fe}\end{array}$ & & \\
\hline G2 & 34 & 17 & $\mathrm{CMQ}$ & 17 & 21 & $\mathrm{~F}$ & 0.65 & 0.08 & 8.1 & Chrysotile & & & \\
\hline $\mathrm{G} 2$ & 35 & $\mathrm{H} 9$ & & & & NSD & & & & & & & \\
\hline
\end{tabular}

Count Categories

AFB $>5,3: 1$

PAS

PCMEF-US

PCMES-US
Asbestos Fibers and Bundles > 5um and 3:1

Primary Asbestos Structures

PCM Equivalent Fibers-NIOSH

PCM Equivalent Structures-NIOSH
AS>5, 3:1 PCMEF-ISO PCMES-ISO TAS
Asbestos Structures $>5$ um and 3:1 PCM Equivalent Fibers-ISO PCM Equivalent Structures-ISO Total Asbestos Structures 
Phone: (206) 781-0155

7619 6th Ave Nw

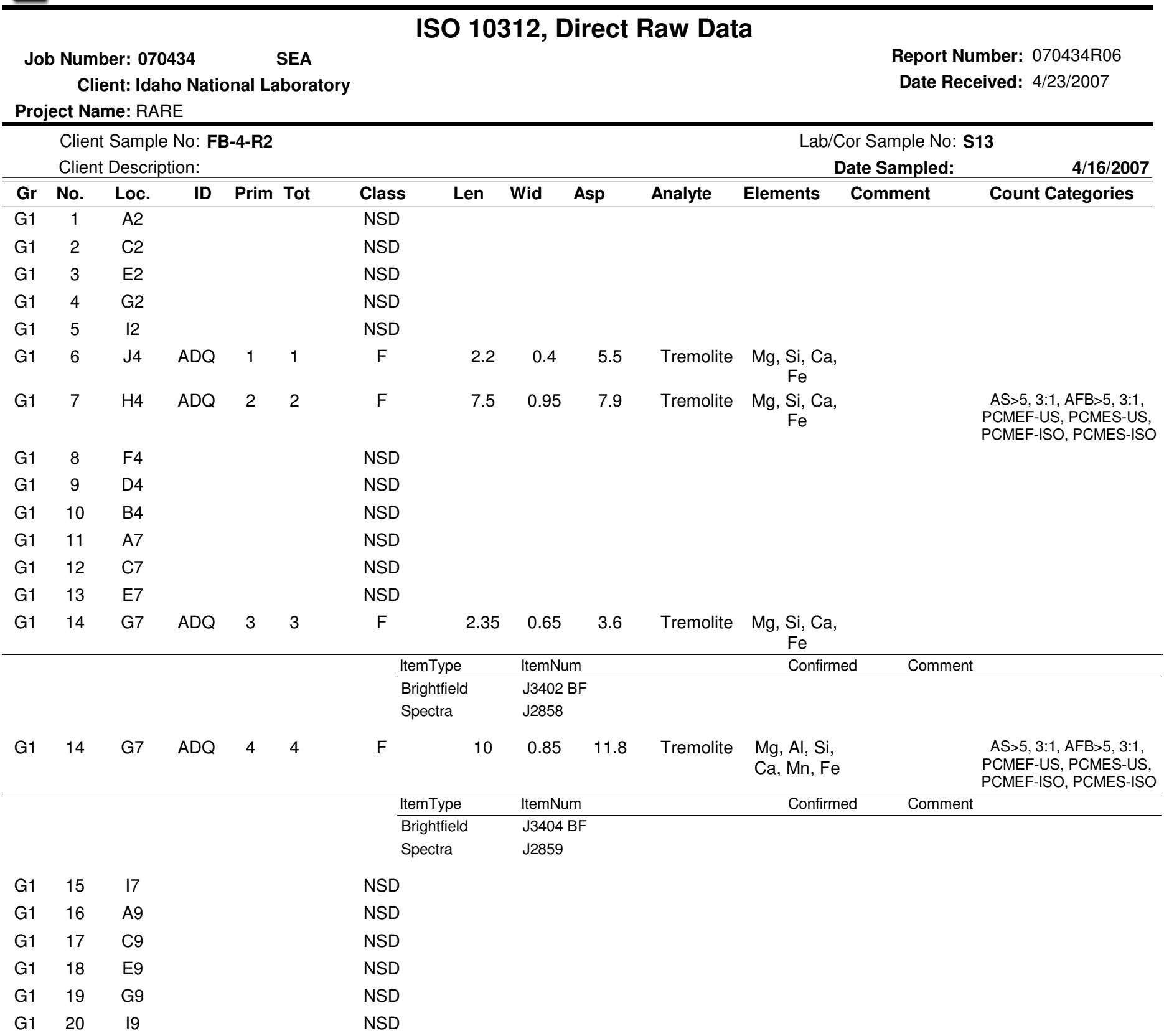

Count Categories

AFB $>5,3: 1$

PAS

PCMEF-US

PCMES-US
Asbestos Fibers and Bundles > 5um and 3:1

Primary Asbestos Structures

PCM Equivalent Fibers-NIOSH

PCM Equivalent Structures-NIOSH
AS $>5,3: 1$ PCMEF-ISO PCMES-ISO TAS
Asbestos Structures $>5$ um and 3:1 PCM Equivalent Fibers-ISO PCM Equivalent Structures-ISO Total Asbestos Structures 
Phone: (206) 781-0155

7619 6th Ave Nw

ISO 10312, Direct Raw Data

Job Number: 070434

SEA

Report Number: 070434R06

Client: Idaho National Laboratory

Date Received: 4/23/2007

Project Name: RARE

Client Sample No: FB-4-R2

Lab/Cor Sample No: S13

Client Description:

Date Sampled:

4/16/2007

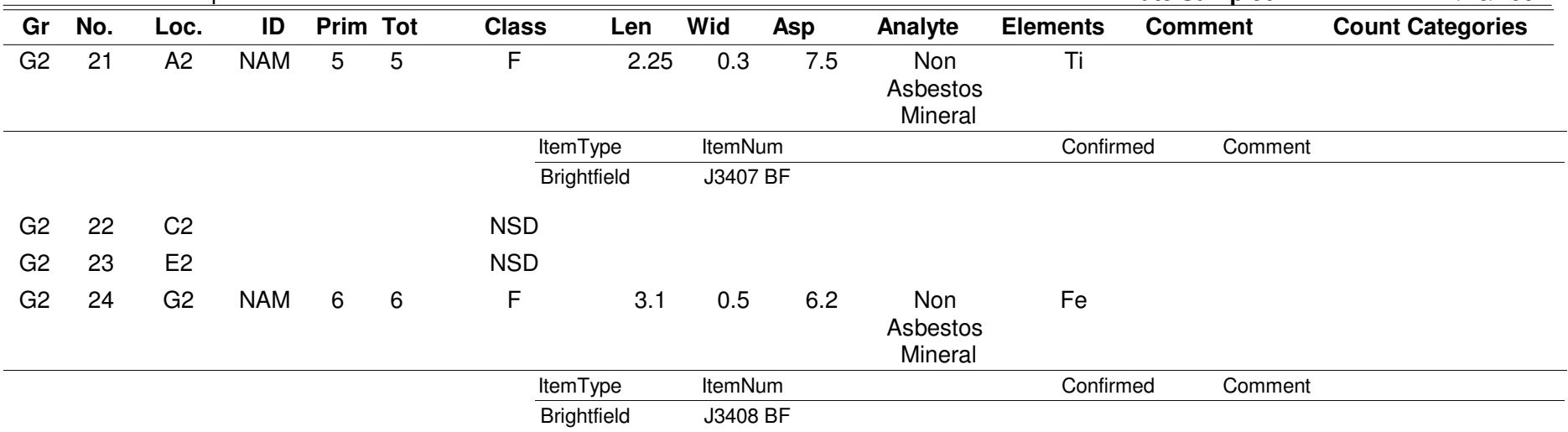

\begin{tabular}{|c|c|c|c|c|c|c|c|c|c|c|c|c|}
\hline G2 & 25 & 12 & $A D Q$ & 7 & & MD 1-0 & 4.2 & 2.7 & 1.6 & Tremolite & $\begin{array}{c}\mathrm{Mg}, \mathrm{Si}, \mathrm{Ca}, \\
\mathrm{Fe}\end{array}$ & \\
\hline & & & & & & \multicolumn{2}{|c|}{ ItemType } & \multicolumn{2}{|l|}{ ItemNum } & & Confirmed & Comment \\
\hline & & & & & & \multicolumn{2}{|r|}{ Brightfield } & \multicolumn{2}{|l|}{ J3409 BF } & & & \\
\hline & & & & & & \multicolumn{2}{|r|}{ Diffraction } & \multicolumn{2}{|l|}{ J3409 } & & KM 5/8/2007 & \multirow[t]{2}{*}{ 5.3A IMAGE } \\
\hline & & & & & & \multicolumn{2}{|c|}{ Spectra } & \multicolumn{2}{|l|}{$\mathrm{J} 2860$} & & & \\
\hline G2 & 25 & 12 & $A D Q$ & & 7 & MF & 4 & 0.5 & 8 & Tremolite & & \\
\hline G2 & 26 & $\mathrm{~J} 4$ & & & & \multicolumn{2}{|l|}{ NSD } & & & & & \\
\hline G2 & 27 & $\mathrm{H} 4$ & & & & \multicolumn{2}{|l|}{ NSD } & & & & & \\
\hline \multirow[t]{5}{*}{ G2 } & 28 & $\mathrm{~F} 4$ & $A Z Q$ & 8 & 8 & $\mathrm{~F}$ & 23.75 & 1.2 & 19.8 & Tremolite & $\begin{array}{l}\mathrm{Mg}, \mathrm{Si}, \mathrm{Ca} \\
\mathrm{Fe}\end{array}$ & $\begin{array}{l}\text { AS }>5,3: 1, \text { AFB }>5,3: 1, \\
\text { PCMEF-US, PCMES-US, } \\
\text { PCMEF-ISO, PCMES-ISO }\end{array}$ \\
\hline & & & & & & & ItemType & \multicolumn{2}{|l|}{ ItemNum } & & Confirmed & Comment \\
\hline & & & & & & \multicolumn{2}{|r|}{ Brightfield } & \multicolumn{2}{|l|}{ J3410 BF } & & & \\
\hline & & & & & & \multicolumn{2}{|r|}{ Diffraction } & J3410 & & & KM 5/8/2007 & ZONE AXIS [ $\left.\begin{array}{llll}-2 & 0 & 3\end{array}\right]$ \\
\hline & & & & & & \multicolumn{2}{|c|}{ Spectra } & $\mathrm{J} 2861$ & & & & \\
\hline G2 & 29 & D4 & & & & NSD & & & & & & \\
\hline G2 & 30 & B4 & & & & NSD & & & & & & \\
\hline G2 & 31 & A7 & & & & NSD & & & & & & \\
\hline G2 & 32 & $\mathrm{C} 7$ & NAM & 9 & 9 & $\mathrm{~F}$ & 6.8 & 1 & 6.8 & $\begin{array}{l}\text { Non } \\
\text { Asbestos } \\
\text { Mineral }\end{array}$ & $\mathrm{S}, \mathrm{Ca}$ & \\
\hline
\end{tabular}

Count Categories

AFB $>5,3: 1$

PAS

PCMEF-US

Asbestos Fibers and Bundles > 5um and 3:1

Primary Asbestos Structures

PCM Equivalent Fibers-NIOSH

PCMES-US
PCM Equivalent Structures-NIOSH
AS $>5,3: 1$ PCMEF-ISO PCMES-ISO TAS
Asbestos Structures $>5$ um and 3:1 PCM Equivalent Fibers-ISO PCM Equivalent Structures-ISO Total Asbestos Structures 
Phone: (206) 781-0155

7619 6th Ave Nw

ISO 10312, Direct Raw Data

Job Number: 070434

SEA

Report Number: 070434R06

Client: Idaho National Laboratory

Date Received: 4/23/2007

Project Name: RARE

Client Sample No: FB-4-R2

Lab/Cor Sample No: S13

Client Description:

Date Sampled:

$4 / 16 / 2007$

\begin{tabular}{|c|c|c|c|c|c|c|c|c|c|c|c|c|c|}
\hline Gr & No. & Loc. & ID & Prim & Tot & Class & Len & Wid & Asp & Analyte & Elements & Comment & Count Categories \\
\hline \multirow[t]{3}{*}{ G2 } & 32 & $\mathrm{C} 7$ & NAM & 10 & & MD 1-C & 2.5 & 0.5 & 5 & $\begin{array}{c}\text { Non } \\
\text { Asbestos } \\
\text { Mineral }\end{array}$ & Al, Si & & \\
\hline & & & & & & \multicolumn{2}{|c|}{ ItemType } & \multicolumn{2}{|c|}{ ItemNum } & & Confir & Com & \\
\hline & & & & & & \multicolumn{2}{|c|}{ Brightfield } & \multicolumn{2}{|c|}{ J3411 BF } & & & & \\
\hline G2 & 32 & $\mathrm{C} 7$ & NAM & & 10 & MF & 1.1 & 0.1 & 11 & $\begin{array}{c}\text { Non } \\
\text { Asbestos } \\
\text { Mineral }\end{array}$ & & & \\
\hline G2 & 33 & E7 & & & & NSD & & & & & & & \\
\hline G2 & 34 & 17 & $A Q$ & 11 & 11 & $\mathrm{~F}$ & 6 & 1.25 & 4.8 & Tremolite & $\begin{array}{r}\mathrm{Mg}, \mathrm{Si} \\
\mathrm{Ca}, \mathrm{Fe}\end{array}$ & & $\begin{array}{l}\text { PCMEF-US, PCMES-US, } \\
\text { PCMEF-ISO, PCMES-ISO }\end{array}$ \\
\hline G2 & 35 & G7 & & & & NSD & & & & & & & \\
\hline
\end{tabular}

Count Categories

$\mathrm{AFB}>5,3: 1$

PAS

PCMEF-US

PCMES-US
Asbestos Fibers and Bundles > 5um and 3:1

Primary Asbestos Structures

PCM Equivalent Fibers-NIOSH

PCM Equivalent Structures-NIOSH
AS $>5,3: 1$ PCMEF-ISO PCMES-ISO TAS
Asbestos Structures $>5$ um and 3:1 PCM Equivalent Fibers-ISO PCM Equivalent Structures-ISO Total Asbestos Structures 
Phone: (206) 781-0155

7619 6th Ave Nw

ISO 10312, Direct Raw Data

Job Number: 070434

SEA

Report Number: 070434R06

Client: Idaho National Laboratory

Date Received: 4/23/2007

Project Name: RARE

Client Sample No: FB-4-R3

Lab/Cor Sample No: S14

Client Description:

Date Sampled:

4/16/2007

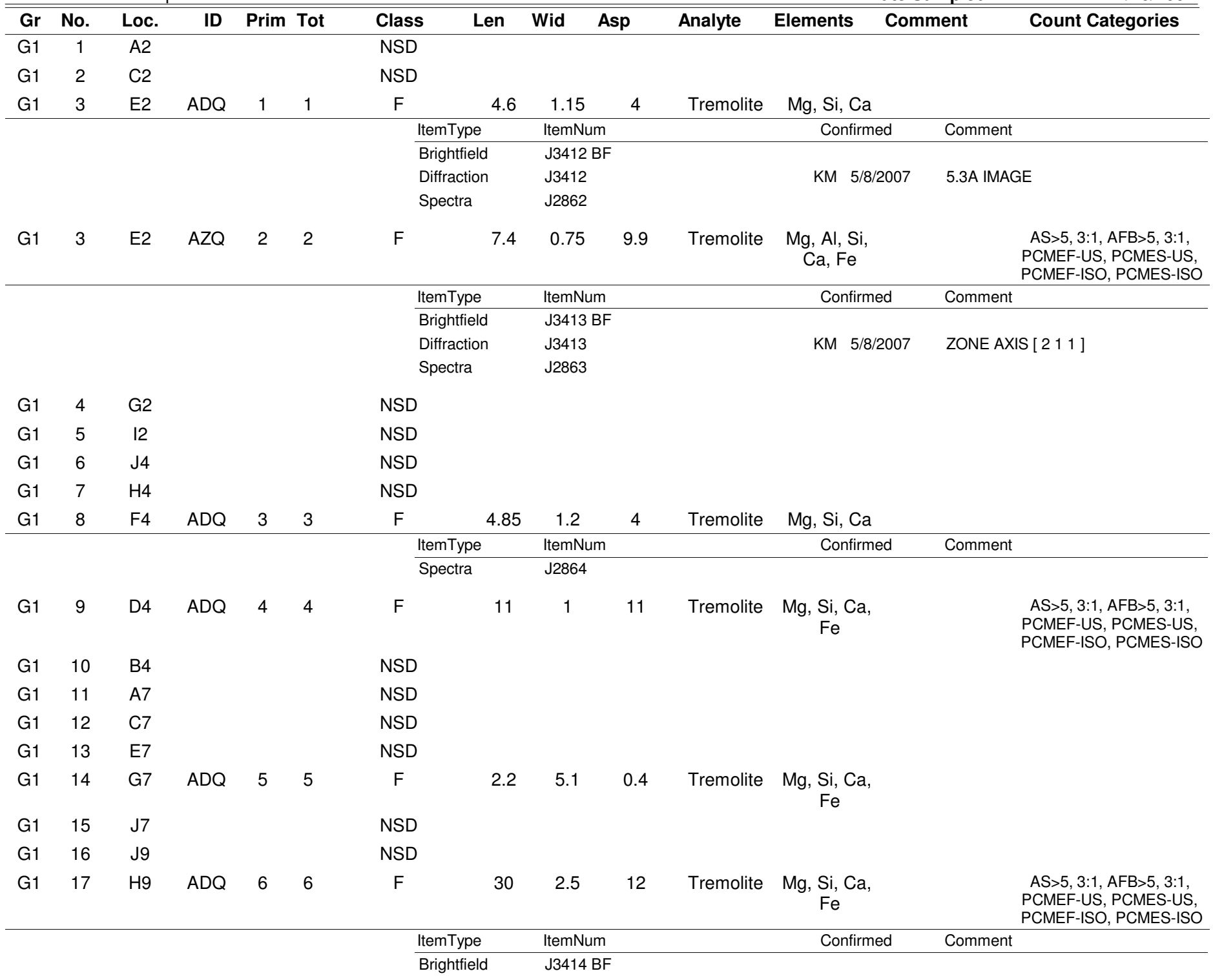

Count Categories

AFB $>5,3: 1$

PAS

PCMEF-US

PCMES-US
Asbestos Fibers and Bundles $>5 u m$ and 3:1

Primary Asbestos Structures

PCM Equivalent Fibers-NIOSH

PCM Equivalent Structures-NIOSH
AS $>5,3: 1$ PCMEF-ISO PCMES-ISO TAS
Asbestos Structures $>5$ um and 3:1 PCM Equivalent Fibers-ISO PCM Equivalent Structures-ISO Total Asbestos Structures 
Job Number: 070434

SEA

Report Number: 070434R06

Client: Idaho National Laboratory

Date Received: 4/23/2007

Project Name: RARE

Client Sample No: FB-4-R3

Lab/Cor Sample No: S14

Client Description:

Date Sampled:

4/16/2007

\begin{tabular}{|c|c|c|c|c|c|c|c|c|c|c|c|c|c|}
\hline $\mathrm{Gr}$ & No. & Loc. & ID & Prim & Tot & Class & Len & Wid & Asp & Analyte & Elements & Comment & Count Categories \\
\hline G1 & 17 & $\mathrm{H} 9$ & $A D Q$ & 7 & & MD 1-1 & 20 & 9 & 2.2 & Tremolite & $\begin{array}{c}\mathrm{Mg}, \mathrm{Si}, \mathrm{Ca} \text {, } \\
\mathrm{Fe}\end{array}$ & & $\mathrm{AS}>5,3: 1$ \\
\hline G1 & 17 & $\mathrm{H} 9$ & $A D Q$ & & 7 & MF & 6.2 & 1.35 & 4.6 & Tremolite & & & PCMEF-US, PCMEF-ISO \\
\hline G1 & 18 & F9 & $A D Q$ & 8 & 8 & $\mathrm{~F}$ & 1.7 & 0.2 & 8.5 & Tremolite & & & \\
\hline G1 & 19 & D9 & & & & NSD & & & & & & & \\
\hline G1 & 20 & B9 & & & & NSD & & & & & & & \\
\hline G2 & 21 & $\mathrm{~A} 2$ & & & & NSD & & & & & & & \\
\hline G2 & 22 & $\mathrm{C} 2$ & & & & NSD & & & & & & & \\
\hline G2 & 23 & E2 & & & & NSD & & & & & & & \\
\hline G2 & 24 & G2 & & & & NSD & & & & & & & \\
\hline G2 & 25 & 12 & & & & NSD & & & & & & & \\
\hline G2 & 26 & 14 & $A D Q$ & 9 & & MD 1-0 & 4 & 1.8 & 2.2 & Tremolite & $\begin{array}{l}\mathrm{Mg}, \mathrm{Si}, \mathrm{Ca} \\
\mathrm{Fe}\end{array}$ & & \\
\hline G2 & 26 & 14 & $A D Q$ & & 9 & MF & 4 & 0.6 & 6.7 & Tremolite & & & \\
\hline G2 & 27 & $\mathrm{H} 4$ & & & & NSD & & & & & & & \\
\hline G2 & 28 & $\mathrm{~F} 4$ & $A D Q$ & 10 & 10 & $\mathrm{~F}$ & 4.35 & 0.65 & 6.7 & Tremolite & $\begin{array}{l}\mathrm{Mg}, \mathrm{Si}, \mathrm{Ca} \\
\mathrm{Fe}\end{array}$ & & \\
\hline G2 & 29 & D4 & NAM & 11 & 11 & $\mathrm{~F}$ & 8.35 & 0.45 & 18.6 & $\begin{array}{c}\text { Non } \\
\text { Asbestos } \\
\text { Mineral }\end{array}$ & $\mathrm{Al}, \mathrm{Si}$ & & \\
\hline & & & & & & \multicolumn{2}{|c|}{ ItemType } & ItemNc & & & Confirm & Com & \\
\hline & & & & & & \multicolumn{2}{|c|}{ Brightfield } & J3415 & & & & & \\
\hline G2 & 30 & B4 & & & & NSD & & & & & & & \\
\hline G2 & 31 & A7 & & & & NSD & & & & & & & \\
\hline G2 & 32 & $\mathrm{C} 7$ & & & & NSD & & & & & & & \\
\hline G2 & 33 & E7 & $A D Q$ & 12 & & MD 1-1 & 8.5 & 7.5 & 1.1 & Tremolite & $\begin{array}{l}\mathrm{Mg}, \mathrm{Si}, \mathrm{Ca} \text {, } \\
\mathrm{Fe}\end{array}$ & & $A S>5,3: 1$ \\
\hline G2 & 33 & E7 & $A D Q$ & & 12 & MF & 5.85 & 0.9 & 6.5 & Tremolite & & & $\begin{array}{c}\text { AFB }>5,3: 1, \text { PCMEF-US, } \\
\text { PCMEF-ISO }\end{array}$ \\
\hline G2 & 34 & G7 & & & & NSD & & & & & & & \\
\hline G2 & 35 & 17 & & & & NSD & & & & & & & \\
\hline
\end{tabular}

Count Categories

AFB $>5,3: 1$

PAS

PCMEF-US

PCMES-US
Asbestos Fibers and Bundles $>5$ um and 3:1

Primary Asbestos Structures

PCM Equivalent Fibers-NIOSH

PCM Equivalent Structures-NIOSH
AS>5, 3:1 PCMEF-ISO PCMES-ISO TAS
Asbestos Structures $>5$ um and 3:1 PCM Equivalent Fibers-ISO PCM Equivalent Structures-ISO Total Asbestos Structures 
Phone: (206) 781-0155

7619 6th Ave Nw

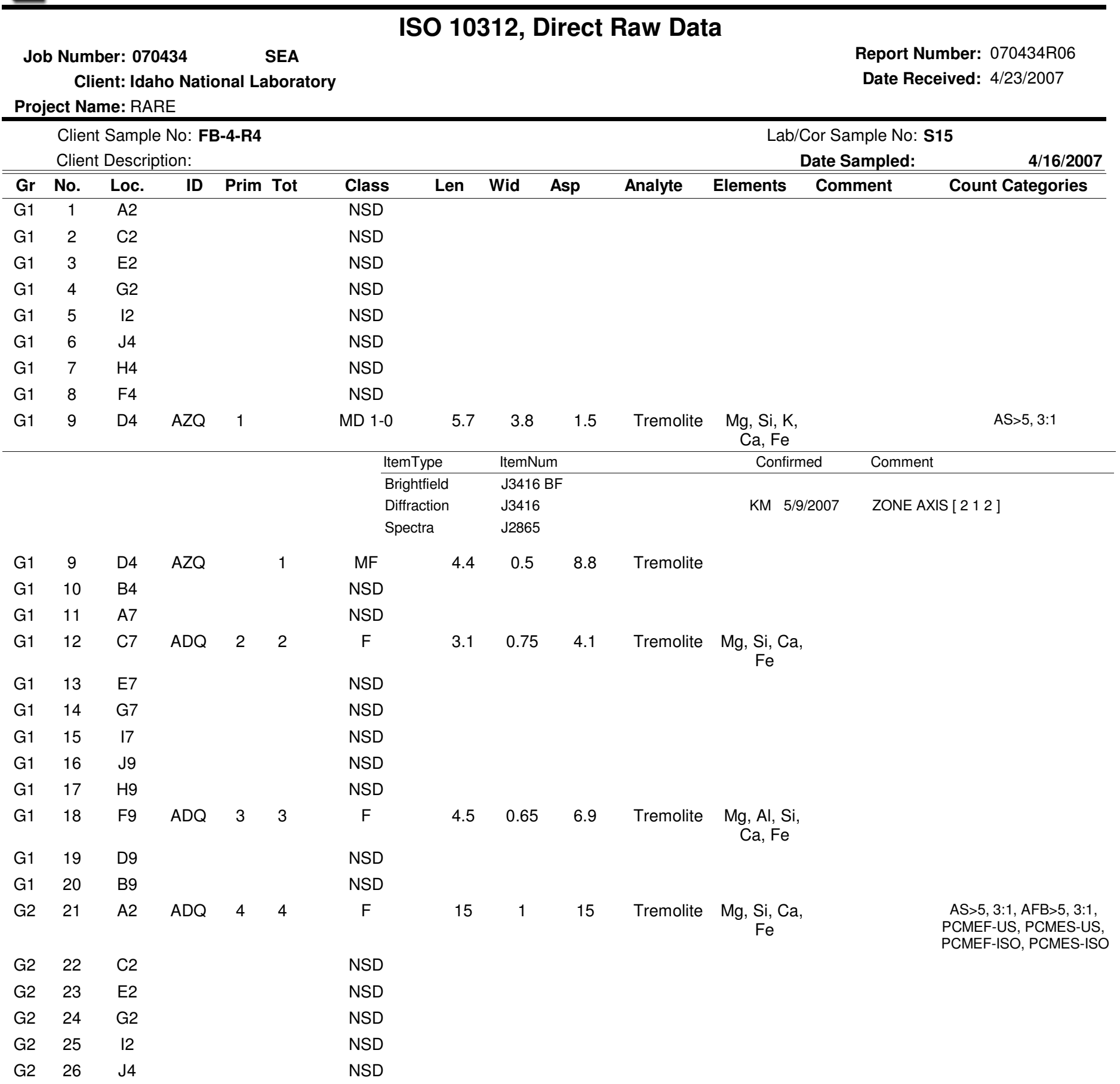

Count Categories

$A F B>5,3: 1$

PAS

PCMEF-US

PCMES-US
Asbestos Fibers and Bundles $>5$ um and 3:1

Primary Asbestos Structures

PCM Equivalent Fibers-NIOSH

PCM Equivalent Structures-NIOSH
AS>5, 3:1 PCMEF-ISO PCMES-ISO TAS
Asbestos Structures $>5$ um and 3:1 PCM Equivalent Fibers-ISO PCM Equivalent Structures-ISO Total Asbestos Structures 
Job Number: 070434

ISO 10312, Direct Raw Data

Client: Idaho National Laboratory

Report Number: 070434R06

ct Name: RARE

Client Sample No: FB-4-R4

Lab/Cor Sample No: S15

Client Description:

Date Sampled:

$4 / 16 / 2007$

\begin{tabular}{|c|c|c|c|c|c|c|c|c|c|c|c|c|}
\hline $\mathbf{G r}$ & No. & Loc. & ID & Prim Tot & Class & Len & Wid & Asp & Analyte & Elements & Comment & Count Categories \\
\hline G2 & 27 & $\mathrm{H} 4$ & & & NSD & & & & & & & \\
\hline G2 & 28 & $\mathrm{~F} 4$ & & & NSD & & & & & & & \\
\hline G2 & 29 & D4 & & & NSD & & & & & & & \\
\hline G2 & 30 & B4 & & & NSD & & & & & & & \\
\hline G2 & 31 & A7 & & & NSD & & & & & & & \\
\hline G2 & 32 & C7 & & & NSD & & & & & & & \\
\hline G2 & 33 & E7 & & & NSD & & & & & & & \\
\hline G2 & 34 & G7 & & & NSD & & & & & & & \\
\hline G2 & 35 & 17 & & & NSD & & & & & & & \\
\hline
\end{tabular}

Asbestos Fibers and Bundles > 5um and 3:1

Primary Asbestos Structures

PCM Equivalent Fibers-NIOSH

PCMEF-US

PCM Equivalent Structures-NIOSH
AS>5, 3:1 PCMEF-ISO PCMES-ISO TAS
Asbestos Structures $>5$ um and 3:1 PCM Equivalent Fibers-ISO PCM Equivalent Structures-ISO Total Asbestos Structures 
Phone: (206) 781-0155

7619 6th Ave Nw

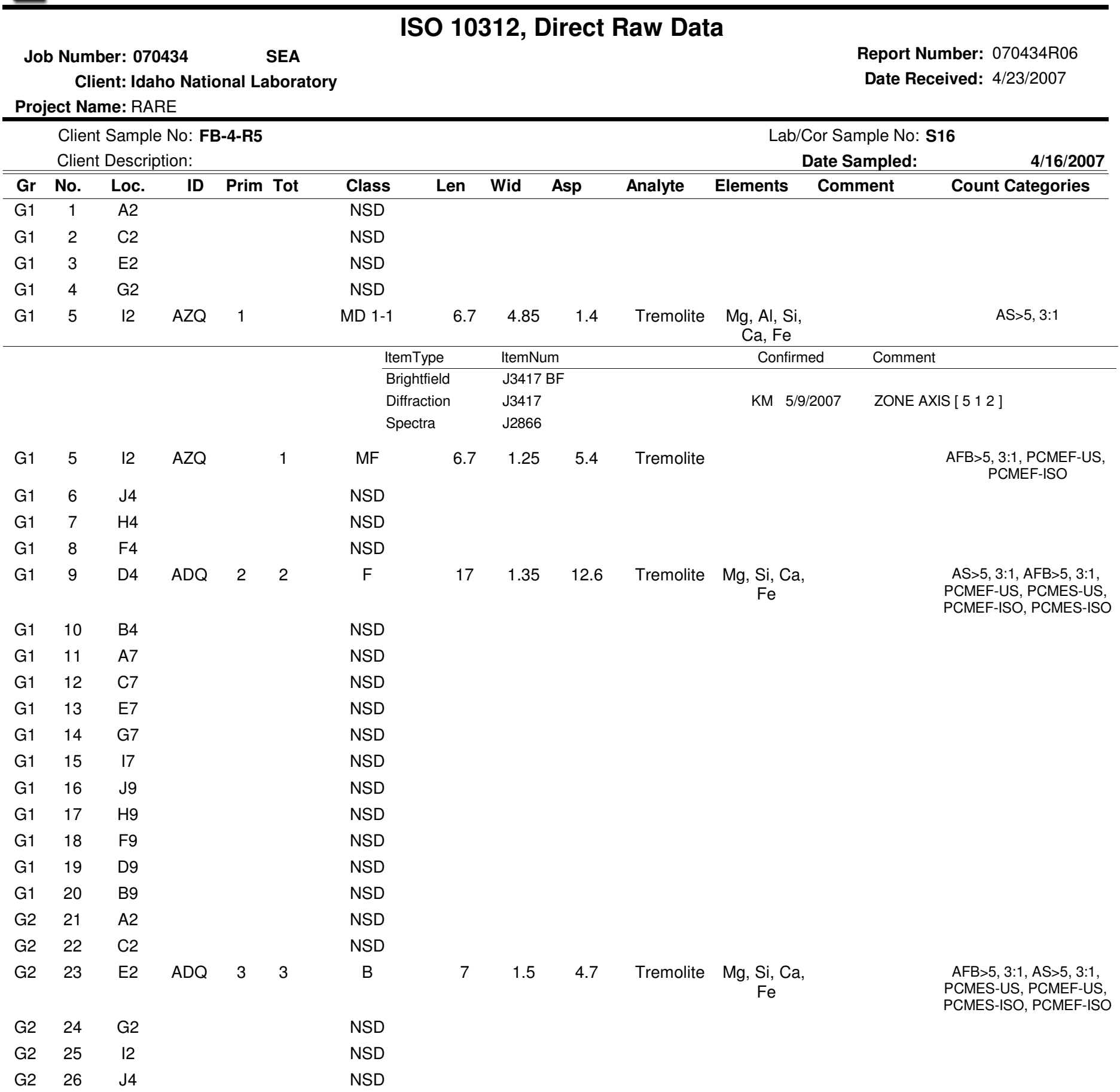

Count Categories

AFB $>5,3: 1$

PAS

PCMEF-US

PCMES-US
Asbestos Fibers and Bundles > 5um and 3:1

Primary Asbestos Structures

PCM Equivalent Fibers-NIOSH

PCM Equivalent Structures-NIOSH
AS $>5,3: 1$ PCMEF-ISO PCMES-ISO TAS
Asbestos Structures $>5$ um and 3:1 PCM Equivalent Fibers-ISO PCM Equivalent Structures-ISO Total Asbestos Structures 
ISO 10312, Direct Raw Data

Job Number: 070434

SEA

Report Number: 070434R06

Client: Idaho National Laboratory

Date Received: 4/23/2007

Project Name: RARE

Client Sample No: FB-4-R5

Lab/Cor Sample No: S16

Client Description:

Date Sampled:

$4 / 16 / 2007$

\begin{tabular}{|c|c|c|c|c|c|c|c|c|c|c|c|c|c|}
\hline $\mathrm{Gr}$ & No. & Loc. & ID & Prim & Tot & Class & Len & Wid & Asp & Analyte & Elements & Comment & Count Categories \\
\hline $\mathrm{G} 2$ & 27 & $\mathrm{H} 4$ & $A D Q$ & 4 & & MD 1-0 & 5.2 & 3.85 & 1.4 & Tremolite & $\begin{array}{c}\mathrm{Mg}, \mathrm{Si}, \mathrm{Ca} \\
\mathrm{Fe}\end{array}$ & & $\mathrm{AS}>5,3: 1$ \\
\hline G2 & 27 & $\mathrm{H} 4$ & $A D Q$ & & 4 & MF & 4.5 & 1.2 & 3.7 & Tremolite & & & \\
\hline $\mathrm{G} 2$ & 28 & $\mathrm{~F} 4$ & $A D Q$ & 5 & 5 & $\mathrm{~F}$ & 3.3 & 0.45 & 7.3 & Tremolite & $\begin{array}{c}\mathrm{Mg}, \mathrm{Si}, \mathrm{Ca} \\
\mathrm{Fe}\end{array}$ & & \\
\hline $\mathrm{G} 2$ & 29 & D4 & & & & NSD & & & & & & & \\
\hline $\mathrm{G} 2$ & 30 & B4 & & & & NSD & & & & & & & \\
\hline $\mathrm{G} 2$ & 31 & A7 & & & & NSD & & & & & & & \\
\hline $\mathrm{G} 2$ & 32 & $\mathrm{C} 7$ & & & & NSD & & & & & & & \\
\hline $\mathrm{G} 2$ & 33 & E7 & & & & NSD & & & & & & & \\
\hline $\mathrm{G} 2$ & 34 & G7 & & & & NSD & & & & & & & \\
\hline G2 & 35 & 17 & & & & NSD & & & & & & & \\
\hline
\end{tabular}

Count Categories

$\mathrm{AFB}>5,3: 1$

PAS

PCMEF-US

PCMES-US
Asbestos Fibers and Bundles > 5um and 3:1

Primary Asbestos Structures

PCM Equivalent Fibers-NIOSH

PCM Equivalent Structures-NIOSH
AS>5, 3:1 PCMEF-ISO PCMES-ISO TAS
Asbestos Structures $>5$ um and 3:1 PCM Equivalent Fibers-ISO PCM Equivalent Structures-ISO Total Asbestos Structures 


\section{ISO 10312, Direct Raw Data}

Job Number: 070434

SEA

Report Number: 070434R06

Client: Idaho National Laboratory

Date Received: 4/23/2007

Project Name: RARE

Client Sample No: FB-4-R6

Lab/Cor Sample No: $\mathbf{S 1 7}$

Client Description:

Date Sampled:

4/16/2007

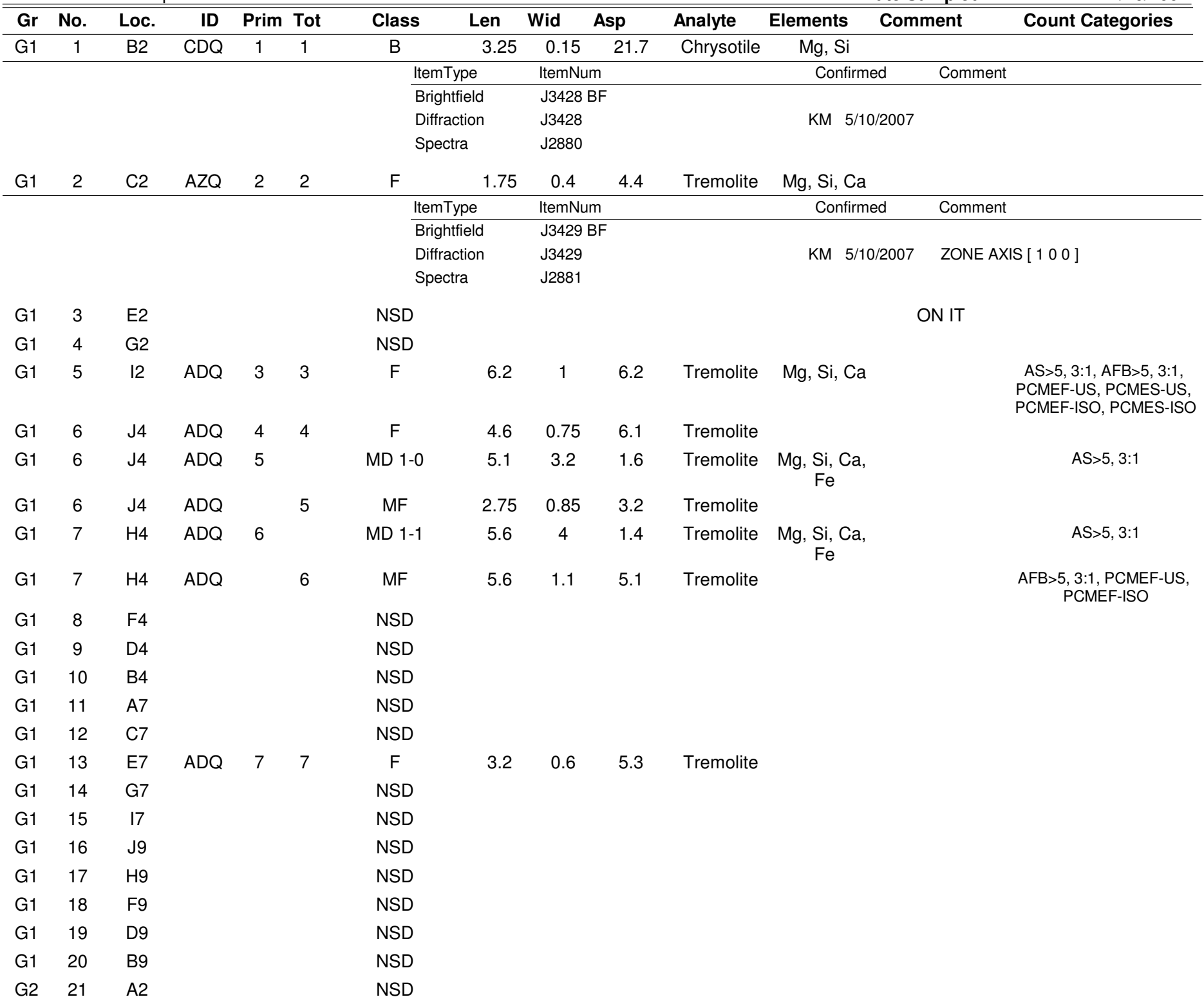

Count Categories

$\mathrm{AFB}>5,3: 1$

PAS

PCMEF-US

Asbestos Fibers and Bundles > 5um and 3:1

Primary Asbestos Structures

PCM Equivalent Fibers-NIOSH

PCMES-US

PCM Equivalent Structures-NIOSH
AS>5, 3:1 PCMEF-ISO PCMES-ISO TAS
Asbestos Structures $>5$ um and 3:1 PCM Equivalent Fibers-ISO PCM Equivalent Structures-ISO Total Asbestos Structures 


\section{ISO 10312, Direct Raw Data}

Job Number: 070434

Client: Idaho National Laboratory

Report Number: 070434R06

Project Name: RARE

Date Received: 4/23/2007

Client Sample No: FB-4-R6

Lab/Cor Sample No: S17

Client Description:

Date Sampled:

4/16/2007

\begin{tabular}{|c|c|c|c|c|c|c|c|c|c|c|c|c|c|}
\hline $\mathrm{Gr}$ & No. & Loc. & ID & Prim & Tot & Class & Len & Wid & Asp & Analyte & Elements & Comment & Count Categories \\
\hline G2 & 22 & $\mathrm{C} 2$ & ADQ & 8 & 8 & $\mathrm{~F}$ & 3.85 & 0.75 & 5.1 & Tremolite & $\mathrm{Mg}, \mathrm{Si}, \mathrm{Ca}$ & & \\
\hline $\mathrm{G} 2$ & 23 & E2 & & & & NSD & & & & & & & \\
\hline G2 & 24 & G2 & & & & NSD & & & & & & & \\
\hline G2 & 25 & 12 & & & & NSD & & & & & & & \\
\hline $\mathrm{G} 2$ & 26 & J4 & & & & NSD & & & & & & & \\
\hline $\mathrm{G} 2$ & 27 & $\mathrm{H} 4$ & & & & NSD & & & & & & & \\
\hline $\mathrm{G} 2$ & 28 & $\mathrm{~F} 4$ & & & & NSD & & & & & & & \\
\hline $\mathrm{G} 2$ & 29 & D4 & & & & NSD & & & & & & & \\
\hline $\mathrm{G} 2$ & 30 & B4 & & & & NSD & & & & & & & \\
\hline $\mathrm{G} 2$ & 31 & A7 & & & & NSD & & & & & & & \\
\hline $\mathrm{G} 2$ & 32 & $\mathrm{C} 7$ & & & & NSD & & & & & & & \\
\hline $\mathrm{G} 2$ & 33 & E7 & & & & NSD & & & & & & & \\
\hline $\mathrm{G} 2$ & 34 & G7 & $A D Q$ & 9 & 9 & $\mathrm{~F}$ & 4.2 & 0.7 & 6 & Tremolite & $\begin{array}{c}\mathrm{Mg}, \mathrm{Si}, \mathrm{Ca}, \\
\mathrm{Fe}\end{array}$ & & \\
\hline $\mathrm{G} 2$ & 35 & 17 & $A D Q$ & 10 & 10 & $\mathrm{~F}$ & 3.75 & 0.85 & 4.4 & Tremolite & $\begin{array}{c}\mathrm{Mg}, \mathrm{Si}, \mathrm{Ca}, \\
\mathrm{Fe}\end{array}$ & & \\
\hline
\end{tabular}

Count Categories

AFB $>5,3: 1$

PAS

PCMEF-US

PCMES-US
Asbestos Fibers and Bundles > 5um and 3:1

Primary Asbestos Structures

PCM Equivalent Fibers-NIOSH

PCM Equivalent Structures-NIOSH
AS $>5,3: 1$ PCMEF-ISO PCMES-ISO TAS
Asbestos Structures $>5$ um and 3:1 PCM Equivalent Fibers-ISO PCM Equivalent Structures-ISO Total Asbestos Structures 
Phone: (206) 781-0155

7619 6th Ave Nw

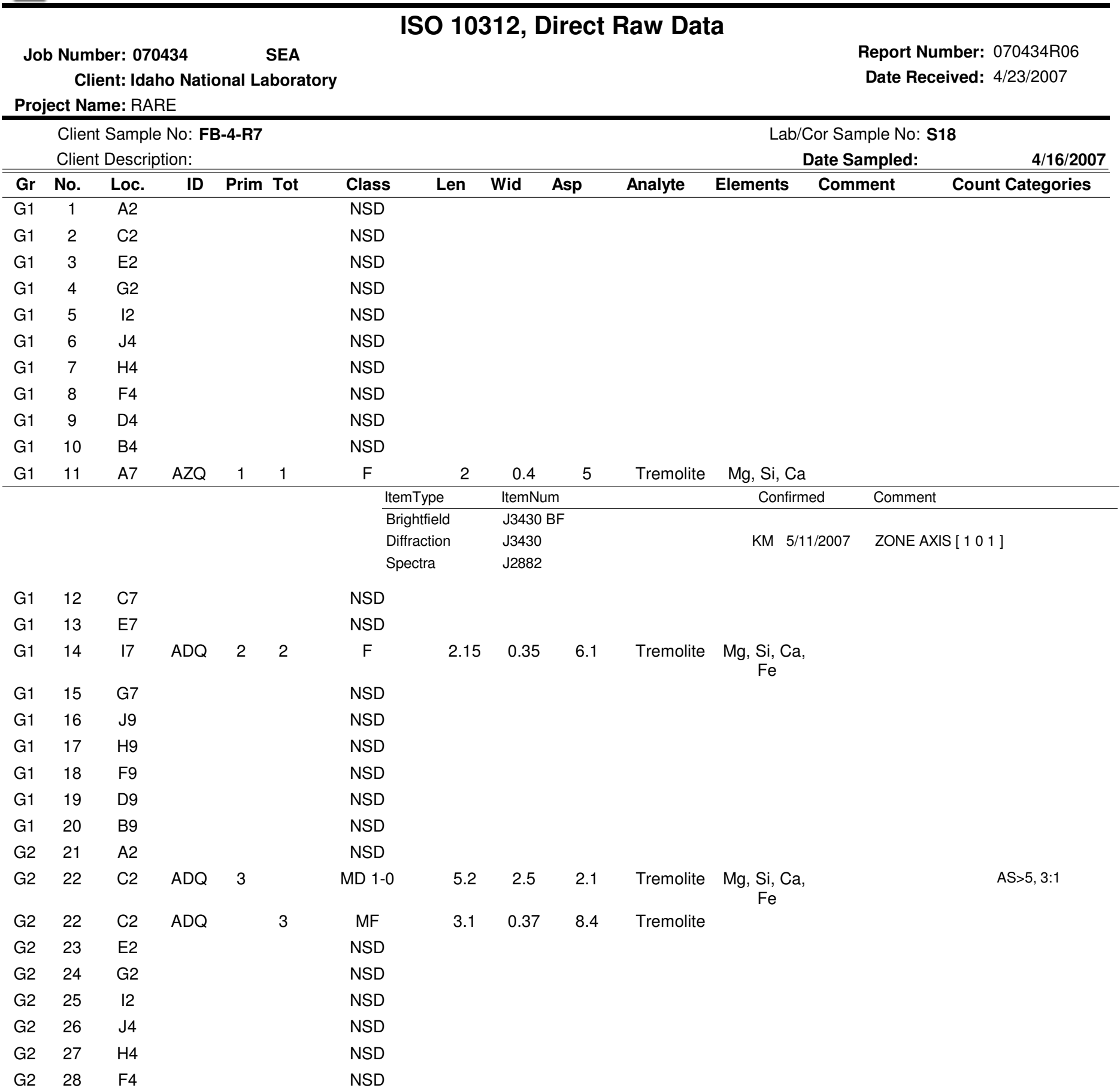

Count Categories

AFB $>5,3: 1$

PAS

PCMEF-US

PCMES-US
Asbestos Fibers and Bundles $>5$ um and 3:1

Primary Asbestos Structures

PCM Equivalent Fibers-NIOSH

PCM Equivalent Structures-NIOSH
AS $>5,3: 1$ PCMEF-ISO PCMES-ISO TAS
Asbestos Structures $>5$ um and 3:1 PCM Equivalent Fibers-ISO PCM Equivalent Structures-ISO Total Asbestos Structures 
Job Number: 070434

ISO 10312, Direct Raw Data

Client: Idaho National Laboratory

Report Number: 070434R06

ct Name: RARE

Client Sample No: FB-4-R7

Lab/Cor Sample No: $\mathbf{S 1 8}$

Client Description:

Date Sampled:

$4 / 16 / 2007$

\begin{tabular}{|c|c|c|c|c|c|c|c|c|c|c|c|c|}
\hline $\mathrm{Gr}$ & No. & Loc. & ID & Prim Tot & Class & Len & Wid & Asp & Analyte & Elements & Comment & Count Categories \\
\hline G2 & 29 & D4 & & & NSD & & & & & & & \\
\hline G2 & 30 & B4 & & & NSD & & & & & & & \\
\hline $\mathrm{G} 2$ & 31 & A7 & & & NSD & & & & & & & \\
\hline $\mathrm{G} 2$ & 32 & $\mathrm{C7}$ & & & NSD & & & & & & & \\
\hline G2 & 33 & E7 & & & NSD & & & & & & & \\
\hline $\mathrm{G} 2$ & 34 & G7 & & & NSD & & & & & & & \\
\hline G2 & 35 & 17 & & & NSD & & & & & & & \\
\hline
\end{tabular}

Asbestos Fibers and Bundles > 5um and 3:1

Primary Asbestos Structures

PCM Equivalent Fibers-NIOSH

PCMEF-US

PCM Equivalent Structures-NIOSH
AS $>5,3: 1$ PCMEF-ISO PCMES-ISO TAS
Asbestos Structures $>5$ um and 3:1 PCM Equivalent Fibers-ISO PCM Equivalent Structures-ISO Total Asbestos Structures 
Phone: (206) 781-0155

Fax: (206) 789-8424

7619 6th Ave Nw

ISO 10312, Direct Raw Data

Job Number: 070434

SEA

Report Number: 070434R06

Client: Idaho National Laboratory

Date Received: 4/23/2007

Project Name: RARE

Client Sample No: FB-3-R1

Lab/Cor Sample No: S19

Client Description:

Date Sampled

4/16/2007

\begin{tabular}{|c|c|c|c|c|c|c|c|c|c|c|c|c|}
\hline Gr & No. & Loc. & ID & Prim Tot & Class & Len & Wid & Asp & Analyte & Elements & Comment & Count Categories \\
\hline G1 & 1 & $\mathrm{~A} 2$ & & & NSD & & & & & & & \\
\hline G1 & 2 & $\mathrm{C} 2$ & & & NSD & & & & & & & \\
\hline G1 & 3 & G2 & & & NSD & & & & & & & \\
\hline G1 & 4 & E2 & & & NSD & & & & & & & \\
\hline G1 & 5 & 12 & & & NSD & & & & & & & \\
\hline G1 & 6 & J4 & & & NSD & & & & & & & \\
\hline G1 & 7 & $\mathrm{H} 4$ & & & NSD & & & & & & & \\
\hline G1 & 8 & $\mathrm{~F} 4$ & & & NSD & & & & & & & \\
\hline G1 & 9 & D4 & & & NSD & & & & & & & \\
\hline G1 & 10 & B4 & & & NSD & & & & & & & \\
\hline G1 & 11 & A7 & & & NSD & & & & & & & \\
\hline G1 & 12 & C7 & & & NSD & & & & & & & \\
\hline G1 & 13 & E7 & & & NSD & & & & & & & \\
\hline G1 & 14 & G7 & & & NSD & & & & & & & \\
\hline G1 & 15 & 17 & & & NSD & & & & & & & \\
\hline G1 & 16 & J9 & & & NSD & & & & & & & \\
\hline G1 & 17 & $\mathrm{H} 9$ & & & NSD & & & & & & & \\
\hline G1 & 18 & F9 & & & NSD & & & & & & & \\
\hline G1 & 19 & D9 & & & NSD & & & & & & & \\
\hline G1 & 20 & B9 & & & NSD & & & & & & & \\
\hline G2 & 21 & $\mathrm{~A} 2$ & & & NSD & & & & & & & \\
\hline G2 & 22 & $\mathrm{C} 2$ & & & NSD & & & & & & & \\
\hline G2 & 23 & E2 & & & NSD & & & & & & & \\
\hline G2 & 24 & G2 & & & NSD & & & & & & & \\
\hline G2 & 25 & 12 & & & NSD & & & & & & & \\
\hline G2 & 26 & J4 & & & NSD & & & & & & & \\
\hline G2 & 27 & $\mathrm{H} 4$ & & & NSD & & & & & & & \\
\hline G2 & 28 & F4 & & & NSD & & & & & & & \\
\hline G2 & 29 & D4 & & & NSD & & & & & & & \\
\hline G2 & 30 & B4 & & & NSD & & & & & & & \\
\hline G2 & 31 & A7 & & & NSD & & & & & & & \\
\hline G2 & 32 & C7 & & & NSD & & & & & & & \\
\hline G2 & 33 & E7 & & & NSD & & & & & & & \\
\hline G2 & 34 & G7 & & & NSD & & & & & & & \\
\hline
\end{tabular}

Count Categories

AFB $>5,3: 1$

PAS

PCMEF-US

Asbestos Fibers and Bundles > 5um and 3:1

AS>5, 3:1 PCMEF-ISO

PCMES-ISO

PCM Equivalent Fibers-NIOSH

PCM Equivalent Structures-NIOSH
TAS
Asbestos Structures $>5$ um and 3:1

PCM Equivalent Fibers-ISO

PCM Equivalent Structures-ISO

Total Asbestos Structures 
Phone: (206) 781-0155

7619 6th Ave Nw

ISO 10312, Direct Raw Data

Job Number: 070434

SEA

Report Number: 070434R06

Client: Idaho National Laboratory

Date Received: 4/23/2007

Project Name: RARE

Client Sample No: FB-3-R1

Lab/Cor Sample No: S19

Client Description:

Date Sampled:

$4 / 16 / 2007$

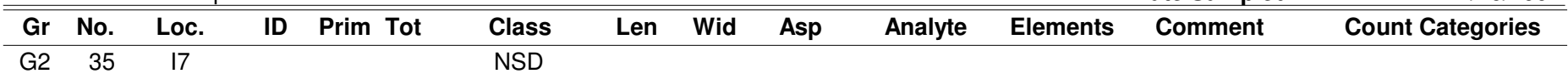

Count Categories

AFB $>5,3: 1$

PAS

PCMEF-US

PCMES-US
Asbestos Fibers and Bundles > 5um and 3:1

Primary Asbestos Structures

PCM Equivalent Fibers-NIOSH

PCM Equivalent Structures-NIOSH
AS>5, 3:1 PCMEF-ISO PCMES-ISO TAS
Asbestos Structures >5um and 3:1 PCM Equivalent Fibers-ISO PCM Equivalent Structures-ISO Total Asbestos Structures 


\section{ISO 10312, Direct Raw Data}

Job Number: 070434

SEA

Report Number: 070434R06

Client: Idaho National Laboratory

Date Received: 4/23/2007

Project Name: RARE

Client Sample No: FB-3-R2

Lab/Cor Sample No: S20

Client Description:

Date Sampled:

4/16/2007

\begin{tabular}{|c|c|c|c|c|c|c|c|c|c|c|c|c|c|}
\hline Gr & No. & Loc. & ID & Prim & Tot & Class & Len & Wid & Asp & Analyte & Elements & nent & Count Categories \\
\hline G1 & 1 & $\mathrm{~A} 2$ & $A Z Q$ & 1 & 1 & $\mathrm{~F}$ & 4 & 0.45 & 8.9 & Tremolite & $\mathrm{Mg}, \mathrm{Si}, \mathrm{Ca}$ & & \\
\hline & & & & & & \multicolumn{2}{|c|}{ ItemType } & ItemNun & & & Confirmed & Comment & \\
\hline & & & & & & \multicolumn{2}{|c|}{ Brightfield } & J3469 B & & & & & \\
\hline & & & & & & \multicolumn{2}{|c|}{ Diffraction } & J3469 & & & KM 5/17/2007 & ZONE AXIS & IS [ $\left.\begin{array}{lll}3 & 1 & 2\end{array}\right]$ \\
\hline & & & & & & \multicolumn{2}{|c|}{ Spectra } & J2921 & & & & & \\
\hline \multirow[t]{4}{*}{ G1 } & 2 & $\mathrm{C} 2$ & $A D Q$ & 2 & 2 & $\mathrm{~F}$ & 19.2 & 1.7 & 11.3 & Tremolite & $\mathrm{Mg}, \mathrm{Si}, \mathrm{Ca}$ & & $\begin{array}{l}\text { AS>5, 3:1, AFB>5, 3:1, } \\
\text { PCMEF-US, PCMES-US, } \\
\text { PCMEF-ISO, PCMES-ISO }\end{array}$ \\
\hline & & & & & & \multicolumn{2}{|c|}{ ItemType } & ItemNun & & & Confirmed & Comment & \\
\hline & & & & & & \multicolumn{2}{|c|}{ Brightfield } & $\mathrm{J} 3470 \mathrm{~B}$ & & & & & \\
\hline & & & & & & \multicolumn{2}{|c|}{ Spectra } & J2922 & & & & & \\
\hline G1 & 3 & E2 & $A D$ & 3 & & MD 1-0 & 5 & 4.8 & 1 & Tremolite & & & \\
\hline G1 & 3 & E2 & $A D$ & & 3 & MF & 1.45 & 0.22 & 6.6 & Tremolite & & & \\
\hline G1 & 4 & G2 & & & & NSD & & & & & & & \\
\hline G1 & 5 & 12 & $A Q$ & 4 & & MD 1-1 & 10 & 4.5 & 2.2 & Tremolite & $\mathrm{Mg}, \mathrm{Si}, \mathrm{Ca}$ & & $A S>5,3: 1$ \\
\hline G1 & 5 & 12 & $A Q$ & & 4 & MF & 7.85 & 1.1 & 7.1 & Tremolite & & & $\begin{array}{c}\text { AFB >5, 3:1, PCMEF-US, } \\
\text { PCMEF-ISO }\end{array}$ \\
\hline G1 & 5 & 12 & $A Q$ & 5 & 5 & $F$ & 9.2 & 0.5 & 18.4 & Tremolite & & & $\begin{array}{l}\text { AS>5, 3:1, AFB }>5,3: 1, \\
\text { PCMEF-US, PCMES-US, } \\
\text { PCMEF-ISO, PCMES-ISO }\end{array}$ \\
\hline G1 & 5 & 12 & $A Q$ & 6 & 6 & $\mathrm{~F}$ & 2.8 & 0.65 & 4.3 & Tremolite & & & \\
\hline G1 & 5 & 12 & $A Q$ & 7 & 7 & $\mathrm{~F}$ & 7.5 & 1.1 & 6.8 & Tremolite & & & $\begin{array}{l}\text { AS }>5,3: 1, \text { AFB }>5,3: 1, \\
\text { PCMEF-US, PCMES-US, } \\
\text { PCMEF-ISO, PCMES-ISO }\end{array}$ \\
\hline G1 & 6 & $\mathrm{H} 4$ & $A Q$ & 8 & 8 & $F$ & 5 & 1.5 & 3.3 & Tremolite & & & \\
\hline G1 & 7 & J4 & & & & NSD & & & & & & & \\
\hline G1 & 8 & $\mathrm{~F} 4$ & $A Q$ & 9 & 9 & $F$ & 2.2 & 0.7 & 3.1 & Tremolite & & & \\
\hline \multirow[t]{3}{*}{ G1 } & 8 & F4 & $A Q$ & 10 & 10 & B & 12 & 2 & 6 & Tremolite & & & $\begin{array}{l}\text { AFB }>5,3: 1, \text { AS }>5,3: 1 \text {, } \\
\text { PCMES-US, PCMEF-US, } \\
\text { PCMES-ISO, PCMEF-ISO }\end{array}$ \\
\hline & & & & & & \multicolumn{2}{|c|}{ ItemType } & ItemNun & & & Confirmed & Comment & \\
\hline & & & & & & \multicolumn{2}{|c|}{ Brightfield } & J3471 B & & & & & \\
\hline G1 & 9 & D4 & & & & NSD & & & & & & & \\
\hline G1 & 10 & B4 & $A Q$ & 11 & 11 & $F$ & 3 & 0.3 & 10 & Tremolite & & & \\
\hline G1 & 10 & B4 & $A Q$ & 12 & 12 & $F$ & 3 & 0.8 & 3.8 & Tremolite & & & \\
\hline G1 & 11 & A7 & & & & NSD & & & & & & & \\
\hline G1 & 12 & C7 & $A Q$ & 13 & 13 & $\mathrm{~F}$ & 6 & 1.7 & 3.5 & Tremolite & & & $\begin{array}{l}\text { PCMEF-US, PCMES-US, } \\
\text { PCMEF-ISO, PCMES-ISO }\end{array}$ \\
\hline
\end{tabular}

Count Categories

AFB $>5,3: 1$

PAS

PCMEF-US

PCMES-US
Asbestos Fibers and Bundles > 5um and 3:1

Primary Asbestos Structures

PCM Equivalent Fibers-NIOSH

PCM Equivalent Structures-NIOSH
AS $>5,3: 1$ PCMEF-ISO PCMES-ISO TAS
Asbestos Structures >5um and 3:1 PCM Equivalent Fibers-ISO PCM Equivalent Structures-ISO Total Asbestos Structures 


\section{ISO 10312, Direct Raw Data}

Job Number: 070434

SEA

Report Number: 070434R06

Client: Idaho National Laboratory

Date Received: 4/23/2007

Project Name: RARE

Client Sample No: FB-3-R2

Lab/Cor Sample No: S20

Client Description:

Date Sampled:

$4 / 16 / 2007$

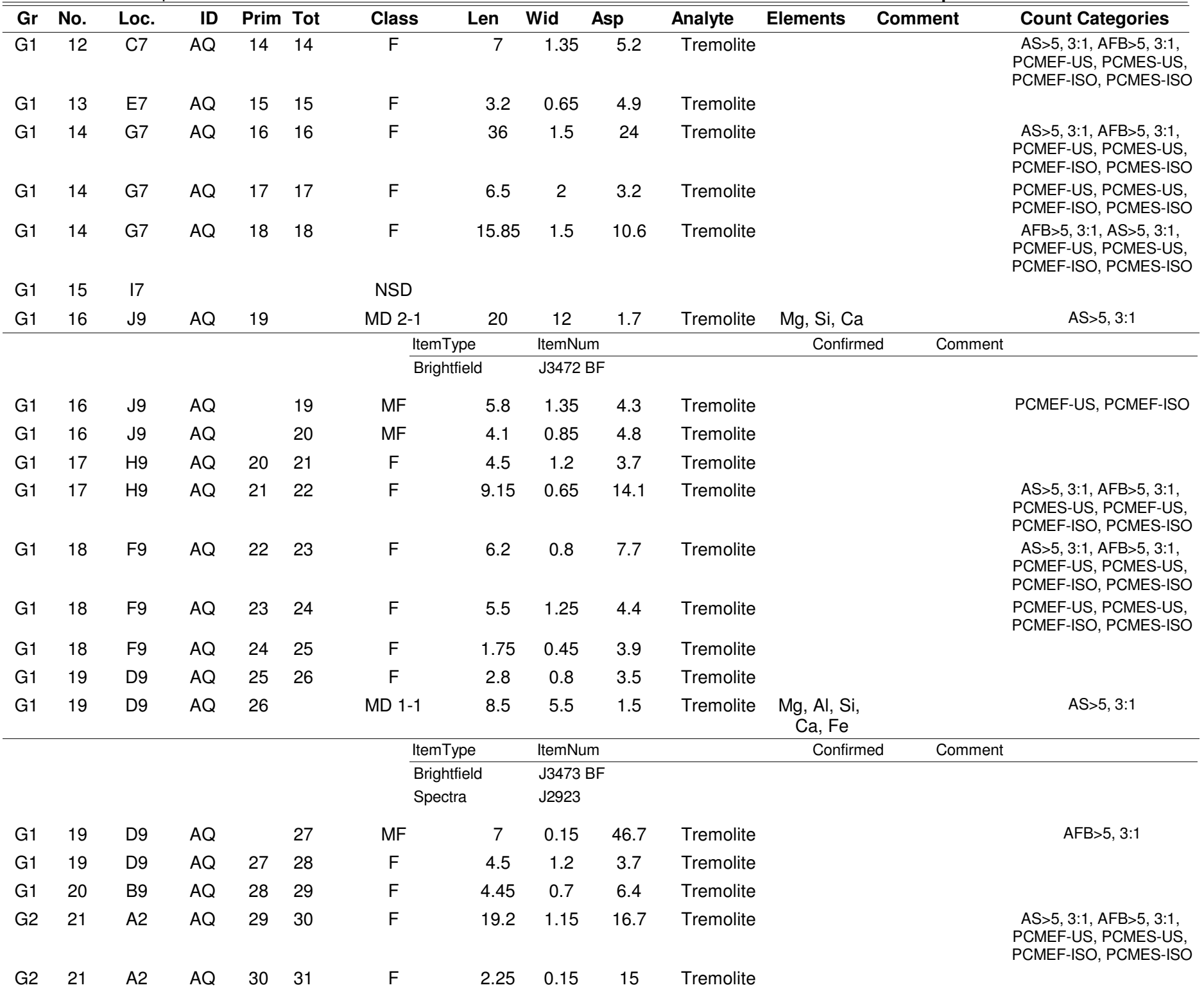

Count Categories

PAS

PCMEF-US

Asbestos Fibers and Bundles $>5 u m$ and 3:1

Primary Asbestos Structures

PCM Equivalent Fibers-NIOSH

PCMES-US

PCM Equivalent Structures-NIOSH
AS $>5,3: 1$ PCMEF-ISO PCMES-ISO TAS
Asbestos Structures $>5$ um and 3:1 PCM Equivalent Fibers-ISO PCM Equivalent Structures-ISO Total Asbestos Structures 


\section{ISO 10312, Direct Raw Data}

Job Number: 070434

SEA

Report Number: 070434R06

Client: Idaho National Laboratory

Date Received: 4/23/2007

Project Name: RARE

Client Sample No: FB-3-R2

Lab/Cor Sample No: S20

Client Description:

Date Sampled:

4/16/2007

\begin{tabular}{|c|c|c|c|c|c|c|c|c|c|c|c|c|c|}
\hline Gr & No. & Loc. & ID & Prim & Tot & Class & Len & Wid & Asp & Analyte & Elements & Comment & Count Categories \\
\hline G2 & 22 & $\mathrm{C} 2$ & $A Q$ & 31 & 32 & $F$ & 3.15 & 0.35 & 9 & Tremolite & & & \\
\hline G2 & 22 & $\mathrm{C} 2$ & $A Q$ & 32 & 33 & $\mathrm{~F}$ & 3.5 & 0.5 & 7 & Tremolite & & & \\
\hline G2 & 23 & E2 & $A Q$ & 33 & 34 & $\mathrm{~F}$ & 4 & 0.6 & 6.7 & Tremolite & & & \\
\hline G2 & 24 & G2 & $A Q$ & 34 & 35 & $\mathrm{~F}$ & 25 & 2.75 & 9.1 & Tremolite & & & $\begin{array}{l}\text { AS>5, 3:1, AFB>5, 3:1, } \\
\text { PCMEF-US, PCMES-US, } \\
\text { PCMEF-ISO, PCMES-ISO }\end{array}$ \\
\hline G2 & 24 & G2 & $A Q$ & 35 & 36 & $\mathrm{~F}$ & 3.5 & 1.15 & 3 & Tremolite & & & \\
\hline G2 & 25 & 12 & $A Q$ & 36 & 37 & $\mathrm{~F}$ & 4.2 & 1 & 4.2 & Tremolite & & & \\
\hline G2 & 25 & 12 & $A Q$ & 37 & 38 & $\mathrm{~F}$ & 3.15 & 0.6 & 5.2 & Tremolite & & & \\
\hline G2 & 26 & J4 & $A Q$ & 38 & & MD 1-0 & 6.85 & 4.2 & 1.6 & Tremolite & & & $A S>5,3: 1$ \\
\hline G2 & 26 & J4 & $A Q$ & & 39 & MF & 3.35 & 0.45 & 7.4 & Tremolite & & & \\
\hline G2 & 27 & $\mathrm{H} 4$ & $A Q$ & 39 & 40 & $F$ & 2.12 & 0.4 & 5.3 & Tremolite & & & \\
\hline G2 & 28 & $\mathrm{~F} 4$ & $A Q$ & 40 & 41 & $F$ & 6.5 & 0.95 & 6.8 & Tremolite & & & $\begin{array}{l}\text { AS }>5,3: 1, \text { AFB }>5,3: 1, \\
\text { PCMEF-US, PCMES-US, } \\
\text { PCMEF-ISO, PCMES-ISO }\end{array}$ \\
\hline G2 & 28 & F4 & $A Q$ & 41 & 42 & $F$ & 4.6 & 1.35 & 3.4 & Tremolite & & & \\
\hline G2 & 28 & $\mathrm{~F} 4$ & $A Q$ & 42 & & MD 1-1 & 8 & 4.5 & 1.8 & Tremolite & $\begin{array}{l}\mathrm{Mg}, \mathrm{Si}, \mathrm{Ca}, \\
\mathrm{Fe}\end{array}$ & & $A S>5,3: 1$ \\
\hline & & & & & & \multicolumn{2}{|c|}{ ItemType } & ItemNc & & & Confirmed & Comment & \\
\hline & & & & & & \multicolumn{2}{|c|}{ Brightfield } & \multicolumn{2}{|c|}{ J3474 BF } & & & & \\
\hline G2 & 28 & $\mathrm{~F} 4$ & $A Q$ & & 43 & MF & 6.75 & 0.75 & 9 & Tremolite & & & $\begin{array}{c}\text { AFB }>5,3: 1, \text { PCMEF-US, } \\
\text { PCMEF-ISO }\end{array}$ \\
\hline G2 & 29 & D4 & $A Q$ & 43 & & MD 2-1 & 30 & 23.5 & 1.3 & Tremolite & $\begin{array}{c}\mathrm{Mg}, \mathrm{Si}, \mathrm{Ca} \\
\mathrm{Fe}\end{array}$ & & $\mathrm{AS}>5,3: 1$ \\
\hline & & & & & & \multicolumn{2}{|c|}{ ItemType } & ItemNc & & & Confirmed & Comment & \\
\hline
\end{tabular}

\begin{tabular}{|c|c|c|c|c|c|c|c|c|c|c|}
\hline G2 & 29 & D4 & $A Q$ & & 44 & MF & 24.5 & 0.5 & 49 & Tremolite \\
\hline G2 & 29 & D4 & $A Q$ & & 45 & MF & 2.12 & 0.2 & 10.6 & Tremolite \\
\hline G2 & 30 & B4 & $A Q$ & 44 & 46 & $\mathrm{~F}$ & 2.85 & 0.75 & 3.8 & Tremolite \\
\hline G2 & 31 & A7 & $A Q$ & 45 & 47 & $F$ & 8 & 1.5 & 5.3 & Tremolite \\
\hline G2 & 31 & A7 & $A Q$ & 46 & 48 & $F$ & 5.8 & 0.75 & 7.7 & Tremolite \\
\hline G2 & 31 & A7 & $A Q$ & 47 & 49 & $F$ & 5.4 & 1.2 & 4.5 & Tremolite \\
\hline G2 & 32 & C7 & $A Q$ & 48 & 50 & $F$ & 5 & 1.5 & 3.3 & Tremolite \\
\hline G2 & 33 & E7 & $A Q$ & 49 & 51 & $\mathrm{~F}$ & 13 & 3 & 4.3 & Tremolite \\
\hline
\end{tabular}

AFB $>5$, 3:1, PCMEF-US, PCMEF-ISO

AS $>5,3: 1, A F B>5,3: 1$ PCMEF-US, PCMES-US PCMEF-ISO, PCMES-ISO AS $>5,3: 1, A F B>5,3: 1$ PCMEF-US, PCMES-US PCMEF-ISO, PCMES-ISO PCMEF-US, PCMES-US, PCMEF-ISO, PCMES-ISO

PCMEF-US, PCMES-US, PCMEF-ISO, PCMES-ISO

Page 65 of 281

\section{Count Categories}

AFB $>5,3: 1$

PAS

PCMEF-US

PCMES-US
Asbestos Fibers and Bundles > 5um and 3:1

Primary Asbestos Structures

PCM Equivalent Fibers-NIOSH

PCM Equivalent Structures-NIOSH
AS $>5,3: 1$ PCMEF-ISO PCMES-ISO TAS
Asbestos Structures $>5$ um and 3:1 PCM Equivalent Fibers-ISO PCM Equivalent Structures-ISO Total Asbestos Structures 
Job Number: 070434

SEA

Report Number: 070434R06

Client: Idaho National Laboratory

Date Received: 4/23/2007

Project Name: RARE

Client Sample No: FB-3-R2

Lab/Cor Sample No: S20

Client Description:

ISO 10312, Direct Raw Data

\begin{tabular}{|c|c|c|c|c|c|c|c|c|c|c|c|c|c|}
\hline Gr & No. & Loc. & ID & Prim & Tot & Class & Len & Wid & Asp & Analyte & Elements & Comment & Count Categories \\
\hline G2 & 33 & E7 & $A Q$ & 50 & 52 & $\mathrm{~F}$ & 5.7 & 0.45 & 12.7 & Tremolite & & & $\begin{array}{l}\text { AS }>5,3: 1, \text { AFB }>5,3: 1, \\
\text { PCMEF-US, PCMES-US, } \\
\text { PCMEF-ISO, PCMES-ISO }\end{array}$ \\
\hline G2 & 34 & G7 & $A Q$ & 51 & & MD 2-2 & 30 & 20 & 1.5 & Tremolite & & & $A S>5,3: 1$ \\
\hline & & & & & & \multicolumn{2}{|c|}{ ItemType } & ItemNu & & & Confir & Com & \\
\hline & & & & & & \multicolumn{2}{|c|}{ Brightfield } & \multicolumn{2}{|c|}{ J3476 BF } & & & & \\
\hline G2 & 34 & G7 & $A Q$ & & 53 & MF & 15 & 0.5 & 30 & Tremolite & & & $\begin{array}{c}\text { AFB }>5,3: 1, \text { PCMEF-US, } \\
\text { PCMEF-ISO }\end{array}$ \\
\hline G2 & 34 & G7 & $A Q$ & & 54 & MF & 10 & 0.55 & 18.2 & Tremolite & & & $\begin{array}{c}\text { AFB }>5,3: 1, \text { PCMEF-US, } \\
\text { PCMEF-ISO }\end{array}$ \\
\hline \multirow[t]{3}{*}{ G2 } & 35 & 17 & $A Q$ & 52 & & MD 1-0 & 6.5 & 5.8 & 1.1 & Tremolite & & & AS $>5,3: 1$ \\
\hline & & & & & & \multicolumn{2}{|c|}{ ItemType } & ItemNu & & & Confir & Com & \\
\hline & & & & & & \multicolumn{2}{|c|}{ Brightfield } & \multicolumn{2}{|c|}{ J3477 BF } & & & & \\
\hline G2 & 35 & 17 & $A Q$ & & 55 & MF & 2.3 & 0.4 & 5.8 & Tremolite & & & \\
\hline G2 & 35 & 17 & $A Q$ & 53 & 56 & $\mathrm{~F}$ & 19 & 1.45 & 13.1 & Tremolite & & & $\begin{array}{l}\text { AS>5, 3:1, AFB>5, 3:1, } \\
\text { PCMES-US, PCMEF-US, } \\
\text { PCMES-ISO, PCMEF-ISO }\end{array}$ \\
\hline
\end{tabular}

Count Categories

$\mathrm{AFB}>5,3: 1$

PAS

PCMEF-US

PCMES-US
Asbestos Fibers and Bundles > 5um and 3:1

Primary Asbestos Structures

PCM Equivalent Fibers-NIOSH

PCM Equivalent Structures-NIOSH
AS $>5,3: 1$

PCMEF-ISO

PCMES-ISO

TAS
Asbestos Structures $>5$ um and 3:1

PCM Equivalent Fibers-ISO

PCM Equivalent Structures-ISO

Total Asbestos Structures 
Phone: (206) 781-0155

Fax: (206) 789-8424

7619 6th Ave Nw

ISO 10312, Direct Raw Data

Job Number: 070434

SEA

Report Number: 070434R06

Client: Idaho National Laboratory

Date Received: 4/23/2007

Project Name: RARE

Client Sample No: FB-3-R3

Lab/Cor Sample No: S21

Client Description:

Date Sampled

$4 / 16 / 2007$

\begin{tabular}{|c|c|c|c|c|c|c|c|c|c|c|c|c|}
\hline $\mathrm{Gr}$ & No. & Loc. & ID & Prim Tot & Class & Len & Wid & Asp & Analyte & Elements & Comment & Count Categories \\
\hline G1 & 1 & $\mathrm{~A} 2$ & & & NSD & & & & & & & \\
\hline G1 & 2 & $\mathrm{C} 2$ & & & NSD & & & & & & & \\
\hline G1 & 3 & E2 & & & NSD & & & & & & & \\
\hline G1 & 4 & G2 & & & NSD & & & & & & & \\
\hline G1 & 5 & 12 & & & NSD & & & & & & & \\
\hline G1 & 6 & J4 & & & NSD & & & & & & & \\
\hline G1 & 7 & $\mathrm{H} 4$ & & & NSD & & & & & & & \\
\hline G1 & 8 & $\mathrm{~F} 4$ & & & NSD & & & & & & & \\
\hline G1 & 9 & D4 & & & NSD & & & & & & & \\
\hline G1 & 10 & B4 & & & NSD & & & & & & & \\
\hline G1 & 11 & A7 & & & NSD & & & & & & & \\
\hline G1 & 12 & C7 & & & NSD & & & & & & & \\
\hline G1 & 13 & E7 & & & NSD & & & & & & & \\
\hline G1 & 14 & G7 & & & NSD & & & & & & & \\
\hline G1 & 15 & 17 & & & NSD & & & & & & & \\
\hline G1 & 16 & J9 & & & NSD & & & & & & & \\
\hline G1 & 17 & $\mathrm{H} 9$ & & & NSD & & & & & & & \\
\hline G1 & 18 & F9 & & & NSD & & & & & & & \\
\hline G1 & 19 & D9 & & & NSD & & & & & & & \\
\hline G1 & 20 & B9 & & & NSD & & & & & & & \\
\hline G2 & 21 & $\mathrm{~A} 2$ & & & NSD & & & & & & & \\
\hline G2 & 22 & $\mathrm{C} 2$ & & & NSD & & & & & & & \\
\hline G2 & 23 & E2 & & & NSD & & & & & & & \\
\hline G2 & 24 & G2 & & & NSD & & & & & & & \\
\hline G2 & 25 & 12 & & & NSD & & & & & & & \\
\hline G2 & 26 & J4 & & & NSD & & & & & & & \\
\hline G2 & 27 & $\mathrm{H} 4$ & & & NSD & & & & & & & \\
\hline G2 & 28 & F4 & & & NSD & & & & & & & \\
\hline G2 & 29 & D4 & & & NSD & & & & & & & \\
\hline G2 & 30 & B4 & & & NSD & & & & & & & \\
\hline G2 & 31 & A7 & & & NSD & & & & & & & \\
\hline G2 & 32 & $\mathrm{C} 7$ & & & NSD & & & & & & & \\
\hline G2 & 33 & E7 & & & NSD & & & & & & & \\
\hline G2 & 34 & G7 & & & NSD & & & & & & & \\
\hline
\end{tabular}

Count Categories

AFB $>5,3: 1$

PAS

PCMEF-US

Asbestos Fibers and Bundles > 5um and 3:1

AS>5, 3:1 PCMEF-ISO

PCMES-ISO

PCM Equivalent Fibers-NIOSH

PCM Equivalent Structures-NIOSH
TAS
Asbestos Structures $>5$ um and 3:1

PCM Equivalent Fibers-ISO

PCM Equivalent Structures-ISO

Total Asbestos Structures 
Phone: (206) 781-0155

7619 6th Ave Nw

ISO 10312, Direct Raw Data

Job Number: 070434

SEA

Report Number: 070434R06

Client: Idaho National Laboratory

Date Received: 4/23/2007

Project Name: RARE

Client Sample No: FB-3-R3

Lab/Cor Sample No: S21

Client Description:

Date Sampled:

$4 / 16 / 2007$

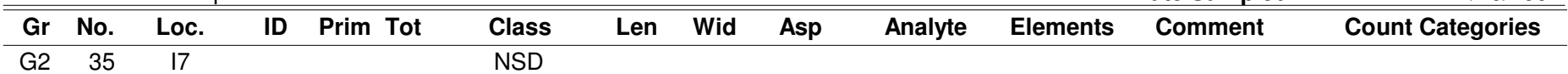

Count Categories

AFB $>5,3: 1$

PAS

PCMEF-US

PCMES-US
Asbestos Fibers and Bundles > 5um and 3:1

Primary Asbestos Structures

PCM Equivalent Fibers-NIOSH

PCM Equivalent Structures-NIOSH
AS>5, 3:1 PCMEF-ISO PCMES-ISO TAS
Asbestos Structures $>5$ um and 3:1 PCM Equivalent Fibers-ISO PCM Equivalent Structures-ISO Total Asbestos Structures 
Phone: (206) 781-0155

Fax: (206) 789-8424

7619 6th Ave Nw

ISO 10312, Direct Raw Data

Job Number: 070434

SEA

Report Number: 070434R06

Client: Idaho National Laboratory

Date Received: 4/23/2007

Project Name: RARE

Client Sample No: FB-3-R4

Lab/Cor Sample No: S22

Client Description:

Date Sampled

$4 / 16 / 2007$

\begin{tabular}{|c|c|c|c|c|c|c|c|c|c|c|c|c|}
\hline $\mathrm{Gr}$ & No. & Loc. & ID & Prim Tot & Class & Len & Wid & Asp & Analyte & Elements & Comment & Count Categories \\
\hline G1 & 1 & $\mathrm{~A} 2$ & & & NSD & & & & & & & \\
\hline G1 & 2 & $\mathrm{C} 2$ & & & NSD & & & & & & & \\
\hline G1 & 3 & E2 & & & NSD & & & & & & & \\
\hline G1 & 4 & G2 & & & NSD & & & & & & & \\
\hline G1 & 5 & 12 & & & NSD & & & & & & & \\
\hline G1 & 6 & J4 & & & NSD & & & & & & & \\
\hline G1 & 7 & $\mathrm{H} 4$ & & & NSD & & & & & & & \\
\hline G1 & 8 & $\mathrm{~F} 4$ & & & NSD & & & & & & & \\
\hline G1 & 9 & D4 & & & NSD & & & & & & & \\
\hline G1 & 10 & B4 & & & NSD & & & & & & & \\
\hline G1 & 11 & A7 & & & NSD & & & & & & & \\
\hline G1 & 12 & C7 & & & NSD & & & & & & & \\
\hline G1 & 13 & E7 & & & NSD & & & & & & & \\
\hline G1 & 14 & G7 & & & NSD & & & & & & & \\
\hline G1 & 15 & 17 & & & NSD & & & & & & & \\
\hline G1 & 16 & J9 & & & NSD & & & & & & & \\
\hline G1 & 17 & $\mathrm{H} 9$ & & & NSD & & & & & & & \\
\hline G1 & 18 & F9 & & & NSD & & & & & & & \\
\hline G1 & 19 & D9 & & & NSD & & & & & & & \\
\hline G1 & 20 & B9 & & & NSD & & & & & & & \\
\hline G2 & 21 & $\mathrm{~A} 2$ & & & NSD & & & & & & & \\
\hline G2 & 22 & $\mathrm{C} 2$ & & & NSD & & & & & & & \\
\hline G2 & 23 & E2 & & & NSD & & & & & & & \\
\hline G2 & 24 & G2 & & & NSD & & & & & & & \\
\hline G2 & 25 & 12 & & & NSD & & & & & & & \\
\hline G2 & 26 & J4 & & & NSD & & & & & & & \\
\hline G2 & 27 & $\mathrm{H} 4$ & & & NSD & & & & & & & \\
\hline G2 & 28 & F4 & & & NSD & & & & & & & \\
\hline G2 & 29 & D4 & & & NSD & & & & & & & \\
\hline G2 & 30 & B4 & & & NSD & & & & & & & \\
\hline G2 & 31 & A7 & & & NSD & & & & & & & \\
\hline G2 & 32 & $\mathrm{C} 7$ & & & NSD & & & & & & & \\
\hline G2 & 33 & E7 & & & NSD & & & & & & & \\
\hline G2 & 34 & G7 & & & NSD & & & & & & & \\
\hline
\end{tabular}

Count Categories

AFB $>5,3: 1$

PAS

PCMEF-US

Asbestos Fibers and Bundles > 5um and 3:1

AS>5, 3:1 PCMEF-ISO

PCMES-ISO

PCM Equivalent Fibers-NIOSH

PCM Equivalent Structures-NIOSH
Asbestos Structures $>5$ um and 3:1

PCM Equivalent Fibers-ISO

PCM Equivalent Structures-ISO

Total Asbestos Structures 
Phone: (206) 781-0155

7619 6th Ave Nw

ISO 10312, Direct Raw Data

Job Number: 070434

SEA

Report Number: 070434R06

Client: Idaho National Laboratory

Date Received: 4/23/2007

Project Name: RARE

Client Sample No: FB-3-R4

Lab/Cor Sample No: S22

Client Description:

Date Sampled:

$4 / 16 / 2007$

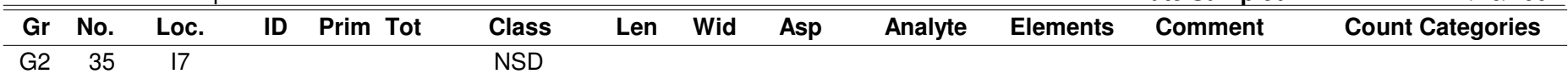

Count Categories

AFB $>5,3: 1$

PAS

PCMEF-US

PCMES-US
Asbestos Fibers and Bundles > 5um and 3:1

Primary Asbestos Structures

PCM Equivalent Fibers-NIOSH

PCM Equivalent Structures-NIOSH
AS>5, 3:1 PCMEF-ISO PCMES-ISO TAS
Asbestos Structures >5um and 3:1 PCM Equivalent Fibers-ISO PCM Equivalent Structures-ISO Total Asbestos Structures 
Job Number: 070434

\section{SEA}

ISO 10312, Direct Raw Data

Client: Idaho National Laboratory

Report Number: 070434R06

Date Received: 4/23/2007

Project Name: RARE

Client Sample No: FB-3-R5

Lab/Cor Sample No: S23

Client Description:

Date Sampled:

$4 / 16 / 2007$

\begin{tabular}{|c|c|c|c|c|c|c|c|c|c|c|c|c|c|}
\hline Gr & No. & Loc. & ID & Prim & Tot & Class & Len & Wid & Asp & Analyte & Elements & Comment & Count Categories \\
\hline G1 & 1 & $\mathrm{~A} 2$ & & & & NSD & & & & & & & \\
\hline G1 & 2 & C2 & & & & NSD & & & & & & & \\
\hline G1 & 3 & E2 & & & & NSD & & & & & & & \\
\hline \multirow[t]{5}{*}{ G1 } & 4 & $\mathrm{G} 2$ & $A Z Q$ & 1 & 1 & $\mathrm{~F}$ & 10 & 0.85 & 11.8 & Tremolite & $\begin{array}{l}\text { Mg, Al, Si, } \\
\text { Ca, Fe }\end{array}$ & & $\begin{array}{l}\text { AS }>5,3: 1, \text { AFB }>5,3: 1, \\
\text { PCMEF-US, PCMES-US, } \\
\text { PCMEF-ISO, PCMES-ISO }\end{array}$ \\
\hline & & & & & & \multicolumn{2}{|c|}{ ItemType } & ItemNum & & & Confirmed & Com & \\
\hline & & & & & & \multicolumn{2}{|c|}{ Brightfield } & $\mathrm{J} 3505 \mathrm{~B}$ & & & & & \\
\hline & & & & & & \multicolumn{2}{|c|}{ Diffraction } & $J 3505$ & & & KM 5/22/2007 & ZON & IS [ $\left.\begin{array}{lll}7 & 1 & 6\end{array}\right]$ \\
\hline & & & & & & \multicolumn{2}{|c|}{ Spectra } & $\mathrm{J} 2950$ & & & & & \\
\hline \multirow[t]{3}{*}{ G1 } & 4 & G2 & $A Q$ & 2 & 2 & $\mathrm{~F}$ & 4.25 & 0.45 & 9.4 & Tremolite & $\begin{array}{l}\text { Mg, Al, Si, } \\
\mathrm{Ca}, \mathrm{Fe}\end{array}$ & & \\
\hline & & & & & & \multicolumn{2}{|c|}{ ItemType } & ItemNum & & & Confirmed & Com & \\
\hline & & & & & & \multicolumn{2}{|c|}{ Brightfield } & J3505 B & & & & & \\
\hline \multirow[t]{5}{*}{ G1 } & 4 & G2 & $A D Q$ & 3 & & MD 1-1 & 7 & 1.75 & 4 & Tremolite & $\begin{array}{l}\mathrm{Mg}, \mathrm{Al}, \mathrm{Si} \\
\mathrm{Ca}, \mathrm{Fe}\end{array}$ & & $\begin{array}{c}\text { AS>5, 3:1, PCMES-US, } \\
\text { PCMES-ISO }\end{array}$ \\
\hline & & & & & & \multicolumn{2}{|c|}{ ItemType } & ItemNum & & & Confirmed & Com & \\
\hline & & & & & & \multicolumn{2}{|c|}{ Brightfield } & J3506 B & & & & & \\
\hline & & & & & & \multicolumn{2}{|c|}{ Diffraction } & J3506 & & & KM 5/22/2007 & $5.3 \mathrm{~A}$ & \\
\hline & & & & & & \multicolumn{2}{|c|}{ Spectra } & J2951 & & & & & \\
\hline G1 & 4 & G2 & $A D Q$ & & 3 & MF & 7 & 0.5 & 14 & Tremolite & & & $\begin{array}{c}\text { AFB }>5,3: 1, \text { PCMEF-US, } \\
\text { PCMEF-ISO }\end{array}$ \\
\hline G1 & 5 & 12 & $A Q$ & 4 & 4 & $\mathrm{~F}$ & 7.75 & 1.15 & 6.7 & Tremolite & $\begin{array}{l}\text { Mg, Al, Si, } \\
\text { Ca, Fe }\end{array}$ & & $\begin{array}{l}\text { AS }>5,3: 1, \text { AFB }>5,3: 1, \\
\text { PCMEF-US, PCMES-US, } \\
\text { PCMEF-ISO, PCMES-ISO }\end{array}$ \\
\hline G1 & 6 & J4 & & & & NSD & & & & & & & \\
\hline G1 & 7 & $\mathrm{H} 4$ & & & & NSD & & & & & & & \\
\hline G1 & 8 & $\mathrm{~F} 4$ & $A Q$ & 5 & 5 & $\mathrm{~F}$ & 23.5 & 1.85 & 12.7 & Tremolite & $\begin{array}{l}\text { Mg, Al, Si, } \\
\text { Ca, Fe }\end{array}$ & & $\begin{array}{l}\text { AS }>5,3: 1, \text { AFB }>5,3: 1, \\
\text { PCMEF-US, PCMES-US, } \\
\text { PCMEF-ISO, PCMES-ISO }\end{array}$ \\
\hline G1 & 8 & F4 & $A Q$ & 6 & 6 & $\mathrm{~F}$ & 4.35 & 0.65 & 6.7 & Tremolite & $\begin{array}{l}\mathrm{Mg}, \mathrm{Al}, \mathrm{Si} \\
\mathrm{Ca}, \mathrm{Fe}\end{array}$ & & \\
\hline G1 & 9 & B4 & $A Q$ & 7 & & MD 1-1 & 17.75 & 4.5 & 3.9 & Tremolite & $\begin{array}{l}\mathrm{Mg}, \mathrm{Al}, \mathrm{Si} \\
\mathrm{Ca}, \mathrm{Fe}\end{array}$ & & AS>5, 3:1, PCMES-US \\
\hline G1 & 9 & B4 & $A Q$ & & 7 & MF & 17.75 & 1.7 & 10.4 & Tremolite & & & $\begin{array}{c}\text { AFB }>5,3: 1, \text { PCMEF-US, } \\
\text { PCMEF-ISO }\end{array}$ \\
\hline
\end{tabular}

\section{Count Categories}

AFB $>5,3: 1$

PAS

PCMEF-US

PCMES-US
Asbestos Fibers and Bundles > 5um and 3:1

Primary Asbestos Structures

PCM Equivalent Fibers-NIOSH

PCM Equivalent Structures-NIOSH
AS $>5,3: 1$ PCMEF-ISO PCMES-ISO TAS
Asbestos Structures $>5$ um and 3:1 PCM Equivalent Fibers-ISO PCM Equivalent Structures-ISO Total Asbestos Structures 


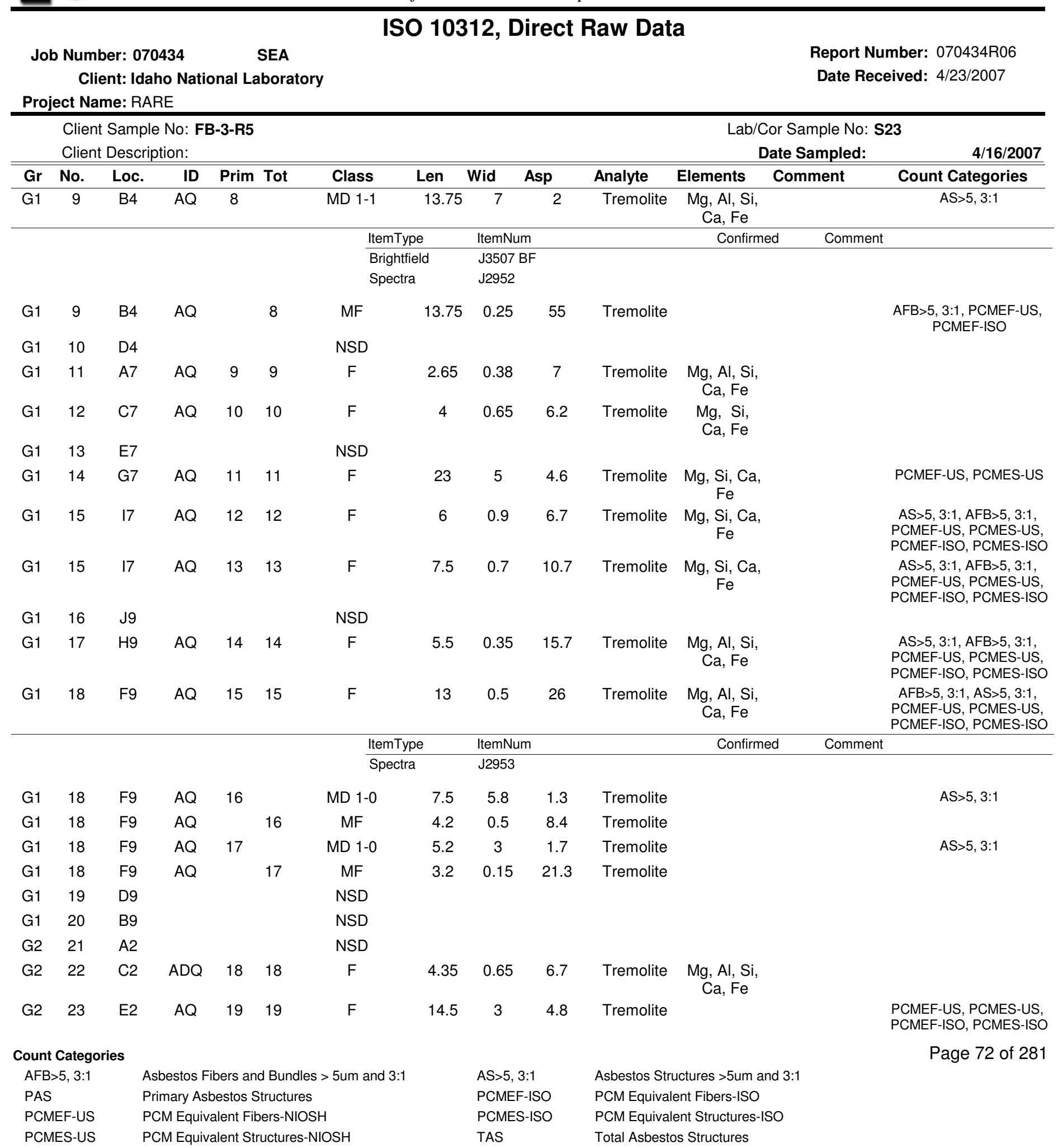


Job Number: 070434

SEA

ISO 10312, Direct Raw Data

Client: Idaho National Laboratory

Report Number: 070434R06

Project Name: RARE

Date Received: 4/23/2007

Client Sample No: FB-3-R5

Lab/Cor Sample No: S23

Client Description:

Date Sampled:

$4 / 16 / 2007$

\begin{tabular}{|c|c|c|c|c|c|c|c|c|c|c|c|c|c|}
\hline Gr & No. & Loc. & ID & Prim & Tot & Class & Len & Wid & Asp & Analyte & Elements & Comment & Count Categories \\
\hline G2 & 24 & G2 & $A D Q$ & 20 & 20 & $F$ & 7 & 0.5 & 14 & Tremolite & & & $\begin{array}{l}\text { AS>5, 3:1, AFB>5, 3:1, } \\
\text { PCMEF-US, PCMES-US, } \\
\text { PCMEF-ISO, PCMES-ISO }\end{array}$ \\
\hline G2 & 25 & 12 & & & & NSD & & & & & & & \\
\hline G2 & 26 & J4 & & & & NSD & & & & & & & \\
\hline G2 & 27 & $\mathrm{H} 4$ & & & & NSD & & & & & & & \\
\hline G2 & 28 & $\mathrm{~F} 4$ & & & & NSD & & & & & & & \\
\hline G2 & 29 & D4 & & & & NSD & & & & & & & \\
\hline G2 & 30 & B4 & $A Q$ & 21 & 21 & $F$ & 13.25 & 3 & 4.4 & Tremolite & & & $\begin{array}{l}\text { PCMEF-US, PCMES-US, } \\
\text { PCMEF-ISO, PCMES-ISO }\end{array}$ \\
\hline G2 & 31 & A7 & & & & NSD & & & & & & & \\
\hline \multirow[t]{2}{*}{ G2 } & 32 & C7 & $A Q$ & 22 & 22 & B & 6.2 & 1.75 & 3.5 & Tremolite & $\begin{array}{l}\text { Mg, Al, Si, } \\
\text { Ca, Fe }\end{array}$ & & $\begin{array}{l}\text { AFB>5, 3:1, AS>5, 3:1, } \\
\text { PCMES-US, PCMEF-US, } \\
\text { PCMES-ISO, PCMEF-ISO }\end{array}$ \\
\hline & & & & & & & & ItemNe & & & Confirm & & \\
\hline
\end{tabular}

G2 $33 \quad$ E7

G2 $34 \quad$ G7

$\begin{array}{lll}A Q & 23 & 23\end{array}$

NSD

G2 34

G7

AQ $\quad 24 \quad 24$

$\mathrm{F}$

5.5

0.75

7.3

F

NSD

G2 $\quad 35 \quad 17$
$\mathrm{AS}>5,3: 1, \mathrm{AFB}>5,3: 1$ PCMEF-US, PCMES-US PCMEF-ISO, PCMES-ISO $A S>5,3: 1, A F B>5,3: 1$ PCMEF-US, PCMES-US, PCMEF-ISO, PCMES-ISO
Count Categories

AFB $>5,3: 1$

PAS

PCMEF-US

PCMES-US
Asbestos Fibers and Bundles $>5 u m$ and 3:1

Primary Asbestos Structures

PCM Equivalent Fibers-NIOSH

PCM Equivalent Structures-NIOSH
AS $>5,3: 1$ PCMEF-ISO PCMES-ISO TAS
Asbestos Structures $>5$ um and 3:1 PCM Equivalent Fibers-ISO PCM Equivalent Structures-ISO Total Asbestos Structures 
Phone: (206) 781-0155

Fax: (206) 789-8424

7619 6th Ave Nw

ISO 10312, Direct Raw Data

Job Number: 070434

SEA

Report Number: 070434R06

Client: Idaho National Laboratory

Date Received: 4/23/2007

Project Name: RARE

Client Sample No: FB-3-R6

Lab/Cor Sample No: S24

Client Description:

Date Sampled

$4 / 16 / 2007$

\begin{tabular}{|c|c|c|c|c|c|c|c|c|c|c|c|c|}
\hline $\mathrm{Gr}$ & No. & Loc. & ID & Prim Tot & Class & Len & Wid & Asp & Analyte & Elements & Comment & Count Categories \\
\hline G1 & 1 & $\mathrm{~A} 2$ & & & NSD & & & & & & & \\
\hline G1 & 2 & $\mathrm{C} 2$ & & & NSD & & & & & & & \\
\hline G1 & 3 & E2 & & & NSD & & & & & & & \\
\hline G1 & 4 & G2 & & & NSD & & & & & & & \\
\hline G1 & 5 & 12 & & & NSD & & & & & & & \\
\hline G1 & 6 & $\mathrm{H} 4$ & & & NSD & & & & & & & \\
\hline G1 & 7 & $\mathrm{~F} 4$ & & & NSD & & & & & & & \\
\hline G1 & 8 & D4 & & & NSD & & & & & & & \\
\hline G1 & 9 & B4 & & & NSD & & & & & & & \\
\hline G1 & 10 & A5 & & & NSD & & & & & & & \\
\hline G1 & 11 & A7 & & & NSD & & & & & & & \\
\hline G1 & 12 & C7 & & & NSD & & & & & & & \\
\hline G1 & 13 & E7 & & & NSD & & & & & & & \\
\hline G1 & 14 & G7 & & & NSD & & & & & & & \\
\hline G1 & 15 & 17 & & & NSD & & & & & & & \\
\hline G1 & 16 & J9 & & & NSD & & & & & & & \\
\hline G1 & 17 & $\mathrm{H} 9$ & & & NSD & & & & & & & \\
\hline G1 & 18 & F9 & & & NSD & & & & & & & \\
\hline G1 & 19 & D9 & & & NSD & & & & & & & \\
\hline G1 & 20 & B9 & & & NSD & & & & & & & \\
\hline G2 & 21 & $\mathrm{~A} 2$ & & & NSD & & & & & & & \\
\hline G2 & 22 & $\mathrm{C} 2$ & & & NSD & & & & & & & \\
\hline G2 & 23 & E2 & & & NSD & & & & & & & \\
\hline G2 & 24 & G2 & & & NSD & & & & & & & \\
\hline G2 & 25 & 12 & & & NSD & & & & & & & \\
\hline G2 & 26 & J4 & & & NSD & & & & & & & \\
\hline G2 & 27 & $\mathrm{H} 4$ & & & NSD & & & & & & & \\
\hline G2 & 28 & F4 & & & NSD & & & & & & & \\
\hline G2 & 29 & D4 & & & NSD & & & & & & & \\
\hline G2 & 30 & B4 & & & NSD & & & & & & & \\
\hline G2 & 31 & A7 & & & NSD & & & & & & & \\
\hline G2 & 32 & $\mathrm{C} 7$ & & & NSD & & & & & & & \\
\hline G2 & 33 & E7 & & & NSD & & & & & & & \\
\hline G2 & 34 & G7 & & & NSD & & & & & & & \\
\hline
\end{tabular}

Count Categories

AFB $>5,3: 1$

PAS

PCMEF-US

Asbestos Fibers and Bundles $>5 \mathrm{um}$ and 3:1

Primary Asbestos Structures

PCM Equivalent Fibers-NIOSH

PCM Equivalent Structures-NIOSH
AS>5, 3:1 PCMEF-ISO PCMES-ISO TAS
Asbestos Structures $>5$ um and 3:1 PCM Equivalent Fibers-ISO PCM Equivalent Structures-ISO Total Asbestos Structures 
Phone: (206) 781-0155

7619 6th Ave Nw

Job Number: 070434

SEA

ISO 10312, Direct Raw Data

Client: Idaho National Laboratory

Report Number: 070434R06

Name: RARE

Client Sample No: FB-3-R6

Lab/Cor Sample No: S24

Client Description:

\begin{tabular}{|c|c|c|c|c|c|c|c|c|c|c|c|c|}
\hline Gr & No. & Loc. & ID & Prim Tot & Class & Len & Wid & Asp & Analyte & Elements & Comment & Count Categories \\
\hline G2 & 35 & 17 & & & NSD & & & & & & & \\
\hline
\end{tabular}

Count Categories

AFB $>5$, 3:1

PAS

PCMEF-US

PCMES-US
Asbestos Fibers and Bundles > 5um and 3:1

Primary Asbestos Structures

PCM Equivalent Fibers-NIOSH

PCM Equivalent Structures-NIOSH
AS>5, 3:1 PCMEF-ISO PCMES-ISO TAS
Fax: (206) 789-8424

http://www.labcor.net 


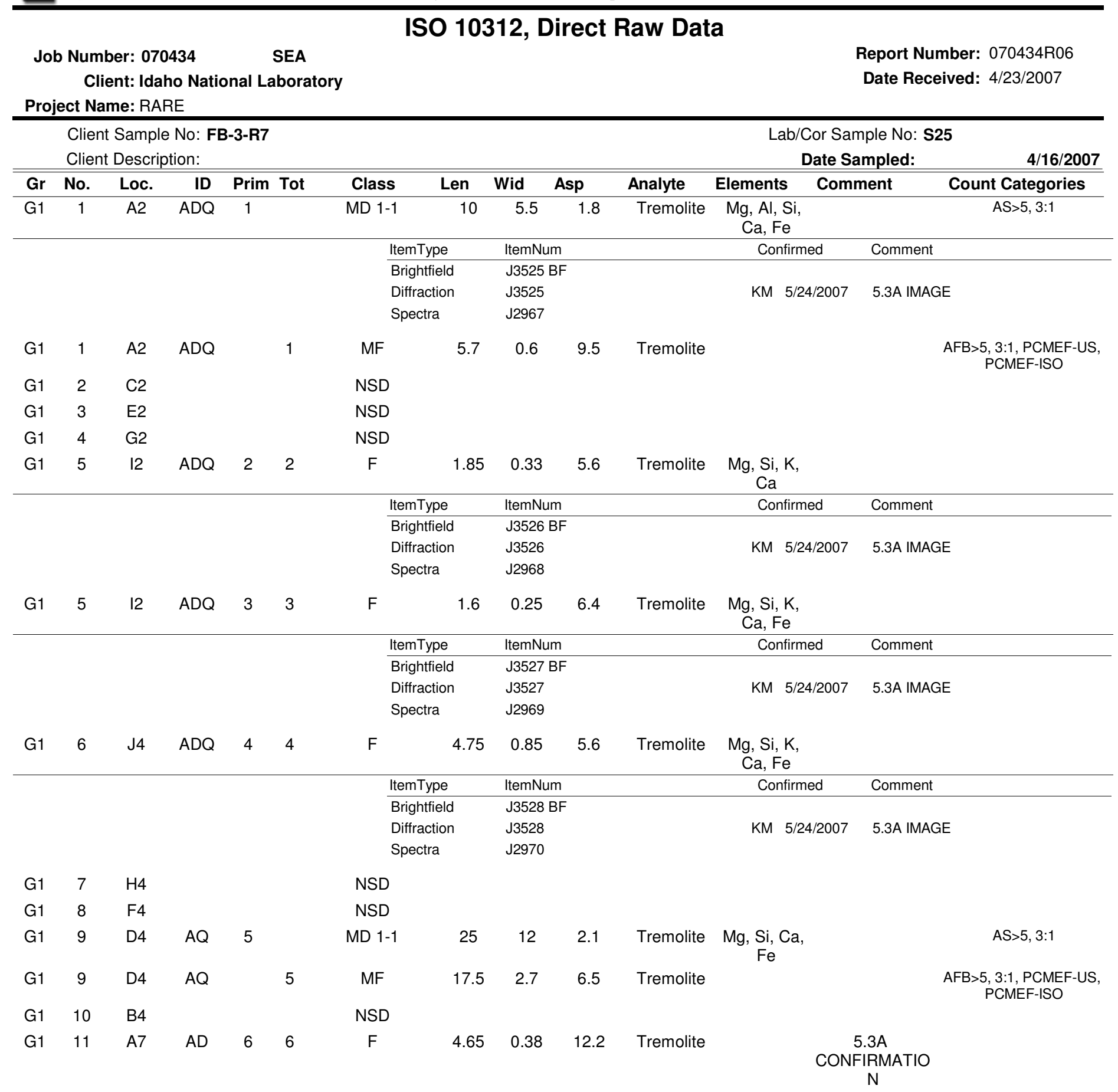

Count Categories

$A F B>5,3: 1$

PAS

PCMEF-US

Asbestos Fibers and Bundles $>$ 5um and 3:1

Primary Asbestos Structures

PCM Equivalent Fibers-NIOSH

PCMES-US
PCM Equivalent Structures-NIOSH
AS $>5,3: 1$ PCMEF-ISO PCMES-ISO TAS
Page 76 of 281 


\section{ISO 10312, Direct Raw Data}

Job Number: 070434

SEA

Report Number: 070434R06

Client: Idaho National Laboratory

Date Received: 4/23/2007

Project Name: RARE

Client Sample No: FB-3-R7

Lab/Cor Sample No: S25

Client Description:

Date Sampled:

$4 / 16 / 2007$

\begin{tabular}{|c|c|c|c|c|c|c|c|c|c|c|c|c|c|}
\hline Gr & No. & Loc. & ID & Prim & Tot & Class & Len & Wid & Asp & Analyte & Elements & ient & Count Categories \\
\hline G1 & 11 & A7 & $A D Q$ & 7 & 7 & $F$ & 5.2 & 0.4 & 13 & Tremolite & $\begin{array}{l}\mathrm{Mg}, \mathrm{Al}, \mathrm{Si} \\
\mathrm{Ca}, \mathrm{Fe}\end{array}$ & & $\begin{array}{l}\text { AS }>5,3: 1, \text { AFB }>5,3: 1, \\
\text { PCMEF-US, PCMES-US, } \\
\text { PCMEF-ISO, PCMES-ISO }\end{array}$ \\
\hline & & & & & & \multicolumn{2}{|c|}{ ItemType } & ItemNum & & & Confirmed & Comr & \\
\hline & & & & & & \multicolumn{2}{|c|}{ Brightfield } & J3529 B & & & & & \\
\hline & & & & & & \multicolumn{2}{|c|}{ Diffraction } & J3529 & & & KM 5/24/2007 & $5.3 \mathrm{~A}$ & \\
\hline & & & & & & \multicolumn{2}{|c|}{ Spectra } & $\mathrm{J} 2971$ & & & & & \\
\hline G1 & 12 & $\mathrm{C} 7$ & & & & \multicolumn{2}{|l|}{ NSD } & & & & & & \\
\hline G1 & 13 & E7 & & & & \multicolumn{2}{|l|}{ NSD } & & & & & & \\
\hline G1 & 14 & G7 & & & & \multicolumn{2}{|l|}{ NSD } & & & & & & \\
\hline G1 & 15 & 17 & $A D Q$ & 8 & 8 & $\mathrm{~F}$ & 18 & 7 & 2.6 & Tremolite & $\begin{array}{c}\mathrm{Mg}, \mathrm{Si}, \mathrm{Ca} \\
\mathrm{Fe}\end{array}$ & & \\
\hline G1 & 15 & 17 & $A Q$ & 9 & 9 & $\mathrm{~F}$ & 3.3 & 0.85 & 3.9 & Tremolite & & & \\
\hline G1 & 16 & J9 & & & & \multicolumn{2}{|l|}{ NSD } & & & & & & \\
\hline G1 & 17 & $\mathrm{H} 9$ & & & & \multicolumn{2}{|l|}{ NSD } & & & & & & \\
\hline G1 & 18 & F9 & $A D Q$ & 10 & & MD 1-1 & 15 & 12 & 1.2 & Tremolite & $\begin{array}{l}\mathrm{Mg}, \mathrm{Si}, \mathrm{Ca} \\
\mathrm{Fe}\end{array}$ & & $A S>5,3: 1$ \\
\hline G1 & 18 & F9 & $A D Q$ & & 10 & MF & 15 & 2 & 7.5 & Tremolite & & & $\begin{array}{c}\text { AFB }>5,3: 1, \text { PCMEF-US, } \\
\text { PCMEF-ISO }\end{array}$ \\
\hline \multirow[t]{5}{*}{ G1 } & 19 & D9 & $A Z Q$ & 11 & 11 & $\mathrm{~F}$ & 5.5 & 0.22 & 25 & Tremolite & $\begin{array}{l}\mathrm{Mg}, \mathrm{Si}, \mathrm{Ca}, \\
\mathrm{Fe}\end{array}$ & & $\begin{array}{l}\text { AS }>5,3: 1, \text { AFB }>5,3: 1, \\
\text { PCMEF-ISO, PCMES-ISO }\end{array}$ \\
\hline & & & & & & \multicolumn{2}{|c|}{ ItemType } & ItemNum & & & Confirmed & Comr & \\
\hline & & & & & & \multicolumn{2}{|c|}{ Brightfield } & J3530 B & & & & & \\
\hline & & & & & & \multicolumn{2}{|c|}{ Diffraction } & $\mathrm{J} 3530$ & & & KM 5/24/2007 & ZON & IS [ $\left.\begin{array}{lll}7 & 1 & 0\end{array}\right]$ \\
\hline & & & & & & \multicolumn{2}{|c|}{ Spectra } & $\mathrm{J} 2972$ & & & & & \\
\hline G1 & 20 & B9 & $A D Q$ & 12 & & MD 1-1 & 13.3 & 4 & 3.3 & Tremolite & $\begin{array}{l}\mathrm{Mg}, \mathrm{Si}, \mathrm{Ca} \\
\mathrm{Fe}\end{array}$ & & AS $>5,3: 1$, PCMES-US \\
\hline G1 & 20 & B9 & $A D Q$ & & 12 & MF & 12.5 & 1 & 12.5 & Tremolite & & & $\begin{array}{c}\text { AFB >5, 3:1, PCMEF-US, } \\
\text { PCMEF-ISO }\end{array}$ \\
\hline G1 & 20 & B9 & $A Q$ & 13 & 13 & $\mathrm{~F}$ & 4.5 & 0.85 & 5.3 & Tremolite & $\begin{array}{l}\mathrm{Mg}, \mathrm{Si}, \mathrm{Ca} \\
\mathrm{Fe}\end{array}$ & & \\
\hline G2 & 21 & A2 & $A Q$ & 14 & 14 & $\mathrm{~F}$ & 5.6 & 0.7 & 8 & Tremolite & & & $\begin{array}{l}\text { AS }>5,3: 1, \text { AFB }>5,3: 1, \\
\text { PCMEF-US, PCMES-US, } \\
\text { PCMEF-ISO, PCMES-ISO }\end{array}$ \\
\hline \multirow[t]{5}{*}{ G2 } & 22 & $\mathrm{C} 2$ & $A Z Q$ & 15 & & CD 2-1 & 7 & 4.5 & 1.6 & Tremolite & $\begin{array}{l}\mathrm{Mg}, \mathrm{Al}, \mathrm{Si} \\
\mathrm{K}, \mathrm{Ca}, \mathrm{Fe}\end{array}$ & & $A S>5,3: 1$ \\
\hline & & & & & & \multicolumn{2}{|c|}{ ItemType } & ItemNum & & & Confirmed & Comr & \\
\hline & & & & & & \multicolumn{2}{|c|}{ Brightfield } & J3531 B & & & & & \\
\hline & & & & & & \multicolumn{2}{|c|}{ Diffraction } & J3531 & & & KM 5/25/2007 & ZON & IS [ $\left.\begin{array}{lll}1 & 1 & 0\end{array}\right]$ \\
\hline & & & & & & \multicolumn{2}{|c|}{ Spectra } & J2973 & & & & & \\
\hline
\end{tabular}

Count Categories

AFB $>5,3: 1$

PAS

PCMEF-US

PCMES-US
Asbestos Fibers and Bundles $>$ 5um and 3:1

Primary Asbestos Structures

PCM Equivalent Fibers-NIOSH

PCM Equivalent Structures-NIOSH
AS $>5,3: 1$ PCMEF-ISO PCMES-ISO TAS
Asbestos Structures $>5$ um and 3:1 PCM Equivalent Fibers-ISO PCM Equivalent Structures-ISO Total Asbestos Structures 
Job Number: 070434

SEA

ISO 10312, Direct Raw Data

Client: Idaho National Laboratory

Project Name: RARE

Client Sample No: FB-3-R7

Lab/Cor Sample No: S25

Client Description:

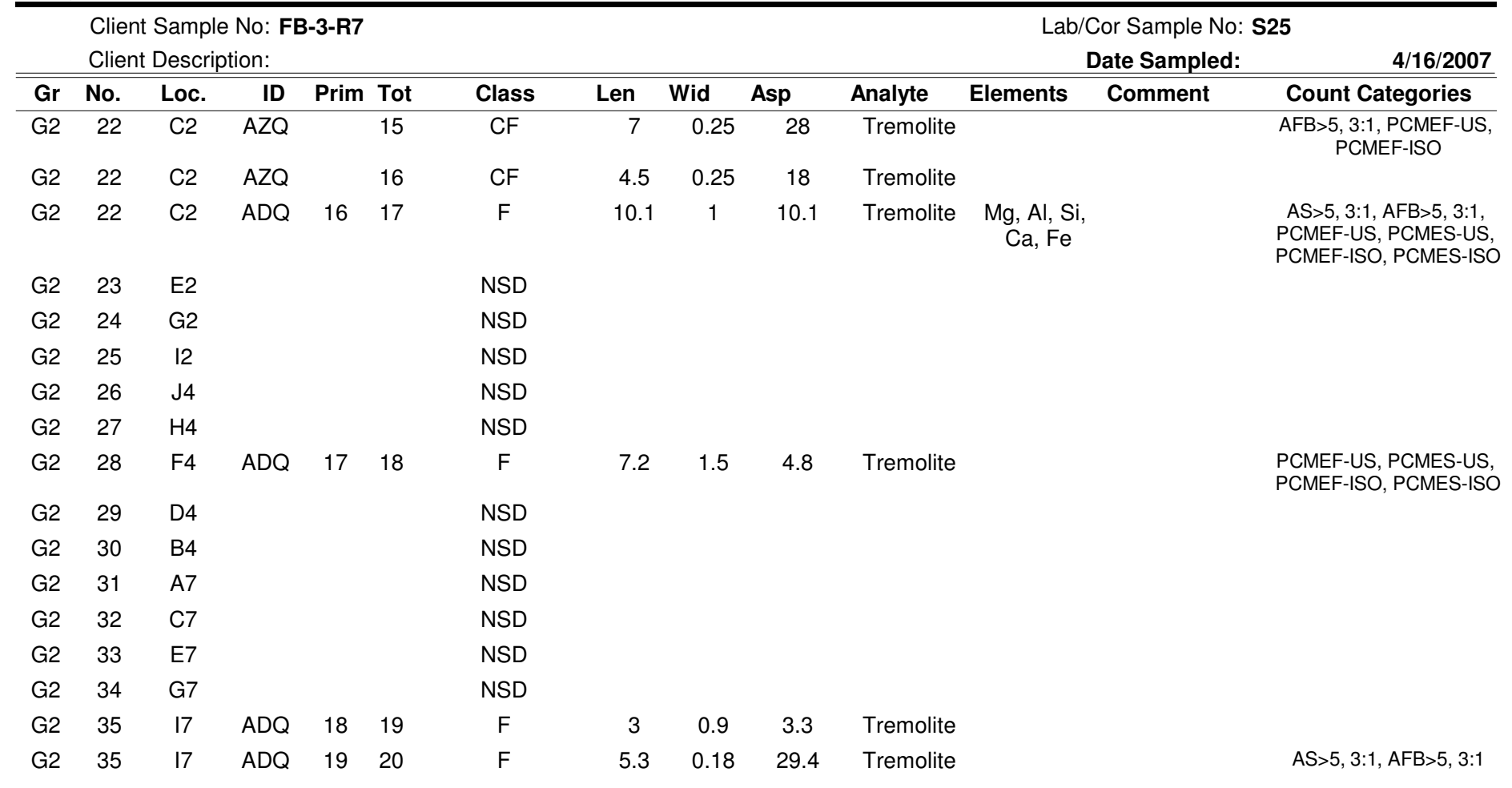

Report Number: 070434R06

Date Received: 4/23/2007
Count Categories

$\mathrm{AFB}>5,3: 1$

PAS

PCMEF-US

PCMES-US
Asbestos Fibers and Bundles > 5um and 3:1

Primary Asbestos Structures

PCM Equivalent Fibers-NIOSH

PCM Equivalent Structures-NIOSH
AS $>5,3: 1$ PCMEF-ISO PCMES-ISO TAS
Asbestos Structures $>5$ um and 3:1 PCM Equivalent Fibers-ISO PCM Equivalent Structures-ISO Total Asbestos Structures 
Job Number: 070434

ISO 10312, Direct Raw Data

Client: Idaho National Laboratory

Report Number: 070434R06

Project Name: RARE

Date Received: 4/23/2007

Client Sample No: FB-2-R1

Lab/Cor Sample No: S26

Client Description:

Date Sampled:

$4 / 16 / 2007$

\begin{tabular}{|c|c|c|c|c|c|c|c|c|c|c|c|c|c|}
\hline Gr & No. & Loc. & ID & Prim & Tot & Class & Len & Wid & Asp & \multirow{2}{*}{$\begin{array}{l}\text { Analyte } \\
\text { Tremolite }\end{array}$} & Elements & Comment & \multirow[t]{2}{*}{ Count Categories } \\
\hline G1 & 1 & $\mathrm{~A} 2$ & $A Q$ & 1 & 1 & $F$ & 4.5 & 1 & 4.5 & & & & \\
\hline G1 & 1 & $\mathrm{~A} 2$ & $A Q$ & 2 & 2 & $\mathrm{~F}$ & 10.5 & 0.75 & 14 & Tremolite & & & $\begin{array}{l}\text { AS>5, 3:1, AFB }>5,3: 1, \\
\text { PCMEF-US, PCMES-US, } \\
\text { PCMEF-ISO, PCMES-ISO }\end{array}$ \\
\hline G1 & 1 & $\mathrm{~A} 2$ & $A Q$ & 3 & 3 & $\mathrm{~F}$ & 4 & 0.45 & 8.9 & Tremolite & & & \\
\hline G1 & 1 & $\mathrm{~A} 2$ & $A Q$ & 4 & 4 & $\mathrm{~F}$ & 9 & 0.75 & 12 & Tremolite & & & $\begin{array}{l}\text { AS>5, 3:1, AFB>5, 3:1, } \\
\text { PCMEF-US, PCMES-US, } \\
\text { PCMEF-ISO, PCMES-ISO }\end{array}$ \\
\hline G1 & 1 & $\mathrm{~A} 2$ & $A Q$ & 5 & 5 & $\mathrm{~F}$ & 20 & 1.5 & 13.3 & Tremolite & & & $\begin{array}{l}\text { AS>5, 3:1, AFB }>5,3: 1, \\
\text { PCMEF-US, PCMES-US, } \\
\text { PCMEF-ISO, PCMES-ISO }\end{array}$ \\
\hline G1 & 1 & $\mathrm{~A} 2$ & $A Q$ & 6 & 6 & $\mathrm{~F}$ & 40 & 1.25 & 32 & Tremolite & & & $\begin{array}{l}\text { AS>5, 3:1, AFB >5, 3:1, } \\
\text { PCMEF-US, PCMES-US, } \\
\text { PCMEF-ISO, PCMES-ISO }\end{array}$ \\
\hline G1 & 1 & $\mathrm{~A} 2$ & $A Q$ & 7 & & MD 1-0 & 5 & 2.5 & 2 & Tremolite & & & \\
\hline G1 & 1 & $\mathrm{~A} 2$ & $A Q$ & & 7 & MF & 5 & 0.85 & 5.9 & Tremolite & & & \\
\hline G1 & 2 & $\mathrm{C} 2$ & $A Q$ & 8 & 8 & $F$ & 8 & 2.12 & 3.8 & Tremolite & & & $\begin{array}{l}\text { PCMEF-US, PCMES-US, } \\
\text { PCMEF-ISO, PCMES-ISO }\end{array}$ \\
\hline \multirow[t]{5}{*}{ G1 } & 2 & $\mathrm{C} 2$ & $A Z Q$ & 9 & & MD 1-0 & 10 & 9 & 1.1 & Tremolite & $\begin{array}{l}\mathrm{Mg}, \mathrm{Al}, \mathrm{Si} \\
\mathrm{Ca}, \mathrm{Fe}\end{array}$ & & $A S>5,3: 1$ \\
\hline & & & & & & \multicolumn{2}{|c|}{ ItemType } & ItemNc & & & Confirmed & Comr & \\
\hline & & & & & & \multicolumn{2}{|c|}{ Brightfield } & J3532 & & & & & \\
\hline & & & & & & \multicolumn{2}{|c|}{ Diffraction } & J3532 & & & KM 5/25/2007 & ZON & IS [ $\left.\begin{array}{lll}1 & 0 & 1\end{array}\right]$ \\
\hline & & & & & & \multicolumn{2}{|c|}{ Spectra } & J2974 & & & & & \\
\hline G1 & 2 & $\mathrm{C} 2$ & $A Z Q$ & & 9 & MF & 4.3 & 0.4 & 10.8 & Tremolite & & & \\
\hline G1 & 2 & $\mathrm{C} 2$ & $A Q$ & 10 & 10 & B & 19.5 & 1.25 & 15.6 & Tremolite & & & $\begin{array}{l}\text { AFB }>5,3: 1, \text { AS }>5,3: 1, \\
\text { PCMES-US, PCMEF-US, } \\
\text { PCMES-ISO, PCMEF-ISO }\end{array}$ \\
\hline G1 & 2 & $\mathrm{C} 2$ & $A Q$ & 11 & 11 & $F$ & 4.45 & 0.8 & 5.6 & Tremolite & & & \\
\hline G1 & 2 & $\mathrm{C} 2$ & $A Q$ & 12 & 12 & $F$ & 4.65 & 1.25 & 3.7 & Tremolite & & & \\
\hline G1 & 3 & E2 & $A Q$ & 13 & 13 & $\mathrm{~F}$ & 2.35 & 0.65 & 3.6 & Tremolite & & & \\
\hline G1 & 3 & E2 & $A Q$ & 14 & & MD 1-0 & 10 & 9 & 1.1 & Tremolite & & & $A S>5,3: 1$ \\
\hline G1 & 3 & E2 & $A Q$ & & 14 & MF & 4.85 & 0.9 & 5.4 & Tremolite & & & \\
\hline G1 & 3 & E2 & $A Q$ & 15 & 15 & B & 5.2 & 1.5 & 3.5 & Tremolite & & & $\begin{array}{l}\text { AFB }>5,3: 1, \text { AS }>5,3: 1, \\
\text { PCMES-US, PCMEF-US, } \\
\text { PCMES-ISO, PCMEF-ISO }\end{array}$ \\
\hline G1 & 3 & E2 & $A Q$ & 16 & 16 & $\mathrm{~F}$ & 7.5 & 1.75 & 4.3 & Tremolite & & & $\begin{array}{l}\text { PCMEF-US, PCMES-US, } \\
\text { PCMEF-ISO, PCMES-ISO }\end{array}$ \\
\hline G1 & 4 & G2 & $A Q$ & 17 & & MD 1-0 & 2.5 & 1.5 & 1.7 & Tremolite & & & \\
\hline G1 & 4 & G2 & $A Q$ & & 17 & MF & 2.2 & 0.38 & 5.8 & Tremolite & & & \\
\hline
\end{tabular}

Count Categories

PAS

PCMEF-US

Asbestos Fibers and Bundles > 5um and 3:1

Primary Asbestos Structures

PCM Equivalent Fibers-NIOSH

PCMES-US
PCM Equivalent Structures-NIOSH
AS $>5,3: 1$ PCMEF-ISO PCMES-ISO TAS
Asbestos Structures $>5$ um and 3:1 PCM Equivalent Fibers-ISO PCM Equivalent Structures-ISO Total Asbestos Structures 
ISO 10312, Direct Raw Data

Job Number: 070434

SEA

Report Number: 070434R06

Client: Idaho National Laboratory

Date Received: 4/23/2007

Project Name: RARE

Client Sample No: FB-2-R1

Lab/Cor Sample No: S26

Client Description:

Date Sampled:

$4 / 16 / 2007$

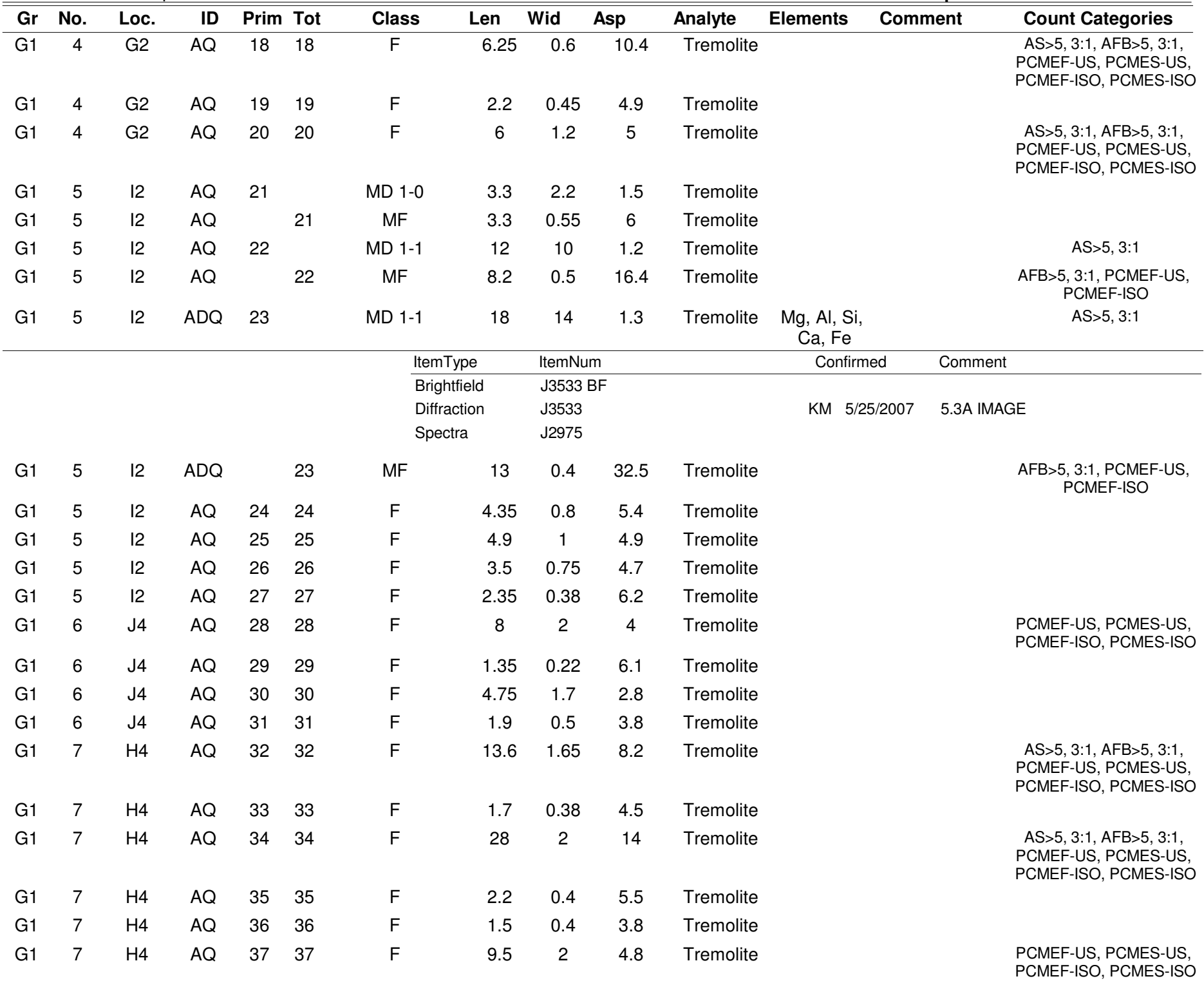

Count Categories

$\mathrm{AFB}>5,3: 1$

PAS

PCMEF-US

Asbestos Fibers and Bundles $>5 \mathrm{um}$ and 3:1

Primary Asbestos Structures

PCM Equivalent Fibers-NIOSH

PCMES-US
AS $>5,3: 1$ PCMEF-ISO PCMES-ISO TAS
Asbestos Structures $>5$ um and 3:1 PCM Equivalent Fibers-ISO PCM Equivalent Structures-ISO Total Asbestos Structures 
ISO 10312, Direct Raw Data

Job Number: 070434

SEA

Report Number: 070434R06

Client: Idaho National Laboratory

Date Received: 4/23/2007

Project Name: RARE

Client Sample No: FB-2-R1

Lab/Cor Sample No: S26

Client Description:

Date Sampled:

4/16/2007

\begin{tabular}{|c|c|c|c|c|c|c|c|c|c|c|c|c|c|}
\hline Gr & No. & Loc. & ID & Prim & Tot & Class & Len & Wid & Asp & Analyte & Elements & Comment & Count Categories \\
\hline G1 & 8 & F4 & $A Q$ & 38 & 38 & $F$ & 70 & 1 & 70 & Tremolite & & & $\begin{array}{l}\text { AS }>5,3: 1, \text { AFB }>5,3: 1, \\
\text { PCMEF-US, PCMES-US, } \\
\text { PCMEF-ISO, PCMES-ISO }\end{array}$ \\
\hline G1 & 8 & $\mathrm{~F} 4$ & $A Q$ & 39 & & MD 1-0 & 3.85 & 1.5 & 2.6 & Tremolite & & & \\
\hline G1 & 8 & $\mathrm{~F} 4$ & $A Q$ & & 39 & MF & 3 & 0.2 & 15 & Tremolite & & & \\
\hline G1 & 8 & $\mathrm{~F} 4$ & $A Q$ & 40 & 40 & $\mathrm{~F}$ & 1 & 0.3 & 3.3 & Tremolite & & & \\
\hline G1 & 8 & $\mathrm{~F} 4$ & $A Q$ & 41 & 41 & $\mathrm{~F}$ & 4.25 & 0.65 & 6.5 & Tremolite & & & \\
\hline G1 & 9 & D4 & $A Q$ & 42 & 42 & $\mathrm{~F}$ & 9.9 & 0.85 & 11.6 & Tremolite & & & $\begin{array}{l}\text { AS }>5,3: 1, \text { AFB }>5,3: 1, \\
\text { PCMEF-US, PCMES-US, } \\
\text { PCMEF-ISO, PCMES-ISO }\end{array}$ \\
\hline G1 & 9 & D4 & $A Q$ & 43 & 43 & $\mathrm{~F}$ & 3 & 0.4 & 7.5 & Tremolite & & & \\
\hline G1 & 9 & D4 & $A Q$ & 44 & 44 & $\mathrm{~F}$ & 3.85 & 0.85 & 4.5 & Tremolite & & & \\
\hline G1 & 9 & D4 & $A Q$ & 45 & 45 & $\mathrm{~F}$ & 4.75 & 0.6 & 7.9 & Tremolite & & & \\
\hline G1 & 9 & D4 & $A Q$ & 46 & 46 & $\mathrm{~F}$ & 2 & 0.75 & 2.7 & Tremolite & & & \\
\hline G1 & 9 & D4 & $A Q$ & 47 & 47 & $\mathrm{~F}$ & 10.2 & 1.25 & 8.2 & Tremolite & & & $\begin{array}{l}\text { AS }>5,3: 1, \text { AFB }>5,3: 1, \\
\text { PCMEF-US, PCMES-US, } \\
\text { PCMEF-ISO, PCMES-ISO }\end{array}$ \\
\hline G1 & 9 & D4 & $A Q$ & 48 & 48 & $\mathrm{~F}$ & 2.1 & 0.2 & 10.5 & Tremolite & & & \\
\hline G1 & 10 & B4 & $A Q$ & 49 & 49 & $\mathrm{~F}$ & 4.75 & 0.55 & 8.6 & Tremolite & & & \\
\hline G1 & 10 & B4 & $A Q$ & 50 & 50 & $\mathrm{~F}$ & 9.5 & 1 & 9.5 & Tremolite & & & $\begin{array}{l}\text { AS }>5,3: 1, \text { AFB }>5,3: 1, \\
\text { PCMEF-US, PCMES-US, } \\
\text { PCMEF-ISO, PCMES-ISO }\end{array}$ \\
\hline G1 & 10 & B4 & $A Q$ & 51 & & MD 1-1 & 18.5 & 4 & 4.6 & Tremolite & & & AS>5, 3:1, PCMES-US \\
\hline G1 & 10 & B4 & $A Q$ & & 51 & MF & 17.5 & 1.1 & 15.9 & Tremolite & & & $\begin{array}{c}\text { AFB }>5,3: 1, \text { PCMEF-US, } \\
\text { PCMEF-ISO }\end{array}$ \\
\hline G1 & 10 & B4 & $A Q$ & 52 & 52 & $\mathrm{~F}$ & 2.9 & 0.4 & 7.2 & Tremolite & & & \\
\hline G1 & 10 & B4 & $A Q$ & 53 & 53 & $F$ & 4.8 & 0.8 & 6 & Tremolite & & & \\
\hline G1 & 10 & B4 & $A Q$ & 54 & 54 & $\mathrm{~F}$ & 8.5 & 0.35 & 24.3 & Tremolite & & & $\begin{array}{l}\text { AS>5, 3:1, AFB>5, 3:1, } \\
\text { PCMEF-US, PCMES-US, } \\
\text { PCMEF-ISO, PCMES-ISO }\end{array}$ \\
\hline G1 & 11 & A7 & $A Q$ & 55 & 55 & $\mathrm{~F}$ & 5.15 & 0.65 & 7.9 & Tremolite & & & $\begin{array}{l}\text { AS }>5,3: 1, \text { AFB }>5,3: 1, \\
\text { PCMEF-US, PCMES-US, } \\
\text { PCMEF-ISO, PCMES-ISO }\end{array}$ \\
\hline G1 & 11 & A7 & $A Q$ & 56 & 56 & $F$ & 1.3 & 0.22 & 5.9 & Tremolite & & & \\
\hline G1 & 11 & A7 & $A Q$ & 57 & 57 & $F$ & 3.2 & 0.6 & 5.3 & Tremolite & & & \\
\hline G1 & 11 & A7 & $A Q$ & 58 & & MD 1-0 & 3 & 1.5 & 2 & Tremolite & & & \\
\hline G1 & 11 & A7 & $A Q$ & & 58 & MF & 2.3 & 0.45 & 5.1 & Tremolite & & & \\
\hline G1 & 11 & A7 & $A Q$ & 59 & 59 & $F$ & 10 & 3 & 3.3 & Tremolite & & & $\begin{array}{l}\text { PCMEF-US, PCMES-US, } \\
\text { PCMEF-ISO, PCMES-ISO }\end{array}$ \\
\hline G1 & 11 & A7 & $A Q$ & 60 & 60 & $F$ & 4.75 & 1.1 & 4.3 & Tremolite & & & \\
\hline G1 & 11 & A7 & $A Q$ & 61 & 61 & $\mathrm{~F}$ & 4.25 & 0.5 & 8.5 & Tremolite & & & \\
\hline
\end{tabular}

Count Categories

AFB $>5,3: 1$

PAS

PCMEF-US

Asbestos Fibers and Bundles $>5 u m$ and 3:1

Primary Asbestos Structures

PCM Equivalent Fibers-NIOSH

PCMES-US
PCM Equivalent Structures-NIOSH
AS $>5,3: 1$ PCMEF-ISO PCMES-ISO TAS
Asbestos Structures $>5$ um and 3:1 PCM Equivalent Fibers-ISO PCM Equivalent Structures-ISO Total Asbestos Structures 
Job Number: 070434

ISO 10312, Direct Raw Data

Client: Idaho National Laboratory

Report Number: 070434R06

Project Name: RARE

Date Received: 4/23/2007

Client Sample No: FB-2-R1

Lab/Cor Sample No: S26

Client Description:

Date Sampled:

$4 / 16 / 2007$

\begin{tabular}{|c|c|c|c|c|c|c|c|c|c|c|c|c|c|}
\hline $\mathbf{G r}$ & No. & Loc. & ID & Prim & Tot & Class & Len & Wid & Asp & Analyte & Elements & Comment & Count Categories \\
\hline G1 & 12 & $\mathrm{C7}$ & $A Q$ & 62 & & MD 1-1 & 8 & 4 & 2 & Tremolite & & & $A S>5,3: 1$ \\
\hline G1 & 12 & $\mathrm{C} 7$ & $A Q$ & & 62 & MF & 5.8 & 0.4 & 14.5 & Tremolite & & & $\begin{array}{c}\text { AFB }>5,3: 1, \text { PCMEF-US, } \\
\text { PCMEF-ISO }\end{array}$ \\
\hline G1 & 12 & $\mathrm{C7}$ & $A Q$ & 63 & & MD 1-1 & 9 & 7 & 1.3 & Tremolite & & & $A S>5,3: 1$ \\
\hline G1 & 12 & $\mathrm{C7}$ & $A Q$ & & 63 & MF & 9 & 0.4 & 22.5 & Tremolite & & & $\begin{array}{c}\text { AFB }>5,3: 1, \text { PCMEF-US, } \\
\text { PCMEF-ISO }\end{array}$ \\
\hline G1 & 12 & $\mathrm{C} 7$ & $A Q$ & 64 & 64 & $\mathrm{~F}$ & 4.7 & 0.7 & 6.7 & Tremolite & & & \\
\hline G1 & 12 & $\mathrm{C7}$ & $A Q$ & 65 & & MD 1-0 & 5.2 & 1.5 & 3.5 & Tremolite & & & $\begin{array}{c}\text { AS }>5,3: 1, \text { PCMES-US, } \\
\text { PCMES-ISO }\end{array}$ \\
\hline G1 & 12 & $\mathrm{C7}$ & $A Q$ & & 65 & MF & 4.8 & 0.2 & 24 & Tremolite & & & \\
\hline G1 & 12 & $\mathrm{C} 7$ & $A Q$ & 66 & 66 & $\mathrm{~F}$ & 3 & 0.38 & 7.9 & Tremolite & & & \\
\hline G1 & 12 & $\mathrm{C} 7$ & $A Q$ & 67 & 67 & $\mathrm{~F}$ & 7.5 & 2.5 & 3 & Tremolite & & & $\begin{array}{l}\text { PCMEF-US, PCMES-US, } \\
\text { PCMEF-ISO, PCMES-ISO }\end{array}$ \\
\hline G1 & 12 & $\mathrm{C7}$ & $A Q$ & 68 & & MD 1-1 & 11.5 & 6 & 1.9 & Tremolite & & & $A S>5,3: 1$ \\
\hline G1 & 12 & $\mathrm{C7}$ & $A Q$ & & 68 & MF & 7.35 & 0.65 & 11.3 & Tremolite & & & $\begin{array}{c}\text { AFB }>5,3: 1, \text { PCMEF-US, } \\
\text { PCMEF-ISO }\end{array}$ \\
\hline G1 & 12 & $\mathrm{C7}$ & $A Q$ & 69 & 69 & $\mathrm{~F}$ & 6.2 & 1.8 & 3.4 & Tremolite & & & $\begin{array}{l}\text { PCMEF-US, PCMES-US, } \\
\text { PCMEF-ISO, PCMES-ISO }\end{array}$ \\
\hline G1 & 13 & E7 & $A Q$ & 70 & 70 & $\mathrm{~F}$ & 4.7 & 0.65 & 7.2 & Tremolite & & & \\
\hline G1 & 13 & E7 & $A Q$ & 71 & 71 & $\mathrm{~F}$ & 3 & 0.7 & 4.3 & Tremolite & & & \\
\hline G1 & 13 & E7 & $A Q$ & 72 & 72 & $\mathrm{~F}$ & 7 & 0.18 & 38.9 & Tremolite & & & $A S>5,3: 1, A F B>5,3: 1$ \\
\hline G1 & 13 & E7 & $A Q$ & 73 & 73 & $\mathrm{~F}$ & 12.2 & 2 & 6.1 & Tremolite & & & $\begin{array}{l}\text { AS }>5,3: 1, \text { AFB }>5,3: 1 \text {, } \\
\text { PCMEF-US, PCMES-US, } \\
\text { PCMES-ISO, PCMEF-ISO }\end{array}$ \\
\hline G1 & 14 & G7 & $A Q$ & 74 & 74 & $\mathrm{~F}$ & 12.5 & 1.25 & 10 & Tremolite & & & $\begin{array}{l}\text { AS }>5,3: 1, \text { AFB }>5,3: 1 \text {, } \\
\text { PCMEF-US, PCMES-US, } \\
\text { PCMEF-ISO, PCMES-ISO }\end{array}$ \\
\hline G1 & 14 & G7 & $A Q$ & 75 & 75 & $\mathrm{~F}$ & 4.2 & 1.8 & 2.3 & Tremolite & & & \\
\hline G1 & 14 & G7 & $A Q$ & 76 & 76 & $F$ & 1.6 & 0.4 & 4 & Tremolite & & & \\
\hline G1 & 14 & G7 & $A Q$ & 77 & 77 & $F$ & 3.4 & 0.4 & 8.5 & Tremolite & & & \\
\hline G1 & 15 & 17 & $A Q$ & 78 & & MD 1-1 & 10.7 & 5 & 2.1 & Tremolite & & & $A S>5,3: 1$ \\
\hline G1 & 15 & 17 & $A Q$ & & 78 & MF & 9.65 & 1 & 9.6 & Tremolite & & & $\begin{array}{c}\text { AFB }>5,3: 1, \text { PCMEF-US, } \\
\text { PCMEF-ISO }\end{array}$ \\
\hline G1 & 15 & 17 & $A Q$ & 79 & 79 & $F$ & 5.1 & 1.2 & 4.2 & Tremolite & & & $\begin{array}{l}\text { PCMEF-US, PCMES-US, } \\
\text { PCMEF-ISO, PCMES-ISO }\end{array}$ \\
\hline G1 & 15 & 17 & $A Q$ & 80 & & MD 1-0 & 4.8 & 4.8 & 1 & Tremolite & & & \\
\hline G1 & 15 & 17 & $A Q$ & & 80 & MF & 4.2 & 0.65 & 6.5 & Tremolite & & & \\
\hline G1 & 15 & 17 & $A Q$ & 81 & 81 & $F$ & 14.4 & 0.4 & 36 & Tremolite & & & $\begin{array}{l}\text { AS }>5,3: 1, \text { AFB }>5,3: 1, \\
\text { PCMEF-US, PCMES-US, } \\
\text { PCMEF-ISO, PCMES-ISO }\end{array}$ \\
\hline
\end{tabular}

Count Categories

AFB $>5,3: 1$

PAS

PCMEF-US

Asbestos Fibers and Bundles > 5um and 3:1

Primary Asbestos Structures

PCM Equivalent Fibers-NIOSH

PCMES-US
AS>5, 3:1 PCMEF-ISO PCMES-ISO TAS
Asbestos Structures >5um and 3:1 PCM Equivalent Fibers-ISO PCM Equivalent Structures-ISO Total Asbestos Structures 
Job Number: 070434

\section{SEA}

ISO 10312, Direct Raw Data

Client: Idaho National Laboratory

Report Number: 070434R06

Date Received: 4/23/2007

Project Name: RARE

Client Sample No: FB-2-R1

Lab/Cor Sample No: S26

Client Description:

Date Sampled:

$4 / 16 / 2007$

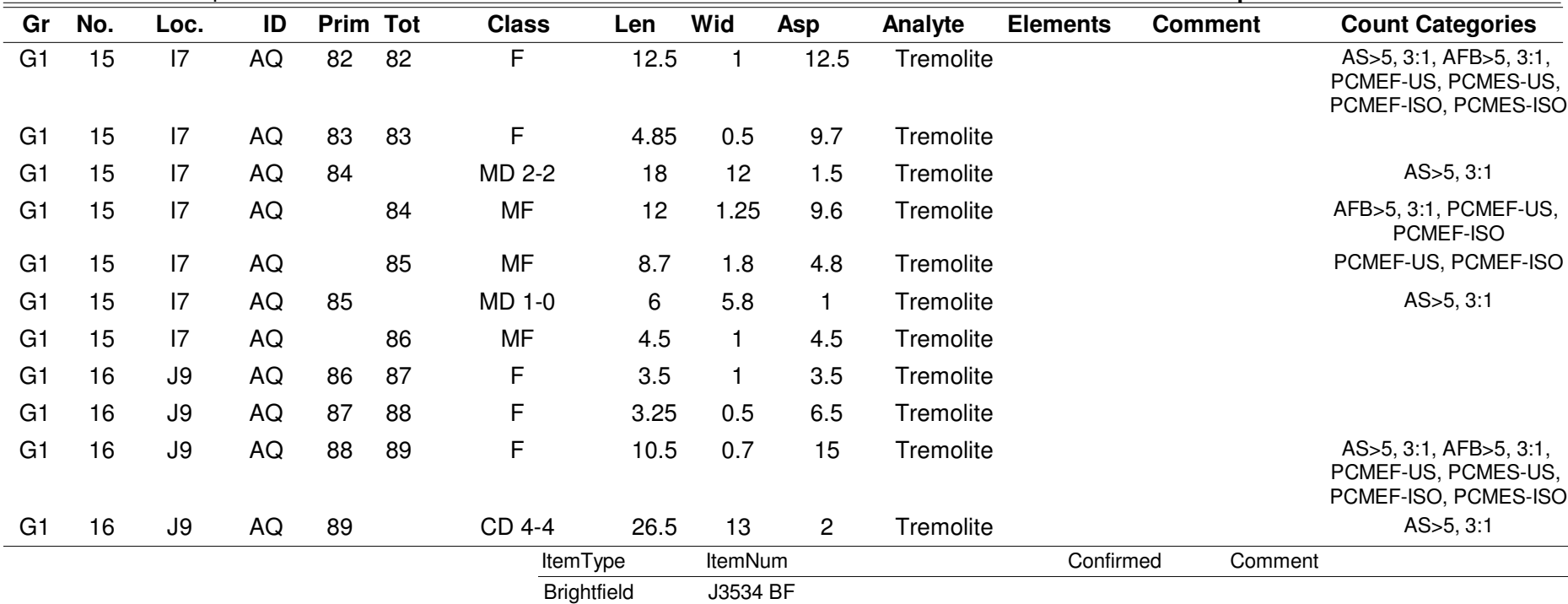

\begin{tabular}{|c|c|c|c|c|c|c|c|c|c|c|}
\hline G1 & 16 & J9 & $A Q$ & & 90 & $\mathrm{CF}$ & 24.35 & 2.85 & 8.5 & Tremolite \\
\hline G1 & 16 & J9 & $A Q$ & & 91 & $\mathrm{CF}$ & 11.5 & 0.75 & 15.3 & Tremolite \\
\hline G1 & 16 & J9 & $A Q$ & & 92 & CF & 7.9 & 1.7 & 4.6 & Tremolite \\
\hline G1 & 16 & J9 & $A Q$ & & 93 & $\mathrm{CF}$ & 5.5 & 0.9 & 6.1 & Tremolite \\
\hline G1 & 17 & $\mathrm{H} 9$ & $A Q$ & 90 & & MD 1-1 & 8 & 5 & 1.6 & Tremolite \\
\hline G1 & 17 & $\mathrm{H} 9$ & $A Q$ & & 94 & MF & 7 & 0.65 & 10.8 & Tremolite \\
\hline G1 & 17 & $\mathrm{H} 9$ & $A Q$ & 91 & 95 & $\mathrm{~F}$ & 3 & 0.65 & 4.6 & Tremolite \\
\hline G1 & 17 & $\mathrm{H} 9$ & $A Q$ & 92 & 96 & $\mathrm{~F}$ & 9.75 & 0.9 & 10.8 & Tremolite \\
\hline G1 & 17 & $\mathrm{H} 9$ & $A Q$ & 93 & & MD 1-0 & 4.5 & 1.5 & 3 & Tremolite \\
\hline G1 & 17 & $\mathrm{H} 9$ & $A Q$ & & 97 & MF & 1.75 & 0.38 & 4.6 & Tremolite \\
\hline G1 & 18 & F9 & $A Q$ & 94 & 98 & $\mathrm{~F}$ & 1.65 & 0.45 & 3.7 & Tremolite \\
\hline G1 & 18 & F9 & $A Q$ & 95 & 99 & $\mathrm{~F}$ & 3.2 & 0.75 & 4.3 & Tremolite \\
\hline G1 & 18 & F9 & $A Q$ & 96 & 100 & $\mathrm{~F}$ & 4 & 0.75 & 5.3 & Tremolite \\
\hline G1 & 18 & F9 & $A Q$ & 97 & 101 & $\mathrm{~F}$ & 2.5 & 0.25 & 10 & Tremolite \\
\hline G1 & 18 & F9 & $A Q$ & 98 & & MD 1-0 & 7.5 & 6 & 1.2 & Tremolite \\
\hline G1 & 18 & F9 & $A Q$ & & 102 & MF & 4.8 & 0.9 & 5.3 & Tremolite \\
\hline
\end{tabular}

AFB $>5,3: 1$, PCMEF-US, PCMEF-ISO

AFB $>5,3: 1$, PCMEF-US, PCMEF-ISO

PCMEF-US, PCMEF-ISO

AFB $>5,3: 1$, PCMEF-US, PCMEF-ISO

AS $>5,3: 1$

AFB $>5,3: 1$, PCMEF-US, PCMEF-ISO

$A S>5,3: 1, A F B>5,3: 1$ PCMEF-US, PCMES-US PCMEF-ISO, PCMES-ISO

Count Categories

$A F B>5,3: 1$

PAS

PCMEF-US

Asbestos Fibers and Bundles $>$ 5um and 3:1

Primary Asbestos Structures

PCM Equivalent Fibers-NIOSH

PCMES-US
AS $>5,3: 1$ PCMEF-ISO PCMES-ISO TAS
Asbestos Structures $>5$ um and 3:1 PCM Equivalent Fibers-ISO PCM Equivalent Structures-ISO Total Asbestos Structures
AS $>5,3: 1$

Page 83 of 281 
ISO 10312, Direct Raw Data

Job Number: 070434

SEA

Report Number: 070434R06

Client: Idaho National Laboratory

Date Received: 4/23/2007

Project Name: RARE

Client Sample No: FB-2-R1

Lab/Cor Sample No: S26

Client Description:

Date Sampled:

4/16/2007

\begin{tabular}{|c|c|c|c|c|c|c|c|c|c|c|c|c|c|}
\hline Gr & No. & Loc. & ID & Prim & Tot & Class & Len & Wid & Asp & Analyte & Elements & Comment & Count Categories \\
\hline G1 & 18 & F9 & $\mathrm{AQ}$ & 99 & 103 & $\mathrm{~F}$ & 4.2 & 0.65 & 6.5 & Tremolite & & & \\
\hline G1 & 19 & D9 & $A Q$ & 100 & 104 & $\mathrm{~F}$ & 35.5 & 2.5 & 14.2 & Tremolite & & & $\begin{array}{l}\text { AS }>5,3: 1, \text { AFB }>5,3: 1 \text {, } \\
\text { PCMEF-US, PCMES-US, } \\
\text { PCMEF-ISO, PCMES-ISO }\end{array}$ \\
\hline G1 & 19 & D9 & $A Q$ & 101 & 105 & $\mathrm{~F}$ & 14 & 1.2 & 11.7 & Tremolite & & & $\begin{array}{l}\text { AS }>5,3: 1, \text { AFB }>5,3: 1, \\
\text { PCMEF-US, PCMES-US, } \\
\text { PCMEF-ISO, PCMES-ISO }\end{array}$ \\
\hline G1 & 19 & D9 & $A Q$ & 102 & 106 & $\mathrm{~F}$ & 11.8 & 3 & 3.9 & Tremolite & & & $\begin{array}{l}\text { PCMEF-US, PCMES-US, } \\
\text { PCMEF-ISO, PCMES-ISO }\end{array}$ \\
\hline G1 & 19 & D9 & $A Q$ & 103 & 107 & $\mathrm{~F}$ & 7.75 & 1.8 & 4.3 & Tremolite & & & $\begin{array}{l}\text { PCMEF-US, PCMES-US, } \\
\text { PCMEF-ISO, PCMES-ISO }\end{array}$ \\
\hline G1 & 19 & D9 & $A Q$ & 104 & 108 & $\mathrm{~F}$ & 2 & 0.35 & 5.7 & Tremolite & & & \\
\hline G1 & 20 & B9 & $A Q$ & 105 & 109 & $\mathrm{~F}$ & 2.8 & 0.45 & 6.2 & Tremolite & & & \\
\hline G1 & 20 & B9 & $A Q$ & 106 & 110 & $F$ & 12 & 0.4 & 30 & Tremolite & & & $\begin{array}{l}\text { AS }>5,3: 1, \text { AFB }>5,3: 1, \\
\text { PCMEF-US, PCMES-US, } \\
\text { PCMEF-ISO, PCMES-ISO }\end{array}$ \\
\hline G1 & 20 & B9 & $A Q$ & 107 & 111 & $\mathrm{~F}$ & 10 & 1.2 & 8.3 & Tremolite & & & $\begin{array}{l}\text { AS }>5,3: 1, \text { AFB }>5,3: 1, \\
\text { PCMEF-US, PCMES-US, } \\
\text { PCMEF-ISO, PCMES-ISO }\end{array}$ \\
\hline G1 & 20 & B9 & $A Q$ & 108 & 112 & $\mathrm{~F}$ & 10.5 & 2 & 5.2 & Tremolite & & & $\begin{array}{l}\text { AS >5, 3:1, AFB }>5,3: 1, \\
\text { PCMEF-US, PCMES-US, } \\
\text { PCMEF-ISO, PCMES-ISO }\end{array}$ \\
\hline G1 & 20 & B9 & $A Q$ & 109 & 113 & $F$ & 8 & 0.5 & 16 & Tremolite & & & $\begin{array}{l}\text { AS }>5,3: 1, \text { AFB }>5,3: 1, \\
\text { PCMEF-US, PCMES-US, } \\
\text { PCMEF-ISO, PCMES-ISO }\end{array}$ \\
\hline G1 & 20 & B9 & $A Q$ & 110 & & MD 2-1 & 22 & 8 & 2.8 & Tremolite & & & $A S>5,3: 1$ \\
\hline G1 & 20 & B9 & $A Q$ & & 114 & MF & 20.7 & 3 & 6.9 & Tremolite & & & $\begin{array}{c}\text { AFB }>5,3: 1, \text { PCMEF-US, } \\
\text { PCMEF-ISO }\end{array}$ \\
\hline G1 & 20 & B9 & $A Q$ & & 115 & MF & 4.75 & 0.2 & 23.8 & Tremolite & & & \\
\hline G1 & 20 & B9 & $A Q$ & 111 & & MD 1-0 & 3.5 & 1.2 & 2.9 & Tremolite & & & \\
\hline G1 & 20 & B9 & $A Q$ & & 116 & MF & 3 & 0.45 & 6.7 & Tremolite & & & \\
\hline G2 & 21 & A2 & $A Q$ & 112 & 117 & $F$ & 23.5 & 3 & 7.8 & Tremolite & & & $\begin{array}{l}\text { AS }>5,3: 1, \text { AFB }>5,3: 1, \\
\text { PCMEF-US, PCMES-US, } \\
\text { PCMEF-ISO, PCMES-ISO }\end{array}$ \\
\hline G2 & 21 & $\mathrm{~A} 2$ & $A Q$ & 113 & 118 & $F$ & 7.5 & 2 & 3.8 & Tremolite & & & $\begin{array}{l}\text { PCMEF-US, PCMES-US, } \\
\text { PCMEF-ISO, PCMES-ISO }\end{array}$ \\
\hline G2 & 21 & $\mathrm{~A} 2$ & $A Q$ & 114 & & MD 1-1 & 8.5 & 6 & 1.4 & Tremolite & & & $A S>5,3: 1$ \\
\hline G2 & 21 & A2 & $A Q$ & & 119 & MF & 6 & 0.8 & 7.5 & Tremolite & & & $\begin{array}{c}\text { AFB }>5,3: 1, \text { PCMEF-US, } \\
\text { PCMEF-ISO }\end{array}$ \\
\hline G2 & 21 & A2 & $A Q$ & 115 & 120 & $\mathrm{~F}$ & 2.5 & 0.5 & 5 & Tremolite & & & \\
\hline G2 & 21 & A2 & $A Q$ & 116 & 121 & $F$ & 1.75 & 0.2 & 8.8 & Tremolite & & & \\
\hline G2 & 21 & A2 & $A Q$ & 117 & & MD 1-0 & 6 & 3 & 2 & Tremolite & & & $A S>5,3: 1$ \\
\hline G2 & 21 & $\mathrm{~A} 2$ & $A Q$ & & 122 & MF & 3.1 & 0.9 & 3.4 & Tremolite & & & \\
\hline
\end{tabular}

Page 84 of 281

\section{Count Categories}

$A F B>5,3: 1$

PAS

PCMEF-US

PCMES-US
Asbestos Fibers and Bundles > 5um and 3:1

Primary Asbestos Structures

PCM Equivalent Fibers-NIOSH

PCM Equivalent Structures-NIOSH
AS>5, $3: 1$ PCMEF-ISO PCMES-ISO TAS
Asbestos Structures >5um and 3:1 PCM Equivalent Fibers-ISO PCM Equivalent Structures-ISO Total Asbestos Structures 
Job Number: 070434

\section{SEA}

ISO 10312, Direct Raw Data

Client: Idaho National Laboratory

Report Number: 070434R06

Date Received: 4/23/2007

Project Name: RARE

Client Sample No: FB-2-R1

Lab/Cor Sample No: S26

Client Description:

Date Sampled:

4/16/2007

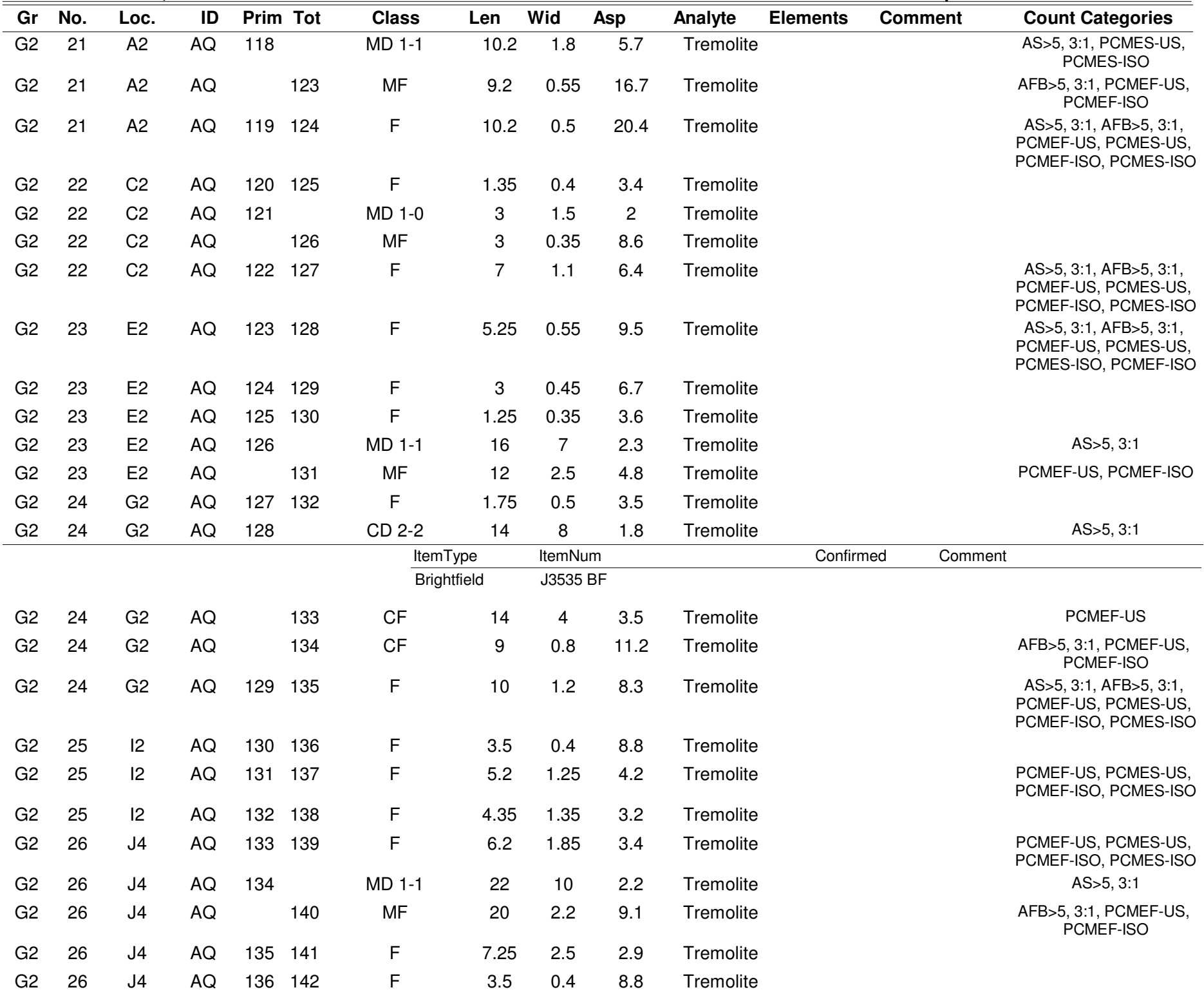

Count Categories

$\mathrm{AFB}>5,3: 1$

PAS

PCMEF-US

Asbestos Fibers and Bundles $>5 u m$ and 3:1

Primary Asbestos Structures

PCM Equivalent Fibers-NIOSH

PCMES-US
PCM Equivalent Structures-NIOSH
AS $>5,3: 1$ PCMEF-ISO PCMES-ISO TAS
Asbestos Structures $>5$ um and 3:1 PCM Equivalent Fibers-ISO PCM Equivalent Structures-ISO Total Asbestos Structures

Page 85 of 281 
Job Number: 070434

ISO 10312, Direct Raw Data

Client: Idaho National Laboratory

Report Number: 070434R06

Project Name: RARE

Date Received: 4/23/2007

Client Sample No: FB-2-R1

Lab/Cor Sample No: S26

Client Description:

Date Sampled:

4/16/2007

\begin{tabular}{|c|c|c|c|c|c|c|c|c|c|c|c|c|c|}
\hline Gr & No. & Loc. & ID & Prim & Tot & Class & Len & Wid & Asp & Analyte & Elements & Comment & Count Categories \\
\hline G2 & 26 & J4 & $A Q$ & 137 & 143 & $\mathrm{~F}$ & 5.12 & 1.6 & 3.2 & Tremolite & & & $\begin{array}{l}\text { PCMEF-US, PCMES-US, } \\
\text { PCMEF-ISO, PCMES-ISO }\end{array}$ \\
\hline G2 & 26 & J4 & $A Q$ & 138 & 144 & $\mathrm{~F}$ & 9.5 & 1.8 & 5.3 & Tremolite & & & $\begin{array}{l}\text { AS }>5,3: 1, \text { AFB }>5,3: 1 \text {, } \\
\text { PCMEF-US, PCMES-US, } \\
\text { PCMEF-ISO, PCMES-ISO }\end{array}$ \\
\hline G2 & 26 & J4 & $A Q$ & 139 & 145 & $\mathrm{~F}$ & 7 & 0.5 & 14 & Tremolite & & & $\begin{array}{l}\text { AS }>5,3: 1, \text { AFB }>5,3: 1, \\
\text { PCMEF-US, PCMES-US, } \\
\text { PCMEF-ISO, PCMES-ISO }\end{array}$ \\
\hline G2 & 26 & J4 & $A Q$ & 140 & 146 & $F$ & 10 & 1.25 & 8 & Tremolite & & & $\begin{array}{l}\text { AS >5, 3:1, AFB >5, 3:1, } \\
\text { PCMEF-US, PCMES-US, } \\
\text { PCMEF-ISO, PCMES-ISO }\end{array}$ \\
\hline G2 & 26 & $\mathrm{~J} 4$ & $A Q$ & 141 & 147 & $\mathrm{~F}$ & 17 & 2 & 8.5 & Tremolite & & & $\begin{array}{l}\text { AS }>5,3: 1, \text { AFB }>5,3: 1, \\
\text { PCMEF-US, PCMES-US, } \\
\text { PCMEF-ISO, PCMES-ISO }\end{array}$ \\
\hline G2 & 26 & J4 & $A Q$ & 142 & & MD 1-1 & 11.5 & 5 & 2.3 & Tremolite & & & $A S>5,3: 1$ \\
\hline G2 & 26 & J4 & $A Q$ & & 148 & MF & 5.35 & 1.2 & 4.5 & Tremolite & & & PCMEF-US, PCMEF-ISO \\
\hline G2 & 27 & $\mathrm{H} 4$ & $A Q$ & 143 & 149 & $\mathrm{~F}$ & 13.5 & 1.2 & 11.2 & Tremolite & & & $\begin{array}{l}\text { AS }>5,3: 1, \text { AFB }>5,3: 1, \\
\text { PCMEF-US, PCMES-US, } \\
\text { PCMEF-ISO, PCMES-ISO }\end{array}$ \\
\hline G2 & 27 & $\mathrm{H} 4$ & $A Q$ & 144 & 150 & $\mathrm{~F}$ & 5.6 & 0.7 & 8 & Tremolite & & & $\begin{array}{l}\text { AFB }>5,3: 1, \text { AS }>5,3: 1, \\
\text { PCMEF-US, PCMES-US, } \\
\text { PCMEF-ISO, PCMES-ISO }\end{array}$ \\
\hline G2 & 27 & $\mathrm{H} 4$ & $A Q$ & 145 & 151 & $\mathrm{~F}$ & 7.75 & 0.8 & 9.7 & Tremolite & & & $\begin{array}{l}\text { AS>5, 3:1, AFB >5, 3:1, } \\
\text { PCMEF-US, PCMES-US, } \\
\text { PCMEF-ISO, PCMES-ISO }\end{array}$ \\
\hline G2 & 27 & $\mathrm{H} 4$ & $A Q$ & 146 & 152 & B & 8 & 2 & 4 & Tremolite & & & $\begin{array}{l}\text { AFB }>5,3: 1, \text { AS }>5,3: 1, \\
\text { PCMEF-US, PCMES-US, } \\
\text { PCMES-ISO, PCMEF-ISO }\end{array}$ \\
\hline G2 & 28 & F4 & $A Q$ & 147 & 153 & $\mathrm{~F}$ & 20 & 1.2 & 16.7 & Tremolite & & & $\begin{array}{l}\text { AS }>5,3: 1, \text { AFB }>5,3: 1, \\
\text { PCMEF-US, PCMES-US, } \\
\text { PCMEF-ISO, PCMES-ISO }\end{array}$ \\
\hline G2 & 28 & $\mathrm{~F} 4$ & $A Q$ & 148 & 154 & $\mathrm{~F}$ & 2.35 & 0.5 & 4.7 & Tremolite & & & \\
\hline G2 & 28 & $\mathrm{~F} 4$ & $A Q$ & 149 & 155 & $\mathrm{~F}$ & 2.75 & 0.18 & 15.3 & Tremolite & & & \\
\hline G2 & 28 & $\mathrm{~F} 4$ & $A Q$ & 150 & 156 & $\mathrm{~F}$ & 18 & 4 & 4.5 & Tremolite & & & PCMEF-US, PCMES-US \\
\hline G2 & 28 & $\mathrm{~F} 4$ & $A Q$ & 151 & 157 & $\mathrm{~F}$ & 6.2 & 1.35 & 4.6 & Tremolite & & & $\begin{array}{l}\text { PCMEF-US, PCMES-US, } \\
\text { PCMEF-ISO, PCMES-ISO }\end{array}$ \\
\hline G2 & 29 & D4 & $A Q$ & 152 & & MD 1-1 & 10 & 4 & 2.5 & Tremolite & & & $A S>5,3: 1$ \\
\hline G2 & 29 & D4 & $A Q$ & & 158 & MF & 9.5 & 0.85 & 11.2 & Tremolite & & & $\begin{array}{c}\text { AFB }>5,3: 1, \text { PCMEF-US, } \\
\text { PCMEF-ISO }\end{array}$ \\
\hline G2 & 29 & D4 & $A Q$ & 153 & 159 & $\mathrm{~F}$ & 10 & 0.7 & 14.3 & Tremolite & & & $\begin{array}{l}\text { AS }>5,3: 1, \text { AFB }>5,3: 1, \\
\text { PCMEF-US, PCMES-US, } \\
\text { PCMEF-ISO, PCMES-ISO }\end{array}$ \\
\hline G2 & 29 & D4 & $A Q$ & 154 & & MD 1-1 & 7 & 2 & 3.5 & Tremolite & & & $\begin{array}{c}\text { AS >5, 3:1, PCMES-US, } \\
\text { PCMES-ISO }\end{array}$ \\
\hline G2 & 29 & D4 & $A Q$ & & 160 & MF & 6 & 0.65 & 9.2 & Tremolite & & & $\begin{array}{c}\text { AFB }>5,3: 1, \text { PCMEF-US, } \\
\text { PCMEF-ISO }\end{array}$ \\
\hline
\end{tabular}

Count Categories

AFB $>5,3: 1$

PAS

PCMEF-US

Asbestos Fibers and Bundles $>5 \mathrm{um}$ and 3:1

Primary Asbestos Structures

PCM Equivalent Fibers-NIOSH

PCMES-US
AS $>5,3: 1$ PCMEF-ISO PCMES-ISO TAS
Asbestos Structures $>5$ um and 3:1 PCM Equivalent Fibers-ISO PCM Equivalent Structures-ISO Total Asbestos Structures 
ISO 10312, Direct Raw Data

Job Number: 070434

SEA

Report Number: 070434R06

Client: Idaho National Laboratory

Date Received: 4/23/2007

Project Name: RARE

Client Sample No: FB-2-R1

Lab/Cor Sample No: S26

Client Description:

Date Sampled:

4/16/2007

\begin{tabular}{|c|c|c|c|c|c|c|c|c|c|c|c|c|c|}
\hline$\overline{\mathrm{Gr}}$ & No. & Loc. & ID & Prim & Tot & Class & Len & Wid & Asp & Analyte & Elements & Comment & Count Categories \\
\hline G2 & 29 & D4 & $A Q$ & 155 & 161 & $\mathrm{~F}$ & 4.25 & 0.65 & 6.5 & Tremolite & & & \\
\hline G2 & 29 & D4 & $A Q$ & 156 & & MD 1-1 & 27 & 10 & 2.7 & Tremolite & & & $A S>5,3: 1$ \\
\hline G2 & 29 & D4 & $A Q$ & & 162 & MF & 20.2 & 0.45 & 44.9 & Tremolite & & & $\begin{array}{c}\text { AFB }>5,3: 1, \text { PCMEF-US, } \\
\text { PCMEF-ISO }\end{array}$ \\
\hline G2 & 29 & D4 & $A Q$ & 157 & 163 & $\mathrm{~F}$ & 8.5 & 1 & 8.5 & Tremolite & & & $\begin{array}{l}\text { AS }>5,3: 1, \text { AFB }>5,3: 1 \text {, } \\
\text { PCMEF-US, PCMES-US, } \\
\text { PCMEF-ISO, PCMES-ISO }\end{array}$ \\
\hline G2 & 30 & B4 & $A Q$ & 158 & 164 & $\mathrm{~F}$ & 13 & 0.6 & 21.7 & Tremolite & & & $\begin{array}{l}\text { AS }>5,3: 1, \text { AFB }>5,3: 1, \\
\text { PCMEF-US, PCMES-US, } \\
\text { PCMEF-ISO, PCMES-ISO }\end{array}$ \\
\hline G2 & 30 & B4 & $A Q$ & 159 & & MD 1-1 & 5.2 & 3 & 1.7 & Tremolite & & & $A S>5,3: 1$ \\
\hline G2 & 30 & B4 & $A Q$ & & 165 & MF & 5.2 & 0.45 & 11.6 & Tremolite & & & $\begin{array}{c}\text { AFB >5, 3:1, PCMEF-US, } \\
\text { PCMEF-ISO }\end{array}$ \\
\hline G2 & 30 & B4 & $A Q$ & 160 & 166 & $\mathrm{~F}$ & 4.9 & 1.2 & 4.1 & Tremolite & & & \\
\hline G2 & 30 & B4 & $A Q$ & 161 & 167 & $\mathrm{~F}$ & 4.75 & 0.7 & 6.8 & Tremolite & & & \\
\hline G2 & 30 & B4 & $A Q$ & 162 & & MD 1-0 & 5 & 4 & 1.2 & Tremolite & & & \\
\hline G2 & 30 & B4 & $A Q$ & & 168 & MF & 2.5 & 0.2 & 12.5 & Tremolite & & & \\
\hline G2 & 30 & B4 & $A Q$ & 163 & 169 & $F$ & 3.8 & 0.8 & 4.8 & Tremolite & & & \\
\hline G2 & 30 & B4 & $A Q$ & 164 & & MD 2-0 & 4.5 & 1.5 & 3 & Tremolite & & & \\
\hline G2 & 30 & B4 & $A Q$ & & 170 & MF & 1.8 & 0.2 & 9 & Tremolite & & & \\
\hline G2 & 30 & B4 & $A Q$ & & 171 & MF & 1.3 & 0.38 & 3.4 & Tremolite & & & \\
\hline G2 & 30 & B4 & $A Q$ & 165 & & MD 1-0 & 4.858 & 3.5 & 1.4 & Tremolite & & & \\
\hline G2 & 30 & B4 & $A Q$ & & 172 & MF & 3.35 & 1 & 3.3 & Tremolite & & & \\
\hline G2 & 31 & A7 & $A Q$ & 166 & 173 & $F$ & 2 & 0.35 & 5.7 & Tremolite & & & \\
\hline G2 & 31 & A7 & $A Q$ & 167 & 174 & $\mathrm{~F}$ & 3 & 0.65 & 4.6 & Tremolite & & & \\
\hline G2 & 31 & A7 & $A Q$ & 168 & 175 & $F$ & 4.9 & 1.5 & 3.3 & Tremolite & & & \\
\hline G2 & 31 & A7 & $A Q$ & 169 & 176 & $F$ & 4.85 & 0.7 & 6.9 & Tremolite & & & \\
\hline G2 & 31 & A7 & $A Q$ & 170 & & CD 3-2 & 13 & 11 & 1.2 & Tremolite & & & $A S>5,3: 1$ \\
\hline G2 & 31 & A7 & $A Q$ & & 177 & $\mathrm{CF}$ & 10.2 & 1.3 & 7.8 & Tremolite & & & $\begin{array}{c}\text { AFB }>5,3: 1, \text { PCMEF-US, } \\
\text { PCMEF-ISO }\end{array}$ \\
\hline G2 & 31 & A7 & $A Q$ & & 178 & CF & 7.2 & 1.8 & 4 & Tremolite & & & PCMEF-US, PCMEF-ISO \\
\hline G2 & 31 & A7 & $A Q$ & & 179 & $\mathrm{CF}$ & 4.35 & 0.8 & 5.4 & Tremolite & & & \\
\hline G2 & 31 & A7 & $A Q$ & 171 & 180 & $\mathrm{~F}$ & 7 & 0.85 & 8.2 & Tremolite & & & $\begin{array}{l}\text { AS >5, 3:1, AFB>5, 3:1, } \\
\text { PCMEF-US, PCMES-US, } \\
\text { PCMEF-ISO, PCMES-ISO }\end{array}$ \\
\hline G2 & 31 & A7 & $A Q$ & 172 & 181 & $\mathrm{~F}$ & 5.75 & 0.35 & 16.4 & Tremolite & & & $\begin{array}{l}\text { AS }>5,3: 1, \text { AFB }>5,3: 1, \\
\text { PCMEF-US, PCMES-US, } \\
\text { PCMEF-ISO, PCMES-ISO }\end{array}$ \\
\hline G2 & 31 & A7 & $A Q$ & 173 & 182 & $\mathrm{~F}$ & 1.7 & 0.18 & 9.4 & Tremolite & & & \\
\hline
\end{tabular}

Count Categories

AFB $>5,3: 1$

PAS

PCMEF-US

Asbestos Fibers and Bundles > 5um and 3:1

Primary Asbestos Structures

PCM Equivalent Fibers-NIOSH

PCMES-US
AS>5, 3:1 PCMEF-ISO PCMES-ISO TAS
Asbestos Structures $>5$ um and 3:1 PCM Equivalent Fibers-ISO PCM Equivalent Structures-ISO Total Asbestos Structures 
ISO 10312, Direct Raw Data

Job Number: 070434

SEA

Report Number: 070434R06

Client: Idaho National Laboratory

Date Received: 4/23/2007

Project Name: RARE

Client Sample No: FB-2-R1

Lab/Cor Sample No: S26

Client Description:

Date Sampled:

4/16/2007

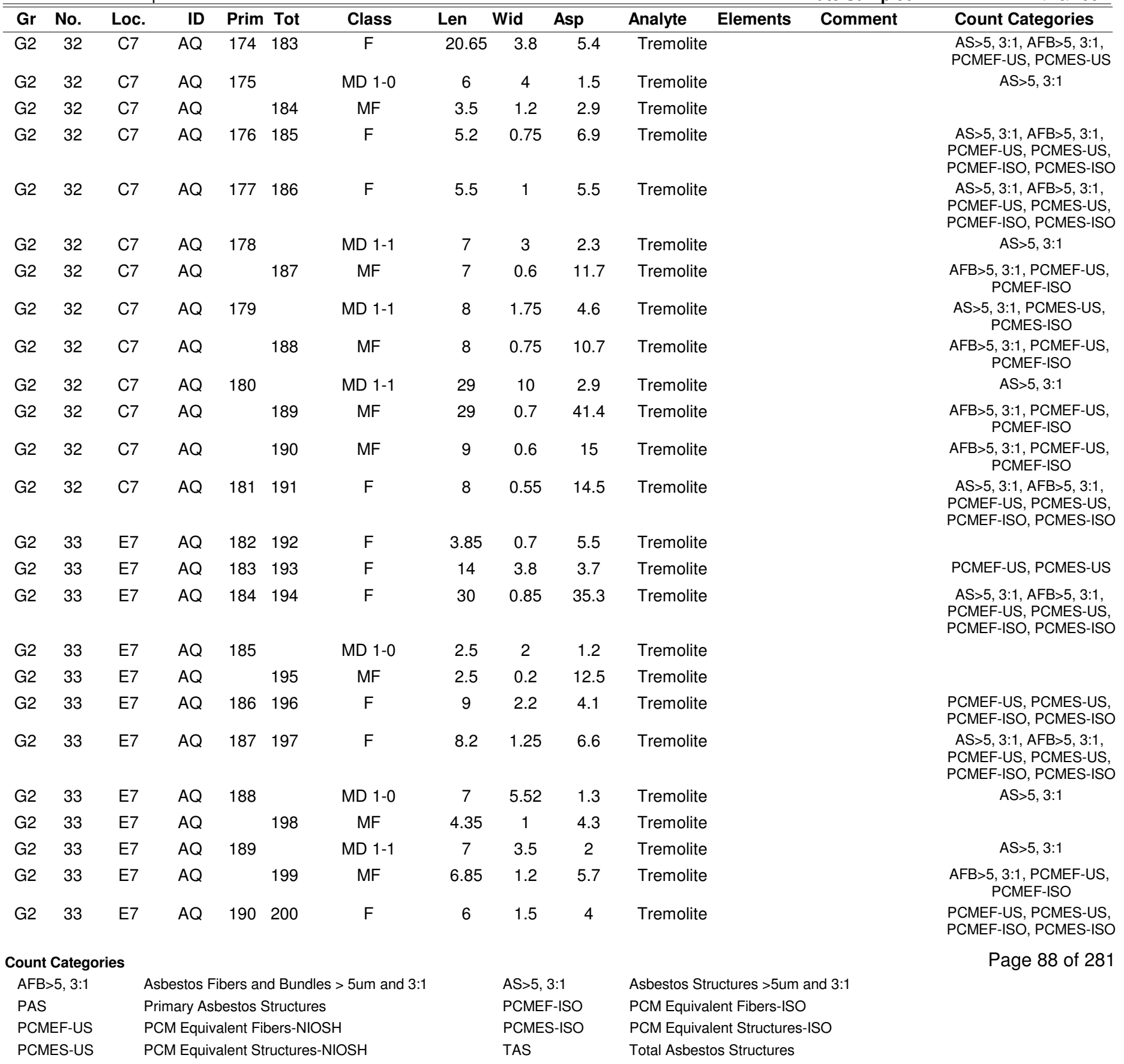


ISO 10312, Direct Raw Data

Job Number: 070434

SEA

Report Number: 070434R06

Client: Idaho National Laboratory

Date Received: 4/23/2007

Project Name: RARE

Client Sample No: FB-2-R1

Lab/Cor Sample No: S26

Client Description:

Date Sampled:

4/16/2007

\begin{tabular}{|c|c|c|c|c|c|c|c|c|c|c|c|c|c|}
\hline$\overline{G r}$ & No. & Loc. & ID & Prim & Tot & Class & Len & Wid & Asp & Analyte & Elements & Comment & Count Categories \\
\hline G2 & 33 & E7 & $\mathrm{AQ}$ & 191 & 201 & $\mathrm{~F}$ & 10 & 1 & 10 & Tremolite & & & $\begin{array}{l}\text { AS>5, 3:1, AFB }>5,3: 1, \\
\text { PCMEF-US, PCMES-US, } \\
\text { PCMEF-ISO, PCMES-ISO }\end{array}$ \\
\hline G2 & 34 & G7 & $\mathrm{AQ}$ & 192 & 202 & $\mathrm{~F}$ & 11.2 & 1.1 & 10.2 & Tremolite & & & $\begin{array}{l}\text { AS }>5,3: 1, \text { AFB }>5,3: 1 \text {, } \\
\text { PCMEF-US, PCMES-US, } \\
\text { PCMEF-ISO, PCMES-ISO }\end{array}$ \\
\hline G2 & 34 & G7 & $A Q$ & 193 & 203 & $\mathrm{~F}$ & 4.35 & 0.7 & 6.2 & Tremolite & & & \\
\hline G2 & 34 & G7 & $A Q$ & 194 & & MD 1-1 & 14 & 9 & 1.6 & Tremolite & & & $A S>5,3: 1$ \\
\hline G2 & 34 & G7 & $\mathrm{AQ}$ & & 204 & MF & 10.3 & 2.2 & 4.7 & Tremolite & & & PCMEF-US, PCMEF-ISO \\
\hline G2 & 34 & G7 & $\mathrm{AQ}$ & 195 & 205 & $\mathrm{~F}$ & 5.12 & 0.55 & 9.3 & Tremolite & & & $\begin{array}{l}\text { AS }>5,3: 1, \text { AFB }>5,3: 1 \text {, } \\
\text { PCMEF-US, PCMES-US, } \\
\text { PCMEF-ISO, PCMES-ISO }\end{array}$ \\
\hline G2 & 35 & 17 & $A Q$ & 196 & & MD 1-0 & 15 & 8 & 1.9 & Tremolite & & & $A S>5,3: 1$ \\
\hline G2 & 35 & 17 & $A Q$ & & 206 & MF & 4.5 & 1.25 & 3.6 & Tremolite & & & \\
\hline G2 & 35 & 17 & $A Q$ & 197 & 207 & $F$ & 7.2 & 0.4 & 18 & Tremolite & & & $\begin{array}{l}\text { AS>5, 3:1, AFB }>5,3: 1, \\
\text { PCMEF-US, PCMES-US, } \\
\text { PCMEF-ISO, PCMES-ISO }\end{array}$ \\
\hline G2 & 35 & 17 & $A Q$ & 198 & & MD 1-1 & 8 & 5.8 & 1.4 & Tremolite & & & $A S>5,3: 1$ \\
\hline G2 & 35 & 17 & $A Q$ & & 208 & MF & 7.8 & 0.6 & 13 & Tremolite & & & $\begin{array}{c}\text { AFB }>5,3: 1, \text { PCMEF-US, } \\
\text { PCMEF-ISO }\end{array}$ \\
\hline G2 & 35 & 17 & $A Q$ & 199 & & MD 1-1 & 19 & 9 & 2.1 & Tremolite & & & $A S>5,3: 1$ \\
\hline G2 & 35 & 17 & $A Q$ & & 209 & MF & 17 & 3.8 & 4.5 & Tremolite & & & PCMEF-US \\
\hline G2 & 35 & 17 & $A Q$ & 200 & & MD 1-1 & 14 & 7 & 2 & Tremolite & & & $A S>5,3: 1$ \\
\hline G2 & 35 & 17 & $A Q$ & & 210 & MF & 14 & 0.7 & 20 & Tremolite & & & $\begin{array}{c}\text { AFB }>5,3: 1, \text { PCMEF-US, } \\
\text { PCMEF-ISO }\end{array}$ \\
\hline
\end{tabular}

Count Categories

AFB $>5,3: 1$

PAS

PCMEF-US

Asbestos Fibers and Bundles > 5um and 3:1

Primary Asbestos Structures

PCM Equivalent Fibers-NIOSH

PCMES-US
AS>5, 3:1 PCMEF-ISO PCMES-ISO TAS
Asbestos Structures >5um and 3:1 PCM Equivalent Fibers-ISO PCM Equivalent Structures-ISO Total Asbestos Structures 


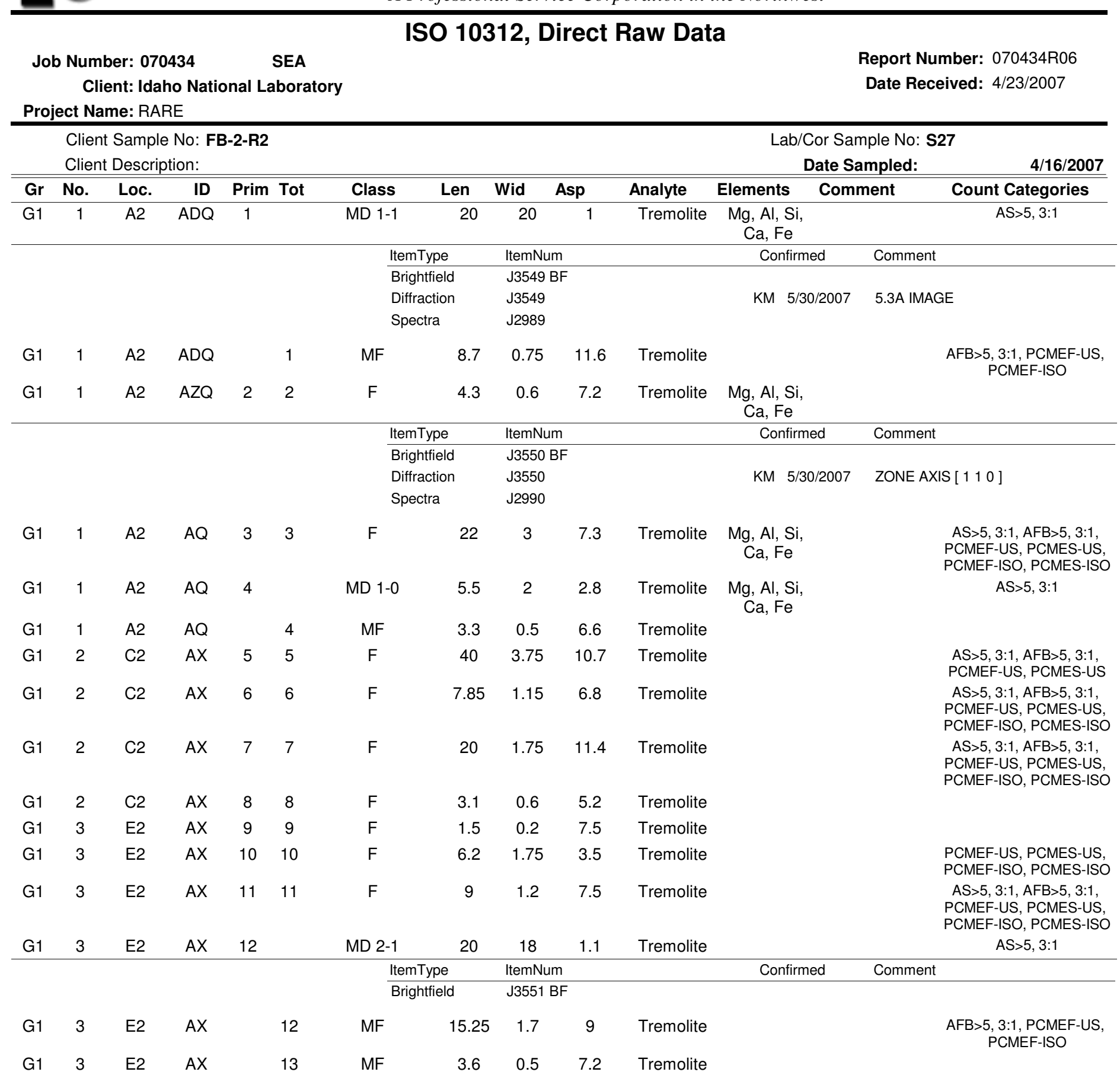

\section{Count Categories}

AFB $>5,3: 1$

PAS

PCMEF-US

PCMES-US
Asbestos Fibers and Bundles > 5um and 3:1

Primary Asbestos Structures

PCM Equivalent Fibers-NIOSH

PCM Equivalent Structures-NIOSH
AS $>5,3: 1$ PCMEF-ISO PCMES-ISO TAS
Asbestos Structures $>5$ um and 3:1 PCM Equivalent Fibers-ISO PCM Equivalent Structures-ISO Total Asbestos Structures 
ISO 10312, Direct Raw Data

Job Number: 070434

SEA

Report Number: 070434R06

Client: Idaho National Laboratory

Date Received: 4/23/2007

Project Name: RARE

Client Sample No: FB-2-R2

Lab/Cor Sample No: S27

Client Description:

Date Sampled:

$4 / 16 / 2007$

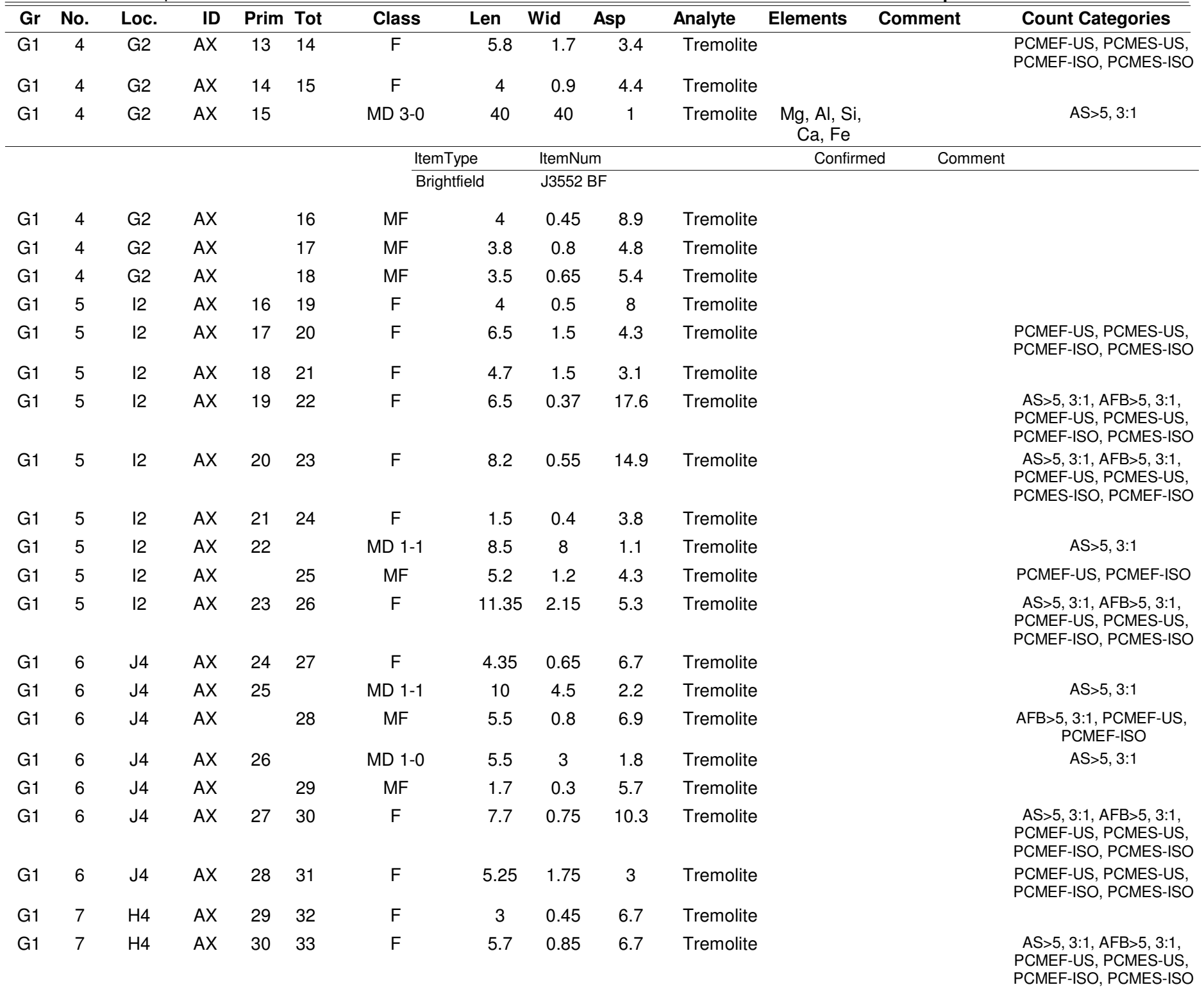

Count Categories

$\mathrm{AFB}>5,3: 1$

PAS

PCMEF-US

Asbestos Fibers and Bundles > 5um and 3:1

Primary Asbestos Structures

PCM Equivalent Fibers-NIOSH

PCMES-US
AS $>5,3: 1$ PCMEF-ISO PCMES-ISO TAS
Asbestos Structures $>5$ um and 3:1 PCM Equivalent Fibers-ISO PCM Equivalent Structures-ISO Total Asbestos Structures

Page 91 of 281 
ISO 10312, Direct Raw Data

Job Number: 070434

SEA

Report Number: 070434R06

Client: Idaho National Laboratory

Date Received: 4/23/2007

Project Name: RARE

Client Sample No: FB-2-R2

Lab/Cor Sample No: S27

Client Description:

Date Sampled:

4/16/2007

\begin{tabular}{|c|c|c|c|c|c|c|c|c|c|c|c|c|c|}
\hline Gr & No. & Loc. & ID & Prim & Tot & Class & Len & Wid & Asp & Analyte & Elements & Comment & Count Categories \\
\hline G1 & 7 & $\mathrm{H} 4$ & $A X$ & 31 & 34 & $\mathrm{~F}$ & 1.75 & 0.45 & 3.9 & Tremolite & & & \\
\hline G1 & 8 & F4 & $A X$ & 32 & 35 & $\mathrm{~F}$ & 10.75 & 1.2 & 9 & Tremolite & & & $\begin{array}{l}\text { AS }>5,3: 1, \text { AFB }>5,3: 1 \text {, } \\
\text { PCMEF-US, PCMES-US, } \\
\text { PCMEF-ISO, PCMES-ISO }\end{array}$ \\
\hline G1 & 8 & $\mathrm{~F} 4$ & $A X$ & 33 & 36 & $\mathrm{~F}$ & 10.7 & 1.2 & 8.9 & Tremolite & & & $\begin{array}{l}\text { AS }>5,3: 1, \text { AFB }>5,3: 1, \\
\text { PCMEF-US, PCMES-US, } \\
\text { PCMEF-ISO, PCMES-ISO }\end{array}$ \\
\hline G1 & 9 & D4 & $A X$ & 34 & 37 & $\mathrm{~F}$ & 6 & 0.35 & 17.1 & Tremolite & & & $\begin{array}{l}\text { AFB>5, 3:1, AS>5, 3:1, } \\
\text { PCMEF-US, PCMES-US, } \\
\text { PCMEF-ISO, PCMES-ISO }\end{array}$ \\
\hline G1 & 9 & D4 & $A X$ & 35 & 38 & $\mathrm{~F}$ & 4.1 & 0.65 & 6.3 & Tremolite & & & \\
\hline G1 & 10 & B4 & $A X$ & 36 & 39 & $\mathrm{~F}$ & 13.4 & 1.35 & 9.9 & Tremolite & & & $\begin{array}{l}\text { AS }>5,3: 1, \text { AFB }>5,3: 1, \\
\text { PCMEF-US, PCMES-US, } \\
\text { PCMEF-ISO, PCMES-ISO }\end{array}$ \\
\hline G1 & 10 & B4 & $A X$ & 37 & 40 & $\mathrm{~F}$ & 7.8 & 0.65 & 12 & Tremolite & & & $\begin{array}{l}\text { AS }>5,3: 1, \text { AFB }>5,3: 1, \\
\text { PCMEF-US, PCMES-US, } \\
\text { PCMEF-ISO, PCMES-ISO }\end{array}$ \\
\hline G1 & 10 & B4 & $A X$ & 38 & & MD 2-1 & 9.35 & 5.65 & 1.7 & Tremolite & & & $A S>5,3: 1$ \\
\hline G1 & 10 & B4 & $A X$ & & 41 & MF & 8.35 & 0.35 & 23.9 & Tremolite & & & $\begin{array}{c}\text { AFB }>5,3: 1, \text { PCMEF-US, } \\
\text { PCMEF-ISO }\end{array}$ \\
\hline G1 & 10 & B4 & $A X$ & & 42 & MF & 3.75 & 0.5 & 7.5 & Tremolite & & & \\
\hline G1 & 10 & B4 & $A X$ & 39 & 43 & $\mathrm{~F}$ & 4.8 & 0.7 & 6.9 & Tremolite & & & \\
\hline G1 & 10 & B4 & $A X$ & 40 & & MD 1-1 & 7 & 5 & 1.4 & Tremolite & & & $A S>5,3: 1$ \\
\hline G1 & 10 & B4 & $A X$ & & 44 & MF & 5.5 & 0.75 & 7.3 & Tremolite & & & $\begin{array}{c}\text { AFB }>5,3: 1, \text { PCMEF-US, } \\
\text { PCMEF-ISO }\end{array}$ \\
\hline G1 & 11 & A7 & $A X$ & 41 & 45 & $F$ & 3.85 & 0.6 & 6.4 & Tremolite & & & \\
\hline G1 & 11 & A7 & $A X$ & 42 & 46 & $\mathrm{~F}$ & 6.5 & 1.8 & 3.6 & Tremolite & & & $\begin{array}{l}\text { PCMEF-US, PCMES-US, } \\
\text { PCMEF-ISO, PCMES-ISO }\end{array}$ \\
\hline G1 & 11 & A7 & $A X$ & 43 & 47 & $\mathrm{~F}$ & 5 & 0.5 & 10 & Tremolite & & & \\
\hline G1 & 11 & A7 & $A X$ & 44 & 48 & $\mathrm{~F}$ & 3 & 0.45 & 6.7 & Tremolite & & & \\
\hline G1 & 11 & A7 & $A X$ & 45 & & MD 1-1 & 8.5 & 5 & 1.7 & Tremolite & & & $A S>5,3: 1$ \\
\hline G1 & 11 & A7 & $A X$ & & 49 & MF & 6.5 & 0.85 & 7.6 & Tremolite & & & $\begin{array}{c}\text { AFB >5, 3:1, PCMEF-US, } \\
\text { PCMEF-ISO }\end{array}$ \\
\hline G1 & 11 & A7 & $A X$ & 46 & 50 & $\mathrm{~F}$ & 5.85 & 1.2 & 4.9 & Tremolite & & & $\begin{array}{l}\text { PCMEF-US, PCMES-US, } \\
\text { PCMEF-ISO, PCMES-ISO }\end{array}$ \\
\hline G1 & 11 & A7 & $A X$ & 47 & 51 & $\mathrm{~F}$ & 1.85 & 0.38 & 4.9 & Tremolite & & & \\
\hline G1 & 12 & $\mathrm{C7}$ & $A X$ & 48 & 52 & $\mathrm{~F}$ & 2.65 & 0.7 & 3.8 & Tremolite & & & \\
\hline G1 & 12 & $\mathrm{C} 7$ & $A X$ & 49 & & MD 2-1 & 14.5 & 12 & 1.2 & Tremolite & & & $A S>5,3: 1$ \\
\hline G1 & 12 & C7 & $A X$ & & 53 & MF & 9 & 0.8 & 11.2 & Tremolite & & & $\begin{array}{c}\text { AFB }>5,3: 1, \text { PCMEF-US, } \\
\text { PCMEF-ISO }\end{array}$ \\
\hline G1 & 12 & C7 & $A X$ & & 54 & MF & 4.75 & 0.6 & 7.9 & Tremolite & & & \\
\hline G1 & 12 & $\mathrm{C} 7$ & $A X$ & 50 & & CD 2-1 & 8.5 & 5 & 1.7 & Tremolite & & & $A S>5,3: 1$ \\
\hline
\end{tabular}

Count Categories

AFB $>5,3: 1$

PAS

PCMEF-US

Asbestos Fibers and Bundles $>5 \mathrm{um}$ and $3:$

Primary Asbestos Structures

PCM Equivalent Fibers-NIOSH

PCMES-US
AS $>5,3: 1$ PCMEF-ISO PCMES-ISO TAS
Asbestos Structures $>5$ um and 3:1 PCM Equivalent Fibers-ISO PCM Equivalent Structures-ISO Total Asbestos Structures 
ISO 10312, Direct Raw Data

Job Number: 070434

SEA

Report Number: 070434R06

Client: Idaho National Laboratory

Date Received: 4/23/2007

Project Name: RARE

Client Sample No: FB-2-R2

Lab/Cor Sample No: $\mathbf{S 2 7}$

Client Description:

Date Sampled:

$4 / 16 / 2007$

\begin{tabular}{|c|c|c|c|c|c|c|c|c|c|c|c|c|c|}
\hline Gr & No. & Loc. & ID & Prim & Tot & Class & Len & Wid & Asp & Analyte & Elements & Comment & Count Categories \\
\hline G1 & 12 & $\mathrm{C} 7$ & $A X$ & & 55 & CF & 8.2 & 1.85 & 4.4 & Tremolite & & & PCMEF-US, PCMEF-ISO \\
\hline G1 & 12 & $\mathrm{C7}$ & $A X$ & & 56 & CF & 4.8 & 0.55 & 8.7 & Tremolite & & & \\
\hline G1 & 12 & $\mathrm{C} 7$ & $A X$ & 51 & 57 & $\mathrm{~F}$ & 24 & 1.5 & 16 & Tremolite & & & $\begin{array}{l}\text { AS }>5,3: 1, \text { AFB }>5,3: 1, \\
\text { PCMEF-US, PCMES-US, } \\
\text { PCMEF-ISO, PCMES-ISO }\end{array}$ \\
\hline G1 & 13 & E7 & $A X$ & 52 & & MD 1-1 & 17.5 & 5 & 3.5 & Tremolite & & & AS $>5,3: 1$, PCMES-US \\
\hline G1 & 13 & E7 & $A X$ & & 58 & MF & 10 & 1.5 & 6.7 & Tremolite & & & $\begin{array}{c}\text { AFB }>5,3: 1, \text { PCMEF-US, } \\
\text { PCMEF-ISO }\end{array}$ \\
\hline G1 & 13 & E7 & $A X$ & 53 & 59 & $\mathrm{~F}$ & 3.15 & 0.25 & 12.6 & Tremolite & & & \\
\hline G1 & 13 & E7 & $A X$ & 54 & 60 & $F$ & 4.8 & 0.85 & 5.6 & Tremolite & & & \\
\hline G1 & 13 & E7 & $A X$ & 55 & 61 & $\mathrm{~F}$ & 4.5 & 1.2 & 3.7 & Tremolite & & & \\
\hline G1 & 14 & G7 & $A X$ & 56 & 62 & $F$ & 6.5 & 1.75 & 3.7 & Tremolite & & & $\begin{array}{l}\text { PCMEF-US, PCMES-US, } \\
\text { PCMEF-ISO, PCMES-ISO }\end{array}$ \\
\hline $\mathrm{G} 1$ & 15 & 17 & $A X$ & 57 & 63 & $F$ & 4.65 & 0.7 & 6.6 & Tremolite & & & \\
\hline G1 & 15 & 17 & $A X$ & 58 & 64 & $F$ & 12 & 0.8 & 15 & Tremolite & & & $\begin{array}{l}\text { AS >5, 3:1, AFB >5, 3:1, } \\
\text { PCMEF-US, PCMES-US, } \\
\text { PCMEF-ISO, PCMES-ISO }\end{array}$ \\
\hline G1 & 15 & 17 & $A X$ & 59 & 65 & $F$ & 11.35 & 2 & 5.7 & Tremolite & & & $\begin{array}{l}\text { AS>5, 3:1, AFB }>5,3: 1 \text {, } \\
\text { PCMEF-US, PCMES-US, } \\
\text { PCMEF-ISO, PCMES-ISO }\end{array}$ \\
\hline G1 & 15 & 17 & $A X$ & 60 & 66 & $F$ & 12 & 1.8 & 6.7 & Tremolite & & & $\begin{array}{l}\text { AS }>5,3: 1, \text { AFB }>5,3: 1, \\
\text { PCMEF-US, PCMES-US, } \\
\text { PCMEF-ISO, PCMES-ISO }\end{array}$ \\
\hline G1 & 15 & 17 & $A X$ & 61 & 67 & $F$ & 3.45 & 0.4 & 8.6 & Tremolite & & & \\
\hline G1 & 15 & 17 & $A X$ & 62 & 68 & $F$ & 4.35 & 0.25 & 17.4 & Tremolite & & & \\
\hline G1 & 16 & J9 & $A X$ & 63 & 69 & $F$ & 16.85 & 1.65 & 10.2 & Tremolite & & & $\begin{array}{l}\text { AS }>5,3: 1, \text { AFB }>5,3: 1, \\
\text { PCMEF-US, PCMES-US, } \\
\text { PCMEF-ISO, PCMES-ISO }\end{array}$ \\
\hline G1 & 16 & J9 & $A X$ & 64 & 70 & $F$ & 7 & 1.5 & 4.7 & Tremolite & & & $\begin{array}{l}\text { PCMEF-US, PCMES-US, } \\
\text { PCMEF-ISO, PCMES-ISO }\end{array}$ \\
\hline G1 & 16 & J9 & $A X$ & 65 & 71 & $F$ & 4 & 0.55 & 7.3 & Tremolite & & & \\
\hline G1 & 16 & J9 & $A X$ & 66 & 72 & $F$ & 3.12 & 0.65 & 4.8 & Tremolite & & & \\
\hline G1 & 16 & J9 & $A X$ & 67 & 73 & $F$ & 4 & 0.6 & 6.7 & Tremolite & & & \\
\hline G1 & 17 & $\mathrm{H} 9$ & $A X$ & 68 & 74 & $F$ & 17.5 & 1.45 & 12.1 & Tremolite & & & $\begin{array}{l}\text { AS }>5,3: 1, \text { AFB }>5,3: 1, \\
\text { PCMEF-US, PCMES-US, } \\
\text { PCMEF-ISO, PCMES-ISO }\end{array}$ \\
\hline G1 & 17 & $\mathrm{H} 9$ & $A X$ & 69 & 75 & $\mathrm{~F}$ & 16 & 4 & 4 & Tremolite & & & PCMEF-US, PCMES-US \\
\hline G1 & 17 & $\mathrm{H} 9$ & $A X$ & 70 & 76 & $\mathrm{~F}$ & 4 & 1.3 & 3.1 & Tremolite & & & \\
\hline G1 & 17 & $\mathrm{H} 9$ & $A X$ & 71 & & MD 1-1 & 6.25 & 3.8 & 1.6 & Tremolite & & & $A S>5,3: 1$ \\
\hline G1 & 17 & H9 & $A X$ & & 77 & MF & 5.12 & 0.5 & 10.2 & Tremolite & & & $\begin{array}{c}\text { AFB }>5,3: 1, \text { PCMEF-US, } \\
\text { PCMEF-ISO }\end{array}$ \\
\hline
\end{tabular}

Count Categories

$\mathrm{AFB}>5,3: 1$

PAS

PCMEF-US

Asbestos Fibers and Bundles > 5um and 3:

Primary Asbestos Structures

PCM Equivalent Fibers-NIOSH

PCMES-US
AS>5, 3:1 PCMEF-ISO PCMES-ISO TAS
Asbestos Structures $>5$ um and 3:1 PCM Equivalent Fibers-ISO PCM Equivalent Structures-ISO Total Asbestos Structures 
Job Number: 070434

ISO 10312, Direct Raw Data

Client: Idaho National Laboratory

Report Number: 070434R06

Project Name: RARE

Date Received: 4/23/2007

Client Sample No: FB-2-R2

Lab/Cor Sample No: S27

Client Description:

Date Sampled:

4/16/2007

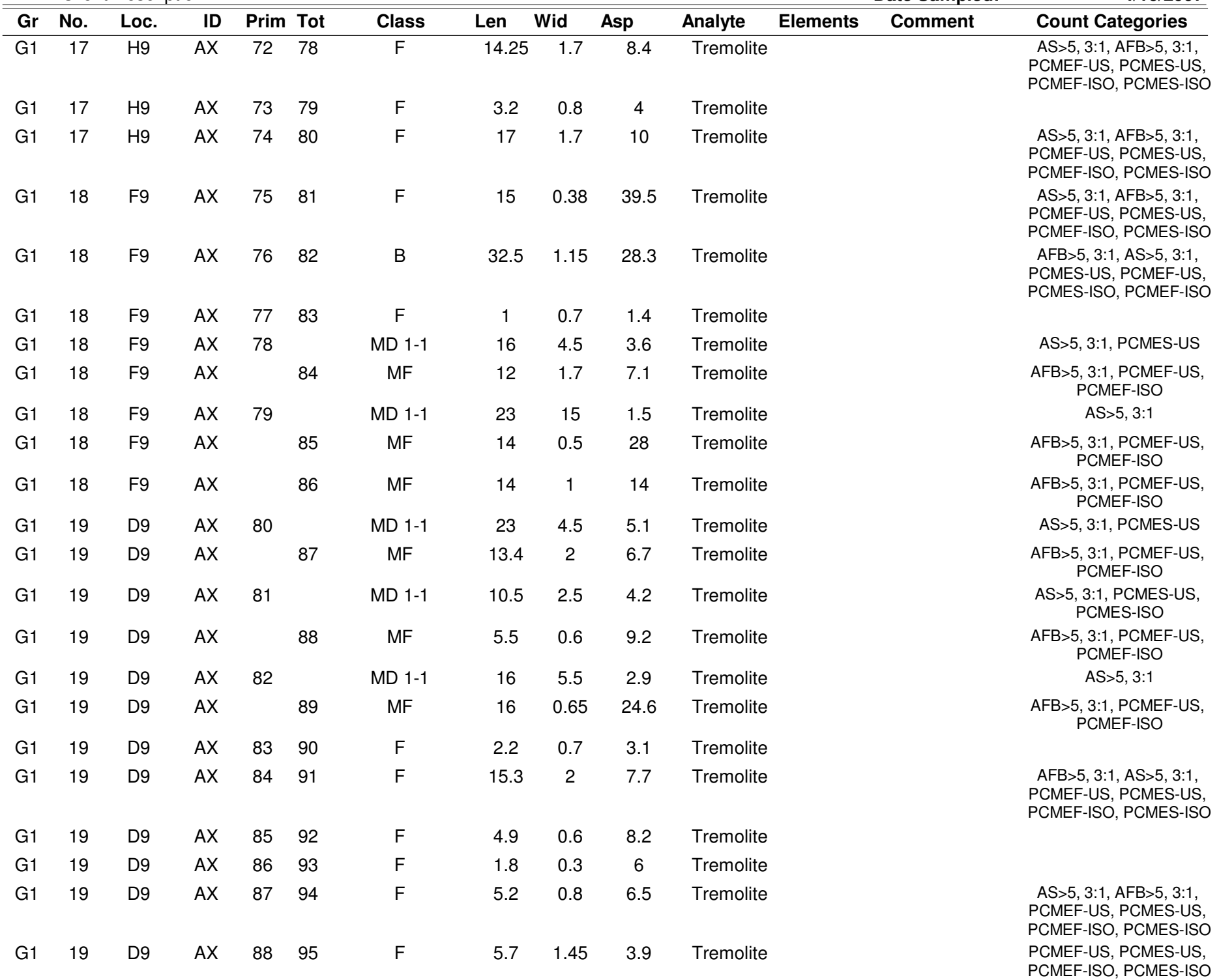

Count Categories

$\mathrm{AFB}>5,3: 1$

PAS

PCMEF-US

Asbestos Fibers and Bundles $>5 u m$ and $3: 1$

Primary Asbestos Structures

PCM Equivalent Fibers-NIOSH

PCMES-US
AS $>5,3: 1$ PCMEF-ISO PCMES-ISO TAS
Asbestos Structures $>5$ um and 3:1 PCM Equivalent Fibers-ISO PCM Equivalent Structures-ISO Total Asbestos Structures 
ISO 10312, Direct Raw Data

Job Number: 070434

SEA

Report Number: 070434R06

Client: Idaho National Laboratory

Date Received: 4/23/2007

Project Name: RARE

Client Sample No: FB-2-R2

Lab/Cor Sample No: $\mathbf{S 2 7}$

Client Description:

Date Sampled:

$4 / 16 / 2007$

\begin{tabular}{|c|c|c|c|c|c|c|c|c|c|c|c|c|c|}
\hline Gr & No. & Loc. & ID & Prim & Tot & Class & Len & Wid & Asp & Analyte & Elements & Comment & Count Categories \\
\hline G1 & 20 & B9 & $A X$ & 89 & 96 & $\mathrm{~F}$ & 10.4 & 1.5 & 6.9 & Tremolite & & & $\begin{array}{l}\text { AS }>5,3: 1, \text { AFB }>5,3: 1, \\
\text { PCMES-US, PCMEF-US, } \\
\text { PCMEF-ISO, PCMES-ISO }\end{array}$ \\
\hline G1 & 20 & B9 & $A X$ & 90 & 97 & $\mathrm{~F}$ & 6.35 & 2 & 3.2 & Tremolite & & & $\begin{array}{l}\text { PCMEF-US, PCMES-US, } \\
\text { PCMEF-ISO, PCMES-ISO }\end{array}$ \\
\hline G1 & 20 & B9 & $A X$ & 91 & 98 & $\mathrm{~F}$ & 6 & 0.5 & 12 & Tremolite & & & $\begin{array}{l}\text { AS>5, 3:1, AFB>5, 3:1, } \\
\text { PCMEF-US, PCMES-US, } \\
\text { PCMEF-ISO, PCMES-ISO }\end{array}$ \\
\hline G2 & 21 & A2 & $A X$ & 92 & 99 & $\mathrm{~F}$ & 4.5 & 1.2 & 3.7 & Tremolite & & & \\
\hline G2 & 21 & $\mathrm{~A} 2$ & $A X$ & 93 & 100 & $\mathrm{~F}$ & 12 & 2.7 & 4.4 & Tremolite & & & $\begin{array}{l}\text { PCMEF-US, PCMES-US, } \\
\text { PCMEF-ISO, PCMES-ISO }\end{array}$ \\
\hline G2 & 21 & A2 & $A X$ & 94 & 101 & $\mathrm{~F}$ & 1.65 & 0.22 & 7.5 & Tremolite & & & \\
\hline G2 & 21 & A2 & $A X$ & 95 & 102 & $\mathrm{~F}$ & 7.8 & 1.5 & 5.2 & Tremolite & & & $\begin{array}{l}\text { AS >5, 3:1, AFB >5, 3:1, } \\
\text { PCMEF-US, PCMES-US, } \\
\text { PCMEF-ISO, PCMES-ISO }\end{array}$ \\
\hline G2 & 21 & A2 & $A X$ & 96 & 103 & $\mathrm{~F}$ & 8 & 0.6 & 13.3 & Tremolite & & & $\begin{array}{l}\text { AS }>5,3: 1, \text { AFB }>5,3: 1, \\
\text { PCMEF-US, PCMES-US, } \\
\text { PCMEF-ISO, PCMES-ISO }\end{array}$ \\
\hline G2 & 21 & A2 & $A X$ & 97 & 104 & $F$ & 4.25 & 0.55 & 7.7 & Tremolite & & & \\
\hline G2 & 22 & $\mathrm{C} 2$ & $A X$ & 98 & 105 & $F$ & 17 & 2 & 8.5 & Tremolite & & & $\begin{array}{l}\text { AS >5, 3:1, AFB >5, 3:1, } \\
\text { PCMEF-US, PCMES-US, } \\
\text { PCMEF-ISO, PCMES-ISO }\end{array}$ \\
\hline G2 & 22 & $\mathrm{C} 2$ & $A X$ & 99 & 106 & $F$ & 27 & 4 & 6.8 & Tremolite & & & $\begin{array}{l}\text { AS }>5,3: 1, \text { AFB }>5,3: 1 \\
\text { PCMEF-US, PCMES-US }\end{array}$ \\
\hline G2 & 22 & $\mathrm{C} 2$ & $A X$ & 100 & 107 & $\mathrm{~F}$ & 27.5 & 2 & 13.8 & Tremolite & & & $\begin{array}{l}\text { AS }>5,3: 1, \text { AFB }>5,3: 1, \\
\text { PCMEF-US, PCMES-US, } \\
\text { PCMEF-ISO, PCMES-ISO }\end{array}$ \\
\hline G2 & 22 & C2 & $A X$ & 101 & 108 & $\mathrm{~F}$ & 19.5 & 1.75 & 11.1 & Tremolite & & & $\begin{array}{l}\text { AS }>5,3: 1, \text { AFB }>5,3: 1, \\
\text { PCMEF-US, PCMES-US, } \\
\text { PCMEF-ISO, PCMES-ISO }\end{array}$ \\
\hline G2 & 22 & $\mathrm{C} 2$ & $A X$ & 102 & 109 & $F$ & 24.75 & 4.5 & 5.5 & Tremolite & & & $\begin{array}{l}\text { AS>5, 3:1, AFB>5, 3:1, } \\
\text { PCMEF-US, PCMES-US }\end{array}$ \\
\hline G2 & 22 & $\mathrm{C} 2$ & $A X$ & 103 & 110 & $\mathrm{~F}$ & 6 & 2 & 3 & Tremolite & & & $\begin{array}{l}\text { PCMEF-US, PCMES-US, } \\
\text { PCMEF-ISO, PCMES-ISO }\end{array}$ \\
\hline G2 & 23 & E2 & $A X$ & 104 & 111 & $F$ & 5.2 & 0.55 & 9.5 & Tremolite & & & $\begin{array}{l}\text { AS }>5,3: 1, \text { AFB }>5,3: 1 \text {, } \\
\text { PCMEF-US, PCMES-US, } \\
\text { PCMEF-ISO, PCMES-ISO }\end{array}$ \\
\hline G2 & 23 & E2 & $A X$ & 105 & 112 & $\mathrm{~F}$ & 1.7 & 0.55 & 3.1 & Tremolite & & & \\
\hline G2 & 23 & E2 & $A X$ & 106 & 113 & $\mathrm{~F}$ & 4.35 & 1.2 & 3.6 & Tremolite & & & \\
\hline G2 & 23 & E2 & $A X$ & 107 & 114 & $\mathrm{~F}$ & 18.63 & 0.75 & 24.8 & Tremolite & & & $\begin{array}{l}\text { AS }>5,3: 1, \text { AFB }>5,3: 1 \text {, } \\
\text { PCMEF-US, PCMES-US, } \\
\text { PCMEF-ISO, PCMES-ISO }\end{array}$ \\
\hline G2 & 24 & G2 & $A X$ & 108 & 115 & $\mathrm{~F}$ & 4.35 & 0.6 & 7.2 & Tremolite & & & \\
\hline G2 & 24 & G2 & $A X$ & 109 & 116 & $\mathrm{~F}$ & 3.85 & 0.7 & 5.5 & Tremolite & & & \\
\hline
\end{tabular}

Count Categories

$\mathrm{AFB}>5,3: 1$

PAS

PCMEF-US

Asbestos Fibers and Bundles $>5 \mathrm{um}$ and $3: 1$

Primary Asbestos Structures

PCM Equivalent Fibers-NIOSH

PCMES-US
AS $>5,3: 1$ PCMEF-ISO PCMES-ISO TAS
Asbestos Structures $>5$ um and 3:1 PCM Equivalent Fibers-ISO PCM Equivalent Structures-ISO Total Asbestos Structures 
Job Number: 070434

ISO 10312, Direct Raw Data

Client: Idaho National Laboratory

Report Number: 070434R06

Project Name: RARE

Date Received: 4/23/2007

Client Sample No: FB-2-R2

Lab/Cor Sample No: S27

Client Description:

Date Sampled:

$4 / 16 / 2007$

\begin{tabular}{|c|c|c|c|c|c|c|c|c|c|c|c|c|c|}
\hline Gr & No. & Loc. & ID & Prim & Tot & Class & Len & Wid & Asp & Analyte & Elements & Comment & Count Categories \\
\hline G2 & 24 & G2 & $A X$ & 110 & 117 & $F$ & 11.2 & 1.2 & 9.3 & Tremolite & & & $\begin{array}{l}\text { AS>5, 3:1, AFB>5, 3:1, } \\
\text { PCMEF-US, PCMES-US, } \\
\text { PCMEF-ISO, PCMES-ISO }\end{array}$ \\
\hline G2 & 24 & G2 & $A X$ & 111 & 118 & $\mathrm{~F}$ & 26 & 3.25 & 8 & Tremolite & & & $\begin{array}{l}\text { AS>5, 3:1, AFB>5, 3:1, } \\
\text { PCMEF-US, PCMES-US }\end{array}$ \\
\hline G2 & 24 & G2 & $A X$ & 112 & 119 & $\mathrm{~F}$ & 3.15 & 0.3 & 10.5 & Tremolite & & & \\
\hline G2 & 24 & G2 & $A X$ & 113 & 120 & $\mathrm{~F}$ & 14.52 & 2.5 & 5.8 & Tremolite & & & $\begin{array}{l}\text { AS>5, 3:1, AFB }>5,3: 1 \text {, } \\
\text { PCMEF-US, PCMES-US, } \\
\text { PCMEF-ISO, PCMES-ISO }\end{array}$ \\
\hline G2 & 24 & G2 & $A X$ & 114 & 121 & $\mathrm{~F}$ & 17 & 1.75 & 9.7 & Tremolite & & & $\begin{array}{l}\text { AS }>5,3: 1, \text { AFB }>5,3: 1, \\
\text { PCMEF-US, PCMES-US, } \\
\text { PCMEF-ISO, PCMES-ISO }\end{array}$ \\
\hline G2 & 24 & G2 & $A X$ & 115 & 122 & $\mathrm{~F}$ & 13.2 & 3 & 4.4 & Tremolite & & & $\begin{array}{l}\text { PCMEF-US, PCMES-US, } \\
\text { PCMEF-ISO, PCMES-ISO }\end{array}$ \\
\hline G2 & 25 & 12 & $A X$ & 116 & 123 & $\mathrm{~F}$ & 25 & 0.65 & 38.5 & Tremolite & & & $\begin{array}{l}\text { AS }>5,3: 1, \text { AFB }>5,3: 1, \\
\text { PCMEF-US, PCMES-US, } \\
\text { PCMEF-ISO, PCMES-ISO }\end{array}$ \\
\hline G2 & 25 & 12 & $A X$ & 117 & 124 & $\mathrm{~F}$ & 10 & 1.2 & 8.3 & Tremolite & & & $\begin{array}{l}\text { AS }>5,3: 1, \text { AFB }>5,3: 1, \\
\text { PCMEF-US, PCMES-US, } \\
\text { PCMEF-ISO, PCMES-ISO }\end{array}$ \\
\hline G2 & 25 & 12 & $A X$ & 118 & 125 & $\mathrm{~F}$ & 4.3 & 0.8 & 5.4 & Tremolite & & & \\
\hline G2 & 25 & 12 & $A X$ & 119 & 126 & $\mathrm{~F}$ & 22 & 1 & 22 & Tremolite & & & $\begin{array}{l}\text { AS>5, 3:1, AFB>5, 3:1, } \\
\text { PCMEF-US, PCMES-US, } \\
\text { PCMEF-ISO, PCMES-ISO }\end{array}$ \\
\hline G2 & 25 & 12 & $A X$ & 120 & 127 & $\mathrm{~F}$ & 9.2 & 0.55 & 16.7 & Tremolite & & & $\begin{array}{l}\text { AS }>5,3: 1, \text { AFB }>5,3: 1, \\
\text { PCMEF-US, PCMES-US, } \\
\text { PCMEF-ISO, PCMES-ISO }\end{array}$ \\
\hline G2 & 25 & 12 & $A X$ & 121 & 128 & $\mathrm{~F}$ & 12.2 & 3.2 & 3.8 & Tremolite & & & PCMEF-US, PCMES-US \\
\hline G2 & 25 & 12 & $A X$ & 122 & 129 & $\mathrm{~F}$ & 18.5 & 1.1 & 16.8 & Tremolite & & & $\begin{array}{l}\text { AS >5, 3:1, AFB>5, 3:1, } \\
\text { PCMEF-US, PCMES-US, } \\
\text { PCMEF-ISO, PCMES-ISO }\end{array}$ \\
\hline G2 & 26 & J4 & $A X$ & 123 & 130 & $\mathrm{~F}$ & 3.2 & 0.7 & 4.6 & Tremolite & & & \\
\hline G2 & 26 & J4 & $A X$ & 124 & 131 & $\mathrm{~F}$ & 3.5 & 0.6 & 5.8 & Tremolite & & & \\
\hline G2 & 26 & J4 & $A X$ & 125 & 132 & $F$ & 4.2 & 0.35 & 12 & Tremolite & & & \\
\hline G2 & 26 & J4 & $A X$ & 126 & 133 & $F$ & 6.5 & 0.45 & 14.4 & Tremolite & & & $\begin{array}{l}\text { AS >5, 3:1, AFB>5, 3:1, } \\
\text { PCMEF-US, PCMES-US, } \\
\text { PCMEF-ISO, PCMES-ISO }\end{array}$ \\
\hline G2 & 27 & $\mathrm{H} 4$ & $A X$ & 127 & 134 & $\mathrm{~F}$ & 4.75 & 0.65 & 7.3 & Tremolite & & & \\
\hline G2 & 27 & $\mathrm{H} 4$ & $A X$ & 128 & 135 & $F$ & 7.75 & 2.5 & 3.1 & Tremolite & & & $\begin{array}{l}\text { PCMES-US, PCMEF-US, } \\
\text { PCMEF-ISO, PCMES-ISO }\end{array}$ \\
\hline G2 & 27 & $\mathrm{H} 4$ & $A X$ & 129 & & MD 2-1 & 12.5 & 9 & 1.4 & Tremolite & & & $A S>5,3: 1$ \\
\hline G2 & 27 & $\mathrm{H} 4$ & $A X$ & & 136 & MF & 12.5 & 0.85 & 14.7 & Tremolite & & & $\begin{array}{c}\text { AFB }>5,3: 1, \text { PCMEF-US, } \\
\text { PCMEF-ISO }\end{array}$ \\
\hline G2 & 27 & $\mathrm{H} 4$ & $A X$ & & 137 & MF & 1.85 & 0.3 & 6.2 & Tremolite & & & \\
\hline G2 & 27 & $\mathrm{H} 4$ & $A X$ & 130 & 138 & $\mathrm{~F}$ & 3 & 0.35 & 8.6 & Tremolite & & & \\
\hline
\end{tabular}

Count Categories

$\mathrm{AFB}>5,3: 1$

PAS

PCMEF-US

Asbestos Fibers and Bundles $>5 \mathrm{um}$ and 3:1

Primary Asbestos Structures

PCM Equivalent Fibers-NIOSH

PCMES-US
AS $>5,3: 1$ PCMEF-ISO PCMES-ISO TAS

Page 96 of 281

Asbestos Structures $>5$ um and 3:1 PCM Equivalent Fibers-ISO PCM Equivalent Structures-ISO Total Asbestos Structures 
Job Number: 070434

ISO 10312, Direct Raw Data

Client: Idaho National Laboratory

Report Number: 070434R06

Project Name: RARE

Date Received: 4/23/2007

Client Sample No: FB-2-R2

Lab/Cor Sample No: S27

Client Description:

Date Sampled:

4/16/2007

\begin{tabular}{|c|c|c|c|c|c|c|c|c|c|c|c|c|c|}
\hline Gr & No. & Loc. & ID & Prim & Tot & Class & Len & Wid & Asp & Analyte & Elements & Comment & Count Categories \\
\hline G2 & 27 & $\mathrm{H} 4$ & $A X$ & 131 & 139 & $\mathrm{~F}$ & 4.35 & 1.12 & 3.9 & Tremolite & & & \\
\hline G2 & 28 & $\mathrm{~F} 4$ & $A X$ & 132 & 140 & $\mathrm{~F}$ & 25 & 0.7 & 35.7 & Tremolite & & & $\begin{array}{l}\text { AS }>5,3: 1, \text { AFB }>5,3: 1 \text {, } \\
\text { PCMEF-US, PCMES-US, } \\
\text { PCMEF-ISO, PCMES-ISO }\end{array}$ \\
\hline G2 & 29 & D4 & $A X$ & 133 & 141 & $\mathrm{~F}$ & 48.5 & 1.85 & 26.2 & Tremolite & & & $\begin{array}{l}\text { AFB }>5,3: 1, \text { AS }>5,3: 1, \\
\text { PCMEF-US, PCMES-US, } \\
\text { PCMEF-ISO, PCMES-ISO }\end{array}$ \\
\hline G2 & 29 & D4 & $A X$ & 134 & 142 & $\mathrm{~F}$ & 10 & 2.1 & 4.8 & Tremolite & & & $\begin{array}{l}\text { PCMEF-US, PCMES-US, } \\
\text { PCMEF-ISO, PCMES-ISO }\end{array}$ \\
\hline G2 & 29 & D4 & $A X$ & 135 & & MD 1-1 & 8.5 & 3.5 & 2.4 & Tremolite & & & $A S>5,3: 1$ \\
\hline G2 & 29 & D4 & $A X$ & & 143 & MF & 5.85 & 0.5 & 11.7 & Tremolite & & & $\begin{array}{c}\text { AFB }>5,3: 1, \text { PCMEF-US, } \\
\text { PCMEF-ISO }\end{array}$ \\
\hline G2 & 29 & D4 & $A X$ & 136 & 144 & $\mathrm{~F}$ & 38 & 1.5 & 25.3 & Tremolite & & & $\begin{array}{l}\text { AS >5, 3:1, AFB>5, 3:1, } \\
\text { PCMEF-US, PCMES-US, } \\
\text { PCMEF-ISO, PCMES-ISO }\end{array}$ \\
\hline G2 & 29 & D4 & $A X$ & 137 & & MD 1-1 & 13 & 10 & 1.3 & Tremolite & & & $A S>5,3: 1$ \\
\hline G2 & 29 & D4 & $A X$ & & 145 & MF & 13 & 0.7 & 18.6 & Tremolite & & & $\begin{array}{c}\text { AFB }>5 \text {, 3:1, PCMEF-US, } \\
\text { PCMEF-ISO }\end{array}$ \\
\hline G2 & 29 & D4 & $A X$ & 138 & & MD 1-0 & 3.5 & 1.25 & 2.8 & Tremolite & & & \\
\hline G2 & 29 & D4 & $A X$ & & 146 & MF & 3.5 & 0.45 & 7.8 & Tremolite & & & \\
\hline G2 & 29 & D4 & $A X$ & 139 & 147 & $F$ & 10.3 & 1 & 10.3 & Tremolite & & & $\begin{array}{l}\text { AS>5, 3:1, AFB>5, 3:1, } \\
\text { PCMEF-US, PCMES-US, } \\
\text { PCMEF-ISO, PCMES-ISO }\end{array}$ \\
\hline G2 & 29 & D4 & $A X$ & 140 & 148 & $\mathrm{~F}$ & 2.7 & 1.35 & 2 & Tremolite & & & \\
\hline G2 & 30 & B4 & $A X$ & 141 & 149 & $F$ & 9.2 & 1.2 & 7.7 & Tremolite & & & $\begin{array}{l}\text { AS }>5,3: 1, \text { AFB }>5,3: 1 \text {, } \\
\text { PCMEF-US, PCMES-US, } \\
\text { PCMEF-ISO, PCMES-ISO }\end{array}$ \\
\hline $\mathrm{G} 2$ & 30 & B4 & $A X$ & 142 & 150 & $F$ & 14 & 1.7 & 8.2 & Tremolite & & & $\begin{array}{l}\text { AS }>5,3: 1, \text { AFB }>5,3: 1, \\
\text { PCMEF-US, PCMES-US, } \\
\text { PCMEF-ISO, PCMES-ISO }\end{array}$ \\
\hline G2 & 31 & A7 & $A X$ & 143 & 151 & $F$ & 5.6 & 0.7 & 8 & Tremolite & & & $\begin{array}{l}\text { AS>5, 3:1, AFB>5, 3:1, } \\
\text { PCMEF-US, PCMES-US, } \\
\text { PCMEF-ISO, PCMES-ISO }\end{array}$ \\
\hline G2 & 31 & A7 & $A X$ & 144 & 152 & $F$ & 7.5 & 0.75 & 10 & Tremolite & & & $\begin{array}{l}\text { AS }>5,3: 1, \text { AFB }>5,3: 1 \text {, } \\
\text { PCMEF-US, PCMES-US, } \\
\text { PCMEF-ISO, PCMES-ISO }\end{array}$ \\
\hline G2 & 31 & A7 & $A X$ & 145 & 153 & $\mathrm{~F}$ & 4.9 & 0.25 & 19.6 & Tremolite & & & \\
\hline G2 & 31 & A7 & $A X$ & 146 & 154 & $F$ & 15.7 & 2 & 7.8 & Tremolite & & & $\begin{array}{l}\text { AS>5, 3:1, AFB }>5,3: 1, \\
\text { PCMEF-US, PCMES-US, } \\
\text { PCMEF-ISO, PCMES-ISO }\end{array}$ \\
\hline $\mathrm{G} 2$ & 31 & A7 & $A X$ & 147 & 155 & $F$ & 5.65 & 0.6 & 9.4 & Tremolite & & & $\begin{array}{l}\text { AS>5, 3:1, AFB>5, 3:1, } \\
\text { PCMEF-US, PCMES-US, } \\
\text { PCMEF-ISO, PCMES-ISO }\end{array}$ \\
\hline G2 & 32 & $\mathrm{C7}$ & $A X$ & 148 & 156 & $F$ & 8.4 & 0.55 & 15.3 & Tremolite & & & $\begin{array}{l}\text { AS }>5,3: 1, \text { AFB }>5,3: 1, \\
\text { PCMEF-US, PCMES-US, } \\
\text { PCMEF-ISO, PCMES-ISO }\end{array}$ \\
\hline
\end{tabular}

Count Categories

AFB $>5,3: 1$

Asbestos Fibers and Bundles $>$ 5um and 3:1

PAS

PCMEF-US

Primary Asbestos Structures

PCM Equivalent Fibers-NIOSH

PCMES-US

PCM Equivalent Structures-NIOSH
AS $>5,3: 1$ PCMEF-ISO PCMES-ISO TAS
Asbestos Structures $>5$ um and 3:1 PCM Equivalent Fibers-ISO PCM Equivalent Structures-ISO Total Asbestos Structures

Page 97 of 281 
Job Number: 070434

SEA

ISO 10312, Direct Raw Data

Client: Idaho National Laboratory
Report Number: 070434R06

Date Received: 4/23/2007

Project Name: RARE

Client Sample No: FB-2-R2

Lab/Cor Sample No: S27

Client Description:

\begin{tabular}{|c|c|c|c|c|c|c|c|c|c|c|c|c|c|}
\hline Gr & No. & Loc. & ID & Prim & Tot & Class & Len & Wid & Asp & Analyte & Elements & Comment & Count Categories \\
\hline G2 & 33 & E7 & $A X$ & 149 & 157 & $\mathrm{~F}$ & 3 & 0.45 & 6.7 & Tremolite & & & \\
\hline G2 & 33 & E7 & $A X$ & 150 & 158 & $\mathrm{~F}$ & 7.8 & 0.8 & 9.8 & Tremolite & & & $\begin{array}{l}\text { AS }>5,3: 1, \text { AFB }>5,3: 1, \\
\text { PCMEF-US, PCMES-US, } \\
\text { PCMEF-ISO, PCMES-ISO }\end{array}$ \\
\hline G2 & 33 & E7 & $A X$ & 151 & 159 & $\mathrm{~F}$ & 16 & 3.75 & 4.3 & Tremolite & & & PCMEF-US, PCMES-US \\
\hline G2 & 33 & E7 & $A X$ & 152 & 160 & $\mathrm{~F}$ & 2.2 & 0.65 & 3.4 & Tremolite & & & \\
\hline G2 & 33 & E7 & $A X$ & 153 & 161 & $\mathrm{~F}$ & 17.5 & 2.25 & 7.8 & Tremolite & & & $\begin{array}{l}\text { AS }>5,3: 1, \text { AFB }>5,3: 1, \\
\text { PCMEF-US, PCMES-US, } \\
\text { PCMEF-ISO, PCMES-ISO }\end{array}$ \\
\hline G2 & 33 & E7 & $A X$ & 154 & 162 & $\mathrm{~F}$ & 4.25 & 0.5 & 8.5 & Tremolite & & & \\
\hline G2 & 34 & G7 & & & & NSD & & & & & & & \\
\hline G2 & 35 & 17 & $A X$ & 155 & 163 & $F$ & 5.5 & 0.6 & 9.2 & Tremolite & & & $\begin{array}{l}\text { AS }>5,3: 1, \text { AFB }>5,3: 1, \\
\text { PCMEF-US, PCMES-US, } \\
\text { PCMEF-ISO, PCMES-ISO }\end{array}$ \\
\hline G2 & 35 & 17 & $A X$ & 156 & & MD 2-0 & 5.8 & 5.25 & 1.1 & Tremolite & & & $A S>5,3: 1$ \\
\hline G2 & 35 & 17 & $A X$ & & 164 & MF & 3.8 & 1.2 & 3.2 & Tremolite & & & \\
\hline G2 & 35 & 17 & $A X$ & & 165 & MF & 3.2 & 0.6 & 5.3 & Tremolite & & & \\
\hline G2 & 35 & 17 & $A X$ & 157 & 166 & $\mathrm{~F}$ & 8.4 & 0.65 & 12.9 & Tremolite & & & $\begin{array}{l}\text { AS }>5,3: 1, \text { AFB }>5,3: 1, \\
\text { PCMEF-US, PCMES-US, } \\
\text { PCMEF-ISO, PCMES-ISO }\end{array}$ \\
\hline
\end{tabular}

Count Categories

AFB $>5,3: 1$

PAS

PCMEF-US

PCMES-US
Asbestos Fibers and Bundles > 5um and 3:1

Primary Asbestos Structures

PCM Equivalent Fibers-NIOSH

PCM Equivalent Structures-NIOSH
AS $>5,3: 1$ PCMEF-ISO PCMES-ISO TAS
Asbestos Structures >5um and 3:1 PCM Equivalent Fibers-ISO PCM Equivalent Structures-ISO Total Asbestos Structures 


\section{ISO 10312, Direct Raw Data}

Job Number: 070434

SEA

Report Number: 070434R06

Client: Idaho National Laboratory

Date Received: 4/23/2007

Project Name: RARE

Client Sample No: FB-2-R3

Lab/Cor Sample No: S28

Client Description:

Date Sampled:

4/16/2007

\begin{tabular}{|c|c|c|c|c|c|c|c|c|c|c|c|c|c|}
\hline $\mathrm{Gr}$ & No. & Loc. & ID & Prim & Tot & Class & Len & Wid & Asp & Analyte & Elements & nent & Count Categories \\
\hline \multirow[t]{5}{*}{$\mathrm{G} 1$} & 1 & $\mathrm{~A} 2$ & NAM & 1 & 1 & $F$ & 19 & 1.1 & 17.3 & $\begin{array}{c}\text { Non } \\
\text { Asbestos } \\
\text { Mineral }\end{array}$ & $\begin{array}{l}\text { Mg, Al, Si, } \\
\text { Fe }\end{array}$ & & \\
\hline & & & & & & \multicolumn{2}{|c|}{ ItemType } & ItemNur & & & Confirmed & Com & \\
\hline & & & & & & \multicolumn{2}{|c|}{ Brightfield } & $\mathrm{J} 3570 \mathrm{E}$ & & & & & \\
\hline & & & & & & \multicolumn{2}{|c|}{ Diffraction } & J3570 & & & & $\begin{array}{l}\text { POS } \\
\text { PAT }\end{array}$ & E TALC - HEXAGONAL \\
\hline & & & & & & \multicolumn{2}{|c|}{ Spectra } & J3008 & & & & & \\
\hline \multirow[t]{3}{*}{ G1 } & 1 & $\mathrm{~A} 2$ & $A Q$ & 2 & 2 & $\mathrm{~F}$ & 4 & 0.45 & 8.9 & Tremolite & $\mathrm{Mg}, \mathrm{Si}, \mathrm{Fe}$ & & \\
\hline & & & & & & \multicolumn{2}{|c|}{ ItemType } & ItemNur & & & Confirmed & Com & \\
\hline & & & & & & \multicolumn{2}{|c|}{ Spectra } & J3009 & & & & & \\
\hline \multirow[t]{5}{*}{ G1 } & 1 & $\mathrm{~A} 2$ & $A Z Q$ & 3 & 3 & $\mathrm{~F}$ & 7 & 0.65 & 10.8 & Tremolite & $\begin{array}{l}\text { Mg, Al, Si, } \\
\text { Ca, Fe }\end{array}$ & & $\begin{array}{l}\text { AS>5, 3:1, AFB>5, 3:1, } \\
\text { PCMEF-US, PCMES-US, } \\
\text { PCMEF-ISO, PCMES-ISO }\end{array}$ \\
\hline & & & & & & \multicolumn{2}{|c|}{ ItemType } & ItemNur & & & Confirmed & Com & \\
\hline & & & & & & \multicolumn{2}{|c|}{ Brightfield } & J3571 & & & & & \\
\hline & & & & & & \multicolumn{2}{|c|}{ Diffraction } & J3571 & & & KM 6/4/2007 & ZON & KIS [ $\left.\begin{array}{lll}1 & 0 & 1\end{array}\right]$ \\
\hline & & & & & & \multicolumn{2}{|c|}{ Spectra } & $\mathrm{J} 3010$ & & & & & \\
\hline G1 & 1 & $\mathrm{~A} 2$ & $A Q$ & 4 & 4 & $\mathrm{~F}$ & 17 & 1.1 & 15.5 & Tremolite & & & $\begin{array}{l}\text { AS>5, 3:1, AFB>5, 3:1, } \\
\text { PCMEF-US, PCMES-US, } \\
\text { PCMEF-ISO, PCMES-ISO }\end{array}$ \\
\hline G1 & 1 & $\mathrm{~A} 2$ & $A Q$ & 5 & 5 & $\mathrm{~F}$ & 4.1 & 1.1 & 3.7 & Tremolite & & & \\
\hline G1 & 1 & $\mathrm{~A} 2$ & $A Q$ & 6 & 6 & $\mathrm{~F}$ & 8 & 0.7 & 11.4 & Tremolite & & & $\begin{array}{l}\text { AS>5, 3:1, AFB>5, 3:1, } \\
\text { PCMEF-US, PCMES-US, } \\
\text { PCMEF-ISO, PCMES-ISO }\end{array}$ \\
\hline G1 & 2 & $\mathrm{C} 2$ & $\mathrm{AQ}$ & 7 & 7 & $\mathrm{~F}$ & 1.7 & 0.15 & 11.3 & Tremolite & & & \\
\hline G1 & 2 & $\mathrm{C} 2$ & $A Q$ & 8 & 8 & $\mathrm{~F}$ & 6.35 & 0.45 & 14.1 & Tremolite & & & $\begin{array}{l}\text { AS }>5,3: 1, \text { AFB }>5,3: 1, \\
\text { PCMEF-US, PCMES-US, } \\
\text { PCMEF-ISO, PCMES-ISO }\end{array}$ \\
\hline G1 & 2 & C2 & $A Q$ & 9 & 9 & $\mathrm{~F}$ & 2.8 & 0.4 & 7 & Tremolite & & & \\
\hline G1 & 2 & $\mathrm{C} 2$ & $A Q$ & 10 & 10 & $\mathrm{~F}$ & 7.75 & 1.12 & 6.9 & Tremolite & & & $\begin{array}{l}\text { AS>5, 3:1, AFB>5, 3:1, } \\
\text { PCMEF-US, PCMES-US, } \\
\text { PCMEF-ISO, PCMES-ISO }\end{array}$ \\
\hline G1 & 2 & $\mathrm{C} 2$ & $A D$ & 11 & 11 & $\mathrm{~F}$ & 10.5 & 0.7 & 15 & Tremolite & & & $\begin{array}{l}\text { AS>5, 3:1, AFB>5, 3:1, } \\
\text { PCMEF-US, PCMES-US, } \\
\text { PCMEF-ISO, PCMES-ISO }\end{array}$ \\
\hline G1 & 2 & $\mathrm{C} 2$ & $A D$ & 12 & & MD 1-0 & 7 & 6 & 1.2 & Tremolite & & & $A S>5,3: 1$ \\
\hline G1 & 2 & $\mathrm{C} 2$ & $A D$ & & 12 & MF & 3.75 & 0.45 & 8.3 & Tremolite & & & \\
\hline G1 & 3 & E2 & $A D$ & 13 & 13 & $F$ & 2.7 & 0.4 & 6.8 & Tremolite & & & \\
\hline G1 & 3 & E2 & $A D$ & 14 & 14 & $F$ & 2.75 & 0.8 & 3.4 & Tremolite & & & \\
\hline
\end{tabular}

Count Categories

$\mathrm{AFB}>5,3: 1$

PAS

PCMEF-US

PCMES-US
Asbestos Fibers and Bundles $>5 u m$ and 3:1

Primary Asbestos Structures

PCM Equivalent Fibers-NIOSH

PCM Equivalent Structures-NIOSH
AS $>5,3: 1$ PCMEF-ISO PCMES-ISO TAS
Asbestos Structures $>5$ um and 3:1 PCM Equivalent Fibers-ISO PCM Equivalent Structures-ISO Total Asbestos Structures 
ISO 10312, Direct Raw Data

Job Number: 070434

SEA

Report Number: 070434R06

Client: Idaho National Laboratory

Date Received: 4/23/2007

Project Name: RARE

Client Sample No: FB-2-R3

Lab/Cor Sample No: S28

Client Description:

Date Sampled:

4/16/2007

\begin{tabular}{|c|c|c|c|c|c|c|c|c|c|c|c|c|c|}
\hline Gr & No. & Loc. & ID & Prim & Tot & Class & Len & Wid & Asp & Analyte & Elements & Comment & Count Categories \\
\hline G1 & 3 & E2 & $A D$ & 15 & 15 & $F$ & 13 & 0.75 & 17.3 & Tremolite & & & $\begin{array}{l}\text { AS }>5,3: 1, \text { AFB }>5,3: 1, \\
\text { PCMEF-US, PCMES-US, } \\
\text { PCMEF-ISO, PCMES-ISO }\end{array}$ \\
\hline G1 & 3 & E2 & $A D$ & 16 & 16 & $\mathrm{~F}$ & 0.7 & 0.15 & 4.7 & Tremolite & & & \\
\hline G1 & 3 & E2 & $A D$ & 17 & 17 & $\mathrm{~F}$ & 7.58 & 1 & 7.6 & Tremolite & & & $\begin{array}{l}\text { AS>5, 3:1, AFB >5, 3:1, } \\
\text { PCMEF-US, PCMES-US, } \\
\text { PCMEF-ISO, PCMES-ISO }\end{array}$ \\
\hline G1 & 3 & E2 & $A D$ & 18 & 18 & $\mathrm{~F}$ & 5.7 & 0.8 & 7.1 & Tremolite & & & $\begin{array}{l}\text { AFB }>5,3: 1, \text { AS }>5,3: 1, \\
\text { PCMEF-US, PCMES-US, } \\
\text { PCMEF-ISO, PCMES-ISO }\end{array}$ \\
\hline G1 & 3 & E2 & $A D$ & 19 & 19 & $\mathrm{~F}$ & 4.75 & 1.1 & 4.3 & Tremolite & & & \\
\hline G1 & 3 & E2 & $A D$ & 20 & 20 & $\mathrm{~F}$ & 7 & 0.45 & 15.6 & Tremolite & & & $\begin{array}{l}\text { AS }>5,3: 1, \text { AFB }>5,3: 1 \text {, } \\
\text { PCMEF-US, PCMES-US, } \\
\text { PCMEF-ISO, PCMES-ISO }\end{array}$ \\
\hline G1 & 4 & G2 & $A D$ & 21 & 21 & $\mathrm{~F}$ & 6 & 1 & 6 & Tremolite & & & $\begin{array}{l}\text { AS }>5,3: 1, \text { AFB }>5,3: 1, \\
\text { PCMEF-US, PCMES-US, } \\
\text { PCMEF-ISO, PCMES-ISO }\end{array}$ \\
\hline G1 & 4 & G2 & $A D$ & 22 & 22 & $F$ & 2.7 & 0.7 & 3.9 & Tremolite & & & \\
\hline G1 & 4 & G2 & $A D$ & 23 & & MD 1-0 & 6 & 1.5 & 4 & Tremolite & & & $\begin{array}{c}\text { AS }>5,3: 1, \text { PCMES-US, } \\
\text { PCMES-ISO }\end{array}$ \\
\hline G1 & 4 & G2 & $A D$ & & 23 & MF & 3.2 & 0.4 & 8 & Tremolite & & & \\
\hline G1 & 4 & G2 & $A D$ & 24 & 24 & $F$ & 9.8 & 1.65 & 5.9 & Tremolite & & & $\begin{array}{l}\text { AS>5, 3:1, AFB }>5,3: 1 \text {, } \\
\text { PCMEF-US, PCMES-US, } \\
\text { PCMEF-ISO, PCMES-ISO }\end{array}$ \\
\hline G1 & 4 & G2 & $A D$ & 25 & 25 & $F$ & 3.2 & 0.7 & 4.6 & Tremolite & & & \\
\hline G1 & 4 & G2 & $A D$ & 26 & 26 & $F$ & 3.2 & 0.45 & 7.1 & Tremolite & & & \\
\hline G1 & 4 & G2 & $A D$ & 27 & 27 & B & 5 & 0.7 & 7.1 & Tremolite & & & \\
\hline G1 & 5 & 12 & $A D$ & 28 & 28 & $\mathrm{~F}$ & 1.75 & 0.3 & 5.8 & Tremolite & & & \\
\hline G1 & 5 & 12 & $A D$ & 29 & 29 & $\mathrm{~F}$ & 2.7 & 0.5 & 5.4 & Tremolite & & & \\
\hline G1 & 5 & 12 & $A D$ & 30 & 30 & $\mathrm{~F}$ & 23 & 4 & 5.8 & Tremolite & & & $\begin{array}{l}\text { AS }>5,3: 1, \text { AFB }>5,3: 1 \\
\text { PCMEF-US, PCMES-US }\end{array}$ \\
\hline G1 & 5 & 12 & $A D$ & 31 & 31 & $\mathrm{~F}$ & 1.25 & 0.35 & 3.6 & Tremolite & & & \\
\hline G1 & 5 & 12 & $A D$ & 32 & 32 & $F$ & 12.5 & 0.85 & 14.7 & Tremolite & & & $\begin{array}{l}\text { AS }>5,3: 1, \text { AFB }>5,3: 1, \\
\text { PCMEF-US, PCMES-US, } \\
\text { PCMEF-ISO, PCMES-ISO }\end{array}$ \\
\hline G1 & 5 & 12 & $A D$ & 33 & 33 & $\mathrm{~F}$ & 5.5 & 0.65 & 8.5 & Tremolite & & & $\begin{array}{l}\text { AS>5, 3:1, AFB>5, 3:1, } \\
\text { PCMEF-US, PCMES-US, } \\
\text { PCMEF-ISO, PCMES-ISO }\end{array}$ \\
\hline G1 & 5 & 12 & $A D$ & 34 & 34 & $\mathrm{~F}$ & 20 & 4.3 & 4.7 & Tremolite & & & PCMEF-US, PCMES-US \\
\hline G1 & 5 & 12 & $A D$ & 35 & 35 & $\mathrm{~F}$ & 1.7 & 0.25 & 6.8 & Tremolite & & & \\
\hline G1 & 5 & 12 & $A D$ & 36 & 36 & $\mathrm{~F}$ & 5 & 1.45 & 3.4 & Tremolite & & & \\
\hline G1 & 6 & J4 & $A D$ & 37 & 37 & $\mathrm{~F}$ & 1.12 & 0.15 & 7.5 & Tremolite & & & \\
\hline G1 & 6 & J4 & $A D$ & 38 & 38 & $\mathrm{~F}$ & 2.7 & 0.5 & 5.4 & Tremolite & & & \\
\hline
\end{tabular}

Count Categories

$A F B>5,3: 1$

PAS

PCMEF-US

Asbestos Fibers and Bundles $>$ 5um and 3:1

Primary Asbestos Structures

PCM Equivalent Fibers-NIOSH

PCMES-US
AS $>5,3: 1$ PCMEF-ISO PCMES-ISO TAS
Asbestos Structures $>5$ um and 3:1 PCM Equivalent Fibers-ISO PCM Equivalent Structures-ISO Total Asbestos Structures 
ISO 10312, Direct Raw Data

Job Number: 070434

SEA

Report Number: 070434R06

Client: Idaho National Laboratory

Date Received: 4/23/2007

Project Name: RARE

Client Sample No: FB-2-R3

Lab/Cor Sample No: S28

Client Description:

Date Sampled:

4/16/2007

\begin{tabular}{|c|c|c|c|c|c|c|c|c|c|c|c|c|c|}
\hline Gr & No. & Loc. & ID & Prim & Tot & Class & Len & Wid & Asp & Analyte & Elements & Comment & Count Categories \\
\hline G1 & 6 & J4 & $A D$ & 39 & 39 & $F$ & 12.5 & 0.65 & 19.2 & Tremolite & & & $\begin{array}{l}\text { AS }>5,3: 1, \text { AFB }>5,3: 1, \\
\text { PCMEF-US, PCMES-US, } \\
\text { PCMEF-ISO, PCMES-ISO }\end{array}$ \\
\hline G1 & 6 & J4 & $A D$ & 40 & 40 & $\mathrm{~F}$ & 3.1 & 0.7 & 4.4 & Tremolite & & & \\
\hline G1 & 6 & $\mathrm{~J} 4$ & $A D$ & 41 & 41 & $\mathrm{~F}$ & 3 & 0.7 & 4.3 & Tremolite & & & \\
\hline G1 & 6 & J4 & $A D$ & 42 & 42 & $\mathrm{~F}$ & 4.5 & 0.35 & 12.9 & Tremolite & & & \\
\hline G1 & 7 & $\mathrm{H} 4$ & $A D$ & 43 & 43 & $\mathrm{~F}$ & 9.5 & 0.65 & 14.6 & Tremolite & & & $\begin{array}{l}\text { AS>5, 3:1, AFB>5, 3:1, } \\
\text { PCMEF-US, PCMES-US, } \\
\text { PCMEF-ISO, PCMES-ISO }\end{array}$ \\
\hline G1 & 7 & $\mathrm{H} 4$ & $A X$ & 44 & 44 & $\mathrm{~F}$ & 15 & 3.5 & 4.3 & Tremolite & & & PCMEF-US, PCMES-US \\
\hline G1 & 7 & $\mathrm{H} 4$ & $A X$ & 45 & 45 & $\mathrm{~F}$ & 4.2 & 0.6 & 7 & Tremolite & & & \\
\hline G1 & 7 & $\mathrm{H} 4$ & $A X$ & 46 & & MD 1-0 & 6.5 & 4 & 1.6 & Tremolite & & & $A S>5,3: 1$ \\
\hline G1 & 7 & $\mathrm{H} 4$ & $A X$ & & 46 & MF & 5 & 0.65 & 7.7 & Tremolite & & & \\
\hline G1 & 7 & $\mathrm{H} 4$ & $A X$ & 47 & 47 & $\mathrm{~F}$ & 15 & 2.75 & 5.5 & Tremolite & & & $\begin{array}{l}\text { AS }>5,3: 1, \text { AFB }>5,3: 1, \\
\text { PCMEF-US, PCMES-US, } \\
\text { PCMEF-ISO, PCMES-ISO }\end{array}$ \\
\hline G1 & 7 & $\mathrm{H} 4$ & $A X$ & 48 & 48 & $\mathrm{~F}$ & 2 & 0.55 & 3.6 & Tremolite & & & \\
\hline G1 & 7 & $\mathrm{H} 4$ & $A X$ & 49 & 49 & $\mathrm{~F}$ & 5.7 & 1.2 & 4.7 & Tremolite & & & $\begin{array}{l}\text { PCMEF-US, PCMES-US, } \\
\text { PCMEF-ISO, PCMES-ISO }\end{array}$ \\
\hline G1 & 8 & $\mathrm{~F} 4$ & $A X$ & 50 & 50 & $\mathrm{~F}$ & 20 & 2.5 & 8 & Tremolite & & & $\begin{array}{l}\text { AS }>5,3: 1, \text { AFB }>5,3: 1, \\
\text { PCMEF-US, PCMES-US, } \\
\text { PCMEF-ISO, PCMES-ISO }\end{array}$ \\
\hline G1 & 8 & $\mathrm{~F} 4$ & $A X$ & 51 & 51 & $\mathrm{~F}$ & 2.2 & 0.6 & 3.7 & Tremolite & & & \\
\hline G1 & 8 & F4 & $A X$ & 52 & 52 & $\mathrm{~F}$ & 4 & 0.6 & 6.7 & Tremolite & & & \\
\hline G1 & 8 & $\mathrm{~F} 4$ & $A X$ & 53 & 53 & $\mathrm{~F}$ & 7.85 & 1 & 7.8 & Tremolite & & & $\begin{array}{l}\text { AS }>5,3: 1, \text { AFB }>5,3: 1, \\
\text { PCMEF-US, PCMES-US, } \\
\text { PCMEF-ISO, PCMES-ISO }\end{array}$ \\
\hline G1 & 9 & D4 & $A X$ & 54 & 54 & $\mathrm{~F}$ & 3.9 & 1.1 & 3.5 & Tremolite & & & \\
\hline G1 & 9 & D4 & $A X$ & 55 & 55 & $\mathrm{~F}$ & 2.5 & 0.6 & 4.2 & Tremolite & & & \\
\hline G1 & 9 & D4 & $A X$ & 56 & 56 & $\mathrm{~F}$ & 7.2 & 1.25 & 5.8 & Tremolite & & & $\begin{array}{l}\text { AS }>5,3: 1, \text { AFB }>5,3: 1 \text {, } \\
\text { PCMEF-US, PCMES-US, } \\
\text { PCMEF-ISO, PCMES-ISO }\end{array}$ \\
\hline G1 & 9 & D4 & $A X$ & 57 & 57 & $\mathrm{~F}$ & 10.7 & 0.6 & 17.8 & Tremolite & & & $\begin{array}{l}\text { AS }>5,3: 1, \text { AFB }>5,3: 1, \\
\text { PCMEF-US, PCMES-US, } \\
\text { PCMEF-ISO, PCMES-ISO }\end{array}$ \\
\hline G1 & 9 & D4 & $A X$ & 58 & 58 & $\mathrm{~F}$ & 9 & 1.2 & 7.5 & Tremolite & & & $\begin{array}{l}\text { AS }>5,3: 1, \text { AFB }>5,3: 1, \\
\text { PCMEF-US, PCMES-US, } \\
\text { PCMEF-ISO, PCMES-ISO }\end{array}$ \\
\hline G1 & 10 & B4 & $A X$ & 59 & 59 & $\mathrm{~F}$ & 3.2 & 0.5 & 6.4 & Tremolite & & & \\
\hline G1 & 10 & B4 & $A X$ & 60 & 60 & $\mathrm{~F}$ & 10.6 & 0.8 & 13.2 & Tremolite & & & $\begin{array}{l}\text { AS }>5,3: 1, \text { AFB }>5,3: 1, \\
\text { PCMEF-US, PCMES-US, } \\
\text { PCMEF-ISO, PCMES-ISO }\end{array}$ \\
\hline G1 & 10 & B4 & $A X$ & 61 & 61 & $\mathrm{~F}$ & 4.75 & 0.5 & 9.5 & Tremolite & & & \\
\hline
\end{tabular}

Count Categories

$A F B>5,3: 1$

Asbestos Fibers and Bundles > 5um and 3:1

PAS

PCMEF-US

Primary Asbestos Structures

PCM Equivalent Fibers-NIOSH

PCMES-US
AS>5, 3:1 PCMEF-ISO PCMES-ISO TAS
Asbestos Structures >5um and 3:1 PCM Equivalent Fibers-ISO PCM Equivalent Structures-ISO Total Asbestos Structures 
ISO 10312, Direct Raw Data

Job Number: 070434

SEA

Report Number: 070434R06

Client: Idaho National Laboratory

Date Received: 4/23/2007

Project Name: RARE

Client Sample No: FB-2-R3

Lab/Cor Sample No: S28

Client Description:

Date Sampled:

4/16/2007

\begin{tabular}{|c|c|c|c|c|c|c|c|c|c|c|c|c|c|}
\hline Gr & No. & Loc. & ID & Prim & Tot & Class & Len & Wid & Asp & Analyte & Elements & Comment & Count Categories \\
\hline G1 & 11 & A7 & $A X$ & 62 & 62 & $\mathrm{~F}$ & 4.5 & 1.1 & 4.1 & Tremolite & & & \\
\hline G1 & 11 & A7 & $A X$ & 63 & 63 & $\mathrm{~F}$ & 4.2 & 0.4 & 10.5 & Tremolite & & & \\
\hline G1 & 11 & A7 & $A X$ & 64 & 64 & $\mathrm{~F}$ & 3.8 & 0.25 & 15.2 & Tremolite & & & \\
\hline G1 & 11 & A7 & $A X$ & 65 & 65 & $\mathrm{~F}$ & 2 & 0.65 & 3.1 & Tremolite & & & \\
\hline G1 & 11 & A7 & $A X$ & 66 & 66 & $\mathrm{~F}$ & 2.5 & 0.65 & 3.8 & Tremolite & & & \\
\hline G1 & 11 & A7 & $A X$ & 67 & 67 & $\mathrm{~F}$ & 3 & 0.55 & 5.5 & Tremolite & & & \\
\hline G1 & 11 & A7 & $A X$ & 68 & 68 & $\mathrm{~F}$ & 5 & 0.7 & 7.1 & Tremolite & & & \\
\hline G1 & 12 & $\mathrm{C7}$ & $A X$ & 69 & & CD 2-1 & 14.3 & 1.5 & 9.5 & Tremolite & & & $\begin{array}{c}\text { AS }>5,3: 1, \text { PCMES-US, } \\
\text { PCMES-ISO }\end{array}$ \\
\hline G1 & 12 & C7 & $A X$ & & 69 & CF & 14.3 & 0.6 & 23.8 & Tremolite & & & $\begin{array}{c}\text { AFB >5, 3:1, PCMEF-US, } \\
\text { PCMEF-ISO }\end{array}$ \\
\hline G1 & 12 & $\mathrm{C7}$ & $A X$ & & 70 & CF & 2.1 & 0.6 & 3.5 & Tremolite & & & \\
\hline G1 & 12 & $\mathrm{C7}$ & $A X$ & 70 & & MD 1-1 & 6 & 2.8 & 2.1 & Tremolite & & & $A S>5,3: 1$ \\
\hline G1 & 12 & $\mathrm{C7}$ & $A X$ & & 71 & MF & 6 & 0.85 & 7.1 & Tremolite & & & $\begin{array}{c}\text { AFB >5, 3:1, PCMEF-US, } \\
\text { PCMEF-ISO }\end{array}$ \\
\hline G1 & 12 & C7 & $A X$ & 71 & & MD 1-1 & 11 & 7 & 1.6 & Tremolite & & & $A S>5,3: 1$ \\
\hline G1 & 12 & C7 & $A X$ & & 72 & MB & 9.8 & 1.35 & 7.3 & Tremolite & & & $\begin{array}{c}\text { AFB >5, 3:1, PCMEF-US, } \\
\text { PCMEF-ISO }\end{array}$ \\
\hline G1 & 12 & C7 & $A X$ & 72 & 73 & $F$ & 1.8 & 0.3 & 6 & Tremolite & & & \\
\hline G1 & 12 & $\mathrm{C7}$ & $A X$ & 73 & 74 & $F$ & 1.2 & 0.22 & 5.5 & Tremolite & & & \\
\hline G1 & 13 & E7 & $A D$ & 74 & & MD 1-0 & 3 & 2.5 & 1.2 & Tremolite & & & \\
\hline G1 & 13 & E7 & $A D$ & & 75 & MF & 3 & 0.35 & 8.6 & Tremolite & & & \\
\hline G1 & 13 & E7 & $A D$ & 75 & 76 & $\mathrm{~F}$ & 1.7 & 0.4 & 4.2 & Tremolite & & & \\
\hline G1 & 13 & E7 & $A X$ & 76 & 77 & $\mathrm{~F}$ & 2.65 & 0.5 & 5.3 & Tremolite & & & \\
\hline G1 & 13 & E7 & $A X$ & 77 & 78 & $\mathrm{~F}$ & 5.25 & 0.5 & 10.5 & Tremolite & & & $\begin{array}{l}\text { AS>5, 3:1, AFB }>5,3: 1, \\
\text { PCMEF-US, PCMES-US, } \\
\text { PCMEF-ISO, PCMES-ISO }\end{array}$ \\
\hline G1 & 13 & E7 & $A X$ & 78 & 79 & $\mathrm{~F}$ & 3.35 & 0.38 & 8.8 & Tremolite & & & \\
\hline $\mathrm{G} 1$ & 13 & E7 & $A X$ & 79 & 80 & $F$ & 4 & 0.7 & 5.7 & Tremolite & & & \\
\hline G1 & 13 & E7 & $A X$ & 80 & 81 & $\mathrm{~F}$ & 2 & 0.65 & 3.1 & Tremolite & & & \\
\hline G1 & 13 & E7 & $A X$ & 81 & 82 & $\mathrm{~F}$ & 2.7 & 0.75 & 3.6 & Tremolite & & & \\
\hline G1 & 14 & G7 & $A X$ & 82 & 83 & $\mathrm{~F}$ & 9.5 & 1.2 & 7.9 & Tremolite & & & $\begin{array}{l}\text { AS }>5,3: 1, \text { AFB }>5,3: 1, \\
\text { PCMEF-US, PCMES-US, } \\
\text { PCMEF-ISO, PCMES-ISO }\end{array}$ \\
\hline G1 & 14 & G7 & $A X$ & 83 & 84 & $\mathrm{~F}$ & 2.2 & 0.7 & 3.1 & Tremolite & & & \\
\hline G1 & 14 & G7 & $A X$ & 84 & 85 & $\mathrm{~F}$ & 8 & 1.25 & 6.4 & Tremolite & & & $\begin{array}{l}\text { AS>5, 3:1, AFB }>5,3: 1 \text {, } \\
\text { PCMEF-US, PCMES-US, } \\
\text { PCMEF-ISO, PCMES-ISO }\end{array}$ \\
\hline G1 & 14 & G7 & $A X$ & 85 & 86 & $\mathrm{~F}$ & 25 & 6.5 & 3.8 & Tremolite & & & PCMES-US, PCMEF-US \\
\hline
\end{tabular}

Count Categories

AFB $>5,3: 1$

PAS

PCMEF-US

Asbestos Fibers and Bundles > 5um and 3:1

Primary Asbestos Structures

PCM Equivalent Fibers-NIOSH

PCMES-US
AS>5, 3:1 PCMEF-ISO PCMES-ISO TAS
Asbestos Structures $>5$ um and 3:1 PCM Equivalent Fibers-ISO PCM Equivalent Structures-ISO Total Asbestos Structures

Page 102 of 281 
ISO 10312, Direct Raw Data

Job Number: 070434

SEA

Report Number: 070434R06

Client: Idaho National Laboratory

Date Received: 4/23/2007

Project Name: RARE

Client Sample No: FB-2-R3

Lab/Cor Sample No: S28

Client Description:

Date Sampled:

4/16/2007

\begin{tabular}{|c|c|c|c|c|c|c|c|c|c|c|c|c|c|}
\hline Gr & No. & Loc. & ID & Prim & Tot & Class & Len & Wid & Asp & Analyte & Elements & Comment & Count Categories \\
\hline G1 & 15 & 17 & $A D$ & 86 & 87 & $\mathrm{~F}$ & 3.35 & 0.85 & 3.9 & Tremolite & & & \\
\hline G1 & 15 & 17 & $A D$ & 87 & 88 & $\mathrm{~F}$ & 3.4 & 0.78 & 4.4 & Tremolite & & & \\
\hline G1 & 15 & 17 & $A X$ & 88 & 89 & $\mathrm{~F}$ & 5.5 & 0.65 & 8.5 & Tremolite & & & $\begin{array}{l}\text { AS }>5,3: 1, \text { AFB }>5,3: 1, \\
\text { PCMEF-US, PCMES-US, } \\
\text { PCMEF-ISO, PCMES-ISO }\end{array}$ \\
\hline G1 & 15 & 17 & $A X$ & 89 & 90 & $\mathrm{~F}$ & 5.3 & 0.35 & 15.1 & Tremolite & & & $\begin{array}{l}\text { AS }>5,3: 1, \text { AFB }>5,3: 1, \\
\text { PCMEF-US, PCMES-US, } \\
\text { PCMEF-ISO, PCMES-ISO }\end{array}$ \\
\hline G1 & 15 & 17 & $A X$ & 90 & 91 & $\mathrm{~F}$ & 6.2 & 1.1 & 5.6 & Tremolite & & & $\begin{array}{l}\text { AFB }>5,3: 1, \text { AS }>5,3: 1, \\
\text { PCMEF-US, PCMES-US, } \\
\text { PCMEF-ISO, PCMES-ISO }\end{array}$ \\
\hline G1 & 15 & 17 & $A X$ & 91 & 92 & $\mathrm{~F}$ & 9.2 & 0.8 & 11.5 & Tremolite & & & $\begin{array}{l}\text { AS }>5,3: 1, \text { AFB }>5,3: 1 \text {, } \\
\text { PCMEF-US, PCMES-US, } \\
\text { PCMEF-ISO, PCMES-ISO }\end{array}$ \\
\hline G1 & 16 & J9 & $A X$ & 92 & & MD 1-0 & 2.25 & 0.8 & 2.8 & Tremolite & & & \\
\hline G1 & 16 & J9 & $A X$ & & 93 & MF & 1.2 & 0.22 & 5.5 & Tremolite & & & \\
\hline G1 & 16 & J9 & $A X$ & 93 & 94 & $\mathrm{~F}$ & 4.35 & 0.65 & 6.7 & Tremolite & & & \\
\hline G1 & 16 & J9 & $A X$ & 94 & 95 & $F$ & 1.75 & 0.25 & 7 & Tremolite & & & \\
\hline G1 & 16 & J9 & $A X$ & 95 & 96 & $F$ & 12.5 & 1.75 & 7.1 & Tremolite & & & $\begin{array}{l}\text { AS }>5,3: 1, \text { AFB }>5,3: 1, \\
\text { PCMEF-US, PCMES-US, } \\
\text { PCMEF-ISO, PCMES-ISO }\end{array}$ \\
\hline G1 & 17 & $\mathrm{H} 9$ & $A X$ & 96 & 97 & $\mathrm{~F}$ & 3.2 & 0.47 & 6.8 & Tremolite & & & \\
\hline G1 & 17 & $\mathrm{H} 9$ & $A X$ & 97 & 98 & $F$ & 2.6 & 0.55 & 4.7 & Tremolite & & & \\
\hline G1 & 17 & $\mathrm{H} 9$ & $A X$ & 98 & 99 & $F$ & 1.65 & 0.4 & 4.1 & Tremolite & & & \\
\hline G1 & 17 & $\mathrm{H} 9$ & $A X$ & 99 & 100 & $\mathrm{~F}$ & 11 & 1.25 & 8.8 & Tremolite & & & $\begin{array}{l}\text { AS>5, 3:1, AFB }>5,3: 1, \\
\text { PCMEF-US, PCMES-US, } \\
\text { PCMEF-ISO, PCMES-ISO }\end{array}$ \\
\hline G1 & 17 & $\mathrm{H} 9$ & $A X$ & 100 & 101 & $\mathrm{~F}$ & 2.2 & 0.55 & 4 & Tremolite & & & \\
\hline G1 & 17 & $\mathrm{H} 9$ & $A X$ & 101 & 102 & $F$ & 2.5 & 0.4 & 6.2 & Tremolite & & & \\
\hline G1 & 17 & $\mathrm{H} 9$ & $A X$ & 102 & & MD 1-1 & 11 & 10 & 1.1 & Tremolite & & & $A S>5,3: 1$ \\
\hline G1 & 17 & $\mathrm{H} 9$ & $A X$ & & 103 & MF & 6.5 & 1.2 & 5.4 & Tremolite & & & $\begin{array}{c}\text { AFB }>5,3: 1, \text { PCMEF-US, } \\
\text { PCMEF-ISO }\end{array}$ \\
\hline G1 & 17 & $\mathrm{H} 9$ & $A X$ & 103 & 104 & $\mathrm{~F}$ & 2.65 & 0.85 & 3.1 & Tremolite & & & \\
\hline G1 & 17 & $\mathrm{H} 9$ & $A X$ & 104 & 105 & $F$ & 1.25 & 0.18 & 6.9 & Tremolite & & & \\
\hline G1 & 17 & $\mathrm{H} 9$ & $A X$ & 105 & 106 & $F$ & 3.85 & 0.33 & 11.7 & Tremolite & & & \\
\hline G1 & 17 & $\mathrm{H} 9$ & $A X$ & 106 & 107 & B & 2.5 & 0.75 & 3.3 & Tremolite & & & \\
\hline G1 & 18 & F9 & $A X$ & 107 & 108 & $\mathrm{~F}$ & 55 & 2.2 & 25 & Tremolite & & & $\begin{array}{l}\text { AS }>5,3: 1, \text { AFB }>5,3: 1, \\
\text { PCMEF-US, PCMES-US, } \\
\text { PCMEF-ISO, PCMES-ISO }\end{array}$ \\
\hline G1 & 18 & F9 & $A X$ & 108 & 109 & $F$ & 3 & 0.55 & 5.5 & Tremolite & & & \\
\hline G1 & 19 & D9 & $A X$ & 109 & & MD 1-0 & 3.5 & 3 & 1.2 & Tremolite & & & \\
\hline
\end{tabular}

Count Categories

$\mathrm{AFB}>5,3: 1$

PAS

PCMEF-US

Asbestos Fibers and Bundles > 5um and 3:1

Primary Asbestos Structures

PCM Equivalent Fibers-NIOSH

PCMES-US
AS $>5,3: 1$ PCMEF-ISO PCMES-ISO TAS
Asbestos Structures $>5$ um and 3:1 PCM Equivalent Fibers-ISO PCM Equivalent Structures-ISO Total Asbestos Structures

Page 103 of 281 
ISO 10312, Direct Raw Data

Job Number: 070434

SEA

Report Number: 070434R06

Client: Idaho National Laboratory

Date Received: 4/23/2007

Project Name: RARE

Client Sample No: FB-2-R3

Lab/Cor Sample No: S28

Client Description:

Date Sampled:

4/16/2007

\begin{tabular}{|c|c|c|c|c|c|c|c|c|c|c|c|c|c|}
\hline Gr & No. & Loc. & ID & Prim & Tot & Class & Len & Wid & Asp & Analyte & Elements & Comment & Count Categories \\
\hline G1 & 19 & D9 & $A X$ & & 110 & MF & 3.5 & 0.4 & 8.8 & Tremolite & & & \\
\hline G1 & 19 & D9 & $A X$ & 110 & 111 & $\mathrm{~F}$ & 1.85 & 0.38 & 4.9 & Tremolite & & & \\
\hline G1 & 19 & D9 & $A X$ & 111 & 112 & $\mathrm{~F}$ & 2.5 & 0.6 & 4.2 & Tremolite & & & \\
\hline G1 & 20 & B9 & $A X$ & 112 & & MD 1-0 & 11 & 9 & 1.2 & Tremolite & & & $A S>5,3: 1$ \\
\hline G1 & 20 & B9 & $A X$ & & 113 & MF & 2.99 & 0.3 & 10 & Tremolite & & & \\
\hline G1 & 20 & B9 & $A X$ & 113 & 114 & $\mathrm{~F}$ & 3.2 & 0.9 & 3.6 & Tremolite & & & \\
\hline G1 & 20 & B9 & $A X$ & 114 & 115 & $\mathrm{~F}$ & 2.9 & 0.3 & 9.7 & Tremolite & & & \\
\hline G1 & 20 & B9 & $A X$ & 115 & 116 & $\mathrm{~F}$ & 2.6 & 0.4 & 6.5 & Tremolite & & & \\
\hline G1 & 20 & B9 & $A X$ & 116 & 117 & $\mathrm{~F}$ & 2.7 & 0.8 & 3.4 & Tremolite & & & \\
\hline G2 & 21 & $\mathrm{~A} 2$ & $A X$ & 117 & & MD 1-0 & 9 & 3 & 3 & Tremolite & & & $\begin{array}{c}\text { AS }>5,3: 1, \text { PCMES-US, } \\
\text { PCMES-ISO }\end{array}$ \\
\hline G2 & 21 & $\mathrm{~A} 2$ & $A X$ & & 118 & MB & 3.5 & 1 & 3.5 & Tremolite & & & \\
\hline G2 & 21 & $\mathrm{~A} 2$ & $A X$ & 118 & 119 & $\mathrm{~F}$ & 1.85 & 0.25 & 7.4 & Tremolite & & & \\
\hline G2 & 21 & $\mathrm{~A} 2$ & $A X$ & 119 & 120 & $\mathrm{~F}$ & 10 & 0.6 & 16.7 & Tremolite & & & $\begin{array}{l}\text { AS>5, 3:1, AFB }>5,3: 1 \text {, } \\
\text { PCMEF-US, PCMES-US, } \\
\text { PCMEF-ISO, PCMES-ISO }\end{array}$ \\
\hline G2 & 21 & $\mathrm{~A} 2$ & $A X$ & 120 & 121 & B & 4.25 & 1.25 & 3.4 & Tremolite & & & \\
\hline G2 & 21 & $\mathrm{~A} 2$ & $A X$ & 121 & 122 & $\mathrm{~F}$ & 2.7 & 0.4 & 6.8 & Tremolite & & & \\
\hline G2 & 21 & $\mathrm{~A} 2$ & $A X$ & 122 & 123 & $\mathrm{~F}$ & 1.75 & 0.35 & 5 & Tremolite & & & \\
\hline G2 & 22 & $\mathrm{C} 2$ & $A X$ & 123 & 124 & $\mathrm{~F}$ & 4.1 & 0.8 & 5.1 & Tremolite & & & \\
\hline G2 & 22 & $\mathrm{C} 2$ & $A X$ & 124 & 125 & $\mathrm{~F}$ & 9 & 1 & 9 & Tremolite & & & $\begin{array}{l}\text { AS>5, 3:1, AFB>5, 3:1, } \\
\text { PCMEF-US, PCMES-US, } \\
\text { PCMEF-ISO, PCMES-ISO }\end{array}$ \\
\hline G2 & 22 & $\mathrm{C} 2$ & $A X$ & 125 & 126 & $\mathrm{~F}$ & 5.4 & 0.6 & 9 & Tremolite & & & $\begin{array}{l}\text { AS>5, 3:1, AFB>5, 3:1, } \\
\text { PCMEF-US, PCMES-US, } \\
\text { PCMEF-ISO, PCMES-ISO }\end{array}$ \\
\hline G2 & 22 & $\mathrm{C} 2$ & $A X$ & 126 & 127 & $\mathrm{~F}$ & 2.6 & 0.2 & 13 & Tremolite & & & \\
\hline G2 & 22 & $\mathrm{C} 2$ & $A X$ & 127 & 128 & $\mathrm{~F}$ & 4.75 & 1.5 & 3.2 & Tremolite & & & \\
\hline G2 & 22 & $\mathrm{C} 2$ & $A X$ & 128 & 129 & $\mathrm{~F}$ & 7.7 & 0.45 & 17.1 & Tremolite & & & $\begin{array}{l}\text { AS }>5,3: 1, \text { AFB }>5,3: 1, \\
\text { PCMEF-US, PCMES-US, } \\
\text { PCMEF-ISO, PCMES-ISO }\end{array}$ \\
\hline G2 & 22 & $\mathrm{C} 2$ & $A X$ & 129 & 130 & $\mathrm{~F}$ & 15 & 2.5 & 6 & Tremolite & & & $\begin{array}{l}\text { AS }>5,3: 1, \text { AFB }>5,3: 1, \\
\text { PCMEF-US, PCMES-US, } \\
\text { PCMEF-ISO, PCMES-ISO }\end{array}$ \\
\hline G2 & 22 & $\mathrm{C} 2$ & $A X$ & 130 & 131 & $\mathrm{~F}$ & 2.75 & 0.6 & 4.6 & Tremolite & & & \\
\hline G2 & 22 & $\mathrm{C} 2$ & $A X$ & 131 & 132 & $\mathrm{~F}$ & 5.12 & 0.45 & 11.4 & Tremolite & & & $\begin{array}{l}\text { AS>5, 3:1, AFB>5, 3:1, } \\
\text { PCMEF-US, PCMES-US, } \\
\text { PCMEF-ISO, PCMES-ISO }\end{array}$ \\
\hline G2 & 22 & $\mathrm{C} 2$ & $A X$ & 132 & 133 & $\mathrm{~F}$ & 4.2 & 0.6 & 7 & Tremolite & & & \\
\hline G2 & 22 & $\mathrm{C} 2$ & $A X$ & 133 & 134 & $\mathrm{~F}$ & 12 & 2.5 & 4.8 & Tremolite & & & $\begin{array}{l}\text { PCMEF-US, PCMES-US, } \\
\text { PCMEF-ISO, PCMES-ISO }\end{array}$ \\
\hline
\end{tabular}

Count Categories

$\mathrm{AFB}>5,3: 1$

PAS

PCMEF-US

Asbestos Fibers and Bundles $>$ 5um and 3:1

Primary Asbestos Structures

PCM Equivalent Fibers-NIOSH

PCMES-US
AS>5, 3:1 PCMEF-ISO PCMES-ISO TAS
Asbestos Structures $>5$ um and 3:1 PCM Equivalent Fibers-ISO PCM Equivalent Structures-ISO Total Asbestos Structures 
ISO 10312, Direct Raw Data

Job Number: 070434

SEA

Report Number: 070434R06

Client: Idaho National Laboratory

Date Received: 4/23/2007

Project Name: RARE

Client Sample No: FB-2-R3

Lab/Cor Sample No: S28

Client Description:

Date Sampled:

4/16/2007

\begin{tabular}{|c|c|c|c|c|c|c|c|c|c|c|c|c|c|}
\hline $\mathbf{G r}$ & No. & Loc. & ID & Prim & Tot & Class & Len & Wid & Asp & Analyte & Elements & Comment & Count Categories \\
\hline G2 & 22 & $\mathrm{C} 2$ & $A X$ & 134 & 135 & $\mathrm{~F}$ & 1.75 & 0.35 & 5 & Tremolite & & & \\
\hline G2 & 23 & E2 & $A X$ & 135 & & MD 1-0 & 3 & 1.2 & 2.5 & Tremolite & & & \\
\hline G2 & 23 & E2 & $A X$ & & 136 & MF & 1.25 & 0.35 & 3.6 & Tremolite & & & \\
\hline G2 & 23 & E2 & $A X$ & 136 & & MD 1-0 & 4 & 3.8 & 1.1 & Tremolite & & & \\
\hline G2 & 23 & E2 & $A X$ & & 137 & MF & 3.1 & 0.4 & 7.7 & Tremolite & & & \\
\hline G2 & 23 & E2 & $A X$ & 137 & 138 & $\mathrm{~F}$ & 5.25 & 1.35 & 3.9 & Tremolite & & & $\begin{array}{l}\text { PCMEF-US, PCMES-US, } \\
\text { PCMEF-ISO, PCMES-ISO }\end{array}$ \\
\hline G2 & 23 & E2 & $A X$ & 138 & & MD 1-1 & 20 & 12 & 1.7 & Tremolite & & & $A S>5,3: 1$ \\
\hline G2 & 23 & E2 & $A X$ & & 139 & MF & 6 & 0.3 & 20 & Tremolite & & & $\begin{array}{c}\text { AFB }>5,3: 1, \text { PCMEF-US, } \\
\text { PCMEF-ISO }\end{array}$ \\
\hline G2 & 23 & E2 & $A X$ & 139 & 140 & $\mathrm{~F}$ & 2.9 & 0.55 & 5.3 & Tremolite & & & \\
\hline G2 & 23 & E2 & $A X$ & 140 & 141 & $F$ & 3 & 0.33 & 9.1 & Tremolite & & & \\
\hline G2 & 23 & E2 & $A X$ & 141 & 142 & $F$ & 3 & 0.56 & 5.4 & Tremolite & & & \\
\hline G2 & 23 & E2 & $A X$ & 142 & 143 & $F$ & 15 & 1.8 & 8.3 & Tremolite & & & $\begin{array}{l}\text { AS >5, 3:1, AFB>5, 3:1, } \\
\text { PCMEF-US, PCMES-US, } \\
\text { PCMEF-ISO, PCMES-ISO }\end{array}$ \\
\hline G2 & 24 & G2 & $A X$ & 143 & & MD 1-0 & 6.5 & 5.8 & 1.1 & Tremolite & & & $A S>5,3: 1$ \\
\hline G2 & 24 & G2 & $A X$ & & 144 & MF & 3.8 & 0.7 & 5.4 & Tremolite & & & \\
\hline G2 & 24 & G2 & $A X$ & 144 & 145 & $\mathrm{~F}$ & 11.85 & 0.885 & 13.4 & Tremolite & & & $\begin{array}{l}\text { AS }>5,3: 1, \text { AFB }>5,3: 1, \\
\text { PCMEF-US, PCMES-US, } \\
\text { PCMEF-ISO, PCMES-ISO }\end{array}$ \\
\hline G2 & 24 & G2 & $A X$ & 145 & 146 & $\mathrm{~F}$ & 16.8 & 0.38 & 44.2 & Tremolite & & & $\begin{array}{l}\text { AS }>5,3: 1, \text { AFB }>5,3: 1, \\
\text { PCMEF-US, PCMES-US, } \\
\text { PCMEF-ISO, PCMES-ISO }\end{array}$ \\
\hline G2 & 25 & 12 & $A X$ & 146 & 147 & $\mathrm{~F}$ & 5 & 0.7 & 7.1 & Tremolite & & & \\
\hline G2 & 25 & 12 & $A X$ & 147 & 148 & $F$ & 1 & 0.25 & 4 & Tremolite & & & \\
\hline G2 & 25 & 12 & $A X$ & 148 & 149 & $F$ & 9.5 & 1.2 & 7.9 & Tremolite & & & $\begin{array}{l}\text { AS }>5,3: 1, \text { AFB }>5,3: 1, \\
\text { PCMEF-US, PCMES-US, } \\
\text { PCMEF-ISO, PCMES-ISO }\end{array}$ \\
\hline G2 & 25 & 12 & $A X$ & 149 & 150 & $F$ & 2.65 & 0.5 & 5.3 & Tremolite & & & \\
\hline G2 & 25 & 12 & $A X$ & 150 & 151 & $\mathrm{~F}$ & 7.2 & 0.85 & 8.5 & Tremolite & & & $\begin{array}{l}\text { AS }>5,3: 1, \text { AFB }>5,3: 1, \\
\text { PCMEF-US, PCMES-US, } \\
\text { PCMEF-ISO, PCMES-ISO }\end{array}$ \\
\hline G2 & 25 & 12 & $A X$ & 151 & 152 & $\mathrm{~F}$ & 10 & 1.7 & 5.9 & Tremolite & & & $\begin{array}{l}\text { AS }>5,3: 1, \text { AFB }>5,3: 1, \\
\text { PCMEF-US, PCMES-US, } \\
\text { PCMES-ISO, PCMEF-ISO }\end{array}$ \\
\hline G2 & 25 & 12 & $A X$ & 152 & 153 & $\mathrm{~F}$ & 1.7 & 0.4 & 4.2 & Tremolite & & & \\
\hline G2 & 25 & 12 & $A X$ & 153 & 154 & $\mathrm{~F}$ & 3.2 & 0.55 & 5.8 & Tremolite & & & \\
\hline G2 & 25 & 12 & $A X$ & 154 & 155 & $F$ & 9 & 3 & 3 & Tremolite & & & $\begin{array}{l}\text { PCMEF-US, PCMES-US, } \\
\text { PCMEF-ISO, PCMES-ISO }\end{array}$ \\
\hline G2 & 25 & 12 & $A X$ & 155 & 156 & $F$ & 1.25 & 0.2 & 6.2 & Tremolite & & & \\
\hline
\end{tabular}

\section{Count Categories}

$\mathrm{AFB}>5,3: 1$

PAS

PCMEF-US

Asbestos Fibers and Bundles > 5um and 3:

Primary Asbestos Structures

PCM Equivalent Fibers-NIOSH

PCMES-US
AS $>5,3: 1$ PCMEF-ISO PCMES-ISO TAS
Asbestos Structures $>5$ um and 3:1 PCM Equivalent Fibers-ISO PCM Equivalent Structures-ISO Total Asbestos Structures 
ISO 10312, Direct Raw Data

Job Number: 070434

SEA

Report Number: 070434R06

Client: Idaho National Laboratory

Date Received: 4/23/2007

Project Name: RARE

Client Sample No: FB-2-R3

Lab/Cor Sample No: S28

Client Description:

Date Sampled:

4/16/2007

\begin{tabular}{|c|c|c|c|c|c|c|c|c|c|c|c|c|c|}
\hline Gr & No. & Loc. & ID & Prim & Tot & Class & Len & Wid & Asp & Analyte & Elements & Comment & Count Categories \\
\hline G2 & 25 & 12 & $A X$ & 156 & 157 & $\mathrm{~F}$ & 5.25 & 0.75 & 7 & Tremolite & & & $\begin{array}{l}\text { AS }>5,3: 1, \text { AFB }>5,3: 1, \\
\text { PCMEF-US, PCMES-US, } \\
\text { PCMEF-ISO, PCMES-ISO }\end{array}$ \\
\hline G2 & 25 & 12 & $A X$ & 157 & 158 & $\mathrm{~F}$ & 4 & 0.5 & 8 & Tremolite & & & \\
\hline G2 & 26 & J4 & $A X$ & 158 & 159 & $\mathrm{~F}$ & 5.7 & 1.2 & 4.7 & Tremolite & & & $\begin{array}{l}\text { PCMEF-US, PCMES-US, } \\
\text { PCMEF-ISO, PCMES-ISO }\end{array}$ \\
\hline G2 & 26 & J4 & $A X$ & 159 & 160 & $\mathrm{~F}$ & 9 & 0.3 & 30 & Tremolite & & & $\begin{array}{l}\text { AS >5, 3:1, AFB >5, 3:1, } \\
\text { PCMEF-US, PCMES-US, } \\
\text { PCMEF-ISO, PCMES-ISO }\end{array}$ \\
\hline G2 & 26 & J4 & $A X$ & 160 & 161 & $\mathrm{~F}$ & 6.2 & 1.12 & 5.5 & Tremolite & & & $\begin{array}{l}\text { AS }>5,3: 1, \text { AFB }>5,3: 1, \\
\text { PCMEF-US, PCMES-US, } \\
\text { PCMEF-ISO, PCMES-ISO }\end{array}$ \\
\hline G2 & 26 & J4 & $A X$ & 161 & 162 & $\mathrm{~F}$ & 4 & 0.65 & 6.2 & Tremolite & & & \\
\hline G2 & 26 & J4 & $A X$ & 162 & & MD 1-0 & 1.8 & 0.6 & 3 & Tremolite & & & \\
\hline G2 & 26 & J4 & $A X$ & & 163 & MF & 1.55 & 0.2 & 7.7 & Tremolite & & & \\
\hline G2 & 27 & $\mathrm{H} 4$ & $A X$ & 163 & 164 & $\mathrm{~F}$ & 8 & 1.2 & 6.7 & Tremolite & & & $\begin{array}{l}\text { AS }>5,3: 1, \text { AFB }>5,3: 1, \\
\text { PCMEF-US, PCMES-US, } \\
\text { PCMEF-ISO, PCMES-ISO }\end{array}$ \\
\hline G2 & 27 & $\mathrm{H} 4$ & $A X$ & 164 & & MD 1-0 & 7 & 2.8 & 2.5 & Tremolite & & & $A S>5,3: 1$ \\
\hline G2 & 27 & $\mathrm{H} 4$ & $A X$ & & 165 & MF & 3.5 & 0.5 & 7 & Tremolite & & & \\
\hline G2 & 27 & $\mathrm{H} 4$ & $A X$ & 165 & 166 & $F$ & 2.2 & 0.7 & 3.1 & Tremolite & & & \\
\hline G2 & 27 & $\mathrm{H} 4$ & $A X$ & 166 & 167 & $F$ & 3.12 & 0.65 & 4.8 & Tremolite & & & \\
\hline G2 & 27 & $\mathrm{H} 4$ & $A X$ & 167 & 168 & $\mathrm{~F}$ & 7.5 & 0.55 & 13.6 & Tremolite & & & $\begin{array}{l}\text { AFB>5, 3:1, AS>5, 3:1, } \\
\text { PCMEF-US, PCMES-US, } \\
\text { PCMEF-ISO, PCMES-ISO }\end{array}$ \\
\hline G2 & 27 & $\mathrm{H} 4$ & $A X$ & 168 & 169 & $F$ & 1.65 & 0.38 & 4.3 & Tremolite & & & \\
\hline G2 & 28 & F4 & $A X$ & 169 & 170 & $\mathrm{~F}$ & 20 & 0.85 & 23.5 & Tremolite & & & $\begin{array}{l}\text { AS }>5,3: 1, \text { AFB }>5,3: 1 \text {, } \\
\text { PCMEF-US, PCMES-US, } \\
\text { PCMEF-ISO, PCMES-ISO }\end{array}$ \\
\hline G2 & 28 & $\mathrm{~F} 4$ & $A X$ & 170 & 171 & $\mathrm{~F}$ & 5.5 & 0.8 & 6.9 & Tremolite & & & $\begin{array}{l}\text { AS }>5,3: 1, \text { AFB }>5,3: 1, \\
\text { PCMEF-US, PCMES-US, } \\
\text { PCMEF-ISO, PCMES-ISO }\end{array}$ \\
\hline G2 & 28 & F4 & $A X$ & 171 & 172 & $F$ & 4.8 & 0.5 & 9.6 & Tremolite & & & \\
\hline G2 & 28 & $\mathrm{~F} 4$ & $A X$ & 172 & 173 & $F$ & 7 & 1.5 & 4.7 & Tremolite & & & $\begin{array}{l}\text { PCMEF-US, PCMES-US, } \\
\text { PCMEF-ISO, PCMES-ISO }\end{array}$ \\
\hline G2 & 28 & F4 & $A X$ & 173 & 174 & $\mathrm{~F}$ & 1.25 & 0.35 & 3.6 & Tremolite & & & \\
\hline G2 & 29 & D4 & $A X$ & 174 & 175 & $F$ & 9.3 & 2.35 & 4 & Tremolite & & & $\begin{array}{l}\text { PCMEF-US, PCMES-US, } \\
\text { PCMEF-ISO, PCMES-ISO }\end{array}$ \\
\hline G2 & 29 & D4 & $A X$ & 175 & 176 & $\mathrm{~F}$ & 12.2 & 1.2 & 10.2 & Tremolite & & & $\begin{array}{l}\text { AS>5, 3:1, AFB>5, 3:1, } \\
\text { PCMEF-US, PCMES-US, } \\
\text { PCMEF-ISO, PCMES-ISO }\end{array}$ \\
\hline G2 & 29 & D4 & $A X$ & 176 & 177 & $\mathrm{~F}$ & 1.25 & 0.2 & 6.2 & Tremolite & & & \\
\hline
\end{tabular}

Count Categories

AFB $>5,3: 1$

PAS

PCMEF-US

Asbestos Fibers and Bundles > 5um and 3:1

Primary Asbestos Structures

PCM Equivalent Fibers-NIOSH

PCMES-US
AS>5, 3:1 PCMEF-ISO PCMES-ISO TAS
Asbestos Structures $>5$ um and 3:1 PCM Equivalent Fibers-ISO PCM Equivalent Structures-ISO Total Asbestos Structures 
ISO 10312, Direct Raw Data

Job Number: 070434

SEA

Report Number: 070434R06

Client: Idaho National Laboratory

Date Received: 4/23/2007

Project Name: RARE

Client Sample No: FB-2-R3

Lab/Cor Sample No: S28

Client Description:

Date Sampled:

4/16/2007

\begin{tabular}{|c|c|c|c|c|c|c|c|c|c|c|c|c|c|}
\hline$\overline{\mathrm{Gr}}$ & No. & Loc. & IID & Prim & Tot & "Class & Len & Wid & Asp & Analyte & Elements & Comment & Count Categories \\
\hline G2 & 29 & D4 & $A X$ & 177 & 178 & $\mathrm{~F}$ & 8 & 1.2 & 6.7 & Tremolite & & & $\begin{array}{l}\text { AS }>5,3: 1, \text { AFB }>5,3: 1, \\
\text { PCMEF-US, PCMES-US, } \\
\text { PCMEF-ISO, PCMES-ISO }\end{array}$ \\
\hline G2 & 29 & D4 & $A X$ & 178 & 179 & $\mathrm{~F}$ & 3.9 & 1.2 & 3.2 & Tremolite & & & \\
\hline G2 & 29 & D4 & $A X$ & 179 & 180 & $\mathrm{~F}$ & 1.35 & 0.4 & 3.4 & Tremolite & & & \\
\hline G2 & 30 & B4 & $A X$ & 180 & 181 & $\mathrm{~F}$ & 10.65 & 1.5 & 7.1 & Tremolite & & & $\begin{array}{l}\text { AS }>5,3: 1, \text { AFB }>5,3: 1, \\
\text { PCMEF-US, PCMES-US, } \\
\text { PCMEF-ISO, PCMES-ISO }\end{array}$ \\
\hline G2 & 30 & B4 & $A X$ & 181 & 182 & $\mathrm{~F}$ & 2.7 & 0.35 & 7.7 & Tremolite & & & \\
\hline G2 & 30 & B4 & $A X$ & 182 & 183 & $\mathrm{~F}$ & 5.2 & 1.75 & 3 & Tremolite & & & \\
\hline G2 & 31 & A7 & $A X$ & 183 & 184 & $\mathrm{~F}$ & 7.15 & 1.1 & 6.5 & Tremolite & & & $\begin{array}{l}\text { AS }>5,3: 1, \text { AFB }>5,3: 1, \\
\text { PCMEF-US, PCMES-US, } \\
\text { PCMEF-ISO, PCMES-ISO }\end{array}$ \\
\hline G2 & 31 & A7 & $A X$ & 184 & 185 & $F$ & 3.8 & 0.5 & 7.6 & Tremolite & & & \\
\hline G2 & 31 & A7 & $A X$ & 185 & 186 & $\mathrm{~F}$ & 3.5 & 0.6 & 5.8 & Tremolite & & & \\
\hline G2 & 31 & A7 & $A X$ & 186 & 187 & $\mathrm{~F}$ & 19 & 1.5 & 12.7 & Tremolite & & & $\begin{array}{l}\text { AS }>5,3: 1, \text { AFB }>5,3: 1, \\
\text { PCMEF-US, PCMES-US, } \\
\text { PCMEF-ISO, PCMES-ISO }\end{array}$ \\
\hline G2 & 31 & A7 & $A X$ & 187 & 188 & $\mathrm{~F}$ & 10 & 1.8 & 5.6 & Tremolite & & & $\begin{array}{l}\text { AS>5, 3:1, AFB>5, 3:1, } \\
\text { PCMEF-US, PCMES-US, } \\
\text { PCMEF-ISO, PCMES-ISO }\end{array}$ \\
\hline G2 & 31 & A7 & $A X$ & 188 & 189 & $\mathrm{~F}$ & 10.1 & 1.25 & 8.1 & Tremolite & & & $\begin{array}{l}\text { AS }>5,3: 1, \text { AFB }>5,3: 1, \\
\text { PCMEF-US, PCMES-US, } \\
\text { PCMEF-ISO, PCMES-ISO }\end{array}$ \\
\hline G2 & 31 & A7 & $A X$ & 189 & 190 & $F$ & 30 & 3 & 10 & Tremolite & & & $\begin{array}{l}\text { AS }>5,3: 1, \text { AFB }>5,3: 1, \\
\text { PCMEF-US, PCMES-US, } \\
\text { PCMEF-ISO, PCMES-ISO }\end{array}$ \\
\hline G2 & 32 & C7 & $A X$ & 190 & 191 & $\mathrm{~F}$ & 4.85 & 0.55 & 8.8 & Tremolite & & & \\
\hline G2 & 32 & C7 & $A X$ & 191 & 192 & $\mathrm{~F}$ & 4.35 & 0.85 & 5.1 & Tremolite & & & \\
\hline G2 & 33 & E7 & $A X$ & 192 & 193 & $\mathrm{~F}$ & 3 & 0.4 & 7.5 & Tremolite & & & \\
\hline G2 & 33 & E7 & $A X$ & 193 & 194 & $\mathrm{~F}$ & 3 & 0.5 & 6 & Tremolite & & & \\
\hline G2 & 33 & E7 & $A X$ & 194 & 195 & $\mathrm{~F}$ & 9.2 & 0.6 & 15.3 & Tremolite & & & $\begin{array}{l}\text { AS>5, 3:1, AFB>5, 3:1, } \\
\text { PCMEF-US, PCMES-US, } \\
\text { PCMEF-ISO, PCMES-ISO }\end{array}$ \\
\hline G2 & 33 & E7 & $A X$ & 195 & 196 & $\mathrm{~F}$ & 14 & 1.2 & 11.7 & Tremolite & & & $\begin{array}{l}\text { AS }>5,3: 1, \text { AFB }>5,3: 1, \\
\text { PCMEF-US, PCMES-US, } \\
\text { PCMEF-ISO, PCMES-ISO }\end{array}$ \\
\hline G2 & 33 & E7 & $A X$ & 196 & 197 & $\mathrm{~F}$ & 12 & 4 & 3 & Tremolite & & & PCMEF-US, PCMES-US \\
\hline G2 & 33 & E7 & $A X$ & 197 & & MD 1-1 & 6.8 & 5.5 & 1.2 & Tremolite & & & $A S>5,3: 1$ \\
\hline G2 & 33 & E7 & $A X$ & & 198 & MF & 5.1 & 0.27 & 18.9 & Tremolite & & & $\begin{array}{c}\text { AFB }>5,3: 1, \text { PCMEF-US, } \\
\text { PCMEF-ISO }\end{array}$ \\
\hline G2 & 33 & E7 & $A X$ & 198 & 199 & $\mathrm{~F}$ & 7 & 0.8 & 8.8 & Tremolite & & & $\begin{array}{l}\text { AS>5, 3:1, AFB>5, 3:1, } \\
\text { PCMEF-US, PCMES-US, } \\
\text { PCMEF-ISO, PCMES-ISO }\end{array}$ \\
\hline
\end{tabular}

Count Categories

AFB $>5,3: 1$

PAS

PCMEF-US

Asbestos Fibers and Bundles > 5um and 3:

Primary Asbestos Structures

PCM Equivalent Fibers-NIOSH

PCMES-US
AS $>5,3: 1$ PCMEF-ISO PCMES-ISO TAS
Asbestos Structures $>5$ um and 3:1 PCM Equivalent Fibers-ISO PCM Equivalent Structures-ISO Total Asbestos Structures 
Job Number: 070434

SEA

ISO 10312, Direct Raw Data

Client: Idaho National Laboratory

Report Number: 070434R06

Project Name: RARE

Date Received: 4/23/2007

Client Sample No: FB-2-R3

Lab/Cor Sample No: S28

Client Description:

Date Sampled:

4/16/2007

\begin{tabular}{|c|c|c|c|c|c|c|c|c|c|c|c|c|c|}
\hline Gr & No. & Loc. & ID & Prim & Tot & Class & Len & Wid & Asp & Analyte & Elements & Comment & Count Categories \\
\hline G2 & 34 & G7 & $A X$ & 199 & 200 & $\mathrm{~F}$ & 7.8 & 1.8 & 4.3 & Tremolite & & & $\begin{array}{l}\text { PCMEF-US, PCMES-US, } \\
\text { PCMEF-ISO, PCMES-ISO }\end{array}$ \\
\hline G2 & 34 & G7 & $A X$ & 200 & 201 & $\mathrm{~F}$ & 7.8 & 0.38 & 20.5 & Tremolite & & & $\begin{array}{l}\text { AS }>5,3: 1, \text { AFB }>5,3: 1, \\
\text { PCMEF-US, PCMES-US, } \\
\text { PCMEF-ISO, PCMES-ISO }\end{array}$ \\
\hline G2 & 34 & G7 & $A X$ & 201 & & MD 1-0 & 11 & 7 & 1.6 & Tremolite & & & $A S>5,3: 1$ \\
\hline G2 & 34 & G7 & $A X$ & & 202 & MF & 4.5 & 0.35 & 12.9 & Tremolite & & & \\
\hline G2 & 34 & G7 & $A X$ & 202 & 203 & $\mathrm{~F}$ & 5.12 & 1.7 & 3 & Tremolite & & & $\begin{array}{l}\text { PCMEF-US, PCMES-US, } \\
\text { PCMEF-ISO, PCMES-ISO }\end{array}$ \\
\hline G2 & 35 & 17 & $A X$ & 203 & 204 & $\mathrm{~F}$ & 2.6 & 0.65 & 4 & Tremolite & & & \\
\hline G2 & 35 & 17 & $A X$ & 204 & 205 & $\mathrm{~F}$ & 4.25 & 1.2 & 3.5 & Tremolite & & & \\
\hline G2 & 35 & 17 & $A X$ & 205 & 206 & $\mathrm{~F}$ & 11.2 & 0.4 & 28 & Tremolite & & & $\begin{array}{l}\text { AS>5, 3:1, AFB>5, 3:1, } \\
\text { PCMEF-US, PCMES-US, } \\
\text { PCMEF-ISO, PCMES-ISO }\end{array}$ \\
\hline G2 & 35 & 17 & $A X$ & 206 & 207 & $\mathrm{~F}$ & 3.8 & 0.7 & 5.4 & Tremolite & & & \\
\hline G2 & 35 & 17 & $A X$ & 207 & 208 & $\mathrm{~F}$ & 3 & 0.65 & 4.6 & Tremolite & & & \\
\hline G2 & 35 & 17 & $A X$ & 208 & 209 & $\mathrm{~F}$ & 4 & 0.65 & 6.2 & Tremolite & & & \\
\hline G2 & 35 & 17 & $A X$ & 209 & & MD 1-1 & 10 & 9 & 1.1 & Tremolite & & & $A S>5,3: 1$ \\
\hline G2 & 35 & 17 & $A X$ & & 210 & MF & 9 & 0.6 & 15 & Tremolite & & & $\begin{array}{c}\text { AFB >5, 3:1, PCMEF-US, } \\
\text { PCMEF-ISO }\end{array}$ \\
\hline
\end{tabular}

Count Categories

AFB $>5,3: 1$

PAS

PCMEF-US

PCMES-US
Asbestos Fibers and Bundles > 5um and 3:1

Primary Asbestos Structures

PCM Equivalent Fibers-NIOSH

PCM Equivalent Structures-NIOSH
AS $>5,3: 1$ PCMEF-ISO PCMES-ISO TAS
Asbestos Structures >5um and 3:1

PCM Equivalent Fibers-ISO

PCM Equivalent Structures-ISO

Total Asbestos Structures 
Job Number: 070434

ISO 10312, Direct Raw Data

Client: Idaho National Laboratory

Report Number: 070434R06

Project Name: RARE

Date Received: 4/23/2007

Client Sample No: FB-2-R4

Lab/Cor Sample No: S29

Client Description:

Date Sampled:

$4 / 16 / 2007$

\begin{tabular}{|c|c|c|c|c|c|c|c|c|c|c|c|c|c|}
\hline Gr & No. & Loc. & ID & Prim & Tot & Class & Len & Wid & Asp & Analyte & Elements & Comment & Count Categories \\
\hline G1 & 1 & A2 & $A X$ & 1 & 1 & $F$ & 1.7 & 0.4 & 4.2 & Tremolite & & & \\
\hline G1 & 1 & $\mathrm{~A} 2$ & $A Q$ & 2 & 2 & $\mathrm{~F}$ & 17.5 & 2 & 8.8 & Tremolite & $\begin{array}{l}\mathrm{Mg}, \mathrm{Al}, \mathrm{Si} \\
\mathrm{Ca}, \mathrm{Fe}\end{array}$ & & $\begin{array}{l}\text { AS }>5,3: 1, \text { AFB }>5,3: 1, \\
\text { PCMEF-US, PCMES-US, } \\
\text { PCMEF-ISO, PCMES-ISO }\end{array}$ \\
\hline & & & & & & \multicolumn{2}{|c|}{ ItemType } & ItemNur & & & Confirmed & Comment & \\
\hline & & & & & & \multicolumn{2}{|c|}{ Spectra } & $\mathrm{J} 3011$ & & & & & \\
\hline G1 & 2 & $\mathrm{C} 2$ & $A X$ & 3 & & MD 1-1 & 7.58 & 6 & 1.3 & Tremolite & & & $A S>5,3: 1$ \\
\hline G1 & 2 & $\mathrm{C} 2$ & $A X$ & & 3 & MF & 5.75 & 1.2 & 4.8 & Tremolite & & & PCMEF-US, PCMEF-ISO \\
\hline G1 & 2 & $\mathrm{C} 2$ & $A X$ & 4 & 4 & $\mathrm{~F}$ & 5.35 & 0.65 & 8.2 & Tremolite & & & $\begin{array}{l}\text { AS }>5,3: 1, \text { AFB }>5,3: 1, \\
\text { PCMEF-US, PCMES-US, } \\
\text { PCMEF-ISO, PCMES-ISO }\end{array}$ \\
\hline G1 & 2 & $\mathrm{C} 2$ & $A X$ & 5 & 5 & $F$ & 3 & 0.3 & 10 & Tremolite & & & \\
\hline G1 & 2 & $\mathrm{C} 2$ & $A X$ & 6 & 6 & $\mathrm{~F}$ & 4 & 0.75 & 5.3 & Tremolite & & & \\
\hline G1 & 2 & $\mathrm{C} 2$ & $A X$ & 7 & & MD 2-2 & 13.8 & 2.75 & 5 & Tremolite & & & $\begin{array}{c}\text { AS }>5,3: 1, \text { PCMES-US, } \\
\text { PCMES-ISO }\end{array}$ \\
\hline G1 & 2 & $\mathrm{C} 2$ & $A X$ & & 7 & MF & 13.2 & 0.5 & 26.4 & Tremolite & & & $\begin{array}{c}\text { AFB }>5,3: 1, \text { PCMEF-US, } \\
\text { PCMEF-ISO }\end{array}$ \\
\hline G1 & 2 & $\mathrm{C} 2$ & $A X$ & & 8 & MF & 5.1 & 0.25 & 20.4 & Tremolite & & & $\begin{array}{c}\text { AFB }>5,3: 1, \text { PCMEF-US, } \\
\text { PCMEF-ISO }\end{array}$ \\
\hline G1 & 2 & $\mathrm{C} 2$ & $A X$ & 8 & 9 & $F$ & 4.5 & 0.65 & 6.9 & Tremolite & & & \\
\hline G1 & 2 & $\mathrm{C} 2$ & $A X$ & 9 & 10 & $F$ & 22.5 & 1.5 & 15 & Tremolite & & & $\begin{array}{l}\text { AS }>5,3: 1, \text { AFB }>5,3: 1 \text {, } \\
\text { PCMEF-US, PCMES-US, } \\
\text { PCMEF-ISO, PCMES-ISO }\end{array}$ \\
\hline G1 & 2 & $\mathrm{C} 2$ & $A X$ & 10 & 11 & $\mathrm{~F}$ & 10.85 & 0.8 & 13.6 & Tremolite & & & $\begin{array}{l}\text { AS }>5,3: 1, \text { AFB }>5,3: 1, \\
\text { PCMEF-US, PCMES-US, } \\
\text { PCMEF-ISO, PCMES-ISO }\end{array}$ \\
\hline G1 & 2 & $\mathrm{C} 2$ & $A X$ & 11 & & MD 1-0 & 6 & 1.85 & 3.2 & Tremolite & & & $\begin{array}{c}\text { AS >5, 3:1, PCMES-US, } \\
\text { PCMES-ISO }\end{array}$ \\
\hline G1 & 2 & $\mathrm{C} 2$ & $A X$ & & 12 & MF & 4.8 & 0.55 & 8.7 & Tremolite & & & \\
\hline G1 & 2 & $\mathrm{C} 2$ & $A X$ & 12 & 13 & $\mathrm{~F}$ & 5.4 & 0.6 & 9 & Tremolite & & & $\begin{array}{l}\text { AFB }>5,3: 1, \text { AS }>5,3: 1, \\
\text { PCMEF-US, PCMES-US, } \\
\text { PCMEF-ISO, PCMES-ISO }\end{array}$ \\
\hline \multirow[t]{3}{*}{ G1 } & 2 & $\mathrm{C} 2$ & $A D Q$ & 13 & 14 & $\mathrm{~F}$ & 9.8 & 1.2 & 8.2 & Tremolite & $\begin{array}{l}\mathrm{Mg}, \mathrm{Al}, \mathrm{Si} \\
\mathrm{Ca}, \mathrm{Fe}\end{array}$ & & $\begin{array}{l}\text { AS>5, 3:1, AFB >5, 3:1, } \\
\text { PCMEF-US, PCMES-US, } \\
\text { PCMEF-ISO, PCMES-ISO }\end{array}$ \\
\hline & & & & & & \multicolumn{2}{|c|}{ ItemType } & ItemNur & & & Confirmed & Comment & \\
\hline & & & & & & \multicolumn{2}{|c|}{ Spectra } & J3012 & & & & & \\
\hline G1 & 2 & $\mathrm{C} 2$ & $A X$ & 14 & 15 & $F$ & 4.35 & 1.2 & 3.6 & Tremolite & & & \\
\hline G1 & 2 & $\mathrm{C} 2$ & $A X$ & 15 & 16 & $F$ & 7 & 0.2 & 35 & Tremolite & & & $\begin{array}{l}\text { AS }>5,3: 1, A F B>5,3: 1, \\
\text { PCMEF-ISO, PCMES-ISO }\end{array}$ \\
\hline
\end{tabular}

Count Categories

$\mathrm{AFB}>5,3: 1$

PAS

PCMEF-US

PCMES-US
Asbestos Fibers and Bundles $>$ 5um and 3:1

Primary Asbestos Structures

PCM Equivalent Fibers-NIOSH

PCM Equivalent Structures-NIOSH
AS $>5,3: 1$ PCMEF-ISO PCMES-ISO TAS
Asbestos Structures $>5$ um and 3:1

PCM Equivalent Fibers-ISO

PCM Equivalent Structures-ISO

Total Asbestos Structures 
ISO 10312, Direct Raw Data

Job Number: 070434

SEA

Report Number: 070434R06

Client: Idaho National Laboratory

Date Received: 4/23/2007

Project Name: RARE

Client Sample No: FB-2-R4

Lab/Cor Sample No: S29

Client Description:

Date Sampled:

4/16/2007

\begin{tabular}{|c|c|c|c|c|c|c|c|c|c|c|c|c|c|}
\hline Gr & No. & Loc. & ID & Prim & Tot & Class & Len & Wid & Asp & Analyte & Elements & nent & Count Categories \\
\hline \multirow[t]{5}{*}{ G1 } & 3 & E2 & $A Z Q$ & 16 & 17 & $F$ & 4.45 & 0.53 & 8.4 & Tremolite & $\begin{array}{l}\text { Mg, Al, Si, } \\
\mathrm{Ca}, \mathrm{Fe}\end{array}$ & & \\
\hline & & & & & & \multicolumn{2}{|c|}{ ItemType } & ItemNun & & & Confirmed & Com & \\
\hline & & & & & & \multicolumn{2}{|c|}{ Brightfield } & J3572 B & & & & & \\
\hline & & & & & & \multicolumn{2}{|c|}{ Diffraction } & J3572 & & & KM 6/4/2007 & ZON & IS [ $\left.\begin{array}{lll}1 & 0 & 0\end{array}\right]$ \\
\hline & & & & & & \multicolumn{2}{|c|}{ Spectra } & J3013 & & & & & \\
\hline G1 & 3 & E2 & $A X$ & 17 & 18 & $\mathrm{~F}$ & 6.5 & 1.5 & 4.3 & Tremolite & & & $\begin{array}{l}\text { PCMEF-US, PCMES-US, } \\
\text { PCMEF-ISO, PCMES-ISO }\end{array}$ \\
\hline G1 & 3 & E2 & $A X$ & 18 & 19 & $\mathrm{~F}$ & 5.85 & 1 & 5.8 & Tremolite & & & $\begin{array}{l}\text { AS >5, 3:1, AFB >5, 3:1, } \\
\text { PCMEF-US, PCMES-US, } \\
\text { PCMEF-ISO, PCMES-ISO }\end{array}$ \\
\hline G1 & 3 & E2 & $A X$ & 19 & 20 & B & 4.5 & 0.75 & 6 & Tremolite & & & \\
\hline G1 & 3 & E2 & $A X$ & 20 & 21 & $\mathrm{~F}$ & 4 & 0.65 & 6.2 & Tremolite & & & \\
\hline G1 & 4 & G2 & $A X$ & 21 & 22 & $\mathrm{~F}$ & 4.8 & 0.4 & 12 & Tremolite & & & \\
\hline G1 & 4 & G2 & $A X$ & 22 & 23 & $\mathrm{~F}$ & 3.25 & 0.65 & 5 & Tremolite & & & \\
\hline G1 & 4 & G2 & $A X$ & 23 & 24 & $\mathrm{~F}$ & 4.2 & 1 & 4.2 & Tremolite & & & \\
\hline G1 & 4 & G2 & $A X$ & 24 & 25 & $\mathrm{~F}$ & 3 & 0.85 & 3.5 & Tremolite & & & \\
\hline G1 & 5 & 12 & $A X$ & 25 & 26 & $\mathrm{~F}$ & 2.2 & 0.18 & 12.2 & Tremolite & & & \\
\hline G1 & 5 & 12 & $A X$ & 26 & 27 & $\mathrm{~F}$ & 3.25 & 0.38 & 8.6 & Tremolite & & & \\
\hline G1 & 5 & 12 & $A X$ & 27 & 28 & $\mathrm{~F}$ & 1.2 & 0.15 & 8 & Tremolite & & & \\
\hline G1 & 5 & 12 & $A X$ & 28 & 29 & $\mathrm{~F}$ & 1.1 & 0.22 & 5 & Tremolite & & & \\
\hline G1 & 5 & 12 & $A X$ & 29 & 30 & $\mathrm{~F}$ & 1.5 & 0.2 & 7.5 & Tremolite & & & \\
\hline G1 & 5 & 12 & $A X$ & 30 & 31 & $\mathrm{~F}$ & 19 & 2 & 9.5 & Tremolite & & & $\begin{array}{l}\text { AS }>5,3: 1, \text { AFB }>5,3: 1, \\
\text { PCMEF-US, PCMES-US, } \\
\text { PCMEF-ISO, PCMES-ISO }\end{array}$ \\
\hline G1 & 5 & 12 & $A X$ & 31 & 32 & $\mathrm{~F}$ & 4 & 0.4 & 10 & Tremolite & & & \\
\hline G1 & 5 & 12 & $A X$ & 32 & 33 & $\mathrm{~F}$ & 15.25 & 1 & 15.2 & Tremolite & & & $\begin{array}{l}\text { AS >5, 3:1, AFB>5, 3:1, } \\
\text { PCMEF-US, PCMES-US, } \\
\text { PCMEF-ISO, PCMES-ISO }\end{array}$ \\
\hline G1 & 5 & 12 & $A X$ & 33 & 34 & $\mathrm{~F}$ & 6 & 0.7 & 8.6 & Tremolite & & & $\begin{array}{l}\text { AS }>5,3: 1, \text { AFB }>5,3: 1, \\
\text { PCMEF-US, PCMES-US, } \\
\text { PCMEF-ISO, PCMES-ISO }\end{array}$ \\
\hline G1 & 6 & J4 & $A X$ & 34 & 35 & $F$ & 1.25 & 0.38 & 3.3 & Tremolite & & & \\
\hline G1 & 6 & J4 & $A X$ & 35 & 36 & $\mathrm{~F}$ & 3.25 & 0.5 & 6.5 & Tremolite & & & \\
\hline G1 & 6 & J4 & $A X$ & 36 & 37 & $\mathrm{~F}$ & 1.65 & 0.35 & 4.7 & Tremolite & & & \\
\hline G1 & 6 & J4 & $A X$ & 37 & 38 & $\mathrm{~F}$ & 5.7 & 0.8 & 7.1 & Tremolite & & & $\begin{array}{l}\text { AS>5, 3:1, AFB>5, 3:1, } \\
\text { PCMEF-US, PCMES-US, } \\
\text { PCMEF-ISO, PCMES-ISO }\end{array}$ \\
\hline G1 & 6 & J4 & $A X$ & 38 & 39 & $\mathrm{~F}$ & 4.5 & 0.8 & 5.6 & Tremolite & & & \\
\hline G1 & 6 & J4 & $A X$ & 39 & 40 & $\mathrm{~F}$ & 1.75 & 0.55 & 3.2 & Tremolite & & & \\
\hline
\end{tabular}

Count Categories

PAS

PCMEF-US

Asbestos Fibers and Bundles $>$ 5um and 3:1

Primary Asbestos Structures

PCM Equivalent Fibers-NIOSH

PCMES-US
AS $>5,3: 1$ PCMEF-ISO PCMES-ISO TAS
Asbestos Structures $>5$ um and 3:1 PCM Equivalent Fibers-ISO PCM Equivalent Structures-ISO Total Asbestos Structures

Page 110 of 281 
Job Number: 070434

ISO 10312, Direct Raw Data

Client: Idaho National Laboratory

Report Number: 070434R06

Project Name: RARE

Date Received: 4/23/2007

Client Sample No: FB-2-R4

Lab/Cor Sample No: $\mathbf{S 2 9}$

Client Description:

Date Sampled:

$4 / 16 / 2007$

\begin{tabular}{|c|c|c|c|c|c|c|c|c|c|c|c|c|c|}
\hline Gr & No. & Loc. & ID & Prim & Tot & Class & Len & Wid & Asp & Analyte & Elements & Comment & Count Categories \\
\hline G1 & 7 & $\mathrm{H} 4$ & $A X$ & 40 & 41 & $F$ & 7 & 0.55 & 12.7 & Tremolite & & & $\begin{array}{l}\text { AS }>5,3: 1, \text { AFB }>5,3: 1, \\
\text { PCMEF-US, PCMES-US, } \\
\text { PCMEF-ISO, PCMES-ISO }\end{array}$ \\
\hline G1 & 7 & $\mathrm{H} 4$ & $A X$ & 41 & 42 & $\mathrm{~F}$ & 54 & 1.2 & 45 & Tremolite & & & $\begin{array}{l}\text { AS }>5,3: 1, \text { AFB }>5,3: 1 \text {, } \\
\text { PCMEF-US, PCMES-US, } \\
\text { PCMEF-ISO, PCMES-ISO }\end{array}$ \\
\hline G1 & 7 & $\mathrm{H} 4$ & $A X$ & 42 & 43 & $\mathrm{~F}$ & 7.5 & 1.35 & 5.6 & Tremolite & & & $\begin{array}{l}\text { AS }>5,3: 1, \text { AFB }>5,3: 1, \\
\text { PCMEF-US, PCMES-US, } \\
\text { PCMEF-ISO, PCMES-ISO }\end{array}$ \\
\hline G1 & 7 & $\mathrm{H} 4$ & $A X$ & 43 & 44 & $\mathrm{~F}$ & 6.1 & 1.2 & 5.1 & Tremolite & & & $\begin{array}{l}\text { AS }>5,3: 1, \text { AFB }>5,3: 1, \\
\text { PCMEF-US, PCMES-US, } \\
\text { PCMEF-ISO, PCMES-ISO }\end{array}$ \\
\hline G1 & 7 & $\mathrm{H} 4$ & $A X$ & 44 & 45 & $\mathrm{~F}$ & 3.75 & 0.8 & 4.7 & Tremolite & & & \\
\hline G1 & 7 & $\mathrm{H} 4$ & $A X$ & 45 & 46 & $\mathrm{~F}$ & 1.7 & 0.38 & 4.5 & Tremolite & & & \\
\hline G1 & 7 & $\mathrm{H} 4$ & $A X$ & 46 & 47 & $\mathrm{~F}$ & 6 & 1.25 & 4.8 & Tremolite & & & $\begin{array}{l}\text { PCMEF-US, PCMES-US, } \\
\text { PCMEF-ISO, PCMES-ISO }\end{array}$ \\
\hline G1 & 7 & $\mathrm{H} 4$ & $A X$ & 47 & 48 & $\mathrm{~F}$ & 1.8 & 0.68 & 2.6 & Tremolite & & & \\
\hline G1 & 7 & $\mathrm{H} 4$ & $A X$ & 48 & 49 & $\mathrm{~F}$ & 1.75 & 0.65 & 2.7 & Tremolite & & & \\
\hline G1 & 8 & $\mathrm{~F} 4$ & $A X$ & 49 & 50 & $\mathrm{~F}$ & 4.85 & 1.5 & 3.2 & Tremolite & & & \\
\hline G1 & 8 & $\mathrm{~F} 4$ & $A X$ & 50 & 51 & $\mathrm{~F}$ & 6.75 & 2 & 3.4 & Tremolite & & & $\begin{array}{l}\text { PCMEF-US, PCMES-US, } \\
\text { PCMEF-ISO, PCMES-ISO }\end{array}$ \\
\hline G1 & 8 & $\mathrm{~F} 4$ & $A X$ & 51 & 52 & $\mathrm{~F}$ & 21.7 & 1.8 & 12.1 & Tremolite & & & $\begin{array}{l}\text { AS }>5,3: 1, \text { AFB }>5,3: 1, \\
\text { PCMEF-US, PCMES-US, } \\
\text { PCMEF-ISO, PCMES-ISO }\end{array}$ \\
\hline G1 & 8 & $\mathrm{~F} 4$ & $A X$ & 52 & & CD 2-2 & 35 & 12.2 & 2.9 & Tremolite & & & $A S>5,3: 1$ \\
\hline G1 & 8 & $\mathrm{~F} 4$ & $A X$ & & 53 & $\mathrm{CF}$ & 27.5 & 3.75 & 7.3 & Tremolite & & & AFB $>5,3: 1$, PCMEF-US \\
\hline G1 & 8 & $\mathrm{~F} 4$ & $A X$ & & 54 & $\mathrm{CF}$ & 9 & 0.75 & 12 & Tremolite & & & $\begin{array}{c}\text { AFB }>5,3: 1, \text { PCMEF-US, } \\
\text { PCMEF-ISO }\end{array}$ \\
\hline G1 & 8 & $\mathrm{~F} 4$ & $A X$ & & 55 & CF & 5.5 & 0.55 & 10 & Tremolite & & & $\begin{array}{c}\text { AFB }>5,3: 1, \text { PCMEF-US, } \\
\text { PCMEF-ISO }\end{array}$ \\
\hline G1 & 8 & $\mathrm{~F} 4$ & $A X$ & & 56 & CB & 4.5 & 0.8 & 5.6 & Tremolite & & & \\
\hline G1 & 8 & $\mathrm{~F} 4$ & $A X$ & 53 & 57 & $\mathrm{~F}$ & 7.75 & 0.65 & 11.9 & Tremolite & & & $\begin{array}{l}\text { AS }>5,3: 1, \text { AFB }>5,3: 1 \text {, } \\
\text { PCMEF-US, PCMES-US, } \\
\text { PCMEF-ISO, PCMES-ISO }\end{array}$ \\
\hline G1 & 8 & $\mathrm{~F} 4$ & $A X$ & 54 & 58 & $\mathrm{~F}$ & 13.5 & 1.8 & 7.5 & Tremolite & & & $\begin{array}{l}\text { AS }>5,3: 1, \text { AFB }>5,3: 1, \\
\text { PCMEF-US, PCMES-US, } \\
\text { PCMEF-ISO, PCMES-ISO }\end{array}$ \\
\hline G1 & 8 & $\mathrm{~F} 4$ & $A X$ & 55 & 59 & $\mathrm{~F}$ & 6.2 & 1.2 & 5.2 & Tremolite & & & $\begin{array}{l}\text { AS }>5,3: 1, \text { AFB }>5,3: 1, \\
\text { PCMEF-US, PCMES-US, } \\
\text { PCMEF-ISO, PCMES-ISO }\end{array}$ \\
\hline G1 & 8 & $\mathrm{~F} 4$ & $A X$ & 56 & 60 & $\mathrm{~F}$ & 5.6 & 0.7 & 8 & Tremolite & & & $\begin{array}{l}\text { AS }>5,3: 1, \text { AFB }>5,3: 1, \\
\text { PCMEF-US, PCMES-US, } \\
\text { PCMEF-ISO, PCMES-ISO }\end{array}$ \\
\hline G1 & 8 & $\mathrm{~F} 4$ & $A X$ & 57 & 61 & $\mathrm{~F}$ & 29 & 4.5 & 6.4 & Tremolite & & & $\begin{array}{l}\text { AS }>5,3: 1, \text { AFB }>5,3: 1 \\
\text { PCMEF-US, PCMES-US }\end{array}$ \\
\hline
\end{tabular}

Count Categories

AFB $>5,3: 1$

Asbestos Fibers and Bundles > 5um and 3:1

PAS

PCMEF-US

Primary Asbestos Structures

PCM Equivalent Fibers-NIOSH

PCMES-US
AS>5, 3:1 PCMEF-ISO PCMES-ISO TAS
Asbestos Structures >5um and 3:1 PCM Equivalent Fibers-ISO PCM Equivalent Structures-ISO Total Asbestos Structures

Page 111 of 281 
ISO 10312, Direct Raw Data

Job Number: 070434

SEA

Report Number: 070434R06

Client: Idaho National Laboratory

Date Received: 4/23/2007

Project Name: RARE

Client Sample No: FB-2-R4

Lab/Cor Sample No: S29

Client Description:

Date Sampled:

$4 / 16 / 2007$

\begin{tabular}{|c|c|c|c|c|c|c|c|c|c|c|c|c|c|}
\hline Gr & No. & Loc. & ID & Prim & Tot & Class & Len & Wid & Asp & Analyte & Elements & Comment & Count Categories \\
\hline G1 & 9 & D4 & $A X$ & 58 & 62 & $\mathrm{~F}$ & 2.7 & 0.22 & 12.3 & Tremolite & & & \\
\hline G1 & 9 & D4 & $A X$ & 59 & 63 & $\mathrm{~F}$ & 7.65 & 1.1 & 7 & Tremolite & & & $\begin{array}{l}\text { AS }>5,3: 1, \text { AFB }>5,3: 1, \\
\text { PCMEF-US, PCMES-US, } \\
\text { PCMEF-ISO, PCMES-ISO }\end{array}$ \\
\hline G1 & 9 & D4 & $A X$ & 60 & 64 & $\mathrm{~F}$ & 13.2 & 2.7 & 4.9 & Tremolite & & & $\begin{array}{l}\text { PCMEF-US, PCMES-US, } \\
\text { PCMEF-ISO, PCMES-ISO }\end{array}$ \\
\hline G1 & 9 & D4 & $A X$ & 61 & 65 & $\mathrm{~F}$ & 4.9 & 0.75 & 6.5 & Tremolite & & & \\
\hline G1 & 9 & D4 & $A X$ & 62 & & MD 1-1 & 10 & 4.5 & 2.2 & Tremolite & & & $A S>5,3: 1$ \\
\hline G1 & 9 & D4 & $A X$ & & 66 & MF & 6 & 0.8 & 7.5 & Tremolite & & & $\begin{array}{c}\text { AFB }>5,3: 1, \text { PCMEF-US, } \\
\text { PCMEF-ISO }\end{array}$ \\
\hline G1 & 10 & B4 & $A X$ & 63 & 67 & $\mathrm{~F}$ & 2 & 0.35 & 5.7 & Tremolite & & & \\
\hline G1 & 10 & B4 & $A X$ & 64 & 68 & $\mathrm{~F}$ & 15 & 0.7 & 21.4 & Tremolite & & & $\begin{array}{l}\text { AS >5, 3:1, AFB>5, 3:1, } \\
\text { PCMEF-US, PCMES-US, } \\
\text { PCMEF-ISO, PCMES-ISO }\end{array}$ \\
\hline G1 & 10 & B4 & $A X$ & 65 & 69 & $F$ & 3.75 & 0.65 & 5.8 & Tremolite & & & \\
\hline G1 & 10 & B4 & $A X$ & 66 & 70 & $F$ & 2.75 & 0.35 & 7.9 & Tremolite & & & \\
\hline G1 & 10 & B4 & $A X$ & 67 & 71 & $F$ & 4.2 & 0.5 & 8.4 & Tremolite & & & \\
\hline G1 & 10 & B4 & $A X$ & 68 & 72 & $F$ & 40 & 4.75 & 8.4 & Tremolite & & & $\begin{array}{l}\text { AS }>5,3: 1, \text { AFB }>5,3: 1, \\
\text { PCMEF-US, PCMES-US }\end{array}$ \\
\hline G1 & 11 & A7 & $A X$ & 69 & & MD 1-1 & 22 & 10 & 2.2 & Tremolite & & & $A S>5,3: 1$ \\
\hline G1 & 11 & A7 & $A X$ & & 73 & MF & 21.5 & 0.5 & 43 & Tremolite & & & $\begin{array}{c}\text { AFB }>5,3: 1, \text { PCMEF-US, } \\
\text { PCMEF-ISO }\end{array}$ \\
\hline G1 & 11 & A7 & $A X$ & 70 & & CD 2-1 & 13 & 5 & 2.6 & Tremolite & & & $A S>5,3: 1$ \\
\hline G1 & 11 & A7 & $A X$ & & 74 & $\mathrm{CF}$ & 13 & 4 & 3.2 & Tremolite & & & PCMEF-US \\
\hline G1 & 11 & A7 & $A X$ & & 75 & CF & 4 & 0.8 & 5 & Tremolite & & & \\
\hline G1 & 11 & A7 & $A X$ & 71 & & MD 1-1 & 11.2 & 3.5 & 3.2 & Tremolite & & & AS $>5,3: 1$, PCMES-US \\
\hline G1 & 11 & A7 & $A X$ & & 76 & MF & 9.7 & 0.5 & 19.4 & Tremolite & & & $\begin{array}{c}\text { AFB >5, 3:1, PCMEF-US, } \\
\text { PCMEF-ISO }\end{array}$ \\
\hline G1 & 11 & A7 & $A X$ & 72 & 77 & $\mathrm{~F}$ & 7 & 0.55 & 12.7 & Tremolite & & & $\begin{array}{l}\text { AS }>5,3: 1, \text { AFB }>5,3: 1, \\
\text { PCMEF-US, PCMES-US, } \\
\text { PCMEF-ISO, PCMES-ISO }\end{array}$ \\
\hline G1 & 11 & A7 & $A X$ & 73 & 78 & $\mathrm{~F}$ & 3 & 0.8 & 3.8 & Tremolite & & & \\
\hline G1 & 12 & $\mathrm{C7}$ & $A X$ & 74 & 79 & $\mathrm{~F}$ & 12 & 1.2 & 10 & Tremolite & & & $\begin{array}{l}\text { AS>5, 3:1, AFB>5, 3:1, } \\
\text { PCMEF-US, PCMES-US, } \\
\text { PCMEF-ISO, PCMES-ISO }\end{array}$ \\
\hline G1 & 12 & $\mathrm{C} 7$ & $A X$ & 75 & 80 & $\mathrm{~F}$ & 6.12 & 0.55 & 11.1 & Tremolite & & & $\begin{array}{l}\text { AS >5, 3:1, AFB >5, 3:1, } \\
\text { PCMEF-US, PCMES-US, } \\
\text { PCMEF-ISO, PCMES-ISO }\end{array}$ \\
\hline G1 & 12 & $\mathrm{C} 7$ & $A X$ & 76 & 81 & $\mathrm{~F}$ & 8.6 & 0.5 & 17.2 & Tremolite & & & $\begin{array}{l}\text { AS>5, 3:1, AFB }>5,3: 1 \text {, } \\
\text { PCMEF-US, PCMES-US, } \\
\text { PCMEF-ISO, PCMES-ISO }\end{array}$ \\
\hline
\end{tabular}

Count Categories

$\mathrm{AFB}>5,3: 1$

PAS

PCMEF-US

PCMES-US
Asbestos Fibers and Bundles > 5um and 3:1

Primary Asbestos Structures

PCM Equivalent Fibers-NIOSH

PCM Equivalent Structures-NIOSH
AS>5, 3:1 PCMEF-ISO PCMES-ISO TAS
Asbestos Structures >5um and 3:1 PCM Equivalent Fibers-ISO PCM Equivalent Structures-ISO Total Asbestos Structures 
ISO 10312, Direct Raw Data

Job Number: 070434

SEA

Report Number: 070434R06

Client: Idaho National Laboratory

Date Received: 4/23/2007

Project Name: RARE

Client Sample No: FB-2-R4

Lab/Cor Sample No: S29

Client Description:

Date Sampled:

$4 / 16 / 2007$

\begin{tabular}{|c|c|c|c|c|c|c|c|c|c|c|c|c|c|}
\hline Gr & No. & Loc. & ID & Prim & Tot & Class & Len & Wid & Asp & Analyte & Elements & Comment & Count Categories \\
\hline G1 & 12 & $\mathrm{C} 7$ & $A X$ & 77 & 82 & $F$ & 9.8 & 0.85 & 11.5 & Tremolite & & & $\begin{array}{l}\text { AS }>5,3: 1, \text { AFB }>5,3: 1, \\
\text { PCMEF-US, PCMES-US, } \\
\text { PCMEF-ISO, PCMES-ISO }\end{array}$ \\
\hline G1 & 13 & E7 & $A X$ & 78 & & MD 1-1 & 16 & 6.5 & 2.5 & Tremolite & & & $A S>5,3: 1$ \\
\hline G1 & 13 & E7 & $A X$ & & 83 & MF & 7 & 0.5 & 14 & Tremolite & & & $\begin{array}{c}\text { AFB }>5,3: 1, \text { PCMEF-US, } \\
\text { PCMEF-ISO }\end{array}$ \\
\hline G1 & 13 & E7 & $A X$ & 79 & 84 & $\mathrm{~F}$ & 2 & 0.65 & 3.1 & Tremolite & & & \\
\hline G1 & 13 & E7 & $A X$ & 80 & 85 & $\mathrm{~F}$ & 2 & 0.5 & 4 & Tremolite & & & \\
\hline G1 & 13 & E7 & $A X$ & 81 & 86 & $\mathrm{~F}$ & 5.1 & 1.5 & 3.4 & Tremolite & & & $\begin{array}{l}\text { PCMEF-US, PCMES-US, } \\
\text { PCMEF-ISO, PCMES-ISO }\end{array}$ \\
\hline G1 & 14 & G7 & $A X$ & 82 & 87 & $\mathrm{~F}$ & 26 & 1.5 & 17.3 & Tremolite & & & $\begin{array}{l}\text { AS }>5,3: 1, \text { AFB }>5,3: 1 \text {, } \\
\text { PCMEF-US, PCMES-US, } \\
\text { PCMEF-ISO, PCMES-ISO }\end{array}$ \\
\hline G1 & 14 & G7 & $A X$ & 83 & 88 & $\mathrm{~F}$ & 12 & 0.6 & 20 & Tremolite & & & $\begin{array}{l}\text { AS>5, 3:1, AFB >5, 3:1, } \\
\text { PCMEF-US, PCMES-US, } \\
\text { PCMEF-ISO, PCMES-ISO }\end{array}$ \\
\hline G1 & 14 & G7 & $A X$ & 84 & 89 & $F$ & 3.65 & 0.6 & 6.1 & Tremolite & & & \\
\hline G1 & 14 & G7 & $A X$ & 85 & 90 & $\mathrm{~F}$ & 7 & 0.75 & 9.3 & Tremolite & & & $\begin{array}{l}\text { AS }>5,3: 1, \text { AFB }>5,3: 1, \\
\text { PCMEF-US, PCMES-US, } \\
\text { PCMEF-ISO, PCMES-ISO }\end{array}$ \\
\hline G1 & 15 & 17 & $A X$ & 86 & 91 & $\mathrm{~F}$ & 5.65 & 1.45 & 3.9 & Tremolite & & & $\begin{array}{l}\text { PCMEF-US, PCMES-US, } \\
\text { PCMEF-ISO, PCMES-ISO }\end{array}$ \\
\hline G1 & 15 & 17 & $A X$ & 87 & 92 & $F$ & 4 & 0.8 & 5 & Tremolite & & & \\
\hline G1 & 15 & 17 & $A X$ & 88 & 93 & $F$ & 1.5 & 0.2 & 7.5 & Tremolite & & & \\
\hline G1 & 15 & 17 & $A X$ & 89 & 94 & $F$ & 2.5 & 0.5 & 5 & Tremolite & & & \\
\hline G1 & 15 & 17 & $A X$ & 90 & 95 & $F$ & 2.8 & 0.7 & 4 & Tremolite & & & \\
\hline G1 & 16 & J9 & $A X$ & 91 & 96 & $F$ & 7.75 & 1.8 & 4.3 & Tremolite & & & $\begin{array}{l}\text { PCMEF-US, PCMES-US, } \\
\text { PCMEF-ISO, PCMES-ISO }\end{array}$ \\
\hline G1 & 16 & J9 & $A X$ & 92 & 97 & $\mathrm{~F}$ & 7 & 0.38 & 18.4 & Tremolite & & & $\begin{array}{l}\text { AS }>5,3: 1, \text { AFB }>5,3: 1, \\
\text { PCMEF-US, PCMES-US, } \\
\text { PCMEF-ISO, PCMES-ISO }\end{array}$ \\
\hline G1 & 16 & J9 & $A X$ & 93 & 98 & $F$ & 6.2 & 1.2 & 5.2 & Tremolite & & & $\begin{array}{l}\text { AS }>5,3: 1, \text { AFB }>5,3: 1, \\
\text { PCMEF-US, PCMES-US, } \\
\text { PCMEF-ISO, PCMES-ISO }\end{array}$ \\
\hline G1 & 16 & J9 & $A X$ & 94 & & CD 2-2 & 20.25 & 3.75 & 5.4 & Tremolite & & & AS>5, 3:1, PCMES-US \\
\hline G1 & 16 & J9 & $A X$ & & 99 & CF & 16.85 & 1.5 & 11.2 & Tremolite & & & $\begin{array}{c}\text { AFB >5, 3:1, PCMEF-US, } \\
\text { PCMEF-ISO }\end{array}$ \\
\hline G1 & 16 & J9 & $A X$ & & 100 & CF & 16.5 & 0.8 & 20.6 & Tremolite & & & $\begin{array}{c}\text { AFB >5, 3:1, PCMEF-US, } \\
\text { PCMEF-ISO }\end{array}$ \\
\hline G1 & 16 & J9 & $A X$ & 95 & 101 & $\mathrm{~F}$ & 14 & 2 & 7 & Tremolite & & & $\begin{array}{l}\text { AS>5, 3:1, AFB }>5,3: 1, \\
\text { PCMEF-US, PCMES-US, } \\
\text { PCMEF-ISO, PCMES-ISO }\end{array}$ \\
\hline G1 & 16 & J9 & $A X$ & 96 & 102 & $\mathrm{~F}$ & 9.5 & 2 & 4.8 & Tremolite & & & $\begin{array}{l}\text { PCMEF-US, PCMES-US, } \\
\text { PCMES-ISO, PCMEF-ISO }\end{array}$ \\
\hline
\end{tabular}

Count Categories

$\mathrm{AFB}>5,3: 1$

PAS

PCMEF-US

Asbestos Fibers and Bundles > 5um and 3:

Primary Asbestos Structures

PCM Equivalent Fibers-NIOSH

PCMES-US
AS>5, 3:1 PCMEF-ISO PCMES-ISO TAS
Asbestos Structures >5um and 3:1 PCM Equivalent Fibers-ISO PCM Equivalent Structures-ISO Total Asbestos Structures

Page 113 of 281 
ISO 10312, Direct Raw Data

Job Number: 070434

SEA

Report Number: 070434R06

Client: Idaho National Laboratory

Date Received: 4/23/2007

Project Name: RARE

Client Sample No: FB-2-R4

Lab/Cor Sample No: S29

Client Description:

Date Sampled:

4/16/2007

\begin{tabular}{|c|c|c|c|c|c|c|c|c|c|c|c|c|c|}
\hline Gr & No. & Loc. & ID & Prim & Tot & Class & Len & Wid & Asp & Analyte & Elements & Comment & Count Categories \\
\hline G1 & 16 & J9 & $A X$ & 97 & 103 & $\mathrm{~F}$ & 3.8 & 0.15 & 25.3 & Tremolite & & & \\
\hline G1 & 16 & J9 & $A X$ & 98 & & MD 2-0 & 8.5 & 5 & 1.7 & Tremolite & & & $A S>5,3: 1$ \\
\hline G1 & 16 & J9 & $A X$ & & 104 & MF & 4.5 & 0.65 & 6.9 & Tremolite & & & \\
\hline G1 & 16 & J9 & $A X$ & & 105 & MF & 3.9 & 0.55 & 7.1 & Tremolite & & & \\
\hline G1 & 16 & J9 & $A X$ & 99 & 106 & $\mathrm{~F}$ & 5.55 & 0.7 & 7.9 & Tremolite & & & $\begin{array}{l}\text { AS>5, 3:1, AFB >5, 3:1, } \\
\text { PCMEF-US, PCMES-US, } \\
\text { PCMEF-ISO, PCMES-ISO }\end{array}$ \\
\hline G1 & 17 & $\mathrm{H} 9$ & $A X$ & 100 & 107 & $\mathrm{~F}$ & 22 & 1.75 & 12.6 & Tremolite & & & $\begin{array}{l}\text { AS }>5,3: 1, \text { AFB }>5,3: 1, \\
\text { PCMES-US, PCMEF-US, } \\
\text { PCMEF-ISO, PCMES-ISO }\end{array}$ \\
\hline G1 & 17 & $\mathrm{H} 9$ & $A X$ & 101 & 108 & $\mathrm{~F}$ & 3.3 & 0.65 & 5.1 & Tremolite & & & \\
\hline G1 & 17 & H9 & $A X$ & 102 & 109 & $\mathrm{~F}$ & 4.35 & 0.75 & 5.8 & Tremolite & & & \\
\hline G1 & 17 & $\mathrm{H} 9$ & $A X$ & 103 & 110 & $\mathrm{~F}$ & 1.35 & 0.4 & 3.4 & Tremolite & & & \\
\hline G1 & 18 & F9 & $A X$ & 104 & 111 & $F$ & 3.1 & 0.68 & 4.6 & Tremolite & & & \\
\hline G1 & 18 & F9 & $A X$ & 105 & 112 & $\mathrm{~F}$ & 1.7 & 0.12 & 14.2 & Tremolite & & & \\
\hline G1 & 18 & F9 & $A X$ & 106 & 113 & $\mathrm{~F}$ & 5 & 0.5 & 10 & Tremolite & & & \\
\hline G1 & 18 & F9 & $A X$ & 107 & 114 & $\mathrm{~F}$ & 14 & 2.6 & 5.4 & Tremolite & & & $\begin{array}{l}\text { AS }>5,3: 1, \text { AFB }>5,3: 1, \\
\text { PCMEF-US, PCMES-US, } \\
\text { PCMEF-ISO, PCMES-ISO }\end{array}$ \\
\hline G1 & 18 & F9 & $A X$ & 108 & & MD 1-0 & 5 & 1.8 & 2.8 & Tremolite & & & \\
\hline G1 & 18 & F9 & $A X$ & & 115 & MF & 3 & 0.6 & 5 & Tremolite & & & \\
\hline G1 & 18 & F9 & $A X$ & 109 & & CD 2-1 & 8.5 & 4 & 2.1 & Tremolite & & & $A S>5,3: 1$ \\
\hline G1 & 18 & F9 & $A X$ & & 116 & $\mathrm{CB}$ & 6.8 & 1.85 & 3.7 & Tremolite & & & $\begin{array}{c}\text { AFB }>5,3: 1, \text { PCMEF-US, } \\
\text { PCMEF-ISO }\end{array}$ \\
\hline G1 & 18 & F9 & $A X$ & & 117 & $\mathrm{CF}$ & 4.35 & 0.8 & 5.4 & Tremolite & & & \\
\hline G1 & 18 & F9 & $A X$ & 110 & 118 & $\mathrm{~F}$ & 2 & 0.6 & 3.3 & Tremolite & & & \\
\hline G1 & 19 & D9 & $A X$ & 111 & 119 & $\mathrm{~F}$ & 3.85 & 0.5 & 7.7 & Tremolite & & & \\
\hline G1 & 19 & D9 & $A X$ & 112 & 120 & $\mathrm{~F}$ & 27 & 3.5 & 7.7 & Tremolite & & & $\begin{array}{l}\text { AS }>5,3: 1, \text { AFB }>5,3: 1 \\
\text { PCMEF-US, PCMES-US }\end{array}$ \\
\hline G1 & 19 & D9 & $A X$ & 113 & 121 & $F$ & 1.2 & 0.35 & 3.4 & Tremolite & & & \\
\hline G1 & 19 & D9 & $A X$ & 114 & 122 & $\mathrm{~F}$ & 5.2 & 0.75 & 6.9 & Tremolite & & & $\begin{array}{l}\text { AS >5, 3:1, AFB >5, 3:1, } \\
\text { PCMEF-US, PCMES-US, } \\
\text { PCMEF-ISO, PCMES-ISO }\end{array}$ \\
\hline G1 & 19 & D9 & $A X$ & 115 & 123 & $\mathrm{~F}$ & 4 & 0.3 & 13.3 & Tremolite & & & \\
\hline G1 & 20 & B9 & $A X$ & 116 & 124 & $\mathrm{~F}$ & 2.5 & 0.45 & 5.6 & Tremolite & & & \\
\hline G1 & 20 & B9 & $A X$ & 117 & 125 & $\mathrm{~F}$ & 6 & 0.55 & 10.9 & Tremolite & & & $\begin{array}{l}\text { AS }>5,3: 1, \text { AFB }>5,3: 1 \text {, } \\
\text { PCMEF-US, PCMES-US, } \\
\text { PCMEF-ISO, PCMES-ISO }\end{array}$ \\
\hline $\mathrm{G} 1$ & 20 & B9 & $A X$ & 118 & 126 & $F$ & 13 & 3 & 4.3 & Tremolite & & & $\begin{array}{l}\text { PCMEF-US, PCMES-US, } \\
\text { PCMEF-ISO, PCMES-ISO }\end{array}$ \\
\hline
\end{tabular}

Count Categories

$\mathrm{AFB}>5,3: 1$

PAS

PCMEF-US

Asbestos Fibers and Bundles > 5um and 3:

Primary Asbestos Structures

PCM Equivalent Fibers-NIOSH

PCMES-US
AS $>5,3: 1$ PCMEF-ISO PCMES-ISO TAS
Asbestos Structures $>5$ um and 3:1 PCM Equivalent Fibers-ISO PCM Equivalent Structures-ISO Total Asbestos Structures

Page 114 of 281 
ISO 10312, Direct Raw Data

Job Number: 070434

SEA

Report Number: 070434R06

Client: Idaho National Laboratory

Date Received: 4/23/2007

Project Name: RARE

Client Sample No: FB-2-R4

Lab/Cor Sample No: S29

Client Description:

Date Sampled:

4/16/2007

\begin{tabular}{|c|c|c|c|c|c|c|c|c|c|c|c|c|}
\hline Gr & No. & Loc. & ID & Prim & Tot & Class & Len & Wid & Asp & Analyte & Comment & Count Categories \\
\hline G2 & 21 & $\mathrm{~A} 2$ & $A X$ & 119 & 127 & $F$ & 7.5 & 0.45 & 16.7 & Tremolite & & $\begin{array}{l}\text { AS }>5,3: 1, \text { AFB }>5,3: 1, \\
\text { PCMEF-US, PCMES-US, } \\
\text { PCMEF-ISO, PCMES-ISO }\end{array}$ \\
\hline G2 & 21 & $\mathrm{~A} 2$ & $A X$ & 120 & & MD 1-0 & 5.2 & 2.5 & 2.1 & Tremolite & & $A S>5,3: 1$ \\
\hline G2 & 21 & $\mathrm{~A} 2$ & $A X$ & & 128 & MF & 3.2 & 0.5 & 6.4 & Tremolite & & \\
\hline G2 & 21 & $\mathrm{~A} 2$ & $A X$ & 121 & 129 & $\mathrm{~F}$ & 1.75 & 0.5 & 3.5 & Tremolite & & \\
\hline G2 & 22 & $\mathrm{C} 2$ & $A X$ & 122 & 130 & $\mathrm{~F}$ & 6 & 1.25 & 4.8 & Tremolite & & $\begin{array}{l}\text { PCMEF-US, PCMES-US, } \\
\text { PCMEF-ISO, PCMES-ISO }\end{array}$ \\
\hline G2 & 22 & $\mathrm{C} 2$ & $A X$ & 123 & 131 & $\mathrm{~F}$ & 2.2 & 0.75 & 2.9 & Tremolite & & \\
\hline G2 & 22 & $\mathrm{C} 2$ & $A X$ & 124 & 132 & $\mathrm{~F}$ & 5.1 & 1 & 5.1 & Tremolite & & $\begin{array}{l}\text { AS }>5,3: 1, \text { AFB }>5,3: 1 \text {, } \\
\text { PCMEF-US, PCMES-US, } \\
\text { PCMEF-ISO, PCMES-ISO }\end{array}$ \\
\hline G2 & 22 & $\mathrm{C} 2$ & $A X$ & 125 & 133 & $\mathrm{~F}$ & 14.2 & 1.8 & 7.9 & Tremolite & & $\begin{array}{l}\text { AFB }>5,3: 1, \text { AS }>5,3: 1, \\
\text { PCMEF-US, PCMES-US, } \\
\text { PCMEF-ISO, PCMES-ISO }\end{array}$ \\
\hline G2 & 22 & $\mathrm{C} 2$ & $A X$ & 126 & 134 & $F$ & 7.7 & 2 & 3.8 & Tremolite & & $\begin{array}{l}\text { PCMEF-US, PCMES-US, } \\
\text { PCMEF-ISO, PCMES-ISO }\end{array}$ \\
\hline G2 & 22 & $\mathrm{C} 2$ & $A X$ & 127 & 135 & $F$ & 7 & 1.1 & 6.4 & Tremolite & & $\begin{array}{l}\text { AS }>5,3: 1, \text { AFB }>5,3: 1, \\
\text { PCMEF-US, PCMES-US, } \\
\text { PCMEF-ISO, PCMES-ISO }\end{array}$ \\
\hline G2 & 22 & $\mathrm{C} 2$ & $A X$ & 128 & 136 & $\mathrm{~F}$ & 12 & 0.65 & 18.5 & Tremolite & & $\begin{array}{l}\text { AS }>5,3: 1, \text { AFB }>5,3: 1 \text {, } \\
\text { PCMEF-US, PCMES-US, } \\
\text { PCMEF-ISO, PCMES-ISO }\end{array}$ \\
\hline G2 & 23 & E2 & $A X$ & 129 & 137 & $F$ & 6.85 & 1.8 & 3.8 & Tremolite & & $\begin{array}{l}\text { PCMEF-US, PCMES-US, } \\
\text { PCMEF-ISO, PCMES-ISO }\end{array}$ \\
\hline G2 & 23 & E2 & $A X$ & 130 & 138 & $F$ & 7.5 & 1.5 & 5 & Tremolite & & $\begin{array}{l}\text { AS >5, 3:1, AFB >5, 3:1, } \\
\text { PCMEF-US, PCMES-US, } \\
\text { PCMEF-ISO, PCMES-ISO }\end{array}$ \\
\hline G2 & 23 & E2 & $A X$ & 131 & 139 & $F$ & 1.75 & 0.2 & 8.8 & Tremolite & & \\
\hline G2 & 23 & E2 & $A X$ & 132 & 140 & $\mathrm{~F}$ & 7.2 & 0.75 & 9.6 & Tremolite & & $\begin{array}{l}\text { AS }>5,3: 1, \text { AFB }>5,3: 1, \\
\text { PCMEF-US, PCMES-US, } \\
\text { PCMEF-ISO, PCMES-ISO }\end{array}$ \\
\hline G2 & 24 & G2 & $A X$ & 133 & 141 & $F$ & 2.1 & 0.7 & 3 & Tremolite & & \\
\hline G2 & 24 & G2 & $A X$ & 134 & 142 & $\mathrm{~F}$ & 4 & 0.55 & 7.3 & Tremolite & & \\
\hline G2 & 24 & G2 & $A X$ & 135 & 143 & $\mathrm{~F}$ & 13 & 0.4 & 32.5 & Tremolite & & $\begin{array}{l}\text { AS }>5,3: 1, \text { AFB }>5,3: 1, \\
\text { PCMEF-US, PCMES-US, } \\
\text { PCMEF-ISO, PCMES-ISO }\end{array}$ \\
\hline G2 & 24 & G2 & $A X$ & 136 & 144 & $F$ & 5 & 0.5 & 10 & Tremolite & & \\
\hline G2 & 24 & G2 & $A X$ & 137 & 145 & $F$ & 5.2 & 1.12 & 4.6 & Tremolite & & $\begin{array}{l}\text { PCMEF-US, PCMES-US, } \\
\text { PCMEF-ISO, PCMES-ISO }\end{array}$ \\
\hline G2 & 24 & G2 & $A X$ & 138 & 146 & $F$ & 17 & 1.5 & 11.3 & Tremolite & & $\begin{array}{l}\text { AS>5, 3:1, AFB>5, 3:1, } \\
\text { PCMEF-US, PCMES-US, } \\
\text { PCMEF-ISO, PCMES-ISO }\end{array}$ \\
\hline G2 & 25 & 12 & $A X$ & 139 & 147 & $F$ & 20.8 & 1.2 & 17.3 & Tremolite & & $\begin{array}{l}\text { AS }>5,3: 1, \text { AFB }>5,3: 1, \\
\text { PCMEF-US, PCMES-US, } \\
\text { PCMEF-ISO, PCMES-ISO }\end{array}$ \\
\hline \multicolumn{12}{|c|}{ Count Categories } & Page 115 of 281 \\
\hline \multicolumn{3}{|c|}{ AFB $>5,3: 1$} & \multicolumn{5}{|c|}{ Asbestos Fibers and Bundles $>5 \mathrm{um}$ and $3: 1$} & \multicolumn{2}{|c|}{$A S>5,3: 1$} & \multicolumn{3}{|c|}{ Asbestos Structures $>5$ um and 3:1 } \\
\hline \multicolumn{3}{|c|}{ PAS } & \multicolumn{5}{|c|}{ Primary Asbestos Structures } & \multicolumn{2}{|c|}{ PCMEF-ISO } & \multicolumn{3}{|c|}{ PCM Equivalent Fibers-ISO } \\
\hline \multicolumn{3}{|c|}{ PCMEF-US } & \multicolumn{5}{|c|}{ PCM Equivalent Fibers-NIOSH } & \multicolumn{2}{|c|}{ PCMES-ISO } & \multicolumn{3}{|c|}{ PCM Equivalent Structures-ISO } \\
\hline \multicolumn{3}{|c|}{ PCMES-US } & \multicolumn{5}{|c|}{ PCM Equivalent Structures-NIOSH } & \multicolumn{2}{|l|}{ TAS } & \multicolumn{3}{|c|}{ Total Asbestos Structures } \\
\hline
\end{tabular}


ISO 10312, Direct Raw Data

Job Number: 070434

SEA

Report Number: 070434R06

Client: Idaho National Laboratory

Date Received: 4/23/2007

Project Name: RARE

Client Sample No: FB-2-R4

Lab/Cor Sample No: S29

Client Description:

Date Sampled:

$4 / 16 / 2007$

\begin{tabular}{|c|c|c|c|c|c|c|c|c|c|c|c|c|c|}
\hline Gr & No. & Loc. & ID & Prim & Tot & Class & Len & Wid & Asp & Analyte & Elements & Comment & Count Categories \\
\hline G2 & 25 & 12 & $A X$ & 140 & 148 & $\mathrm{~F}$ & 7.2 & 0.8 & 9 & Tremolite & & & $\begin{array}{l}\text { AS }>5,3: 1, \text { AFB }>5,3: 1, \\
\text { PCMEF-US, PCMES-US, } \\
\text { PCMEF-ISO, PCMES-ISO }\end{array}$ \\
\hline G2 & 25 & 12 & $A X$ & 141 & 149 & $\mathrm{~F}$ & 4.35 & 0.75 & 5.8 & Tremolite & & & \\
\hline G2 & 25 & 12 & $A X$ & 142 & 150 & $\mathrm{~F}$ & 12.7 & 0.65 & 19.5 & Tremolite & & & $\begin{array}{l}\text { AS }>5,3: 1, \text { AFB }>5,3: 1, \\
\text { PCMEF-US, PCMES-US, } \\
\text { PCMEF-ISO, PCMES-ISO }\end{array}$ \\
\hline G2 & 26 & J4 & $A X$ & 143 & 151 & $\mathrm{~F}$ & 6.5 & 1.2 & 5.4 & Tremolite & & & $\begin{array}{l}\text { AS >5, 3:1, AFB >5, 3:1, } \\
\text { PCMEF-US, PCMES-US, } \\
\text { PCMEF-ISO, PCMES-ISO }\end{array}$ \\
\hline G2 & 26 & J4 & $A X$ & 144 & 152 & $\mathrm{~F}$ & 2.2 & 0.55 & 4 & Tremolite & & & \\
\hline G2 & 26 & J4 & $A X$ & 145 & 153 & $\mathrm{~F}$ & 10.5 & 1.2 & 8.8 & Tremolite & & & $\begin{array}{l}\text { AS>5, 3:1, AFB>5, 3:1, } \\
\text { PCMEF-US, PCMES-US, } \\
\text { PCMEF-ISO, PCMES-ISO }\end{array}$ \\
\hline G2 & 26 & J4 & $A X$ & 146 & 154 & $\mathrm{~F}$ & 2.75 & 0.9 & 3.1 & Tremolite & & & \\
\hline G2 & 26 & J4 & $A X$ & 147 & 155 & $F$ & 23.2 & 1.2 & 19.3 & Tremolite & & & $\begin{array}{l}\text { AS >5, 3:1, AFB>5, 3:1, } \\
\text { PCMEF-US, PCMES-US, } \\
\text { PCMEF-ISO, PCMES-ISO }\end{array}$ \\
\hline G2 & 27 & $\mathrm{H} 4$ & $A X$ & 148 & 156 & $F$ & 11 & 0.38 & 28.9 & Tremolite & & & $\begin{array}{l}\text { AS }>5,3: 1, \text { AFB }>5,3: 1 \text {, } \\
\text { PCMEF-US, PCMES-US, } \\
\text { PCMEF-ISO, PCMES-ISO }\end{array}$ \\
\hline G2 & 27 & $\mathrm{H} 4$ & $A X$ & 149 & 157 & $F$ & 14.35 & 1.28 & 11.2 & Tremolite & & & $\begin{array}{l}\text { AS }>5,3: 1, \text { AFB }>5,3: 1, \\
\text { PCMEF-US, PCMES-US, } \\
\text { PCMEF-ISO, PCMES-ISO }\end{array}$ \\
\hline G2 & 27 & $\mathrm{H} 4$ & $A X$ & 150 & 158 & $F$ & 2.65 & 0.3 & 8.8 & Tremolite & & & \\
\hline G2 & 27 & $\mathrm{H} 4$ & $A X$ & 151 & 159 & $F$ & 1.65 & 0.35 & 4.7 & Tremolite & & & \\
\hline G2 & 27 & $\mathrm{H} 4$ & $A X$ & 152 & 160 & $F$ & 10.7 & 0.65 & 16.5 & Tremolite & & & $\begin{array}{l}\text { AS>5, 3:1, AFB>5, 3:1, } \\
\text { PCMEF-US, PCMES-US, } \\
\text { PCMEF-ISO, PCMES-ISO }\end{array}$ \\
\hline G2 & 27 & $\mathrm{H} 4$ & $A X$ & 153 & 161 & $F$ & 10.65 & 1.2 & 8.9 & Tremolite & & & $\begin{array}{l}\text { AS }>5,3: 1, \text { AFB }>5,3: 1 \text {, } \\
\text { PCMEF-US, PCMES-US, } \\
\text { PCMEF-ISO, PCMES-ISO }\end{array}$ \\
\hline G2 & 28 & F4 & $A X$ & 154 & 162 & $F$ & 4 & 0.85 & 4.7 & Tremolite & & & \\
\hline G2 & 28 & $\mathrm{~F} 4$ & $A X$ & 155 & 163 & $F$ & 3.85 & 0.6 & 6.4 & Tremolite & & & \\
\hline G2 & 28 & $\mathrm{~F} 4$ & $A X$ & 156 & 164 & $\mathrm{~F}$ & 12 & 2 & 6 & Tremolite & & & $\begin{array}{l}\text { AS>5, 3:1, AFB>5, 3:1, } \\
\text { PCMEF-US, PCMES-US, } \\
\text { PCMEF-ISO, PCMES-ISO }\end{array}$ \\
\hline G2 & 28 & $\mathrm{~F} 4$ & $A X$ & 157 & 165 & $F$ & 3.15 & 0.45 & 7 & Tremolite & & & \\
\hline G2 & 29 & D4 & $A X$ & 158 & 166 & $F$ & 5.8 & 1 & 5.8 & Tremolite & & & $\begin{array}{l}\text { AS }>5,3: 1, \text { AFB }>5,3: 1, \\
\text { PCMEF-US, PCMES-US, } \\
\text { PCMEF-ISO, PCMES-ISO }\end{array}$ \\
\hline G2 & 29 & D4 & $A X$ & 159 & 167 & $F$ & 5 & 1.1 & 4.5 & Tremolite & & & \\
\hline G2 & 29 & D4 & $A X$ & 160 & 168 & $\mathrm{~F}$ & 18.5 & 1.2 & 15.4 & Tremolite & & & $\begin{array}{l}\text { AS>5, 3:1, AFB>5, 3:1, } \\
\text { PCMEF-US, PCMES-US, } \\
\text { PCMEF-ISO, PCMES-ISO }\end{array}$ \\
\hline
\end{tabular}

Count Categories

$\mathrm{AFB}>5,3: 1$

PAS

PCMEF-US

Asbestos Fibers and Bundles > 5um and 3:1

Primary Asbestos Structures

PCM Equivalent Fibers-NIOSH

PCMES-US
AS>5, 3:1 PCMEF-ISO PCMES-ISO TAS
Asbestos Structures >5um and 3:1 PCM Equivalent Fibers-ISO PCM Equivalent Structures-ISO Total Asbestos Structures

Page 116 of 281 
ISO 10312, Direct Raw Data

Job Number: 070434

SEA

Report Number: 070434R06

Client: Idaho National Laboratory

Date Received: 4/23/2007

Project Name: RARE

Client Sample No: FB-2-R4

Lab/Cor Sample No: S29

Client Description:

Date Sampled:

4/16/2007

\begin{tabular}{|c|c|c|c|c|c|c|c|c|c|c|c|c|c|}
\hline Gr & No. & Loc. & ID & Prim & Tot & Class & Len & Wid & Asp & Analyte & Elements & Comment & Count Categories \\
\hline G2 & 29 & D4 & $A X$ & 161 & 169 & $\mathrm{~F}$ & 2.5 & 0.45 & 5.6 & Tremolite & & & \\
\hline G2 & 29 & D4 & $A X$ & 162 & 170 & $\mathrm{~F}$ & 10.5 & 1.2 & 8.8 & Tremolite & & & $\begin{array}{l}\text { AS }>5,3: 1, \text { AFB }>5,3: 1, \\
\text { PCMEF-US, PCMES-US, } \\
\text { PCMEF-ISO, PCMES-ISO }\end{array}$ \\
\hline G2 & 29 & D4 & $A X$ & 163 & 171 & $\mathrm{~F}$ & 10 & 2 & 5 & Tremolite & & & $\begin{array}{l}\text { AS>5, 3:1, AFB>5, 3:1, } \\
\text { PCMEF-US, PCMES-US, } \\
\text { PCMEF-ISO, PCMES-ISO }\end{array}$ \\
\hline
\end{tabular}

$\begin{array}{lllllllcccr}\text { G2 } & 29 & \text { D4 } & \text { AX } & 164 & 172 & \text { F } & 3 & 1 & 3 & \text { Tremolite } \\ \text { G2 } & 30 & \text { B4 } & \text { AX } & 165 & 173 & \text { F } & 7.7 & 1.5 & 5.1 & \text { Tremolite }\end{array}$

$\begin{array}{lllllllll}B & A X & 165 & 173 & F & 7.7 & 1.5 & 5.1 & \text { Tremolte }\end{array}$

$\begin{array}{lllllllclll}\text { G2 } & 30 & \mathrm{~B} 4 & \mathrm{AX} & 166 & 174 & \mathrm{~F} & 2 & 0.6 & 3.3 & \text { Tremolite } \\ \text { G2 } & 30 & \mathrm{~B} 4 & \mathrm{AX} & 167 & 175 & \mathrm{~F} & 4.25 & 0.6 & 7.1 & \text { Tremolite } \\ \text { G2 } & 30 & \mathrm{~B} 4 & \mathrm{AX} & 168 & 176 & \mathrm{~F} & 5.6 & 1 & 5.6 & \text { Tremolite } \\ & & & & & & & & & & \\ \text { G2 } & 31 & \mathrm{~A} 7 & \mathrm{AX} & 169 & 177 & \mathrm{~F} & 2.7 & 0.2 & 13.5 & \text { Tremolite } \\ \mathrm{G} 2 & 31 & \mathrm{~A} 7 & \mathrm{AX} & 170 & 178 & \mathrm{~F} & 4 & 0.4 & 10 & \text { Tremolite } \\ \mathrm{G} 2 & 31 & \mathrm{~A} 7 & \mathrm{AX} & 171 & 179 & \mathrm{~F} & 5.8 & 1.25 & 4.6 & \text { Tremolite } \\ \mathrm{G} 2 & 32 & \mathrm{C} 7 & \mathrm{AX} & 172 & 180 & \mathrm{~F} & 6.55 & 0.4 & 16.4 & \text { Tremolite } \\ & & & & & & & & & & \\ \mathrm{G} 2 & 32 & \mathrm{C} 7 & \mathrm{AX} & 173 & 181 & \mathrm{~F} & 3.35 & 0.38 & 8.8 & \text { Tremolite } \\ \mathrm{G} 2 & 32 & \mathrm{C} 7 & \mathrm{AX} & 174 & & \mathrm{CD} 2-1 & 7 & 4 & 1.8 & \text { Tremolite } \\ \mathrm{G} 2 & 32 & \mathrm{C} 7 & \mathrm{AX} & & 182 & \mathrm{CF} & 5.75 & 1 & 5.8 & \text { Tremolite } \\ \mathrm{G} 2 & 32 & \mathrm{C} 7 & \mathrm{AX} & & 183 & \mathrm{CF} & 4.3 & 0.38 & 11.3 & \text { Tremolite } \\ \mathrm{G} 2 & 32 & \mathrm{C} 7 & \mathrm{AX} & 175 & 184 & \mathrm{~F} & 3.85 & 0.4 & 9.6 & \text { Tremolite } \\ \mathrm{G} 2 & 32 & \mathrm{C} 7 & \mathrm{AX} & 176 & 185 & \mathrm{~F} & 6.2 & 1.85 & 3.4 & \text { Tremolite } \\ \mathrm{G} 2 & 32 & \mathrm{C} 7 & \mathrm{AX} & 177 & 186 & \mathrm{~F} & 5.6 & 1.2 & 4.7 & \text { Tremolite } \\ \mathrm{G} 2 & 32 & \mathrm{C} 7 & \mathrm{AX} & 178 & 187 & \mathrm{~F} & 2.6 & 0.7 & 3.7 & \text { Tremolite } \\ \mathrm{G} 2 & 33 & \mathrm{E} 7 & \mathrm{AX} & 179 & 188 & \mathrm{~F} & 4 & 0.5 & 8 & \text { Tremolite } \\ \mathrm{G} 2 & 33 & \mathrm{E} 7 & \mathrm{AX} & 180 & 189 & \mathrm{~F} & 3.2 & 0.75 & 4.3 & \text { Tremolite } \\ \mathrm{G} 2 & 33 & \mathrm{E} 7 & \mathrm{AX} & 181 & 190 & \mathrm{~F} & 4.2 & 0.6 & 7 & \text { Tremolite } \\ \mathrm{G} 2 & 33 & \mathrm{E} 7 & \mathrm{AX} & 182 & 191 & \mathrm{~F} & 1.3 & 0.38 & 3.4 & \text { Tremolite } \\ \mathrm{G} 2 & 33 & \mathrm{E} 7 & \mathrm{AX} & 183 & 192 & \mathrm{~F} & 17.2 & 1.8 & 9.6 & \text { Tremolite }\end{array}$

AS $>5,3: 1, A F B>5,3: 1$ PCMEF-US, PCMES-US, PCMEF-ISO, PCMES-ISO

$A S>5,3: 1, A F B>5,3: 1$ PCMEF-US, PCMES-US PCMEF-ISO, PCMES-ISO

PCMEF-US, PCMES-US PCMEF-ISO, PCMES-ISO

$A S>5,3: 1, A F B>5,3: 1$ PCMEF-US, PCMES-US,

PCMES-ISO, PCMEF-ISO

AS $>5,3: 1$

AFB $>5,3: 1$, PCMEF-US, PCMEF-ISO

PCMEF-US, PCMES-US PCMEF-ISO, PCMES-ISO PCMEF-US, PCMES-US, PCMEF-ISO, PCMES-ISO

AS $>5,3: 1, A F B>5,3: 1$ PCMEF-US, PCMES-US, PCMEF-ISO, PCMES-ISO

Count Categories

$\mathrm{AFB}>5,3: 1$

PAS

PCMEF-US

Asbestos Fibers and Bundles $>5 u m$ and 3:1

Primary Asbestos Structures

PCM Equivalent Fibers-NIOSH

PCMES-US
AS>5, 3:1 PCMEF-ISO PCMES-ISO TAS
Asbestos Structures $>5$ um and 3:1 PCM Equivalent Fibers-ISO PCM Equivalent Structures-ISO Total Asbestos Structures 
ISO 10312, Direct Raw Data

Job Number: 070434

SEA

Report Number: 070434R06

Client: Idaho National Laboratory

Date Received: 4/23/2007

Project Name: RARE

Client Sample No: FB-2-R4

Lab/Cor Sample No: S29

Client Description:

Date Sampled:

4/16/2007

\begin{tabular}{|c|c|c|c|c|c|c|c|c|c|c|c|c|c|}
\hline $\mathbf{G r}$ & No. & Loc. & ID & Prim & Tot & Class & Len & Wid & Asp & Analyte & Elements & Comment & Count Categories \\
\hline G2 & 33 & E7 & $A X$ & 184 & 193 & $F$ & 5.5 & 0.8 & 6.9 & Tremolite & & & $\begin{array}{l}\text { AS }>5,3: 1, \text { AFB }>5,3: 1, \\
\text { PCMEF-US, PCMES-US, } \\
\text { PCMEF-ISO, PCMES-ISO }\end{array}$ \\
\hline G2 & 33 & E7 & $A X$ & 185 & 194 & B & 40 & 3 & 13.3 & Tremolite & & & $\begin{array}{l}\text { AFB }>5,3: 1, \text { AS }>5,3: 1, \\
\text { PCMES-US, PCMEF-US, } \\
\text { PCMES-ISO, PCMEF-ISO }\end{array}$ \\
\hline G2 & 33 & E7 & $A X$ & 186 & 195 & $\mathrm{~F}$ & 11.2 & 0.85 & 13.2 & Tremolite & & & $\begin{array}{l}\text { AS >5, 3:1, AFB >5, 3:1, } \\
\text { PCMEF-US, PCMES-US, } \\
\text { PCMEF-ISO, PCMES-ISO }\end{array}$ \\
\hline G2 & 33 & E7 & $A X$ & 187 & 196 & $\mathrm{~F}$ & 3.2 & 0.7 & 4.6 & Tremolite & & & \\
\hline G2 & 34 & G7 & $A X$ & 188 & 197 & $\mathrm{~F}$ & 3 & 0.8 & 3.8 & Tremolite & & & \\
\hline G2 & 34 & G7 & $A X$ & 189 & 198 & $\mathrm{~F}$ & 14 & 0.75 & 18.7 & Tremolite & & & $\begin{array}{l}\text { AS }>5,3: 1, \text { AFB }>5,3: 1, \\
\text { PCMEF-US, PCMES-US, } \\
\text { PCMEF-ISO, PCMES-ISO }\end{array}$ \\
\hline G2 & 34 & G7 & $A X$ & 190 & 199 & $\mathrm{~F}$ & 60 & 4 & 15 & Tremolite & & & $\begin{array}{l}\text { AS }>5,3: 1, \text { AFB }>5,3: 1 \\
\text { PCMEF-US, PCMES-US }\end{array}$ \\
\hline G2 & 34 & G7 & $A X$ & 191 & 200 & $\mathrm{~F}$ & 10.5 & 1.85 & 5.7 & Tremolite & & & $\begin{array}{l}\text { AS>5, 3:1, AFB>5, 3:1, } \\
\text { PCMEF-US, PCMES-US, } \\
\text { PCMEF-ISO, PCMES-ISO }\end{array}$ \\
\hline G2 & 34 & G7 & $A X$ & 192 & 201 & $\mathrm{~F}$ & 5 & 1.2 & 4.2 & Tremolite & & & \\
\hline G2 & 34 & G7 & $A X$ & 193 & & MD 1-0 & 7.5 & 3 & 2.5 & Tremolite & & & $A S>5,3: 1$ \\
\hline G2 & 34 & G7 & $A X$ & & 202 & MF & 5 & 0.4 & 12.5 & Tremolite & & & \\
\hline G2 & 35 & 17 & $A X$ & 194 & 203 & $\mathrm{~F}$ & 5.25 & 0.7 & 7.5 & Tremolite & & & $\begin{array}{l}\text { AS }>5,3: 1, \text { AFB }>5,3: 1, \\
\text { PCMEF-US, PCMES-US, } \\
\text { PCMEF-ISO, PCMES-ISO }\end{array}$ \\
\hline G2 & 35 & 17 & $A X$ & 195 & 204 & $\mathrm{~F}$ & 18 & 4.5 & 4 & Tremolite & & & PCMEF-US, PCMES-US \\
\hline
\end{tabular}

Count Categories

AFB $>5,3: 1$

PAS

PCMEF-US

PCMES-US
Asbestos Fibers and Bundles > 5um and 3:1

Primary Asbestos Structures

PCM Equivalent Fibers-NIOSH

PCM Equivalent Structures-NIOSH
AS $>5,3: 1$ PCMEF-ISO PCMES-ISO TAS
Asbestos Structures $>5$ um and 3:1

PCM Equivalent Fibers-ISO

PCM Equivalent Structures-ISO

Total Asbestos Structures 
ISO 10312, Direct Raw Data

Job Number: 070434

SEA

Report Number: 070434R06

Client: Idaho National Laboratory

Date Received: 4/23/2007

Project Name: RARE

Client Sample No: FB-2-R5

Lab/Cor Sample No: S30

Client Description:

Date Sampled:

4/16/2007

\begin{tabular}{|c|c|c|c|c|c|c|c|c|c|c|c|c|c|}
\hline Gr & No. & Loc. & ID & Prim & Tot & Class & Len & Wid & Asp & Analyte & Elements & Comment & Count Categories \\
\hline G1 & 1 & $\mathrm{~A} 2$ & $A X$ & 1 & 1 & $\mathrm{~F}$ & 1.7 & 0.22 & 7.7 & Tremolite & & & \\
\hline G1 & 1 & $\mathrm{~A} 2$ & $A X$ & 2 & 2 & $\mathrm{~F}$ & 1.6 & 0.2 & 8 & Tremolite & & & \\
\hline \multirow[t]{5}{*}{ G1 } & 1 & $\mathrm{~A} 2$ & $A D Q$ & 3 & 3 & $\mathrm{~F}$ & 13 & 0.65 & 20 & Tremolite & $\begin{array}{c}\mathrm{Mg}, \mathrm{Al}, \mathrm{Si}, \\
\mathrm{Ca}, \mathrm{Fe}\end{array}$ & & $\begin{array}{l}\text { AS }>5,3: 1, \text { AFB }>5,3: 1, \\
\text { PCMEF-US, PCMES-US, } \\
\text { PCMEF-ISO, PCMES-ISO }\end{array}$ \\
\hline & & & & & & \multicolumn{2}{|c|}{ ItemType } & ItemNc & & & Confirn & Com & \\
\hline & & & & & & \multicolumn{2}{|c|}{ Brightfield } & J3579 & & & & & \\
\hline & & & & & & \multicolumn{2}{|c|}{ Diffraction } & J3579 & & & $\mathrm{KM} \quad 6 / 5$ & 2007 & \\
\hline & & & & & & \multicolumn{2}{|c|}{ Spectra } & J3020 & & & & & \\
\hline
\end{tabular}

\begin{tabular}{|c|c|c|c|c|c|c|c|c|c|c|}
\hline G1 & 1 & $\mathrm{~A} 2$ & $A X$ & 4 & & MD 1-1 & 5.8 & 3 & 1.9 & Tremolite \\
\hline G1 & 1 & $\mathrm{~A} 2$ & $A X$ & & 4 & MF & 5.8 & 0.38 & 15.3 & Tremolite \\
\hline G1 & 1 & A2 & $A X$ & 5 & 5 & $\mathrm{~F}$ & 16.35 & 1.85 & 8.8 & Tremolite \\
\hline G1 & 1 & $\mathrm{~A} 2$ & $A X$ & 6 & 6 & $F$ & 4 & 0.5 & 8 & Tremolite \\
\hline G1 & 1 & $\mathrm{~A} 2$ & $A X$ & 7 & 7 & $\mathrm{~F}$ & 13.6 & 1.2 & 11.3 & Tremolite \\
\hline G1 & 2 & $\mathrm{C} 2$ & $A X$ & 8 & & MD 1-1 & 7.5 & 5.2 & 1.4 & Tremolite \\
\hline G1 & 2 & $\mathrm{C} 2$ & $A X$ & & 8 & MF & 5.5 & 0.18 & 30.6 & Tremolite \\
\hline G1 & 2 & $\mathrm{C} 2$ & $A X$ & 9 & 9 & $\mathrm{~F}$ & 2.5 & 0.35 & 7.1 & Tremolite \\
\hline G1 & 2 & $\mathrm{C} 2$ & $A X$ & 10 & 10 & $\mathrm{~F}$ & 3 & 0.5 & 6 & Tremolite \\
\hline G1 & 2 & $\mathrm{C} 2$ & $A X$ & 11 & 11 & $F$ & 1.8 & 0.38 & 4.7 & Tremolite \\
\hline G1 & 3 & E2 & $A X$ & 12 & 12 & $F$ & 2 & 0.4 & 5 & Tremolite \\
\hline G1 & 3 & E2 & $A X$ & 13 & 13 & $F$ & 8.25 & 0.85 & 9.7 & Tremolite \\
\hline G1 & 3 & E2 & $A X$ & 14 & 14 & $\mathrm{~F}$ & 4.5 & 1 & 4.5 & Tremolite \\
\hline G1 & 3 & E2 & $A X$ & 15 & 15 & $F$ & 12.5 & 1.8 & 6.9 & Tremolite \\
\hline G1 & 3 & E2 & $A X$ & 16 & 16 & $\mathrm{~F}$ & 8.75 & 0.55 & 15.9 & Tremolite \\
\hline G1 & 3 & E2 & $A X$ & 17 & & MD 1-0 & 4 & 4 & 1 & Tremolite \\
\hline G1 & 3 & E2 & $A X$ & & 17 & MF & 2.5 & 0.3 & 8.3 & Tremolite \\
\hline G1 & 4 & G2 & $A X$ & 18 & 18 & $F$ & 3.25 & 1.1 & 3 & Tremolite \\
\hline G1 & 4 & G2 & $A X$ & 19 & 19 & $\mathrm{~F}$ & 4.25 & 0.7 & 6.1 & Tremolite \\
\hline G1 & 4 & G2 & $A X$ & 20 & 20 & $F$ & 4.8 & 1 & 4.8 & Tremolite \\
\hline
\end{tabular}

AS>5, $3: 1$

AFB $>5,3: 1$, PCMEF-US, PCMEF-ISO

$A S>5,3: 1, A F B>5,3: 1$ PCMEF-US, PCMES-US PCMEF-ISO, PCMES-ISO

AS $>5,3: 1, A F B>5,3: 1$ PCMEF-US, PCMES-US, PCMEF-ISO, PCMES-ISO $A S>5,3: 1$

$\mathrm{AFB}>5,3: 1$

$A S>5,3: 1, A F B>5,3: 1$ PCMEF-US, PCMES-US PCMEF-ISO, PCMES-ISO

AS $>5,3: 1, A F B>5,3: 1$ PCMEF-US, PCMES-US PCMEF-ISO, PCMES-ISO $A S>5,3: 1, A F B>5,3: 1$ PCMEF-US, PCMES-US, PCMEF-ISO, PCMES-ISO

Page 119 of 281

\section{Count Categories}

AFB $>5,3: 1$

PAS

PCMEF-US

PCMES-US
Asbestos Fibers and Bundles > 5um and 3:1

Primary Asbestos Structures

PCM Equivalent Fibers-NIOSH

PCM Equivalent Structures-NIOSH
AS>5, 3:1 PCMEF-ISO PCMES-ISO TAS
Asbestos Structures >5um and 3:1 PCM Equivalent Fibers-ISO PCM Equivalent Structures-ISO Total Asbestos Structures 
ISO 10312, Direct Raw Data

Job Number: 070434

SEA

Report Number: 070434R06

Client: Idaho National Laboratory

Date Received: 4/23/2007

Project Name: RARE

Client Sample No: FB-2-R5

Lab/Cor Sample No: S30

Client Description:

Date Sampled:

4/16/2007

\begin{tabular}{|c|c|c|c|c|c|c|c|c|c|c|c|c|c|}
\hline$\overline{\mathrm{Gr}}$ & No. & Loc. & ID & Prim & Tot & Class & Len & Wid & Asp & Analyte & Elements & Comment & Count Categories \\
\hline G1 & 4 & G2 & $A X$ & 21 & 21 & $\mathrm{~F}$ & 2.65 & 0.38 & 7 & Tremolite & & & \\
\hline G1 & 4 & G2 & $A X$ & 22 & 22 & $\mathrm{~F}$ & 2.4 & 0.18 & 13.3 & Tremolite & & & \\
\hline G1 & 5 & 12 & $A X$ & 23 & 23 & $\mathrm{~F}$ & 5 & 0.65 & 7.7 & Tremolite & & & \\
\hline G1 & 5 & 12 & $A X$ & 24 & 24 & $\mathrm{~F}$ & 3 & 0.65 & 4.6 & Tremolite & & & \\
\hline G1 & 5 & 12 & $A X$ & 25 & 25 & $\mathrm{~F}$ & 4 & 0.9 & 4.4 & Tremolite & & & \\
\hline G1 & 5 & 12 & $A X$ & 26 & 26 & $\mathrm{~F}$ & 3 & 0.5 & 6 & Tremolite & & & \\
\hline G1 & 5 & 12 & $A X$ & 27 & 27 & $\mathrm{~F}$ & 1.65 & 0.38 & 4.3 & Tremolite & & & \\
\hline G1 & 5 & 12 & $A X$ & 28 & 28 & $\mathrm{~F}$ & 9.65 & 2.5 & 3.9 & Tremolite & & & $\begin{array}{l}\text { PCMEF-US, PCMES-US, } \\
\text { PCMEF-ISO, PCMES-ISO }\end{array}$ \\
\hline G1 & 6 & J4 & $A X$ & 29 & 29 & $\mathrm{~F}$ & 9.25 & 0.6 & 15.4 & Tremolite & & & $\begin{array}{l}\text { AS }>5,3: 1, \text { AFB }>5,3: 1, \\
\text { PCMEF-US, PCMES-US, } \\
\text { PCMEF-ISO, PCMES-ISO }\end{array}$ \\
\hline G1 & 6 & J4 & $A X$ & 30 & 30 & $\mathrm{~F}$ & 6.12 & 0.7 & 8.7 & Tremolite & & & $\begin{array}{l}\text { AS }>5,3: 1, \text { AFB }>5,3: 1 \text {, } \\
\text { PCMEF-US, PCMES-US, } \\
\text { PCMEF-ISO, PCMES-ISO }\end{array}$ \\
\hline G1 & 6 & $\mathrm{~J} 4$ & $A X$ & 31 & & CD 2-1 & 5.5 & 4 & 1.4 & Tremolite & & & $A S>5,3: 1$ \\
\hline G1 & 6 & J4 & $A X$ & & 31 & $\mathrm{CF}$ & 5.5 & 0.3 & 18.3 & Tremolite & & & $\begin{array}{c}\text { AFB }>5,3: 1, \text { PCMEF-US, } \\
\text { PCMEF-ISO }\end{array}$ \\
\hline G1 & 6 & J4 & $A X$ & & 32 & CF & 4 & 0.75 & 5.3 & Tremolite & & & \\
\hline G1 & 7 & $\mathrm{H} 4$ & $A X$ & 32 & 33 & $\mathrm{~F}$ & 8.8 & 0.8 & 11 & Tremolite & & & $\begin{array}{l}\text { AS }>5,3: 1, \text { AFB }>5,3: 1 \text {, } \\
\text { PCMEF-US, PCMES-US, } \\
\text { PCMEF-ISO, PCMES-ISO }\end{array}$ \\
\hline G1 & 7 & $\mathrm{H} 4$ & $A X$ & 33 & 34 & $\mathrm{~F}$ & 5.4 & 0.5 & 10.8 & Tremolite & & & $\begin{array}{l}\text { AS }>5,3: 1, \text { AFB }>5,3: 1, \\
\text { PCMEF-US, PCMES-US, } \\
\text { PCMEF-ISO, PCMES-ISO }\end{array}$ \\
\hline G1 & 8 & $\mathrm{~F} 4$ & $A X$ & 34 & 35 & $\mathrm{~F}$ & 5.25 & 0.8 & 6.6 & Tremolite & & & $\begin{array}{l}\text { AS >5, 3:1, AFB>5, 3:1, } \\
\text { PCMEF-US, PCMES-US, } \\
\text { PCMEF-ISO, PCMES-ISO }\end{array}$ \\
\hline G1 & 8 & $\mathrm{~F} 4$ & $A X$ & 35 & 36 & $\mathrm{~F}$ & 4 & 0.8 & 5 & Tremolite & & & \\
\hline G1 & 8 & $\mathrm{~F} 4$ & $A X$ & 36 & 37 & $\mathrm{~F}$ & 4.8 & 0.75 & 6.4 & Tremolite & & & \\
\hline G1 & 9 & D4 & $A X$ & 37 & 38 & $\mathrm{~F}$ & 2.5 & 0.6 & 4.2 & Tremolite & & & \\
\hline G1 & 9 & D4 & $A X$ & 38 & 39 & B & 9 & 2.8 & 3.2 & Tremolite & & & $\begin{array}{l}\text { AFB }>5,3: 1, \text { AS }>5,3: 1, \\
\text { PCMES-US, PCMEF-US, } \\
\text { PCMES-ISO, PCMEF-ISO }\end{array}$ \\
\hline G1 & 9 & D4 & $A X$ & 39 & 40 & $\mathrm{~F}$ & 1.65 & 0.3 & 5.5 & Tremolite & & & \\
\hline G1 & 9 & D4 & $A X$ & 40 & 41 & $\mathrm{~F}$ & 2 & 0.3 & 6.7 & Tremolite & & & \\
\hline G1 & 9 & D4 & $A X$ & 41 & 42 & $\mathrm{~F}$ & 3.1 & 0.35 & 8.9 & Tremolite & & & \\
\hline G1 & 9 & D4 & $A X$ & 42 & 43 & $\mathrm{~F}$ & 1 & 0.2 & 5 & Tremolite & & & \\
\hline G1 & 9 & D4 & $A X$ & 43 & 44 & $F$ & 5.3 & 0.8 & 6.6 & Tremolite & & & $\begin{array}{l}\text { AS }>5,3: 1, \text { AFB }>5,3: 1, \\
\text { PCMEF-US, PCMES-US, } \\
\text { PCMEF-ISO, PCMES-ISO }\end{array}$ \\
\hline
\end{tabular}

Count Categories

$\mathrm{AFB}>5,3: 1$

PAS

PCMEF-US

Asbestos Fibers and Bundles > 5um and 3:

Primary Asbestos Structures

PCM Equivalent Fibers-NIOSH

PCMES-US
AS>5, 3:1 PCMEF-ISO PCMES-ISO TAS
Asbestos Structures $>5$ um and 3:1 PCM Equivalent Fibers-ISO PCM Equivalent Structures-ISO Total Asbestos Structures 


\section{ISO 10312, Direct Raw Data}

Job Number: 070434

SEA

Report Number: 070434R06

Client: Idaho National Laboratory

Date Received: 4/23/2007

Project Name: RARE

Client Sample No: FB-2-R5

Lab/Cor Sample No: S30

Client Description:

Date Sampled:

$4 / 16 / 2007$

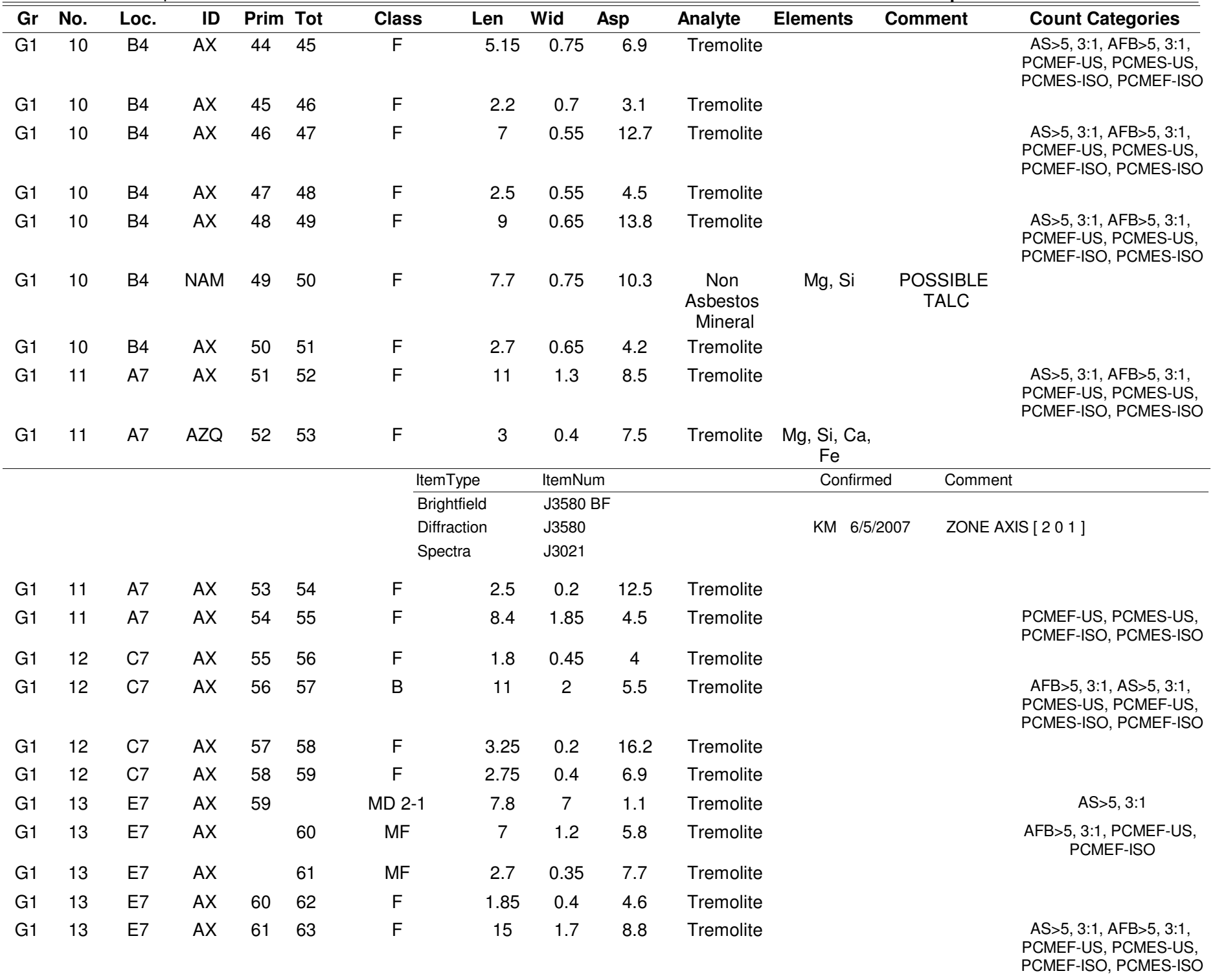

Count Categories

$\mathrm{AFB}>5,3: 1$

PAS

PCMEF-US

Asbestos Fibers and Bundles > 5um and 3:1

Primary Asbestos Structures

PCM Equivalent Fibers-NIOSH

PCMES-US
AS>5, 3:1 PCMEF-ISO PCMES-ISO TAS
Asbestos Structures $>5$ um and 3:1 PCM Equivalent Fibers-ISO PCM Equivalent Structures-ISO Total Asbestos Structures 
ISO 10312, Direct Raw Data

Job Number: 070434

SEA

Report Number: 070434R06

Client: Idaho National Laboratory

Date Received: 4/23/2007

Project Name: RARE

Client Sample No: FB-2-R5

Lab/Cor Sample No: S30

Client Description:

Date Sampled:

$4 / 16 / 2007$

\begin{tabular}{|c|c|c|c|c|c|c|c|c|c|c|c|c|c|}
\hline$\overline{\mathrm{Gr}}$ & No. & Loc. & IID & Prim & Tot & Class & Len & Wid & Asp & Analyte & Elements & Comment & Count Categories \\
\hline G1 & 13 & E7 & $A X$ & 62 & 64 & $\mathrm{~F}$ & 6.5 & 0.8 & 8.1 & Tremolite & & & $\begin{array}{l}\text { AS>5, 3:1, AFB }>5,3: 1, \\
\text { PCMEF-US, PCMES-US, } \\
\text { PCMEF-ISO, PCMES-ISO }\end{array}$ \\
\hline G1 & 13 & E7 & $A X$ & 63 & 65 & $\mathrm{~F}$ & 3 & 0.7 & 4.3 & Tremolite & & & \\
\hline G1 & 13 & E7 & $A X$ & 64 & 66 & $\mathrm{~F}$ & 15.8 & 2 & 7.9 & Tremolite & & & $\begin{array}{l}\text { AS }>5,3: 1, \text { AFB }>5,3: 1, \\
\text { PCMEF-US, PCMES-US, } \\
\text { PCMEF-ISO, PCMES-ISO }\end{array}$ \\
\hline G1 & 13 & E7 & $A X$ & 65 & 67 & $\mathrm{~F}$ & 4.2 & 0.55 & 7.6 & Tremolite & & & \\
\hline G1 & 13 & E7 & $A X$ & 66 & 68 & $\mathrm{~F}$ & 4.5 & 0.4 & 11.2 & Tremolite & & & \\
\hline G1 & 13 & E7 & $A X$ & 67 & 69 & $\mathrm{~F}$ & 5 & 0.55 & 9.1 & Tremolite & & & \\
\hline G1 & 13 & E7 & $A X$ & 68 & 70 & $\mathrm{~F}$ & 9.75 & 0.7 & 13.9 & Tremolite & & & $\begin{array}{l}\text { AS }>5,3: 1, \text { AFB }>5,3: 1, \\
\text { PCMEF-US, PCMES-US, } \\
\text { PCMEF-ISO, PCMES-ISO }\end{array}$ \\
\hline G1 & 14 & G7 & $A X$ & 69 & 71 & $\mathrm{~F}$ & 3 & 0.4 & 7.5 & Tremolite & & & \\
\hline G1 & 15 & 17 & $A X$ & 70 & 72 & $\mathrm{~F}$ & 2.5 & 0.5 & 5 & Tremolite & & & \\
\hline G1 & 15 & 17 & $A X$ & 71 & 73 & $\mathrm{~F}$ & 4.2 & 0.5 & 8.4 & Tremolite & & & \\
\hline G1 & 15 & 17 & $A X$ & 72 & 74 & $\mathrm{~F}$ & 11.1 & 0.7 & 15.9 & Tremolite & & & $\begin{array}{l}\text { AS }>5,3: 1, \text { AFB }>5,3: 1, \\
\text { PCMEF-US, PCMES-US, } \\
\text { PCMEF-ISO, PCMES-ISO }\end{array}$ \\
\hline G1 & 16 & J9 & $A X$ & 73 & 75 & $\mathrm{~F}$ & 1.75 & 0.22 & 8 & Tremolite & & & \\
\hline G1 & 16 & J9 & $A X$ & 74 & & MD 1-0 & 4 & 3.8 & 1.1 & Tremolite & & & \\
\hline G1 & 16 & J9 & $A X$ & & 76 & MF & 4 & 1 & 4 & Tremolite & & & \\
\hline G1 & 16 & J9 & $A X$ & 75 & 77 & $\mathrm{~F}$ & 11.8 & 1.75 & 6.7 & Tremolite & & & $\begin{array}{l}\text { AS>5, 3:1, AFB }>5,3: 1 \text {, } \\
\text { PCMEF-US, PCMES-US, } \\
\text { PCMEF-ISO, PCMES-ISO }\end{array}$ \\
\hline G1 & 16 & J9 & $A X$ & 76 & 78 & $F$ & 2 & 0.35 & 5.7 & Tremolite & & & \\
\hline G1 & 17 & $\mathrm{H} 9$ & $A X$ & 77 & 79 & $\mathrm{~F}$ & 14.35 & 1.8 & 8 & Tremolite & & & $\begin{array}{l}\text { AS }>5,3: 1, \text { AFB }>5,3: 1, \\
\text { PCMEF-US, PCMES-US, } \\
\text { PCMEF-ISO, PCMES-ISO }\end{array}$ \\
\hline G1 & 17 & $\mathrm{H} 9$ & $A X$ & 78 & 80 & $\mathrm{~F}$ & 24.2 & 0.68 & 35.6 & Tremolite & & & $\begin{array}{l}\text { AS }>5,3: 1, \text { AFB }>5,3: 1, \\
\text { PCMEF-US, PCMES-US, } \\
\text { PCMEF-ISO, PCMES-ISO }\end{array}$ \\
\hline G1 & 17 & $\mathrm{H} 9$ & $A X$ & 79 & 81 & $\mathrm{~F}$ & 2.5 & 0.35 & 7.1 & Tremolite & & & \\
\hline G1 & 17 & $\mathrm{H} 9$ & $A X$ & 80 & 82 & $\mathrm{~F}$ & 2.65 & 0.7 & 3.8 & Tremolite & & & \\
\hline G1 & 17 & $\mathrm{H} 9$ & $A X$ & 81 & & MD 1-0 & 4.5 & 3.8 & 1.2 & Tremolite & & & \\
\hline G1 & 17 & $\mathrm{H} 9$ & $A X$ & & 83 & MF & 2.7 & 0.4 & 6.8 & Tremolite & & & \\
\hline G1 & 17 & $\mathrm{H} 9$ & $A X$ & 82 & 84 & $\mathrm{~F}$ & 20 & 3 & 6.7 & Tremolite & & & $\begin{array}{l}\text { AS>5, 3:1, AFB >5, 3:1, } \\
\text { PCMEF-US, PCMES-US, } \\
\text { PCMEF-ISO, PCMES-ISO }\end{array}$ \\
\hline G1 & 17 & $\mathrm{H} 9$ & $A X$ & 83 & 85 & $\mathrm{~F}$ & 5.12 & 0.6 & 8.5 & Tremolite & & & $\begin{array}{l}\text { AS }>5,3: 1, \text { AFB }>5,3: 1, \\
\text { PCMEF-US, PCMES-US, } \\
\text { PCMEF-ISO, PCMES-ISO }\end{array}$ \\
\hline
\end{tabular}

Count Categories

$\mathrm{AFB}>5,3: 1$

PAS

PCMEF-US

Asbestos Fibers and Bundles $>5 u m$ and $3: 1$

Primary Asbestos Structures

PCM Equivalent Fibers-NIOSH

PCMES-US
AS $>5,3: 1$ PCMEF-ISO PCMES-ISO TAS
Asbestos Structures $>5$ um and 3:1 PCM Equivalent Fibers-ISO PCM Equivalent Structures-ISO Total Asbestos Structures

Page 122 of 281 
ISO 10312, Direct Raw Data

Job Number: 070434

SEA

Report Number: 070434R06

Client: Idaho National Laboratory

Date Received: 4/23/2007

Project Name: RARE

Client Sample No: FB-2-R5

Lab/Cor Sample No: S30

Client Description:

Date Sampled:

4/16/2007

\begin{tabular}{|c|c|c|c|c|c|c|c|c|c|c|c|c|c|}
\hline Gr & No. & Loc. & ID & Prim & Tot & Class & Len & Wid & Asp & Analyte & Elements & Comment & Count Categories \\
\hline G1 & 18 & F9 & $A X$ & 84 & 86 & $\mathrm{~F}$ & 8.65 & 0.75 & 11.5 & Tremolite & & & $\begin{array}{l}\text { AS }>5,3: 1, \text { AFB }>5,3: 1, \\
\text { PCMEF-US, PCMES-US, } \\
\text { PCMEF-ISO, PCMES-ISO }\end{array}$ \\
\hline G1 & 18 & F9 & $A X$ & 85 & 87 & $\mathrm{~F}$ & 1.75 & 0.2 & 8.8 & Tremolite & & & \\
\hline G1 & 18 & F9 & $A X$ & 86 & 88 & $\mathrm{~F}$ & 3.2 & 0.2 & 16 & Tremolite & & & \\
\hline G1 & 19 & D9 & $A X$ & 87 & 89 & $\mathrm{~F}$ & 15 & 0.75 & 20 & Tremolite & & & $\begin{array}{l}\text { AS }>5,3: 1, \text { AFB }>5,3: 1, \\
\text { PCMEF-US, PCMES-US, } \\
\text { PCMEF-ISO, PCMES-ISO }\end{array}$ \\
\hline G1 & 19 & D9 & $A X$ & 88 & 90 & $\mathrm{~F}$ & 5.75 & 0.8 & 7.2 & Tremolite & & & $\begin{array}{l}\text { AS }>5,3: 1, \text { AFB }>5,3: 1, \\
\text { PCMEF-US, PCMES-US, } \\
\text { PCMEF-ISO, PCMES-ISO }\end{array}$ \\
\hline G1 & 19 & D9 & $A X$ & 89 & 91 & $\mathrm{~F}$ & 1.25 & 0.25 & 5 & Tremolite & & & \\
\hline G1 & 19 & D9 & $A X$ & 90 & & MD 1-0 & 5 & 3 & 1.7 & Tremolite & & & \\
\hline G1 & 19 & D9 & $A X$ & & 92 & MF & 5 & 0.85 & 5.9 & Tremolite & & & \\
\hline G1 & 20 & B9 & $A X$ & 91 & 93 & $F$ & 4.9 & 0.75 & 6.5 & Tremolite & & & \\
\hline G1 & 20 & B9 & $A X$ & 92 & 94 & $\mathrm{~F}$ & 27.5 & 0.8 & 34.4 & Tremolite & & & $\begin{array}{l}\text { AS }>5,3: 1, \text { AFB }>5,3: 1, \\
\text { PCMEF-US, PCMES-US, } \\
\text { PCMEF-ISO, PCMES-ISO }\end{array}$ \\
\hline G1 & 20 & B9 & $A X$ & 93 & 95 & $F$ & 4.5 & 0.75 & 6 & Tremolite & & & \\
\hline G1 & 20 & B9 & $A X$ & 94 & 96 & $F$ & 4.2 & 0.38 & 11.1 & Tremolite & & & \\
\hline G2 & 21 & A2 & $A X$ & 95 & 97 & $\mathrm{~F}$ & 9.75 & 0.8 & 12.2 & Tremolite & & & $\begin{array}{l}\text { AS }>5,3: 1, \text { AFB }>5,3: 1, \\
\text { PCMEF-US, PCMES-US, } \\
\text { PCMEF-ISO, PCMES-ISO }\end{array}$ \\
\hline G2 & 21 & $\mathrm{~A} 2$ & $A X$ & 96 & 98 & $F$ & 4.35 & 0.25 & 17.4 & Tremolite & & & \\
\hline G2 & 21 & $\mathrm{~A} 2$ & $A X$ & 97 & 99 & $\mathrm{~F}$ & 2.65 & 0.52 & 5.1 & Tremolite & & & \\
\hline G2 & 21 & $\mathrm{~A} 2$ & $A X$ & 98 & 100 & $F$ & 5.3 & 0.9 & 5.9 & Tremolite & & & $\begin{array}{l}\text { AS>5, 3:1, AFB>5, 3:1, } \\
\text { PCMEF-US, PCMES-US, } \\
\text { PCMEF-ISO, PCMES-ISO }\end{array}$ \\
\hline G2 & 22 & $\mathrm{C} 2$ & $A X$ & 99 & 101 & $F$ & 18 & 0.7 & 25.7 & Tremolite & & & $\begin{array}{l}\text { AS>5, 3:1, AFB>5, 3:1, } \\
\text { PCMEF-US, PCMES-US, } \\
\text { PCMEF-ISO, PCMES-ISO }\end{array}$ \\
\hline G2 & 22 & $\mathrm{C} 2$ & $A X$ & 100 & 102 & $\mathrm{~F}$ & 2.75 & 0.6 & 4.6 & Tremolite & & & \\
\hline G2 & 22 & $\mathrm{C} 2$ & $A X$ & 101 & 103 & $F$ & 2.85 & 0.35 & 8.1 & Tremolite & & & \\
\hline G2 & 22 & $\mathrm{C} 2$ & $A X$ & 102 & 104 & $F$ & 3.1 & 0.85 & 3.6 & Tremolite & & & \\
\hline G2 & 22 & $\mathrm{C} 2$ & $A X$ & 103 & 105 & $F$ & 4 & 0.4 & 10 & Tremolite & & & \\
\hline G2 & 22 & $\mathrm{C} 2$ & $A X$ & 104 & 106 & $F$ & 11.5 & 1.1 & 10.5 & Tremolite & & & $\begin{array}{l}\text { AS >5, 3:1, AFB>5, 3:1, } \\
\text { PCMEF-US, PCMES-US, } \\
\text { PCMEF-ISO, PCMES-ISO }\end{array}$ \\
\hline G2 & 23 & E2 & $A X$ & 105 & 107 & $F$ & 5.75 & 1.25 & 4.6 & Tremolite & & & $\begin{array}{l}\text { PCMEF-US, PCMES-US, } \\
\text { PCMEF-ISO, PCMES-ISO }\end{array}$ \\
\hline G2 & 23 & E2 & $A X$ & 106 & 108 & $\mathrm{~F}$ & 11 & 1 & 11 & Tremolite & & & $\begin{array}{l}\text { AS }>5,3: 1, \text { AFB }>5,3: 1 \text {, } \\
\text { PCMEF-US, PCMES-US, } \\
\text { PCMEF-ISO, PCMES-ISO }\end{array}$ \\
\hline
\end{tabular}

Count Categories

AFB $>5,3: 1$

Asbestos Fibers and Bundles $>$ 5um and 3:1

PAS

PCMEF-US

Primary Asbestos Structures

PCM Equivalent Fibers-NIOSH

PCMES-US
AS $>5,3: 1$ PCMEF-ISO PCMES-ISO TAS
Asbestos Structures $>5$ um and 3:1 PCM Equivalent Fibers-ISO PCM Equivalent Structures-ISO Total Asbestos Structures

Page 123 of 281 
ISO 10312, Direct Raw Data

Job Number: 070434

SEA

Report Number: 070434R06

Client: Idaho National Laboratory

Date Received: 4/23/2007

Project Name: RARE

Client Sample No: FB-2-R5

Lab/Cor Sample No: S30

Client Description:

Date Sampled:

4/16/2007

\begin{tabular}{|c|c|c|c|c|c|c|c|c|c|c|c|c|c|}
\hline Gr & No. & Loc. & ID & Prim & Tot & Class & Len & Wid & Asp & Analyte & Elements & Comment & Count Categories \\
\hline G2 & 23 & E2 & $A X$ & 107 & 109 & $\mathrm{~F}$ & 2.85 & 0.8 & 3.6 & Tremolite & & & \\
\hline G2 & 23 & E2 & $A X$ & 108 & 110 & $\mathrm{~F}$ & 3.75 & 0.85 & 4.4 & Tremolite & & & \\
\hline G2 & 23 & E2 & $A X$ & 109 & 111 & $\mathrm{~F}$ & 1.7 & 0.25 & 6.8 & Tremolite & & & \\
\hline G2 & 23 & E2 & $A X$ & 110 & 112 & $\mathrm{~F}$ & 7 & 0.7 & 10 & Tremolite & & & $\begin{array}{l}\text { AS }>5,3: 1, \text { AFB }>5,3: 1, \\
\text { PCMEF-US, PCMES-US, } \\
\text { PCMEF-ISO, PCMES-ISO }\end{array}$ \\
\hline G2 & 24 & G2 & $A X$ & 111 & & MD 1-1 & 9 & 2 & 4.5 & Tremolite & & & $\begin{array}{c}\text { AS }>5,3: 1, \text { PCMES-US, } \\
\text { PCMES-ISO }\end{array}$ \\
\hline G2 & 24 & G2 & $A X$ & & 113 & MF & 8 & 0.75 & 10.7 & Tremolite & & & $\begin{array}{c}\text { AFB }>5,3: 1, \text { PCMEF-US, } \\
\text { PCMEF-ISO }\end{array}$ \\
\hline G2 & 24 & G2 & $A X$ & 112 & 114 & $\mathrm{~F}$ & 9.5 & 0.65 & 14.6 & Tremolite & & & $\begin{array}{l}\text { AS >5, 3:1, AFB>5, 3:1, } \\
\text { PCMEF-US, PCMES-US, } \\
\text { PCMEF-ISO, PCMES-ISO }\end{array}$ \\
\hline G2 & 25 & 12 & $A X$ & 113 & 115 & $\mathrm{~F}$ & 7.5 & 0.65 & 11.5 & Tremolite & & & $\begin{array}{l}\text { AS }>5,3: 1, \text { AFB }>5,3: 1, \\
\text { PCMEF-US, PCMES-US, } \\
\text { PCMEF-ISO, PCMES-ISO }\end{array}$ \\
\hline G2 & 25 & 12 & $A X$ & 114 & 116 & $F$ & 1.7 & 0.3 & 5.7 & Tremolite & & & \\
\hline G2 & 25 & 12 & $A X$ & 115 & 117 & $F$ & 4.75 & 0.8 & 5.9 & Tremolite & & & \\
\hline G2 & 26 & J4 & $A X$ & 116 & 118 & $F$ & 4.6 & 0.55 & 8.4 & Tremolite & & & \\
\hline G2 & 26 & J4 & $A X$ & 117 & 119 & $F$ & 4 & 0.8 & 5 & Tremolite & & & \\
\hline G2 & 26 & J4 & $A X$ & 118 & 120 & $F$ & 6 & 1.2 & 5 & Tremolite & & & $\begin{array}{l}\text { AS }>5,3: 1, \text { AFB }>5,3: 1, \\
\text { PCMEF-US, PCMES-US, } \\
\text { PCMEF-ISO, PCMES-ISO }\end{array}$ \\
\hline G2 & 26 & J4 & $A X$ & 119 & 121 & $F$ & 4.2 & 0.65 & 6.5 & Tremolite & & & \\
\hline G2 & 26 & J4 & $A X$ & 120 & 122 & $F$ & 22.5 & 1 & 22.5 & Tremolite & & & $\begin{array}{l}\text { AS }>5,3: 1, \text { AFB }>5,3: 1 \text {, } \\
\text { PCMEF-US, PCMES-US, } \\
\text { PCMEF-ISO, PCMES-ISO }\end{array}$ \\
\hline G2 & 27 & $\mathrm{H} 4$ & $A X$ & 121 & 123 & $\mathrm{~F}$ & 4.2 & 0.8 & 5.2 & Tremolite & & & \\
\hline G2 & 27 & $\mathrm{H} 4$ & $A X$ & 122 & 124 & $\mathrm{~F}$ & 3.8 & 0.65 & 5.8 & Tremolite & & & \\
\hline G2 & 27 & $\mathrm{H} 4$ & $A X$ & 123 & 125 & $\mathrm{~F}$ & 4.35 & 0.6 & 7.2 & Tremolite & & & \\
\hline G2 & 27 & $\mathrm{H} 4$ & $A X$ & 124 & 126 & $F$ & 5.2 & 1.25 & 4.2 & Tremolite & & & $\begin{array}{l}\text { PCMEF-US, PCMES-US, } \\
\text { PCMEF-ISO, PCMES-ISO }\end{array}$ \\
\hline G2 & 28 & F4 & $A X$ & 125 & 127 & $F$ & 7 & 0.6 & 11.7 & Tremolite & & & $\begin{array}{l}\text { AS>5, 3:1, AFB>5, 3:1, } \\
\text { PCMEF-US, PCMES-US, } \\
\text { PCMEF-ISO, PCMES-ISO }\end{array}$ \\
\hline G2 & 29 & D4 & $A X$ & 126 & 128 & $F$ & 7 & 1.75 & 4 & Tremolite & & & $\begin{array}{l}\text { PCMEF-US, PCMES-US, } \\
\text { PCMEF-ISO, PCMES-ISO }\end{array}$ \\
\hline G2 & 29 & D4 & $A X$ & 127 & 129 & $F$ & 7.85 & 1.85 & 4.2 & Tremolite & & & $\begin{array}{l}\text { PCMEF-US, PCMES-US, } \\
\text { PCMEF-ISO, PCMES-ISO }\end{array}$ \\
\hline G2 & 29 & D4 & $A X$ & 128 & 130 & $F$ & 4.35 & 1.2 & 3.6 & Tremolite & & & \\
\hline G2 & 29 & D4 & $A X$ & 129 & 131 & $F$ & 20 & 3 & 6.7 & Tremolite & & & $\begin{array}{l}\text { AS }>5,3: 1, \text { AFB }>5,3: 1, \\
\text { PCMEF-US, PCMES-US, } \\
\text { PCMEF-ISO, PCMES-ISO }\end{array}$ \\
\hline
\end{tabular}

\section{Count Categories}

$\mathrm{AFB}>5,3: 1$

PAS

PCMEF-US

PCMES-US
Asbestos Fibers and Bundles > 5um and 3:1

Primary Asbestos Structures

PCM Equivalent Fibers-NIOSH

PCM Equivalent Structures-NIOSH
AS $>5,3: 1$ PCMEF-ISO PCMES-ISO TAS
Asbestos Structures $>5$ um and 3:1 PCM Equivalent Fibers-ISO PCM Equivalent Structures-ISO Total Asbestos Structures

Page 124 of 281 
ISO 10312, Direct Raw Data

Job Number: 070434

SEA

Report Number: 070434R06

Client: Idaho National Laboratory

Date Received: 4/23/2007

Project Name: RARE

Client Sample No: FB-2-R5

Lab/Cor Sample No: S30

Client Description:

Date Sampled:

4/16/2007

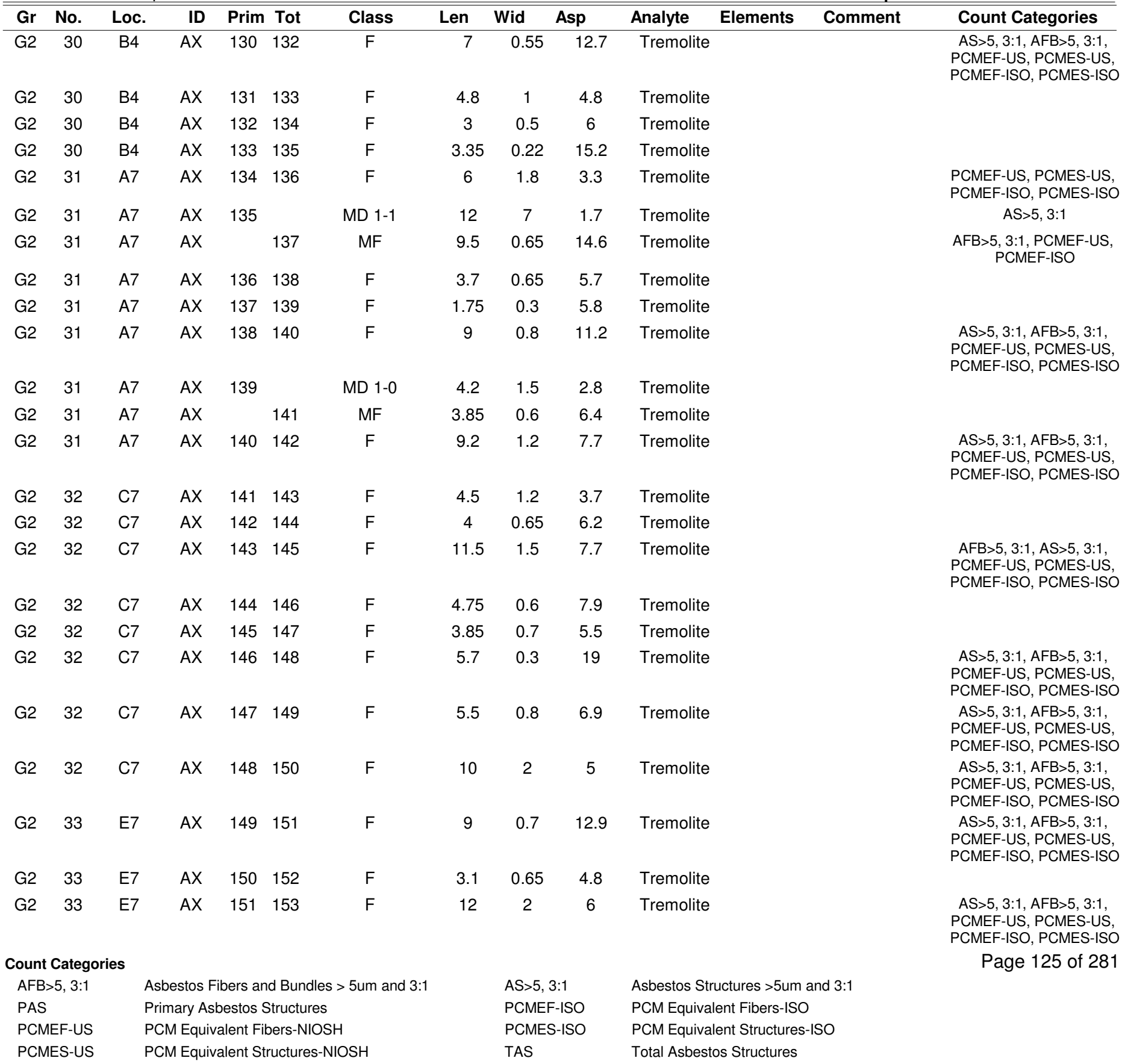


ISO 10312, Direct Raw Data

Job Number: 070434

SEA

Report Number: 070434R06

Client: Idaho National Laboratory

Date Received: 4/23/2007

Project Name: RARE

Client Sample No: FB-2-R5

Lab/Cor Sample No: S30

Client Description:

Date Sampled:

4/16/2007

\begin{tabular}{|c|c|c|c|c|c|c|c|c|c|c|c|c|c|}
\hline Gr & No. & Loc. & ID & Prim & Tot & Class & Len & Wid & Asp & Analyte & Elements & Comment & Count Categories \\
\hline G2 & 33 & E7 & $A X$ & 152 & 154 & $F$ & 11.5 & 0.55 & 20.9 & Tremolite & & & $\begin{array}{l}\text { AS }>5,3: 1, \text { AFB }>5,3: 1, \\
\text { PCMEF-US, PCMES-US, } \\
\text { PCMEF-ISO, PCMES-ISO }\end{array}$ \\
\hline G2 & 33 & E7 & $A X$ & 153 & 155 & $\mathrm{~F}$ & 11.35 & 1.5 & 7.6 & Tremolite & & & $\begin{array}{l}\text { AS>5, 3:1, AFB>5, 3:1, } \\
\text { PCMEF-US, PCMES-US, } \\
\text { PCMEF-ISO, PCMES-ISO }\end{array}$ \\
\hline G2 & 33 & E7 & $A X$ & 154 & 156 & $\mathrm{~F}$ & 4.15 & 0.7 & 5.9 & Tremolite & & & \\
\hline G2 & 34 & G7 & $A X$ & 155 & 157 & $\mathrm{~F}$ & 14.7 & 1.8 & 8.2 & Tremolite & & & $\begin{array}{l}\text { AS }>5,3: 1, \text { AFB }>5,3: 1, \\
\text { PCMEF-US, PCMES-US, } \\
\text { PCMEF-ISO, PCMES-ISO }\end{array}$ \\
\hline G2 & 34 & G7 & $A X$ & 156 & 158 & $\mathrm{~F}$ & 11 & 0.7 & 15.7 & Tremolite & & & $\begin{array}{l}\text { AS }>5,3: 1, \text { AFB }>5,3: 1, \\
\text { PCMEF-US, PCMES-US, } \\
\text { PCMES-ISO, PCMEF-ISO }\end{array}$ \\
\hline G2 & 34 & G7 & $A X$ & 157 & 159 & $\mathrm{~F}$ & 4.75 & 0.5 & 9.5 & Tremolite & & & \\
\hline G2 & 35 & 17 & $A X$ & 158 & 160 & $F$ & 8.5 & 0.22 & 38.6 & Tremolite & & & $\begin{array}{l}\text { AS }>5,3: 1, \text { AFB }>5,3: 1, \\
\text { PCMEF-ISO, PCMES-ISO }\end{array}$ \\
\hline G2 & 35 & 17 & $A X$ & 159 & 161 & $F$ & 5.2 & 0.75 & 6.9 & Tremolite & & & $\begin{array}{l}\text { AS>5, 3:1, AFB>5, 3:1, } \\
\text { PCMEF-US, PCMES-US, } \\
\text { PCMEF-ISO, PCMES-ISO }\end{array}$ \\
\hline G2 & 35 & 17 & $A X$ & 160 & 162 & $F$ & 9.45 & 0.38 & 24.9 & Tremolite & & & $\begin{array}{l}\text { AS>5, 3:1, AFB }>5,3: 1, \\
\text { PCMEF-US, PCMES-US, } \\
\text { PCMEF-ISO, PCMES-ISO }\end{array}$ \\
\hline
\end{tabular}

Count Categories

AFB $>5,3: 1$

PAS

PCMEF-US

Asbestos Fibers and Bundles > 5um and 3:1

Primary Asbestos Structures

PCM Equivalent Fibers-NIOSH

PCMES-US
PCM Equivalent Structures-NIOSH
AS>5, 3:1 PCMEF-ISO PCMES-ISO TAS
Asbestos Structures $>5$ um and 3:1 PCM Equivalent Fibers-ISO PCM Equivalent Structures-ISO Total Asbestos Structures 
ISO 10312, Direct Raw Data

Job Number: 070434

SEA

Report Number: 070434R06

Client: Idaho National Laboratory

Date Received: 4/23/2007

Project Name: RARE

Client Sample No: FB-2-R6

Lab/Cor Sample No: S31

Client Description:

Date Sampled:

$4 / 16 / 2007$

\begin{tabular}{|c|c|c|c|c|c|c|c|c|c|c|c|c|c|}
\hline Gr & No. & Loc. & ID & Prim & Tot & Class & Len & Wid & Asp & Analyte & Elements & Comment & Count Categories \\
\hline G1 & 1 & $\mathrm{~A} 2$ & $A X$ & 1 & 1 & $F$ & 1.75 & 0.38 & 4.6 & Tremolite & & & \\
\hline G1 & 1 & $\mathrm{~A} 2$ & $A X$ & 2 & 2 & $\mathrm{~F}$ & 2.6 & 0.3 & 8.7 & Tremolite & & & \\
\hline G1 & 1 & $\mathrm{~A} 2$ & $A X$ & 3 & 3 & $\mathrm{~F}$ & 3.1 & 0.75 & 4.1 & Tremolite & & & \\
\hline G1 & 1 & $\mathrm{~A} 2$ & $A X$ & 4 & 4 & $\mathrm{~F}$ & 5.2 & 1.1 & 4.7 & Tremolite & & & $\begin{array}{l}\text { PCMEF-US, PCMES-US, } \\
\text { PCMEF-ISO, PCMES-ISO }\end{array}$ \\
\hline G1 & 1 & $\mathrm{~A} 2$ & $A X$ & 5 & 5 & $\mathrm{~F}$ & 1.7 & 0.65 & 2.6 & Tremolite & & & \\
\hline G1 & 1 & $\mathrm{~A} 2$ & $A X$ & 6 & 6 & $\mathrm{~F}$ & 11 & 1.75 & 6.3 & Tremolite & & & $\begin{array}{l}\text { AS }>5,3: 1, \text { AFB }>5,3: 1, \\
\text { PCMEF-US, PCMES-US, } \\
\text { PCMEF-ISO, PCMES-ISO }\end{array}$ \\
\hline G1 & 1 & $\mathrm{~A} 2$ & $A X$ & 7 & 7 & $\mathrm{~F}$ & 4.5 & 0.65 & 6.9 & Tremolite & & & \\
\hline G1 & 1 & $\mathrm{~A} 2$ & $A X$ & 8 & & MD 1-0 & 4.35 & 1.5 & 2.9 & Tremolite & & & \\
\hline G1 & 1 & $\mathrm{~A} 2$ & $A X$ & & 8 & MF & 4.2 & 0.3 & 14 & Tremolite & & & \\
\hline G1 & 2 & $\mathrm{C} 2$ & $A X$ & 9 & 9 & $\mathrm{~F}$ & 11.65 & 1.3 & 9 & Tremolite & & & $\begin{array}{l}\text { AS }>5,3: 1, \text { AFB }>5,3: 1 \text {, } \\
\text { PCMEF-US, PCMES-US, } \\
\text { PCMEF-ISO, PCMES-ISO }\end{array}$ \\
\hline G1 & 2 & $\mathrm{C} 2$ & $A X$ & 10 & & MD 1-1 & 8.5 & 4 & 2.1 & Tremolite & & & $A S>5,3: 1$ \\
\hline G1 & 2 & $\mathrm{C} 2$ & $A X$ & & 10 & MF & 5.7 & 0.7 & 8.1 & Tremolite & & & $\begin{array}{c}\text { AFB >5, 3:1, PCMEF-US, } \\
\text { PCMEF-ISO }\end{array}$ \\
\hline G1 & 2 & $\mathrm{C} 2$ & $A X$ & 11 & 11 & $\mathrm{~F}$ & 1.75 & 0.38 & 4.6 & Tremolite & & & \\
\hline G1 & 2 & $\mathrm{C} 2$ & $A X$ & 12 & 12 & $\mathrm{~F}$ & 55 & 1.15 & 47.8 & Tremolite & & & $\begin{array}{l}\text { AS }>5,3: 1, \text { AFB }>5,3: 1, \\
\text { PCMEF-US, PCMES-US, } \\
\text { PCMEF-ISO, PCMES-ISO }\end{array}$ \\
\hline G1 & 3 & $\mathrm{H} 4$ & $A X$ & 13 & 13 & $\mathrm{~F}$ & 1.75 & 0.45 & 3.9 & Tremolite & & & \\
\hline G1 & 3 & $\mathrm{H} 4$ & $A X$ & 14 & 14 & $\mathrm{~F}$ & 25.38 & 1.35 & 18.8 & Tremolite & & & $\begin{array}{l}\text { AS }>5,3: 1, \text { AFB }>5,3: 1, \\
\text { PCMEF-US, PCMES-US, } \\
\text { PCMEF-ISO, PCMES-ISO }\end{array}$ \\
\hline G1 & 3 & $\mathrm{H} 4$ & $A X$ & 15 & 15 & $\mathrm{~F}$ & 2.22 & 0.6 & 3.7 & Tremolite & & & \\
\hline G1 & 3 & $\mathrm{H} 4$ & $A X$ & 16 & 16 & $\mathrm{~F}$ & 4 & 0.38 & 10.5 & Tremolite & & & \\
\hline G1 & 3 & $\mathrm{H} 4$ & $A X$ & 17 & & MD 1-0 & 5.85 & 4 & 1.5 & Tremolite & & & $A S>5,3: 1$ \\
\hline G1 & 3 & $\mathrm{H} 4$ & $A X$ & & 17 & MF & 3.85 & 1.12 & 3.4 & Tremolite & & & \\
\hline G1 & 4 & D7 & $A X$ & 18 & 18 & $F$ & 2 & 0.5 & 4 & Tremolite & & & \\
\hline G1 & 4 & D7 & $A X$ & 19 & 19 & $\mathrm{~F}$ & 5.75 & 1.12 & 5.1 & Tremolite & & & $\begin{array}{l}\text { AS >5, 3:1, AFB>5, 3:1, } \\
\text { PCMEF-US, PCMES-US, } \\
\text { PCMEF-ISO, PCMES-ISO }\end{array}$ \\
\hline G1 & 4 & D7 & $A X$ & 20 & 20 & $\mathrm{~F}$ & 1.85 & 0.45 & 4.1 & Tremolite & & & \\
\hline G1 & 4 & D7 & $A X$ & 21 & 21 & $\mathrm{~F}$ & 6.35 & 1.5 & 4.2 & Tremolite & & & $\begin{array}{l}\text { PCMEF-US, PCMES-US, } \\
\text { PCMEF-ISO, PCMES-ISO }\end{array}$ \\
\hline G1 & 4 & D7 & $A X$ & 22 & 22 & $\mathrm{~F}$ & 2 & 0.55 & 3.6 & Tremolite & & & \\
\hline G1 & 5 & B5 & $A X$ & 23 & 23 & $F$ & 4 & 0.3 & 13.3 & Tremolite & & & \\
\hline G1 & 5 & B5 & $A X$ & 24 & & MD 1-1 & 12.5 & 7 & 1.8 & Tremolite & & & $A S>5,3: 1$ \\
\hline
\end{tabular}

Count Categories

PAS

PCMEF-US

Asbestos Fibers and Bundles > 5um and 3:1

Primary Asbestos Structures

PCM Equivalent Fibers-NIOSH

PCMES-US
AS $>5,3: 1$ PCMEF-ISO PCMES-ISO TAS
Asbestos Structures $>5$ um and 3:1 PCM Equivalent Fibers-ISO PCM Equivalent Structures-ISO Total Asbestos Structures 
Job Number: 070434

\section{SEA}

ISO 10312, Direct Raw Data

Client: Idaho National Laboratory

Report Number: 070434R06

Date Received: 4/23/2007

Project Name: RARE

Client Sample No: FB-2-R6

Lab/Cor Sample No: S31

Client Description:

Date Sampled:

$4 / 16 / 2007$

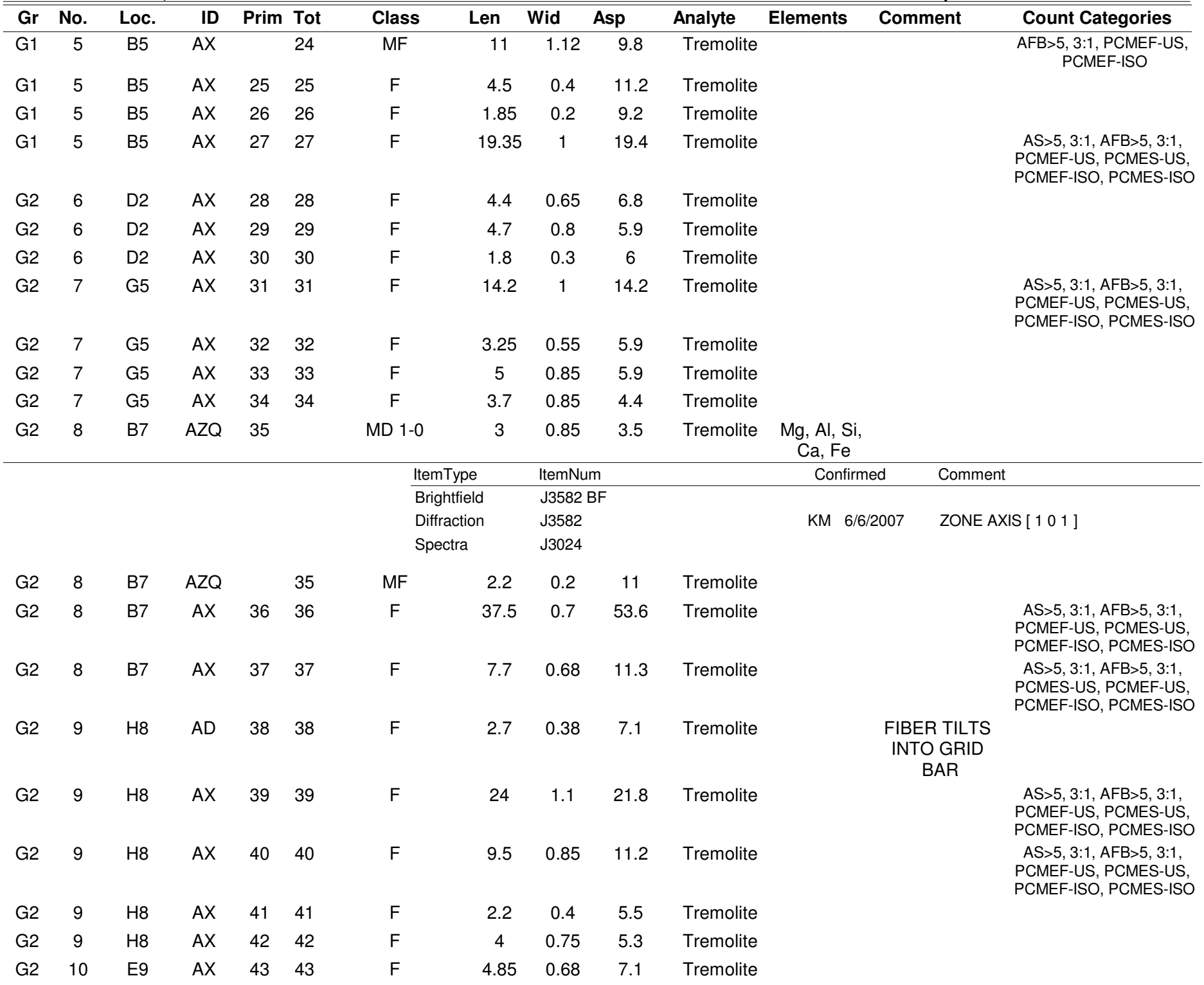

Count Categories

$\mathrm{AFB}>5,3: 1$

PAS

PCMEF-US

Asbestos Fibers and Bundles $>5 u m$ and 3:1

Primary Asbestos Structures

PCM Equivalent Fibers-NIOSH

PCMES-US
AS $>5,3: 1$ PCMEF-ISO PCMES-ISO TAS
Asbestos Structures $>5$ um and 3:1 PCM Equivalent Fibers-ISO PCM Equivalent Structures-ISO Total Asbestos Structures 
Phone: (206) 781-0155

7619 6th Ave Nw

Job Number: 070434

ISO 10312, Direct Raw Data

Client: Idaho National Laboratory

Report Number: 070434R06

Name: RARE

Client Sample No: FB-2-R6

Lab/Cor Sample No: S31

Client Description:

\begin{tabular}{|c|c|c|c|c|c|c|c|c|c|c|c|c|c|}
\hline Gr & No. & Loc. & ID & Prim & Tot & Class & Len & Wid & Asp & Analyte & Elements & Comment & Count Categories \\
\hline G2 & 10 & E9 & $A X$ & 44 & 44 & $F$ & 5 & 0.65 & 7.7 & Tremolite & & & \\
\hline G2 & 10 & E9 & $A X$ & 45 & 45 & $F$ & 15 & 1.85 & 8.1 & Tremolite & & & $\begin{array}{l}\text { AS }>5,3: 1, \text { AFB }>5,3: 1, \\
\text { PCMEF-US, PCMES-US, } \\
\text { PCMEF-ISO, PCMES-ISO }\end{array}$ \\
\hline
\end{tabular}

Count Categories

AFB $>5,3: 1$

PAS

PCMEF-US

PCMES-US
Asbestos Fibers and Bundles > 5um and 3:1

Primary Asbestos Structures

PCM Equivalent Fibers-NIOSH

PCM Equivalent Structures-NIOSH
AS $>5,3: 1$ PCMEF-ISO PCMES-ISO TAS
Asbestos Structures $>5$ um and 3:1 PCM Equivalent Fibers-ISO PCM Equivalent Structures-ISO Total Asbestos Structures 
Job Number: 070434

ISO 10312, Direct Raw Data

Client: Idaho National Laboratory

Report Number: 070434R06

Project Name: RARE

Date Received: 4/23/2007

Client Sample No: FB-2-R7

Lab/Cor Sample No: S32

Client Description:

Date Sampled:

4/16/2007

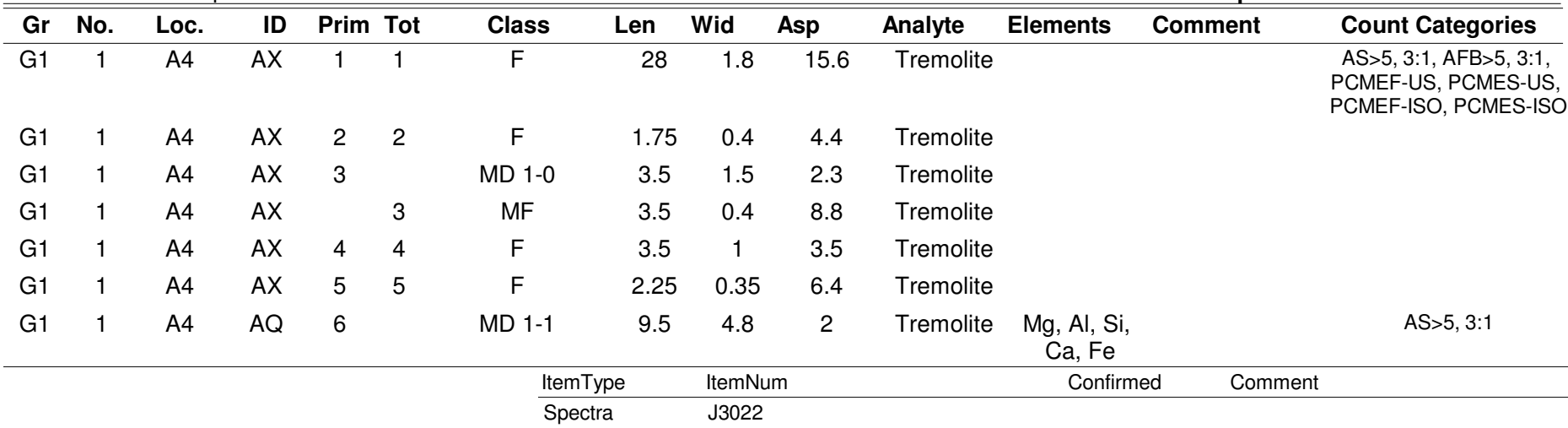

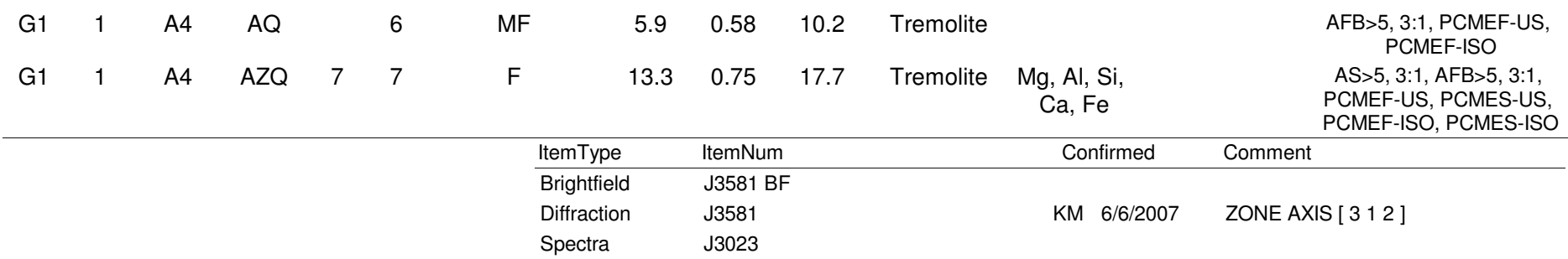

$\begin{array}{llllccccccc}\text { G1 } & 1 & \text { A4 } & \text { AX } & 8 & 8 & \text { F } & 19.7 & 1.75 & 11.3 & \text { Tremolite } \\ \text { G1 } & 1 & \text { A4 } & \text { AX } & 9 & 9 & \text { F } & 11.2 & 1.2 & 9.3 & \text { Tremolite } \\ & & & & & & & & & & \\ \text { G1 } & 1 & \text { A4 } & \text { AX } & 10 & 10 & \text { F } & 3.75 & 0.38 & 9.9 & \text { Tremolite } \\ \text { G1 } & 2 & \text { D1 } & \text { AX } & 11 & 11 & \text { F } & 1.7 & 0.35 & 4.9 & \text { Tremolite } \\ \text { G1 } & 2 & \text { D1 } & \text { AX } & 12 & 12 & \text { F } & 5.8 & 0.8 & 7.2 & \text { Tremolite }\end{array}$
PCMEF-US, PCMES-US PCMEF-ISO, PCMES-ISO

$A S>5,3: 1, A F B>5,3: 1$ PCMEF-US, PCMES-US, PCMEF-ISO, PCMES-ISO

$\begin{array}{llllllccccc}\text { G1 } & 2 & \text { D1 } & \text { AX } & 13 & & \text { MD 1-0 } & 5.8 & 5 & 1.2 & \text { Tremolite } \\ \text { G1 } & 2 & \text { D1 } & \text { AX } & & 13 & \text { MF } & 4.85 & 0.38 & 12.8 & \text { Tremolite } \\ \text { G1 } & 2 & \text { D1 } & \text { AX } & 14 & 14 & \text { F } & 3.5 & 0.7 & 5 & \text { Tremolite } \\ \text { G1 } & 2 & \text { D1 } & \text { AX } & 15 & 15 & \text { F } & 5.25 & 0.4 & 13.1 & \text { Tremolite } \\ & & & & & & & & & & \\ \text { G1 } & 3 & \text { G3 } & \text { AX } & 16 & 16 & \text { F } & 6 & 1.5 & 4 & \text { Tremolite } \\ \text { G1 } & 3 & \text { G3 } & \text { AX } & 17 & 17 & \text { F } & 2 & 0.55 & 3.6 & \text { Tremolite }\end{array}$

$A S>5,3: 1, A F B>5,3: 1$, PCMEF-US, PCMES-US, PCMEF-ISO, PCMES-ISO AS $>5,3: 1$

$A S>5,3: 1, A F B>5,3: 1$ PCMEF-US, PCMES-US, PCMEF-ISO, PCMES-ISO PCMEF-US, PCMES-US, PCMES-ISO, PCMEF-ISO

Count Categories

AFB $>5,3: 1$

PAS

PCMEF-US

Asbestos Fibers and Bundles $>5 \mathrm{um}$ and $3: 1$

Primary Asbestos Structures

PCM Equivalent Fibers-NIOSH

PCMES-US
AS $>5,3: 1$ PCMEF-ISO PCMES-ISO TAS
Asbestos Structures $>5$ um and 3:1 PCM Equivalent Fibers-ISO PCM Equivalent Structures-ISO Total Asbestos Structures 
ISO 10312, Direct Raw Data

Job Number: 070434

SEA

Report Number: 070434R06

Client: Idaho National Laboratory

Date Received: 4/23/2007

Project Name: RARE

Client Sample No: FB-2-R7

Lab/Cor Sample No: S32

Client Description:

Date Sampled:

$4 / 16 / 2007$

\begin{tabular}{|c|c|c|c|c|c|c|c|c|c|c|c|c|c|}
\hline Gr & No. & Loc. & ID & Prim & Tot & Class & Len & Wid & Asp & Analyte & Elements & Comment & Count Categories \\
\hline G1 & 3 & G3 & $A X$ & 18 & 18 & $F$ & 4.5 & 1.2 & 3.7 & Tremolite & & & \\
\hline G1 & 3 & G3 & $A X$ & 19 & 19 & $\mathrm{~F}$ & 3.2 & 0.65 & 4.9 & Tremolite & & & \\
\hline G1 & 3 & G3 & $A X$ & 20 & 20 & $\mathrm{~F}$ & 44 & 1.2 & 36.7 & Tremolite & & & $\begin{array}{l}\text { AS>5, 3:1, AFB>5, 3:1, } \\
\text { PCMEF-US, PCMES-US, } \\
\text { PCMEF-ISO, PCMES-ISO }\end{array}$ \\
\hline G1 & 3 & G3 & $A X$ & 21 & 21 & $\mathrm{~F}$ & 1.35 & 0.33 & 4.1 & Tremolite & & & \\
\hline G1 & 3 & G3 & $A X$ & 22 & 22 & $\mathrm{~F}$ & 2.8 & 0.5 & 5.6 & Tremolite & & & \\
\hline G1 & 3 & G3 & $A X$ & 23 & 23 & $\mathrm{~F}$ & 6 & 0.4 & 15 & Tremolite & & & $\begin{array}{l}\text { AS>5, 3:1, AFB>5, 3:1, } \\
\text { PCMEF-US, PCMES-US, } \\
\text { PCMEF-ISO, PCMES-ISO }\end{array}$ \\
\hline G1 & 4 & 16 & $A X$ & 24 & 24 & $\mathrm{~F}$ & 5.5 & 1.1 & 5 & Tremolite & & & $\begin{array}{l}\text { AS }>5,3: 1, \text { AFB }>5,3: 1, \\
\text { PCMEF-US, PCMES-US, } \\
\text { PCMEF-ISO, PCMES-ISO }\end{array}$ \\
\hline G1 & 4 & 16 & $A X$ & 25 & 25 & $\mathrm{~F}$ & 3.7 & 0.75 & 4.9 & Tremolite & & & \\
\hline G1 & 4 & 16 & $A X$ & 26 & 26 & $F$ & 19 & 2 & 9.5 & Tremolite & & & $\begin{array}{l}\text { AS }>5,3: 1, \text { AFB }>5,3: 1 \text {, } \\
\text { PCMEF-US, PCMES-US, } \\
\text { PCMEF-ISO, PCMES-ISO }\end{array}$ \\
\hline G1 & 4 & 16 & $A X$ & 27 & 27 & $F$ & 6.2 & 1.2 & 5.2 & Tremolite & & & $\begin{array}{l}\text { AS>5, 3:1, AFB }>5,3: 1, \\
\text { PCMEF-US, PCMES-US, } \\
\text { PCMEF-ISO, PCMES-ISO }\end{array}$ \\
\hline G1 & 4 & 16 & $A X$ & 28 & 28 & $\mathrm{~F}$ & 3.7 & 0.7 & 5.3 & Tremolite & & & \\
\hline G1 & 4 & 16 & $A X$ & 29 & 29 & $\mathrm{~F}$ & 6.5 & 1.1 & 5.9 & Tremolite & & & $\begin{array}{l}\text { AS >5, 3:1, AFB>5, 3:1, } \\
\text { PCMEF-US, PCMES-US, } \\
\text { PCMEF-ISO, PCMES-ISO }\end{array}$ \\
\hline G1 & 5 & D8 & $A X$ & 30 & 30 & $\mathrm{~F}$ & 10 & 0.9 & 11.1 & Tremolite & & & $\begin{array}{l}\text { AS }>5,3: 1, \text { AFB }>5,3: 1, \\
\text { PCMEF-US, PCMES-US, } \\
\text { PCMEF-ISO, PCMES-ISO }\end{array}$ \\
\hline G1 & 5 & D8 & $A X$ & 31 & 31 & $\mathrm{~F}$ & 5.1 & 0.65 & 7.8 & Tremolite & & & $\begin{array}{l}\text { AS }>5,3: 1, \text { AFB }>5,3: 1, \\
\text { PCMEF-US, PCMES-US, } \\
\text { PCMEF-ISO, PCMES-ISO }\end{array}$ \\
\hline G1 & 5 & D8 & $A X$ & 32 & 32 & $\mathrm{~F}$ & 28 & 1.8 & 15.6 & Tremolite & & & $\begin{array}{l}\text { AS }>5,3: 1, \text { AFB }>5,3: 1, \\
\text { PCMEF-US, PCMES-US, } \\
\text { PCMEF-ISO, PCMES-ISO }\end{array}$ \\
\hline G1 & 5 & D8 & $A X$ & 33 & 33 & $\mathrm{~F}$ & 5.2 & 0.65 & 8 & Tremolite & & & $\begin{array}{l}\text { AS }>5,3: 1, \text { AFB }>5,3: 1, \\
\text { PCMEF-US, PCMES-US, } \\
\text { PCMEF-ISO, PCMES-ISO }\end{array}$ \\
\hline G1 & 5 & D8 & $A X$ & 34 & 34 & $\mathrm{~F}$ & 56 & 4 & 14 & Tremolite & & & $\begin{array}{l}\text { AS }>5,3: 1, \text { AFB }>5,3: 1 \\
\text { PCMEF-US, PCMES-US }\end{array}$ \\
\hline G1 & 5 & D8 & $A X$ & 35 & 35 & $\mathrm{~F}$ & 3.75 & 0.33 & 11.4 & Tremolite & & & \\
\hline G2 & 6 & D3 & $A X$ & 36 & 36 & $F$ & 2.3 & 0.3 & 7.7 & Tremolite & & & \\
\hline G2 & 6 & D3 & $A X$ & 37 & & MD 1-0 & 11 & 5 & 2.2 & Tremolite & & & $A S>5,3: 1$ \\
\hline G2 & 6 & D3 & $A X$ & & 37 & MF & 5 & 0.38 & 13.2 & Tremolite & & & \\
\hline G2 & 6 & D3 & $A X$ & 38 & 38 & $F$ & 4 & 0.45 & 8.9 & Tremolite & & & \\
\hline
\end{tabular}

Count Categories

$\mathrm{AFB}>5,3: 1$

PAS

PCMEF-US

Asbestos Fibers and Bundles > 5um and 3:1

Primary Asbestos Structures

PCM Equivalent Fibers-NIOSH

PCMES-US
AS $>5,3: 1$ PCMEF-ISO PCMES-ISO TAS
Asbestos Structures $>5$ um and 3:1 PCM Equivalent Fibers-ISO PCM Equivalent Structures-ISO Total Asbestos Structures 
ISO 10312, Direct Raw Data

Job Number: 070434

SEA

Report Number: 070434R06

Client: Idaho National Laboratory

Date Received: 4/23/2007

Project Name: RARE

Client Sample No: FB-2-R7

Lab/Cor Sample No: S32

Client Description:

Date Sampled:

$4 / 16 / 2007$

\begin{tabular}{|c|c|c|c|c|c|c|c|c|c|c|c|c|c|}
\hline Gr & No. & Loc. & ID & Prim & Tot & Class & Len & Wid & Asp & Analyte & Elements & Comment & Count Categories \\
\hline G2 & 6 & D3 & $A X$ & 39 & 39 & $\mathrm{~F}$ & 17 & 1.2 & 14.2 & Tremolite & & & $\begin{array}{l}\text { AS }>5,3: 1, \text { AFB }>5,3: 1, \\
\text { PCMEF-US, PCMES-US, } \\
\text { PCMEF-ISO, PCMES-ISO }\end{array}$ \\
\hline G2 & 6 & D3 & $A X$ & 40 & 40 & $\mathrm{~F}$ & 11.2 & 1.2 & 9.3 & Tremolite & & & $\begin{array}{l}\text { AS }>5,3: 1, \text { AFB }>5,3: 1 \text {, } \\
\text { PCMEF-US, PCMES-US, } \\
\text { PCMEF-ISO, PCMES-ISO }\end{array}$ \\
\hline G2 & 6 & D3 & $A X$ & 41 & 41 & $\mathrm{~F}$ & 4.2 & 0.5 & 8.4 & Tremolite & & & \\
\hline G2 & 6 & D3 & $A X$ & 42 & 42 & $\mathrm{~F}$ & 3.3 & 0.5 & 6.6 & Tremolite & & & \\
\hline G2 & 7 & G1 & $A X$ & 43 & 43 & $\mathrm{~F}$ & 7 & 0.5 & 14 & Tremolite & & & $\begin{array}{l}\text { AS }>5,3: 1, \text { AFB }>5,3: 1 \text {, } \\
\text { PCMEF-US, PCMES-US, } \\
\text { PCMES-ISO, PCMEF-ISO }\end{array}$ \\
\hline G2 & 7 & G1 & $A X$ & 44 & 44 & $\mathrm{~F}$ & 8 & 0.2 & 40 & Tremolite & & & $\begin{array}{l}\text { AS>5, 3:1, AFB }>5,3: 1, \\
\text { PCMEF-ISO, PCMES-ISO }\end{array}$ \\
\hline G2 & 7 & G1 & $A X$ & 45 & 45 & $\mathrm{~F}$ & 10.2 & 1 & 10.2 & Tremolite & & & $\begin{array}{l}\text { AS }>5,3: 1, \text { AFB }>5,3: 1 \text {, } \\
\text { PCMEF-US, PCMES-US, } \\
\text { PCMEF-ISO, PCMES-ISO }\end{array}$ \\
\hline G2 & 7 & G1 & $A X$ & 46 & 46 & $\mathrm{~F}$ & 2.2 & 0.4 & 5.5 & Tremolite & & & \\
\hline G2 & 7 & G1 & $A X$ & 47 & 47 & $\mathrm{~F}$ & 11.5 & 0.85 & 13.5 & Tremolite & & & $\begin{array}{l}\text { AS }>5,3: 1, \text { AFB }>5,3: 1, \\
\text { PCMEF-US, PCMES-US, } \\
\text { PCMEF-ISO, PCMES-ISO }\end{array}$ \\
\hline G2 & 7 & G1 & $A X$ & 48 & 48 & $\mathrm{~F}$ & 14 & 2.2 & 6.4 & Tremolite & & & $\begin{array}{l}\text { AS }>5,3: 1, \text { AFB }>5,3: 1, \\
\text { PCMEF-US, PCMES-US, } \\
\text { PCMEF-ISO, PCMES-ISO }\end{array}$ \\
\hline G2 & 7 & G1 & $A X$ & 49 & 49 & $\mathrm{~F}$ & 4 & 0.7 & 5.7 & Tremolite & & & \\
\hline G2 & 7 & G1 & $A X$ & 50 & 50 & $\mathrm{~F}$ & 5.8 & 0.6 & 9.7 & Tremolite & & & $\begin{array}{l}\text { AS >5, 3:1, AFB >5, 3:1, } \\
\text { PCMEF-US, PCMES-US, } \\
\text { PCMEF-ISO, PCMES-ISO }\end{array}$ \\
\hline G2 & 8 & 13 & $A X$ & 51 & & MD 1-1 & 23.8 & 10 & 2.4 & Tremolite & & & $A S>5,3: 1$ \\
\hline G2 & 8 & 13 & $A X$ & & 51 & MF & 19.5 & 0.55 & 35.5 & Tremolite & & & $\begin{array}{c}\text { AFB }>5,3: 1, \text { PCMEF-US, } \\
\text { PCMEF-ISO }\end{array}$ \\
\hline G2 & 8 & 13 & $A X$ & 52 & 52 & $F$ & 4.5 & 0.9 & 5 & Tremolite & & & \\
\hline G2 & 8 & 13 & $A X$ & 53 & 53 & $\mathrm{~F}$ & 6.2 & 0.35 & 17.7 & Tremolite & & & $\begin{array}{l}\text { AS>5, 3:1, AFB>5, 3:1, } \\
\text { PCMEF-US, PCMES-US, } \\
\text { PCMEF-ISO, PCMES-ISO }\end{array}$ \\
\hline G2 & 9 & F7 & $A X$ & 54 & 54 & $F$ & 16 & 0.6 & 26.7 & Tremolite & & & $\begin{array}{l}\text { AS>5, 3:1, AFB }>5,3: 1 \text {, } \\
\text { PCMEF-US, PCMES-US, } \\
\text { PCMEF-ISO, PCMES-ISO }\end{array}$ \\
\hline G2 & 9 & F7 & $A X$ & 55 & 55 & $F$ & 12 & 1.2 & 10 & Tremolite & & & $\begin{array}{l}\text { AS>5, 3:1, AFB>5, 3:1, } \\
\text { PCMEF-US, PCMES-US, } \\
\text { PCMEF-ISO, PCMES-ISO }\end{array}$ \\
\hline G2 & 9 & F7 & $A X$ & 56 & 56 & $\mathrm{~F}$ & 4.35 & 0.65 & 6.7 & Tremolite & & & \\
\hline G2 & 9 & F7 & $A X$ & 57 & 57 & $\mathrm{~F}$ & 3 & 0.6 & 5 & Tremolite & & & \\
\hline G2 & 9 & F7 & $A X$ & 58 & & MD 1-1 & 20 & 12 & 1.7 & Tremolite & & & $A S>5,3: 1$ \\
\hline G2 & 9 & F7 & $A X$ & & 58 & MF & 8.75 & 0.9 & 9.7 & Tremolite & & & $\begin{array}{c}\text { AFB }>5,3: 1, \text { PCMEF-US, } \\
\text { PCMEF-ISO }\end{array}$ \\
\hline
\end{tabular}

Count Categories

AFB $>5,3: 1$

Asbestos Fibers and Bundles > 5um and 3:1

Primary Asbestos Structures

PCM Equivalent Fibers-NIOSH

PCMEF-US

$\mathrm{PCM}$ Equivalent Structures-NIOSH
AS>5, 3:1 PCMEF-ISO PCMES-ISO TAS
Asbestos Structures $>5$ um and 3:1 PCM Equivalent Fibers-ISO PCM Equivalent Structures-ISO Total Asbestos Structures 
ISO 10312, Direct Raw Data

Job Number: 070434

SEA

Report Number: 070434R06

Client: Idaho National Laboratory

Date Received: 4/23/2007

Project Name: RARE

Client Sample No: FB-2-R7

Lab/Cor Sample No: S32

Client Description:

Date Sampled:

4/16/2007

\begin{tabular}{|c|c|c|c|c|c|c|c|c|c|c|c|c|c|}
\hline$\overline{\mathrm{Gr}}$ & No. & Loc. & IID & Prim & Tot & Class & Len & Wid & Asp & Analyte & Elements & Comment & Count Categories \\
\hline G2 & 9 & F7 & $A X$ & 59 & 59 & $\mathrm{~F}$ & 3.75 & 0.55 & 6.8 & Tremolite & & & \\
\hline G2 & 9 & F7 & $A X$ & 60 & 60 & $\mathrm{~F}$ & 1.75 & 0.4 & 4.4 & Tremolite & & & \\
\hline G2 & 10 & B9 & $A X$ & 61 & 61 & $\mathrm{~F}$ & 10.5 & 0.7 & 15 & Tremolite & & & $\begin{array}{l}\text { AS }>5,3: 1, \text { AFB }>5,3: 1, \\
\text { PCMEF-US, PCMES-US, } \\
\text { PCMEF-ISO, PCMES-ISO }\end{array}$ \\
\hline G2 & 10 & B9 & $A X$ & 62 & 62 & $\mathrm{~F}$ & 7 & 0.5 & 14 & Tremolite & & & $\begin{array}{l}\text { AS }>5,3: 1, \text { AFB }>5,3: 1, \\
\text { PCMEF-US, PCMES-US, } \\
\text { PCMEF-ISO, PCMES-ISO }\end{array}$ \\
\hline G2 & 10 & B9 & $A X$ & 63 & 63 & $\mathrm{~F}$ & 9 & 1.2 & 7.5 & Tremolite & & & $\begin{array}{l}\text { AS>5, 3:1, AFB>5, 3:1, } \\
\text { PCMEF-US, PCMES-US, } \\
\text { PCMEF-ISO, PCMES-ISO }\end{array}$ \\
\hline G2 & 10 & B9 & $A X$ & 64 & 64 & $\mathrm{~F}$ & 12 & 2.5 & 4.8 & Tremolite & & & $\begin{array}{l}\text { PCMEF-US, PCMES-US, } \\
\text { PCMEF-ISO, PCMES-ISO }\end{array}$ \\
\hline G2 & 10 & B9 & $A X$ & 65 & 65 & $\mathrm{~F}$ & 12 & 1.85 & 6.5 & Tremolite & & & $\begin{array}{l}\text { AFB>5, 3:1, AS>5, 3:1, } \\
\text { PCMEF-US, PCMES-US, } \\
\text { PCMEF-ISO, PCMES-ISO }\end{array}$ \\
\hline G2 & 10 & B9 & $A X$ & 66 & 66 & $F$ & 20 & 1.85 & 10.8 & Tremolite & & & $\begin{array}{l}\text { AS }>5,3: 1, \text { AFB }>5,3: 1, \\
\text { PCMEF-US, PCMES-US, } \\
\text { PCMEF-ISO, PCMES-ISO }\end{array}$ \\
\hline G2 & 10 & B9 & $A X$ & 67 & 67 & $\mathrm{~F}$ & 3.5 & 0.55 & 6.4 & Tremolite & & & \\
\hline G2 & 10 & B9 & $A X$ & 68 & 68 & $\mathrm{~F}$ & 15.35 & 0.7 & 21.9 & Tremolite & & & $\begin{array}{l}\text { AS }>5,3: 1, \text { AFB }>5,3: 1, \\
\text { PCMEF-US, PCMES-US, } \\
\text { PCMEF-ISO, PCMES-ISO }\end{array}$ \\
\hline
\end{tabular}

Count Categories

AFB $>5,3: 1$

PAS

PCMEF-US

Asbestos Fibers and Bundles > 5um and 3:1

Primary Asbestos Structures

PCM Equivalent Fibers-NIOSH

PCMES-US
PCM Equivalent Structures-NIOSH
AS $>5,3: 1$ PCMEF-ISO PCMES-ISO TAS
Asbestos Structures $>5$ um and 3:1 PCM Equivalent Fibers-ISO PCM Equivalent Structures-ISO Total Asbestos Structures 
ISO 10312, Direct Raw Data

Job Number: 070434

SEA

Report Number: 070434R06

Client: Idaho National Laboratory

Date Received: 4/23/2007

Project Name: RARE

Client Sample No: FB-1-R1

Lab/Cor Sample No: S33

Client Description:

Date Sampled:

$4 / 16 / 2007$

\begin{tabular}{|c|c|c|c|c|c|c|c|c|c|c|c|c|c|}
\hline Gr & No. & Loc. & ID & Prim & Tot & Class & Len & Wid & Asp & Analyte & Elements & Comment & Count Categories \\
\hline G1 & 1 & B4 & $A X$ & 1 & 1 & $F$ & 6.8 & 1.15 & 5.9 & Tremolite & & & $\begin{array}{l}\text { AS }>5,3: 1, \text { AFB }>5,3: 1, \\
\text { PCMEF-US, PCMES-US, } \\
\text { PCMEF-ISO, PCMES-ISO }\end{array}$ \\
\hline G1 & 2 & D4 & $A X$ & 2 & 2 & $\mathrm{~F}$ & 4.2 & 0.5 & 8.4 & Tremolite & & & \\
\hline G1 & 2 & D4 & $A X$ & 3 & 3 & $\mathrm{~F}$ & 7.5 & 2 & 3.8 & Tremolite & & & $\begin{array}{l}\text { PCMEF-US, PCMES-US, } \\
\text { PCMEF-ISO, PCMES-ISO }\end{array}$ \\
\hline G1 & 2 & D4 & $A X$ & 4 & 4 & $\mathrm{~F}$ & 4 & 0.5 & 8 & Tremolite & & & \\
\hline G1 & 2 & D4 & $A X$ & 5 & 5 & $\mathrm{~F}$ & 4 & 1.1 & 3.6 & Tremolite & & & \\
\hline G1 & 2 & D4 & $A X$ & 6 & & MD 1-0 & 7 & 7 & 1 & Tremolite & & & $A S>5,3: 1$ \\
\hline G1 & 2 & D4 & $A X$ & & 6 & MF & 4.5 & 1 & 4.5 & Tremolite & & & \\
\hline G1 & 2 & D4 & $A X$ & 7 & & CD 2-0 & 5 & 2 & 2.5 & Tremolite & & & \\
\hline G1 & 2 & D4 & $A X$ & & 7 & $\mathrm{CF}$ & 5 & 0.75 & 6.7 & Tremolite & & & \\
\hline G1 & 2 & D4 & $A X$ & & 8 & $\mathrm{CF}$ & 1.7 & 0.35 & 4.9 & Tremolite & & & \\
\hline G1 & 3 & 15 & $A X$ & 8 & 9 & $\mathrm{~F}$ & 24.5 & 0.6 & 40.8 & Tremolite & & & $\begin{array}{l}\text { AS }>5,3: 1, \text { AFB }>5,3: 1, \\
\text { PCMEF-US, PCMES-US, } \\
\text { PCMEF-ISO, PCMES-ISO }\end{array}$ \\
\hline G1 & 3 & 15 & $A X$ & 9 & 10 & $\mathrm{~F}$ & 1.75 & 0.55 & 3.2 & Tremolite & & & \\
\hline G1 & 4 & $\mathrm{H} 8$ & $A X$ & 10 & 11 & $\mathrm{~F}$ & 32.5 & 0.6 & 54.2 & Tremolite & & & $\begin{array}{l}\text { AS }>5,3: 1, \text { AFB }>5,3: 1, \\
\text { PCMEF-US, PCMES-US, } \\
\text { PCMEF-ISO, PCMES-ISO }\end{array}$ \\
\hline G1 & 4 & $\mathrm{H} 8$ & $A X$ & 11 & 12 & $\mathrm{~F}$ & 14 & 2 & 7 & Tremolite & & & $\begin{array}{l}\text { AS }>5,3: 1, \text { AFB }>5,3: 1, \\
\text { PCMEF-US, PCMES-US, } \\
\text { PCMEF-ISO, PCMES-ISO }\end{array}$ \\
\hline \multirow[t]{5}{*}{ G1 } & 4 & $\mathrm{H} 8$ & $A Z Q$ & 12 & & MD 3-1 & 25 & 6 & 4.2 & Tremolite & $\begin{array}{l}\mathrm{Mg}, \mathrm{Si}, \mathrm{Ca} \\
\mathrm{Fe}\end{array}$ & & AS >5, 3:1, PCMES-US \\
\hline & & & & & & \multicolumn{2}{|c|}{ ItemType } & ItemNc & & & Confirmed & Com & \\
\hline & & & & & & \multicolumn{2}{|c|}{ Brightfield } & J3583 & & & & & \\
\hline & & & & & & \multicolumn{2}{|c|}{ Diffraction } & J3583 & & & $\mathrm{KM} \quad 6 / 6 / 2007$ & ZON & IS [ [ $\left.\begin{array}{lll}2 & 0 & 1\end{array}\right]$ \\
\hline & & & & & & \multicolumn{2}{|c|}{ Spectra } & J3025 & & & & & \\
\hline G1 & 4 & $\mathrm{H} 8$ & $A Z Q$ & & 13 & MF & 20 & 1 & 20 & Tremolite & & & $\begin{array}{c}\text { AFB >5, 3:1, PCMEF-US, } \\
\text { PCMEF-ISO }\end{array}$ \\
\hline G1 & 4 & $\mathrm{H} 8$ & $A Z Q$ & & 14 & MF & 4 & 0.66 & 6.1 & Tremolite & & & \\
\hline G1 & 4 & $\mathrm{H} 8$ & $A Z Q$ & & 15 & MF & 3.2 & 0.5 & 6.4 & Tremolite & & & \\
\hline G1 & 4 & $\mathrm{H} 8$ & $A X$ & 13 & & MD 1-1 & 10 & 5 & 2 & Tremolite & & & $A S>5,3: 1$ \\
\hline G1 & 4 & $\mathrm{H} 8$ & $A X$ & & 16 & MF & 10 & 0.5 & 20 & Tremolite & & & $\begin{array}{c}\text { AFB }>5,3: 1, \text { PCMEF-US, } \\
\text { PCMEF-ISO }\end{array}$ \\
\hline G1 & 5 & D7 & $A X$ & 14 & 17 & $\mathrm{~F}$ & 10.8 & 0.8 & 13.5 & Tremolite & & & $\begin{array}{l}\text { AS >5, 3:1, AFB>5, 3:1, } \\
\text { PCMEF-US, PCMES-US, } \\
\text { PCMEF-ISO, PCMES-ISO }\end{array}$ \\
\hline G1 & 5 & D7 & $A X$ & 15 & 18 & $\mathrm{~F}$ & 1.5 & 0.3 & 5 & Tremolite & & & \\
\hline
\end{tabular}

Count Categories

$A F B>5,3: 1$

PAS

PCMEF-US

Asbestos Fibers and Bundles > 5um and 3:

Primary Asbestos Structures

PCM Equivalent Fibers-NIOSH

PCMES-US
AS $>5,3: 1$ PCMEF-ISO PCMES-ISO TAS
Asbestos Structures $>5$ um and 3:1 PCM Equivalent Fibers-ISO PCM Equivalent Structures-ISO Total Asbestos Structures 
ISO 10312, Direct Raw Data

Job Number: 070434

SEA

Report Number: 070434R06

Client: Idaho National Laboratory

Date Received: 4/23/2007

Project Name: RARE

Client Sample No: FB-1-R1

Lab/Cor Sample No: S33

Client Description:

Date Sampled:

$4 / 16 / 2007$

\begin{tabular}{|c|c|c|c|c|c|c|c|c|c|c|c|c|c|}
\hline Gr & No. & Loc. & ID & Prim & Tot & Class & Len & Wid & Asp & Analyte & Elements & Comment & Count Categories \\
\hline G1 & 5 & D7 & $A X$ & 16 & 19 & $F$ & 8.85 & 0.6 & 14.8 & Tremolite & & & $\begin{array}{l}\text { AS>5, 3:1, AFB>5, 3:1, } \\
\text { PCMEF-US, PCMES-US, } \\
\text { PCMEF-ISO, PCMES-ISO }\end{array}$ \\
\hline G2 & 6 & B3 & $A X$ & 17 & 20 & $\mathrm{~F}$ & 15 & 0.7 & 21.4 & Tremolite & & & $\begin{array}{l}\text { AS }>5,3: 1, \text { AFB }>5,3: 1 \text {, } \\
\text { PCMEF-US, PCMES-US, } \\
\text { PCMEF-ISO, PCMES-ISO }\end{array}$ \\
\hline G2 & 6 & B3 & $A X$ & 18 & 21 & $\mathrm{~F}$ & 7.7 & 1.2 & 6.4 & Tremolite & & & $\begin{array}{l}\text { AS }>5,3: 1, \text { AFB }>5,3: 1, \\
\text { PCMEF-US, PCMES-US, } \\
\text { PCMEF-ISO, PCMES-ISO }\end{array}$ \\
\hline G2 & 7 & E1 & $A X$ & 19 & 22 & $\mathrm{~F}$ & 5 & 0.65 & 7.7 & Tremolite & & & \\
\hline G2 & 8 & G5 & $A X$ & 20 & 23 & $\mathrm{~F}$ & 6 & 0.5 & 12 & Tremolite & & & $\begin{array}{l}\text { AS }>5,3: 1, \text { AFB }>5,3: 1 \text {, } \\
\text { PCMEF-US, PCMES-US, } \\
\text { PCMEF-ISO, PCMES-ISO }\end{array}$ \\
\hline G2 & 8 & G5 & $A X$ & 21 & 24 & $\mathrm{~F}$ & 13 & 1.2 & 10.8 & Tremolite & & & $\begin{array}{l}\text { AS }>5,3: 1, \text { AFB }>5,3: 1, \\
\text { PCMEF-US, PCMES-US, } \\
\text { PCMEF-ISO, PCMES-ISO }\end{array}$ \\
\hline G2 & 9 & J8 & $A X$ & 22 & 25 & $F$ & 10.1 & 1.1 & 9.2 & Tremolite & & & $\begin{array}{l}\text { AS >5, 3:1, AFB>5, 3:1, } \\
\text { PCMEF-US, PCMES-US, } \\
\text { PCMEF-ISO, PCMES-ISO }\end{array}$ \\
\hline G2 & 9 & J8 & $A X$ & 23 & 26 & $F$ & 13.5 & 0.75 & 18 & Tremolite & & & $\begin{array}{l}\text { AS>5, 3:1, AFB>5, 3:1, } \\
\text { PCMEF-US, PCMES-US, } \\
\text { PCMEF-ISO, PCMES-ISO }\end{array}$ \\
\hline G2 & 10 & D8 & $A X$ & 24 & 27 & $\mathrm{~F}$ & 6.5 & 0.4 & 16.2 & Tremolite & & & $\begin{array}{l}\text { AS }>5,3: 1, \text { AFB }>5,3: 1, \\
\text { PCMEF-US, PCMES-US, } \\
\text { PCMEF-ISO, PCMES-ISO }\end{array}$ \\
\hline G2 & 10 & D8 & $A X$ & 25 & & MD 1-1 & 15 & 5 & 3 & Tremolite & & & AS>5, 3:1, PCMES-US \\
\hline G2 & 10 & D8 & $A X$ & & 28 & MF & 12.5 & 1 & 12.5 & Tremolite & & & $\begin{array}{c}\text { AFB }>5,3: 1, \text { PCMEF-US, } \\
\text { PCMEF-ISO }\end{array}$ \\
\hline G2 & 10 & D8 & $A X$ & 26 & 29 & $\mathrm{~F}$ & 7.5 & 0.85 & 8.8 & Tremolite & & & $\begin{array}{l}\text { AS }>5,3: 1, \text { AFB }>5,3: 1, \\
\text { PCMEF-US, PCMES-US, } \\
\text { PCMEF-ISO, PCMES-ISO }\end{array}$ \\
\hline G2 & 10 & D8 & $A X$ & 27 & 30 & $\mathrm{~F}$ & 17.75 & 1.75 & 10.1 & Tremolite & & & $\begin{array}{l}\text { AS }>5,3: 1, \text { AFB }>5,3: 1 \text {, } \\
\text { PCMEF-US, PCMES-US, } \\
\text { PCMEF-ISO, PCMES-ISO }\end{array}$ \\
\hline G2 & 10 & D8 & $A X$ & 28 & 31 & $\mathrm{~F}$ & 6 & 0.6 & 10 & Tremolite & & & $\begin{array}{l}\text { AS }>5,3: 1, \text { AFB }>5,3: 1, \\
\text { PCMEF-US, PCMES-US, } \\
\text { PCMEF-ISO, PCMES-ISO }\end{array}$ \\
\hline
\end{tabular}

Count Categories

AFB $>5,3: 1$

PAS

PCMEF-US

Asbestos Fibers and Bundles > 5um and 3:1

Primary Asbestos Structures

PCM Equivalent Fibers-NIOSH

PCMES-US
AS>5, 3:1 PCMEF-ISO PCMES-ISO TAS
Asbestos Structures >5um and 3:1 PCM Equivalent Fibers-ISO PCM Equivalent Structures-ISO Total Asbestos Structures 
Phone: (206) 781-0155

Fax: (206) 789-8424

7619 6th Ave Nw

ISO 10312, Direct Raw Data

Job Number: 070434

SEA

Report Number: 070434R06

Client: Idaho National Laboratory

Date Received: 4/23/2007

Project Name: RARE

Client Sample No: FB-1-R2

Lab/Cor Sample No: S34

Client Description:

Date Sampled

$4 / 16 / 2007$

\begin{tabular}{|c|c|c|c|c|c|c|c|c|c|c|c|c|}
\hline $\mathrm{Gr}$ & No. & Loc. & ID & Prim Tot & Class & Len & Wid & Asp & Analyte & Elements & Comment & Count Categories \\
\hline G1 & 1 & $\mathrm{~A} 2$ & & & NSD & & & & & & & \\
\hline G1 & 2 & $\mathrm{C} 2$ & & & NSD & & & & & & & \\
\hline G1 & 3 & E2 & & & NSD & & & & & & & \\
\hline G1 & 4 & G2 & & & NSD & & & & & & & \\
\hline G1 & 5 & 12 & & & NSD & & & & & & & \\
\hline G1 & 6 & J4 & & & NSD & & & & & & & \\
\hline G1 & 7 & $\mathrm{H} 4$ & & & NSD & & & & & & & \\
\hline G1 & 8 & $\mathrm{~F} 4$ & & & NSD & & & & & & & \\
\hline G1 & 9 & D4 & & & NSD & & & & & & & \\
\hline G1 & 10 & B4 & & & NSD & & & & & & & \\
\hline G1 & 11 & A7 & & & NSD & & & & & & & \\
\hline G1 & 12 & C7 & & & NSD & & & & & & & \\
\hline G1 & 13 & E7 & & & NSD & & & & & & & \\
\hline G1 & 14 & G7 & & & NSD & & & & & & & \\
\hline G1 & 15 & 17 & & & NSD & & & & & & & \\
\hline G1 & 16 & J9 & & & NSD & & & & & & & \\
\hline G1 & 17 & $\mathrm{H} 9$ & & & NSD & & & & & & & \\
\hline G1 & 18 & F9 & & & NSD & & & & & & & \\
\hline G1 & 19 & D9 & & & NSD & & & & & & & \\
\hline G1 & 20 & B9 & & & NSD & & & & & & & \\
\hline G2 & 21 & $\mathrm{~A} 2$ & & & NSD & & & & & & & \\
\hline G2 & 22 & $\mathrm{C} 2$ & & & NSD & & & & & & & \\
\hline G2 & 23 & E2 & & & NSD & & & & & & & \\
\hline G2 & 24 & G2 & & & NSD & & & & & & & \\
\hline G2 & 25 & 12 & & & NSD & & & & & & & \\
\hline G2 & 26 & J4 & & & NSD & & & & & & & \\
\hline G2 & 27 & $\mathrm{H} 4$ & & & NSD & & & & & & & \\
\hline G2 & 28 & F4 & & & NSD & & & & & & & \\
\hline G2 & 29 & D4 & & & NSD & & & & & & & \\
\hline G2 & 30 & B4 & & & NSD & & & & & & & \\
\hline G2 & 31 & A7 & & & NSD & & & & & & & \\
\hline G2 & 32 & $\mathrm{C} 7$ & & & NSD & & & & & & & \\
\hline G2 & 33 & E7 & & & NSD & & & & & & & \\
\hline G2 & 34 & G7 & & & NSD & & & & & & & \\
\hline
\end{tabular}

Count Categories

AFB $>5,3: 1$

PAS

PCMEF-US

PCMES-US
Asbestos Fibers and Bundles > 5um and 3:1

Primary Asbestos Structures

PCM Equivalent Fibers-NIOSH

PCM Equivalent Structures-NIOSH
AS $>5,3: 1$ PCMEF-ISO PCMES-ISO TAS
Asbestos Structures $>5$ um and 3:1 PCM Equivalent Fibers-ISO PCM Equivalent Structures-ISO Total Asbestos Structures 
Phone: (206) 781-0155

7619 6th Ave Nw

ISO 10312, Direct Raw Data

Job Number: 070434

SEA

Report Number: 070434R06

Client: Idaho National Laboratory

Date Received: 4/23/2007

Project Name: RARE

Client Sample No: FB-1-R2

Lab/Cor Sample No: S34

Client Description:

Date Sampled:

$4 / 16 / 2007$

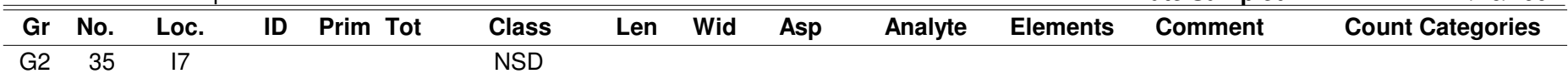

Count Categories

AFB $>5$, 3:1

PAS

PCMEF-US

PCMES-US
Asbestos Fibers and Bundles > 5um and 3:1

Primary Asbestos Structures

PCM Equivalent Fibers-NIOSH

PCM Equivalent Structures-NIOSH
AS>5, 3:1 PCMEF-ISO PCMES-ISO TAS
Asbestos Structures >5um and 3:1 PCM Equivalent Fibers-ISO PCM Equivalent Structures-ISO Total Asbestos Structures 
ISO 10312, Direct Raw Data

Job Number: 070434

SEA

Report Number: 070434R06

Client: Idaho National Laboratory

Date Received: 4/23/2007

Project Name: RARE

Client Sample No: FB-1-R3

Lab/Cor Sample No: S35

Client Description:

Date Sampled:

4/16/2007

\begin{tabular}{|c|c|c|c|c|c|c|c|c|c|c|c|c|}
\hline Gr & No. & Loc. & ID & Prim & Tot & Class & Len & Wid & Asp & Analyte & Elements & Count Categories \\
\hline G1 & 1 & E1 & AZQ & 1 & 1 & $F$ & 9.8 & 0.6 & 16.3 & Tremolite & $\begin{array}{l}\mathrm{Mg}, \mathrm{Al}, \mathrm{Si} \\
\mathrm{Ca}, \mathrm{Fe}\end{array}$ & $\begin{array}{l}\text { AS>5, 3:1, AFB>5, 3:1, } \\
\text { PCMEF-US, PCMES-US, } \\
\text { PCMEF-ISO, PCMES-ISO }\end{array}$ \\
\hline & & & & & & \multicolumn{2}{|c|}{ ItemType } & ItemNu & & & Confirmed & Comment \\
\hline & & & & & & \multicolumn{2}{|c|}{ Brightfield } & J3584 & & & & \\
\hline & & & & & & \multicolumn{2}{|c|}{ Diffraction } & $\mathrm{J} 3584$ & & & KM 6/6/2007 & ZONE AXIS [ $\left.3 \begin{array}{lll}3 & 1 & 4\end{array}\right]$ \\
\hline & & & & & & \multicolumn{2}{|c|}{ Spectra } & J3026 & & & & \\
\hline G1 & 1 & E1 & $A X$ & 2 & 2 & $\mathrm{~F}$ & 3.85 & 0.65 & 5.9 & Tremolite & & \\
\hline G1 & 1 & E1 & $A X$ & 3 & 3 & $\mathrm{~F}$ & 3.8 & 0.8 & 4.8 & Tremolite & & \\
\hline G1 & 1 & E1 & $A X$ & 4 & 4 & $\mathrm{~F}$ & 8.8 & 1.2 & 7.3 & Tremolite & & $\begin{array}{l}\text { AS }>5,3: 1, \text { AFB }>5,3: 1, \\
\text { PCMEF-US, PCMES-US, } \\
\text { PCMEF-ISO, PCMES-ISO }\end{array}$ \\
\hline G1 & 1 & E1 & $A X$ & 5 & 5 & $\mathrm{~F}$ & 3.2 & 0.65 & 4.9 & Tremolite & & \\
\hline G1 & 1 & E1 & $A X$ & 6 & 6 & $\mathrm{~F}$ & 5.5 & 1.25 & 4.4 & Tremolite & & $\begin{array}{l}\text { PCMEF-US, PCMES-US, } \\
\text { PCMEF-ISO, PCMES-ISO }\end{array}$ \\
\hline G1 & 2 & 13 & $A X$ & 7 & 7 & $\mathrm{~F}$ & 7.25 & 0.4 & 18.1 & Tremolite & & $\begin{array}{l}\text { AS }>5,3: 1, \text { AFB }>5,3: 1, \\
\text { PCMEF-US, PCMES-US, } \\
\text { PCMEF-ISO, PCMES-ISO }\end{array}$ \\
\hline G1 & 2 & 13 & $A X$ & 8 & 8 & $F$ & 4.9 & 1.1 & 4.5 & Tremolite & & \\
\hline G1 & 2 & 13 & $A X$ & 9 & 9 & $\mathrm{~F}$ & 45 & 0.9 & 50 & Tremolite & & $\begin{array}{l}\text { AS>5, 3:1, AFB >5, 3:1, } \\
\text { PCMEF-US, PCMES-US, } \\
\text { PCMEF-ISO, PCMES-ISO }\end{array}$ \\
\hline G1 & 2 & 13 & $A X$ & 10 & 10 & $F$ & 2 & 0.4 & 5 & Tremolite & & \\
\hline G1 & 3 & G6 & $A X$ & 11 & 11 & $F$ & 4.85 & 0.85 & 5.7 & Tremolite & & \\
\hline G1 & 3 & G6 & $A X$ & 12 & 12 & $F$ & 15.5 & 1.2 & 12.9 & Tremolite & & $\begin{array}{l}\text { AS }>5,3: 1, \text { AFB }>5,3: 1, \\
\text { PCMES-US, PCMEF-US, } \\
\text { PCMEF-ISO, PCMES-ISO }\end{array}$ \\
\hline G1 & 4 & J8 & $A X$ & 13 & 13 & $F$ & 1.1 & 0.35 & 3.1 & Tremolite & & \\
\hline G1 & 4 & J8 & $A X$ & 14 & 14 & $F$ & 48 & 1.85 & 25.9 & Tremolite & & $\begin{array}{l}\text { AS>5, 3:1, AFB>5, 3:1, } \\
\text { PCMEF-US, PCMES-US, } \\
\text { PCMEF-ISO, PCMES-ISO }\end{array}$ \\
\hline G1 & 4 & J8 & $A X$ & 15 & & MD 1-0 & 2 & 1.5 & 1.3 & Tremolite & & \\
\hline G1 & 4 & J8 & $A X$ & & 15 & MF & 1.75 & 0.5 & 3.5 & Tremolite & & \\
\hline G1 & 4 & J8 & $A X$ & 16 & 16 & $F$ & 12 & 1.75 & 6.9 & Tremolite & & $\begin{array}{l}\text { AS>5, 3:1, AFB>5, 3:1, } \\
\text { PCMEF-US, PCMES-US, } \\
\text { PCMEF-ISO, PCMES-ISO }\end{array}$ \\
\hline G1 & 4 & J8 & $A X$ & 17 & 17 & $F$ & 12.5 & 0.55 & 22.7 & Tremolite & & $\begin{array}{l}\text { AS>5, 3:1, AFB >5, 3:1, } \\
\text { PCMEF-US, PCMES-US, } \\
\text { PCMEF-ISO, PCMES-ISO }\end{array}$ \\
\hline G1 & 4 & J8 & $A X$ & 18 & 18 & $F$ & 5.8 & 0.85 & 6.8 & Tremolite & & $\begin{array}{l}\text { AS }>5,3: 1, \text { AFB }>5,3: 1, \\
\text { PCMEF-US, PCMES-US, } \\
\text { PCMEF-ISO, PCMES-ISO }\end{array}$ \\
\hline
\end{tabular}

Count Categories

AFB $>5,3: 1$

PAS

PCMEF-US

PCMES-US
Asbestos Fibers and Bundles > 5um and 3:1

Primary Asbestos Structures

PCM Equivalent Fibers-NIOSH

PCM Equivalent Structures-NIOSH
AS>5, 3:1 PCMEF-ISO PCMES-ISO TAS
Asbestos Structures $>5$ um and 3:1 PCM Equivalent Fibers-ISO PCM Equivalent Structures-ISO Total Asbestos Structures 
ISO 10312, Direct Raw Data

Job Number: 070434

SEA

Report Number: 070434R06

Client: Idaho National Laboratory

Date Received: 4/23/2007

Project Name: RARE

Client Sample No: FB-1-R3

Lab/Cor Sample No: S35

Client Description:

Date Sampled:

$4 / 16 / 2007$

\begin{tabular}{|c|c|c|c|c|c|c|c|c|c|c|c|c|c|}
\hline Gr & No. & Loc. & ID & Prim & Tot & Class & Len & Wid & Asp & Analyte & Elements & Comment & Count Categories \\
\hline G1 & 5 & E7 & $A X$ & 19 & 19 & $F$ & 20.5 & 2 & 10.2 & Tremolite & & & $\begin{array}{l}\text { AS>5, 3:1, AFB>5, 3:1, } \\
\text { PCMEF-US, PCMES-US, } \\
\text { PCMEF-ISO, PCMES-ISO }\end{array}$ \\
\hline G1 & 5 & E7 & $A X$ & 20 & 20 & $\mathrm{~F}$ & 6.2 & 0.55 & 11.3 & Tremolite & & & $\begin{array}{l}\text { AS }>5,3: 1, \text { AFB }>5,3: 1 \text {, } \\
\text { PCMEF-US, PCMES-US, } \\
\text { PCMEF-ISO, PCMES-ISO }\end{array}$ \\
\hline G1 & 5 & E7 & $A X$ & 21 & 21 & $\mathrm{~F}$ & 10.6 & 1.1 & 9.6 & Tremolite & & & $\begin{array}{l}\text { AS }>5,3: 1, \text { AFB }>5,3: 1, \\
\text { PCMEF-US, PCMES-US, } \\
\text { PCMEF-ISO, PCMES-ISO }\end{array}$ \\
\hline G1 & 5 & E7 & $A X$ & 22 & 22 & $\mathrm{~F}$ & 6.2 & 0.4 & 15.5 & Tremolite & & & $\begin{array}{l}\text { AS }>5,3: 1, \text { AFB }>5,3: 1, \\
\text { PCMEF-US, PCMES-US, } \\
\text { PCMEF-ISO, PCMES-ISO }\end{array}$ \\
\hline G1 & 5 & E7 & $A X$ & 23 & 23 & $\mathrm{~F}$ & 3.35 & 1 & 3.3 & Tremolite & & & \\
\hline G2 & 6 & B3 & $A X$ & 24 & 24 & $\mathrm{~F}$ & 2.2 & 0.7 & 3.1 & Tremolite & & & \\
\hline G2 & 7 & $\mathrm{~F} 2$ & $A X$ & 25 & 25 & $\mathrm{~F}$ & 35 & 2.25 & 15.6 & Tremolite & & & $\begin{array}{l}\text { AS }>5,3: 1, \text { AFB }>5,3: 1, \\
\text { PCMEF-US, PCMES-US, } \\
\text { PCMEF-ISO, PCMES-ISO }\end{array}$ \\
\hline G2 & 7 & $\mathrm{~F} 2$ & $A X$ & 26 & & MD 1-0 & 4.8 & 4.2 & 1.1 & Tremolite & & & \\
\hline G2 & 7 & $\mathrm{~F} 2$ & $A X$ & & 26 & MF & 3.85 & 0.4 & 9.6 & Tremolite & & & \\
\hline G2 & 8 & G4 & $A X$ & 27 & 27 & $\mathrm{~F}$ & 4 & 0.55 & 7.3 & Tremolite & & & \\
\hline G2 & 9 & 18 & $A X$ & 28 & 28 & $\mathrm{~F}$ & 10 & 1 & 10 & Tremolite & & & $\begin{array}{l}\text { AS }>5,3: 1, \text { AFB }>5,3: 1, \\
\text { PCMEF-US, PCMES-US, } \\
\text { PCMES-ISO, PCMEF-ISO }\end{array}$ \\
\hline G2 & 9 & 18 & $A X$ & 29 & 29 & $\mathrm{~F}$ & 9 & 0.45 & 20 & Tremolite & & & $\begin{array}{l}\text { AS }>5,3: 1, \text { AFB }>5,3: 1, \\
\text { PCMEF-US, PCMES-US, } \\
\text { PCMEF-ISO, PCMES-ISO }\end{array}$ \\
\hline G2 & 10 & D7 & $A X$ & 30 & 30 & $\mathrm{~F}$ & 8.85 & 0.8 & 11.1 & Tremolite & & & $\begin{array}{l}\text { AS }>5,3: 1, \text { AFB }>5,3: 1, \\
\text { PCMEF-US, PCMES-US, } \\
\text { PCMEF-ISO, PCMES-ISO }\end{array}$ \\
\hline G2 & 10 & D7 & $A X$ & 31 & 31 & $\mathrm{~F}$ & 10 & 1.5 & 6.7 & Tremolite & & & $\begin{array}{l}\text { AS }>5,3: 1, \text { AFB }>5,3: 1 \text {, } \\
\text { PCMEF-US, PCMES-US, } \\
\text { PCMEF-ISO, PCMES-ISO }\end{array}$ \\
\hline G2 & 10 & D7 & $A X$ & 32 & 32 & $\mathrm{~F}$ & 15.5 & 4.35 & 3.6 & Tremolite & & & PCMEF-US, PCMES-US \\
\hline
\end{tabular}

Count Categories

AFB $>5,3: 1$

PAS

PCMEF-US

Asbestos Fibers and Bundles > 5um and 3:1

Primary Asbestos Structures

PCM Equivalent Fibers-NIOSH

PCMES-US
PCM Equivalent Structures-NIOSH
AS $>5,3: 1$ PCMEF-ISO PCMES-ISO TAS
Asbestos Structures $>5$ um and 3:1 PCM Equivalent Fibers-ISO PCM Equivalent Structures-ISO Total Asbestos Structures 
ISO 10312, Direct Raw Data

Job Number: 070434

SEA

Report Number: 070434R06

Client: Idaho National Laboratory

Date Received: 4/23/2007

Project Name: RARE

Client Sample No: FB-1-R4

Lab/Cor Sample No: S36

Client Description:

Date Sampled:

4/16/2007

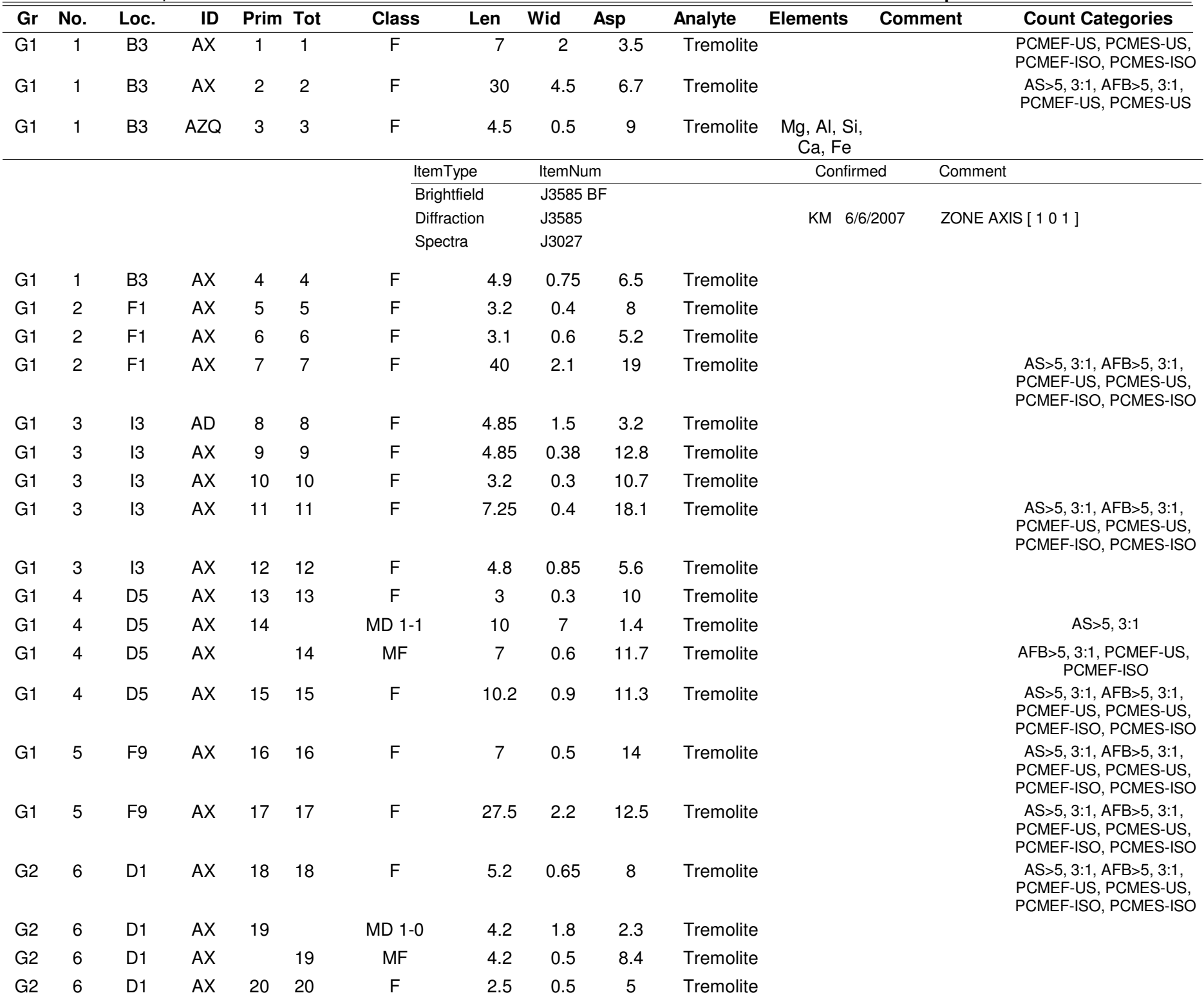

Count Categories

$\mathrm{AFB}>5,3: 1$

PAS

PCMEF-US

Asbestos Fibers and Bundles $>$ 5um and 3:1

Primary Asbestos Structures

PCM Equivalent Fibers-NIOSH

PCMES-US
AS $>5,3: 1$ PCMEF-ISO PCMES-ISO TAS

Page 140 of 281

Asbestos Structures $>5$ um and 3:1 PCM Equivalent Fibers-ISO PCM Equivalent Structures-ISO Total Asbestos Structures 
ISO 10312, Direct Raw Data

Job Number: 070434

SEA

Report Number: 070434R06

Client: Idaho National Laboratory

Date Received: 4/23/2007

Project Name: RARE

Client Sample No: FB-1-R4

Lab/Cor Sample No: S36

Client Description:

Date Sampled:

$4 / 16 / 2007$

\begin{tabular}{|c|c|c|c|c|c|c|c|c|c|c|c|c|c|}
\hline$\overline{\mathrm{Gr}}$ & No. & Loc. & IID & Prim & Tot & Class & Len & Wid & Asp & Analyte & Elements & Comment & "Count Categories \\
\hline G2 & 7 & 14 & $A X$ & 21 & 21 & $\mathrm{~F}$ & 15.75 & 2.8 & 5.6 & Tremolite & & & $\begin{array}{l}\text { AS }>5,3: 1, \text { AFB }>5,3: 1, \\
\text { PCMEF-US, PCMES-US, } \\
\text { PCMEF-ISO, PCMES-ISO }\end{array}$ \\
\hline G2 & 7 & 14 & $A X$ & 22 & 22 & $\mathrm{~F}$ & 4.85 & 1.15 & 4.2 & Tremolite & & & \\
\hline G2 & 7 & 14 & $A X$ & 23 & 23 & $\mathrm{~F}$ & 17 & 0.5 & 34 & Tremolite & & & $\begin{array}{l}\text { AS }>5,3: 1, \text { AFB }>5,3: 1, \\
\text { PCMEF-US, PCMES-US, } \\
\text { PCMEF-ISO, PCMES-ISO }\end{array}$ \\
\hline G2 & 7 & 14 & $A X$ & 24 & 24 & $\mathrm{~F}$ & 4.5 & 0.5 & 9 & Tremolite & & & \\
\hline G2 & 7 & 14 & $A X$ & 25 & 25 & $\mathrm{~F}$ & 5.7 & 1.5 & 3.8 & Tremolite & & & $\begin{array}{l}\text { PCMEF-US, PCMES-US, } \\
\text { PCMEF-ISO, PCMES-ISO }\end{array}$ \\
\hline G2 & 8 & G6 & $A X$ & 26 & & MD 1-1 & 11.5 & 3.5 & 3.3 & Tremolite & & & AS $>5,3: 1$, PCMES-US \\
\hline G2 & 8 & G6 & $A X$ & & 26 & MF & 7.2 & 1 & 7.2 & Tremolite & & & $\begin{array}{c}\text { AFB }>5,3: 1, \text { PCMEF-US, } \\
\text { PCMEF-ISO }\end{array}$ \\
\hline G2 & 8 & G6 & $A X$ & 27 & & MD 1-1 & 13 & 10 & 1.3 & Tremolite & & & $A S>5,3: 1$ \\
\hline G2 & 8 & G6 & $A X$ & & 27 & MF & 5.4 & 0.4 & 13.5 & Tremolite & & & $\begin{array}{c}\text { AFB }>5,3: 1, \text { PCMEF-US, } \\
\text { PCMEF-ISO }\end{array}$ \\
\hline G2 & 9 & $\mathrm{H} 9$ & $A X$ & 28 & 28 & $\mathrm{~F}$ & 2.6 & 0.8 & 3.2 & Tremolite & & & \\
\hline G2 & 9 & $\mathrm{H} 9$ & $A X$ & 29 & 29 & $\mathrm{~F}$ & 10.1 & 1.8 & 5.6 & Tremolite & & & $\begin{array}{l}\text { AS }>5,3: 1, \text { AFB }>5,3: 1, \\
\text { PCMEF-US, PCMES-US, } \\
\text { PCMEF-ISO, PCMES-ISO }\end{array}$ \\
\hline $\mathrm{G} 2$ & 10 & $\mathrm{C7}$ & $A X$ & 30 & 30 & $\mathrm{~F}$ & 21.5 & 1.8 & 11.9 & Tremolite & & & $\begin{array}{l}\text { AS }>5,3: 1, \text { AFB }>5,3: 1 \text {, } \\
\text { PCMEF-US, PCMES-US, } \\
\text { PCMEF-ISO, PCMES-ISO }\end{array}$ \\
\hline G2 & 10 & C7 & $A X$ & 31 & 31 & $\mathrm{~F}$ & 3.35 & 0.6 & 5.6 & Tremolite & & & \\
\hline G2 & 10 & $\mathrm{C7}$ & $A X$ & 32 & 32 & $\mathrm{~F}$ & 4.6 & 0.75 & 6.1 & Tremolite & & & \\
\hline G2 & 10 & C7 & $A X$ & 33 & 33 & $\mathrm{~F}$ & 30 & 5 & 6 & Tremolite & & & $\begin{array}{l}\text { AS }>5,3: 1, \text { AFB }>5,3: 1 \\
\text { PCMEF-US, PCMES-US }\end{array}$ \\
\hline
\end{tabular}

Count Categories

AFB $>5,3: 1$

PAS

PCMEF-US

PCMES-US
Asbestos Fibers and Bundles > 5um and 3:1

Primary Asbestos Structures

PCM Equivalent Fibers-NIOSH

PCM Equivalent Structures-NIOSH
AS>5, 3:1 PCMEF-ISO PCMES-ISO TAS
Asbestos Structures $>5$ um and 3:1

PCM Equivalent Fibers-ISO

PCM Equivalent Structures-ISO

Total Asbestos Structures 
Job Number: 070434

ISO 10312, Direct Raw Data

Client: Idaho National Laboratory

Report Number: 070434R06

Project Name: RARE

Date Received: 4/23/2007

Client Sample No: FB-1-R5

Lab/Cor Sample No: S37

Client Description:

Date Sampled:

4/16/2007

\begin{tabular}{|c|c|c|c|c|c|c|c|c|c|c|c|c|c|}
\hline Gr & No. & Loc. & ID & Prim & Tot & Class & Len & Wid & Asp & Analyte & Elements & Comment & Count Categories \\
\hline G1 & 1 & B2 & $A X$ & 1 & 1 & $\mathrm{~F}$ & 4.5 & 0.3 & 15 & Tremolite & & & \\
\hline G1 & 1 & B2 & $A X$ & 2 & 2 & $\mathrm{~F}$ & 5.1 & 0.5 & 10.2 & Tremolite & & & $\begin{array}{l}\text { AS }>5,3: 1, \text { AFB }>5,3: 1, \\
\text { PCMEF-US, PCMES-US, } \\
\text { PCMEF-ISO, PCMES-ISO }\end{array}$ \\
\hline G1 & 1 & B2 & $A X$ & 3 & 3 & $\mathrm{~F}$ & 4.8 & 0.35 & 13.7 & Tremolite & & & \\
\hline G1 & 2 & $\mathrm{~F} 1$ & $A X$ & 4 & 4 & $\mathrm{~F}$ & 20.3 & 1 & 20.3 & Tremolite & & & $\begin{array}{l}\text { AS>5, 3:1, AFB }>5,3: 1 \text {, } \\
\text { PCMEF-US, PCMES-US, } \\
\text { PCMEF-ISO, PCMES-ISO }\end{array}$ \\
\hline G1 & 2 & $\mathrm{~F} 1$ & $A X$ & 5 & 5 & $\mathrm{~F}$ & 4.8 & 0.85 & 5.6 & Tremolite & & & \\
\hline \multirow[t]{2}{*}{ G1 } & 3 & 13 & $A Q$ & 6 & 6 & $\mathrm{~F}$ & 10 & 1.5 & 6.7 & Tremolite & $\begin{array}{c}\mathrm{Mg}, \mathrm{Si}, \mathrm{Ca} \\
\mathrm{Fe}\end{array}$ & & $\begin{array}{l}\text { AS }>5,3: 1, \text { AFB }>5,3: 1, \\
\text { PCMEF-US, PCMES-US, } \\
\text { PCMEF-ISO, PCMES-ISO }\end{array}$ \\
\hline & & & & & & & & Item Nu & & & Confirm & Com & \\
\hline
\end{tabular}

$\begin{array}{llllllllll}\text { G1 } & 4 & \mathrm{G} 5 & \mathrm{AX} & 7 & \mathrm{MD} \mathrm{1-1} & 9 & 5 & 1.8 & \text { Tremolite }\end{array}$

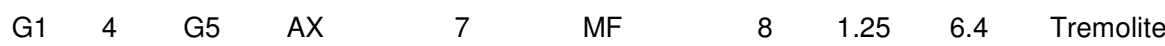

$\begin{array}{llllll}\text { G1 } & 4 & \mathrm{G} 5 & \mathrm{AX} & 8 & 8\end{array}$

G1 4 G5 AX 9

G1 4 G5

AX

MD 1-1

F

G1 $5 \quad$ F10

AZQ $\quad 10 \quad 10$

F

$\begin{array}{llll}4.5 & 0.65 & 6.9 & \text { Tremolite }\end{array}$

$\begin{array}{llll}44 & 10 & 4.4 & \text { Tremolite }\end{array}$

$\begin{array}{llll}44 & 0.7 & 62.9 & \text { Tremolite }\end{array}$

$A S>5,3: 1$

AFB $>5,3: 1$, PCMEF-US, PCMEF-ISO

AS $>5,3: 1$, PCMES-US

AFB $>5,3: 1$, PCMEF-US, PCMEF-ISO

AS $>5,3: 1, A F B>5,3: 1$ PCMEF-US, PCMES-US, PCMEF-ISO, PCMES-ISO

\begin{tabular}{|c|c|c|c|c|c|c|c|c|c|c|c|c|}
\hline & & & & & & & ItemType & ItemNur & & & Confirmed & Comment \\
\hline & & & & & & & Brightfield & $\mathrm{J} 3586 \mathrm{E}$ & & & & \\
\hline & & & & & & & Diffraction & J3586 & & & KM 6/7/2007 & ZONE AXIS [ $\left.\begin{array}{lll}3 & 1 & 4\end{array}\right]$ \\
\hline & & & & & & & Spectra & J3029 & & & & \\
\hline G1 & 5 & $\mathrm{~F} 10$ & $A X$ & 11 & 11 & $\mathrm{~F}$ & 5 & 0.3 & 16.7 & Tremolite & & \\
\hline G2 & 6 & B5 & $A X$ & 12 & 12 & $\mathrm{~F}$ & 5.8 & 1 & 5.8 & Tremolite & & $\begin{array}{l}\text { AS }>5,3: 1, \text { AFB }>5,3: 1, \\
\text { PCMEF-US, PCMES-US, } \\
\text { PCMEF-ISO, PCMES-ISO }\end{array}$ \\
\hline G2 & 6 & B5 & $A X$ & 13 & 13 & $\mathrm{~F}$ & 7.65 & 0.6 & 12.8 & Tremolite & & $\begin{array}{l}\text { AS }>5,3: 1, \text { AFB }>5,3: 1, \\
\text { PCMEF-US, PCMES-US, } \\
\text { PCMEF-ISO, PCMES-ISO }\end{array}$ \\
\hline G2 & 6 & B5 & $A X$ & 14 & 14 & $\mathrm{~F}$ & 20 & 2.85 & 7 & Tremolite & & $\begin{array}{l}\text { AS }>5,3: 1, \text { AFB }>5,3: 1, \\
\text { PCMEF-US, PCMES-US, } \\
\text { PCMEF-ISO, PCMES-ISO }\end{array}$ \\
\hline G2 & 7 & E2 & $A X$ & 15 & 15 & $\mathrm{~F}$ & 5.7 & 1 & 5.7 & Tremolite & & $\begin{array}{l}\text { AS>5, 3:1, AFB }>5,3: 1, \\
\text { PCMEF-US, PCMES-US, } \\
\text { PCMEF-ISO, PCMES-ISO }\end{array}$ \\
\hline G2 & 7 & E2 & $A X$ & 16 & 16 & $\mathrm{~F}$ & 3.1 & 0.85 & 3.6 & Tremolite & & \\
\hline G2 & 7 & E2 & $A X$ & 17 & 17 & $\mathrm{~F}$ & 3.12 & 0.4 & 7.8 & Tremolite & & \\
\hline
\end{tabular}

Count Categories

$A F B>5,3: 1$

PAS

PCMEF-US

Asbestos Fibers and Bundles > 5um and 3:1

Primary Asbestos Structures

PCM Equivalent Fibers-NIOSH

PCMES-US
AS $>5,3: 1$ PCMEF-ISO PCMES-ISO TAS

Page 142 of 281

Asbestos Structures $>5$ um and 3:1 PCM Equivalent Fibers-ISO PCM Equivalent Structures-ISO Total Asbestos Structures 
Job Number: 070434

ISO 10312, Direct Raw Data

Client: Idaho National Laboratory

Report Number: 070434R06

Project Name: RARE

Date Received: 4/23/2007

Client Sample No: FB-1-R5

Lab/Cor Sample No: S37

Client Description:

Date Sampled:

4/16/2007

\begin{tabular}{|c|c|c|c|c|c|c|c|c|c|c|c|c|c|}
\hline $\mathrm{Gr}$ & No. & Loc. & 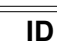 & Prim & Tot & Class & Len & Wid & Asp & Analyte & Elements & Comment & Count Categories \\
\hline G2 & 7 & E2 & $A X$ & 18 & 18 & $\mathrm{~F}$ & 4.9 & 0.25 & 19.6 & Tremolite & & & \\
\hline G2 & 8 & $\mathrm{H} 4$ & $A X$ & 19 & 19 & $\mathrm{~F}$ & 1.2 & 0.2 & 6 & Tremolite & & & \\
\hline G2 & 8 & $\mathrm{H} 4$ & $A X$ & 20 & 20 & $\mathrm{~F}$ & 4.2 & 1.2 & 3.5 & Tremolite & & & \\
\hline G2 & 8 & $\mathrm{H} 4$ & $A X$ & 21 & 21 & $\mathrm{~F}$ & 42 & 1 & 42 & Tremolite & & & $\begin{array}{l}\text { AS }>5,3: 1, \text { AFB }>5,3: 1 \text {, } \\
\text { PCMEF-US, PCMES-US, } \\
\text { PCMEF-ISO, PCMES-ISO }\end{array}$ \\
\hline G2 & 9 & J8 & $A X$ & 22 & 22 & $\mathrm{~F}$ & 1.1 & 0.3 & 3.7 & Tremolite & & & \\
\hline G2 & 9 & J8 & $A X$ & 23 & 23 & $\mathrm{~F}$ & 4.5 & 0.8 & 5.6 & Tremolite & & & \\
\hline G2 & 10 & D8 & $A X$ & 24 & & MD 1-1 & 13 & 3 & 4.3 & Tremolite & & & $\begin{array}{c}\text { AS }>5,3: 1, \text { PCMES-US, } \\
\text { PCMES-ISO }\end{array}$ \\
\hline G2 & 10 & D8 & $A X$ & & 24 & MF & 10.12 & 0.6 & 16.9 & Tremolite & & & $\begin{array}{c}\text { AFB }>5,3: 1, \text { PCMEF-US, } \\
\text { PCMEF-ISO }\end{array}$ \\
\hline G2 & 10 & D8 & $A X$ & 25 & 25 & $\mathrm{~F}$ & 12.5 & 0.45 & 27.8 & Tremolite & & & $\begin{array}{l}\text { AS }>5,3: 1, \text { AFB }>5,3: 1 \text {, } \\
\text { PCMEF-US, PCMES-US, } \\
\text { PCMEF-ISO, PCMES-ISO }\end{array}$ \\
\hline G2 & 10 & D8 & $A X$ & 26 & 26 & $\mathrm{~F}$ & 3.2 & 0.7 & 4.6 & Tremolite & & & \\
\hline
\end{tabular}

Count Categories

AFB $>5,3: 1$

PAS

PCMEF-US

PCMES-US
Asbestos Fibers and Bundles > 5um and 3:1

Primary Asbestos Structures

PCM Equivalent Fibers-NIOSH

PCM Equivalent Structures-NIOSH
AS $>5,3: 1$ PCMEF-ISO PCMES-ISO TAS
Asbestos Structures $>5$ um and 3:1

PCM Equivalent Fibers-ISO

PCM Equivalent Structures-ISO

Total Asbestos Structures 


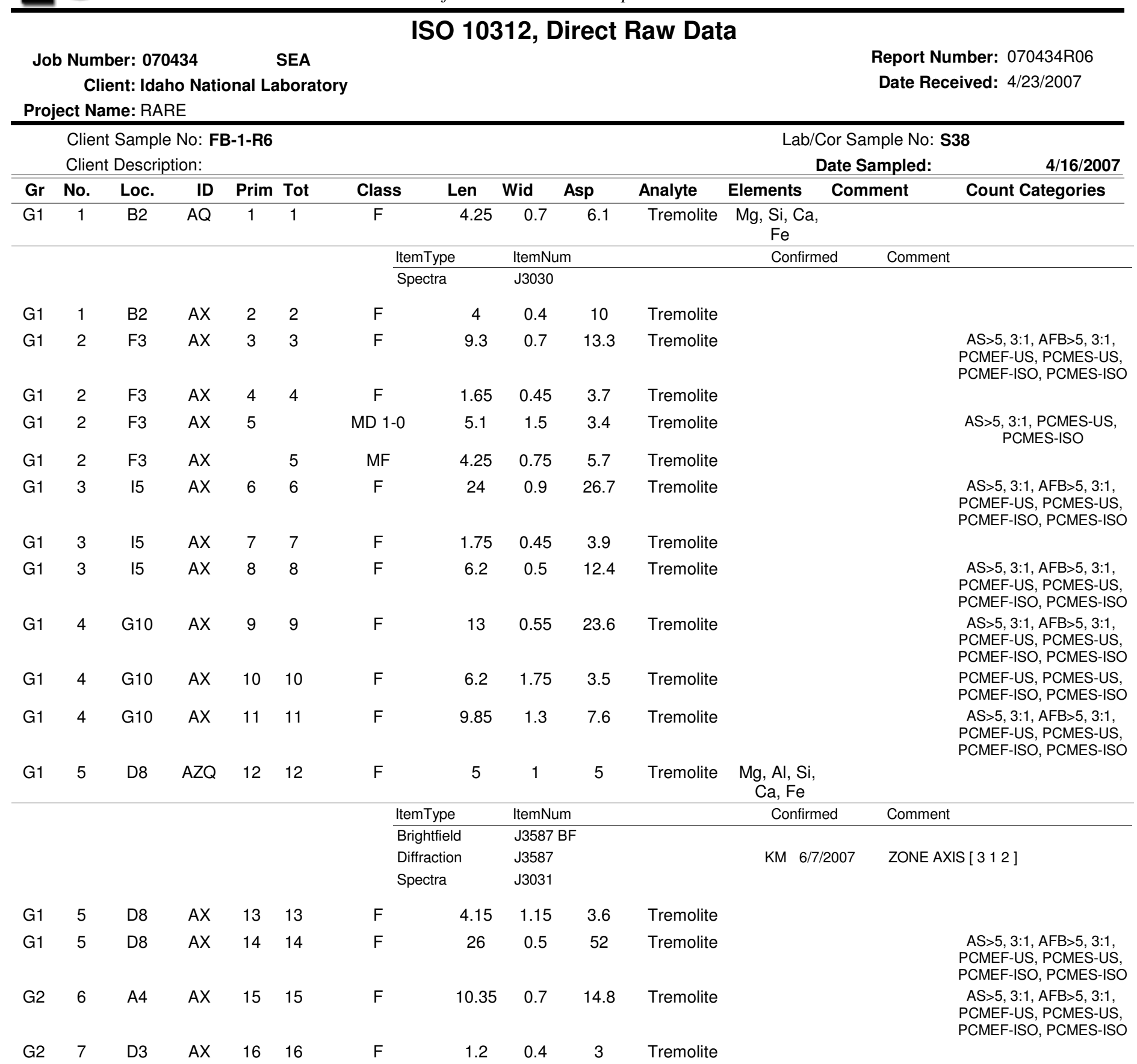

\section{Count Categories}

\section{AFB $>5,3: 1$}

PAS

PCMEF-US

PCMES-US
Asbestos Fibers and Bundles $>$ 5um and 3:1

Primary Asbestos Structures

PCM Equivalent Fibers-NIOSH

PCM Equivalent Structures-NIOSH
AS $>5,3: 1$ PCMEF-ISO PCMES-ISO TAS
Asbestos Structures $>5$ um and 3:1 PCM Equivalent Fibers-ISO PCM Equivalent Structures-ISO Total Asbestos Structures 
Job Number: 070434

SEA

Report Number: 070434R06

Client: Idaho National Laboratory

Date Received: 4/23/2007

Project Name: RARE

Client Sample No: FB-1-R6

Lab/Cor Sample No: S38

Client Description:

ISO 10312, Direct Raw Data

\begin{tabular}{|c|c|c|c|c|c|c|c|c|c|c|c|c|c|}
\hline Gr & No. & Loc. & ID & Prim & Tot & Class & Len & Wid & Asp & Analyte & Elements & Comment & Count Categories \\
\hline G2 & 7 & D3 & $A X$ & 17 & 17 & $\mathrm{~F}$ & 7.5 & 0.8 & 9.4 & Tremolite & & & $\begin{array}{l}\text { AS }>5,3: 1, \text { AFB }>5,3: 1, \\
\text { PCMEF-US, PCMES-US, } \\
\text { PCMEF-ISO, PCMES-ISO }\end{array}$ \\
\hline G2 & 7 & D3 & $A X$ & 18 & 18 & $\mathrm{~F}$ & 1.35 & 0.3 & 4.5 & Tremolite & & & \\
\hline G2 & 8 & 14 & $A X$ & 19 & & MD 1-0 & 10 & 5 & 2 & Tremolite & & & $A S>5,3: 1$ \\
\hline G2 & 8 & 14 & $A X$ & & 19 & MF & 2 & 0.35 & 5.7 & Tremolite & & & \\
\hline G2 & 8 & 14 & $A X$ & 20 & 20 & $\mathrm{~F}$ & 5.65 & 0.75 & 7.5 & Tremolite & & & $\begin{array}{l}\text { AS }>5,3: 1, \text { AFB }>5,3: 1, \\
\text { PCMEF-US, PCMES-US, } \\
\text { PCMEF-ISO, PCMES-ISO }\end{array}$ \\
\hline G2 & 8 & 14 & $A X$ & 21 & 21 & $\mathrm{~F}$ & 9 & 0.7 & 12.9 & Tremolite & & & $\begin{array}{l}\text { AS>5, 3:1, AFB>5, 3:1, } \\
\text { PCMEF-US, PCMES-US, } \\
\text { PCMEF-ISO, PCMES-ISO }\end{array}$ \\
\hline G2 & 9 & G7 & $A X$ & 22 & 22 & $\mathrm{~F}$ & 5.15 & 0.6 & 8.6 & Tremolite & & & $\begin{array}{l}\text { AS }>5,3: 1, \text { AFB }>5,3: 1, \\
\text { PCMEF-US, PCMES-US, } \\
\text { PCMEF-ISO, PCMES-ISO }\end{array}$ \\
\hline G2 & 9 & G7 & $A X$ & 23 & 23 & $\mathrm{~F}$ & 12.5 & 0.4 & 31.2 & Tremolite & & & $\begin{array}{l}\text { AS }>5,3: 1, \text { AFB }>5,3: 1, \\
\text { PCMEF-US, PCMES-US, } \\
\text { PCMEF-ISO, PCMES-ISO }\end{array}$ \\
\hline G2 & 9 & G7 & $A X$ & 24 & 24 & $F$ & 8 & 0.85 & 9.4 & Tremolite & & & $\begin{array}{l}\text { AS>5, 3:1, AFB }>5,3: 1, \\
\text { PCMEF-US, PCMES-US, } \\
\text { PCMEF-ISO, PCMES-ISO }\end{array}$ \\
\hline G2 & 10 & D7 & $A X$ & 25 & 25 & $F$ & 24 & 0.4 & 60 & Tremolite & & & $\begin{array}{l}\text { AFB>5, 3:1, AS>5, 3:1, } \\
\text { PCMEF-US, PCMES-US, } \\
\text { PCMEF-ISO, PCMES-ISO }\end{array}$ \\
\hline
\end{tabular}

Count Categories

AFB $>5,3: 1$

PAS

PCMEF-US

PCMES-US
Asbestos Fibers and Bundles > 5um and 3:1

Primary Asbestos Structures

PCM Equivalent Fibers-NIOSH

PCM Equivalent Structures-NIOSH
AS $>5,3: 1$ PCMEF-ISO PCMES-ISO TAS
Asbestos Structures >5um and 3:1 PCM Equivalent Fibers-ISO PCM Equivalent Structures-ISO Total Asbestos Structures 
ISO 10312, Direct Raw Data

Job Number: 070434

SEA

Report Number: 070434R06

Client: Idaho National Laboratory

Date Received: 4/23/2007

Project Name: RARE

Client Sample No: FB-1-R7

Lab/Cor Sample No: S39

Client Description:

Date Sampled:

$4 / 16 / 2007$

\begin{tabular}{|c|c|c|c|c|c|c|c|c|c|c|c|c|c|}
\hline$\overline{\mathrm{Gr}}$ & No. & Loc. & ID & Prim & Tot & Class & Len & Wid & Asp & Analyte & Elements & nent & Count Categories \\
\hline G1 & 1 & $\mathrm{C} 2$ & $A Z Q$ & 1 & 1 & $\mathrm{~F}$ & 4 & 0.38 & 10.5 & Tremolite & $\begin{array}{l}\text { Mg, Al, Si, } \\
\text { Ca, Fe }\end{array}$ & & \\
\hline & & & & & & \multicolumn{2}{|c|}{ ItemType } & ItemNu & & & Confirmed & Com & \\
\hline & & & & & & \multicolumn{2}{|c|}{ Brightfield } & $\mathrm{J} 3588$ & & & & & \\
\hline & & & & & & \multicolumn{2}{|c|}{ Diffraction } & J3588 & & & KM 6/7/2007 & ZON & XIS [ $\left.\begin{array}{lll}3 & 1 & 0\end{array}\right]$ \\
\hline & & & & & & \multicolumn{2}{|c|}{ Spectra } & J3032 & & & & & \\
\hline G1 & 1 & $\mathrm{C} 2$ & $A X$ & 2 & 2 & $\mathrm{~F}$ & 12 & 1.35 & 8.9 & Tremolite & & & $\begin{array}{l}\text { AS>5, 3:1, AFB >5, 3:1, } \\
\text { PCMEF-US, PCMES-US, } \\
\text { PCMEF-ISO, PCMES-ISO }\end{array}$ \\
\hline G1 & 1 & $\mathrm{C} 2$ & $A X$ & 3 & 3 & $\mathrm{~F}$ & 3.15 & 0.3 & 10.5 & Tremolite & & & \\
\hline G1 & 2 & $\mathrm{~F} 1$ & $A X$ & 4 & 4 & $\mathrm{~F}$ & 5.6 & 0.4 & 14 & Tremolite & & & $\begin{array}{l}\text { AS }>5,3: 1, \text { AFB }>5,3: 1 \text {, } \\
\text { PCMEF-US, PCMES-US, } \\
\text { PCMEF-ISO, PCMES-ISO }\end{array}$ \\
\hline G1 & 2 & $\mathrm{~F} 1$ & $A X$ & 5 & 5 & $\mathrm{~F}$ & 9 & 1.25 & 7.2 & Tremolite & & & $\begin{array}{l}\text { AS }>5,3: 1, \text { AFB }>5,3: 1 \text {, } \\
\text { PCMEF-US, PCMES-US, } \\
\text { PCMEF-ISO, PCMES-ISO }\end{array}$ \\
\hline G1 & 3 & 13 & $A X$ & 6 & & MD 2-1 & 15 & 12 & 1.2 & Tremolite & & & $A S>5,3: 1$ \\
\hline G1 & 3 & 13 & $A X$ & & 6 & MF & 5.2 & 0.38 & 13.7 & Tremolite & & & $\begin{array}{c}\text { AFB }>5,3: 1, \text { PCMEF-US, } \\
\text { PCMEF-ISO }\end{array}$ \\
\hline G1 & 3 & 13 & $A X$ & & 7 & MF & 2.3 & 0.4 & 5.8 & Tremolite & & & \\
\hline G1 & 3 & 13 & $A X$ & 7 & & MD 1-1 & 20 & 10 & 2 & Tremolite & & & $A S>5,3: 1$ \\
\hline G1 & 3 & 13 & $A X$ & & 8 & MF & 18.5 & 0.85 & 21.8 & Tremolite & & & $\begin{array}{c}\text { AFB }>5,3: 1, \text { PCMEF-US, } \\
\text { PCMEF-ISO }\end{array}$ \\
\hline G1 & 4 & G7 & $A X$ & 8 & 9 & $\mathrm{~F}$ & 2.35 & 0.25 & 9.4 & Tremolite & & & \\
\hline G1 & 4 & G7 & $A X$ & 9 & 10 & $\mathrm{~F}$ & 10.15 & 1.2 & 8.5 & Tremolite & & & $\begin{array}{l}\text { AS }>5,3: 1, \text { AFB }>5,3: 1 \text {, } \\
\text { PCMEF-US, PCMES-US, } \\
\text { PCMEF-ISO, PCMES-ISO }\end{array}$ \\
\hline G1 & 4 & G7 & $A X$ & 10 & 11 & $\mathrm{~F}$ & 5.35 & 0.9 & 5.9 & Tremolite & & & $\begin{array}{l}\text { AS>5, 3:1, AFB }>5,3: 1, \\
\text { PCMEF-US, PCMES-US, } \\
\text { PCMEF-ISO, PCMES-ISO }\end{array}$ \\
\hline G1 & 5 & D6 & & & & NSD & & & & & & & \\
\hline G2 & 6 & A4 & $A X$ & 11 & 12 & $\mathrm{~F}$ & 14.5 & 0.78 & 18.6 & Tremolite & & & $\begin{array}{l}\text { AS >5, 3:1, AFB>5, 3:1, } \\
\text { PCMES-US, PCMEF-US, } \\
\text { PCMEF-ISO, PCMES-ISO }\end{array}$ \\
\hline G2 & 6 & A4 & $A X$ & 12 & 13 & $\mathrm{~F}$ & 3.1 & 0.8 & 3.9 & Tremolite & & & \\
\hline G2 & 6 & A4 & $A X$ & 13 & & MD 1-0 & 7 & 4 & 1.8 & Tremolite & & & $A S>5,3: 1$ \\
\hline G2 & 6 & A4 & $A X$ & & 14 & MF & 4.2 & 0.5 & 8.4 & Tremolite & & & \\
\hline G2 & 7 & $\mathrm{C} 1$ & $A X$ & 14 & 15 & $\mathrm{~F}$ & 11 & 0.5 & 22 & Tremolite & & & $\begin{array}{l}\text { AS >5, 3:1, AFB>5, 3:1, } \\
\text { PCMEF-US, PCMES-US, } \\
\text { PCMEF-ISO, PCMES-ISO }\end{array}$ \\
\hline G2 & 7 & $\mathrm{C} 1$ & $A X$ & 15 & 16 & $\mathrm{~F}$ & 1.75 & 0.4 & 4.4 & Tremolite & & & \\
\hline
\end{tabular}

Count Categories

$\mathrm{AFB}>5,3: 1$

PAS

PCMEF-US

Asbestos Fibers and Bundles $>5 \mathrm{um}$ and $3: 1$

Primary Asbestos Structures

PCM Equivalent Fibers-NIOSH

PCMES-US
AS $>5,3: 1$ PCMEF-ISO PCMES-ISO TAS
Asbestos Structures $>5$ um and 3:1 PCM Equivalent Fibers-ISO PCM Equivalent Structures-ISO Total Asbestos Structures 
ISO 10312, Direct Raw Data

Job Number: 070434

SEA

Report Number: 070434R06

Client: Idaho National Laboratory

Date Received: 4/23/2007

Project Name: RARE

Client Sample No: FB-1-R7

Lab/Cor Sample No: S39

Client Description:

Date Sampled:

4/16/2007

\begin{tabular}{|c|c|c|c|c|c|c|c|c|c|c|c|c|c|}
\hline$\overline{\mathrm{Gr}}$ & No. & Loc. & 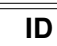 & Prim & Tot & Class & Len & Wid & Asp & Analyte & Elements & Comment & Count Categories \\
\hline G2 & 7 & C1 & $A X$ & 16 & 17 & $\mathrm{~F}$ & 8.58 & 0.88 & 9.8 & Tremolite & & & $\begin{array}{l}\text { AS }>5,3: 1, \text { AFB }>5,3: 1, \\
\text { PCMEF-US, PCMES-US, } \\
\text { PCMEF-ISO, PCMES-ISO }\end{array}$ \\
\hline G2 & 8 & G2 & $A X$ & 17 & 18 & $\mathrm{~F}$ & 5 & 0.6 & 8.3 & Tremolite & & & \\
\hline G2 & 8 & G2 & $A X$ & 18 & 19 & $\mathrm{~F}$ & 6.2 & 1.75 & 3.5 & Tremolite & & & $\begin{array}{l}\text { PCMEF-US, PCMES-US, } \\
\text { PCMEF-ISO, PCMES-ISO }\end{array}$ \\
\hline G2 & 8 & G2 & $A X$ & 19 & 20 & $\mathrm{~F}$ & 6.2 & 1.75 & 3.5 & Tremolite & & & $\begin{array}{l}\text { PCMEF-US, PCMES-US, } \\
\text { PCMEF-ISO, PCMES-ISO }\end{array}$ \\
\hline G2 & 8 & G2 & $A X$ & 20 & 21 & $\mathrm{~F}$ & 7.7 & 0.8 & 9.6 & Tremolite & & & $\begin{array}{l}\text { AS }>5,3: 1, \text { AFB }>5,3: 1, \\
\text { PCMEF-US, PCMES-US, } \\
\text { PCMEF-ISO, PCMES-ISO }\end{array}$ \\
\hline G2 & 8 & G2 & $A X$ & 21 & 22 & $\mathrm{~F}$ & 4.25 & 0.8 & 5.3 & Tremolite & & & \\
\hline G2 & 9 & J5 & $A X$ & 22 & 23 & $\mathrm{~F}$ & 2.4 & 0.3 & 8 & Tremolite & & & \\
\hline G2 & 9 & J5 & $A X$ & 23 & & MD 1-0 & 2.75 & 1.1 & 2.5 & Tremolite & & & \\
\hline G2 & 9 & J5 & $A X$ & & 24 & MF & 2.35 & 0.25 & 9.4 & Tremolite & & & \\
\hline G2 & 10 & $\mathrm{~F} 10$ & $A X$ & 24 & 25 & $\mathrm{~F}$ & 2.5 & 0.65 & 3.8 & Tremolite & & & \\
\hline G2 & 10 & $\mathrm{~F} 10$ & $A X$ & 25 & 26 & $\mathrm{~F}$ & 8.2 & 2 & 4.1 & Tremolite & & & $\begin{array}{l}\text { PCMEF-US, PCMES-US, } \\
\text { PCMEF-ISO, PCMES-ISO }\end{array}$ \\
\hline G2 & 10 & $\mathrm{~F} 10$ & $A X$ & 26 & 27 & $\mathrm{~F}$ & 7.5 & 0.65 & 11.5 & Tremolite & & & $\begin{array}{l}\text { AS }>5,3: 1, \text { AFB }>5,3: 1, \\
\text { PCMEF-US, PCMES-US, } \\
\text { PCMEF-ISO, PCMES-ISO }\end{array}$ \\
\hline
\end{tabular}

Count Categories

AFB $>5,3: 1$

PAS

PCMEF-US

Asbestos Fibers and Bundles > 5um and 3:1

Primary Asbestos Structures

PCM Equivalent Fibers-NIOSH

PCMES-US
AS $>5,3: 1$ PCMEF-ISO PCMES-ISO TAS
Asbestos Structures $>5$ um and 3:1 PCM Equivalent Fibers-ISO PCM Equivalent Structures-ISO Total Asbestos Structures 
Phone: (206) 781-0155

7619 6th Ave Nw

\section{ISO 10312, Direct Raw Data}

Job Number: 070434

SEA

Report Number: 070434R06

Client: Idaho National Laboratory

Date Received: 4/23/2007

Project Name: RARE

Reviewed by:

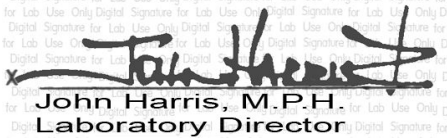

John Harris, M. Pir.

Asbestos Fibers and Bundles > 5um and 3:1

Primary Asbestos Structures

PCM Equivalent Fibers-NIOSH

PCMEF-US

PCM Equivalent Structures-NIOSH
AS $>5,3: 1$ PCMEF-ISO PCMES-ISO TAS
Asbestos Structures >5um and 3:1 PCM Equivalent Fibers-ISO

PCM Equivalent Structures-ISO

Total Asbestos Structures 
Job Number: 070434

SEA

Client: Idaho National Laboratory
A Professional Service Corporation in the Northwest

\section{ISO 10312, Direct Count Categories}

Report Number: 070434R06

Date Received: 4/23/2007

Project Name: RARE

Lab/Cor Sample No.: S3

Client Sample No.: cleaning blank \#3

Description:

Filter Fraction: 1

Residual Ash Vol:
Aliquot Dilution: 0

Final Dilution: 0
Volume (L): 0

Lab Filter Area (mm2): 385

Grid Openings Analyzed: 35

Average Grid Opening Area: 0.009

Area Analyzed (mm2): 0.315

Analytical Sens. (struc/cc): 0

Dectection Limit. (struc/cc): 0

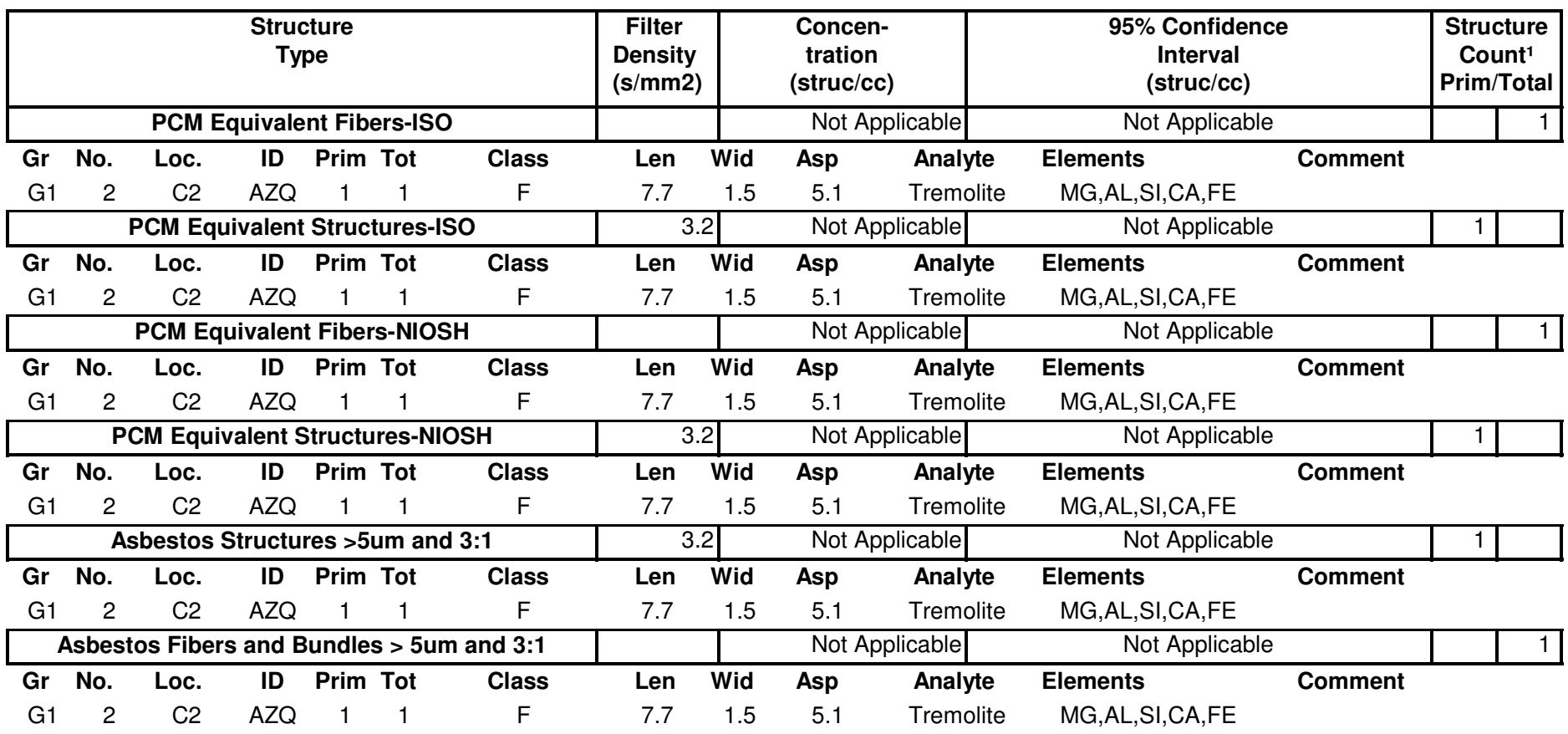


Job Number: 070434

\section{SEA}

\section{ISO 10312, Direct Count Categories}

Client: Idaho National Laboratory

Project Name: RARE

\section{Lab/Cor Sample No.: S10}

Client Sample No.: cleaning protocol 2

\section{Description:}

Filter Fraction: 1

Residual Ash Vol:
Aliquot Dilution: 0

Final Dilution: 0
Report Number: 070434R06

Date Received: 4/23/2007
Volume (L): 0

Lab Filter Area (mm2): 385

Grid Openings Analyzed: 35

Average Grid Opening Area: 0.009

Area Analyzed (mm2): 0.315

Analytical Sens. (struc/cc): 0

Dectection Limit. (struc/cc): 0

\begin{tabular}{|c|c|c|c|c|c|c|c|c|c|c|c|c|c|c|}
\hline \multicolumn{7}{|c|}{$\begin{array}{c}\text { Structure } \\
\text { Type }\end{array}$} & \multirow{2}{*}{$\begin{array}{c}\begin{array}{c}\text { Filter } \\
\text { Density } \\
\text { (s/mm2) }\end{array} \\
\end{array}$} & \multirow{2}{*}{\multicolumn{3}{|c|}{\begin{tabular}{c|}
$\begin{array}{c}\text { Concen- } \\
\text { tration } \\
\text { (struc/cc) }\end{array}$ \\
Not Applicable
\end{tabular}}} & \multirow{2}{*}{\multicolumn{2}{|c|}{$\begin{array}{c}\begin{array}{c}95 \% \text { Confidence } \\
\text { Interval } \\
\text { (struc/cc) }\end{array} \\
\text { Not Applicable } \\
\end{array}$}} & \multicolumn{2}{|c|}{\begin{tabular}{|c|} 
Structure \\
Count $^{1}$ \\
Prim/Total
\end{tabular}} \\
\hline \multicolumn{7}{|c|}{ PCM Equivalent Fibers-ISO } & & & & & & & & 2 \\
\hline Gr & No. & Loc. & ID & Prim & Tot & Class & Len & Wid & Asp & Analy & Elements & Comment & & \\
\hline G1 & 1 & $\mathrm{~A} 2$ & $A Z Q$ & 1 & 1 & $\mathrm{~F}$ & 5.5 & 1.1 & 5 & Tremo & $\mathrm{MG}, \mathrm{SI}, \mathrm{CA}, \mathrm{FE}$ & & & \\
\hline G2 & 21 & $\mathrm{~A} 2$ & $A D Q$ & & 2 & MF & 5.2 & 0.53 & 9.8 & Tremo & & & & \\
\hline \multicolumn{7}{|c|}{ PCM Equivalent Structures-ISO } & \multicolumn{2}{|c|}{3.2} & \multicolumn{2}{|c|}{ Not Applicable } & \multicolumn{2}{|l|}{ Not Applicable } & 1 & \\
\hline $\mathbf{G r}$ & No. & Loc. & ID & Prim & Tot & Class & Len & Wid & Asp & Analy & Elements & Comment & & \\
\hline G1 & 1 & $\mathrm{~A} 2$ & $A Z Q$ & 1 & 1 & $\mathrm{~F}$ & 5.5 & 1.1 & 5 & Tremo & $\mathrm{MG}, \mathrm{SI}, \mathrm{CA}, \mathrm{FE}$ & & & \\
\hline \multicolumn{7}{|c|}{ PCM Equivalent Fibers-NIOSH } & & & \multicolumn{2}{|c|}{ Not Applicable } & \multicolumn{2}{|l|}{ Not Applicable } & & 2 \\
\hline Gr & No. & Loc. & ID & Prim & Tot & Class & Len & Wid & Asp & Analy & Elements & Comment & & \\
\hline G1 & 1 & $\mathrm{~A} 2$ & $A Z Q$ & 1 & 1 & $\mathrm{~F}$ & 5.5 & 1.1 & 5 & Tremol & $\mathrm{MG}, \mathrm{SI}, \mathrm{CA}, \mathrm{FE}$ & & & \\
\hline G2 & 21 & $\mathrm{~A} 2$ & $A D Q$ & & 2 & MF & 5.2 & 0.53 & 9.8 & Tremol & & & & \\
\hline \multicolumn{7}{|c|}{ PCM Equivalent Structures-NIOSH } & \multicolumn{2}{|c|}{3.2} & \multicolumn{2}{|c|}{ Not Applicable } & \multicolumn{2}{|l|}{ Not Applicable } & 1 & \\
\hline Gr & No. & Loc. & ID & Prim & Tot & Class & Len & Wid & Asp & Analyt & Elements & Comment & & \\
\hline G1 & 1 & $\mathrm{~A} 2$ & $A Z Q$ & 1 & 1 & $\mathrm{~F}$ & 5.5 & 1.1 & 5 & Tremol & MG,SI,CA,FE & & & \\
\hline \multicolumn{7}{|c|}{ Asbestos Structures $>5$ um and 3:1 } & \multicolumn{2}{|c|}{6.3} & \multicolumn{2}{|c|}{ Not Applicable } & \multicolumn{2}{|l|}{ Not Applicable } & 2 & \\
\hline Gr & No. & Loc. & ID & Prim & Tot & Class & Len & Wid & Asp & Analyt & Elements & Comment & & \\
\hline G1 & 1 & A2 & $A Z Q$ & 1 & 1 & $\mathrm{~F}$ & 5.5 & 1.1 & 5 & Tremol & MG,SI,CA,FE & & & \\
\hline G2 & 21 & $\mathrm{~A} 2$ & $A D Q$ & 2 & & MD 1-1 & 8 & 6 & 1.3 & Tremol & MG,SI,CA,FE & & & \\
\hline \multicolumn{7}{|c|}{ Asbestos Fibers and Bundles > 5um and 3:1 } & & & \multicolumn{2}{|c|}{ Not Applicable } & \multicolumn{2}{|l|}{ Not Applicable } & & 2 \\
\hline Gr & No. & Loc. & ID & Prim & Tot & Class & Len & Wid & Asp & Analyt & Elements & Comment & & \\
\hline G1 & 1 & $\mathrm{~A} 2$ & $A Z Q$ & 1 & 1 & $\mathrm{~F}$ & 5.5 & 1.1 & 5 & Tremol & MG,SI,CA,FE & & & \\
\hline G2 & 21 & $\mathrm{~A} 2$ & $A D Q$ & & 2 & MF & 5.2 & 0.53 & 9.8 & Tremol & & & & \\
\hline
\end{tabular}


A Professional Service Corporation in the Northwest

Job Number: 070434

SEA

ISO 10312, Direct Count Categories

Client: Idaho National Laboratory

Project Name: RARE

Lab/Cor Sample No.: S12

Client Sample No.: FB-4-R1

Description:

Filter Fraction: 1

Residual Ash Vol:
Aliquot Dilution: 0

Final Dilution: 0
Report Number: 070434R06

Date Received: 4/23/2007
Volume (L): 0

Lab Filter Area (mm2): 385

Grid Openings Analyzed: 35

Average Grid Opening Area: 0.009

Area Analyzed (mm2): 0.315

Analytical Sens. (struc/cc): 0

Dectection Limit. (struc/cc): 0

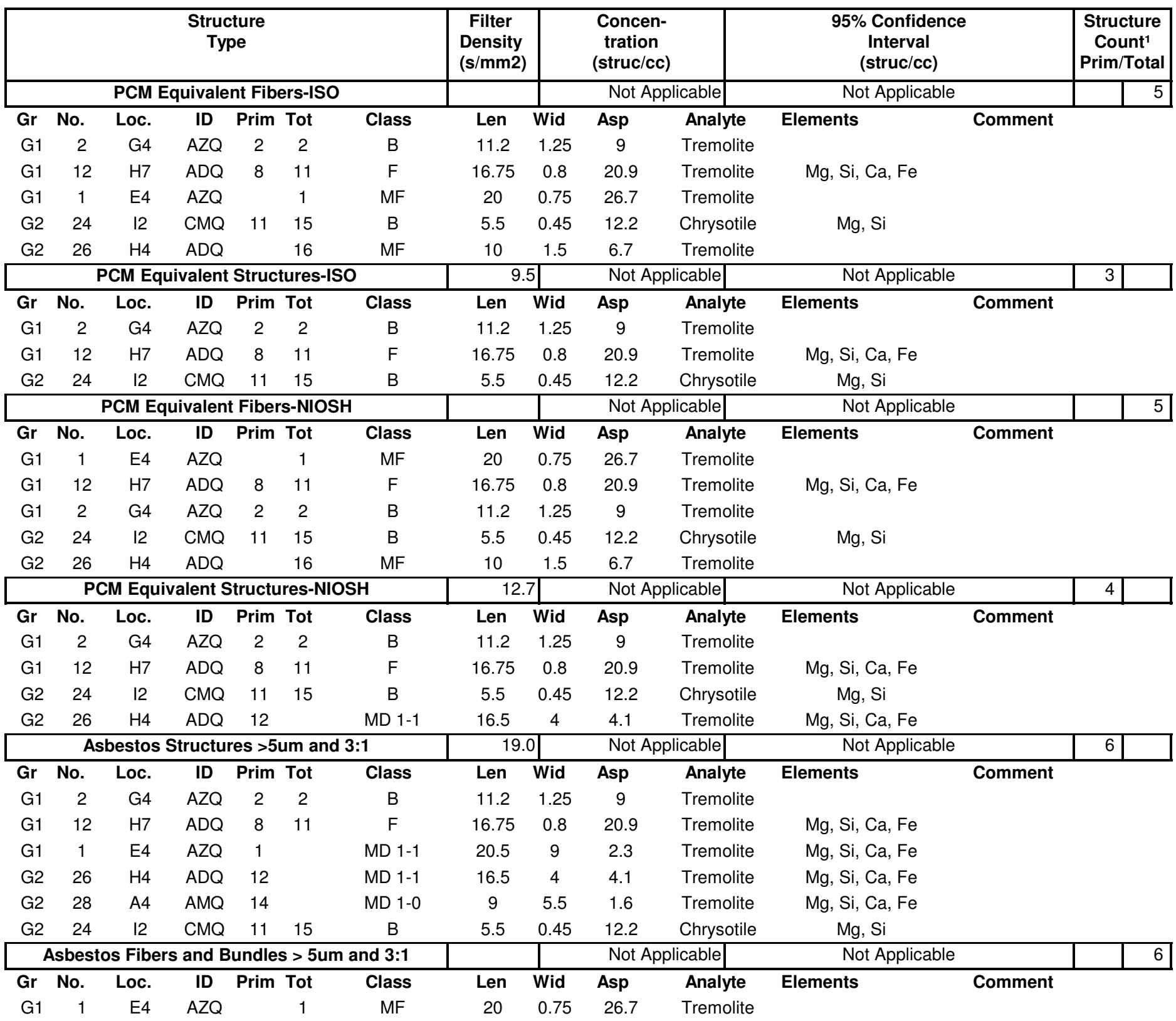


A Professional Service Corporation in the Northwest

ISO 10312, Direct Count Categories

Job Number: 070434

SEA

Report Number: 070434R06

Client: Idaho National Laboratory

Date Received: 4/23/2007

Project Name: RARE

Lab/Cor Sample No.: S12

Client Sample No.: FB-4-R1

Description:

Filter Fraction: 1

Residual Ash Vol:
Aliquot Dilution: 0

Final Dilution: 0
Volume (L): 0

Lab Filter Area (mm2): 385

Grid Openings Analyzed: 35

Average Grid Opening Area: 0.009

Area Analyzed (mm2): 0.315

Analytical Sens. (struc/cc): 0

Dectection Limit. (struc/cc): 0

\begin{tabular}{|c|c|c|c|c|c|c|c|c|c|c|c|c|}
\hline \multicolumn{7}{|c|}{$\begin{array}{c}\text { Structure } \\
\text { Type }\end{array}$} & $\begin{array}{c}\text { Filter } \\
\text { Density } \\
(\mathrm{s} / \mathrm{mm} 2)\end{array}$ & \multicolumn{3}{|c|}{$\begin{array}{l}\text { Concen- } \\
\text { tration } \\
\text { (struc/cc) }\end{array}$} & $\begin{array}{c}\text { 95\% Confidence } \\
\text { Interval } \\
\text { (struc/cc) }\end{array}$ & \begin{tabular}{|c} 
Structure \\
Count ${ }^{1}$ \\
Prim/Total
\end{tabular} \\
\hline \multicolumn{7}{|c|}{ Asbestos Fibers and Bundles > 5um and 3:1 } & & & \multicolumn{2}{|c|}{ Not Applicable } & Not Applicable & 6 \\
\hline Gr & No. & Loc. & ID & Prim & Tot & Class & Len & Wid & Asp & Anal & Elements & \\
\hline G1 & 2 & G4 & $A Z Q$ & 2 & 2 & B & 11.2 & 1.25 & 9 & Trem & & \\
\hline G1 & 8 & E2 & $\mathrm{CDQ}$ & & 5 & $\mathrm{CF}$ & 8 & 0.1 & 80 & Chrys & & \\
\hline G1 & 12 & $\mathrm{H} 7$ & $A D Q$ & 8 & 11 & $\mathrm{~F}$ & 16.75 & 0.8 & 20.9 & Trem & $\mathrm{Mg}, \mathrm{Si}, \mathrm{Ca}, \mathrm{Fe}$ & \\
\hline G2 & 26 & $\mathrm{H} 4$ & $A D Q$ & & 16 & MF & 10 & 1.5 & 6.7 & Trem & & \\
\hline $\mathrm{G} 2$ & 24 & 12 & $\mathrm{CMQ}$ & 11 & 15 & B & 5.5 & 0.45 & 12.2 & Chrys & $\mathrm{Mg}, \mathrm{Si}$ & \\
\hline
\end{tabular}


Job Number: 070434

SEA

ISO 10312, Direct Count Categories

Client: Idaho National Laboratory

Project Name: RARE

Lab/Cor Sample No.: S13

Client Sample No.: FB-4-R2

Description:

Filter Fraction: 1

Residual Ash Vol:
Aliquot Dilution: 0

Final Dilution: 0
Report Number: 070434R06

Date Received: 4/23/2007
Volume (L): 0

Lab Filter Area (mm2): 385

Grid Openings Analyzed: 35

Average Grid Opening Area: 0.009

Area Analyzed (mm2): 0.315

Analytical Sens. (struc/cc): 0

Dectection Limit. (struc/cc): 0

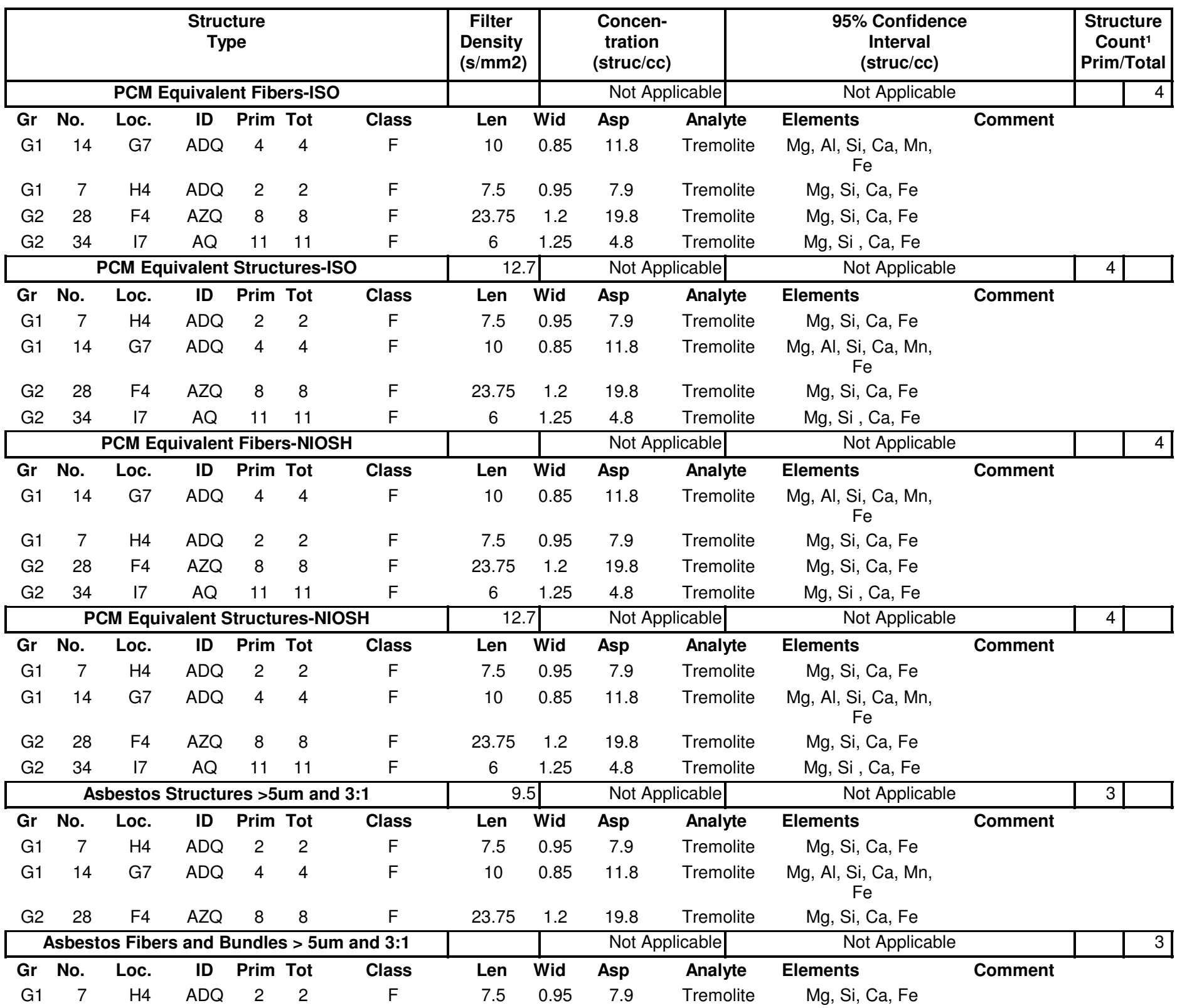


ISO 10312, Direct Count Categories

Job Number: 070434

SEA

Client: Idaho National Laboratory

Project Name: RARE

Lab/Cor Sample No.: S13

Client Sample No.: FB-4-R2

Description:

Filter Fraction: 1

Residual Ash Vol:
Aliquot Dilution: 0

Final Dilution: 0
Report Number: 070434R06

Date Received: 4/23/2007
Volume (L): 0

Lab Filter Area (mm2): 385

Grid Openings Analyzed: 35

Average Grid Opening Area: 0.009

Area Analyzed (mm2): 0.315

Analytical Sens. (struc/cc): 0

Dectection Limit. (struc/cc): 0

\begin{tabular}{|c|c|c|c|c|c|c|c|c|c|c|c|c|}
\hline \multicolumn{7}{|c|}{$\begin{array}{c}\text { Structure } \\
\text { Type }\end{array}$} & $\begin{array}{c}\text { Filter } \\
\text { Density } \\
(\mathrm{s} / \mathrm{mm} 2)\end{array}$ & \multicolumn{3}{|c|}{$\begin{array}{l}\text { Concen- } \\
\text { tration } \\
\text { (struc/cc) }\end{array}$} & $\begin{array}{c}\text { 95\% Confidence } \\
\text { Interval } \\
\text { (struc/cc) }\end{array}$ & $\begin{array}{c}\text { Structure } \\
\text { Count }{ }^{1} \\
\text { Prim/Total }\end{array}$ \\
\hline \multicolumn{7}{|c|}{ Asbestos Fibers and Bundles > 5um and 3:1 } & & & Not & licable & Not Applicable & 3 \\
\hline $\mathrm{Gr}$ & No. & Loc. & ID & Prim & Tot & Class & Len & Wid & Asp & Anal & Elements & \\
\hline G1 & 14 & G7 & $A D Q$ & 4 & 4 & $\mathrm{~F}$ & 10 & 0.85 & 11.8 & Trem & $\begin{array}{c}\mathrm{Mg}, \mathrm{Al}, \mathrm{Si}, \mathrm{Ca}, \mathrm{Mn}, \\
\mathrm{Fe}\end{array}$ & \\
\hline G2 & 28 & $\mathrm{~F} 4$ & $\mathrm{AZQ}$ & 8 & 8 & $\mathrm{~F}$ & 23.75 & 1.2 & 19.8 & Trem & $\mathrm{Mg}, \mathrm{Si}, \mathrm{Ca}, \mathrm{Fe}$ & \\
\hline
\end{tabular}


A Professional Service Corporation in the Northwest

Job Number: 070434

Client: Idaho National Laboratory

ISO 10312, Direct Count Categories

Project Name: RARE

Lab/Cor Sample No.: S14

Client Sample No.: FB-4-R3

Description:

Filter Fraction: 1

Residual Ash Vol:
SEA

Report Number: 070434R06

Date Received: 4/23/2007
Volume (L): 0

Lab Filter Area (mm2): 385

Grid Openings Analyzed: 35

Average Grid Opening Area: 0.009

Area Analyzed (mm2): 0.315

Final Dilution: 0

Analytical Sens. (struc/cc): 0

Dectection Limit. (struc/cc): 0

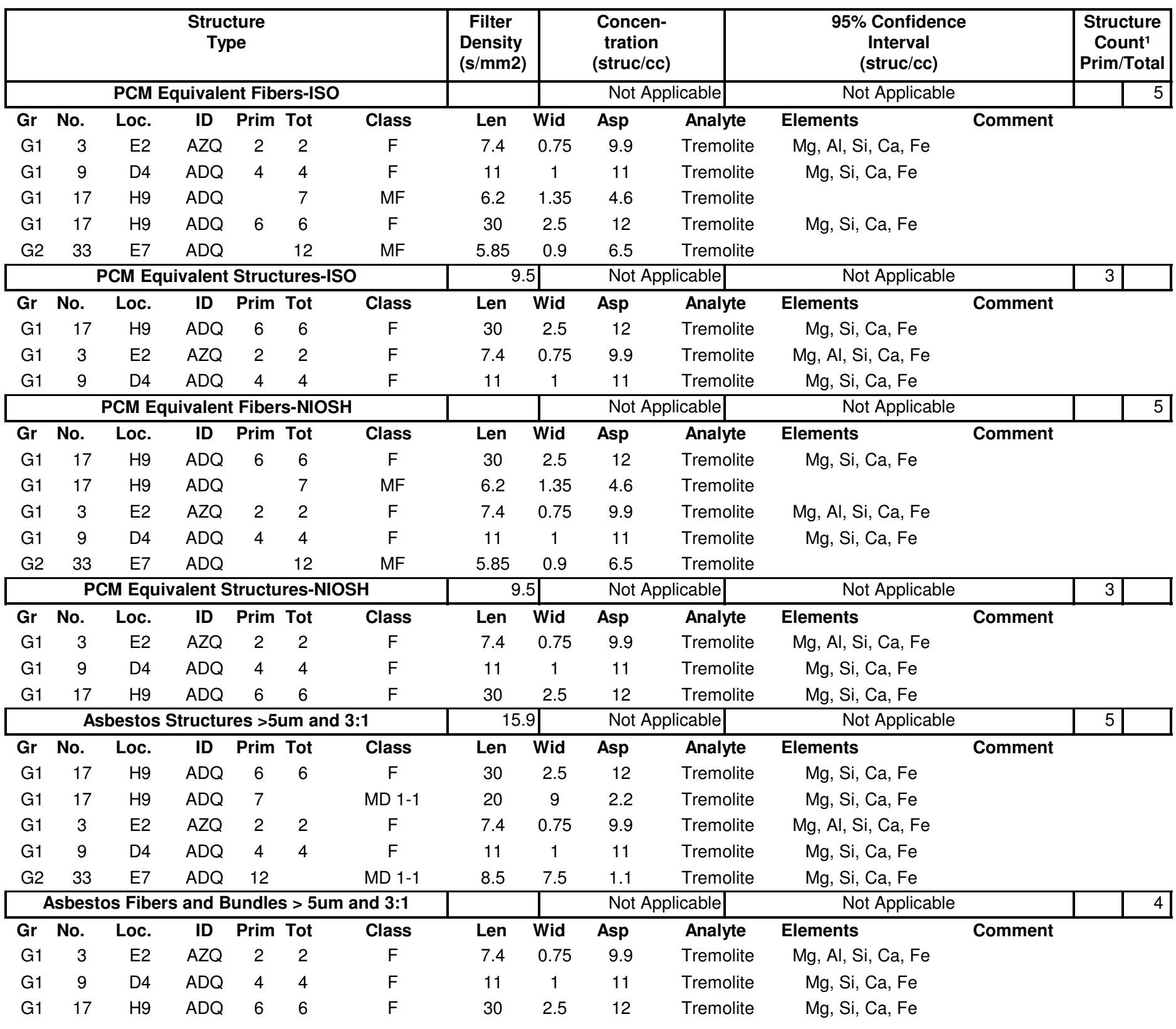


Phone: (206) 781-0155

7619 6th Ave Nw

ISO 10312, Direct Count Categories

Job Number: 070434

SEA

Report Number: 070434R06

Client: Idaho National Laboratory

Date Received: 4/23/2007

Project Name: RARE

Lab/Cor Sample No.: S14

Client Sample No.: FB-4-R3

Description:

Filter Fraction: 1

Aliquot Dilution: 0

Final Dilution: 0

Volume (L): 0

Lab Filter Area (mm2): 385

Grid Openings Analyzed: 35

Residual Ash Vol:

Area Analyzed (mm2): 0.315

Analytical Sens. (struc/cc): 0

Dectection Limit. (struc/cc): 0

\begin{tabular}{|c|c|c|c|c|c|c|c|c|c|c|}
\hline \multicolumn{6}{|c|}{$\begin{array}{c}\text { Structure } \\
\text { Type }\end{array}$} & $\begin{array}{c}\text { Filter } \\
\text { Density } \\
(\mathrm{s} / \mathrm{mm} 2)\end{array}$ & & $\begin{array}{l}\text { Concen- } \\
\text { tration } \\
\text { (struc/cc) }\end{array}$ & $\begin{array}{c}\text { 95\% Confidence } \\
\text { Interval } \\
\text { (struc/cc) }\end{array}$ & $\begin{array}{c}\text { Structure } \\
\text { Count }^{1} \\
\text { Prim/Total }\end{array}$ \\
\hline \multicolumn{6}{|c|}{ Asbestos Fibers and Bundles $>5$ um and 3:1 } & & & Not Applicable & Not Applicable & 4 \\
\hline Gr & No. & Loc. & ID & Prim Tot & Class & Len & Wid & Asp & Elements & \\
\hline G2 & 33 & E7 & $A D Q$ & 12 & MF & 5.85 & 0.9 & Trem & & \\
\hline
\end{tabular}


Job Number: 070434

SEA

Client: Idaho National Laboratory

\section{ISO 10312, Direct Count Categories}

Report Number: 070434R06

Date Received: 4/23/2007

Project Name: RARE

Lab/Cor Sample No.: S15

Client Sample No.: FB-4-R4

Description:

Filter Fraction: 1

Residual Ash Vol:
Aliquot Dilution: 0

Final Dilution: 0
Volume (L): 0

Lab Filter Area (mm2): 385

Grid Openings Analyzed: 35

Average Grid Opening Area: 0.009

Area Analyzed (mm2): 0.315

Analytical Sens. (struc/cc): 0

Dectection Limit. (struc/cc): 0

\begin{tabular}{|c|c|c|c|c|c|c|c|c|c|c|c|c|c|}
\hline \multicolumn{6}{|c|}{$\begin{array}{c}\text { Structure } \\
\text { Type }\end{array}$} & \multirow{2}{*}{$\begin{array}{c}\begin{array}{c}\text { Filter } \\
\text { Density } \\
\text { (s/mm2) }\end{array} \\
\end{array}$} & \multirow{2}{*}{\multicolumn{3}{|c|}{\begin{tabular}{c|}
$\begin{array}{c}\text { Concen- } \\
\text { tration } \\
\text { (struc/cc) }\end{array}$ \\
Not Applicable
\end{tabular}}} & \multirow{2}{*}{\multicolumn{2}{|c|}{$\begin{array}{c}\begin{array}{c}95 \% \text { Confidence } \\
\text { Interval } \\
\text { (struc/cc) }\end{array} \\
\text { Not Applicable }\end{array}$}} & \multicolumn{2}{|c|}{$\begin{array}{l}\text { Structure } \\
\text { Count }^{1} \\
\text { Prim/Total }\end{array}$} \\
\hline \multicolumn{6}{|c|}{ PCM Equivalent Fibers-ISO } & & & & & & & & 1 \\
\hline $\mathrm{Gr}$ & No. & Loc. & ID & Prim Tot & Class & Len & Wid & Asp & Analyte & Elements & Comment & & \\
\hline G2 & 21 & $\mathrm{~A} 2$ & $A D Q$ & 4 & $\mathrm{~F}$ & 15 & 1 & 15 & Tremolite & $\mathrm{Mg}, \mathrm{Si}, \mathrm{Ca}, \mathrm{Fe}$ & & & \\
\hline \multicolumn{6}{|c|}{ PCM Equivalent Structures-ISO } & \multicolumn{2}{|c|}{3.2} & \multicolumn{2}{|c|}{ Not Applicable } & \multicolumn{2}{|l|}{ Not Applicable } & 1 & \\
\hline $\mathrm{Gr}$ & No. & Loc. & ID & Prim Tot & Class & Len & Wid & Asp & Analyte & Elements & Comment & & \\
\hline G2 & 21 & $\mathrm{~A} 2$ & $A D Q$ & 4 & $\mathrm{~F}$ & 15 & 1 & 15 & Tremolite & $\mathrm{Mg}, \mathrm{Si}, \mathrm{Ca}, \mathrm{Fe}$ & & & \\
\hline \multicolumn{6}{|c|}{ PCM Equivalent Fibers-NIOSH } & & & \multicolumn{2}{|c|}{ Not Applicable } & \multicolumn{2}{|l|}{ Not Applicable } & & 1 \\
\hline $\mathrm{Gr}$ & No. & Loc. & ID & Prim Tot & Class & Len & Wid & Asp & Analyte & Elements & Comment & & \\
\hline G2 & 21 & $\mathrm{~A} 2$ & $A D Q$ & 4 & $\mathrm{~F}$ & 15 & 1 & 15 & Tremolite & $\mathrm{Mg}, \mathrm{Si}, \mathrm{Ca}, \mathrm{Fe}$ & & & \\
\hline \multicolumn{6}{|c|}{ PCM Equivalent Structures-NIOSH } & \multicolumn{2}{|c|}{3.2} & \multicolumn{2}{|c|}{ Not Applicable } & \multicolumn{2}{|l|}{ Not Applicable } & 1 & \\
\hline $\mathbf{G r}$ & No. & Loc. & ID & Prim Tot & Class & Len & Wid & Asp & Analyte & Elements & Comment & & \\
\hline G2 & 21 & $\mathrm{~A} 2$ & $A D Q$ & 4 & $\mathrm{~F}$ & 15 & 1 & 15 & Tremolite & $\mathrm{Mg}, \mathrm{Si}, \mathrm{Ca}, \mathrm{Fe}$ & & & \\
\hline \multicolumn{6}{|c|}{ Asbestos Structures $>5$ um and 3:1 } & \multicolumn{2}{|c|}{6.3} & \multicolumn{2}{|c|}{ Not Applicable } & \multicolumn{2}{|l|}{ Not Applicable } & 2 & \\
\hline $\mathrm{Gr}$ & No. & Loc. & ID & Prim Tot & Class & Len & Wid & Asp & Analyte & Elements & Comment & & \\
\hline G1 & 9 & D4 & $A Z Q$ & 1 & MD 1-0 & 5.7 & 3.8 & 1.5 & Tremolite & $\mathrm{Mg}, \mathrm{Si}, \mathrm{K}, \mathrm{Ca}, \mathrm{Fe}$ & & & \\
\hline G2 & 21 & $\mathrm{~A} 2$ & $A D Q$ & 4 & $\mathrm{~F}$ & 15 & 1 & 15 & Tremolite & $\mathrm{Mg}, \mathrm{Si}, \mathrm{Ca}, \mathrm{Fe}$ & & & \\
\hline \multicolumn{6}{|c|}{ Asbestos Fibers and Bundles > 5um and 3:1 } & & & \multicolumn{2}{|c|}{ Not Applicable } & \multicolumn{2}{|l|}{ Not Applicable } & & 1 \\
\hline $\mathbf{G r}$ & No. & Loc. & ID & Prim Tot & Class & Len & Wid & Asp & Analyte & Elements & ient & & \\
\hline $\mathrm{G} 2$ & 21 & $\mathrm{~A} 2$ & $A D Q$ & 4 & $\mathrm{~F}$ & 15 & 1 & 15 & Tremolite & $\mathrm{Mg}, \mathrm{Si}, \mathrm{Ca}, \mathrm{Fe}$ & & & \\
\hline
\end{tabular}


Job Number: 070434

Client: Idaho National Laboratory

ISO 10312, Direct Count Categories

Project Name: RARE

Lab/Cor Sample No.: S16

Client Sample No.: FB-4-R5

Description:

Filter Fraction: 1

Residual Ash Vol:
SEA

Report Number: 070434R06

Date Received: 4/23/2007
Volume (L): 0

Lab Filter Area (mm2): 385

Grid Openings Analyzed: 35

Average Grid Opening Area: 0.009

Area Analyzed (mm2): 0.315

Final Dilution: 0
Analytical Sens. (struc/cc): 0

Dectection Limit. (struc/cc): 0

\begin{tabular}{|c|c|c|c|c|c|c|c|c|c|c|c|c|c|c|}
\hline \multicolumn{7}{|c|}{$\begin{array}{c}\text { Structure } \\
\text { Type }\end{array}$} & \multirow[t]{2}{*}{$\begin{array}{c}\text { Filter } \\
\text { Density } \\
(\mathrm{s} / \mathrm{mm} 2)\end{array}$} & \multirow{2}{*}{\multicolumn{3}{|c|}{\begin{tabular}{|c|}
$\begin{array}{l}\text { Concen- } \\
\text { tration } \\
\text { (struc/cc) }\end{array}$ \\
Not Applicable \\
\end{tabular}}} & \multirow{2}{*}{\multicolumn{2}{|c|}{$\begin{array}{c}\begin{array}{c}95 \% \text { Confidence } \\
\text { Interval } \\
\text { (struc/cc) }\end{array} \\
\text { Not Applicable } \\
\end{array}$}} & \multicolumn{2}{|c|}{$\begin{array}{c}\text { Structure } \\
\text { Count }^{1} \\
\text { Prim/Total }\end{array}$} \\
\hline \multicolumn{7}{|c|}{ PCM Equivalent Fibers-ISO } & & & & & & & & 3 \\
\hline Gr & No. & Loc. & ID & Prim & Tot & Class & Len & Wid & Asp & Analy & Elements & Comment & & \\
\hline G1 & 5 & 12 & $A Z Q$ & & 1 & MF & 6.7 & 1.25 & 5.4 & Tremo & & & & \\
\hline G1 & 9 & D4 & $A D Q$ & 2 & 2 & $\mathrm{~F}$ & 17 & 1.35 & 12.6 & Tremo & $\mathrm{Mg}, \mathrm{Si}, \mathrm{Ca}, \mathrm{Fe}$ & & & \\
\hline G2 & 23 & E2 & $A D Q$ & 3 & 3 & $\mathrm{~B}$ & 7 & 1.5 & 4.7 & Tremo & $\mathrm{Mg}, \mathrm{Si}, \mathrm{Ca}, \mathrm{Fe}$ & & & \\
\hline \multicolumn{7}{|c|}{ PCM Equivalent Structures-ISO } & \multicolumn{2}{|c|}{6.3} & \multicolumn{2}{|c|}{ Not Applicable } & \multicolumn{2}{|l|}{ Not Applicable } & 2 & \\
\hline Gr & No. & Loc. & ID & Prim & Tot & Class & Len & Wid & Asp & Analy & Elements & Comment & & \\
\hline G1 & 9 & D4 & $A D Q$ & 2 & 2 & $\mathrm{~F}$ & 17 & 1.35 & 12.6 & Tremo & $\mathrm{Mg}, \mathrm{Si}, \mathrm{Ca}, \mathrm{Fe}$ & & & \\
\hline G2 & 23 & E2 & $A D Q$ & 3 & 3 & B & 7 & 1.5 & 4.7 & Tremo & $\mathrm{Mg}, \mathrm{Si}, \mathrm{Ca}, \mathrm{Fe}$ & & & \\
\hline \multicolumn{7}{|c|}{ PCM Equivalent Fibers-NIOSH } & & & \multicolumn{2}{|c|}{ Not Applicable } & \multicolumn{2}{|l|}{ Not Applicable } & & 3 \\
\hline Gr & No. & Loc. & ID & Prim & Tot & Class & Len & Wid & Asp & Analy & Elements & Comment & & \\
\hline G1 & 9 & D4 & $A D Q$ & 2 & 2 & $\mathrm{~F}$ & 17 & 1.35 & 12.6 & Tremo & $\mathrm{Mg}, \mathrm{Si}, \mathrm{Ca}, \mathrm{Fe}$ & & & \\
\hline G1 & 5 & 12 & $A Z Q$ & & 1 & MF & 6.7 & 1.25 & 5.4 & Tremo & & & & \\
\hline G2 & 23 & E2 & $A D Q$ & 3 & 3 & B & 7 & 1.5 & 4.7 & Tremo & $\mathrm{Mg}, \mathrm{Si}, \mathrm{Ca}, \mathrm{Fe}$ & & & \\
\hline \multicolumn{7}{|c|}{ PCM Equivalent Structures-NIOSH } & \multicolumn{2}{|c|}{6.3} & \multicolumn{2}{|c|}{ Not Applicable } & \multicolumn{2}{|l|}{ Not Applicable } & 2 & \\
\hline Gr & No. & Loc. & ID & Prim & Tot & Class & Len & Wid & Asp & Analy & Elements & Comment & & \\
\hline G1 & 9 & D4 & $A D Q$ & 2 & 2 & $\mathrm{~F}$ & 17 & 1.35 & 12.6 & Tremo & $\mathrm{Mg}, \mathrm{Si}, \mathrm{Ca}, \mathrm{Fe}$ & & & \\
\hline G2 & 23 & E2 & $A D Q$ & 3 & 3 & B & 7 & 1.5 & 4.7 & Tremo & $\mathrm{Mg}, \mathrm{Si}, \mathrm{Ca}, \mathrm{Fe}$ & & & \\
\hline \multicolumn{7}{|c|}{ Asbestos Structures $>5 \mathrm{um}$ and 3:1 } & \multicolumn{2}{|c|}{12.7} & \multicolumn{2}{|c|}{ Not Applicable } & \multicolumn{2}{|l|}{ Not Applicable } & 4 & \\
\hline Gr & No. & Loc. & ID & Prim & Tot & Class & Len & Wid & Asp & Analy & Elements & Comment & & \\
\hline G1 & 5 & 12 & $A Z Q$ & 1 & & MD 1-1 & 6.7 & 4.85 & 1.4 & Tremo & $\mathrm{Mg}, \mathrm{Al}, \mathrm{Si}, \mathrm{Ca}, \mathrm{Fe}$ & & & \\
\hline G1 & 9 & D4 & $A D Q$ & 2 & 2 & $\mathrm{~F}$ & 17 & 1.35 & 12.6 & Tremo & $\mathrm{Mg}, \mathrm{Si}, \mathrm{Ca}, \mathrm{Fe}$ & & & \\
\hline $\mathrm{G} 2$ & 27 & $\mathrm{H} 4$ & $A D Q$ & 4 & & MD 1-0 & 5.2 & 3.85 & 1.4 & Tremo & $\mathrm{Mg}, \mathrm{Si}, \mathrm{Ca}, \mathrm{Fe}$ & & & \\
\hline $\mathrm{G} 2$ & 23 & E2 & $A D Q$ & 3 & 3 & B & 7 & 1.5 & 4.7 & Tremo & $\mathrm{Mg}, \mathrm{Si}, \mathrm{Ca}, \mathrm{Fe}$ & & & \\
\hline \multicolumn{7}{|c|}{ Asbestos Fibers and Bundles > 5um and 3:1 } & & & \multicolumn{2}{|c|}{\begin{tabular}{l|l|} 
Not Applicable \\
\end{tabular}} & \multicolumn{2}{|l|}{ Not Applicable } & & 3 \\
\hline Gr & No. & Loc. & ID & Prim & Tot & Class & Len & Wid & Asp & Analy & Elements & Comment & & \\
\hline G1 & 5 & 12 & $A Z Q$ & & 1 & MF & 6.7 & 1.25 & 5.4 & Tremo & & & & \\
\hline G1 & 9 & D4 & $A D Q$ & 2 & 2 & $\mathrm{~F}$ & 17 & 1.35 & 12.6 & Tremo & $\mathrm{Mg}, \mathrm{Si}, \mathrm{Ca}, \mathrm{Fe}$ & & & \\
\hline G2 & 23 & E2 & $A D Q$ & 3 & 3 & B & 7 & 1.5 & 4.7 & Tremo & $\mathrm{Mg}, \mathrm{Si}, \mathrm{Ca}, \mathrm{Fe}$ & & & \\
\hline
\end{tabular}


Job Number: 070434

SEA

ISO 10312, Direct Count Categories

Client: Idaho National Laboratory

Project Name: RARE

Lab/Cor Sample No.: S17

Client Sample No.: FB-4-R6

Description:

Filter Fraction: 1

Residual Ash Vol:
Aliquot Dilution: 0

Final Dilution: 0
Report Number: 070434R06

Date Received: 4/23/2007
Volume (L): 0

Lab Filter Area (mm2): 385

Grid Openings Analyzed: 35

Average Grid Opening Area: 0.009

Area Analyzed (mm2): 0.315

Analytical Sens. (struc/cc): 0

Dectection Limit. (struc/cc): 0

\begin{tabular}{|c|c|c|c|c|c|c|c|c|c|c|c|c|c|c|}
\hline \multicolumn{7}{|c|}{$\begin{array}{c}\text { Structure } \\
\text { Type }\end{array}$} & \multirow{2}{*}{$\begin{array}{c}\begin{array}{c}\text { Filter } \\
\text { Density } \\
\text { (s/mm2) }\end{array} \\
\end{array}$} & \multirow{2}{*}{\multicolumn{3}{|c|}{\begin{tabular}{c|}
$\begin{array}{c}\text { Concen- } \\
\text { tration } \\
\text { (struc/cc) }\end{array}$ \\
Not Applicable
\end{tabular}}} & \multirow{2}{*}{\multicolumn{2}{|c|}{$\begin{array}{c}\begin{array}{c}95 \% \text { Confidence } \\
\text { Interval } \\
\text { (struc/cc) }\end{array} \\
\text { Not Applicable }\end{array}$}} & \multicolumn{2}{|c|}{\begin{tabular}{|c|} 
Structure \\
Count $^{1}$ \\
Prim/Total
\end{tabular}} \\
\hline \multicolumn{7}{|c|}{ PCM Equivalent Fibers-ISO } & & & & & & & & 2 \\
\hline Gr & No. & Loc. & ID & Prim & Tot & Class & Len & Wid & Asp & Analyte & Elements & Comment & & \\
\hline G1 & 5 & 12 & $A D Q$ & 3 & 3 & $\mathrm{~F}$ & 6.2 & 1 & 6.2 & Tremolite & $\mathrm{Mg}, \mathrm{Si}, \mathrm{Ca}$ & & & \\
\hline G1 & 7 & $\mathrm{H} 4$ & $A D Q$ & & 6 & MF & 5.6 & 1.1 & 5.1 & Tremolite & & & & \\
\hline \multicolumn{7}{|c|}{ PCM Equivalent Structures-ISO } & \multicolumn{2}{|c|}{3.2} & \multicolumn{2}{|c|}{ Not Applicable } & \multicolumn{2}{|l|}{ Not Applicable } & 1 & \\
\hline Gr & No. & Loc. & ID & Prim & Tot & Class & Len & Wid & Asp & Analyte & Elements & Comment & & \\
\hline G1 & 5 & 12 & ADQ & 3 & 3 & $\mathrm{~F}$ & 6.2 & 1 & 6.2 & Tremolite & $\mathrm{Mg}, \mathrm{Si}, \mathrm{Ca}$ & & & \\
\hline \multicolumn{7}{|c|}{ PCM Equivalent Fibers-NIOSH } & & & \multicolumn{2}{|c|}{ Not Applicable } & \multicolumn{2}{|l|}{ Not Applicable } & & 2 \\
\hline Gr & No. & Loc. & ID & Prim & Tot & Class & Len & Wid & Asp & Analyte & Elements & Comment & & \\
\hline G1 & 5 & 12 & $A D Q$ & 3 & 3 & $\mathrm{~F}$ & 6.2 & 1 & 6.2 & Tremolite & $\mathrm{Mg}, \mathrm{Si}, \mathrm{Ca}$ & & & \\
\hline G1 & 7 & $\mathrm{H} 4$ & $A D Q$ & & 6 & MF & 5.6 & 1.1 & 5.1 & Tremolite & & & & \\
\hline \multicolumn{7}{|c|}{ PCM Equivalent Structures-NIOSH } & \multicolumn{2}{|c|}{3.2} & \multicolumn{2}{|c|}{ Not Applicable } & \multicolumn{2}{|l|}{ Not Applicable } & 1 & \\
\hline Gr & No. & Loc. & ID & Prim & Tot & Class & Len & Wid & Asp & Analyte & Elements & Comment & & \\
\hline G1 & 5 & 12 & ADQ & 3 & 3 & $\mathrm{~F}$ & 6.2 & 1 & 6.2 & Tremolite & $\mathrm{Mg}, \mathrm{Si}, \mathrm{Ca}$ & & & \\
\hline \multicolumn{7}{|c|}{ Asbestos Structures >5um and 3:1 } & \multicolumn{2}{|c|}{9.5} & \multicolumn{2}{|c|}{ Not Applicable } & \multicolumn{2}{|l|}{ Not Applicable } & 3 & \\
\hline Gr & No. & Loc. & ID & Prim & Tot & Class & Len & Wid & Asp & Analyte & Elements & Comment & & \\
\hline G1 & 7 & $\mathrm{H} 4$ & $A D Q$ & 6 & & MD 1-1 & 5.6 & 4 & 1.4 & Tremolite & $\mathrm{Mg}, \mathrm{Si}, \mathrm{Ca}, \mathrm{Fe}$ & & & \\
\hline G1 & 6 & J4 & ADQ & 5 & & MD 1-0 & 5.1 & 3.2 & 1.6 & Tremolite & $\mathrm{Mg}, \mathrm{Si}, \mathrm{Ca}, \mathrm{Fe}$ & & & \\
\hline G1 & 5 & 12 & ADQ & 3 & 3 & $\mathrm{~F}$ & 6.2 & 1 & 6.2 & Tremolite & $\mathrm{Mg}, \mathrm{Si}, \mathrm{Ca}$ & & & \\
\hline \multicolumn{7}{|c|}{ Asbestos Fibers and Bundles $>5 u m$ and 3:1 } & & & \multicolumn{2}{|c|}{ Not Applicable } & Not Applicable & & & 2 \\
\hline Gr & No. & Loc. & ID & Prim & Tot & Class & Len & Wid & Asp & Analyte & Elements & Comment & & \\
\hline G1 & 5 & 12 & ADQ & 3 & 3 & $\mathrm{~F}$ & 6.2 & 1 & 6.2 & Tremolite & $\mathrm{Mg}, \mathrm{Si}, \mathrm{Ca}$ & & & \\
\hline G1 & 7 & $\mathrm{H} 4$ & $A D Q$ & & 6 & MF & 5.6 & 1.1 & 5.1 & Tremolite & & & & \\
\hline
\end{tabular}


Phone: (206) 781-0155

7619 6th Ave Nw

ISO 10312, Direct Count Categories

Job Number: 070434

SEA

Report Number: 070434R06

Client: Idaho National Laboratory

Date Received: 4/23/2007

Project Name: RARE

Lab/Cor Sample No.: S18

Client Sample No.: FB-4-R7

Description:

Filter Fraction: 1

Residual Ash Vol:
Aliquot Dilution: 0

Final Dilution: 0
Volume (L): 0

Lab Filter Area (mm2): 385

Grid Openings Analyzed: 35

Average Grid Opening Area: 0.009

Area Analyzed (mm2): 0.315

Analytical Sens. (struc/cc): 0

Dectection Limit. (struc/cc): 0

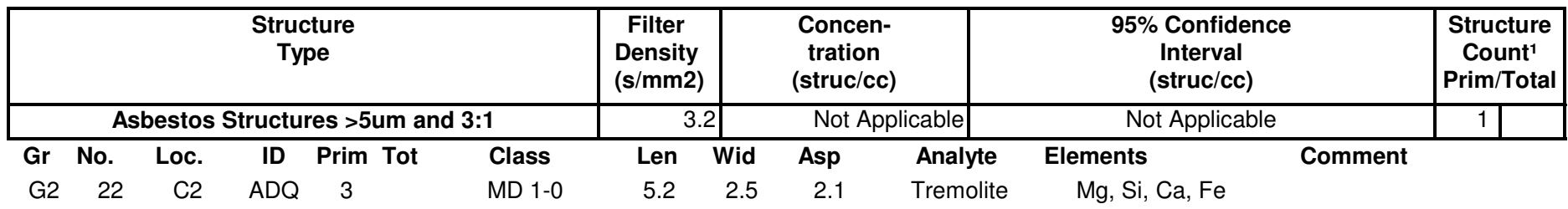


Job Number: 070434

SEA

ISO 10312, Direct Count Categories

Client: Idaho National Laboratory

Report Number: 070434R06

Date Received: 4/23/2007

Project Name: RARE

Lab/Cor Sample No.: S20

Client Sample No.: FB-3-R2

Description:

Filter Fraction: 1

Residual Ash Vol:
Aliquot Dilution: 0

Final Dilution: 0
Volume (L): 0

Lab Filter Area (mm2): 385

Grid Openings Analyzed: 35

Average Grid Opening Area: 0.009

Area Analyzed (mm2): 0.315

Analytical Sens. (struc/cc): 0

Dectection Limit. (struc/cc): 0

\begin{tabular}{|c|c|c|c|c|c|c|c|c|c|c|c|c|c|c|c|}
\hline & & & $\begin{array}{c}\text { Struc } \\
\text { Ty }\end{array}$ & $\begin{array}{l}\text { ture } \\
\text { pe }\end{array}$ & & & $\begin{array}{c}\text { Filter } \\
\text { Density } \\
(\mathrm{s} / \mathrm{mm} 2)\end{array}$ & & $\begin{array}{r}\text { Conce } \\
\text { tratic } \\
\text { (struc) }\end{array}$ & & & $\begin{array}{c}95 \% \text { Confidence } \\
\text { Interval } \\
\text { (struc/cc) }\end{array}$ & & $\begin{array}{r}\text { Stru } \\
\text { Co } \\
\text { Prim }\end{array}$ & $\begin{array}{l}\text { ture } \\
\text { nt }{ }^{1} \\
\text { otal }\end{array}$ \\
\hline & & PCM & quivale & nt Fib & ers-IS & & & & Not & licable & & Not Applicable & & & 27 \\
\hline $\mathrm{Gr}$ & No. & Loc. & ID & Prim & Tot & Class & Len & Wid & Asp & Anal & & Elements & Comment & & \\
\hline G1 & 18 & F9 & $\mathrm{AQ}$ & 22 & 23 & $\mathrm{~F}$ & 6.2 & 0.8 & 7.7 & Trem & lite & & & & \\
\hline G1 & 18 & F9 & $A Q$ & 23 & 24 & $\mathrm{~F}$ & 5.5 & 1.25 & 4.4 & Trem & lite & & & & \\
\hline G1 & 17 & $\mathrm{H} 9$ & $A Q$ & 21 & 22 & $\mathrm{~F}$ & 9.15 & 0.65 & 14.1 & Trem & lite & & & & \\
\hline G1 & 16 & J9 & $A Q$ & & 19 & MF & 5.8 & 1.35 & 4.3 & Trem & lite & & & & \\
\hline G1 & 14 & G7 & $A Q$ & 18 & 18 & $\mathrm{~F}$ & 15.85 & 1.5 & 10.6 & Trem & blite & & & & \\
\hline G1 & 14 & G7 & $\mathrm{AQ}$ & 17 & 17 & $\mathrm{~F}$ & 6.5 & 2 & 3.2 & Trem & lite & & & & \\
\hline G1 & 14 & G7 & $A Q$ & 16 & 16 & $\mathrm{~F}$ & 36 & 1.5 & 24 & Trem & lite & & & & \\
\hline G1 & 12 & $\mathrm{C} 7$ & $\mathrm{AQ}$ & 13 & 13 & $\mathrm{~F}$ & 6 & 1.7 & 3.5 & Trem & lite & & & & \\
\hline $\mathrm{G} 1$ & 8 & $\mathrm{~F} 4$ & $A Q$ & 10 & 10 & B & 12 & 2 & 6 & Trem & lite & & & & \\
\hline $\mathrm{G} 1$ & 5 & 12 & $A Q$ & 7 & 7 & $\mathrm{~F}$ & 7.5 & 1.1 & 6.8 & Trem & lite & & & & \\
\hline $\mathrm{G} 1$ & 5 & 12 & $A Q$ & 5 & 5 & $\mathrm{~F}$ & 9.2 & 0.5 & 18.4 & Trem & lite & & & & \\
\hline $\mathrm{G} 1$ & 5 & 12 & $A Q$ & & 4 & MF & 7.85 & 1.1 & 7.1 & Trem & lite & & & & \\
\hline G1 & 2 & $\mathrm{C} 2$ & $A D Q$ & 2 & 2 & $\mathrm{~F}$ & 19.2 & 1.7 & 11.3 & Trem & olite & $\mathrm{Mg}, \mathrm{Si}, \mathrm{Ca}$ & & & \\
\hline G1 & 12 & $\mathrm{C} 7$ & $A Q$ & 14 & 14 & $\mathrm{~F}$ & 7 & 1.35 & 5.2 & Trem & blite & & & & \\
\hline G2 & 29 & D4 & $A Q$ & & 44 & MF & 24.5 & 0.5 & 49 & Trem & lite & & & & \\
\hline G2 & 35 & 17 & $A Q$ & 53 & 56 & $\mathrm{~F}$ & 19 & 1.45 & 13.1 & Trem & plite & & & & \\
\hline $\mathrm{G} 2$ & 34 & G7 & $A Q$ & & 54 & MF & 10 & 0.55 & 18.2 & Trem & olite & & & & \\
\hline G2 & 34 & G7 & $A Q$ & & 53 & MF & 15 & 0.5 & 30 & Trem & lite & & & & \\
\hline G2 & 33 & E7 & $A Q$ & 50 & 52 & $\mathrm{~F}$ & 5.7 & 0.45 & 12.7 & Trem & lite & & & & \\
\hline G2 & 33 & E7 & $A Q$ & 49 & 51 & $F$ & 13 & 3 & 4.3 & Trem & lite & & & & \\
\hline G2 & 31 & A7 & $A Q$ & 47 & 49 & $\mathrm{~F}$ & 5.4 & 1.2 & 4.5 & Trem & blite & & & & \\
\hline G2 & 21 & $\mathrm{~A} 2$ & $A Q$ & 29 & 30 & $\mathrm{~F}$ & 19.2 & 1.15 & 16.7 & Trem & lite & & & & \\
\hline G2 & 31 & A7 & $A Q$ & 45 & 47 & $\mathrm{~F}$ & 8 & 1.5 & 5.3 & Trem & lite & & & & \\
\hline G2 & 28 & $\mathrm{~F} 4$ & $\mathrm{AQ}$ & 40 & 41 & $\mathrm{~F}$ & 6.5 & 0.95 & 6.8 & Trem & lite & & & & \\
\hline $\mathrm{G} 2$ & 28 & $\mathrm{~F} 4$ & $A Q$ & & 43 & MF & 6.75 & 0.75 & 9 & Trem & lite & & & & \\
\hline G2 & 24 & G2 & $A Q$ & 34 & 35 & $\mathrm{~F}$ & 25 & 2.75 & 9.1 & Trem & lite & & & & \\
\hline $\mathrm{G} 2$ & 31 & A7 & $A Q$ & 46 & 48 & $\mathrm{~F}$ & 5.8 & 0.75 & 7.7 & Trem & lite & & & & \\
\hline & & $\mathrm{CM} \mathrm{Ec}$ & ivalent & Struc & tures & & 66.7 & & Not & licable & & Not Applicable & & 21 & \\
\hline $\mathrm{Gr}$ & No. & Loc. & ID & Prim & Tot & Class & Len & Wid & Asp & Anal & & Elements & Comment & & \\
\hline G1 & 14 & G7 & $A Q$ & 16 & 16 & $\mathrm{~F}$ & 36 & 1.5 & 24 & Trem & lite & & & & \\
\hline $\mathrm{G} 1$ & 14 & G7 & $A Q$ & 18 & 18 & $\mathrm{~F}$ & 15.85 & 1.5 & 10.6 & Trem & lite & & & & \\
\hline G1 & 18 & F9 & $A Q$ & 23 & 24 & $\mathrm{~F}$ & 5.5 & 1.25 & 4.4 & Trem & lite & & & & \\
\hline G1 & 17 & $\mathrm{H} 9$ & $\mathrm{AQ}$ & 21 & 22 & $\mathrm{~F}$ & 9.15 & 0.65 & 14.1 & Trem & olite & & & & \\
\hline G1 & 14 & G7 & $A Q$ & 17 & 17 & $F$ & 6.5 & 2 & 3.2 & Trem & lite & & & & \\
\hline
\end{tabular}


Job Number: 070434

SEA

ISO 10312, Direct Count Categories

Client: Idaho National Laboratory

Report Number: 070434R06

Date Received: 4/23/2007

Project Name: RARE

Lab/Cor Sample No.: S20

Client Sample No.: FB-3-R2

Description:

Filter Fraction: 1

Residual Ash Vol:
Aliquot Dilution: 0

Final Dilution: 0
Volume (L): 0

Lab Filter Area (mm2): 385

Grid Openings Analyzed: 35

Average Grid Opening Area: 0.009

Area Analyzed (mm2): 0.315

Analytical Sens. (struc/cc): 0

Dectection Limit. (struc/cc): 0

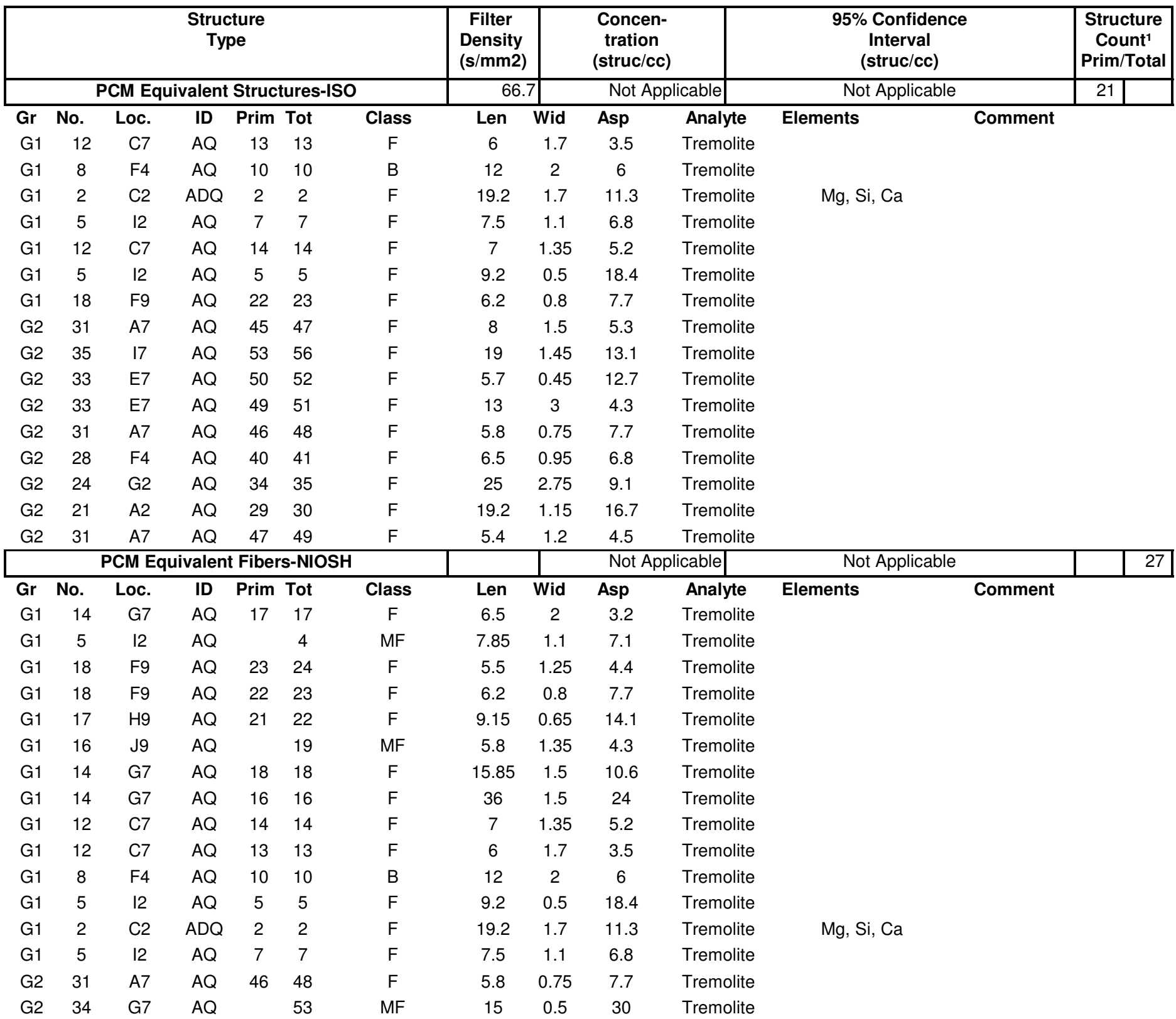


Job Number: 070434

SEA

ISO 10312, Direct Count Categories

Client: Idaho National Laboratory

Report Number: 070434R06

Date Received: 4/23/2007

Project Name: RARE

Lab/Cor Sample No.: S20

Client Sample No.: FB-3-R2

Description:

Filter Fraction: 1

Residual Ash Vol:
Aliquot Dilution: 0

Final Dilution: 0
Volume (L): 0

Lab Filter Area (mm2): 385

Grid Openings Analyzed: 35

Average Grid Opening Area: 0.009

Area Analyzed (mm2): 0.315

Analytical Sens. (struc/cc): 0

Dectection Limit. (struc/cc): 0

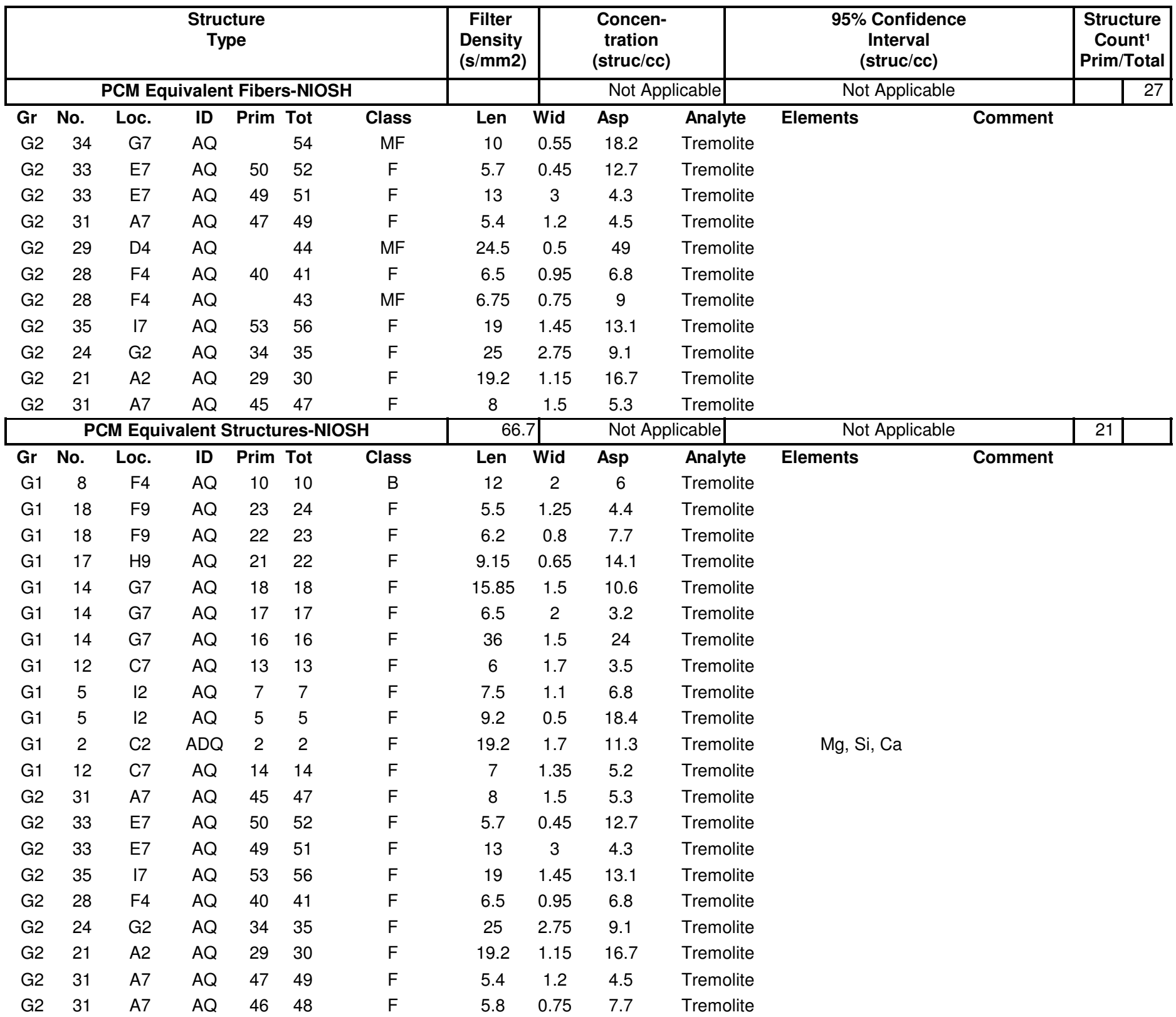


Job Number: 070434

SEA

ISO 10312, Direct Count Categories

Client: Idaho National Laboratory

Project Name: RARE

Lab/Cor Sample No.: S20

Client Sample No.: FB-3-R2

Description:

Filter Fraction: 1

Residual Ash Vol:
Aliquot Dilution: 0

Final Dilution: 0
Report Number: 070434R06

Date Received: 4/23/2007
Volume (L): 0

Lab Filter Area (mm2): 385

Grid Openings Analyzed: 35

Average Grid Opening Area: 0.009

Area Analyzed (mm2): 0.315

Analytical Sens. (struc/cc): 0

Dectection Limit. (struc/cc): 0

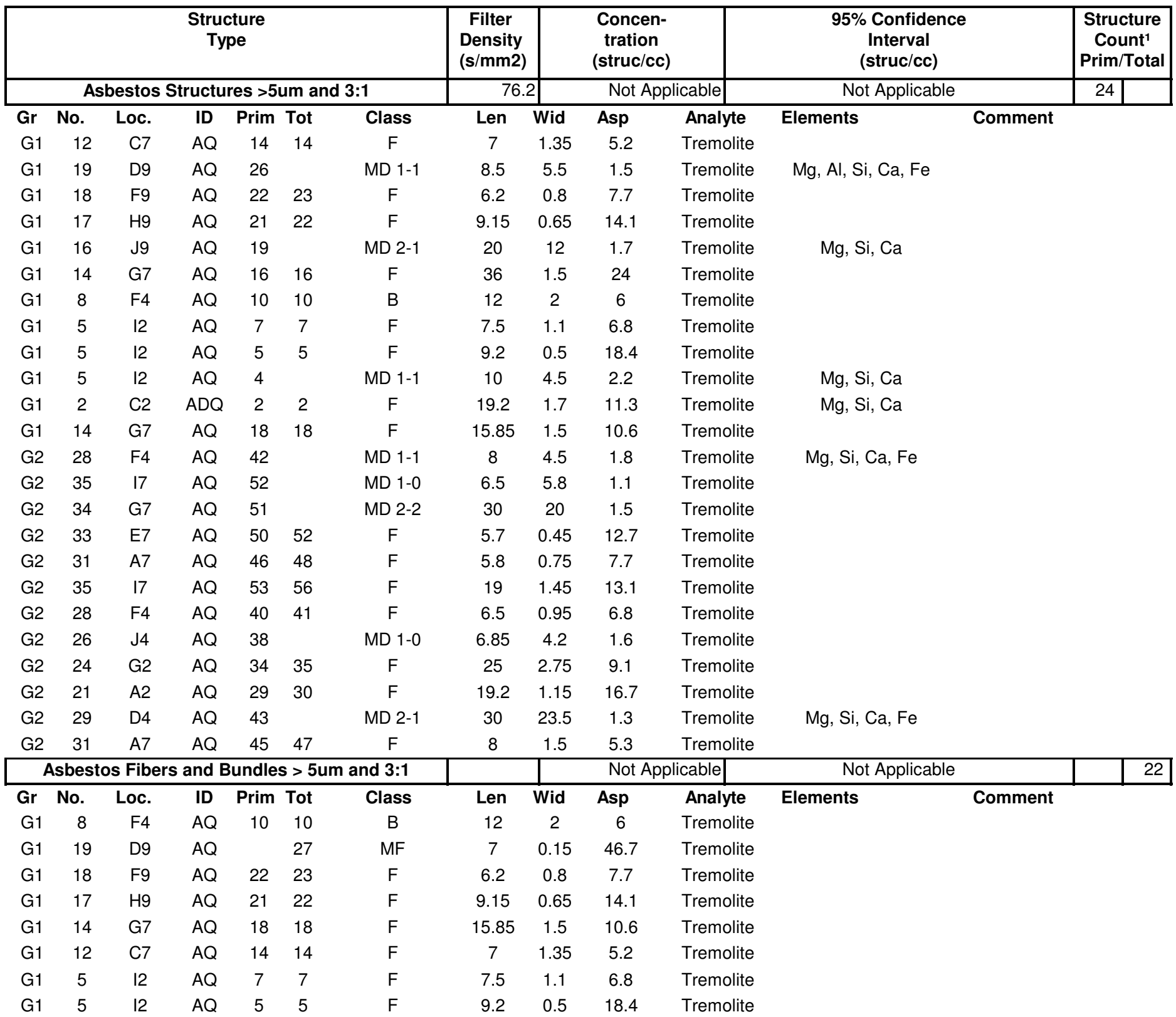


A Professional Service Corporation in the Northwest

ISO 10312, Direct Count Categories

Job Number: 070434

SEA

Report Number: 070434R06

Client: Idaho National Laboratory

Date Received: 4/23/2007

Project Name: RARE

Lab/Cor Sample No.: S20

Client Sample No.: FB-3-R2

Description:

Filter Fraction: 1

Residual Ash Vol:
Aliquot Dilution: 0

Final Dilution: 0
Volume (L): 0

Lab Filter Area (mm2): 385

Grid Openings Analyzed: 35

Average Grid Opening Area: 0.009

Area Analyzed (mm2): 0.315

Analytical Sens. (struc/cc): 0

Dectection Limit. (struc/cc): 0

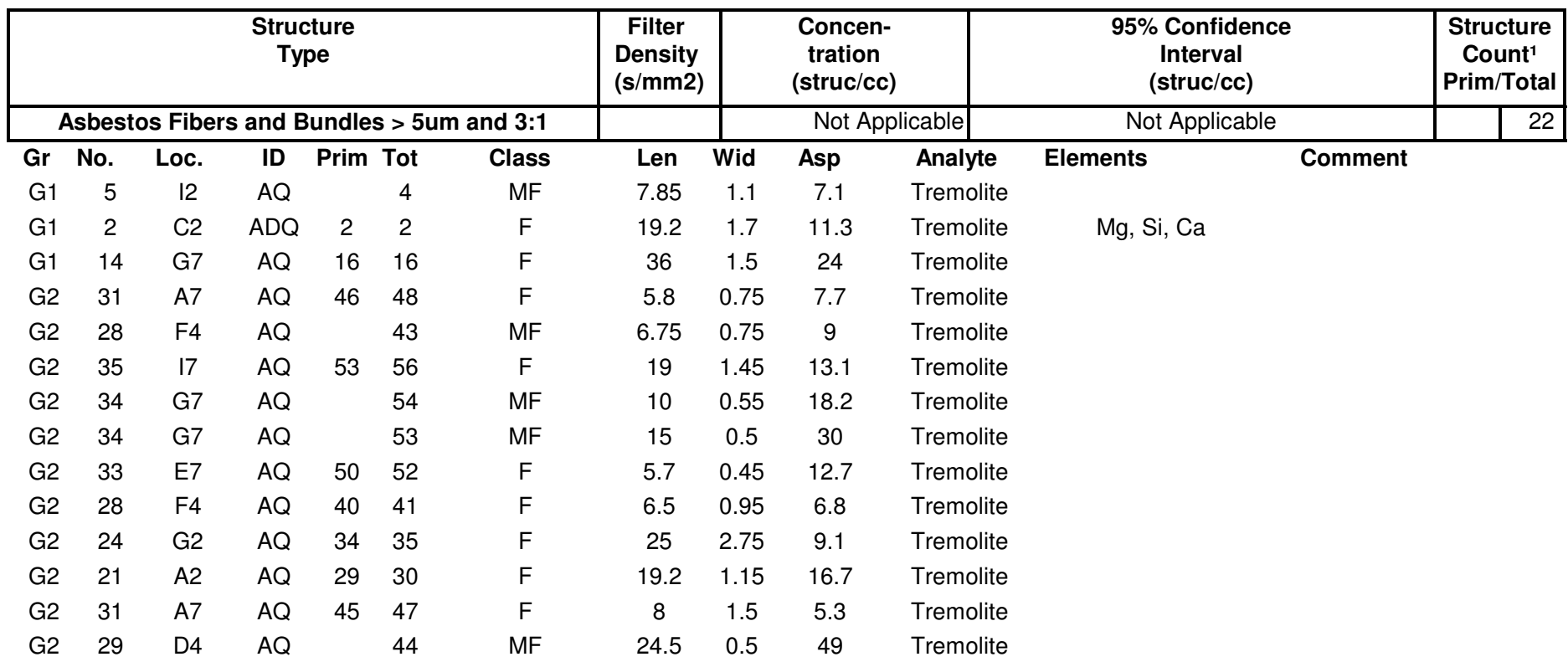


A Professional Service Corporation in the Northwest

Job Number: 070434

SEA

ISO 10312, Direct Count Categories

Client: Idaho National Laboratory

Report Number: 070434R06

Date Received: 4/23/2007

Project Name: RARE

Lab/Cor Sample No.: S23

Client Sample No.: FB-3-R5

Description:

Filter Fraction: 1

Residual Ash Vol:
Aliquot Dilution: 0

Final Dilution: 0
Volume (L): 0

Lab Filter Area (mm2): 385

Grid Openings Analyzed: 35

Average Grid Opening Area: 0.009

Area Analyzed (mm2): 0.315

Analytical Sens. (struc/cc): 0

Dectection Limit. (struc/cc): 0

\begin{tabular}{|c|c|c|c|c|c|c|c|c|c|c|c|c|c|c|}
\hline \multicolumn{7}{|c|}{$\begin{array}{c}\text { Structure } \\
\text { Type }\end{array}$} & \multirow{3}{*}{$\begin{array}{c}\begin{array}{c}\text { Filter } \\
\text { Density } \\
(\mathrm{s} / \mathrm{mm} 2)\end{array} \\
\text { Len }\end{array}$} & \multirow{2}{*}{\multicolumn{3}{|c|}{\begin{tabular}{c|}
$\begin{array}{c}\text { Concen- } \\
\text { tration } \\
\text { (struc/cc) }\end{array}$ \\
Not Applicable
\end{tabular}}} & \multirow{2}{*}{\multicolumn{2}{|c|}{$\begin{array}{c}\begin{array}{c}95 \% \text { Confidence } \\
\text { Interval } \\
\text { (struc/cc) }\end{array} \\
\text { Not Applicable } \\
\end{array}$}} & \multicolumn{2}{|c|}{\begin{tabular}{|c|} 
Structure \\
Count $^{1}$ \\
Prim/Total
\end{tabular}} \\
\hline \multicolumn{7}{|c|}{ PCM Equivalent Fibers-ISO } & & & & & & & & 16 \\
\hline $\mathrm{Gr}$ & No. & Loc. & ID & Prim & Tot & Class & & Wid & Asp & Analyte & Elements & Comment & & \\
\hline G1 & 8 & $\mathrm{~F} 4$ & $A Q$ & 5 & 5 & $\mathrm{~F}$ & 23.5 & 1.85 & 12.7 & Tremolite & $\mathrm{Mg}, \mathrm{Al}, \mathrm{Si}, \mathrm{Ca}, \mathrm{Fe}$ & & & \\
\hline G1 & 18 & F9 & $A Q$ & 15 & 15 & $\mathrm{~F}$ & 13 & 0.5 & 26 & Tremolite & $\mathrm{Mg}, \mathrm{Al}, \mathrm{Si}, \mathrm{Ca}, \mathrm{Fe}$ & & & \\
\hline G1 & 17 & $\mathrm{H} 9$ & $A Q$ & 14 & 14 & $\mathrm{~F}$ & 5.5 & 0.35 & 15.7 & Tremolite & $\mathrm{Mg}, \mathrm{Al}, \mathrm{Si}, \mathrm{Ca}, \mathrm{Fe}$ & & & \\
\hline G1 & 15 & 17 & $A Q$ & 13 & 13 & $\mathrm{~F}$ & 7.5 & 0.7 & 10.7 & Tremolite & $\mathrm{Mg}, \mathrm{Si}, \mathrm{Ca}, \mathrm{Fe}$ & & & \\
\hline G1 & 15 & 17 & $A Q$ & 12 & 12 & $\mathrm{~F}$ & 6 & 0.9 & 6.7 & Tremolite & $\mathrm{Mg}, \mathrm{Si}, \mathrm{Ca}, \mathrm{Fe}$ & & & \\
\hline G1 & 9 & B4 & $A Q$ & & 7 & MF & 17.75 & 1.7 & 10.4 & Tremolite & & & & \\
\hline G1 & 5 & 12 & $A Q$ & 4 & 4 & $\mathrm{~F}$ & 7.75 & 1.15 & 6.7 & Tremolite & $\mathrm{Mg}, \mathrm{Al}, \mathrm{Si}, \mathrm{Ca}, \mathrm{Fe}$ & & & \\
\hline G1 & 4 & G2 & $A Z Q$ & 1 & 1 & $\mathrm{~F}$ & 10 & 0.85 & 11.8 & Tremolite & $\mathrm{Mg}, \mathrm{Al}, \mathrm{Si}, \mathrm{Ca}, \mathrm{Fe}$ & & & \\
\hline G1 & 4 & G2 & ADQ & & 3 & MF & 7 & 0.5 & 14 & Tremolite & & & & \\
\hline G1 & 9 & B4 & $A Q$ & & 8 & MF & 13.75 & 0.25 & 55 & Tremolite & & & & \\
\hline G2 & 24 & G2 & $A D Q$ & 20 & 20 & $\mathrm{~F}$ & 7 & 0.5 & 14 & Tremolite & & & & \\
\hline G2 & 34 & G7 & $A Q$ & 23 & 23 & $\mathrm{~F}$ & 5.5 & 0.75 & 7.3 & Tremolite & & & & \\
\hline $\mathrm{G} 2$ & 34 & G7 & $A Q$ & 24 & 24 & $\mathrm{~F}$ & 25 & 1 & 25 & Tremolite & & & & \\
\hline $\mathrm{G} 2$ & 23 & E2 & $A Q$ & 19 & 19 & $\mathrm{~F}$ & 14.5 & 3 & 4.8 & Tremolite & & & & \\
\hline G2 & 32 & $\mathrm{C} 7$ & $A Q$ & 22 & 22 & B & 6.2 & 1.75 & 3.5 & Tremolite & $\mathrm{Mg}, \mathrm{Al}, \mathrm{Si}, \mathrm{Ca}, \mathrm{Fe}$ & & & \\
\hline G2 & 30 & B4 & $A Q$ & 21 & 21 & $\mathrm{~F}$ & 13.25 & 3 & 4.4 & Tremolite & & & & \\
\hline \multicolumn{7}{|c|}{ PCM Equivalent Structures-ISO } & 44. & & Not & icable & Not Applicable & & 14 & \\
\hline $\mathrm{Gr}$ & No. & Loc. & ID & Prim & Tot & Class & Len & Wid & Asp & Analyte & Elements & Comment & & \\
\hline G1 & 18 & F9 & $A Q$ & 15 & 15 & $\mathrm{~F}$ & 13 & 0.5 & 26 & Tremolite & $\mathrm{Mg}, \mathrm{Al}, \mathrm{Si}, \mathrm{Ca}, \mathrm{Fe}$ & & & \\
\hline G1 & 4 & G2 & ADQ & 3 & & MD 1-1 & 7 & 1.75 & 4 & Tremolite & $\mathrm{Mg}, \mathrm{Al}, \mathrm{Si}, \mathrm{Ca}, \mathrm{Fe}$ & & & \\
\hline G1 & 4 & G2 & $A Z Q$ & 1 & 1 & $\mathrm{~F}$ & 10 & 0.85 & 11.8 & Tremolite & $\mathrm{Mg}, \mathrm{Al}, \mathrm{Si}, \mathrm{Ca}, \mathrm{Fe}$ & & & \\
\hline G1 & 5 & 12 & $A Q$ & 4 & 4 & $\mathrm{~F}$ & 7.75 & 1.15 & 6.7 & Tremolite & $\mathrm{Mg}, \mathrm{Al}, \mathrm{Si}, \mathrm{Ca}, \mathrm{Fe}$ & & & \\
\hline G1 & 8 & $\mathrm{~F} 4$ & $A Q$ & 5 & 5 & $\mathrm{~F}$ & 23.5 & 1.85 & 12.7 & Tremolite & $\mathrm{Mg}, \mathrm{Al}, \mathrm{Si}, \mathrm{Ca}, \mathrm{Fe}$ & & & \\
\hline G1 & 15 & 17 & $A Q$ & 12 & 12 & $\mathrm{~F}$ & 6 & 0.9 & 6.7 & Tremolite & $\mathrm{Mg}, \mathrm{Si}, \mathrm{Ca}, \mathrm{Fe}$ & & & \\
\hline G1 & 15 & 17 & $A Q$ & 13 & 13 & $\mathrm{~F}$ & 7.5 & 0.7 & 10.7 & Tremolite & $\mathrm{Mg}, \mathrm{Si}, \mathrm{Ca}, \mathrm{Fe}$ & & & \\
\hline G1 & 17 & $\mathrm{H} 9$ & $A Q$ & 14 & 14 & $\mathrm{~F}$ & 5.5 & 0.35 & 15.7 & Tremolite & $\mathrm{Mg}, \mathrm{Al}, \mathrm{Si}, \mathrm{Ca}, \mathrm{Fe}$ & & & \\
\hline G2 & 34 & G7 & $A Q$ & 24 & 24 & $\mathrm{~F}$ & 25 & 1 & 25 & Tremolite & & & & \\
\hline $\mathrm{G} 2$ & 34 & G7 & $A Q$ & 23 & 23 & $\mathrm{~F}$ & 5.5 & 0.75 & 7.3 & Tremolite & & & & \\
\hline G2 & 32 & $\mathrm{C} 7$ & $A Q$ & 22 & 22 & B & 6.2 & 1.75 & 3.5 & Tremolite & $\mathrm{Mg}, \mathrm{Al}, \mathrm{Si}, \mathrm{Ca}, \mathrm{Fe}$ & & & \\
\hline $\mathrm{G} 2$ & 30 & B4 & $A Q$ & 21 & 21 & $\mathrm{~F}$ & 13.25 & 3 & 4.4 & Tremolite & & & & \\
\hline G2 & 23 & E2 & $A Q$ & 19 & 19 & $\mathrm{~F}$ & 14.5 & 3 & 4.8 & Tremolite & & & & \\
\hline G2 & 24 & G2 & $A D Q$ & 20 & 20 & $\mathrm{~F}$ & 7 & 0.5 & 14 & Tremolite & & & & \\
\hline & & CM E & ivalent & Fiber & rs-NIC & & & & Not & icable & Not Applicable & & & 17 \\
\hline
\end{tabular}


A Professional Service Corporation in the Northwest

Job Number: 070434

SEA

ISO 10312, Direct Count Categories

Client: Idaho National Laboratory

Report Number: 070434R06

Date Received: 4/23/2007

Project Name: RARE

Lab/Cor Sample No.: S23

Client Sample No.: FB-3-R5

Description:

Filter Fraction: 1

Residual Ash Vol:
Aliquot Dilution: 0

Final Dilution: 0
Volume (L): 0

Lab Filter Area (mm2): 385

Grid Openings Analyzed: 35

Average Grid Opening Area: 0.009

Area Analyzed (mm2): 0.315

Analytical Sens. (struc/cc): 0

Dectection Limit. (struc/cc): 0

\begin{tabular}{|c|c|c|c|c|c|c|c|c|c|c|c|c|c|c|c|}
\hline & & & $\begin{array}{r}\text { Struo } \\
\text { Ty }\end{array}$ & $\begin{array}{l}\text { ture } \\
\text { pe }\end{array}$ & & & $\begin{array}{c}\text { Filter } \\
\text { Density } \\
(\mathrm{s} / \mathrm{mm} 2)\end{array}$ & & $\begin{array}{r}\text { Conce } \\
\text { tratic } \\
\text { (struc) }\end{array}$ & & & $\begin{array}{c}\text { 95\% Confidence } \\
\text { Interval } \\
\text { (struc/cc) }\end{array}$ & & $\begin{array}{r}\text { Struc } \\
\text { Col } \\
\text { Prim }\end{array}$ & \\
\hline & & $\mathrm{CM} \mathrm{EC}$ & ivalen & Fiber & rs-NIC & & & & Not & licable & & Not Applicable & & & 17 \\
\hline Gr & No. & Loc. & ID & Prim & Tot & Class & Len & Wid & Asp & Analy & & Elements & Comment & & \\
\hline G1 & 14 & G7 & $\mathrm{AQ}$ & 11 & 11 & $\mathrm{~F}$ & 23 & 5 & 4.6 & Tremo & lite & $\mathrm{Mg}, \mathrm{Si}, \mathrm{Ca}, \mathrm{Fe}$ & & & \\
\hline G1 & 17 & H9 & $A Q$ & 14 & 14 & $\mathrm{~F}$ & 5.5 & 0.35 & 15.7 & Tremc & lite & $\mathrm{Mg}, \mathrm{Al}, \mathrm{Si}, \mathrm{Ca}, \mathrm{Fe}$ & & & \\
\hline G1 & 4 & G2 & $A Z Q$ & 1 & 1 & $\mathrm{~F}$ & 10 & 0.85 & 11.8 & Tremc & lite & $\mathrm{Mg}, \mathrm{Al}, \mathrm{Si}, \mathrm{Ca}, \mathrm{Fe}$ & & & \\
\hline G1 & 15 & 17 & $A Q$ & 13 & 13 & $\mathrm{~F}$ & 7.5 & 0.7 & 10.7 & Tremc & lite & $\mathrm{Mg}, \mathrm{Si}, \mathrm{Ca}, \mathrm{Fe}$ & & & \\
\hline G1 & 18 & F9 & $\mathrm{AQ}$ & 15 & 15 & $\mathrm{~F}$ & 13 & 0.5 & 26 & Tremo & lite & $\mathrm{Mg}, \mathrm{Al}, \mathrm{Si}, \mathrm{Ca}, \mathrm{Fe}$ & & & \\
\hline G1 & 15 & 17 & $\mathrm{AQ}$ & 12 & 12 & $\mathrm{~F}$ & 6 & 0.9 & 6.7 & Tremo & lite & $\mathrm{Mg}, \mathrm{Si}, \mathrm{Ca}, \mathrm{Fe}$ & & & \\
\hline G1 & 9 & B4 & $A Q$ & & 7 & MF & 17.75 & 1.7 & 10.4 & Tremc & lite & & & & \\
\hline G1 & 5 & 12 & $\mathrm{AQ}$ & 4 & 4 & $\mathrm{~F}$ & 7.75 & 1.15 & 6.7 & Tremo & lite & $\mathrm{Mg}, \mathrm{Al}, \mathrm{Si}, \mathrm{Ca}, \mathrm{Fe}$ & & & \\
\hline $\mathrm{G} 1$ & 4 & G2 & $A D Q$ & & 3 & MF & 7 & 0.5 & 14 & Tremc & lite & & & & \\
\hline $\mathrm{G} 1$ & 8 & $\mathrm{~F} 4$ & $A Q$ & 5 & 5 & $\mathrm{~F}$ & 23.5 & 1.85 & 12.7 & Tremc & lite & $\mathrm{Mg}, \mathrm{Al}, \mathrm{Si}, \mathrm{Ca}, \mathrm{Fe}$ & & & \\
\hline G1 & 9 & B4 & $A Q$ & & 8 & MF & 13.75 & 0.25 & 55 & Tremc & lite & & & & \\
\hline G2 & 30 & B4 & $A Q$ & 21 & 21 & $\mathrm{~F}$ & 13.25 & 3 & 4.4 & Tremc & lite & & & & \\
\hline G2 & 34 & G7 & $\mathrm{AQ}$ & 24 & 24 & $\mathrm{~F}$ & 25 & 1 & 25 & Tremc & lite & & & & \\
\hline G2 & 32 & $\mathrm{C7}$ & $A Q$ & 22 & 22 & B & 6.2 & 1.75 & 3.5 & Tremc & lite & $\mathrm{Mg}, \mathrm{Al}, \mathrm{Si}, \mathrm{Ca}, \mathrm{Fe}$ & & & \\
\hline G2 & 24 & G2 & $A D Q$ & 20 & 20 & $\mathrm{~F}$ & 7 & 0.5 & 14 & Tremc & lite & & & & \\
\hline G2 & 23 & E2 & $A Q$ & 19 & 19 & $\mathrm{~F}$ & 14.5 & 3 & 4.8 & Tremc & lite & & & & \\
\hline $\mathrm{G} 2$ & 34 & G7 & $A Q$ & 23 & 23 & $\mathrm{~F}$ & 5.5 & 0.75 & 7.3 & Tremc & lite & & & & \\
\hline & & M Equ & alent S & tructu & Ires- 1 & & 50.8 & & Not & licable & & Not Applicable & & 16 & \\
\hline $\mathrm{Gr}$ & No. & Loc. & ID & Prim & Tot & Class & Len & Wid & Asp & Analy & & Elements & Comment & & \\
\hline G1 & 14 & G7 & $A Q$ & 11 & 11 & $\mathrm{~F}$ & 23 & 5 & 4.6 & Tremc & lite & $\mathrm{Mg}, \mathrm{Si}, \mathrm{Ca}, \mathrm{Fe}$ & & & \\
\hline G1 & 17 & H9 & $A Q$ & 14 & 14 & $\mathrm{~F}$ & 5.5 & 0.35 & 15.7 & Tremc & lite & $\mathrm{Mg}, \mathrm{Al}, \mathrm{Si}, \mathrm{Ca}, \mathrm{Fe}$ & & & \\
\hline G1 & 18 & F9 & $\mathrm{AQ}$ & 15 & 15 & $\mathrm{~F}$ & 13 & 0.5 & 26 & Tremo & lite & $\mathrm{Mg}, \mathrm{Al}, \mathrm{Si}, \mathrm{Ca}, \mathrm{Fe}$ & & & \\
\hline G1 & 15 & 17 & $\mathrm{AQ}$ & 13 & 13 & $\mathrm{~F}$ & 7.5 & 0.7 & 10.7 & Tremc & lite & $\mathrm{Mg}, \mathrm{Si}, \mathrm{Ca}, \mathrm{Fe}$ & & & \\
\hline G1 & 15 & 17 & $A Q$ & 12 & 12 & $\mathrm{~F}$ & 6 & 0.9 & 6.7 & Tremc & lite & $\mathrm{Mg}, \mathrm{Si}, \mathrm{Ca}, \mathrm{Fe}$ & & & \\
\hline G1 & 8 & $\mathrm{~F} 4$ & $A Q$ & 5 & 5 & $\mathrm{~F}$ & 23.5 & 1.85 & 12.7 & Tremc & lite & $\mathrm{Mg}, \mathrm{Al}, \mathrm{Si}, \mathrm{Ca}, \mathrm{Fe}$ & & & \\
\hline G1 & 5 & 12 & $A Q$ & 4 & 4 & $\mathrm{~F}$ & 7.75 & 1.15 & 6.7 & Tremc & lite & $\mathrm{Mg}, \mathrm{Al}, \mathrm{Si}, \mathrm{Ca}, \mathrm{Fe}$ & & & \\
\hline G1 & 9 & B4 & $A Q$ & 7 & & MD 1-1 & 17.75 & 4.5 & 3.9 & Tremc & lite & $\mathrm{Mg}, \mathrm{Al}, \mathrm{Si}, \mathrm{Ca}, \mathrm{Fe}$ & & & \\
\hline G1 & 4 & G2 & $A Z Q$ & 1 & 1 & $\mathrm{~F}$ & 10 & 0.85 & 11.8 & Tremc & lite & $\mathrm{Mg}, \mathrm{Al}, \mathrm{Si}, \mathrm{Ca}, \mathrm{Fe}$ & & & \\
\hline G1 & 4 & G2 & $A D Q$ & 3 & & MD 1-1 & 7 & 1.75 & 4 & Tremo & lite & $\mathrm{Mg}, \mathrm{Al}, \mathrm{Si}, \mathrm{Ca}, \mathrm{Fe}$ & & & \\
\hline G2 & 30 & B4 & $A Q$ & 21 & 21 & $\mathrm{~F}$ & 13.25 & 3 & 4.4 & Tremc & lite & & & & \\
\hline $\mathrm{G} 2$ & 34 & $\mathrm{G} 7$ & $A Q$ & 24 & 24 & $\mathrm{~F}$ & 25 & 1 & 25 & Tremc & lite & & & & \\
\hline $\mathrm{G} 2$ & 32 & $\mathrm{C} 7$ & $A Q$ & 22 & 22 & B & 6.2 & 1.75 & 3.5 & Tremc & lite & $\mathrm{Mg}, \mathrm{Al}, \mathrm{Si}, \mathrm{Ca}, \mathrm{Fe}$ & & & \\
\hline G2 & 24 & G2 & $A D Q$ & 20 & 20 & $\mathrm{~F}$ & 7 & 0.5 & 14 & Tremc & lite & & & & \\
\hline $\mathrm{G} 2$ & 23 & E2 & $A Q$ & 19 & 19 & $\mathrm{~F}$ & 14.5 & 3 & 4.8 & Tremc & lite & & & & \\
\hline
\end{tabular}


Job Number: 070434

SEA

ISO 10312, Direct Count Categories

Client: Idaho National Laboratory

Report Number: 070434R06

Date Received: 4/23/2007

Project Name: RARE

Lab/Cor Sample No.: S23

Client Sample No.: FB-3-R5

Description:

Filter Fraction: 1

Residual Ash Vol:
Aliquot Dilution: 0

Final Dilution: 0
Volume (L): 0

Lab Filter Area (mm2): 385

Grid Openings Analyzed: 35

Average Grid Opening Area: 0.009

Area Analyzed (mm2): 0.315

Analytical Sens. (struc/cc): 0

Dectection Limit. (struc/cc): 0

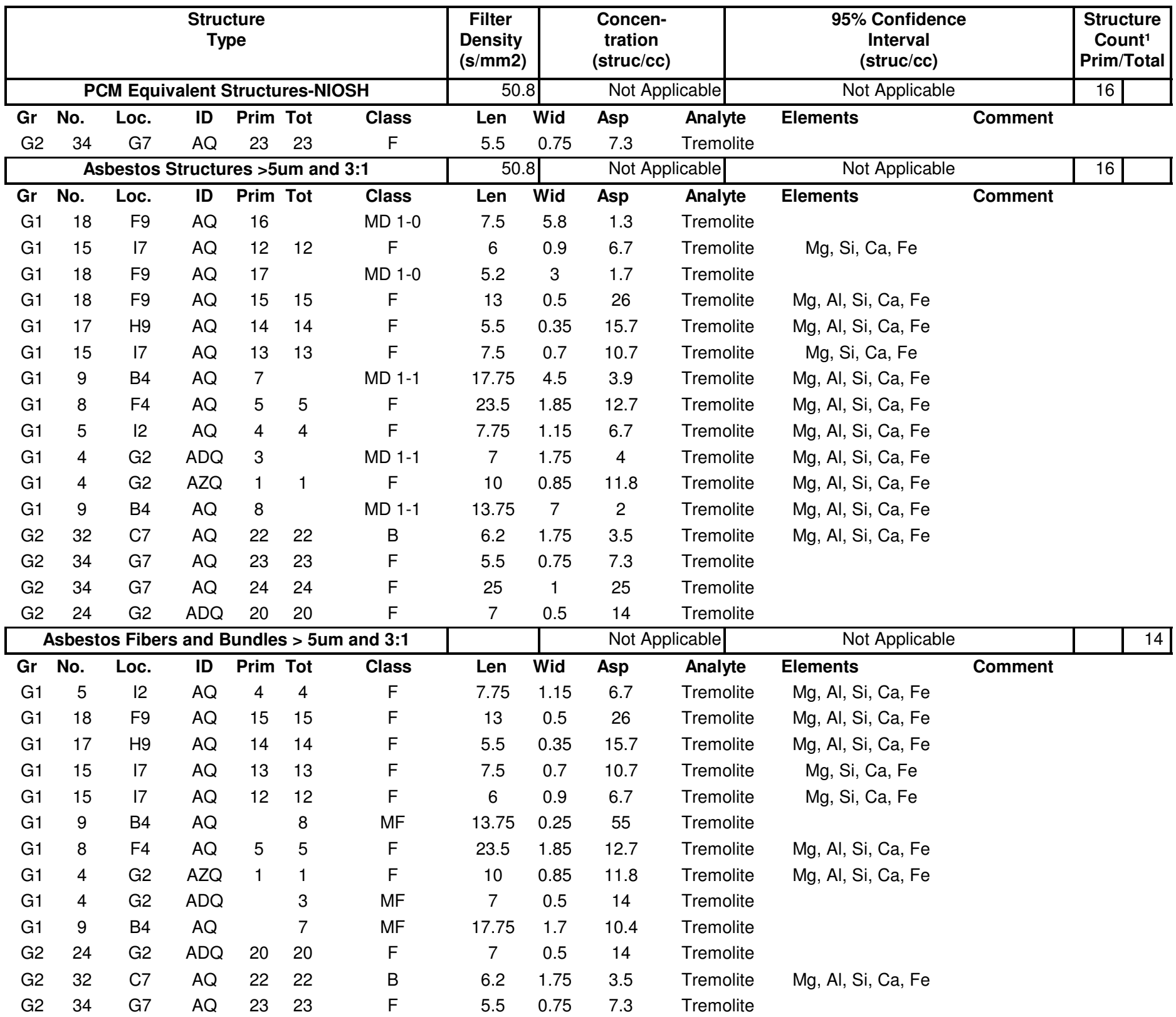


Phone: (206) 781-0155

7619 6th Ave Nw

A Professional Service Corporation in the Northwest

ISO 10312, Direct Count Categories

Job Number: 070434

SEA

Report Number: 070434R06

Client: Idaho National Laboratory

Date Received: 4/23/2007

Project Name: RARE

Lab/Cor Sample No.: S23

Client Sample No.: FB-3-R5

Description:

Filter Fraction: 1

Aliquot Dilution: 0

Final Dilution: 0

Volume (L): 0

Lab Filter Area (mm2): 385

Grid Openings Analyzed: 35

Residual Ash Vol:

Area Analyzed (mm2): 0.315

Analytical Sens. (struc/cc): 0

Dectection Limit. (struc/cc): 0

\begin{tabular}{|c|c|c|c|c|c|c|c|c|c|c|}
\hline \multicolumn{6}{|c|}{$\begin{array}{c}\text { Structure } \\
\text { Type }\end{array}$} & $\begin{array}{c}\text { Filter } \\
\text { Density } \\
(\mathbf{s} / \mathrm{mm} 2)\end{array}$ & & $\begin{array}{l}\text { Concen- } \\
\text { tration } \\
\text { (struc/cc) }\end{array}$ & $\begin{array}{c}\text { 95\% Confidence } \\
\text { Interval } \\
\text { (struc/cc) }\end{array}$ & $\begin{array}{c}\text { Structure } \\
\text { Count }^{1} \\
\text { Prim/Total }\end{array}$ \\
\hline \multicolumn{6}{|c|}{ Asbestos Fibers and Bundles > 5um and 3:1 } & & & Not Applicable & Not Applicable & 14 \\
\hline Gr & No. & Loc. & ID & Prim Tot & Class & Len & Wid & Asp & Elements & \\
\hline G2 & 34 & G7 & $A Q$ & 24 & $F$ & 25 & 1 & Trem & & \\
\hline
\end{tabular}


A Professional Service Corporation in the Northwest

Job Number: 070434

\section{SEA}

ISO 10312, Direct Count Categories

Client: Idaho National Laboratory

Report Number: 070434R06

Date Received: 4/23/2007

Project Name: RARE

Lab/Cor Sample No.: S25

Client Sample No.: FB-3-R7

Description:

Filter Fraction: 1

Residual Ash Vol:
Aliquot Dilution: 0

Final Dilution: 0
Volume (L): 0

Lab Filter Area (mm2): 385

Grid Openings Analyzed: 35

Average Grid Opening Area: 0.009

Area Analyzed (mm2): 0.315

Analytical Sens. (struc/cc): 0

Dectection Limit. (struc/cc): 0

\begin{tabular}{|c|c|c|c|c|c|c|c|c|c|c|c|c|c|c|c|}
\hline & & & $\begin{array}{c}\text { Struc } \\
\text { Typ }\end{array}$ & $\begin{array}{l}\text { ture } \\
\text { pe }\end{array}$ & & & $\begin{array}{c}\text { Filter } \\
\text { Density } \\
(\mathrm{s} / \mathrm{mm} 2)\end{array}$ & & $\begin{array}{r}\text { Conce } \\
\text { tratic } \\
\text { (struc/ }\end{array}$ & & & $\begin{array}{c}95 \% \text { Confidence } \\
\text { Interval } \\
\text { (struc/cc) }\end{array}$ & & $\begin{array}{r}\text { Struc } \\
\text { Col } \\
\text { Prim }\end{array}$ & $\begin{array}{l}\text { ture } \\
\text { nt } \\
\text { "otal }\end{array}$ \\
\hline & & PCM & quivale & nt Fib & ers-IS & & & & Not & licable & & Not Applicable & & & 10 \\
\hline $\mathrm{Gr}$ & No. & Loc. & ID & Prim & Tot & Class & Len & Wid & Asp & Anal & & Elements & Comment & & \\
\hline G1 & 18 & F9 & $A D Q$ & & 10 & MF & 15 & 2 & 7.5 & Trem & olite & & & & \\
\hline G1 & 20 & B9 & $A D Q$ & & 12 & MF & 12.5 & 1 & 12.5 & Trem & olite & & & & \\
\hline G1 & 19 & D9 & $\mathrm{AZQ}$ & 11 & 11 & $\mathrm{~F}$ & 5.5 & 0.22 & 25 & Trem & olite & $\mathrm{Mg}, \mathrm{Si}, \mathrm{Ca}, \mathrm{Fe}$ & & & \\
\hline G1 & 9 & D4 & $A Q$ & & 5 & MF & 17.5 & 2.7 & 6.5 & Trem & olite & & & & \\
\hline G1 & 1 & $\mathrm{~A} 2$ & $A D Q$ & & 1 & MF & 5.7 & 0.6 & 9.5 & Trem & olite & & & & \\
\hline G1 & 11 & A7 & $A D Q$ & 7 & 7 & $\mathrm{~F}$ & 5.2 & 0.4 & 13 & Trem & olite & $\mathrm{Mg}, \mathrm{Al}, \mathrm{Si}, \mathrm{Ca}, \mathrm{Fe}$ & & & \\
\hline G2 & 21 & $\mathrm{~A} 2$ & $A Q$ & 14 & 14 & $\mathrm{~F}$ & 5.6 & 0.7 & 8 & Trem & olite & & & & \\
\hline $\mathrm{G} 2$ & 22 & $\mathrm{C} 2$ & $A D Q$ & 16 & 17 & $\mathrm{~F}$ & 10.1 & 1 & 10.1 & Trem & olite & $\mathrm{Mg}, \mathrm{Al}, \mathrm{Si}, \mathrm{Ca}, \mathrm{Fe}$ & & & \\
\hline G2 & 22 & $\mathrm{C} 2$ & $\mathrm{AZQ}$ & & 15 & CF & 7 & 0.25 & 28 & Trem & olite & & & & \\
\hline G2 & 28 & $\mathrm{~F} 4$ & ADQ & 17 & 18 & $\mathrm{~F}$ & 7.2 & 1.5 & 4.8 & Trem & olite & & & & \\
\hline & & $\mathrm{CM} \mathrm{Ec}$ & ivalent & Struc & tures & & 15.9 & & Not & licable & & Not Applicable & & 5 & \\
\hline $\mathrm{Gr}$ & No. & Loc. & ID & Prim & Tot & Class & Len & Wid & Asp & Anal & & Elements & Comment & & \\
\hline G1 & 19 & D9 & $\mathrm{AZQ}$ & 11 & 11 & $\mathrm{~F}$ & 5.5 & 0.22 & 25 & Trem & olite & $\mathrm{Mg}, \mathrm{Si}, \mathrm{Ca}, \mathrm{Fe}$ & & & \\
\hline G1 & 11 & A7 & $A D Q$ & 7 & 7 & $\mathrm{~F}$ & 5.2 & 0.4 & 13 & Trem & olite & $\mathrm{Mg}, \mathrm{Al}, \mathrm{Si}, \mathrm{Ca}, \mathrm{Fe}$ & & & \\
\hline G2 & 28 & $\mathrm{~F} 4$ & $A D Q$ & 17 & 18 & $\mathrm{~F}$ & 7.2 & 1.5 & 4.8 & Trem & olite & & & & \\
\hline G2 & 21 & $\mathrm{~A} 2$ & $A Q$ & 14 & 14 & $\mathrm{~F}$ & 5.6 & 0.7 & 8 & Trem & lite & & & & \\
\hline $\mathrm{G} 2$ & 22 & $\mathrm{C} 2$ & ADQ & 16 & 17 & $\mathrm{~F}$ & 10.1 & 1 & 10.1 & Trem & lite & $\mathrm{Mg}, \mathrm{Al}, \mathrm{Si}, \mathrm{Ca}, \mathrm{Fe}$ & & & \\
\hline & & CM E & ivalent & t Fiber & $s-\mathrm{NIC}$ & & & & Not & licable & & Not Applicable & & & 9 \\
\hline $\mathrm{Gr}$ & No. & Loc. & ID & Prim & Tot & Class & Len & Wid & Asp & Anal & & Elements & Comment & & \\
\hline G1 & 1 & $\mathrm{~A} 2$ & $A D Q$ & & 1 & MF & 5.7 & 0.6 & 9.5 & Trem & blite & & & & \\
\hline G1 & 9 & D4 & $A Q$ & & 5 & MF & 17.5 & 2.7 & 6.5 & Trem & olite & & & & \\
\hline G1 & 11 & A7 & $A D Q$ & 7 & 7 & $\mathrm{~F}$ & 5.2 & 0.4 & 13 & Trem & lite & $\mathrm{Mg}, \mathrm{Al}, \mathrm{Si}, \mathrm{Ca}, \mathrm{Fe}$ & & & \\
\hline G1 & 18 & F9 & $A D Q$ & & 10 & MF & 15 & 2 & 7.5 & Trem & lite & & & & \\
\hline G1 & 20 & B9 & $A D Q$ & & 12 & MF & 12.5 & 1 & 12.5 & Trem & blite & & & & \\
\hline G2 & 22 & $\mathrm{C} 2$ & $A D Q$ & 16 & 17 & $\mathrm{~F}$ & 10.1 & 1 & 10.1 & Trem & blite & $\mathrm{Mg}, \mathrm{Al}, \mathrm{Si}, \mathrm{Ca}, \mathrm{Fe}$ & & & \\
\hline G2 & 22 & $\mathrm{C} 2$ & $A Z Q$ & & 15 & $\mathrm{CF}$ & 7 & 0.25 & 28 & Trem & lite & & & & \\
\hline G2 & 28 & $\mathrm{~F} 4$ & ADQ & 17 & 18 & $\mathrm{~F}$ & 7.2 & 1.5 & 4.8 & Trem & lite & & & & \\
\hline G2 & 21 & $\mathrm{~A} 2$ & $A Q$ & 14 & 14 & $\mathrm{~F}$ & 5.6 & 0.7 & 8 & Trem & lite & & & & \\
\hline & & M Equ & alent S & tructu & Ires-I & & 15.9 & & Not & licable & & Not Applicable & & 5 & \\
\hline $\mathrm{Gr}$ & No. & Loc. & ID & Prim & Tot & Class & Len & Wid & Asp & Anal & & Elements & Comment & & \\
\hline G1 & 11 & A7 & $A D Q$ & 7 & 7 & $\mathrm{~F}$ & 5.2 & 0.4 & 13 & Trem & blite & $\mathrm{Mg}, \mathrm{Al}, \mathrm{Si}, \mathrm{Ca}, \mathrm{Fe}$ & & & \\
\hline G1 & 20 & B9 & $A D Q$ & 12 & & MD 1-1 & 13.3 & 4 & 3.3 & Trem & lite & $\mathrm{Mg}, \mathrm{Si}, \mathrm{Ca}, \mathrm{Fe}$ & & & \\
\hline G2 & 21 & $\mathrm{~A} 2$ & $A Q$ & 14 & 14 & $\mathrm{~F}$ & 5.6 & 0.7 & 8 & Trem & lite & & & & \\
\hline G2 & 22 & $\mathrm{C} 2$ & $A D Q$ & 16 & 17 & $\mathrm{~F}$ & 10.1 & 1 & 10.1 & Trem & lite & $\mathrm{Mg}, \mathrm{Al}, \mathrm{Si}, \mathrm{Ca}, \mathrm{Fe}$ & & & \\
\hline
\end{tabular}


Job Number: 070434

SEA

ISO 10312, Direct Count Categories

Client: Idaho National Laboratory

Report Number: 070434R06

Date Received: 4/23/2007

Project Name: RARE

Lab/Cor Sample No.: S25

Client Sample No.: FB-3-R7

Description:

Filter Fraction: 1

Residual Ash Vol:
Aliquot Dilution: 0

Final Dilution: 0
Volume (L): 0

Lab Filter Area (mm2): 385

Grid Openings Analyzed: 35

Average Grid Opening Area: 0.009

Area Analyzed (mm2): 0.315

Analytical Sens. (struc/cc): 0

Dectection Limit. (struc/cc): 0

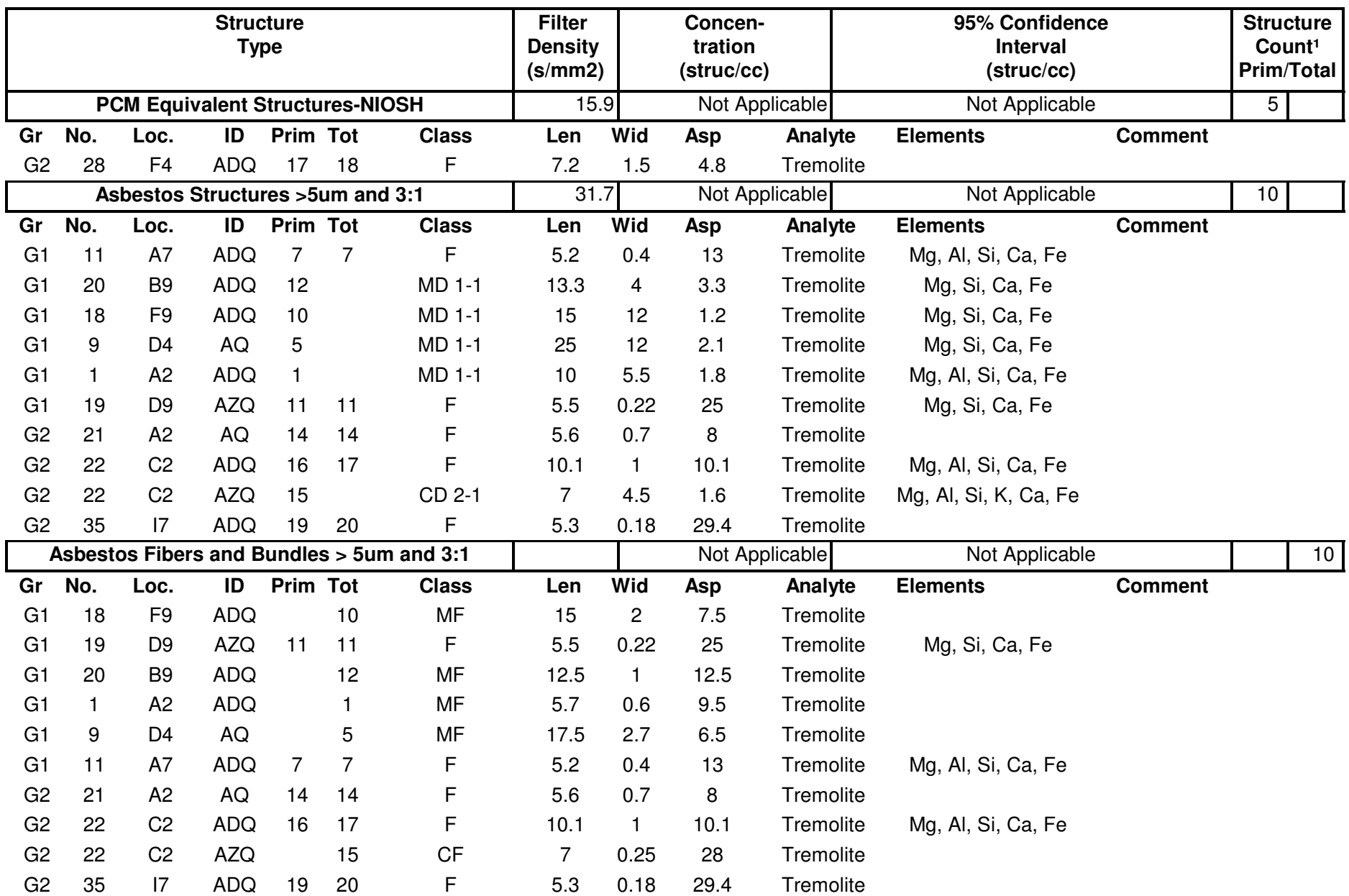


Job Number: 070434

SEA

ISO 10312, Direct Count Categories

Client: Idaho National Laboratory

Report Number: 070434R06

ct Name: RARE

Lab/Cor Sample No.: S26

Client Sample No.: FB-2-R1

Description:

Filter Fraction: 1

Residual Ash Vol:
Aliquot Dilution: 0

Final Dilution: 0
Date Received: 4/23/2007
Volume (L): 0

Lab Filter Area (mm2): 385

Grid Openings Analyzed: 35

Average Grid Opening Area: 0.009

Area Analyzed (mm2): 0.315

Analytical Sens. (struc/cc): 0

Dectection Limit. (struc/cc): 0

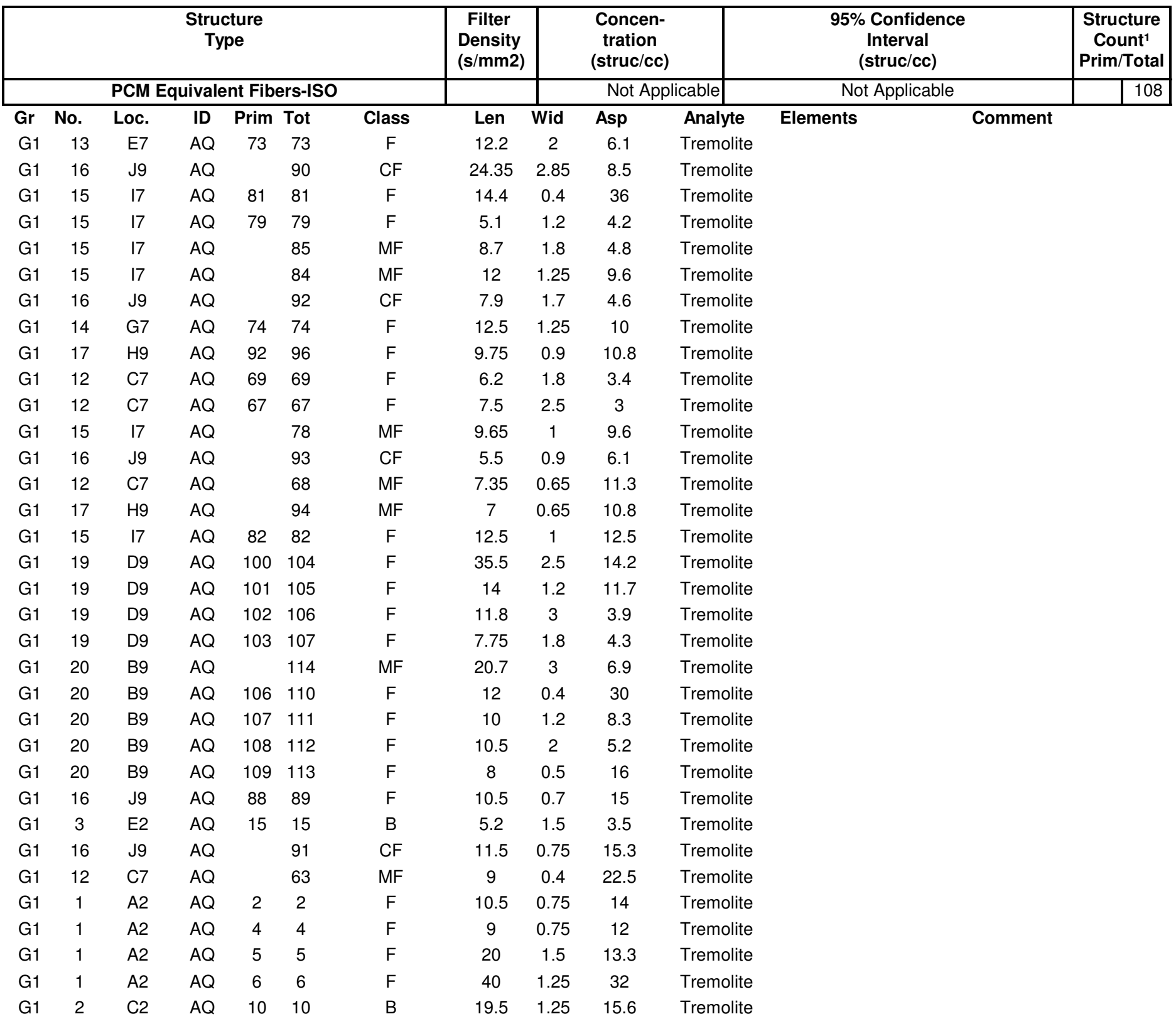


Job Number: 070434

SEA

ISO 10312, Direct Count Categories

Client: Idaho National Laboratory

Report Number: 070434R06

Date Received: 4/23/2007

Project Name: RARE

Lab/Cor Sample No.: S26

Client Sample No.: FB-2-R1

Description:

Filter Fraction: 1

Residual Ash Vol:
Aliquot Dilution: 0

Final Dilution: 0
Volume (L): 0

Lab Filter Area (mm2): 385

Grid Openings Analyzed: 35

Average Grid Opening Area: 0.009

Area Analyzed (mm2): 0.315

Analytical Sens. (struc/cc): 0

Dectection Limit. (struc/cc): 0

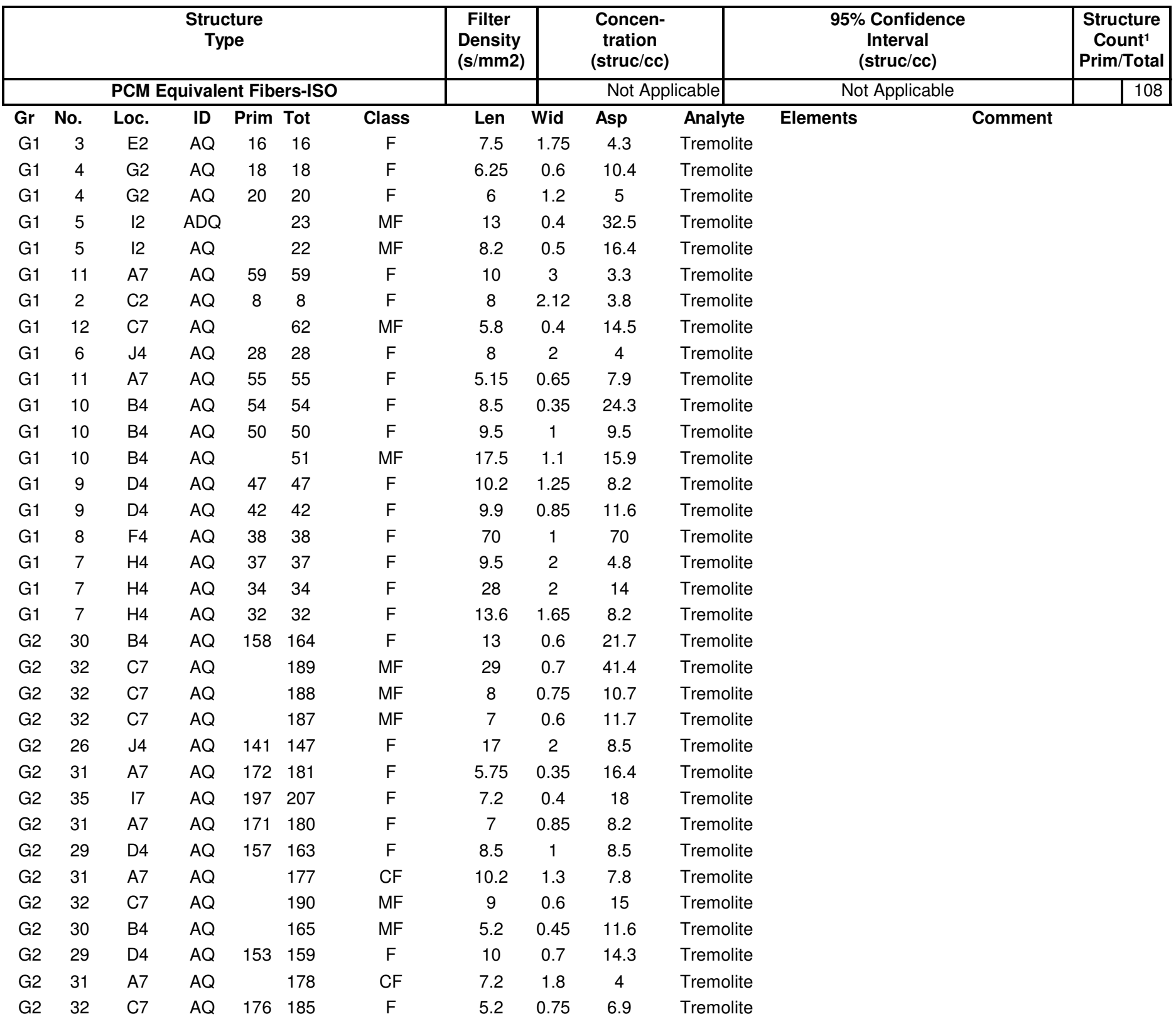


Job Number: 070434

SEA

ISO 10312, Direct Count Categories

Client: Idaho National Laboratory

Report Number: 070434R06

Date Received: 4/23/2007

Project Name: RARE

Lab/Cor Sample No.: S26

Client Sample No.: FB-2-R1

Description:

Filter Fraction: 1

Residual Ash Vol:
Aliquot Dilution: 0

Final Dilution: 0
Volume (L): 0

Lab Filter Area (mm2): 385

Grid Openings Analyzed: 35

Average Grid Opening Area: 0.009

Area Analyzed (mm2): 0.315

Analytical Sens. (struc/cc): 0

Dectection Limit. (struc/cc): 0

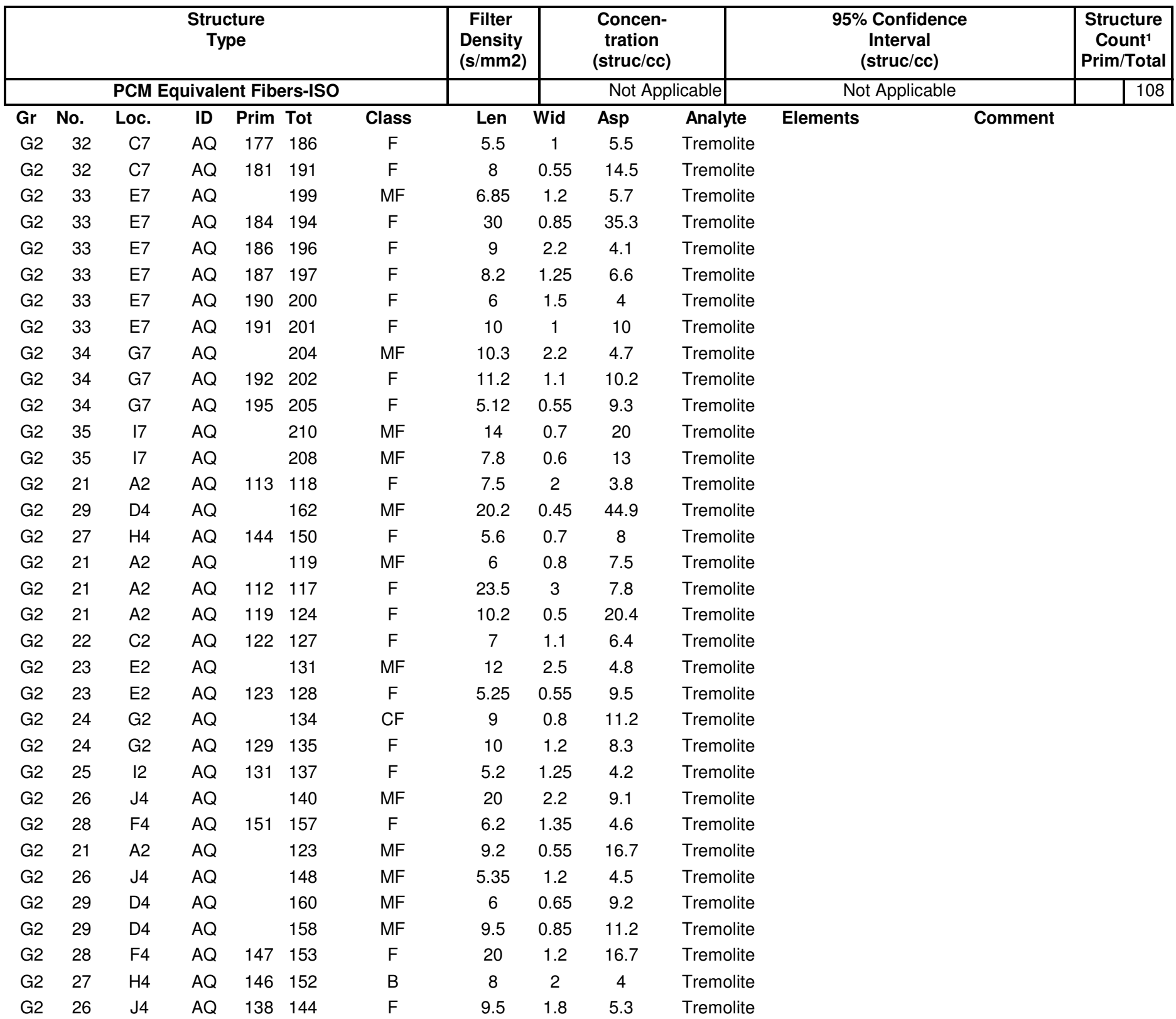


Job Number: 070434

SEA

ISO 10312, Direct Count Categories

Client: Idaho National Laboratory

Report Number: 070434R06

Date Received: 4/23/2007

Project Name: RARE

Lab/Cor Sample No.: S26

Client Sample No.: FB-2-R1

Description:

Filter Fraction: 1

Residual Ash Vol:
Aliquot Dilution: 0

Final Dilution: 0
Volume (L): 0

Lab Filter Area (mm2): 385

Grid Openings Analyzed: 35

Average Grid Opening Area: 0.009

Area Analyzed (mm2): 0.315

Analytical Sens. (struc/cc): 0

Dectection Limit. (struc/cc): 0

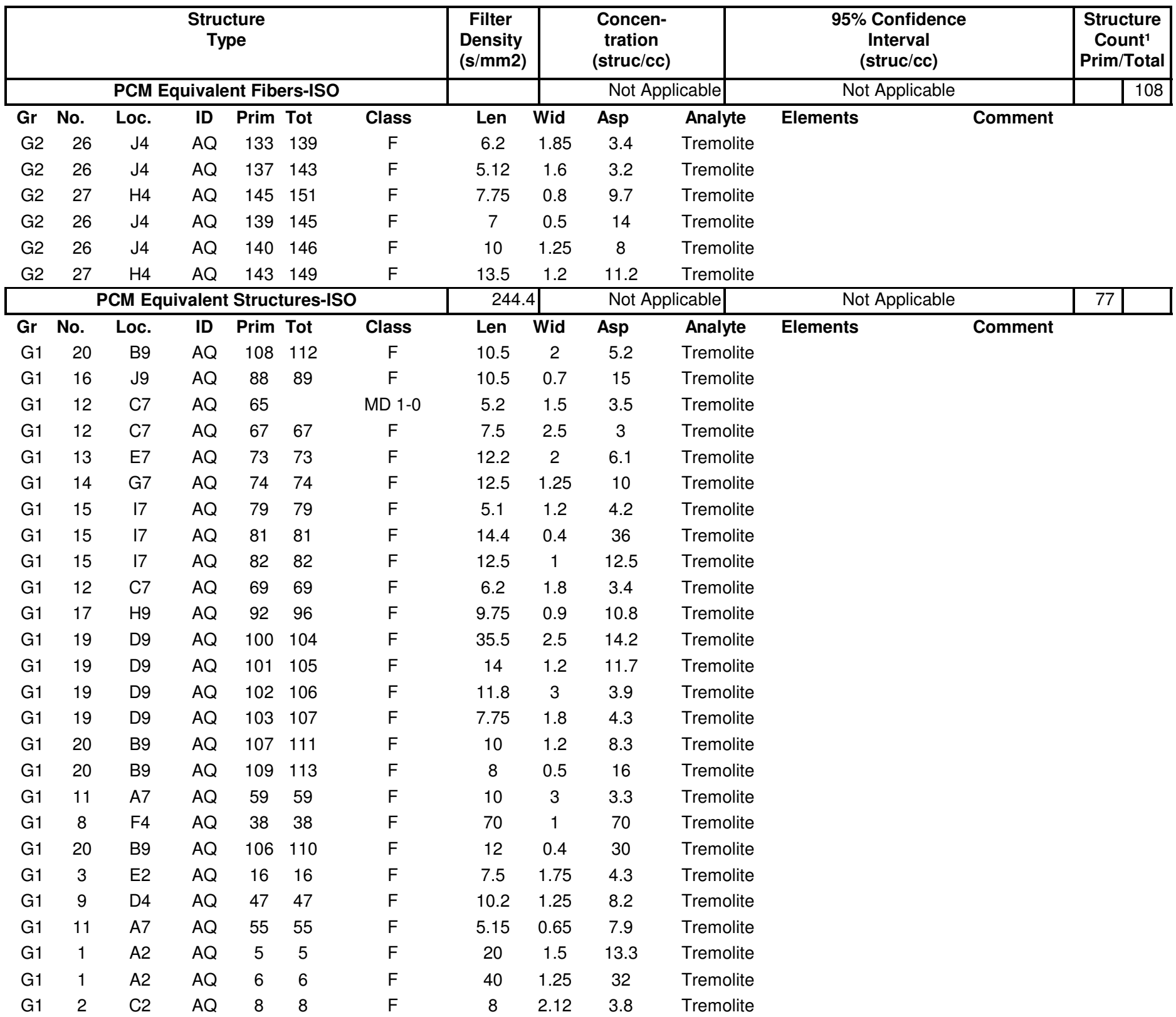


Job Number: 070434

SEA

ISO 10312, Direct Count Categories

Client: Idaho National Laboratory

Report Number: 070434R06

Date Received: 4/23/2007

Project Name: RARE

Lab/Cor Sample No.: S26

Client Sample No.: FB-2-R1

Description:

Filter Fraction: 1

Residual Ash Vol:
Aliquot Dilution: 0

Final Dilution: 0
Volume (L): 0

Lab Filter Area (mm2): 385

Grid Openings Analyzed: 35

Average Grid Opening Area: 0.009

Area Analyzed (mm2): 0.315

Analytical Sens. (struc/cc): 0

Dectection Limit. (struc/cc): 0

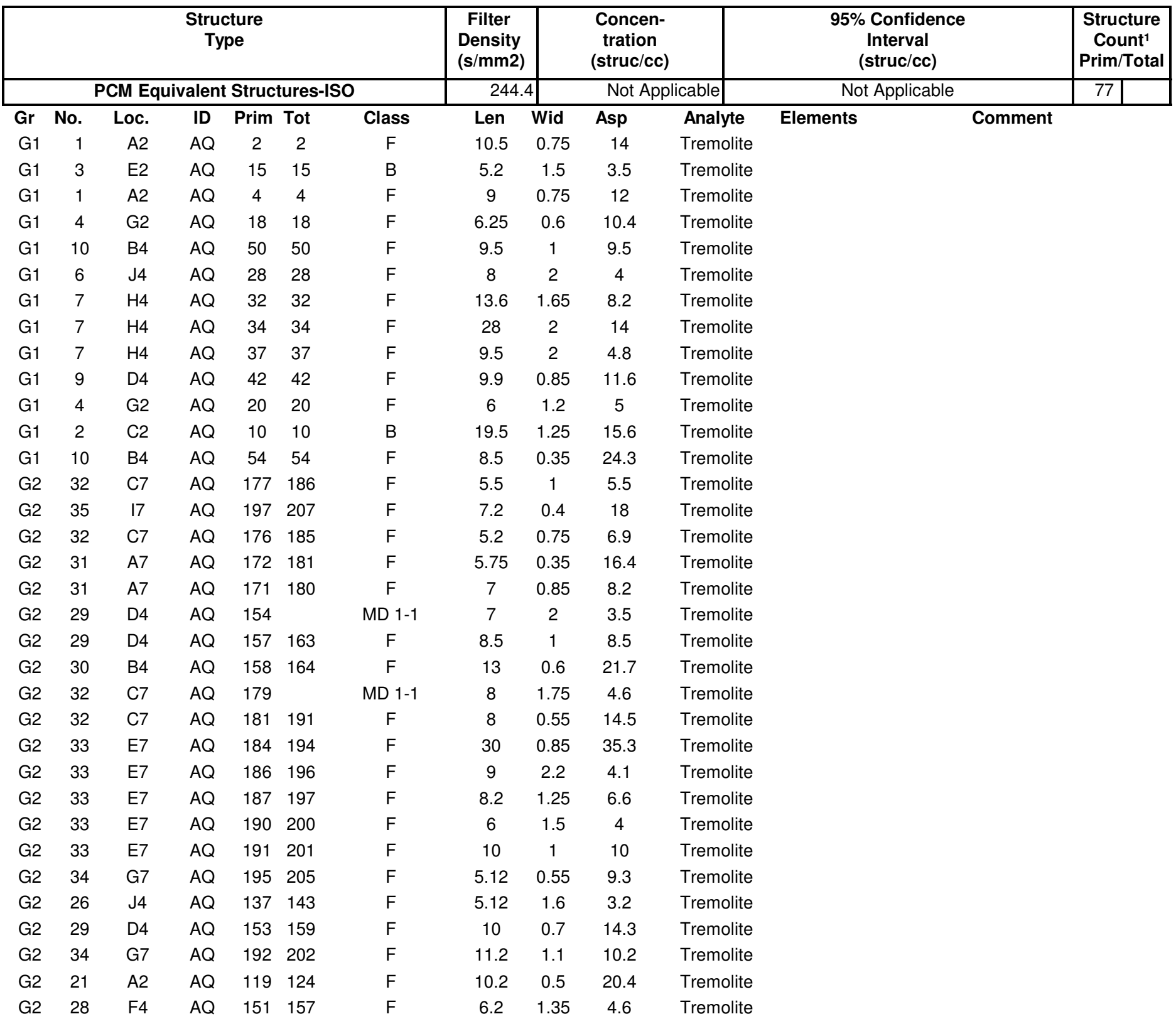


A Professional Service Corporation in the Northwest

Job Number: 070434

SEA

ISO 10312, Direct Count Categories

Client: Idaho National Laboratory

Report Number: 070434R06

Date Received: 4/23/2007

Project Name: RARE

Lab/Cor Sample No.: S26

Client Sample No.: FB-2-R1

Description:

Filter Fraction: 1

Residual Ash Vol:
Aliquot Dilution: 0

Final Dilution: 0
Volume (L): 0

Lab Filter Area (mm2): 385

Grid Openings Analyzed: 35

Average Grid Opening Area: 0.009

Area Analyzed (mm2): 0.315

Analytical Sens. (struc/cc): 0

Dectection Limit. (struc/cc): 0

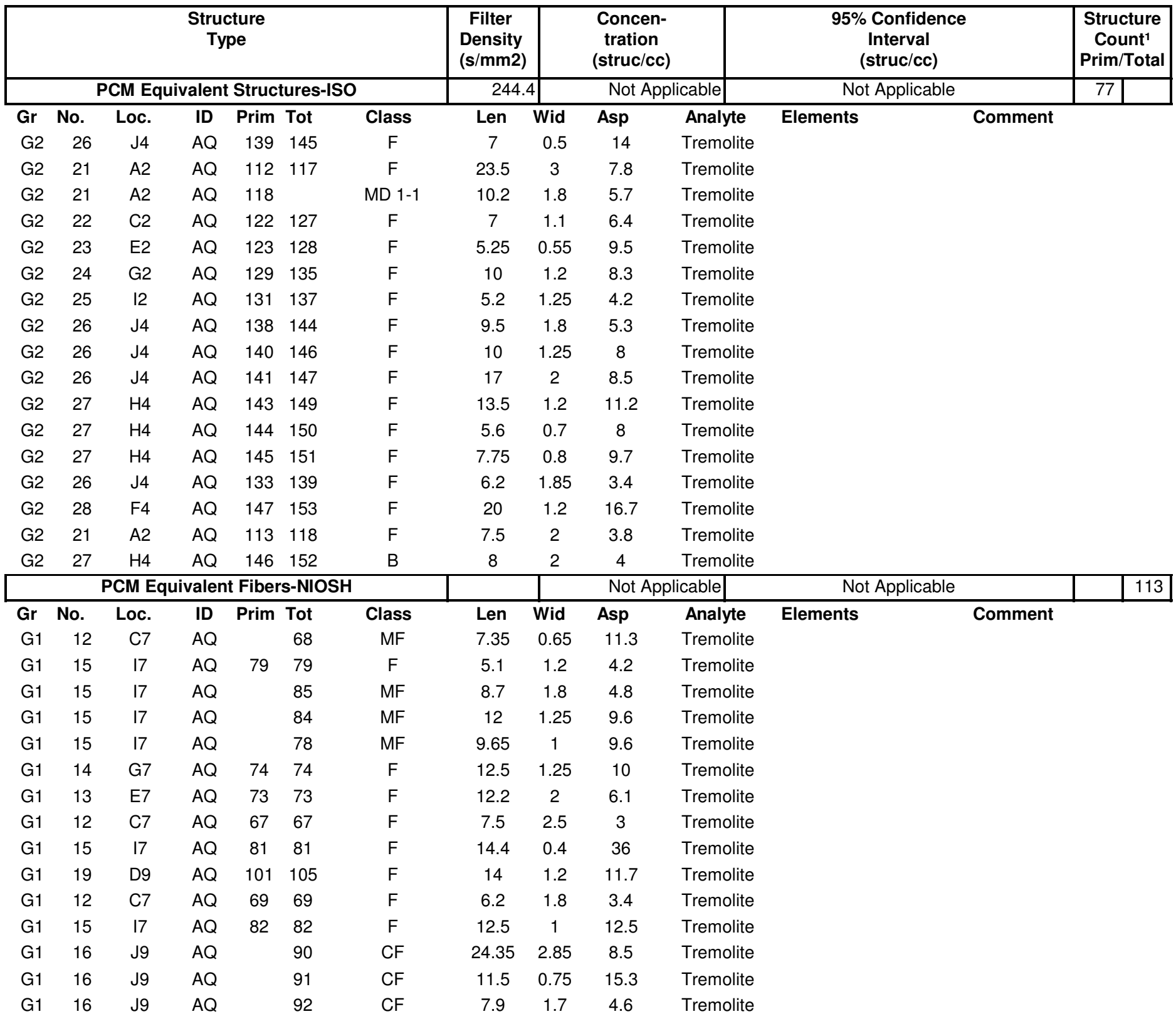


Job Number: 070434

SEA

ISO 10312, Direct Count Categories

Client: Idaho National Laboratory

Report Number: 070434R06

ct Name: RARE

Lab/Cor Sample No.: S26

Client Sample No.: FB-2-R1

Description:

Filter Fraction: 1

Residual Ash Vol:
Aliquot Dilution: 0

Final Dilution: 0
Date Received: 4/23/2007
Volume (L): 0

Lab Filter Area (mm2): 385

Grid Openings Analyzed: 35

Average Grid Opening Area: 0.009

Area Analyzed (mm2): 0.315

Analytical Sens. (struc/cc): 0

Dectection Limit. (struc/cc): 0

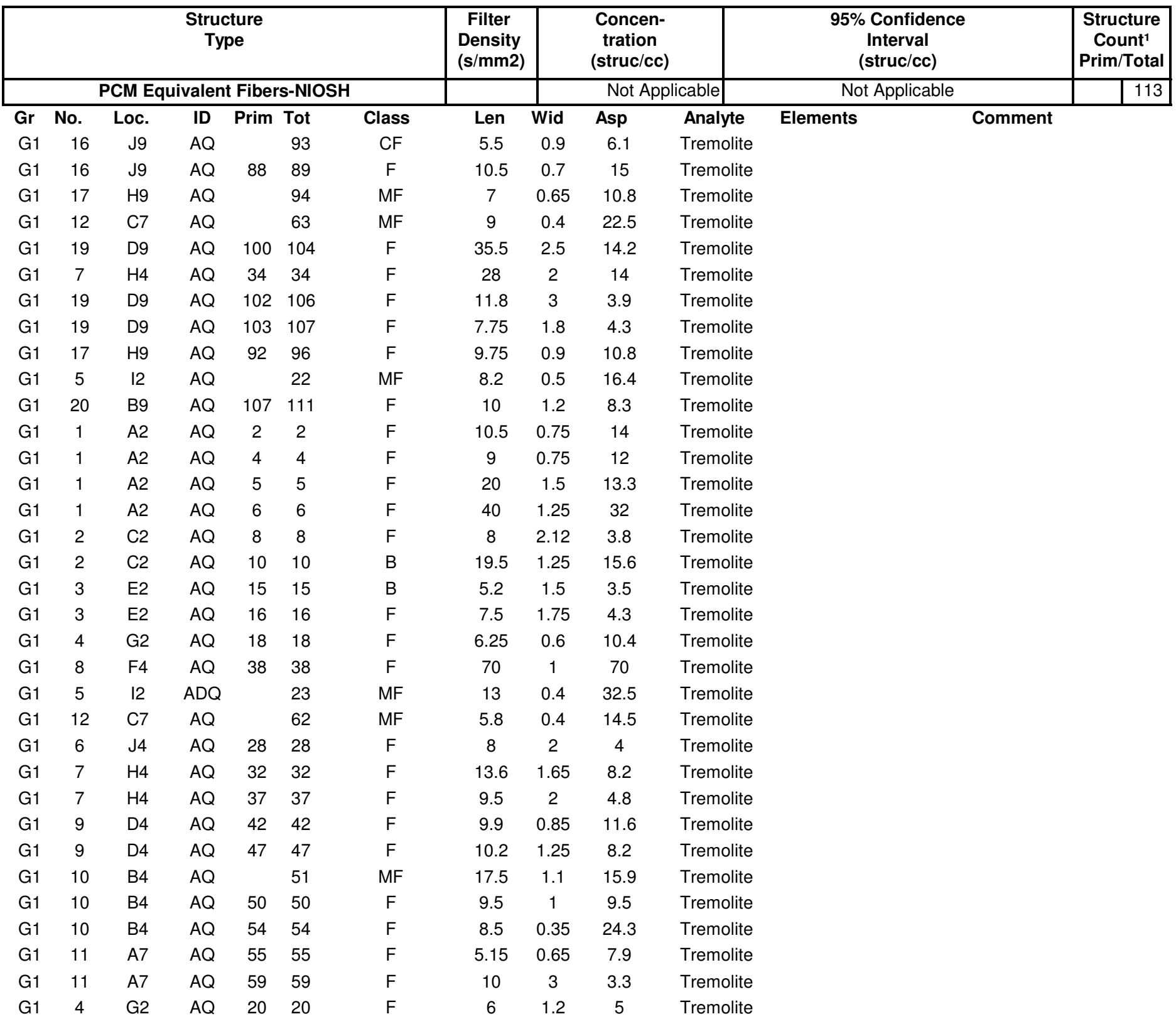


Job Number: 070434

SEA

ISO 10312, Direct Count Categories

Client: Idaho National Laboratory

Report Number: 070434R06

Date Received: 4/23/2007

Project Name: RARE

Lab/Cor Sample No.: S26

Client Sample No.: FB-2-R1

Description:

Filter Fraction: 1

Residual Ash Vol:
Aliquot Dilution: 0

Final Dilution: 0
Volume (L): 0

Lab Filter Area (mm2): 385

Grid Openings Analyzed: 35

Average Grid Opening Area: 0.009

Area Analyzed (mm2): 0.315

Analytical Sens. (struc/cc): 0

Dectection Limit. (struc/cc): 0

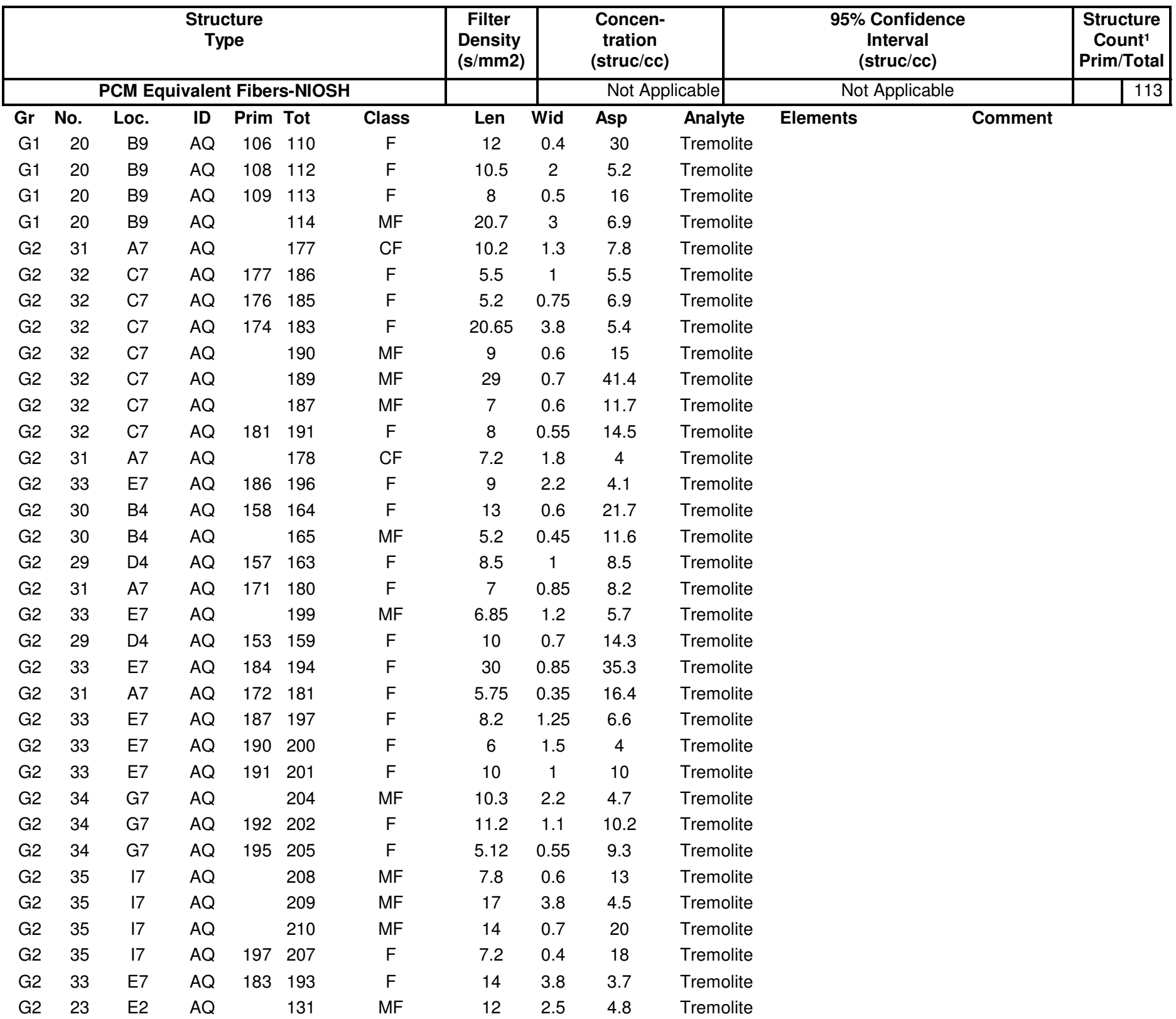


Job Number: 070434

SEA

ISO 10312, Direct Count Categories

Client: Idaho National Laboratory

Report Number: 070434R06

Date Received: 4/23/2007

Project Name: RARE

Lab/Cor Sample No.: S26

Client Sample No.: FB-2-R1

Description:

Filter Fraction: 1

Residual Ash Vol:
Aliquot Dilution: 0

Final Dilution: 0
Volume (L): 0

Lab Filter Area (mm2): 385

Grid Openings Analyzed: 35

Average Grid Opening Area: 0.009

Area Analyzed (mm2): 0.315

Analytical Sens. (struc/cc): 0

Dectection Limit. (struc/cc): 0

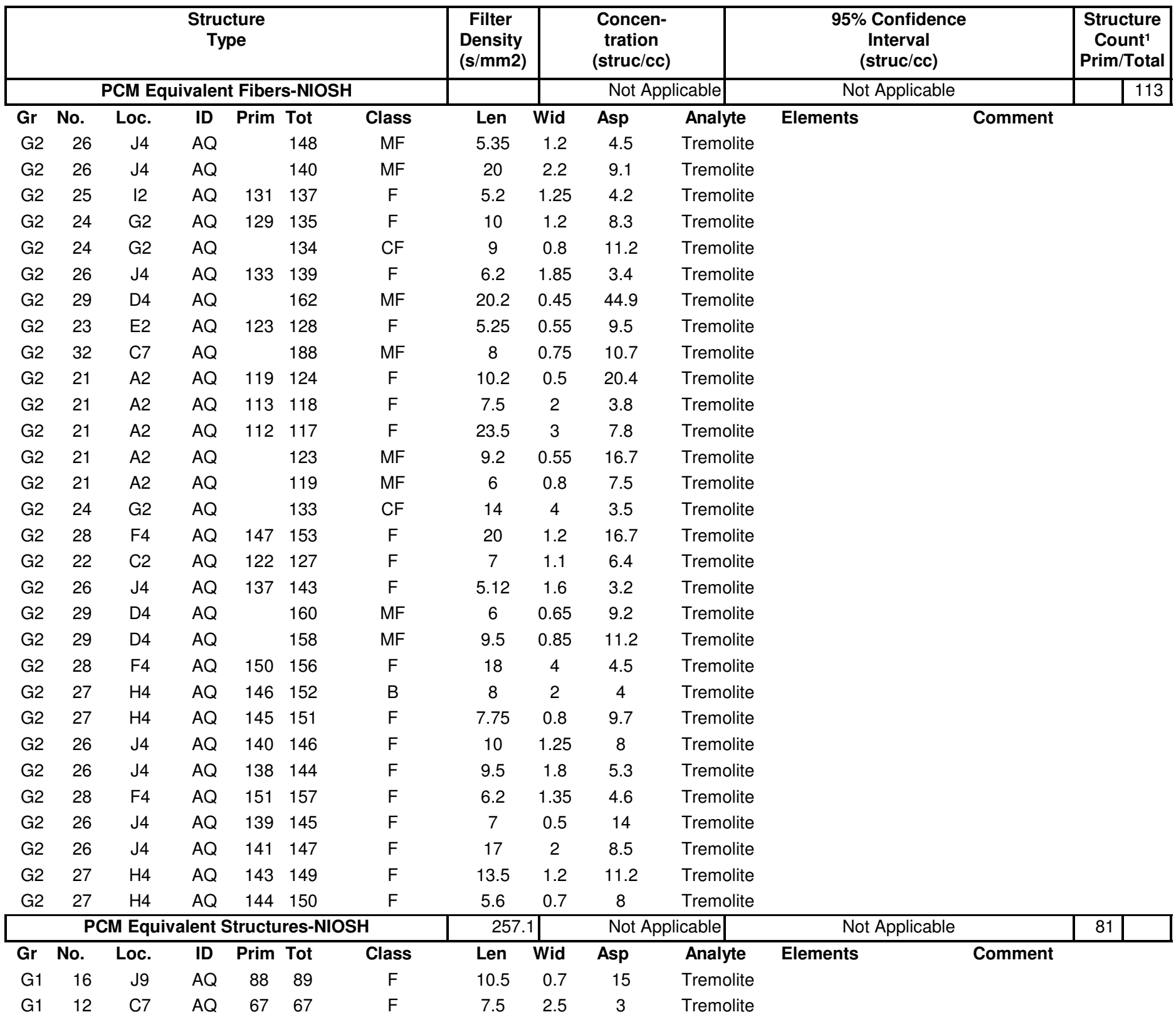


Job Number: 070434

SEA

ISO 10312, Direct Count Categories

Client: Idaho National Laboratory

Report Number: 070434R06

ct Name: RARE

Lab/Cor Sample No.: S26

Client Sample No.: FB-2-R1

Description:

Filter Fraction: 1

Residual Ash Vol:
Aliquot Dilution: 0

Final Dilution: 0
Date Received: 4/23/2007
Volume (L): 0

Lab Filter Area (mm2): 385

Grid Openings Analyzed: 35

Average Grid Opening Area: 0.009

Area Analyzed (mm2): 0.315

Analytical Sens. (struc/cc): 0

Dectection Limit. (struc/cc): 0

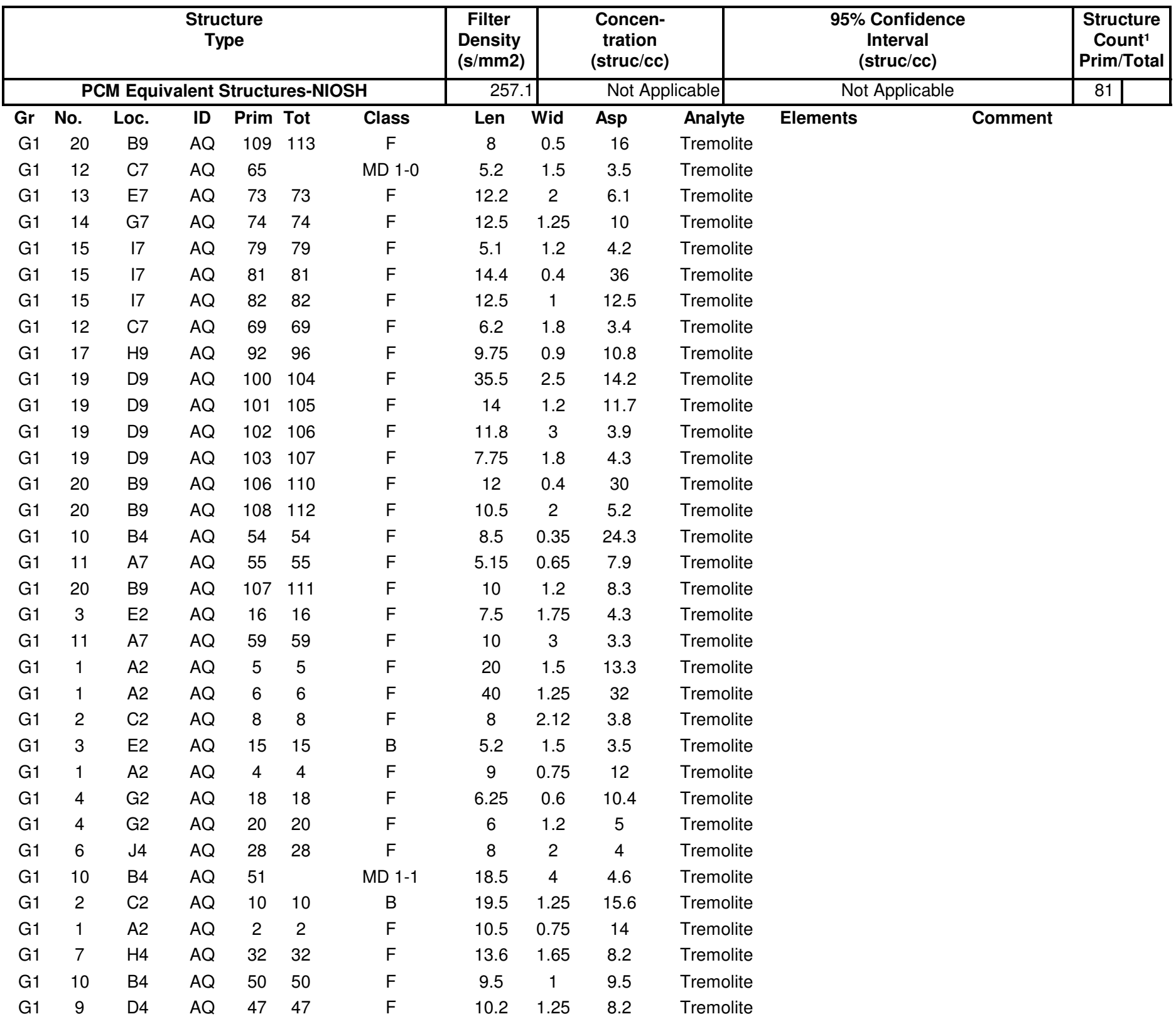


Job Number: 070434

SEA

ISO 10312, Direct Count Categories

Client: Idaho National Laboratory

Report Number: 070434R06

Date Received: 4/23/2007

Project Name: RARE

Lab/Cor Sample No.: S26

Client Sample No.: FB-2-R1

Description:

Filter Fraction: 1

Residual Ash Vol:
Aliquot Dilution: 0

Final Dilution: 0
Volume (L): 0

Lab Filter Area (mm2): 385

Grid Openings Analyzed: 35

Average Grid Opening Area: 0.009

Area Analyzed (mm2): 0.315

Analytical Sens. (struc/cc): 0

Dectection Limit. (struc/cc): 0

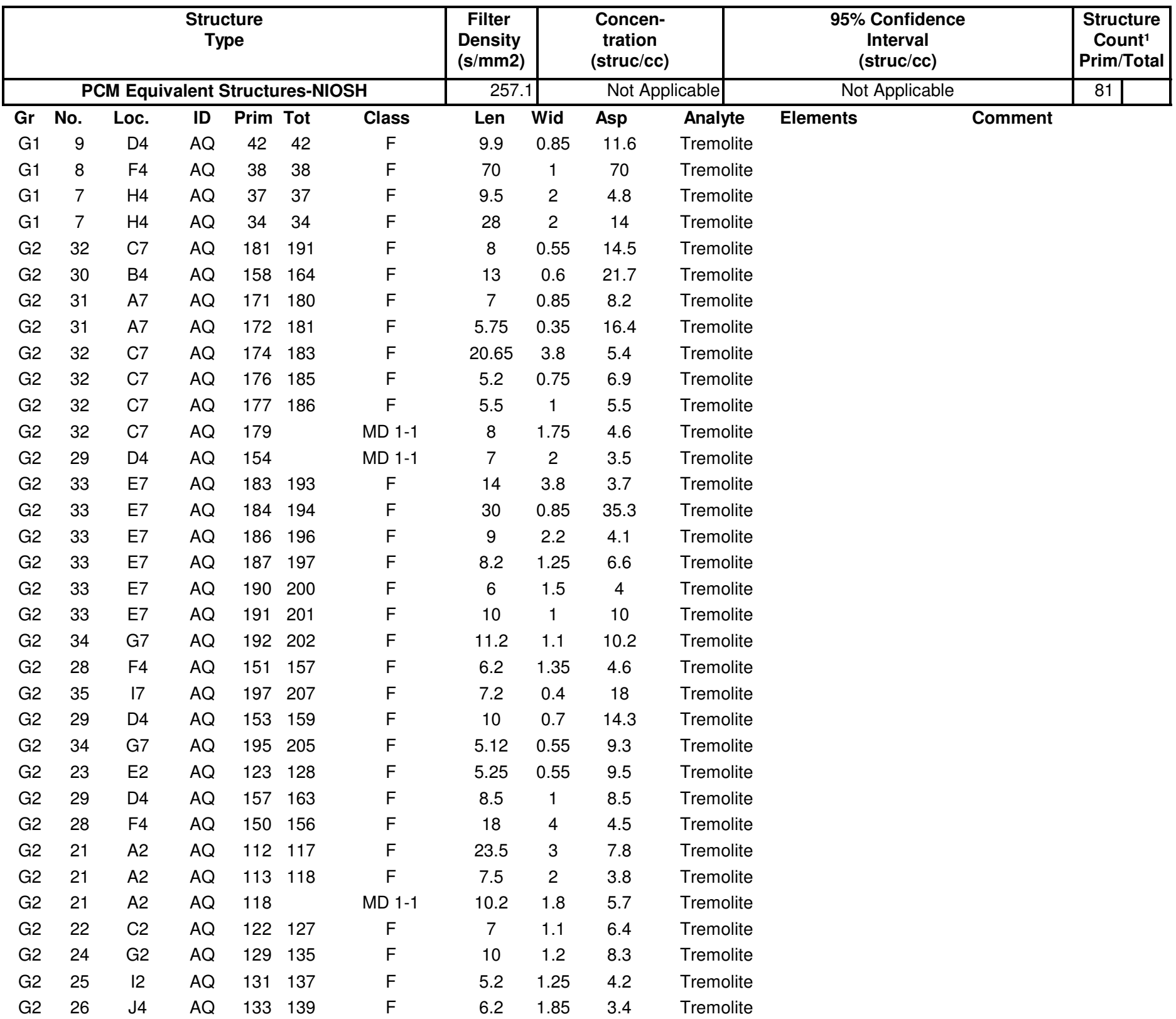


Job Number: 070434

SEA

ISO 10312, Direct Count Categories

Client: Idaho National Laboratory

Report Number: 070434R06

Date Received: 4/23/2007

Project Name: RARE

Lab/Cor Sample No.: S26

Client Sample No.: FB-2-R1

Description:

Filter Fraction: 1

Residual Ash Vol:
Aliquot Dilution: 0

Final Dilution: 0
Volume (L): 0

Lab Filter Area (mm2): 385

Grid Openings Analyzed: 35

Average Grid Opening Area: 0.009

Area Analyzed (mm2): 0.315

Analytical Sens. (struc/cc): 0

Dectection Limit. (struc/cc): 0

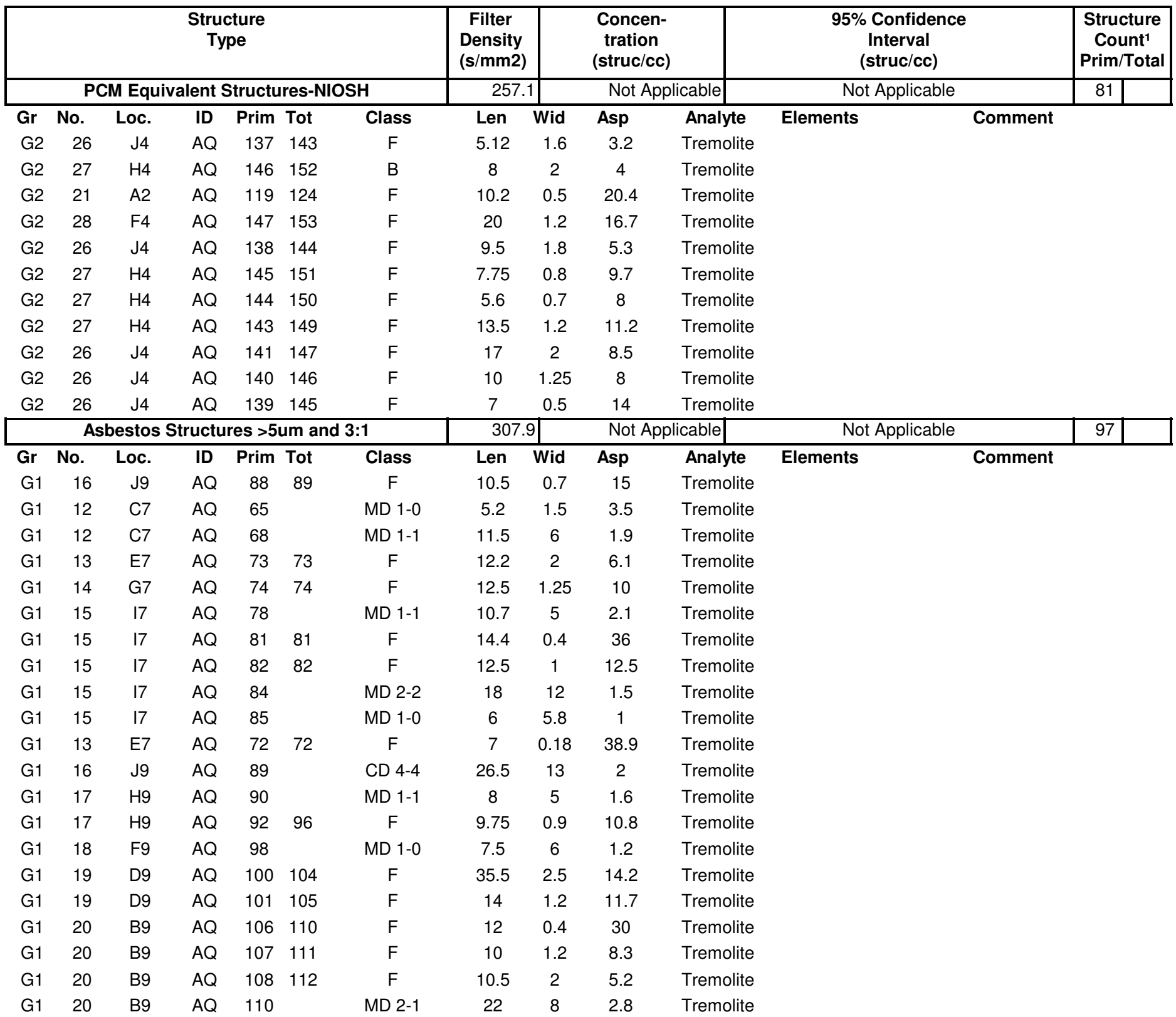


Job Number: 070434

SEA

ISO 10312, Direct Count Categories

Client: Idaho National Laboratory

Report Number: 070434R06

Date Received: 4/23/2007

Project Name: RARE

Lab/Cor Sample No.: S26

Client Sample No.: FB-2-R1

Description:

Filter Fraction: 1

Residual Ash Vol:
Aliquot Dilution: 0

Final Dilution: 0
Volume (L): 0

Lab Filter Area (mm2): 385

Grid Openings Analyzed: 35

Average Grid Opening Area: 0.009

Area Analyzed (mm2): 0.315

Analytical Sens. (struc/cc): 0

Dectection Limit. (struc/cc): 0

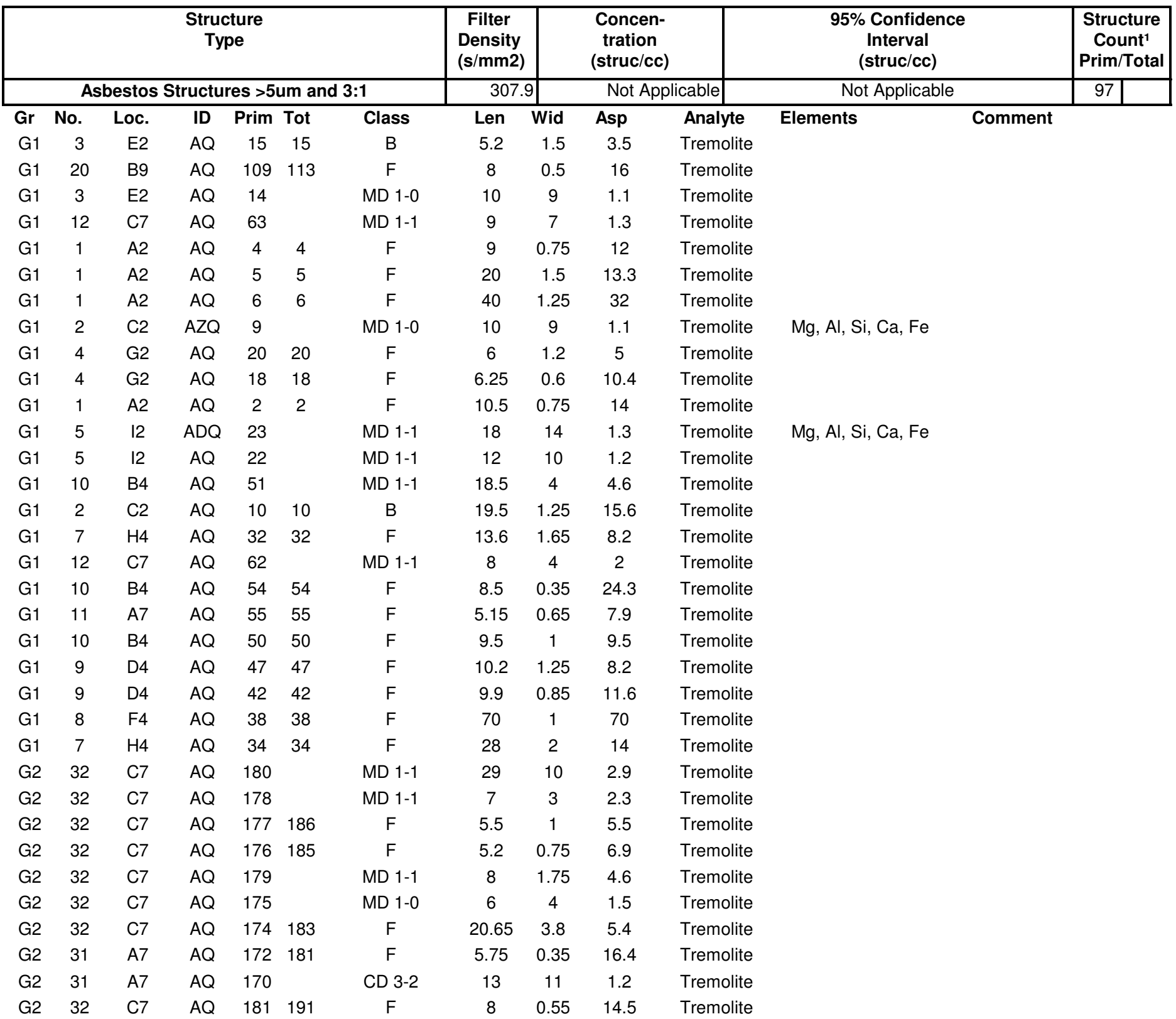


Job Number: 070434

SEA

ISO 10312, Direct Count Categories

Client: Idaho National Laboratory

Report Number: 070434R06

Date Received: 4/23/2007

Project Name: RARE

Lab/Cor Sample No.: S26

Client Sample No.: FB-2-R1

Description:

Filter Fraction: 1

Residual Ash Vol:
Aliquot Dilution: 0

Final Dilution: 0
Volume (L): 0

Lab Filter Area (mm2): 385

Grid Openings Analyzed: 35

Average Grid Opening Area: 0.009

Area Analyzed (mm2): 0.315

Analytical Sens. (struc/cc): 0

Dectection Limit. (struc/cc): 0

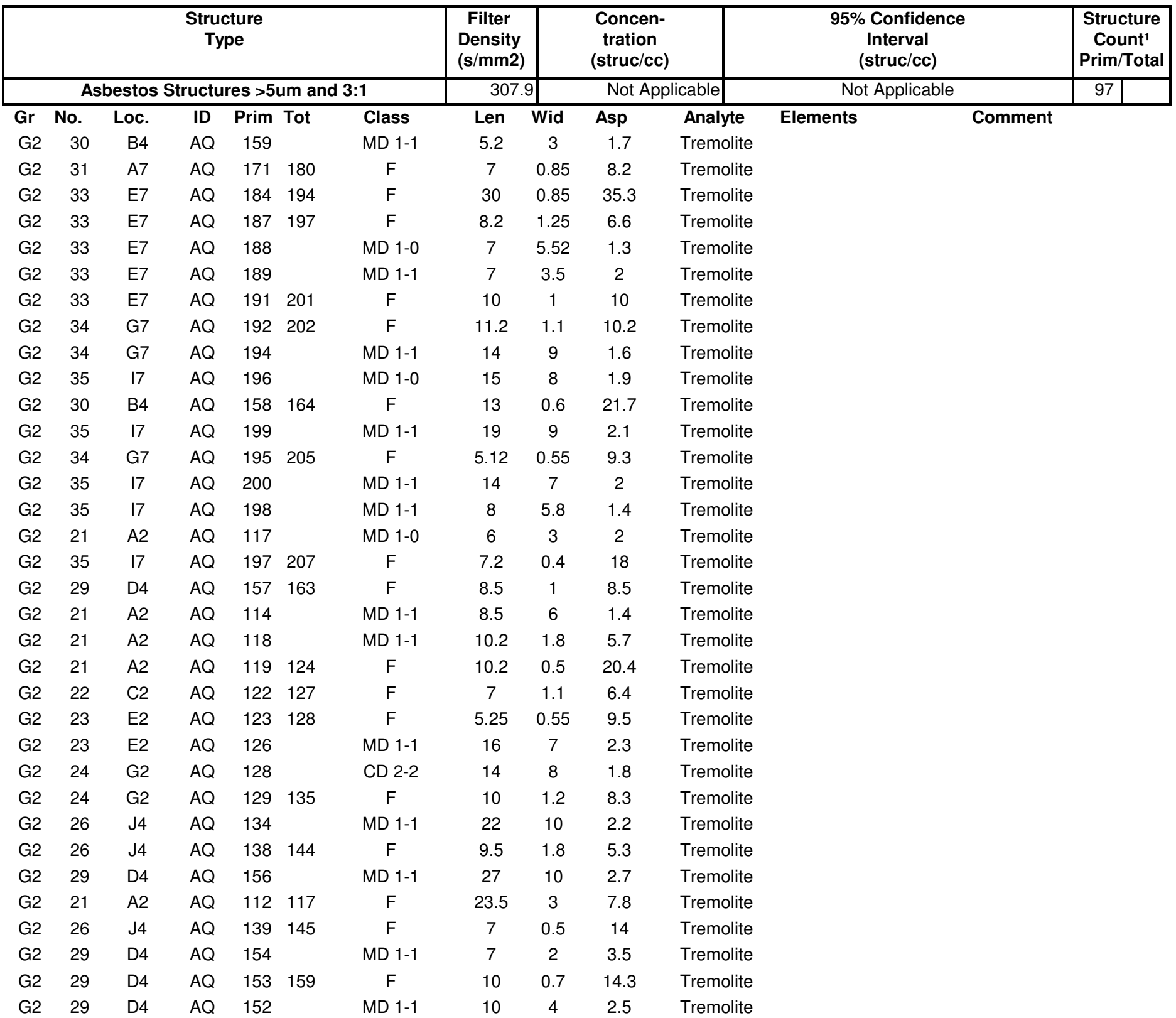


Job Number: 070434

SEA

ISO 10312, Direct Count Categories

Client: Idaho National Laboratory

Report Number: 070434R06

Date Received: 4/23/2007

Project Name: RARE

Lab/Cor Sample No.: S26

Client Sample No.: FB-2-R1

Description:

Filter Fraction: 1

Residual Ash Vol:
Aliquot Dilution: 0

Final Dilution: 0
Volume (L): 0

Lab Filter Area (mm2): 385

Grid Openings Analyzed: 35

Average Grid Opening Area: 0.009

Area Analyzed (mm2): 0.315

Analytical Sens. (struc/cc): 0

Dectection Limit. (struc/cc): 0

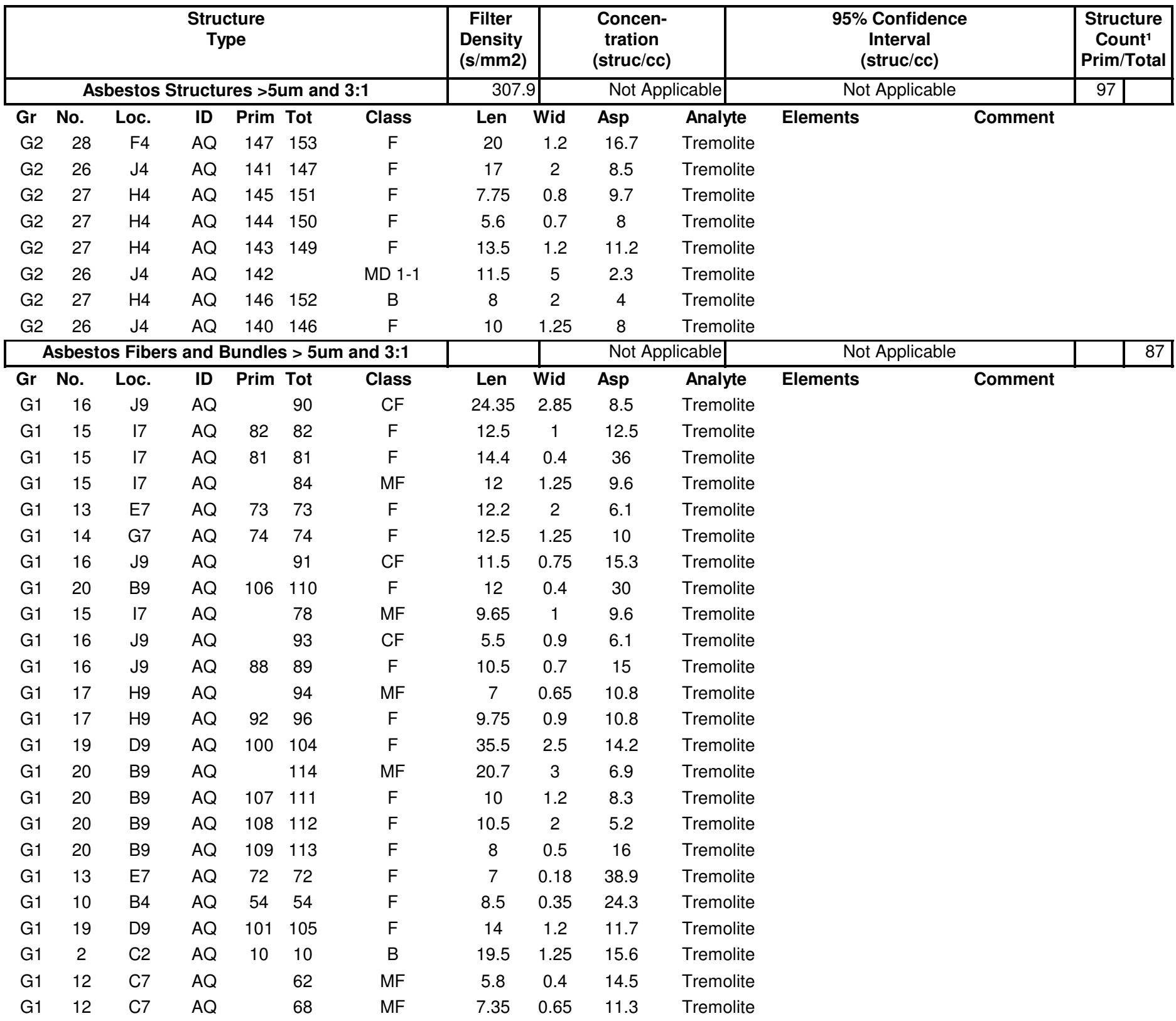


Job Number: 070434

SEA

ISO 10312, Direct Count Categories

Client: Idaho National Laboratory

Report Number: 070434R06

Date Received: 4/23/2007

Project Name: RARE

Lab/Cor Sample No.: S26

Client Sample No.: FB-2-R1

Description:

Filter Fraction: 1

Residual Ash Vol:
Aliquot Dilution: 0

Final Dilution: 0
Volume (L): 0

Lab Filter Area (mm2): 385

Grid Openings Analyzed: 35

Average Grid Opening Area: 0.009

Area Analyzed (mm2): 0.315

Analytical Sens. (struc/cc): 0

Dectection Limit. (struc/cc): 0

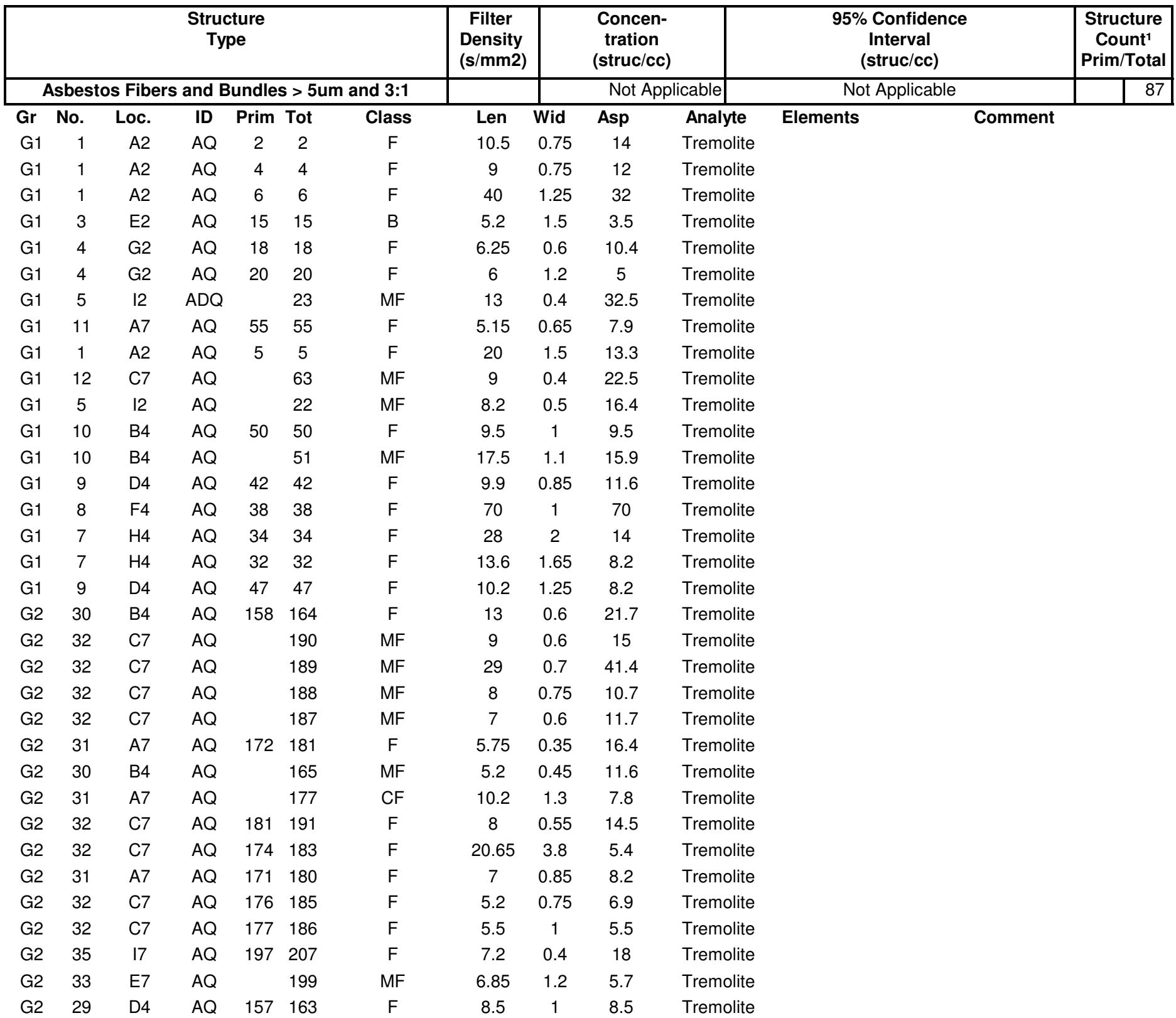


Job Number: 070434

SEA

ISO 10312, Direct Count Categories

Client: Idaho National Laboratory

Report Number: 070434R06

Date Received: 4/23/2007

Project Name: RARE

Lab/Cor Sample No.: S26

Client Sample No.: FB-2-R1

Description:

Filter Fraction: 1

Residual Ash Vol:
Aliquot Dilution: 0

Final Dilution: 0
Volume (L): 0

Lab Filter Area (mm2): 385

Grid Openings Analyzed: 35

Average Grid Opening Area: 0.009

Area Analyzed (mm2): 0.315

Analytical Sens. (struc/cc): 0

Dectection Limit. (struc/cc): 0

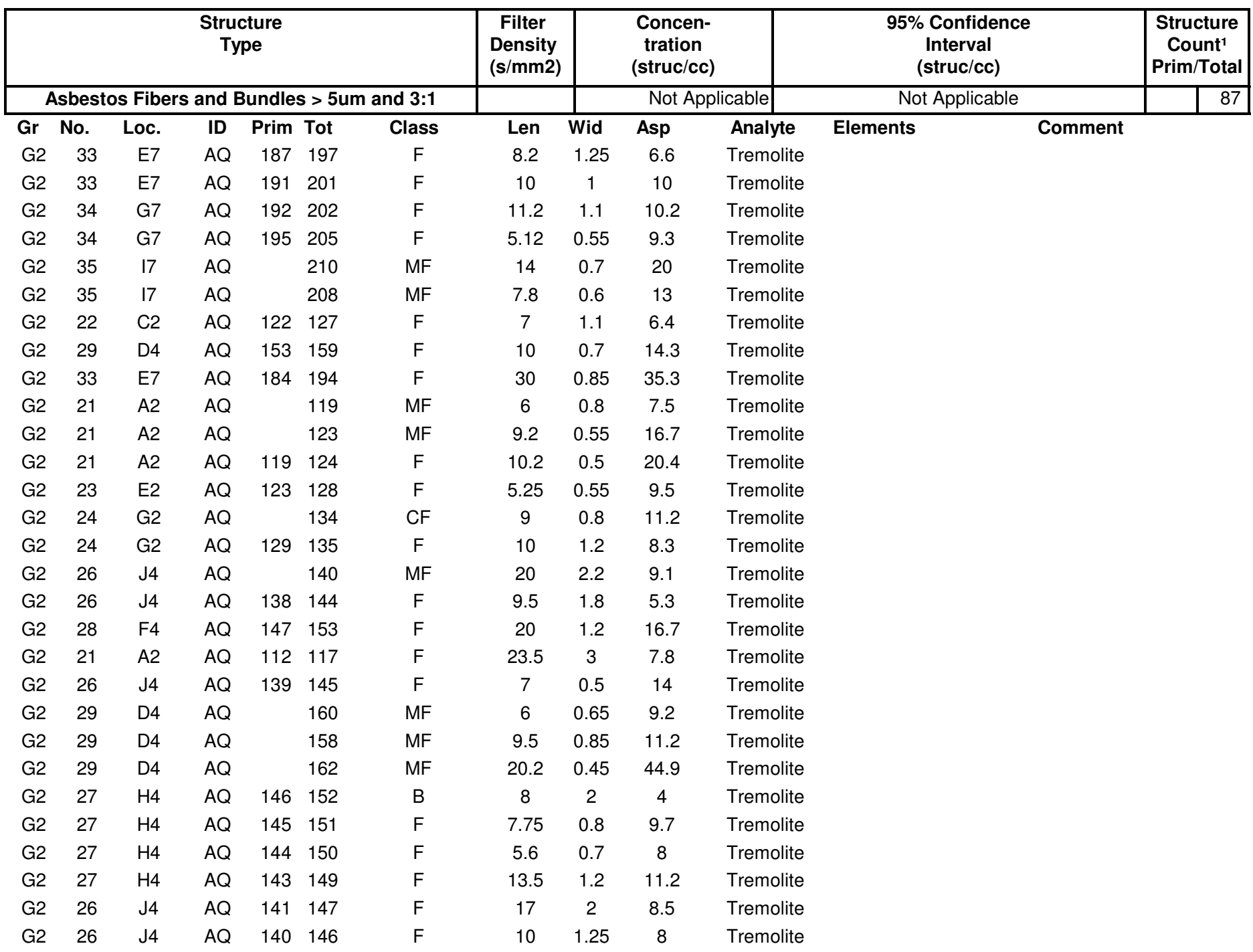


Job Number: 070434

SEA

ISO 10312, Direct Count Categories

Client: Idaho National Laboratory

Report Number: 070434R06

ct Name: RARE

Lab/Cor Sample No.: S27

Client Sample No.: FB-2-R2

Description:

Filter Fraction: 1

Residual Ash Vol:
Aliquot Dilution: 0

Final Dilution: 0
Date Received: 4/23/2007
Volume (L): 0

Lab Filter Area (mm2): 385

Grid Openings Analyzed: 35

Average Grid Opening Area: 0.009

Area Analyzed (mm2): 0.315

Analytical Sens. (struc/cc): 0

Dectection Limit. (struc/cc): 0

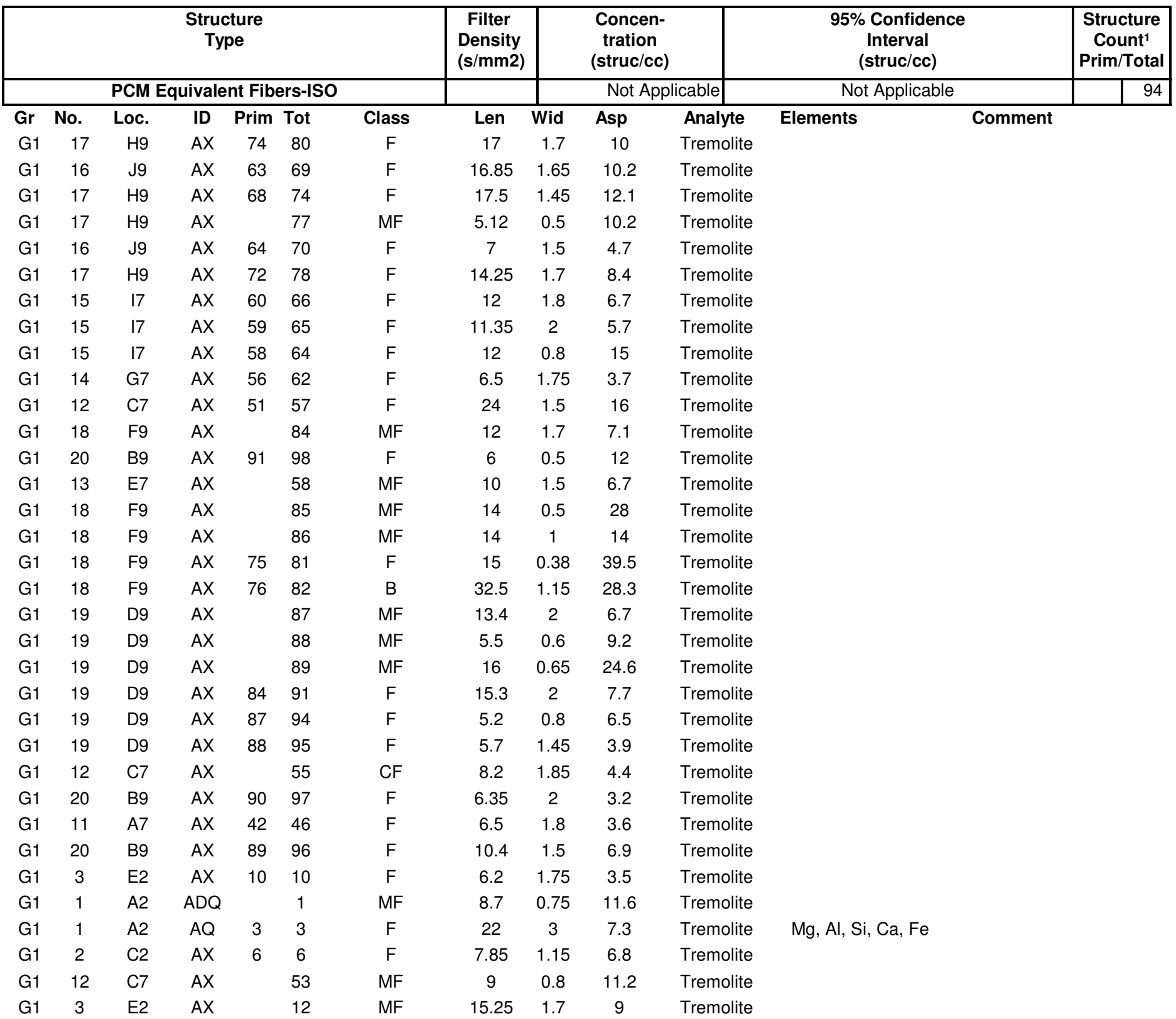


Job Number: 070434

SEA

ISO 10312, Direct Count Categories

Client: Idaho National Laboratory

Report Number: 070434R06

Date Received: 4/23/2007

Project Name: RARE

Lab/Cor Sample No.: S27

Client Sample No.: FB-2-R2

Description:

Filter Fraction: 1

Residual Ash Vol:
Aliquot Dilution: 0

Final Dilution: 0
Volume (L): 0

Lab Filter Area (mm2): 385

Grid Openings Analyzed: 35

Average Grid Opening Area: 0.009

Area Analyzed (mm2): 0.315

Analytical Sens. (struc/cc): 0

Dectection Limit. (struc/cc): 0

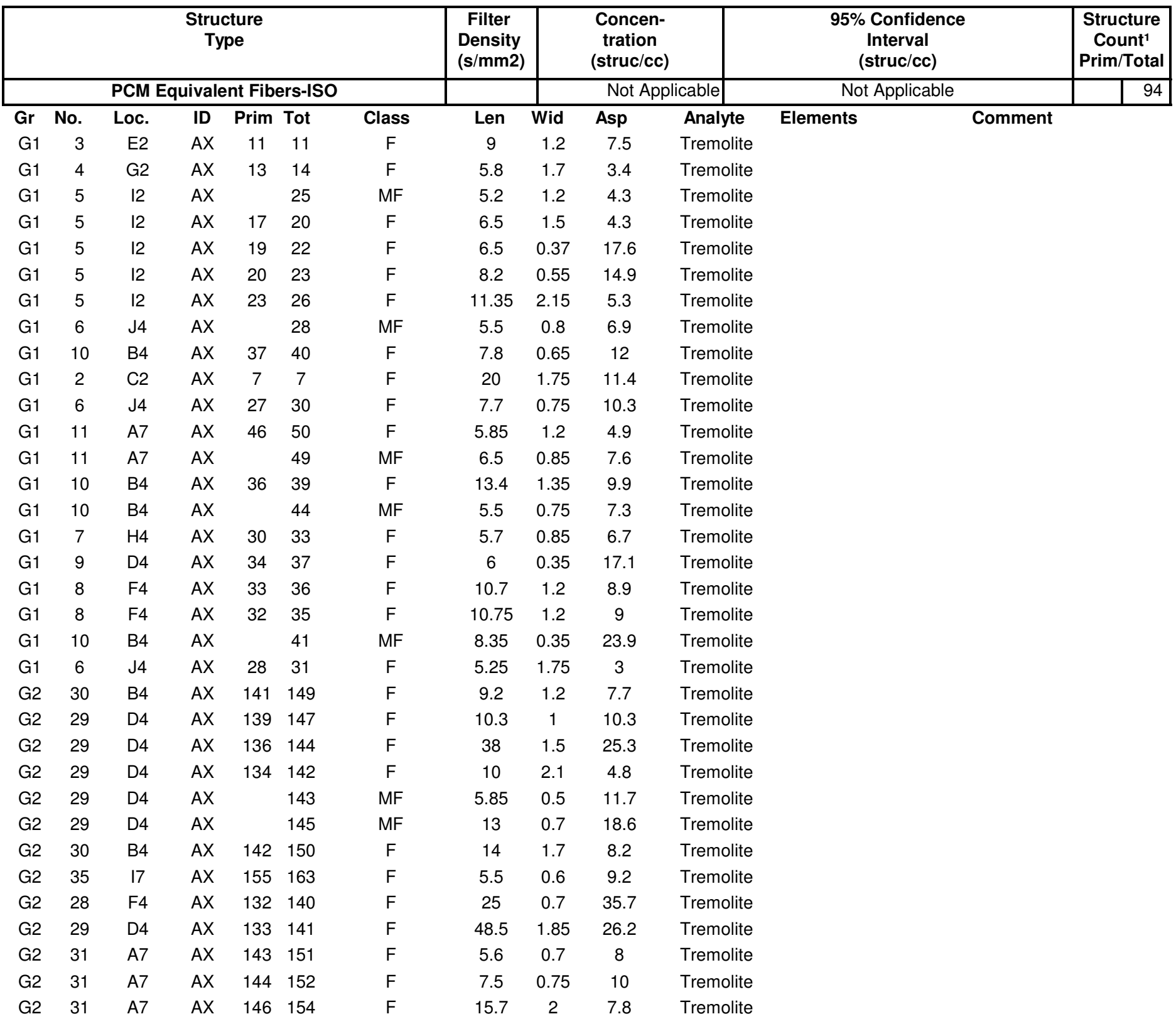


Job Number: 070434

SEA

ISO 10312, Direct Count Categories

Client: Idaho National Laboratory

Report Number: 070434R06

Date Received: 4/23/2007

Project Name: RARE

Lab/Cor Sample No.: S27

Client Sample No.: FB-2-R2

Description:

Filter Fraction: 1

Residual Ash Vol:
Aliquot Dilution: 0

Final Dilution: 0
Volume (L): 0

Lab Filter Area (mm2): 385

Grid Openings Analyzed: 35

Average Grid Opening Area: 0.009

Area Analyzed (mm2): 0.315

Analytical Sens. (struc/cc): 0

Dectection Limit. (struc/cc): 0

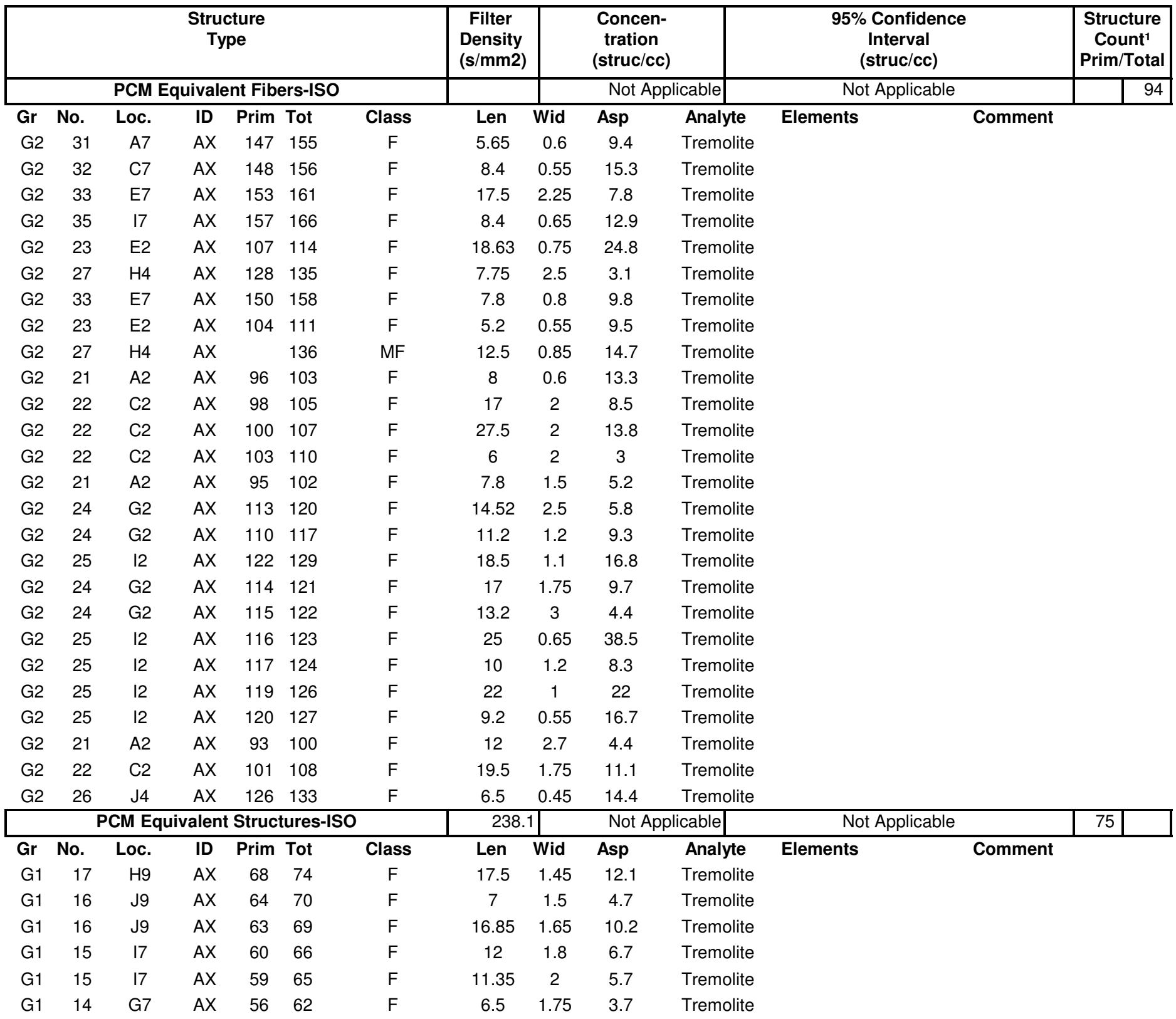


Job Number: 070434

SEA

ISO 10312, Direct Count Categories

Client: Idaho National Laboratory

Report Number: 070434R06

Date Received: 4/23/2007

Project Name: RARE

Lab/Cor Sample No.: S27

Client Sample No.: FB-2-R2

Description:

Filter Fraction: 1

Residual Ash Vol:
Aliquot Dilution: 0

Final Dilution: 0
Volume (L): 0

Lab Filter Area (mm2): 385

Grid Openings Analyzed: 35

Average Grid Opening Area: 0.009

Area Analyzed (mm2): 0.315

Analytical Sens. (struc/cc): 0

Dectection Limit. (struc/cc): 0

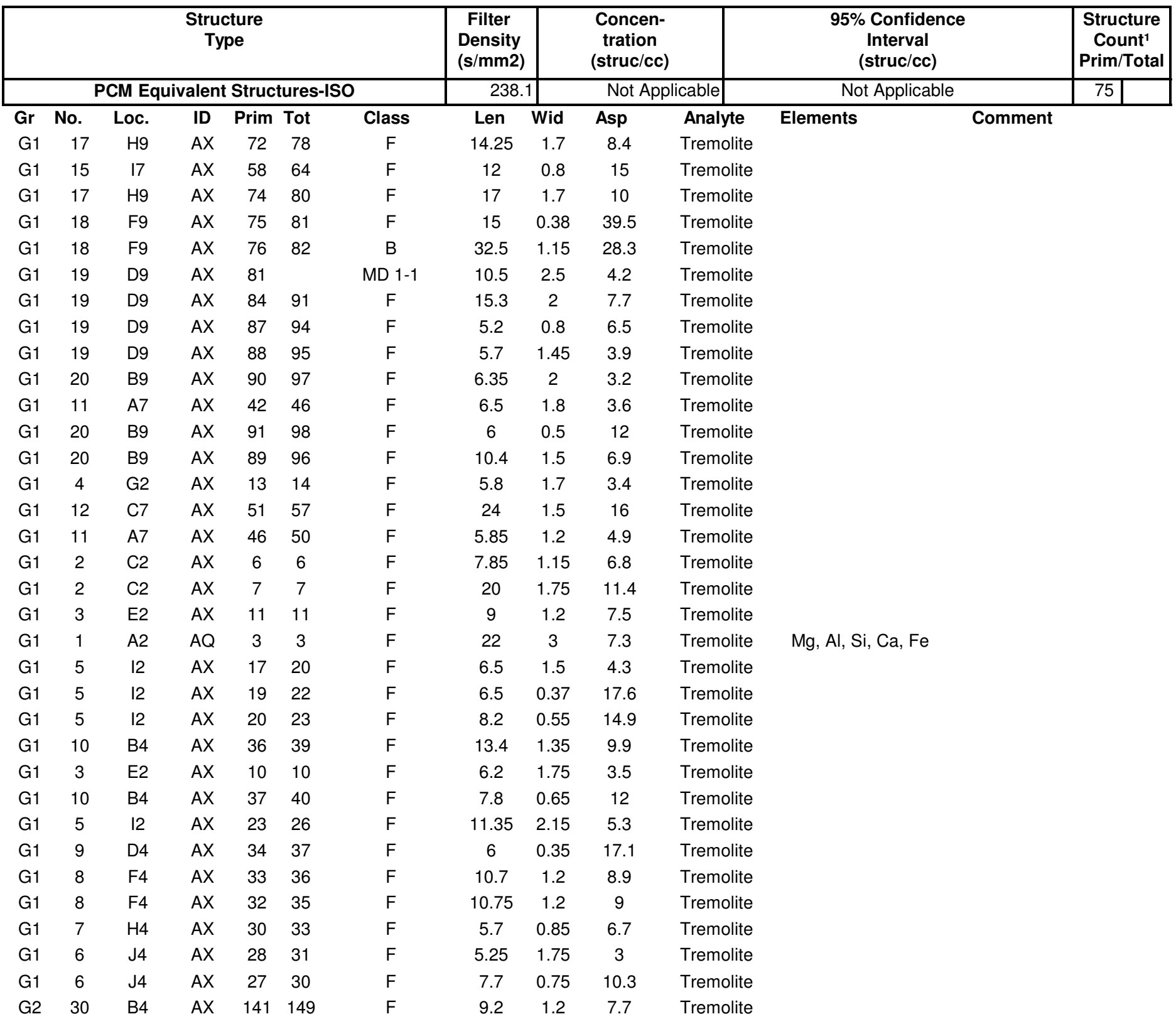


Job Number: 070434

SEA

ISO 10312, Direct Count Categories

Client: Idaho National Laboratory

Report Number: 070434R06

Date Received: 4/23/2007

Project Name: RARE

Lab/Cor Sample No.: S27

Client Sample No.: FB-2-R2

Description:

Filter Fraction: 1

Residual Ash Vol:
Aliquot Dilution: 0

Final Dilution: 0
Volume (L): 0

Lab Filter Area (mm2): 385

Grid Openings Analyzed: 35

Average Grid Opening Area: 0.009

Area Analyzed (mm2): 0.315

Analytical Sens. (struc/cc): 0

Dectection Limit. (struc/cc): 0

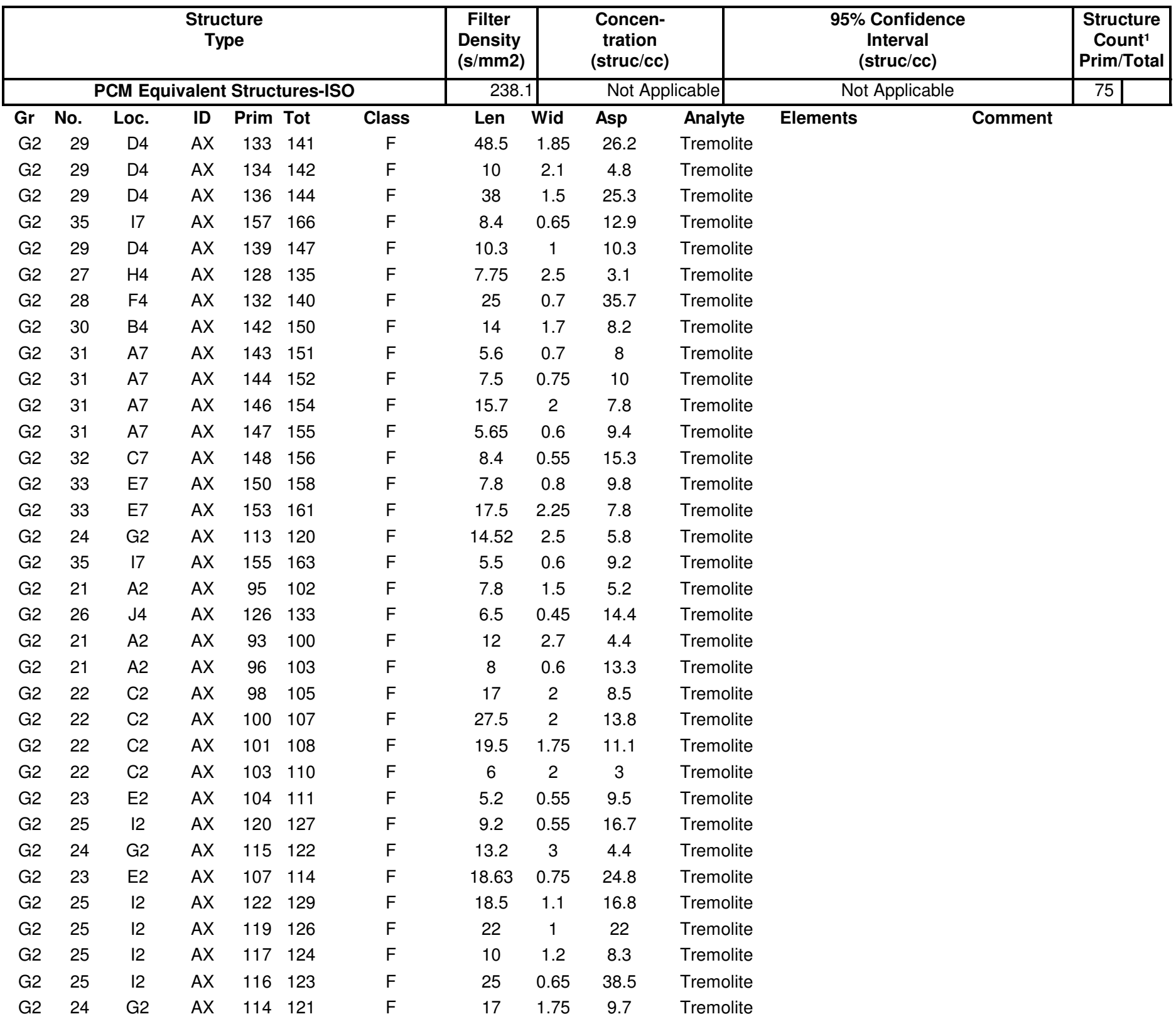


Job Number: 070434

SEA

ISO 10312, Direct Count Categories

Client: Idaho National Laboratory

Report Number: 070434R06

Date Received: 4/23/2007

Project Name: RARE

Lab/Cor Sample No.: S27

Client Sample No.: FB-2-R2

Description:

Filter Fraction: 1

Residual Ash Vol:
Aliquot Dilution: 0

Final Dilution: 0
Volume (L): 0

Lab Filter Area (mm2): 385

Grid Openings Analyzed: 35

Average Grid Opening Area: 0.009

Area Analyzed (mm2): 0.315

Analytical Sens. (struc/cc): 0

Dectection Limit. (struc/cc): 0

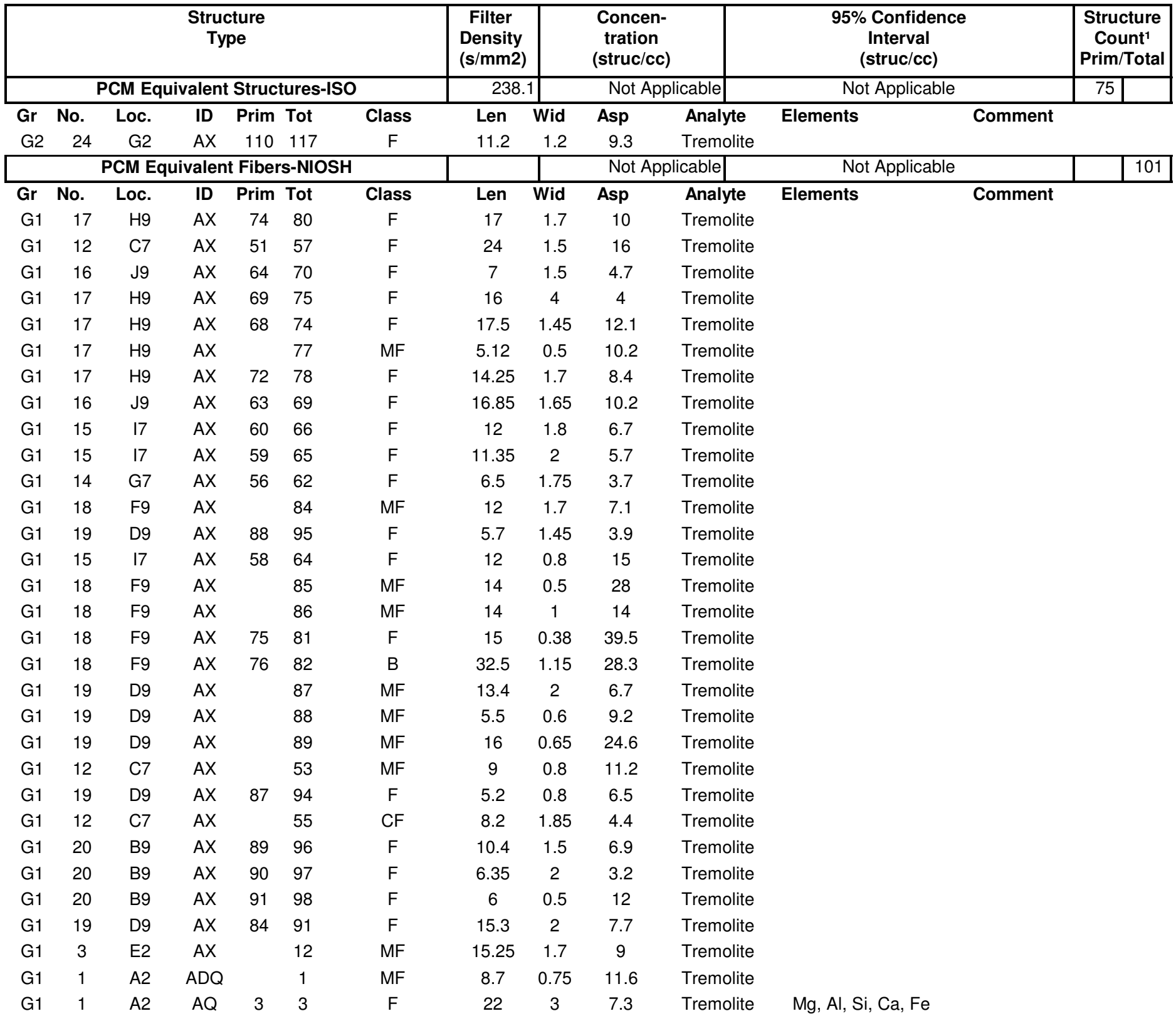


Job Number: 070434

SEA

ISO 10312, Direct Count Categories

Client: Idaho National Laboratory

Report Number: 070434R06

Date Received: 4/23/2007

Project Name: RARE

Lab/Cor Sample No.: S27

Client Sample No.: FB-2-R2

Description:

Filter Fraction: 1

Residual Ash Vol:
Aliquot Dilution: 0

Final Dilution: 0
Volume (L): 0

Lab Filter Area (mm2): 385

Grid Openings Analyzed: 35

Average Grid Opening Area: 0.009

Area Analyzed (mm2): 0.315

Analytical Sens. (struc/cc): 0

Dectection Limit. (struc/cc): 0

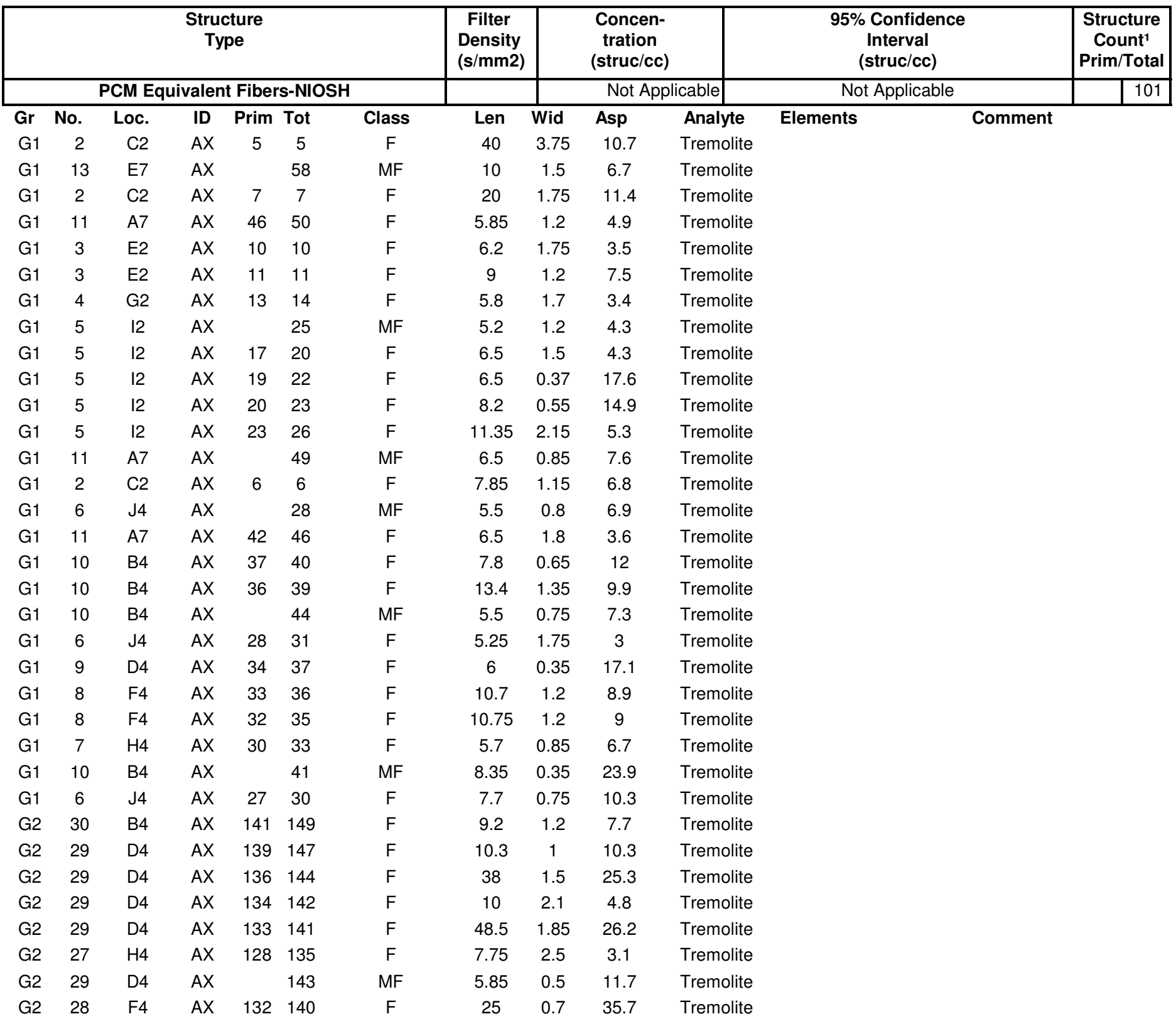


Job Number: 070434

SEA

ISO 10312, Direct Count Categories

Client: Idaho National Laboratory

Report Number: 070434R06

Date Received: 4/23/2007

Project Name: RARE

Lab/Cor Sample No.: S27

Client Sample No.: FB-2-R2

Description:

Filter Fraction: 1

Residual Ash Vol:
Aliquot Dilution: 0

Final Dilution: 0
Volume (L): 0

Lab Filter Area (mm2): 385

Grid Openings Analyzed: 35

Average Grid Opening Area: 0.009

Area Analyzed (mm2): 0.315

Analytical Sens. (struc/cc): 0

Dectection Limit. (struc/cc): 0

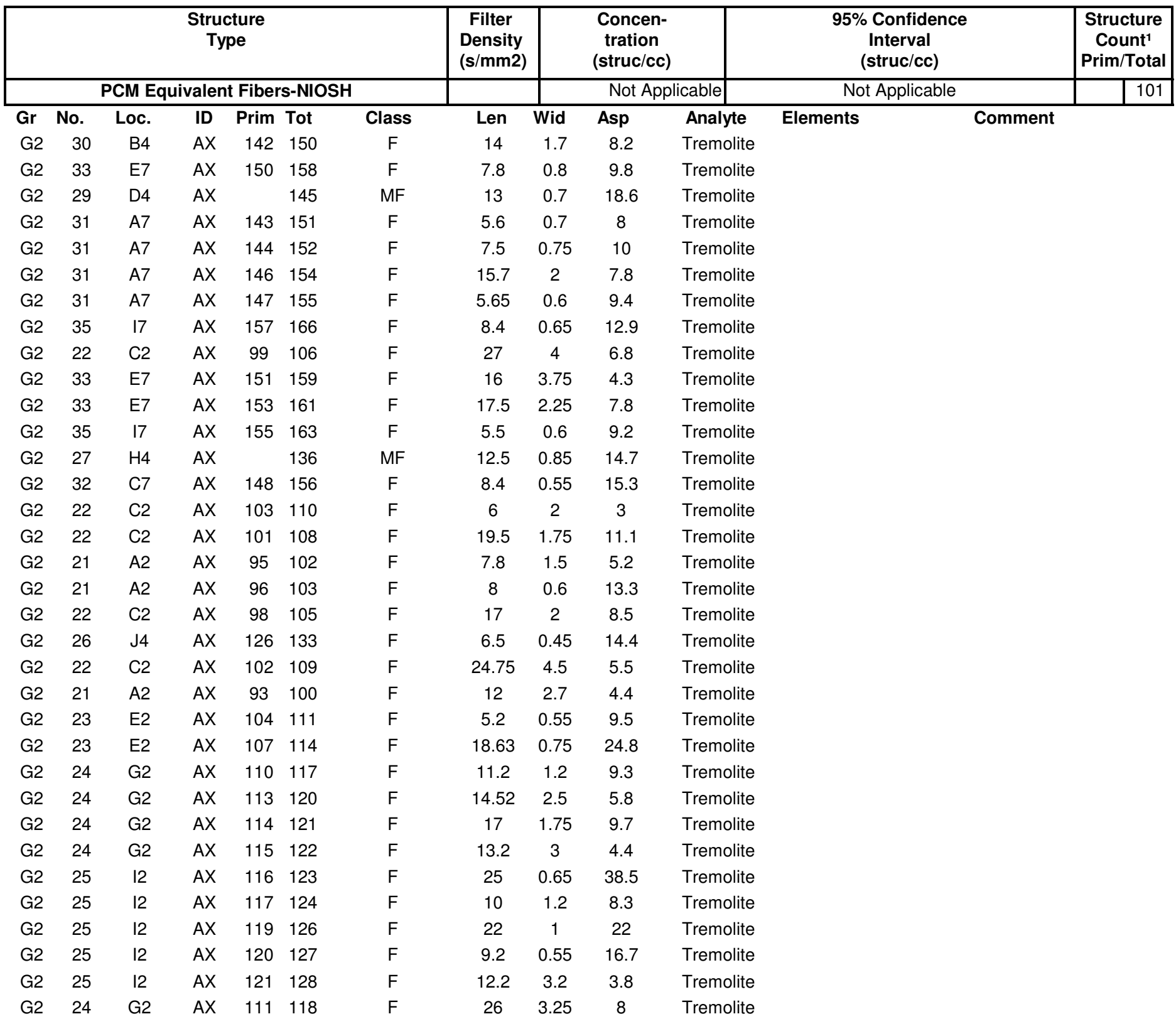


Job Number: 070434

SEA

ISO 10312, Direct Count Categories

Client: Idaho National Laboratory

Report Number: 070434R06

Date Received: 4/23/2007

Project Name: RARE

Lab/Cor Sample No.: S27

Client Sample No.: FB-2-R2

Description:

Filter Fraction: 1

Residual Ash Vol:
Aliquot Dilution: 0

Final Dilution: 0
Volume (L): 0

Lab Filter Area (mm2): 385

Grid Openings Analyzed: 35

Average Grid Opening Area: 0.009

Area Analyzed (mm2): 0.315

Analytical Sens. (struc/cc): 0

Dectection Limit. (struc/cc): 0

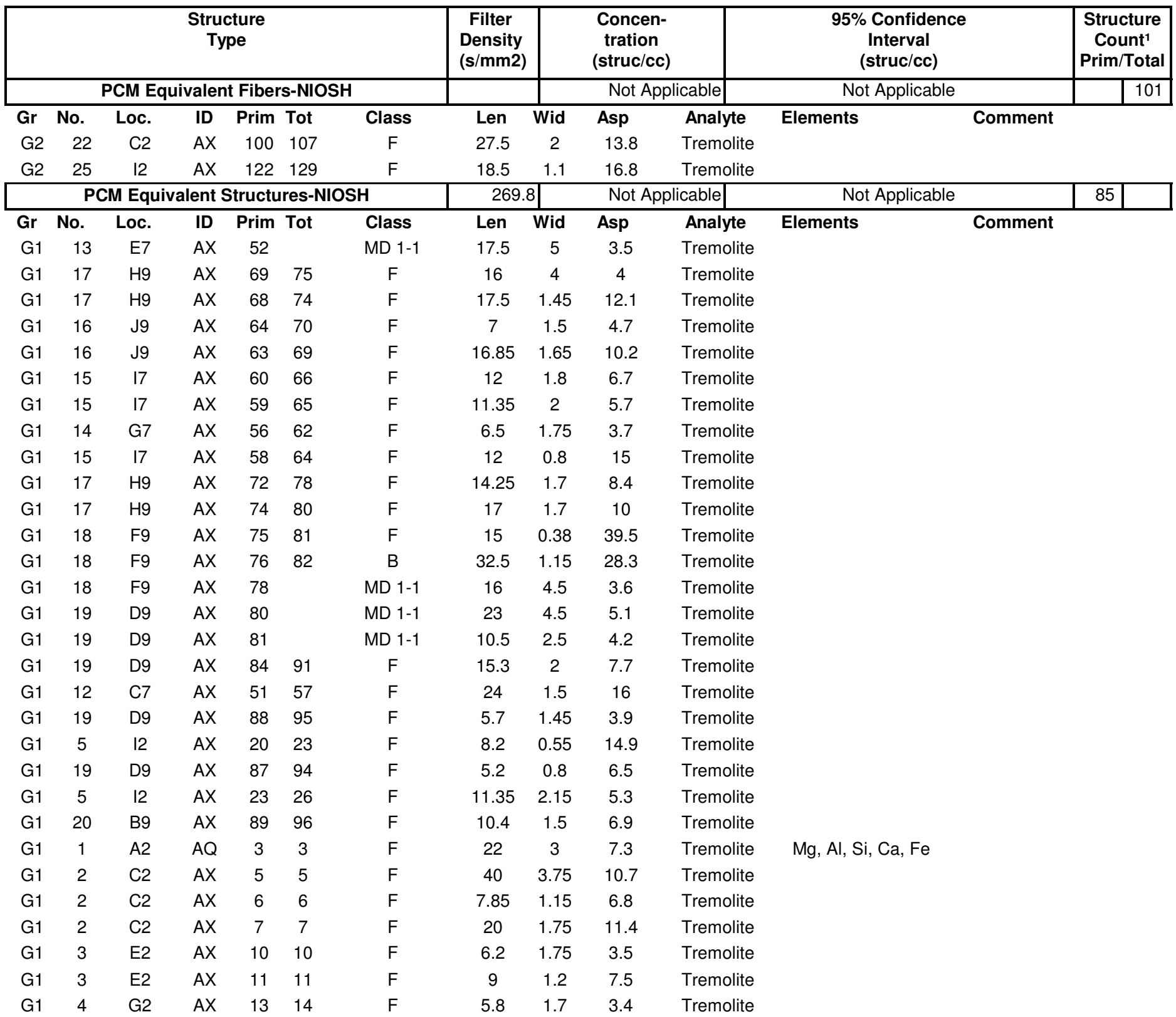


Job Number: 070434

SEA

ISO 10312, Direct Count Categories

Client: Idaho National Laboratory

Report Number: 070434R06

Date Received: 4/23/2007

Project Name: RARE

Lab/Cor Sample No.: S27

Client Sample No.: FB-2-R2

Description:

Filter Fraction: 1

Residual Ash Vol:
Aliquot Dilution: 0

Final Dilution: 0
Volume (L): 0

Lab Filter Area (mm2): 385

Grid Openings Analyzed: 35

Average Grid Opening Area: 0.009

Area Analyzed (mm2): 0.315

Analytical Sens. (struc/cc): 0

Dectection Limit. (struc/cc): 0

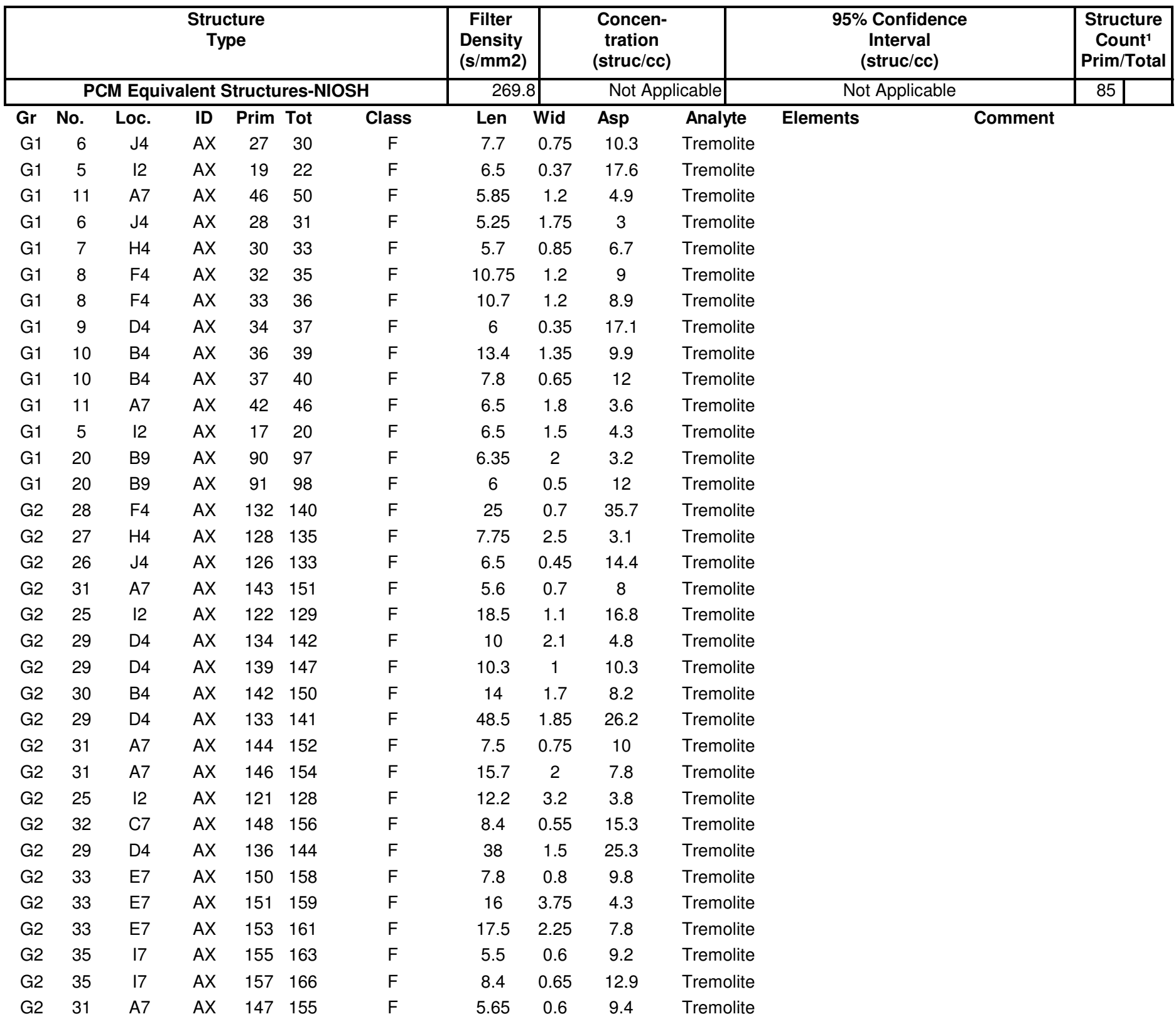


Job Number: 070434

SEA

ISO 10312, Direct Count Categories

Client: Idaho National Laboratory

Report Number: 070434R06

Date Received: 4/23/2007

Project Name: RARE

Lab/Cor Sample No.: S27

Client Sample No.: FB-2-R2

Description:

Filter Fraction: 1

Residual Ash Vol:
Aliquot Dilution: 0

Final Dilution: 0
Volume (L): 0

Lab Filter Area (mm2): 385

Grid Openings Analyzed: 35

Average Grid Opening Area: 0.009

Area Analyzed (mm2): 0.315

Analytical Sens. (struc/cc): 0

Dectection Limit. (struc/cc): 0

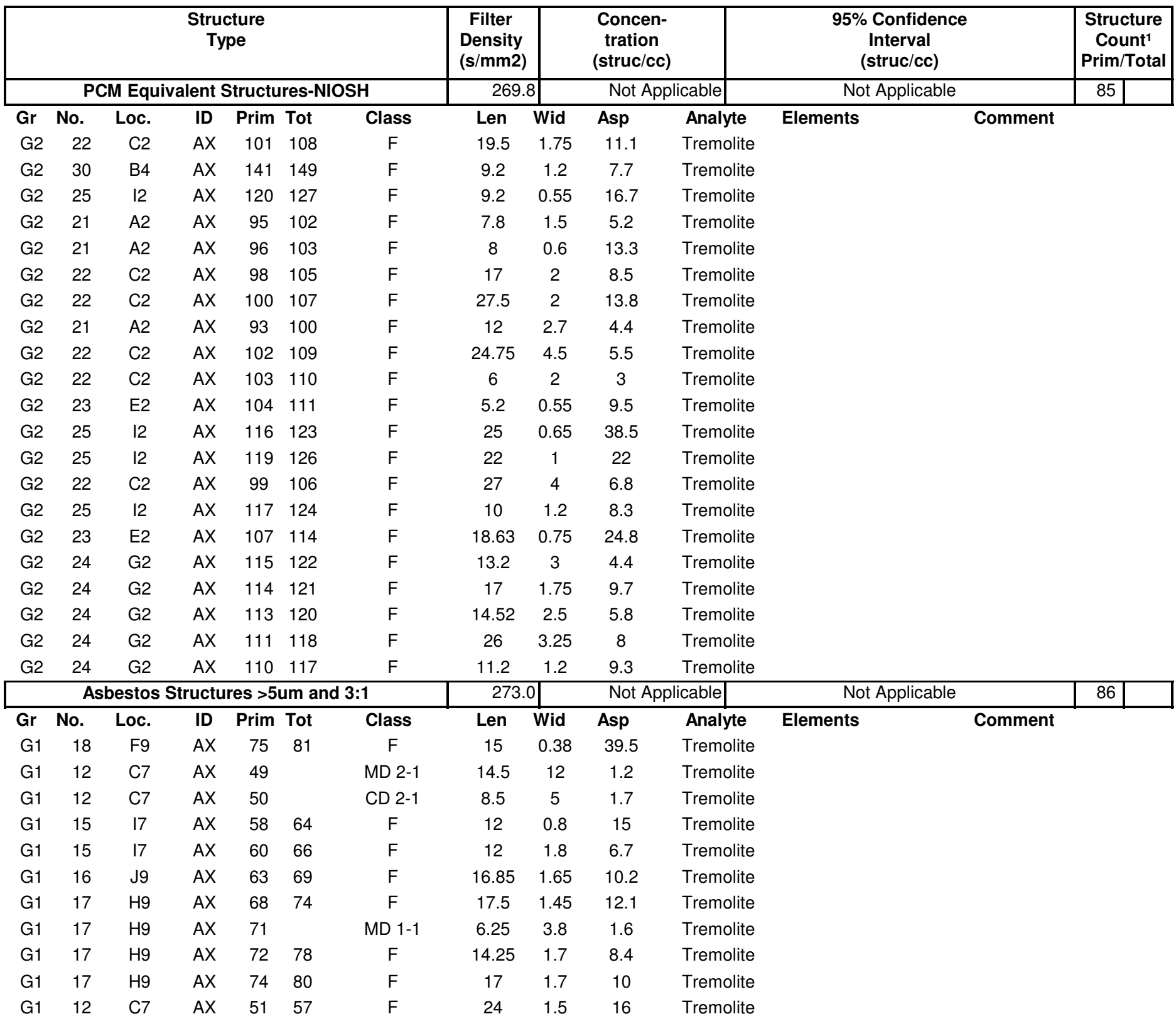


Job Number: 070434

SEA

ISO 10312, Direct Count Categories

Client: Idaho National Laboratory

Report Number: 070434R06

Date Received: 4/23/2007

Project Name: RARE

Lab/Cor Sample No.: S27

Client Sample No.: FB-2-R2

Description:

Filter Fraction: 1

Residual Ash Vol:
Aliquot Dilution: 0

Final Dilution: 0
Volume (L): 0

Lab Filter Area (mm2): 385

Grid Openings Analyzed: 35

Average Grid Opening Area: 0.009

Area Analyzed (mm2): 0.315

Analytical Sens. (struc/cc): 0

Dectection Limit. (struc/cc): 0

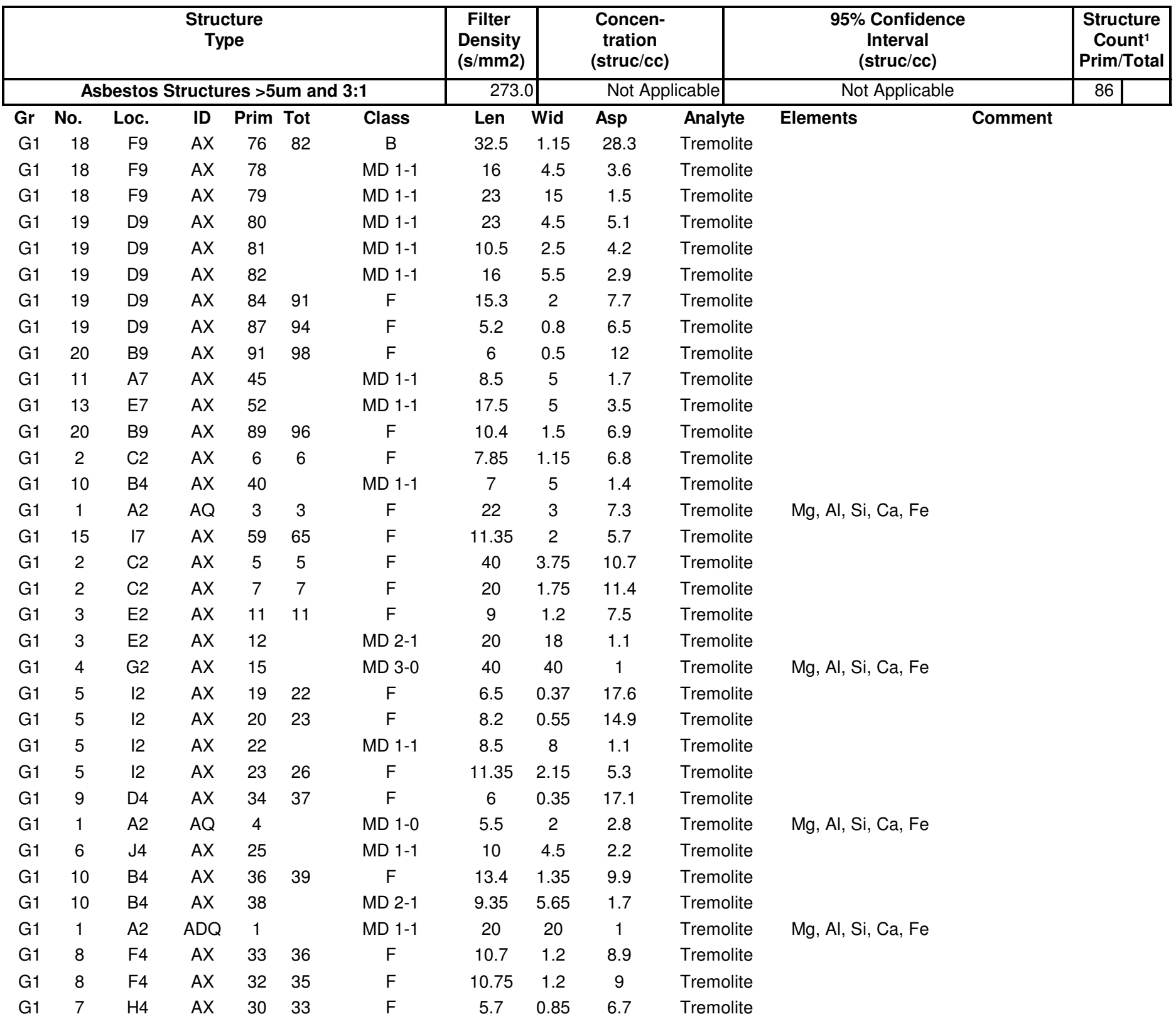


Job Number: 070434

SEA

ISO 10312, Direct Count Categories

Client: Idaho National Laboratory

Report Number: 070434R06

Date Received: 4/23/2007

Project Name: RARE

Lab/Cor Sample No.: S27

Client Sample No.: FB-2-R2

Description:

Filter Fraction: 1

Residual Ash Vol:
Aliquot Dilution: 0

Final Dilution: 0
Volume (L): 0

Lab Filter Area (mm2): 385

Grid Openings Analyzed: 35

Average Grid Opening Area: 0.009

Area Analyzed (mm2): 0.315

Analytical Sens. (struc/cc): 0

Dectection Limit. (struc/cc): 0

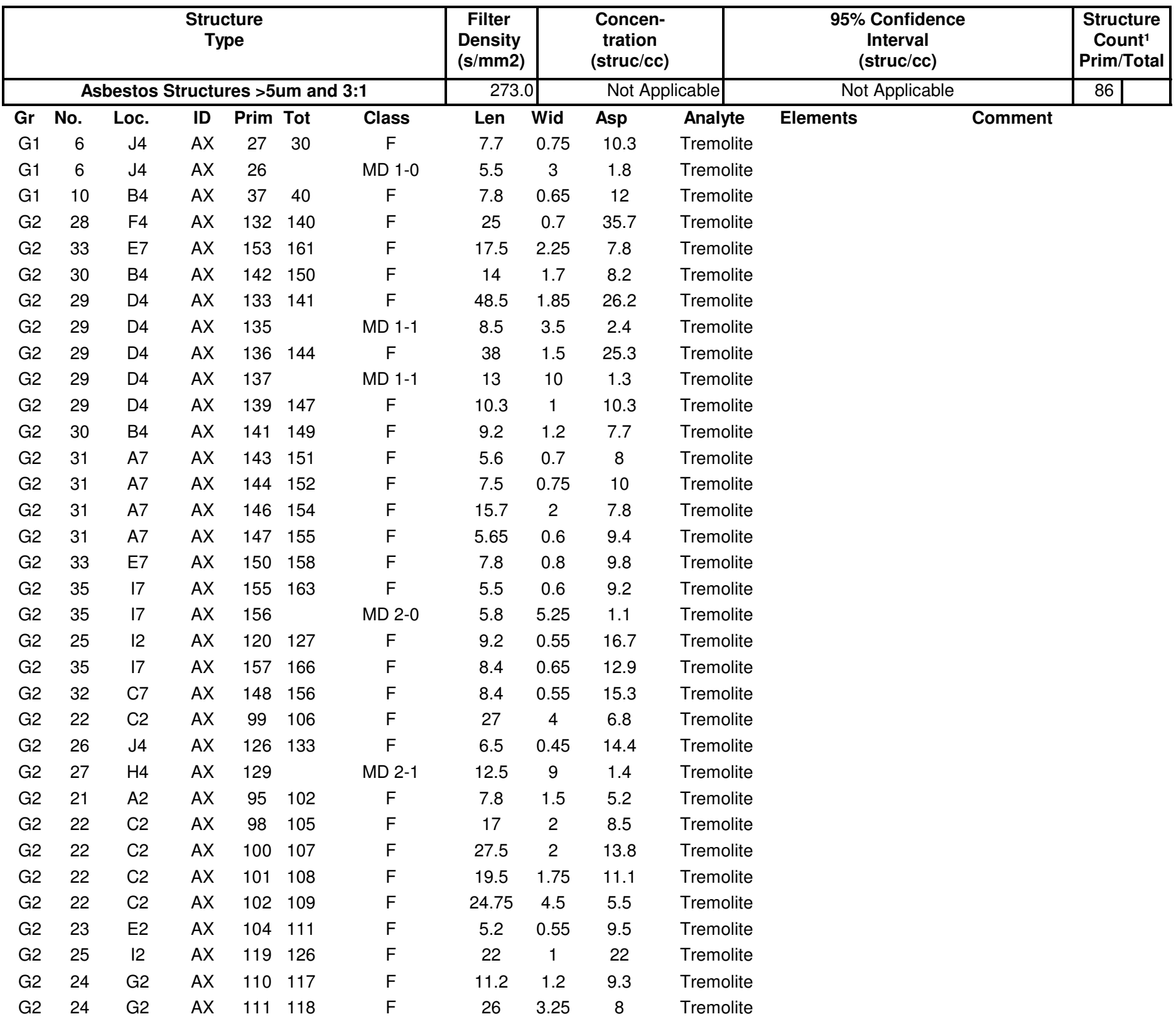


Job Number: 070434

SEA

ISO 10312, Direct Count Categories

Client: Idaho National Laboratory

Report Number: 070434R06

Date Received: 4/23/2007

Project Name: RARE

Lab/Cor Sample No.: S27

Client Sample No.: FB-2-R2

Description:

Filter Fraction: 1

Residual Ash Vol:
Aliquot Dilution: 0

Final Dilution: 0
Volume (L): 0

Lab Filter Area (mm2): 385

Grid Openings Analyzed: 35

Average Grid Opening Area: 0.009

Area Analyzed (mm2): 0.315

Analytical Sens. (struc/cc): 0

Dectection Limit. (struc/cc): 0

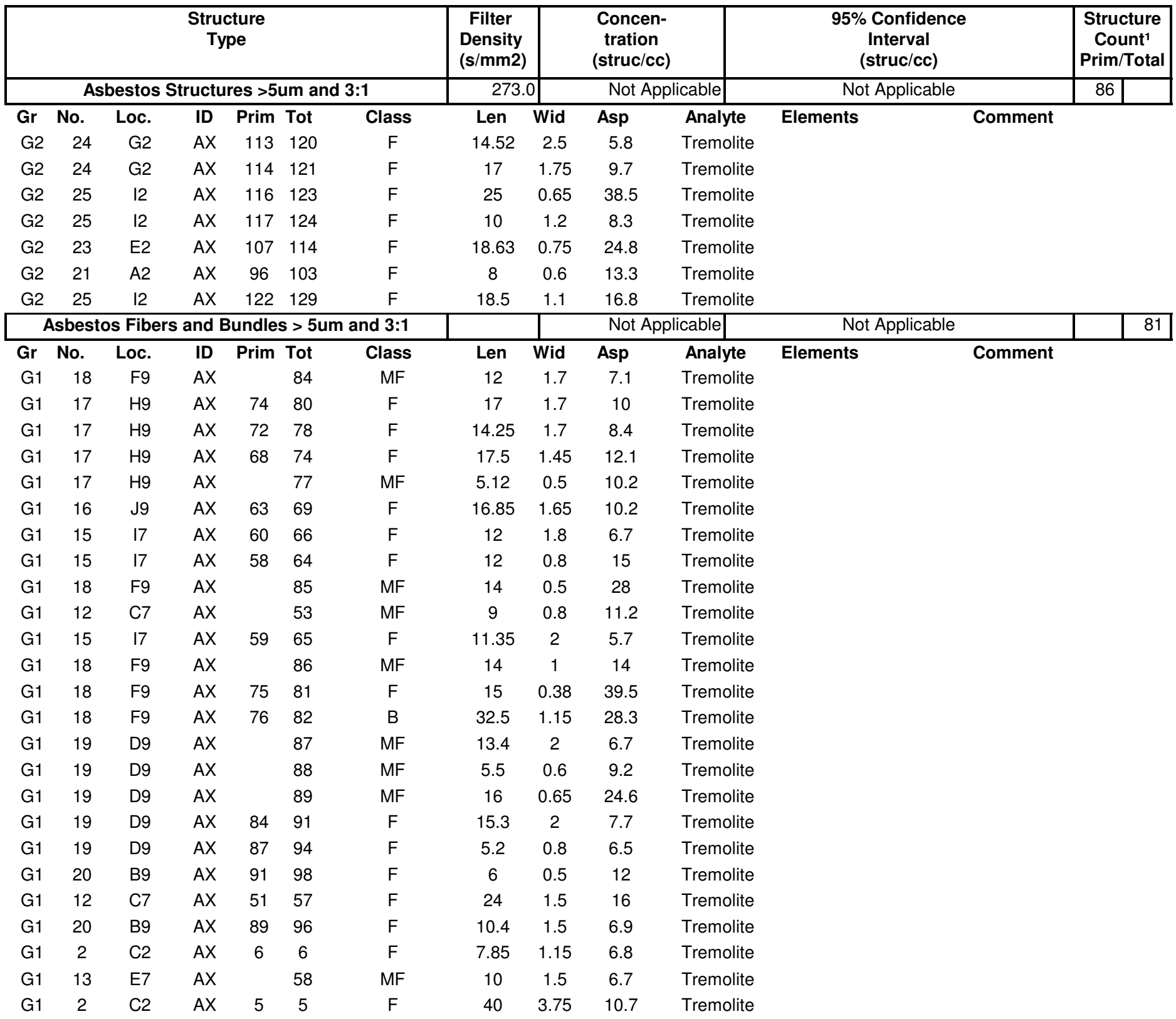


Job Number: 070434

SEA

ISO 10312, Direct Count Categories

Client: Idaho National Laboratory

Report Number: 070434R06

Date Received: 4/23/2007

Project Name: RARE

Lab/Cor Sample No.: S27

Client Sample No.: FB-2-R2

Description:

Filter Fraction: 1

Residual Ash Vol:
Aliquot Dilution: 0

Final Dilution: 0
Volume (L): 0

Lab Filter Area (mm2): 385

Grid Openings Analyzed: 35

Average Grid Opening Area: 0.009

Area Analyzed (mm2): 0.315

Analytical Sens. (struc/cc): 0

Dectection Limit. (struc/cc): 0

\begin{tabular}{|c|c|c|c|c|c|c|c|c|c|c|c|c|}
\hline & & & $\begin{array}{r}\text { Struc } \\
\text { Ty }\end{array}$ & $\begin{array}{l}\text { ture } \\
\text { oe }\end{array}$ & & & $\begin{array}{c}\text { Filter } \\
\text { Density } \\
(\mathrm{s} / \mathrm{mm} 2)\end{array}$ & & $\begin{array}{r}\text { Conce } \\
\text { tratio } \\
\text { (struc/ }\end{array}$ & & $\begin{array}{c}\text { 95\% Confidence } \\
\text { Interval } \\
\text { (struc/cc) }\end{array}$ & $\begin{array}{l}\text { Structure } \\
\text { Count }^{1} \\
\text { Prim/Total }\end{array}$ \\
\hline & Asbest & s Fibe & and $B$ & undles & $s>5 t$ & nd $3: 1$ & & & Not $A$ & licable & Not Applicable & 81 \\
\hline Gr & No. & Loc. & ID & Prim & Tot & Class & Len & Wid & Asp & Analyte & Elements & \\
\hline G1 & 2 & $\mathrm{C} 2$ & $A X$ & 7 & 7 & $\mathrm{~F}$ & 20 & 1.75 & 11.4 & Tremolite & & \\
\hline G1 & 3 & E2 & $A X$ & & 12 & MF & 15.25 & 1.7 & 9 & Tremolite & & \\
\hline G1 & 3 & E2 & $A X$ & 11 & 11 & $F$ & 9 & 1.2 & 7.5 & Tremolite & & \\
\hline G1 & 5 & 12 & $A X$ & 19 & 22 & $F$ & 6.5 & 0.37 & 17.6 & Tremolite & & \\
\hline G1 & 5 & 12 & $A X$ & 20 & 23 & $F$ & 8.2 & 0.55 & 14.9 & Tremolite & & \\
\hline G1 & 5 & 12 & $A X$ & 23 & 26 & $\mathrm{~F}$ & 11.35 & 2.15 & 5.3 & Tremolite & & \\
\hline G1 & 6 & $\mathrm{~J} 4$ & $A X$ & & 28 & MF & 5.5 & 0.8 & 6.9 & Tremolite & & \\
\hline G1 & 6 & $\mathrm{~J} 4$ & $A X$ & 27 & 30 & $F$ & 7.7 & 0.75 & 10.3 & Tremolite & & \\
\hline G1 & 11 & A7 & $A X$ & & 49 & MF & 6.5 & 0.85 & 7.6 & Tremolite & & \\
\hline G1 & 1 & $\mathrm{~A} 2$ & $A Q$ & 3 & 3 & $\mathrm{~F}$ & 22 & 3 & 7.3 & Tremolite & $\mathrm{Mg}, \mathrm{Al}, \mathrm{Si}, \mathrm{Ca}, \mathrm{Fe}$ & \\
\hline G1 & 7 & $\mathrm{H} 4$ & $A X$ & 30 & 33 & $F$ & 5.7 & 0.85 & 6.7 & Tremolite & & \\
\hline G1 & 1 & $\mathrm{~A} 2$ & $A D Q$ & & 1 & MF & 8.7 & 0.75 & 11.6 & Tremolite & & \\
\hline G1 & 10 & B4 & $A X$ & 37 & 40 & $F$ & 7.8 & 0.65 & 12 & Tremolite & & \\
\hline G1 & 10 & B4 & $A X$ & 36 & 39 & $\mathrm{~F}$ & 13.4 & 1.35 & 9.9 & Tremolite & & \\
\hline G1 & 10 & B4 & $A X$ & & 44 & MF & 5.5 & 0.75 & 7.3 & Tremolite & & \\
\hline G1 & 10 & B4 & $A X$ & & 41 & MF & 8.35 & 0.35 & 23.9 & Tremolite & & \\
\hline G1 & 9 & D4 & $A X$ & 34 & 37 & $\mathrm{~F}$ & 6 & 0.35 & 17.1 & Tremolite & & \\
\hline G1 & 8 & $\mathrm{~F} 4$ & $A X$ & 33 & 36 & $F$ & 10.7 & 1.2 & 8.9 & Tremolite & & \\
\hline G1 & 8 & $\mathrm{~F} 4$ & $A X$ & 32 & 35 & $F$ & 10.75 & 1.2 & 9 & Tremolite & & \\
\hline G2 & 28 & $\mathrm{~F} 4$ & $A X$ & 132 & 140 & $F$ & 25 & 0.7 & 35.7 & Tremolite & & \\
\hline G2 & 33 & E7 & $A X$ & 153 & 161 & $\mathrm{~F}$ & 17.5 & 2.25 & 7.8 & Tremolite & & \\
\hline G2 & 30 & B4 & $A X$ & 142 & 150 & $F$ & 14 & 1.7 & 8.2 & Tremolite & & \\
\hline G2 & 29 & D4 & $A X$ & & 145 & MF & 13 & 0.7 & 18.6 & Tremolite & & \\
\hline G2 & 29 & D4 & $A X$ & 133 & 141 & $F$ & 48.5 & 1.85 & 26.2 & Tremolite & & \\
\hline G2 & 29 & D4 & $A X$ & 136 & 144 & $F$ & 38 & 1.5 & 25.3 & Tremolite & & \\
\hline G2 & 29 & D4 & $A X$ & 139 & 147 & $F$ & 10.3 & 1 & 10.3 & Tremolite & & \\
\hline G2 & 30 & B4 & $A X$ & 141 & 149 & $F$ & 9.2 & 1.2 & 7.7 & Tremolite & & \\
\hline G2 & 29 & D4 & $A X$ & & 143 & MF & 5.85 & 0.5 & 11.7 & Tremolite & & \\
\hline G2 & 31 & A7 & $A X$ & 143 & 151 & $F$ & 5.6 & 0.7 & 8 & Tremolite & & \\
\hline G2 & 31 & A7 & $A X$ & 144 & 152 & $\mathrm{~F}$ & 7.5 & 0.75 & 10 & Tremolite & & \\
\hline G2 & 31 & A7 & $A X$ & 146 & 154 & $F$ & 15.7 & 2 & 7.8 & Tremolite & & \\
\hline G2 & 31 & A7 & $A X$ & 147 & 155 & $F$ & 5.65 & 0.6 & 9.4 & Tremolite & & \\
\hline G2 & 33 & E7 & $A X$ & 150 & 158 & $F$ & 7.8 & 0.8 & 9.8 & Tremolite & & \\
\hline G2 & 35 & 17 & $A X$ & 155 & 163 & $F$ & 5.5 & 0.6 & 9.2 & Tremolite & & \\
\hline
\end{tabular}


Job Number: 070434

SEA

Client: Idaho National Laboratory

Project Name: RARE

Lab/Cor Sample No.: S27

Client Sample No.: FB-2-R2

Description:

Filter Fraction: 1

Residual Ash Vol:
Report Number: 070434R06

Date Received: 4/23/2007

ISO 10312, Direct Count Categories
Aliquot Dilution: 0

Final Dilution: 0
Volume (L): 0

Lab Filter Area (mm2): 385

Grid Openings Analyzed: 35

Average Grid Opening Area: 0.009

Area Analyzed (mm2): 0.315

Analytical Sens. (struc/cc): 0

Dectection Limit. (struc/cc): 0

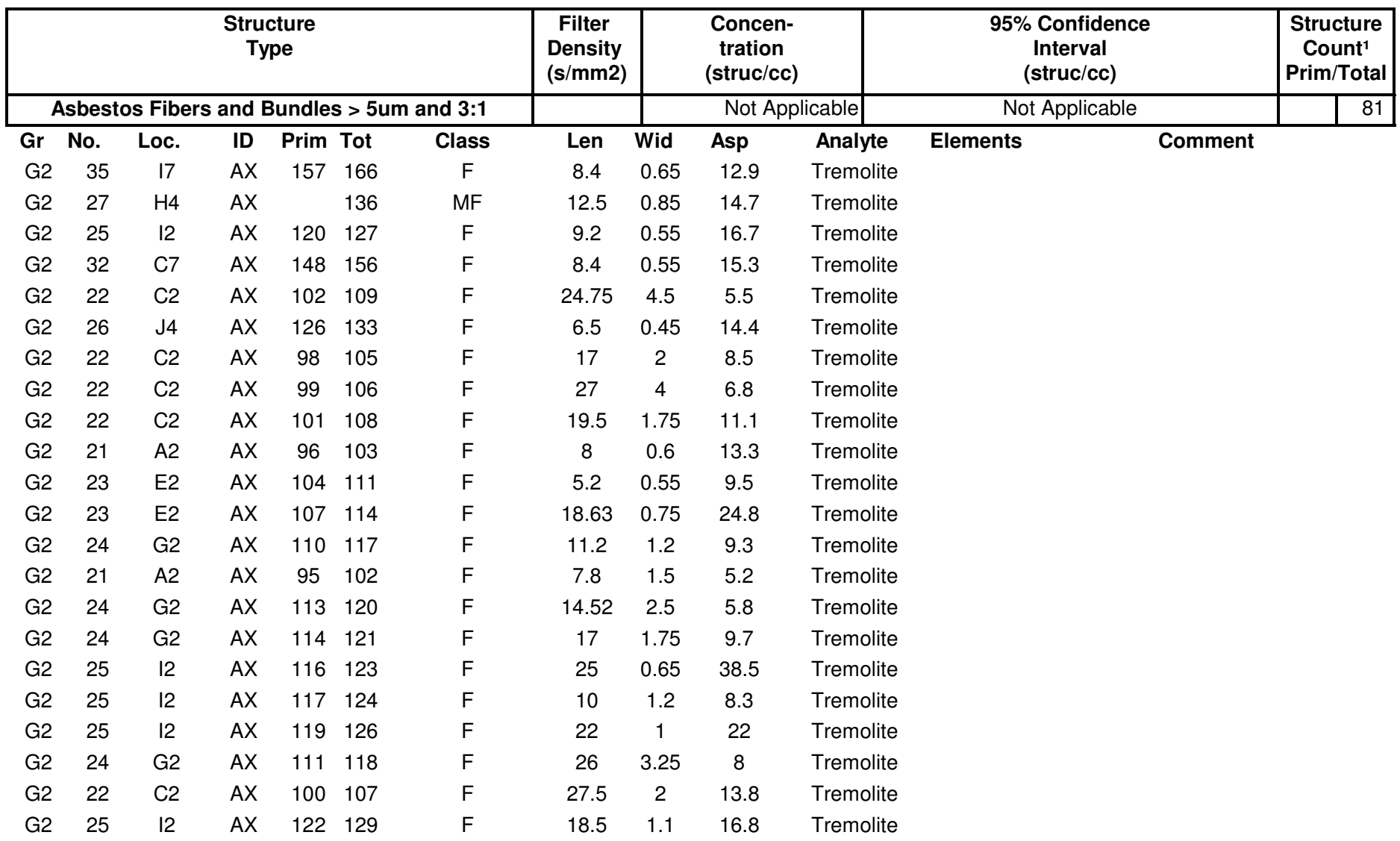


Job Number: 070434

SEA

ISO 10312, Direct Count Categories

Client: Idaho National Laboratory

Report Number: 070434R06

Date Received: 4/23/2007

Project Name: RARE

Lab/Cor Sample No.: S28

Client Sample No.: FB-2-R3

Description:

Filter Fraction: 1

Residual Ash Vol:
Aliquot Dilution: 0

Final Dilution: 0
Volume (L): 0

Lab Filter Area (mm2): 385

Grid Openings Analyzed: 35

Average Grid Opening Area: 0.009

Area Analyzed (mm2): 0.315

Analytical Sens. (struc/cc): 0

Dectection Limit. (struc/cc): 0

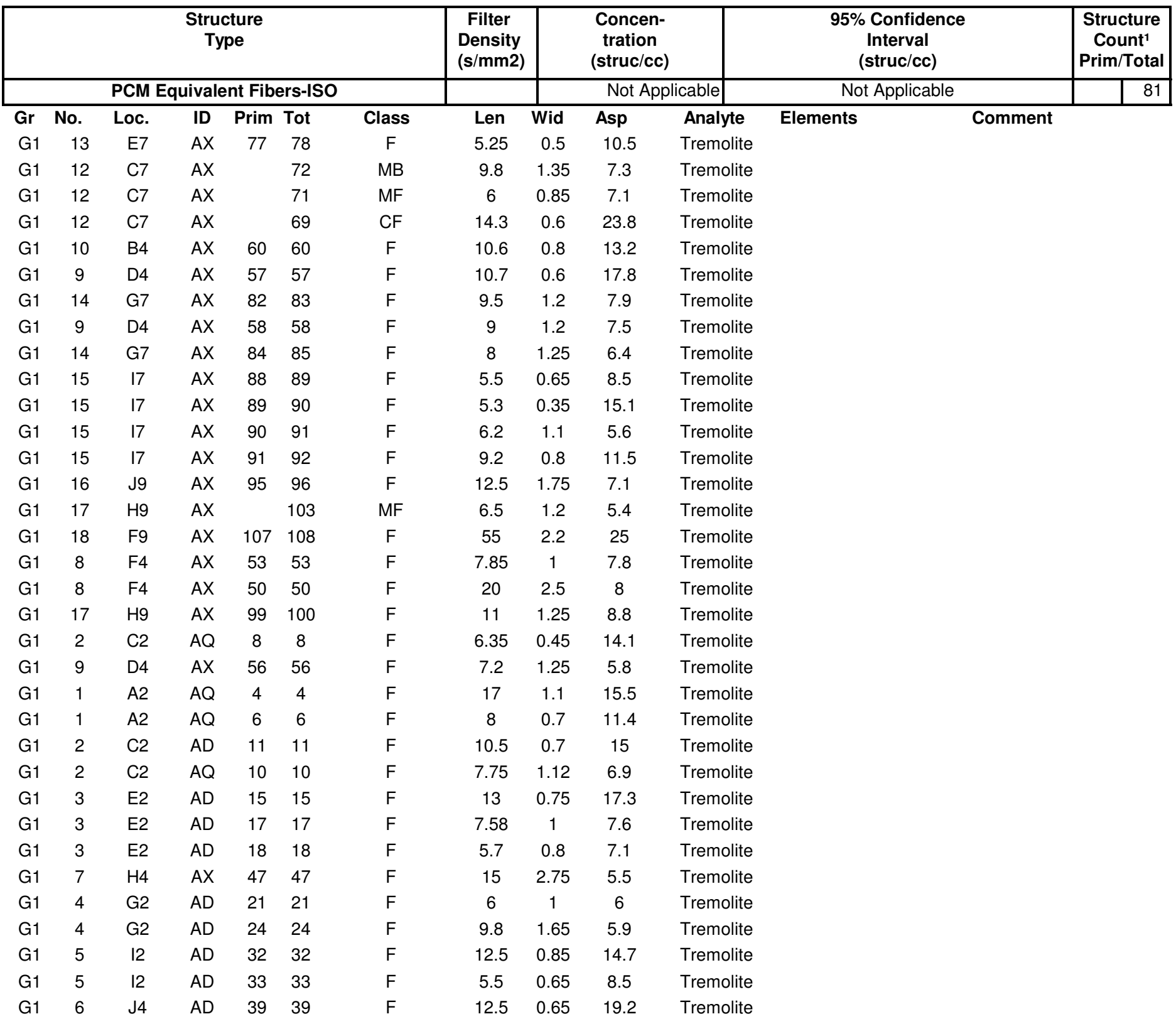


Job Number: 070434

SEA

ISO 10312, Direct Count Categories

Client: Idaho National Laboratory

Report Number: 070434R06

Date Received: 4/23/2007

Project Name: RARE

Lab/Cor Sample No.: S28

Client Sample No.: FB-2-R3

Description:

Filter Fraction: 1

Residual Ash Vol:
Aliquot Dilution: 0

Final Dilution: 0
Volume (L): 0

Lab Filter Area (mm2): 385

Grid Openings Analyzed: 35

Average Grid Opening Area: 0.009

Area Analyzed (mm2): 0.315

Analytical Sens. (struc/cc): 0

Dectection Limit. (struc/cc): 0

\begin{tabular}{|c|c|c|c|c|c|c|c|c|c|c|c|c|}
\hline & & & $\begin{array}{r}\text { Struc } \\
\text { Ty }\end{array}$ & $\begin{array}{l}\text { ture } \\
\text { pe }\end{array}$ & & & $\begin{array}{c}\text { Filter } \\
\text { Density } \\
(\mathrm{s} / \mathrm{mm} 2)\end{array}$ & & $\begin{array}{r}\text { Conce } \\
\text { tratio } \\
\text { (struc/ }\end{array}$ & & $\begin{array}{c}\text { 95\% Confidence } \\
\text { Interval } \\
\text { (struc/cc) }\end{array}$ & $\begin{array}{c}\text { Structure } \\
\text { Count }^{1} \\
\text { Prim/Total }\end{array}$ \\
\hline & & PCM & quivale & nt Fib & ers-IS & & & & Not & licable & Not Applicable & 81 \\
\hline Gr & No. & Loc. & ID & Prim & Tot & Class & Len & Wid & Asp & Analyte & Comment & \\
\hline G1 & 7 & $\mathrm{H} 4$ & $A D$ & 43 & 43 & $\mathrm{~F}$ & 9.5 & 0.65 & 14.6 & Tremolite & & \\
\hline G1 & 3 & E2 & $A D$ & 20 & 20 & $\mathrm{~F}$ & 7 & 0.45 & 15.6 & Tremolite & & \\
\hline G1 & 1 & $\mathrm{~A} 2$ & $A Z Q$ & 3 & 3 & $\mathrm{~F}$ & 7 & 0.65 & 10.8 & Tremolite & $\mathrm{Mg}, \mathrm{Al}, \mathrm{Si}, \mathrm{Ca}, \mathrm{Fe}$ & \\
\hline G1 & 7 & $\mathrm{H} 4$ & $A X$ & 49 & 49 & $\mathrm{~F}$ & 5.7 & 1.2 & 4.7 & Tremolite & & \\
\hline G2 & 31 & A7 & $A X$ & 186 & 187 & $\mathrm{~F}$ & 19 & 1.5 & 12.7 & Tremolite & & \\
\hline G2 & 31 & A7 & $A X$ & 183 & 184 & $\mathrm{~F}$ & 7.15 & 1.1 & 6.5 & Tremolite & & \\
\hline G2 & 30 & B4 & $A X$ & 180 & 181 & $\mathrm{~F}$ & 10.65 & 1.5 & 7.1 & Tremolite & & \\
\hline G2 & 29 & D4 & $A X$ & 177 & 178 & $\mathrm{~F}$ & 8 & 1.2 & 6.7 & Tremolite & & \\
\hline G2 & 29 & D4 & $A X$ & 175 & 176 & $\mathrm{~F}$ & 12.2 & 1.2 & 10.2 & Tremolite & & \\
\hline G2 & 29 & D4 & $A X$ & 174 & 175 & $\mathrm{~F}$ & 9.3 & 2.35 & 4 & Tremolite & & \\
\hline G2 & 28 & $\mathrm{~F} 4$ & $A X$ & 172 & 173 & $F$ & 7 & 1.5 & 4.7 & Tremolite & & \\
\hline G2 & 28 & $\mathrm{~F} 4$ & $A X$ & 169 & 170 & $\mathrm{~F}$ & 20 & 0.85 & 23.5 & Tremolite & & \\
\hline G2 & 31 & A7 & $A X$ & 187 & 188 & $\mathrm{~F}$ & 10 & 1.8 & 5.6 & Tremolite & & \\
\hline G2 & 35 & 17 & $A X$ & 205 & 206 & $\mathrm{~F}$ & 11.2 & 0.4 & 28 & Tremolite & & \\
\hline G2 & 28 & $\mathrm{~F} 4$ & $A X$ & 170 & 171 & $\mathrm{~F}$ & 5.5 & 0.8 & 6.9 & Tremolite & & \\
\hline G2 & 31 & A7 & $A X$ & 188 & 189 & $\mathrm{~F}$ & 10.1 & 1.25 & 8.1 & Tremolite & & \\
\hline G2 & 31 & A7 & $A X$ & 189 & 190 & $\mathrm{~F}$ & 30 & 3 & 10 & Tremolite & & \\
\hline G2 & 33 & E7 & $A X$ & & 198 & MF & 5.1 & 0.27 & 18.9 & Tremolite & & \\
\hline G2 & 33 & E7 & $A X$ & 194 & 195 & $\mathrm{~F}$ & 9.2 & 0.6 & 15.3 & Tremolite & & \\
\hline G2 & 33 & E7 & $A X$ & 195 & 196 & $F$ & 14 & 1.2 & 11.7 & Tremolite & & \\
\hline G2 & 33 & E7 & $A X$ & 198 & 199 & $\mathrm{~F}$ & 7 & 0.8 & 8.8 & Tremolite & & \\
\hline G2 & 34 & G7 & $A X$ & 199 & 200 & $\mathrm{~F}$ & 7.8 & 1.8 & 4.3 & Tremolite & & \\
\hline G2 & 34 & G7 & $A X$ & 200 & 201 & $\mathrm{~F}$ & 7.8 & 0.38 & 20.5 & Tremolite & & \\
\hline G2 & 35 & 17 & $A X$ & & 210 & MF & 9 & 0.6 & 15 & Tremolite & & \\
\hline G2 & 26 & $\mathrm{~J} 4$ & $A X$ & 160 & 161 & $\mathrm{~F}$ & 6.2 & 1.12 & 5.5 & Tremolite & & \\
\hline G2 & 34 & G7 & $A X$ & 202 & 203 & $\mathrm{~F}$ & 5.12 & 1.7 & 3 & Tremolite & & \\
\hline G2 & 22 & $\mathrm{C} 2$ & $A X$ & 125 & 126 & $\mathrm{~F}$ & 5.4 & 0.6 & 9 & Tremolite & & \\
\hline G2 & 27 & $\mathrm{H} 4$ & $A X$ & 167 & 168 & $\mathrm{~F}$ & 7.5 & 0.55 & 13.6 & Tremolite & & \\
\hline G2 & 22 & $\mathrm{C} 2$ & $A X$ & 124 & 125 & $\mathrm{~F}$ & 9 & 1 & 9 & Tremolite & & \\
\hline G2 & 27 & $\mathrm{H} 4$ & $A X$ & 163 & 164 & $\mathrm{~F}$ & 8 & 1.2 & 6.7 & Tremolite & & \\
\hline G2 & 22 & $\mathrm{C} 2$ & $A X$ & 128 & 129 & $\mathrm{~F}$ & 7.7 & 0.45 & 17.1 & Tremolite & & \\
\hline G2 & 22 & $\mathrm{C} 2$ & $A X$ & 129 & 130 & $\mathrm{~F}$ & 15 & 2.5 & 6 & Tremolite & & \\
\hline G2 & 22 & $\mathrm{C} 2$ & $A X$ & 131 & 132 & $\mathrm{~F}$ & 5.12 & 0.45 & 11.4 & Tremolite & & \\
\hline G2 & 22 & $\mathrm{C} 2$ & $A X$ & 133 & 134 & $F$ & 12 & 2.5 & 4.8 & Tremolite & & \\
\hline
\end{tabular}


Job Number: 070434

SEA

ISO 10312, Direct Count Categories

Client: Idaho National Laboratory

Report Number: 070434R06

Date Received: 4/23/2007

Project Name: RARE

Lab/Cor Sample No.: S28

Client Sample No.: FB-2-R3

Description:

Filter Fraction: 1

Residual Ash Vol:
Aliquot Dilution: 0

Final Dilution: 0
Volume (L): 0

Lab Filter Area (mm2): 385

Grid Openings Analyzed: 35

Average Grid Opening Area: 0.009

Area Analyzed (mm2): 0.315

Analytical Sens. (struc/cc): 0

Dectection Limit. (struc/cc): 0

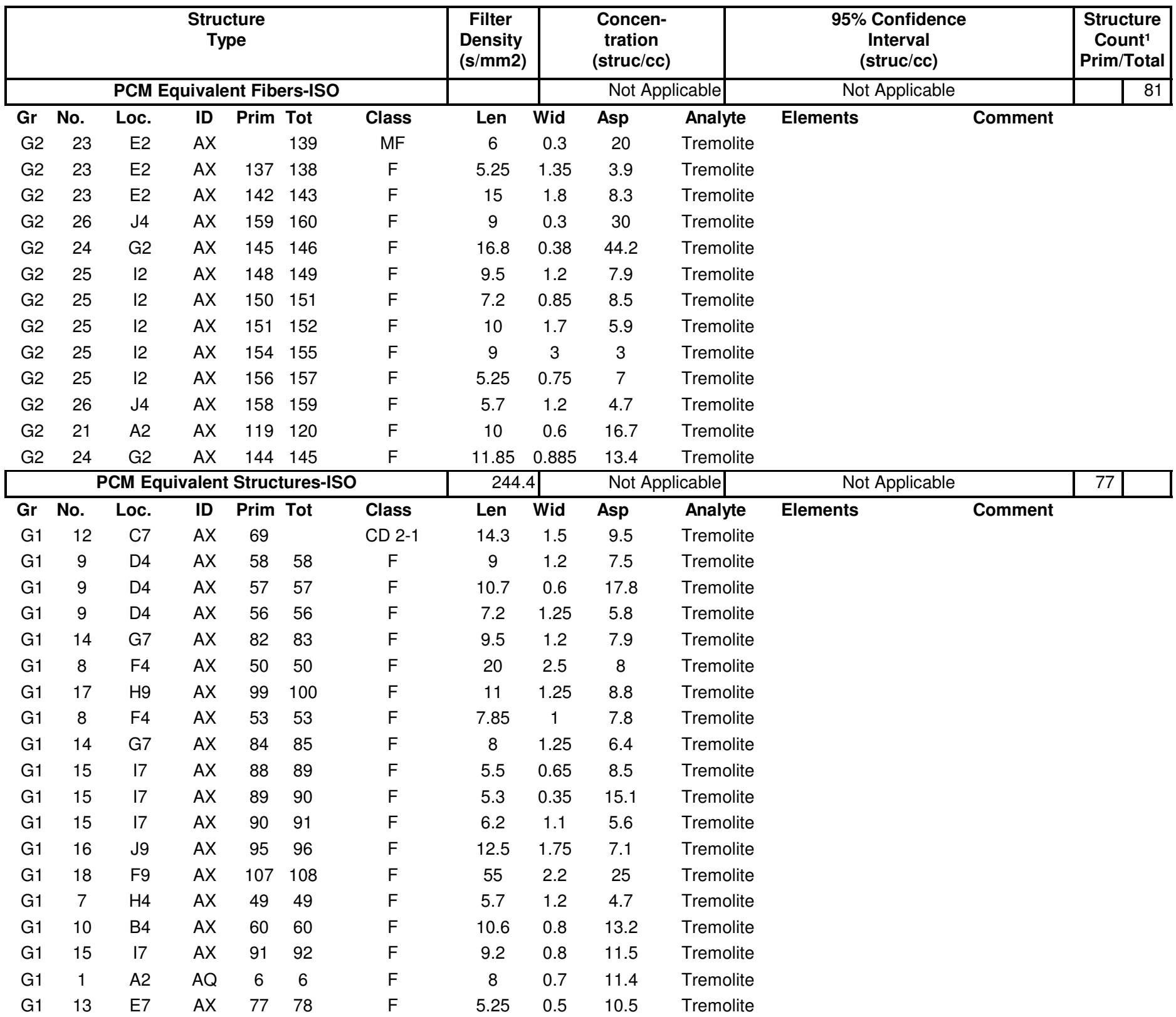


Job Number: 070434

SEA

ISO 10312, Direct Count Categories

Client: Idaho National Laboratory

Report Number: 070434R06

Date Received: 4/23/2007

Project Name: RARE

Lab/Cor Sample No.: S28

Client Sample No.: FB-2-R3

Description:

Filter Fraction: 1

Residual Ash Vol:
Aliquot Dilution: 0

Final Dilution: 0
Volume (L): 0

Lab Filter Area (mm2): 385

Grid Openings Analyzed: 35

Average Grid Opening Area: 0.009

Area Analyzed (mm2): 0.315

Analytical Sens. (struc/cc): 0

Dectection Limit. (struc/cc): 0

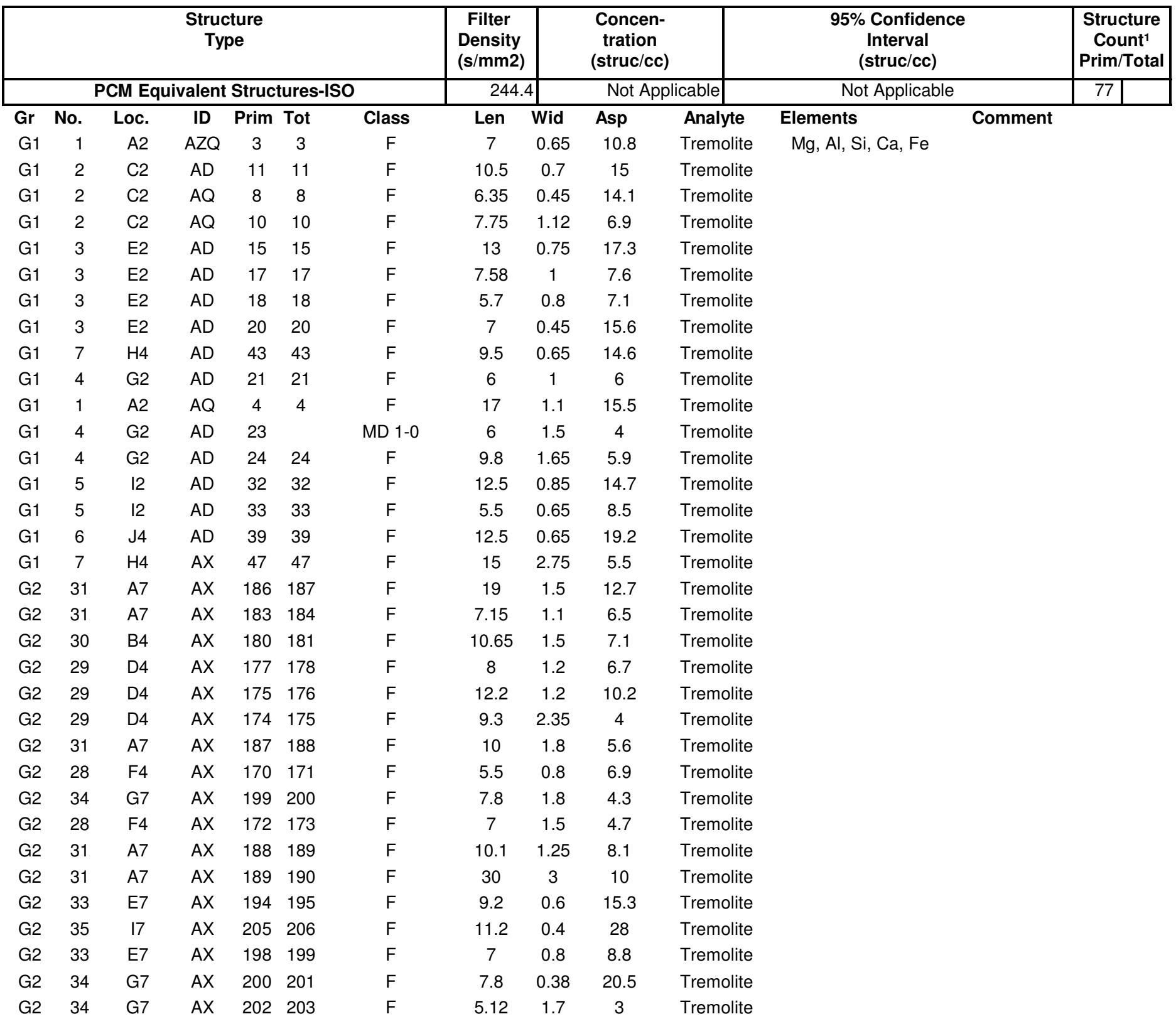


Job Number: 070434

SEA

ISO 10312, Direct Count Categories

Client: Idaho National Laboratory

Report Number: 070434R06

Date Received: 4/23/2007

Project Name: RARE

Lab/Cor Sample No.: S28

Client Sample No.: FB-2-R3

Description:

Filter Fraction: 1

Residual Ash Vol:
Aliquot Dilution: 0

Final Dilution: 0
Volume (L): 0

Lab Filter Area (mm2): 385

Grid Openings Analyzed: 35

Average Grid Opening Area: 0.009

Area Analyzed (mm2): 0.315

Analytical Sens. (struc/cc): 0

Dectection Limit. (struc/cc): 0

\begin{tabular}{|c|c|c|c|c|c|c|c|c|c|c|c|c|c|}
\hline \multicolumn{7}{|c|}{$\begin{array}{c}\text { Structure } \\
\text { Type }\end{array}$} & \multirow{2}{*}{\multicolumn{2}{|c|}{\begin{tabular}{r|}
$\begin{array}{c}\text { Filter } \\
\text { Density } \\
\text { (s/mm2) }\end{array}$ \\
244.4 \\
\end{tabular}}} & \multirow{2}{*}{\multicolumn{2}{|c|}{\begin{tabular}{c|}
$\begin{array}{c}\text { Concen- } \\
\text { tration } \\
\text { (struc/cc) }\end{array}$ \\
Not Applicable
\end{tabular}}} & \multirow{2}{*}{$\begin{array}{c}\begin{array}{c}95 \% \text { Confidence } \\
\text { Interval } \\
\text { (struc/cc) }\end{array} \\
\text { Not Applicable }\end{array}$} & \multicolumn{2}{|c|}{$\begin{array}{c}\text { Structure } \\
\text { Count }^{1} \\
\text { Prim/Total }\end{array}$} \\
\hline \multicolumn{7}{|c|}{ PCM Equivalent Structures-ISO } & & & & & & 77 & \\
\hline Gr & No. & Loc. & ID & Prim & Tot & Class & Len & Wid & Asp & Analyte & Elements & & \\
\hline $\mathrm{G} 2$ & 27 & $\mathrm{H} 4$ & $A X$ & 167 & 168 & $\mathrm{~F}$ & 7.5 & 0.55 & 13.6 & Tremolite & & & \\
\hline G2 & 27 & $\mathrm{H} 4$ & $A X$ & 163 & 164 & $\mathrm{~F}$ & 8 & 1.2 & 6.7 & Tremolite & & & \\
\hline $\mathrm{G} 2$ & 33 & E7 & $A X$ & 195 & 196 & $\mathrm{~F}$ & 14 & 1.2 & 11.7 & Tremolite & & & \\
\hline $\mathrm{G} 2$ & 22 & $\mathrm{C} 2$ & $A X$ & 124 & 125 & $\mathrm{~F}$ & 9 & 1 & 9 & Tremolite & & & \\
\hline G2 & 21 & A2 & $A X$ & 117 & & MD 1-0 & 9 & 3 & 3 & Tremolite & & & \\
\hline G2 & 28 & $\mathrm{~F} 4$ & $A X$ & 169 & 170 & $\mathrm{~F}$ & 20 & 0.85 & 23.5 & Tremolite & & & \\
\hline $\mathrm{G} 2$ & 21 & $\mathrm{~A} 2$ & $A X$ & 119 & 120 & $\mathrm{~F}$ & 10 & 0.6 & 16.7 & Tremolite & & & \\
\hline G2 & 22 & $\mathrm{C} 2$ & $A X$ & 125 & 126 & $\mathrm{~F}$ & 5.4 & 0.6 & 9 & Tremolite & & & \\
\hline $\mathrm{G} 2$ & 22 & $\mathrm{C} 2$ & $A X$ & 128 & 129 & $\mathrm{~F}$ & 7.7 & 0.45 & 17.1 & Tremolite & & & \\
\hline G2 & 22 & $\mathrm{C} 2$ & $A X$ & 129 & 130 & $F$ & 15 & 2.5 & 6 & Tremolite & & & \\
\hline $\mathrm{G} 2$ & 22 & $\mathrm{C} 2$ & $A X$ & 131 & 132 & $\mathrm{~F}$ & 5.12 & 0.45 & 11.4 & Tremolite & & & \\
\hline G2 & 22 & $\mathrm{C} 2$ & $A X$ & 133 & 134 & $\mathrm{~F}$ & 12 & 2.5 & 4.8 & Tremolite & & & \\
\hline G2 & 23 & E2 & $A X$ & 137 & 138 & $\mathrm{~F}$ & 5.25 & 1.35 & 3.9 & Tremolite & & & \\
\hline $\mathrm{G} 2$ & 23 & E2 & $A X$ & 142 & 143 & $\mathrm{~F}$ & 15 & 1.8 & 8.3 & Tremolite & & & \\
\hline G2 & 26 & $\mathrm{~J} 4$ & $A X$ & 159 & 160 & $F$ & 9 & 0.3 & 30 & Tremolite & & & \\
\hline G2 & 24 & G2 & $A X$ & 144 & 145 & $\mathrm{~F}$ & 11.85 & 0.885 & 13.4 & Tremolite & & & \\
\hline G2 & 26 & $\mathrm{~J} 4$ & $A X$ & 160 & 161 & $F$ & 6.2 & 1.12 & 5.5 & Tremolite & & & \\
\hline G2 & 26 & $\mathrm{~J} 4$ & $A X$ & 158 & 159 & $F$ & 5.7 & 1.2 & 4.7 & Tremolite & & & \\
\hline G2 & 25 & 12 & $A X$ & 156 & 157 & $\mathrm{~F}$ & 5.25 & 0.75 & 7 & Tremolite & & & \\
\hline $\mathrm{G} 2$ & 25 & 12 & $A X$ & 154 & 155 & $\mathrm{~F}$ & 9 & 3 & 3 & Tremolite & & & \\
\hline G2 & 25 & 12 & $\mathrm{AX}$ & 151 & 152 & $\mathrm{~F}$ & 10 & 1.7 & 5.9 & Tremolite & & & \\
\hline G2 & 25 & 12 & $A X$ & 150 & 151 & $\mathrm{~F}$ & 7.2 & 0.85 & 8.5 & Tremolite & & & \\
\hline G2 & 25 & 12 & $A X$ & 148 & 149 & $\mathrm{~F}$ & 9.5 & 1.2 & 7.9 & Tremolite & & & \\
\hline $\mathrm{G} 2$ & 24 & G2 & $A X$ & 145 & 146 & $\mathrm{~F}$ & 16.8 & 0.38 & 44.2 & Tremolite & & & \\
\hline \multicolumn{7}{|c|}{ PCM Equivalent Fibers-NIOSH } & & & \multicolumn{2}{|c|}{ Not Applicable } & Not Applicable & & 86 \\
\hline Gr & No. & Loc. & ID & Prim & Tot & Class & Len & Wid & Asp & Analyte & Elements & & \\
\hline G1 & 15 & 17 & $A X$ & 91 & 92 & $\mathrm{~F}$ & 9.2 & 0.8 & 11.5 & Tremolite & & & \\
\hline G1 & 14 & G7 & $A X$ & 82 & 83 & $\mathrm{~F}$ & 9.5 & 1.2 & 7.9 & Tremolite & & & \\
\hline G1 & 9 & D4 & $A X$ & 58 & 58 & $\mathrm{~F}$ & 9 & 1.2 & 7.5 & Tremolite & & & \\
\hline G1 & 10 & B4 & $A X$ & 60 & 60 & $\mathrm{~F}$ & 10.6 & 0.8 & 13.2 & Tremolite & & & \\
\hline G1 & 12 & $\mathrm{C} 7$ & $A X$ & & 69 & CF & 14.3 & 0.6 & 23.8 & Tremolite & & & \\
\hline G1 & 12 & $\mathrm{C} 7$ & $A X$ & & 71 & MF & 6 & 0.85 & 7.1 & Tremolite & & & \\
\hline G1 & 12 & $\mathrm{C} 7$ & $A X$ & & 72 & MB & 9.8 & 1.35 & 7.3 & Tremolite & & & \\
\hline G1 & 13 & E7 & $A X$ & 77 & 78 & $\mathrm{~F}$ & 5.25 & 0.5 & 10.5 & Tremolite & & & \\
\hline
\end{tabular}


Job Number: 070434

SEA

ISO 10312, Direct Count Categories

Client: Idaho National Laboratory

Report Number: 070434R06

ct Name: RARE

Lab/Cor Sample No.: S28

Client Sample No.: FB-2-R3

Description:

Filter Fraction: 1

Residual Ash Vol:
Aliquot Dilution: 0

Final Dilution: 0
Date Received: 4/23/2007
Volume (L): 0

Lab Filter Area (mm2): 385

Grid Openings Analyzed: 35

Average Grid Opening Area: 0.009

Area Analyzed (mm2): 0.315

Analytical Sens. (struc/cc): 0

Dectection Limit. (struc/cc): 0

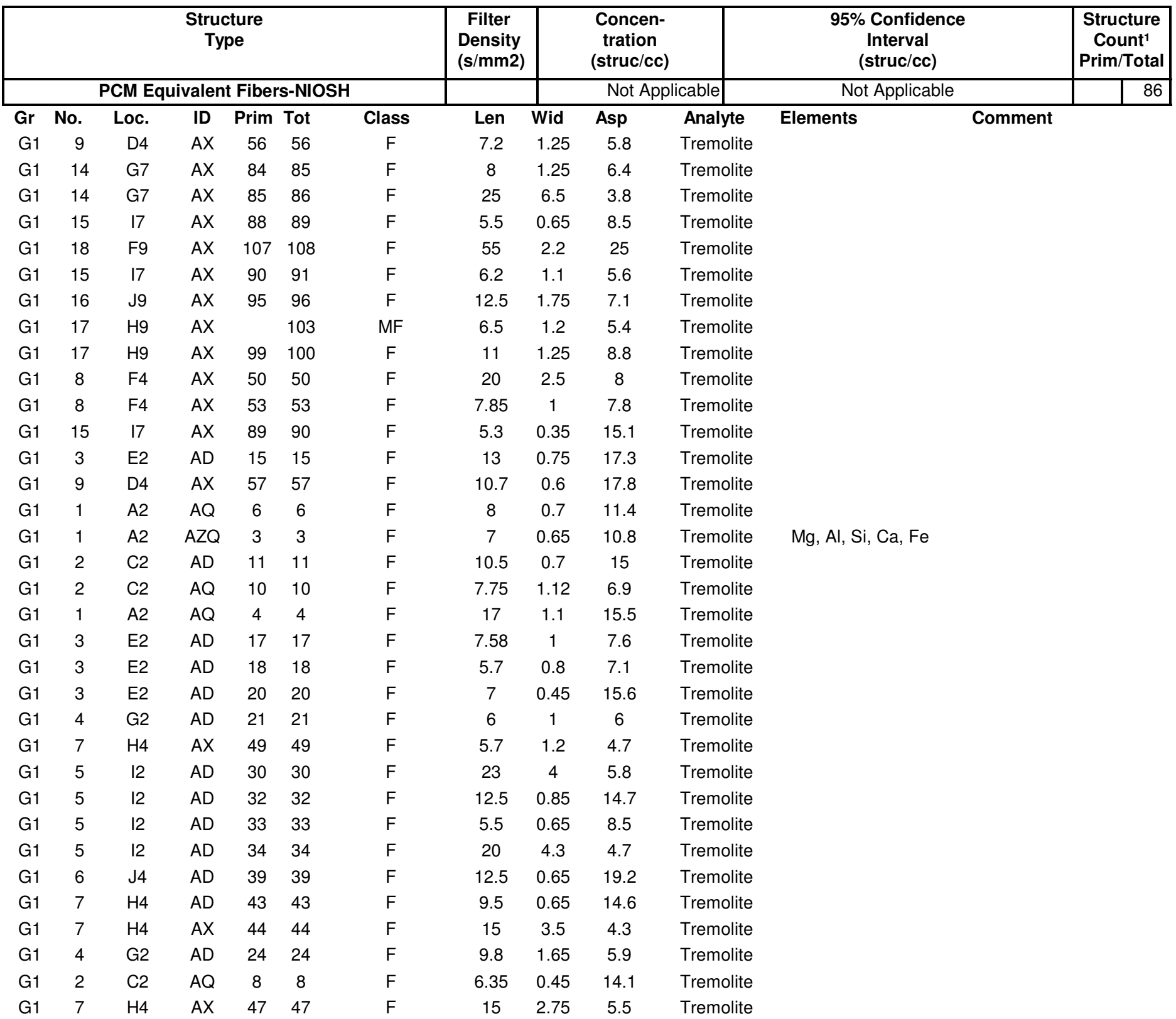


Job Number: 070434

SEA

ISO 10312, Direct Count Categories

Client: Idaho National Laboratory

Report Number: 070434R06

ct Name: RARE

Lab/Cor Sample No.: S28

Client Sample No.: FB-2-R3

Description:

Filter Fraction: 1

Residual Ash Vol:
Aliquot Dilution: 0

Final Dilution: 0
Date Received: 4/23/2007
Volume (L): 0

Lab Filter Area (mm2): 385

Grid Openings Analyzed: 35

Average Grid Opening Area: 0.009

Area Analyzed (mm2): 0.315

Analytical Sens. (struc/cc): 0

Dectection Limit. (struc/cc): 0

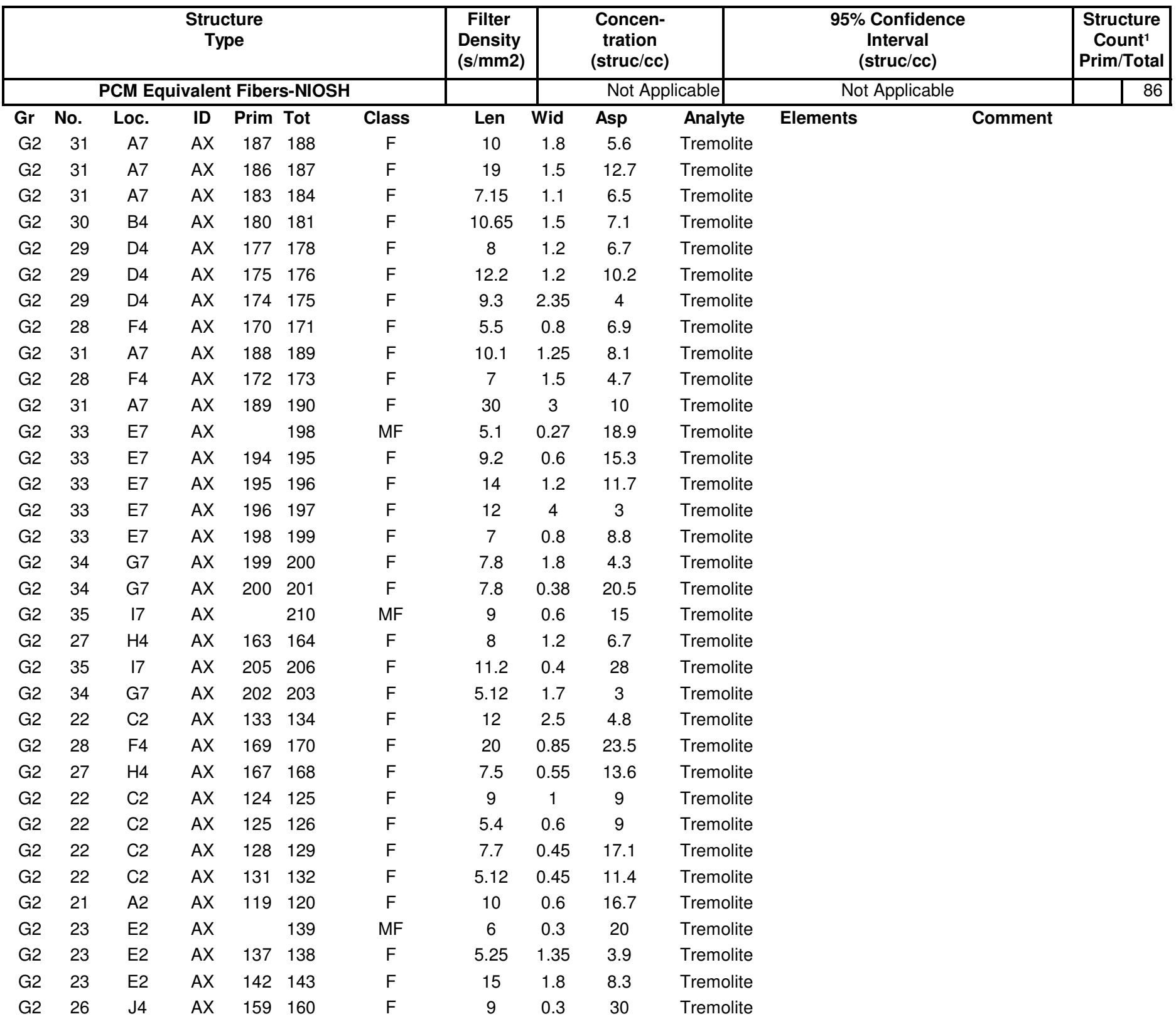


Job Number: 070434

SEA

ISO 10312, Direct Count Categories

Client: Idaho National Laboratory

Report Number: 070434R06

Date Received: 4/23/2007

Project Name: RARE

Lab/Cor Sample No.: S28

Client Sample No.: FB-2-R3

Description:

Filter Fraction: 1

Residual Ash Vol:
Aliquot Dilution: 0

Final Dilution: 0
Volume (L): 0

Lab Filter Area (mm2): 385

Grid Openings Analyzed: 35

Average Grid Opening Area: 0.009

Area Analyzed (mm2): 0.315

Analytical Sens. (struc/cc): 0

Dectection Limit. (struc/cc): 0

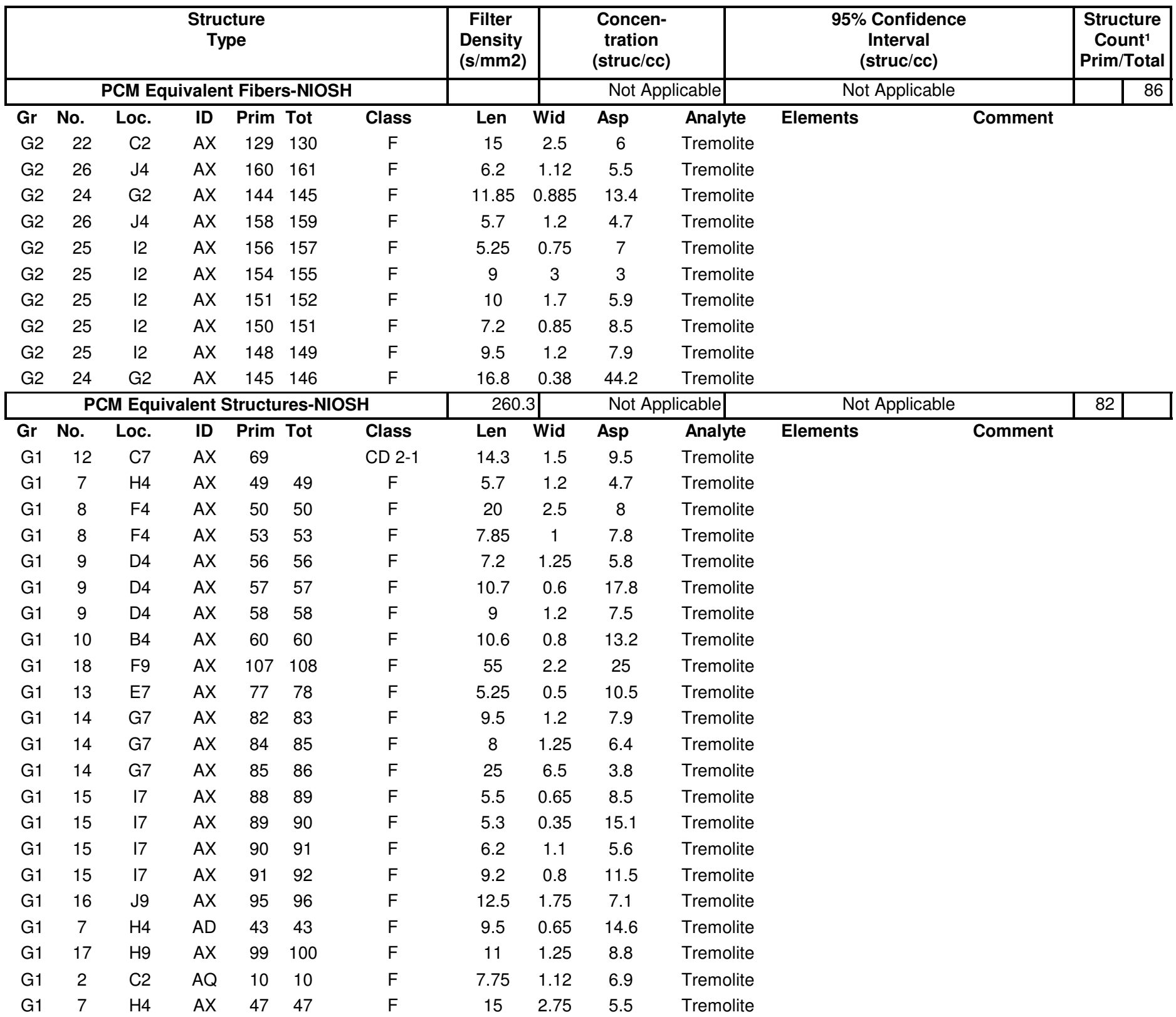


Job Number: 070434

SEA

ISO 10312, Direct Count Categories

Client: Idaho National Laboratory

Report Number: 070434R06

ct Name: RARE

Lab/Cor Sample No.: S28

Client Sample No.: FB-2-R3

Description:

Filter Fraction: 1

Residual Ash Vol:
Aliquot Dilution: 0

Final Dilution: 0
Date Received: 4/23/2007
Volume (L): 0

Lab Filter Area (mm2): 385

Grid Openings Analyzed: 35

Average Grid Opening Area: 0.009

Area Analyzed (mm2): 0.315

Analytical Sens. (struc/cc): 0

Dectection Limit. (struc/cc): 0

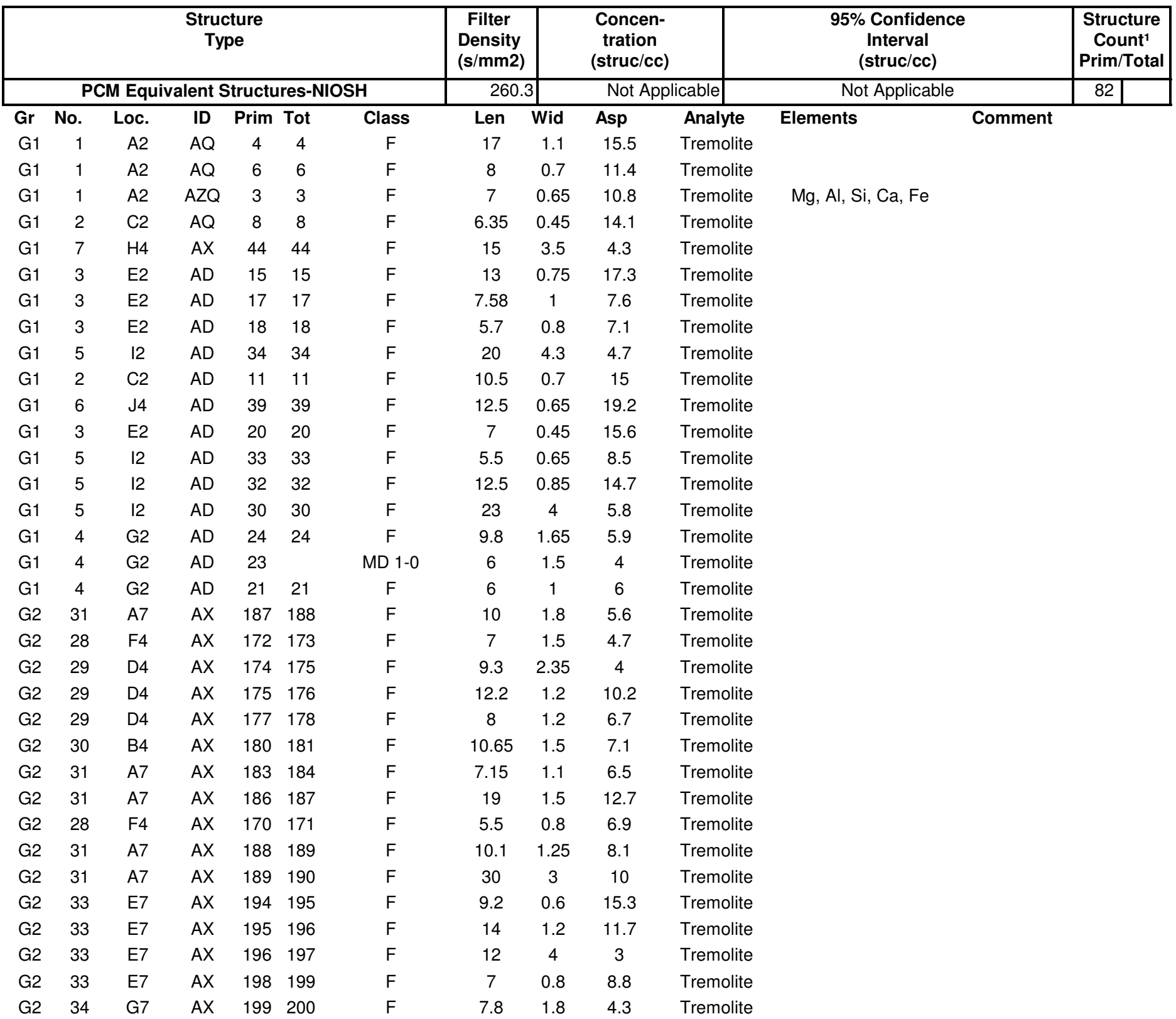


Job Number: 070434

SEA

ISO 10312, Direct Count Categories

Client: Idaho National Laboratory

Report Number: 070434R06

Date Received: 4/23/2007

Project Name: RARE

Lab/Cor Sample No.: S28

Client Sample No.: FB-2-R3

Description:

Filter Fraction: 1

Residual Ash Vol:
Aliquot Dilution: 0

Final Dilution: 0
Volume (L): 0

Lab Filter Area (mm2): 385

Grid Openings Analyzed: 35

Average Grid Opening Area: 0.009

Area Analyzed (mm2): 0.315

Analytical Sens. (struc/cc): 0

Dectection Limit. (struc/cc): 0

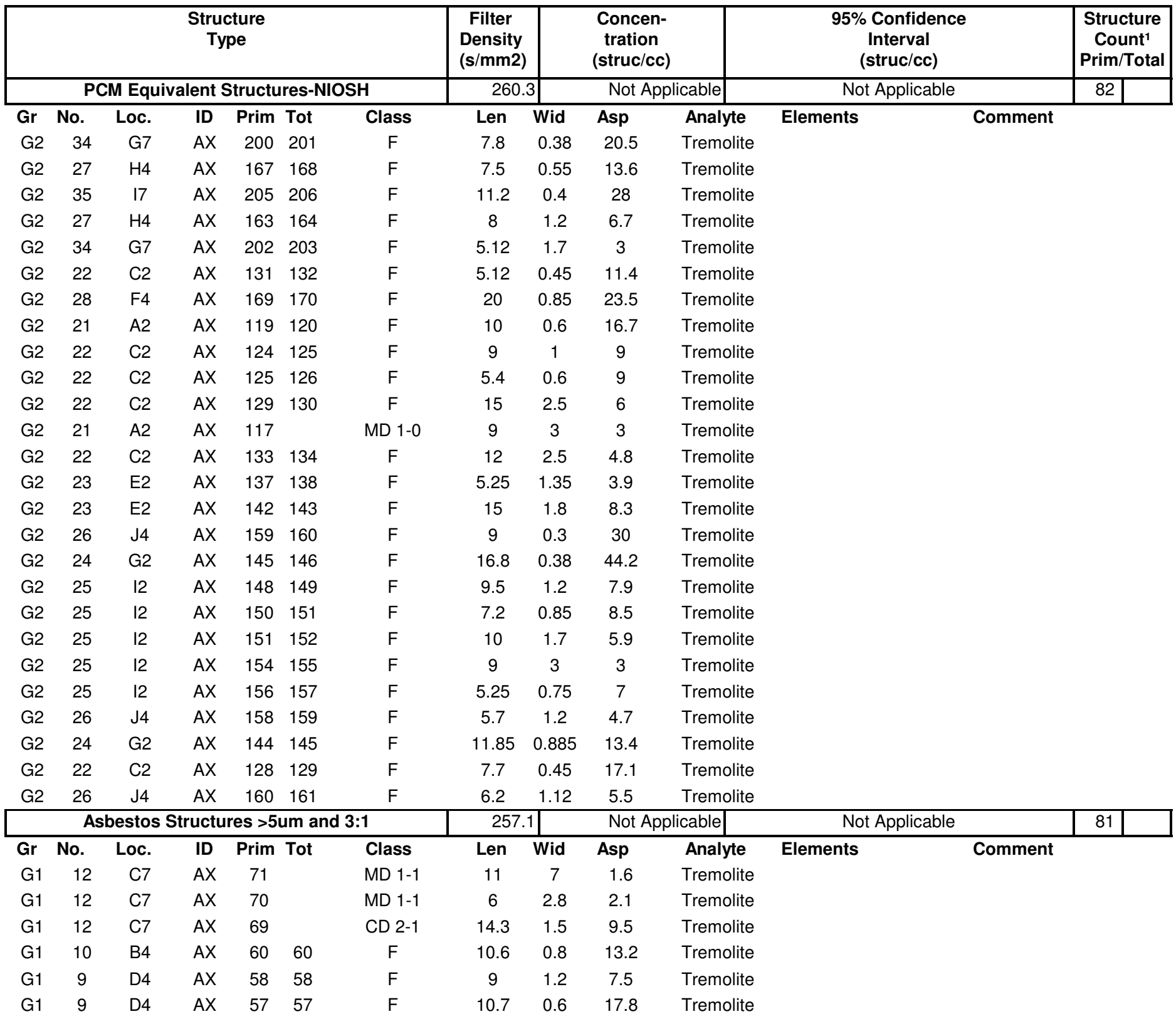


Job Number: 070434

SEA

ISO 10312, Direct Count Categories

Client: Idaho National Laboratory

Report Number: 070434R06

ct Name: RARE

Lab/Cor Sample No.: S28

Client Sample No.: FB-2-R3

Description:

Filter Fraction: 1

Residual Ash Vol:
Aliquot Dilution: 0

Final Dilution: 0
Date Received: 4/23/2007
Volume (L): 0

Lab Filter Area (mm2): 385

Grid Openings Analyzed: 35

Average Grid Opening Area: 0.009

Area Analyzed (mm2): 0.315

Analytical Sens. (struc/cc): 0

Dectection Limit. (struc/cc): 0

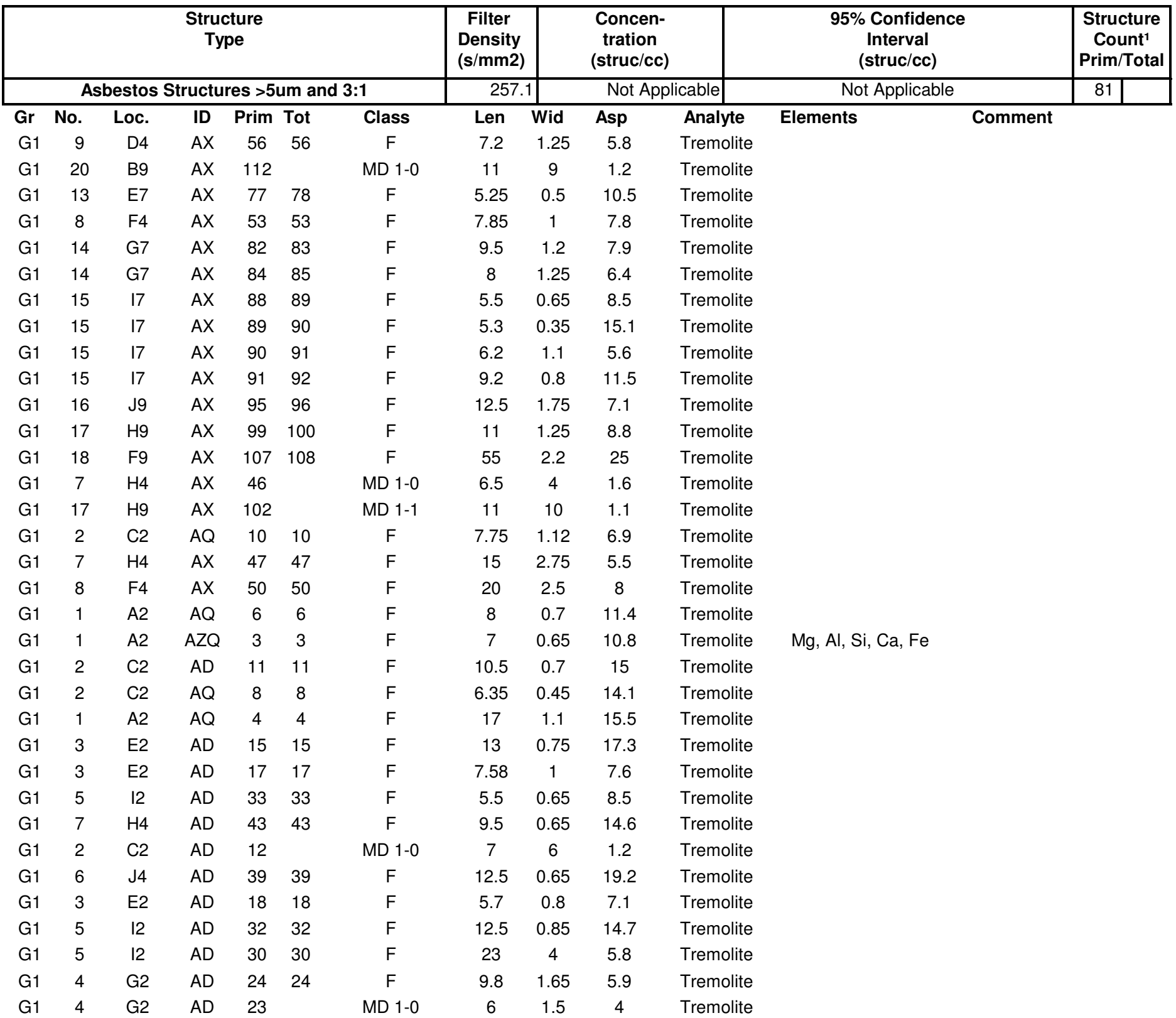


Job Number: 070434

SEA

ISO 10312, Direct Count Categories

Client: Idaho National Laboratory

Report Number: 070434R06

ct Name: RARE

Lab/Cor Sample No.: S28

Client Sample No.: FB-2-R3

Description:

Filter Fraction: 1

Residual Ash Vol:
Aliquot Dilution: 0

Final Dilution: 0
Date Received: 4/23/2007
Volume (L): 0

Lab Filter Area (mm2): 385

Grid Openings Analyzed: 35

Average Grid Opening Area: 0.009

Area Analyzed (mm2): 0.315

Analytical Sens. (struc/cc): 0

Dectection Limit. (struc/cc): 0

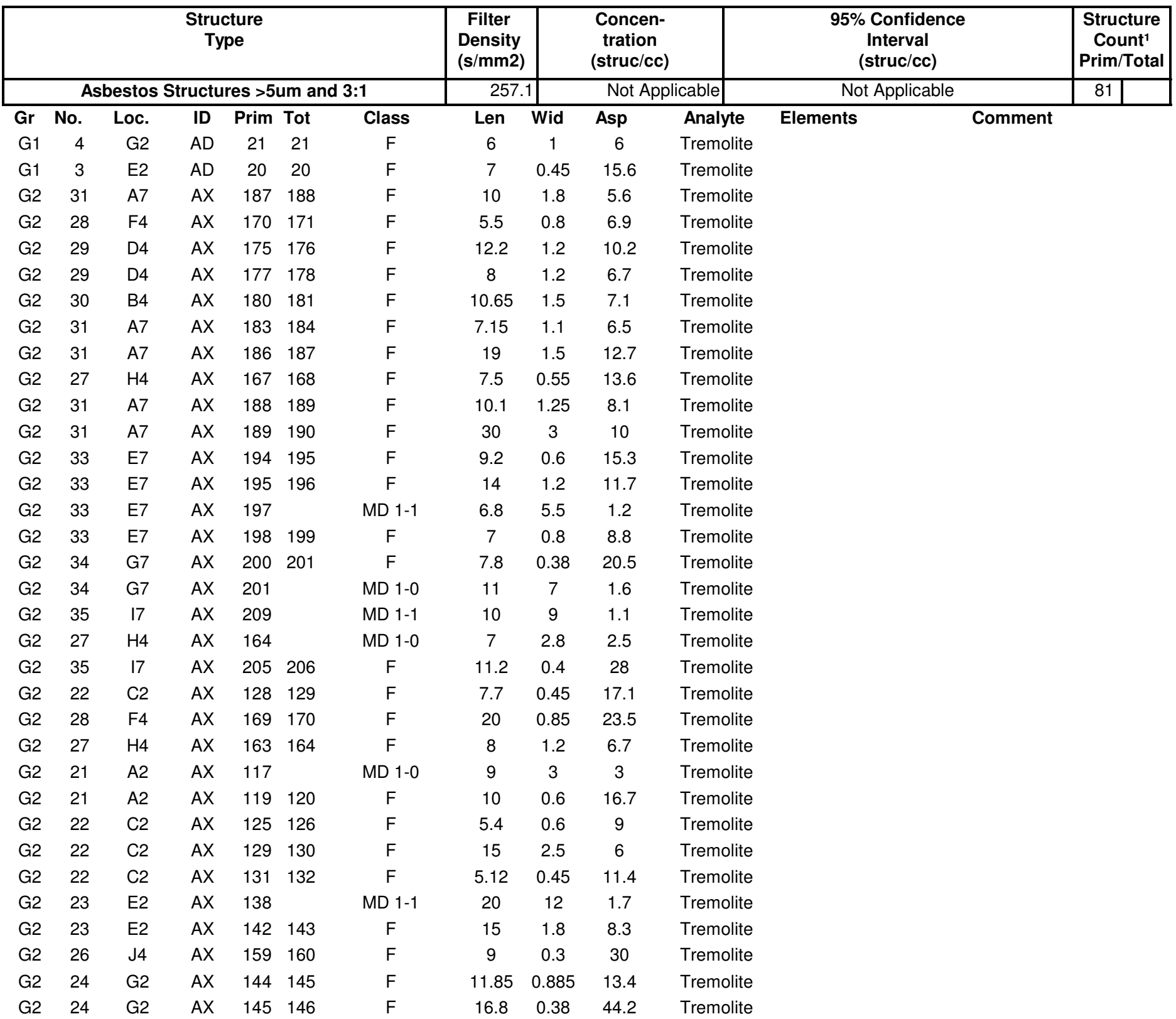


Job Number: 070434

SEA

ISO 10312, Direct Count Categories

Client: Idaho National Laboratory

Report Number: 070434R06

Date Received: 4/23/2007

Project Name: RARE

Lab/Cor Sample No.: S28

Client Sample No.: FB-2-R3

Description:

Filter Fraction: 1

Residual Ash Vol:
Aliquot Dilution: 0

Final Dilution: 0
Volume (L): 0

Lab Filter Area (mm2): 385

Grid Openings Analyzed: 35

Average Grid Opening Area: 0.009

Area Analyzed (mm2): 0.315

Analytical Sens. (struc/cc): 0

Dectection Limit. (struc/cc): 0

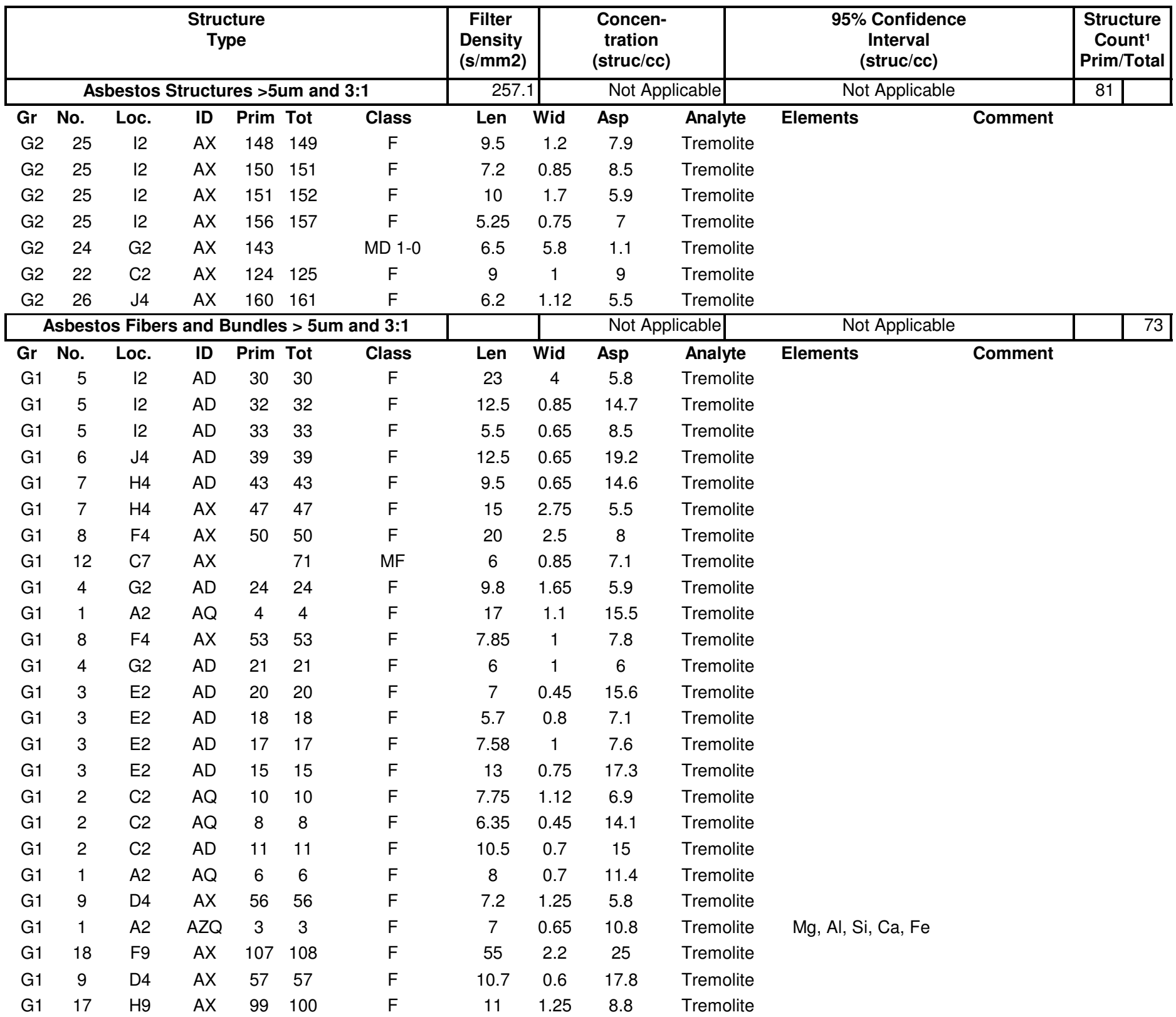


Job Number: 070434

SEA

ISO 10312, Direct Count Categories

Client: Idaho National Laboratory

Report Number: 070434R06

Date Received: 4/23/2007

Project Name: RARE

Lab/Cor Sample No.: S28

Client Sample No.: FB-2-R3

Description:

Filter Fraction: 1

Residual Ash Vol:
Aliquot Dilution: 0

Final Dilution: 0
Volume (L): 0

Lab Filter Area (mm2): 385

Grid Openings Analyzed: 35

Average Grid Opening Area: 0.009

Area Analyzed (mm2): 0.315

Analytical Sens. (struc/cc): 0

Dectection Limit. (struc/cc): 0

\begin{tabular}{|c|c|c|c|c|c|c|c|c|c|c|c|c|}
\hline & & & $\begin{array}{r}\text { Struc } \\
\text { Ty }\end{array}$ & $\begin{array}{l}\text { ture } \\
\text { oe }\end{array}$ & & & $\begin{array}{c}\text { Filter } \\
\text { Density } \\
(\mathrm{s} / \mathrm{mm} 2)\end{array}$ & & $\begin{array}{r}\text { Conce } \\
\text { tratio } \\
\text { (struc/ }\end{array}$ & & $\begin{array}{c}\text { 95\% Confidence } \\
\text { Interval } \\
\text { (struc/cc) }\end{array}$ & $\begin{array}{l}\text { Structure } \\
\text { Count }^{1} \\
\text { Prim/Total }\end{array}$ \\
\hline & Asbest & s Fibe & and $B$ & undles & $s>5 t$ & nd $3: 1$ & & & Not $A$ & licable & Not Applicable & 73 \\
\hline Gr & No. & Loc. & ID & Prim & Tot & Class & Len & Wid & Asp & Analyte & Elements & \\
\hline G1 & 17 & $\mathrm{H} 9$ & $A X$ & & 103 & MF & 6.5 & 1.2 & 5.4 & Tremolite & & \\
\hline G1 & 16 & J9 & $A X$ & 95 & 96 & $F$ & 12.5 & 1.75 & 7.1 & Tremolite & & \\
\hline G1 & 15 & 17 & $A X$ & 91 & 92 & $F$ & 9.2 & 0.8 & 11.5 & Tremolite & & \\
\hline G1 & 15 & 17 & $A X$ & 90 & 91 & $F$ & 6.2 & 1.1 & 5.6 & Tremolite & & \\
\hline G1 & 15 & 17 & $A X$ & 89 & 90 & $\mathrm{~F}$ & 5.3 & 0.35 & 15.1 & Tremolite & & \\
\hline G1 & 9 & D4 & $A X$ & 58 & 58 & $\mathrm{~F}$ & 9 & 1.2 & 7.5 & Tremolite & & \\
\hline G1 & 14 & G7 & $A X$ & 84 & 85 & $\mathrm{~F}$ & 8 & 1.25 & 6.4 & Tremolite & & \\
\hline G1 & 14 & G7 & $A X$ & 82 & 83 & $F$ & 9.5 & 1.2 & 7.9 & Tremolite & & \\
\hline G1 & 13 & E7 & $A X$ & 77 & 78 & $\mathrm{~F}$ & 5.25 & 0.5 & 10.5 & Tremolite & & \\
\hline G1 & 12 & $\mathrm{C7}$ & $A X$ & & 72 & MB & 9.8 & 1.35 & 7.3 & Tremolite & & \\
\hline G1 & 10 & B4 & $A X$ & 60 & 60 & $F$ & 10.6 & 0.8 & 13.2 & Tremolite & & \\
\hline G1 & 12 & $\mathrm{C7}$ & $A X$ & & 69 & CF & 14.3 & 0.6 & 23.8 & Tremolite & & \\
\hline G1 & 15 & 17 & $A X$ & 88 & 89 & $F$ & 5.5 & 0.65 & 8.5 & Tremolite & & \\
\hline G2 & 31 & A7 & $A X$ & 186 & 187 & $\mathrm{~F}$ & 19 & 1.5 & 12.7 & Tremolite & & \\
\hline G2 & 30 & B4 & $A X$ & 180 & 181 & $F$ & 10.65 & 1.5 & 7.1 & Tremolite & & \\
\hline G2 & 29 & D4 & $A X$ & 177 & 178 & $F$ & 8 & 1.2 & 6.7 & Tremolite & & \\
\hline G2 & 29 & D4 & $A X$ & 175 & 176 & $F$ & 12.2 & 1.2 & 10.2 & Tremolite & & \\
\hline G2 & 31 & A7 & $A X$ & 188 & 189 & $F$ & 10.1 & 1.25 & 8.1 & Tremolite & & \\
\hline G2 & 28 & $\mathrm{~F} 4$ & $A X$ & 169 & 170 & $F$ & 20 & 0.85 & 23.5 & Tremolite & & \\
\hline G2 & 34 & G7 & $A X$ & 200 & 201 & $F$ & 7.8 & 0.38 & 20.5 & Tremolite & & \\
\hline G2 & 28 & $\mathrm{~F} 4$ & $A X$ & 170 & 171 & $\mathrm{~F}$ & 5.5 & 0.8 & 6.9 & Tremolite & & \\
\hline G2 & 31 & A7 & $A X$ & 189 & 190 & $F$ & 30 & 3 & 10 & Tremolite & & \\
\hline G2 & 33 & E7 & $A X$ & & 198 & MF & 5.1 & 0.27 & 18.9 & Tremolite & & \\
\hline G2 & 33 & E7 & $A X$ & 194 & 195 & $F$ & 9.2 & 0.6 & 15.3 & Tremolite & & \\
\hline G2 & 35 & 17 & $A X$ & 205 & 206 & $F$ & 11.2 & 0.4 & 28 & Tremolite & & \\
\hline G2 & 33 & E7 & $A X$ & 198 & 199 & $F$ & 7 & 0.8 & 8.8 & Tremolite & & \\
\hline G2 & 35 & 17 & $A X$ & & 210 & MF & 9 & 0.6 & 15 & Tremolite & & \\
\hline G2 & 27 & $\mathrm{H} 4$ & $A X$ & 167 & 168 & $\mathrm{~F}$ & 7.5 & 0.55 & 13.6 & Tremolite & & \\
\hline G2 & 31 & A7 & $A X$ & 183 & 184 & $F$ & 7.15 & 1.1 & 6.5 & Tremolite & & \\
\hline G2 & 33 & E7 & $A X$ & 195 & 196 & $\mathrm{~F}$ & 14 & 1.2 & 11.7 & Tremolite & & \\
\hline G2 & 22 & $\mathrm{C} 2$ & $A X$ & 128 & 129 & $F$ & 7.7 & 0.45 & 17.1 & Tremolite & & \\
\hline G2 & 31 & A7 & $A X$ & 187 & 188 & $F$ & 10 & 1.8 & 5.6 & Tremolite & & \\
\hline G2 & 27 & $\mathrm{H} 4$ & $A X$ & 163 & 164 & $F$ & 8 & 1.2 & 6.7 & Tremolite & & \\
\hline G2 & 21 & $\mathrm{~A} 2$ & $A X$ & 119 & 120 & $F$ & 10 & 0.6 & 16.7 & Tremolite & & \\
\hline
\end{tabular}


A Professional Service Corporation in the Northwest

ISO 10312, Direct Count Categories

Job Number: 070434

SEA

Report Number: 070434R06

Client: Idaho National Laboratory

Date Received: 4/23/2007

Project Name: RARE

Lab/Cor Sample No.: S28

Client Sample No.: FB-2-R3

Description:

Filter Fraction: 1

Residual Ash Vol:
Aliquot Dilution: 0

Final Dilution: 0
Volume (L): 0

Lab Filter Area (mm2): 385

Grid Openings Analyzed: 35

Average Grid Opening Area: 0.009

Area Analyzed (mm2): 0.315

Analytical Sens. (struc/cc): 0

Dectection Limit. (struc/cc): 0

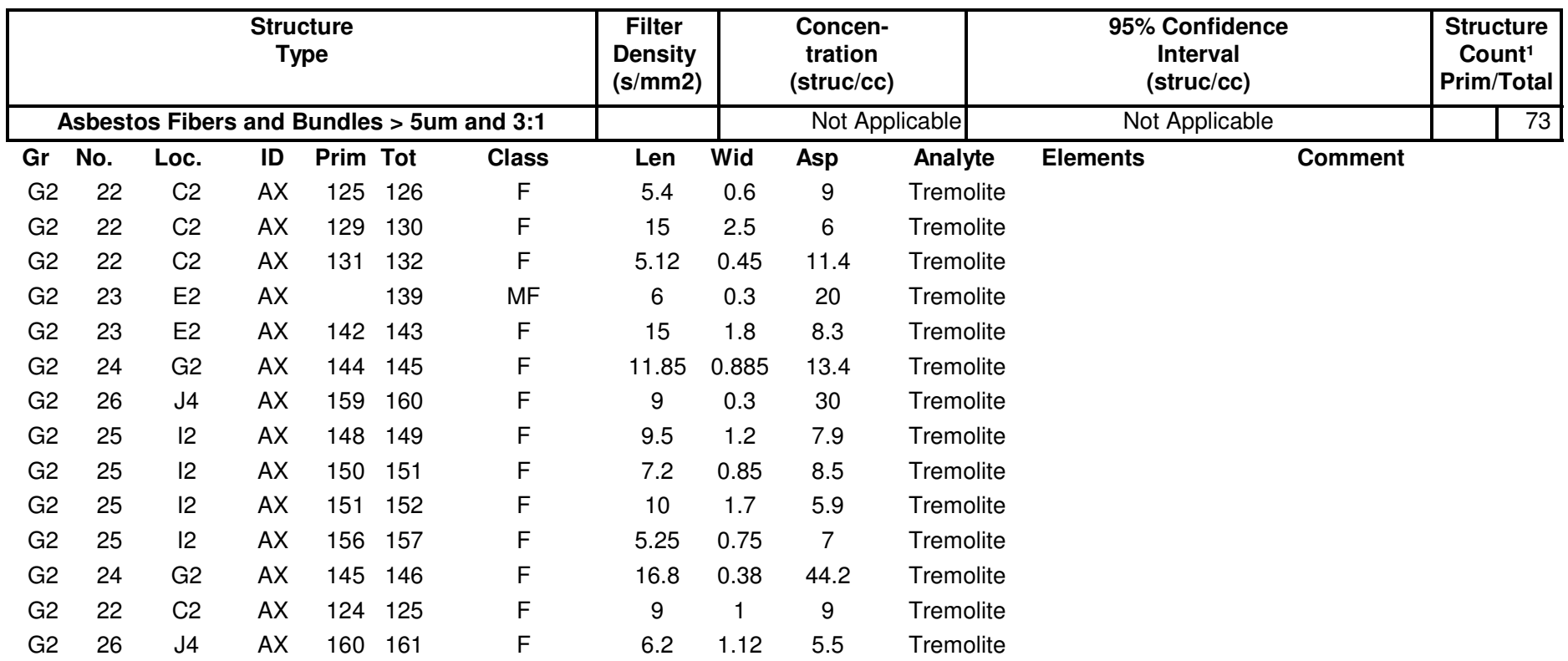


Job Number: 070434

SEA

ISO 10312, Direct Count Categories

Client: Idaho National Laboratory

Report Number: 070434R06

ct Name: RARE

Lab/Cor Sample No.: S29

Client Sample No.: FB-2-R4

Description:

Filter Fraction: 1

Residual Ash Vol:
Aliquot Dilution: 0

Final Dilution: 0
Date Received: 4/23/2007
Volume (L): 0

Lab Filter Area (mm2): 385

Grid Openings Analyzed: 35

Average Grid Opening Area: 0.009

Area Analyzed (mm2): 0.315

Analytical Sens. (struc/cc): 0

Dectection Limit. (struc/cc): 0

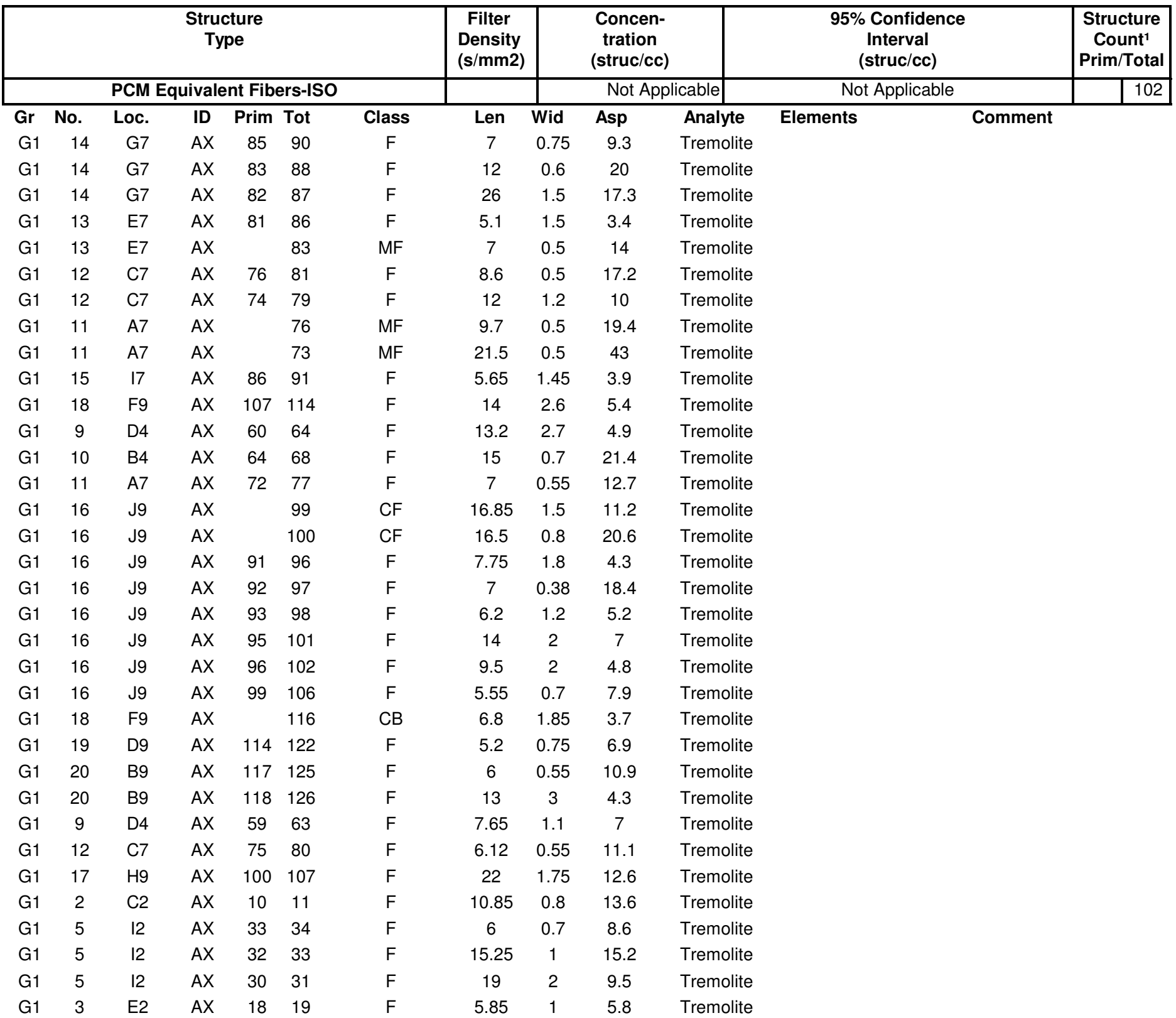


Job Number: 070434

SEA

ISO 10312, Direct Count Categories

Client: Idaho National Laboratory

Report Number: 070434R06

Date Received: 4/23/2007

Project Name: RARE

Lab/Cor Sample No.: S29

Client Sample No.: FB-2-R4

Description:

Filter Fraction: 1

Residual Ash Vol:
Aliquot Dilution: 0

Final Dilution: 0
Volume (L): 0

Lab Filter Area (mm2): 385

Grid Openings Analyzed: 35

Average Grid Opening Area: 0.009

Area Analyzed (mm2): 0.315

Analytical Sens. (struc/cc): 0

Dectection Limit. (struc/cc): 0

\begin{tabular}{|c|c|c|c|c|c|c|c|c|c|c|c|c|}
\hline & & & $\begin{array}{r}\text { Struc } \\
\text { Ty }\end{array}$ & $\begin{array}{l}\text { ture } \\
\text { pe }\end{array}$ & & & $\begin{array}{c}\text { Filter } \\
\text { Density } \\
(\mathrm{s} / \mathrm{mm} 2)\end{array}$ & & $\begin{array}{r}\text { Conc } \\
\text { tratic } \\
\text { (struc }\end{array}$ & & $\begin{array}{c}\text { 95\% Confidence } \\
\text { Interval } \\
\text { (struc/cc) }\end{array}$ & $\begin{array}{c}\text { Structure } \\
\text { Count }^{1} \\
\text { Prim/Total }\end{array}$ \\
\hline & & PCM & quivale & nt Fib & ers-IS & & & & Not & licable & Not Applicable & 102 \\
\hline Gr & No. & Loc. & ID & Prim & Tot & Class & Len & Wid & Asp & Analyte & Comment & \\
\hline G1 & 3 & E2 & $A X$ & 17 & 18 & $\mathrm{~F}$ & 6.5 & 1.5 & 4.3 & Tremolite & & \\
\hline G1 & 6 & J4 & $A X$ & 37 & 38 & $\mathrm{~F}$ & 5.7 & 0.8 & 7.1 & Tremolite & & \\
\hline G1 & 2 & $\mathrm{C} 2$ & $A X$ & 12 & 13 & $\mathrm{~F}$ & 5.4 & 0.6 & 9 & Tremolite & & \\
\hline G1 & 2 & $\mathrm{C} 2$ & $A X$ & 9 & 10 & $\mathrm{~F}$ & 22.5 & 1.5 & 15 & Tremolite & & \\
\hline G1 & 2 & $\mathrm{C} 2$ & $A X$ & 4 & 4 & $\mathrm{~F}$ & 5.35 & 0.65 & 8.2 & Tremolite & & \\
\hline G1 & 2 & $\mathrm{C} 2$ & $A X$ & & 7 & MF & 13.2 & 0.5 & 26.4 & Tremolite & & \\
\hline G1 & 2 & $\mathrm{C} 2$ & $A X$ & & 3 & MF & 5.75 & 1.2 & 4.8 & Tremolite & & \\
\hline G1 & 2 & $\mathrm{C} 2$ & $A D Q$ & 13 & 14 & $\mathrm{~F}$ & 9.8 & 1.2 & 8.2 & Tremolite & $\mathrm{Mg}, \mathrm{Al}, \mathrm{Si}, \mathrm{Ca}, \mathrm{Fe}$ & \\
\hline G1 & 9 & D4 & $A X$ & & 66 & MF & 6 & 0.8 & 7.5 & Tremolite & & \\
\hline G1 & 12 & C7 & $A X$ & 77 & 82 & $\mathrm{~F}$ & 9.8 & 0.85 & 11.5 & Tremolite & & \\
\hline G1 & 2 & $\mathrm{C} 2$ & $A X$ & 15 & 16 & $\mathrm{~F}$ & 7 & 0.2 & 35 & Tremolite & & \\
\hline G1 & 8 & $\mathrm{~F} 4$ & $A X$ & 55 & 59 & $\mathrm{~F}$ & 6.2 & 1.2 & 5.2 & Tremolite & & \\
\hline G1 & 2 & $\mathrm{C} 2$ & $A X$ & & 8 & MF & 5.1 & 0.25 & 20.4 & Tremolite & & \\
\hline G1 & 7 & $\mathrm{H} 4$ & $A X$ & 40 & 41 & $\mathrm{~F}$ & 7 & 0.55 & 12.7 & Tremolite & & \\
\hline G1 & 8 & $\mathrm{~F} 4$ & $A X$ & 54 & 58 & $\mathrm{~F}$ & 13.5 & 1.8 & 7.5 & Tremolite & & \\
\hline G1 & 8 & $\mathrm{~F} 4$ & $A X$ & 51 & 52 & $\mathrm{~F}$ & 21.7 & 1.8 & 12.1 & Tremolite & & \\
\hline G1 & 8 & $\mathrm{~F} 4$ & $A X$ & 50 & 51 & $\mathrm{~F}$ & 6.75 & 2 & 3.4 & Tremolite & & \\
\hline G1 & 8 & $\mathrm{~F} 4$ & $A X$ & & 55 & $\mathrm{CF}$ & 5.5 & 0.55 & 10 & Tremolite & & \\
\hline G1 & 7 & $\mathrm{H} 4$ & $A X$ & 43 & 44 & $\mathrm{~F}$ & 6.1 & 1.2 & 5.1 & Tremolite & & \\
\hline G1 & 8 & $\mathrm{~F} 4$ & $A X$ & & 54 & CF & 9 & 0.75 & 12 & Tremolite & & \\
\hline G1 & 8 & $\mathrm{~F} 4$ & $A X$ & 56 & 60 & $\mathrm{~F}$ & 5.6 & 0.7 & 8 & Tremolite & & \\
\hline G1 & 7 & $\mathrm{H} 4$ & $A X$ & 46 & 47 & $\mathrm{~F}$ & 6 & 1.25 & 4.8 & Tremolite & & \\
\hline G1 & 1 & A2 & $A Q$ & 2 & 2 & $\mathrm{~F}$ & 17.5 & 2 & 8.8 & Tremolite & $\mathrm{Mg}, \mathrm{Al}, \mathrm{Si}, \mathrm{Ca}, \mathrm{Fe}$ & \\
\hline G1 & 7 & $\mathrm{H} 4$ & $A X$ & 41 & 42 & $\mathrm{~F}$ & 54 & 1.2 & 45 & Tremolite & & \\
\hline G1 & 8 & $\mathrm{~F} 4$ & $A X$ & 53 & 57 & $\mathrm{~F}$ & 7.75 & 0.65 & 11.9 & Tremolite & & \\
\hline G1 & 7 & $\mathrm{H} 4$ & $A X$ & 42 & 43 & $\mathrm{~F}$ & 7.5 & 1.35 & 5.6 & Tremolite & & \\
\hline G2 & 30 & B4 & $A X$ & 168 & 176 & $\mathrm{~F}$ & 5.6 & 1 & 5.6 & Tremolite & & \\
\hline G2 & 30 & B4 & $A X$ & 165 & 173 & $\mathrm{~F}$ & 7.7 & 1.5 & 5.1 & Tremolite & & \\
\hline G2 & 29 & D4 & $A X$ & 163 & 171 & $\mathrm{~F}$ & 10 & 2 & 5 & Tremolite & & \\
\hline G2 & 29 & D4 & $A X$ & 162 & 170 & $\mathrm{~F}$ & 10.5 & 1.2 & 8.8 & Tremolite & & \\
\hline G2 & 29 & D4 & $A X$ & 158 & 166 & $\mathrm{~F}$ & 5.8 & 1 & 5.8 & Tremolite & & \\
\hline G2 & 31 & A7 & $A X$ & 171 & 179 & $\mathrm{~F}$ & 5.8 & 1.25 & 4.6 & Tremolite & & \\
\hline G2 & 33 & E7 & $A X$ & 186 & 195 & $\mathrm{~F}$ & 11.2 & 0.85 & 13.2 & Tremolite & & \\
\hline G2 & 28 & $\mathrm{~F} 4$ & $A X$ & 156 & 164 & $F$ & 12 & 2 & 6 & Tremolite & & \\
\hline
\end{tabular}


Job Number: 070434

SEA

ISO 10312, Direct Count Categories

Client: Idaho National Laboratory

Report Number: 070434R06

Date Received: 4/23/2007

Project Name: RARE

Lab/Cor Sample No.: S29

Client Sample No.: FB-2-R4

Description:

Filter Fraction: 1

Residual Ash Vol:
Aliquot Dilution: 0

Final Dilution: 0
Volume (L): 0

Lab Filter Area (mm2): 385

Grid Openings Analyzed: 35

Average Grid Opening Area: 0.009

Area Analyzed (mm2): 0.315

Analytical Sens. (struc/cc): 0

Dectection Limit. (struc/cc): 0

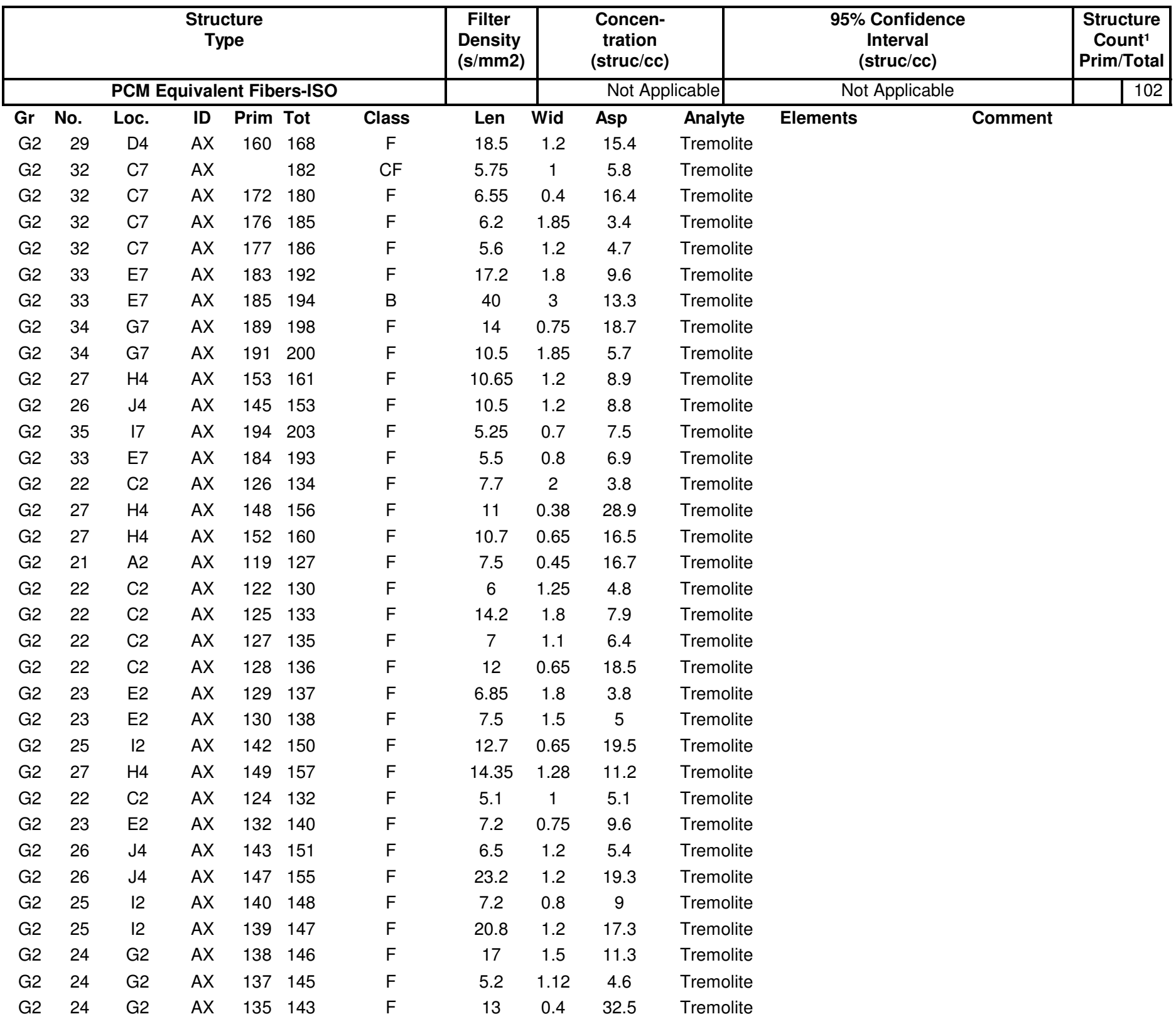


Job Number: 070434

SEA

ISO 10312, Direct Count Categories

Client: Idaho National Laboratory

Report Number: 070434R06

ct Name: RARE

Lab/Cor Sample No.: S29

Client Sample No.: FB-2-R4

Description:

Filter Fraction: 1

Residual Ash Vol:
Aliquot Dilution: 0

Final Dilution: 0
Date Received: 4/23/2007
Volume (L): 0

Lab Filter Area (mm2): 385

Grid Openings Analyzed: 35

Average Grid Opening Area: 0.009

Area Analyzed (mm2): 0.315

Analytical Sens. (struc/cc): 0

Dectection Limit. (struc/cc): 0

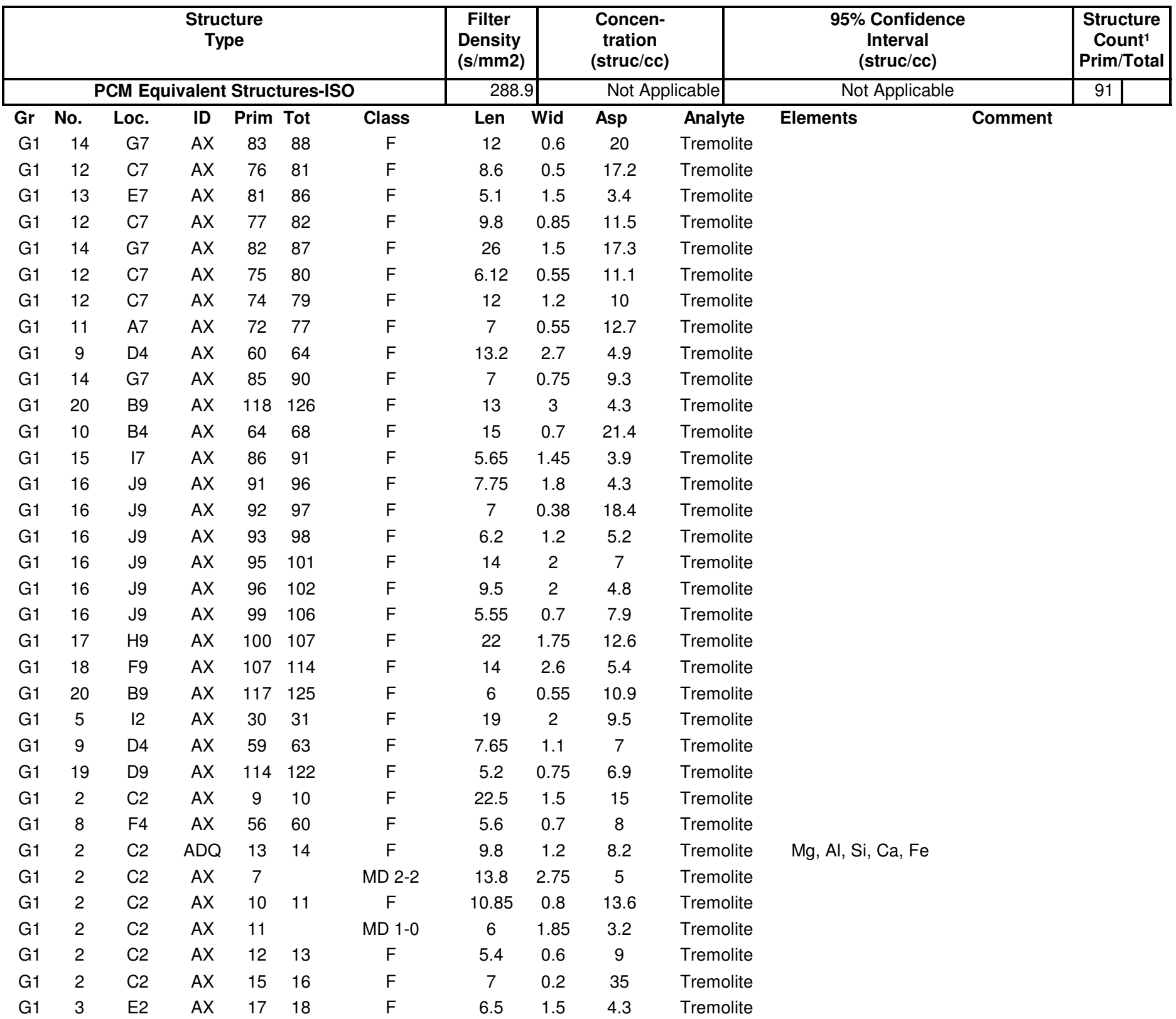


Job Number: 070434

SEA

ISO 10312, Direct Count Categories

Client: Idaho National Laboratory

Report Number: 070434R06

Date Received: 4/23/2007

Project Name: RARE

Lab/Cor Sample No.: S29

Client Sample No.: FB-2-R4

Description:

Filter Fraction: 1

Residual Ash Vol:
Aliquot Dilution: 0

Final Dilution: 0
Volume (L): 0

Lab Filter Area (mm2): 385

Grid Openings Analyzed: 35

Average Grid Opening Area: 0.009

Area Analyzed (mm2): 0.315

Analytical Sens. (struc/cc): 0

Dectection Limit. (struc/cc): 0

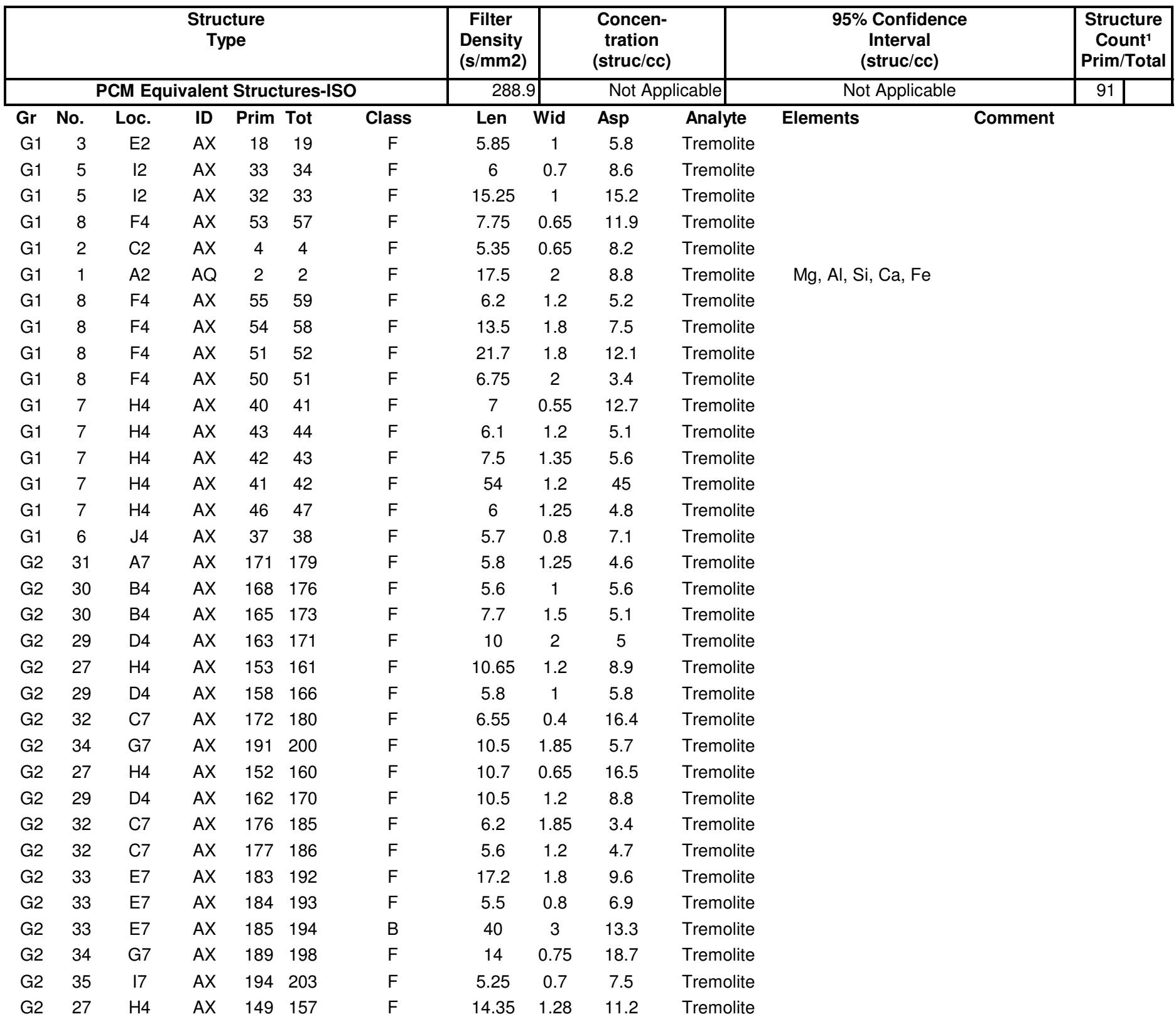


Job Number: 070434

SEA

ISO 10312, Direct Count Categories

Client: Idaho National Laboratory

Report Number: 070434R06

Date Received: 4/23/2007

Project Name: RARE

Lab/Cor Sample No.: S29

Client Sample No.: FB-2-R4

Description:

Filter Fraction: 1

Residual Ash Vol:
Aliquot Dilution: 0

Final Dilution: 0
Volume (L): 0

Lab Filter Area (mm2): 385

Grid Openings Analyzed: 35

Average Grid Opening Area: 0.009

Area Analyzed (mm2): 0.315

Analytical Sens. (struc/cc): 0

Dectection Limit. (struc/cc): 0

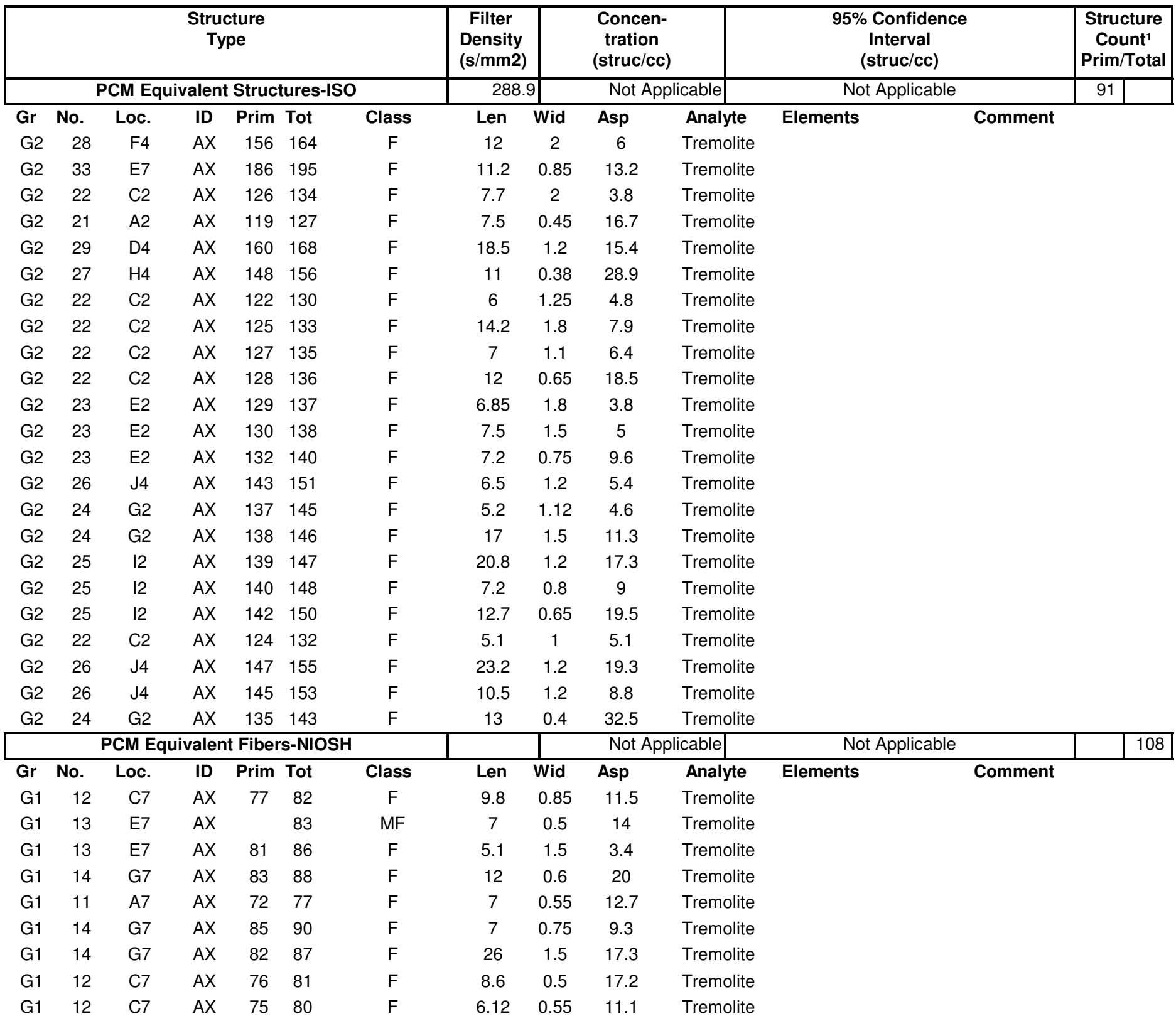


Job Number: 070434

SEA

ISO 10312, Direct Count Categories

Client: Idaho National Laboratory

Report Number: 070434R06

ct Name: RARE

Lab/Cor Sample No.: S29

Client Sample No.: FB-2-R4

Description:

Filter Fraction: 1

Residual Ash Vol:
Aliquot Dilution: 0

Final Dilution: 0
Date Received: 4/23/2007
Volume (L): 0

Lab Filter Area (mm2): 385

Grid Openings Analyzed: 35

Average Grid Opening Area: 0.009

Area Analyzed (mm2): 0.315

Analytical Sens. (struc/cc): 0

Dectection Limit. (struc/cc): 0

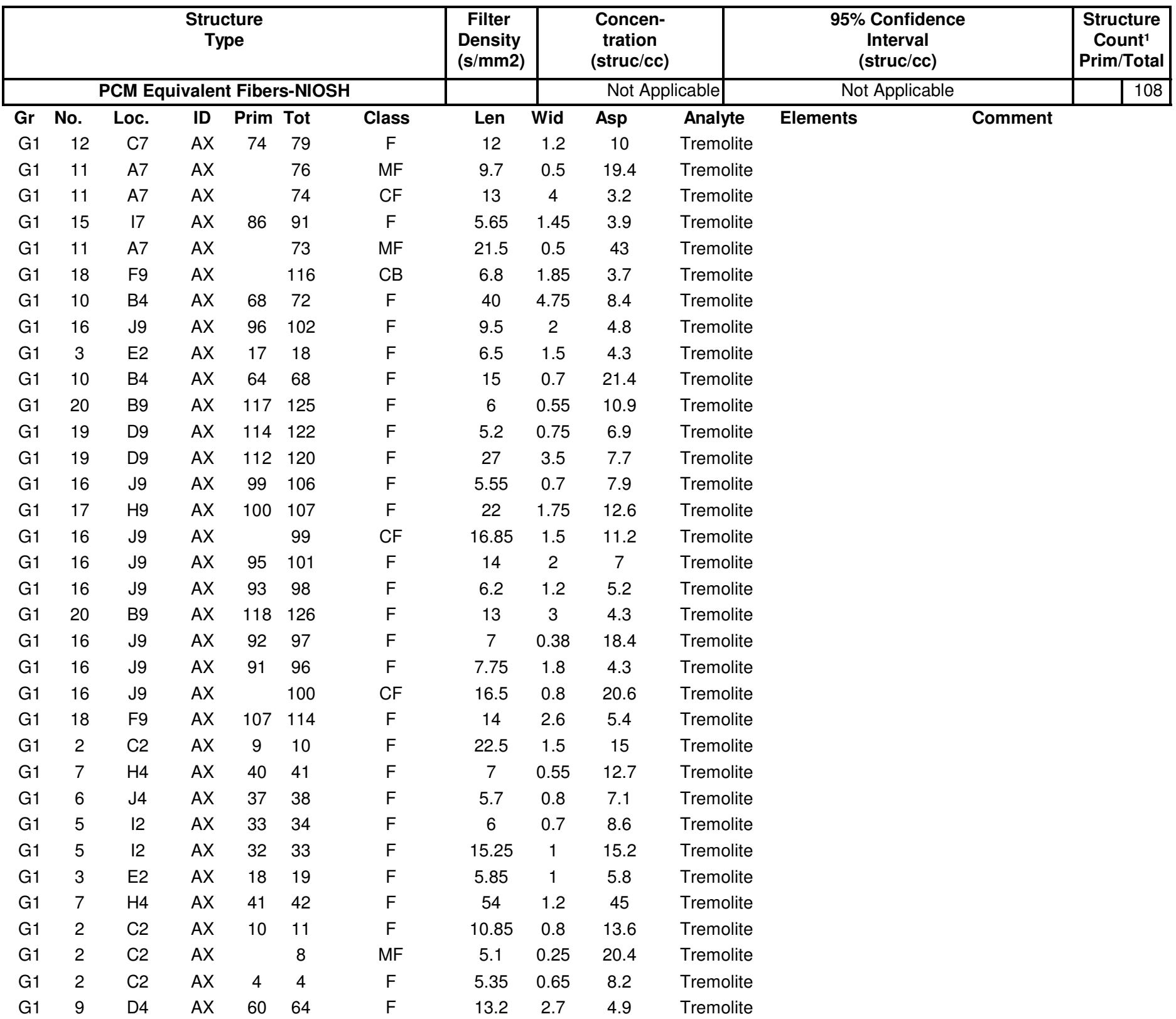


Job Number: 070434

SEA

ISO 10312, Direct Count Categories

Client: Idaho National Laboratory

Report Number: 070434R06

Date Received: 4/23/2007

Project Name: RARE

Lab/Cor Sample No.: S29

Client Sample No.: FB-2-R4

Description:

Filter Fraction: 1

Residual Ash Vol:
Aliquot Dilution: 0

Final Dilution: 0
Volume (L): 0

Lab Filter Area (mm2): 385

Grid Openings Analyzed: 35

Average Grid Opening Area: 0.009

Area Analyzed (mm2): 0.315

Analytical Sens. (struc/cc): 0

Dectection Limit. (struc/cc): 0

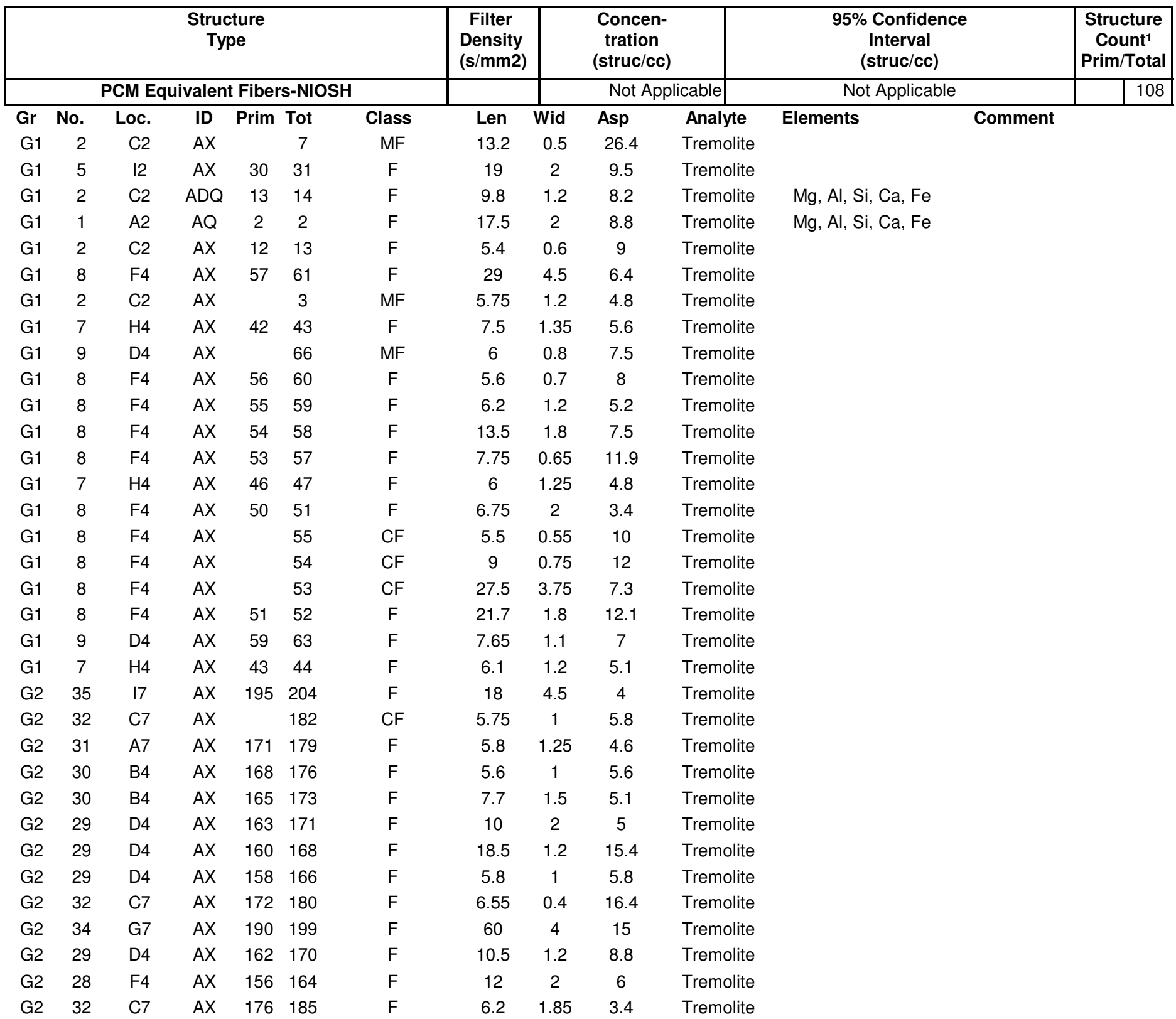


Job Number: 070434

SEA

ISO 10312, Direct Count Categories

Client: Idaho National Laboratory

Report Number: 070434R06

Date Received: 4/23/2007

Project Name: RARE

Lab/Cor Sample No.: S29

Client Sample No.: FB-2-R4

Description:

Filter Fraction: 1

Residual Ash Vol:
Aliquot Dilution: 0

Final Dilution: 0
Volume (L): 0

Lab Filter Area (mm2): 385

Grid Openings Analyzed: 35

Average Grid Opening Area: 0.009

Area Analyzed (mm2): 0.315

Analytical Sens. (struc/cc): 0

Dectection Limit. (struc/cc): 0

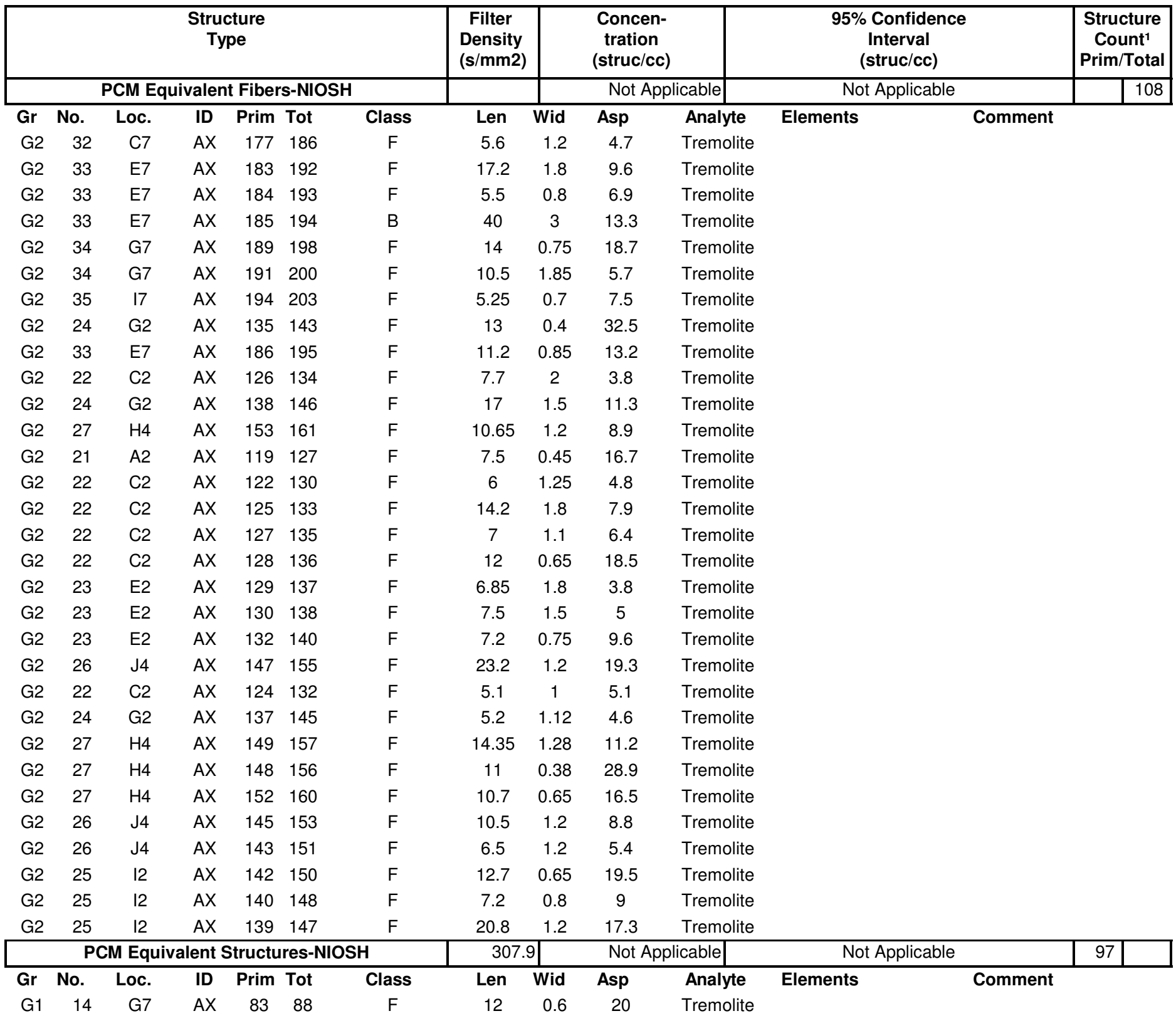


Job Number: 070434

SEA

ISO 10312, Direct Count Categories

Client: Idaho National Laboratory

Report Number: 070434R06

Date Received: 4/23/2007

Project Name: RARE

Lab/Cor Sample No.: S29

Client Sample No.: FB-2-R4

Description:

Filter Fraction: 1

Residual Ash Vol:
Aliquot Dilution: 0

Final Dilution: 0
Volume (L): 0

Lab Filter Area (mm2): 385

Grid Openings Analyzed: 35

Average Grid Opening Area: 0.009

Area Analyzed (mm2): 0.315

Analytical Sens. (struc/cc): 0

Dectection Limit. (struc/cc): 0

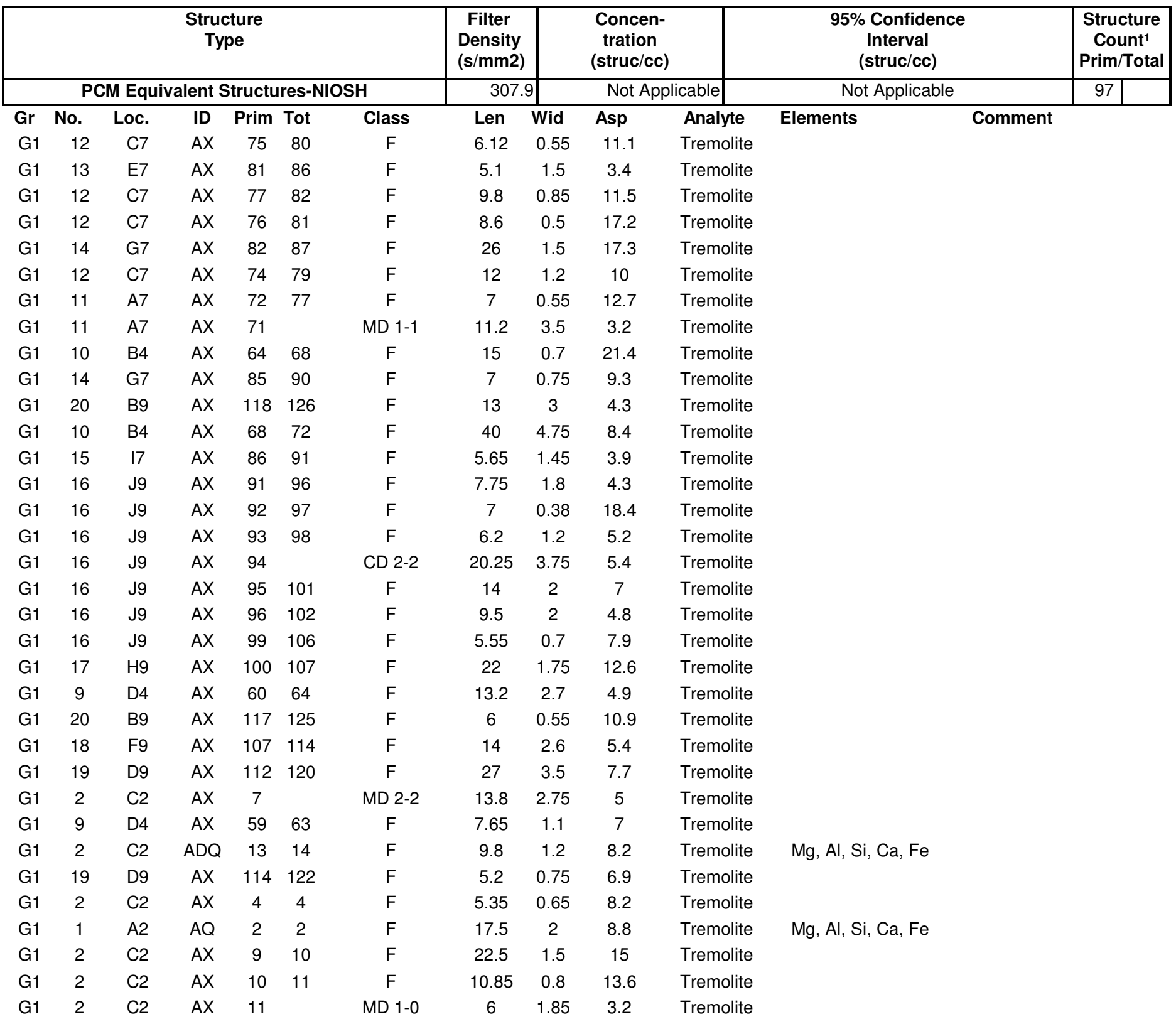


Job Number: 070434

SEA

ISO 10312, Direct Count Categories

Client: Idaho National Laboratory

Report Number: 070434R06

Date Received: 4/23/2007

Project Name: RARE

Lab/Cor Sample No.: S29

Client Sample No.: FB-2-R4

Description:

Filter Fraction: 1

Residual Ash Vol:
Aliquot Dilution: 0

Final Dilution: 0
Volume (L): 0

Lab Filter Area (mm2): 385

Grid Openings Analyzed: 35

Average Grid Opening Area: 0.009

Area Analyzed (mm2): 0.315

Analytical Sens. (struc/cc): 0

Dectection Limit. (struc/cc): 0

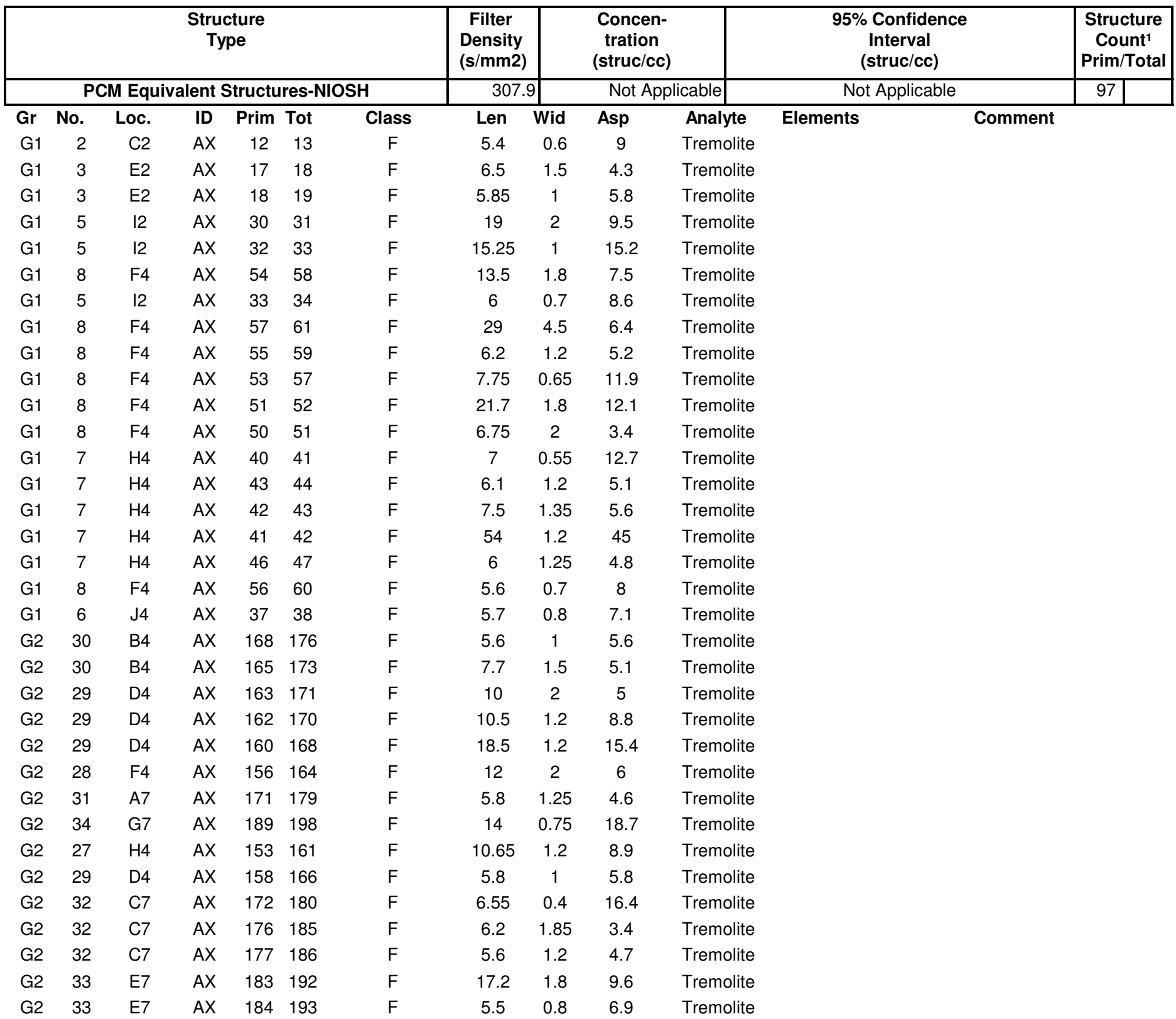


Job Number: 070434

SEA

ISO 10312, Direct Count Categories

Client: Idaho National Laboratory

Report Number: 070434R06

Date Received: 4/23/2007

Project Name: RARE

Lab/Cor Sample No.: S29

Client Sample No.: FB-2-R4

Description:

Filter Fraction: 1

Residual Ash Vol:
Aliquot Dilution: 0

Final Dilution: 0
Volume (L): 0

Lab Filter Area (mm2): 385

Grid Openings Analyzed: 35

Average Grid Opening Area: 0.009

Area Analyzed (mm2): 0.315

Analytical Sens. (struc/cc): 0

Dectection Limit. (struc/cc): 0

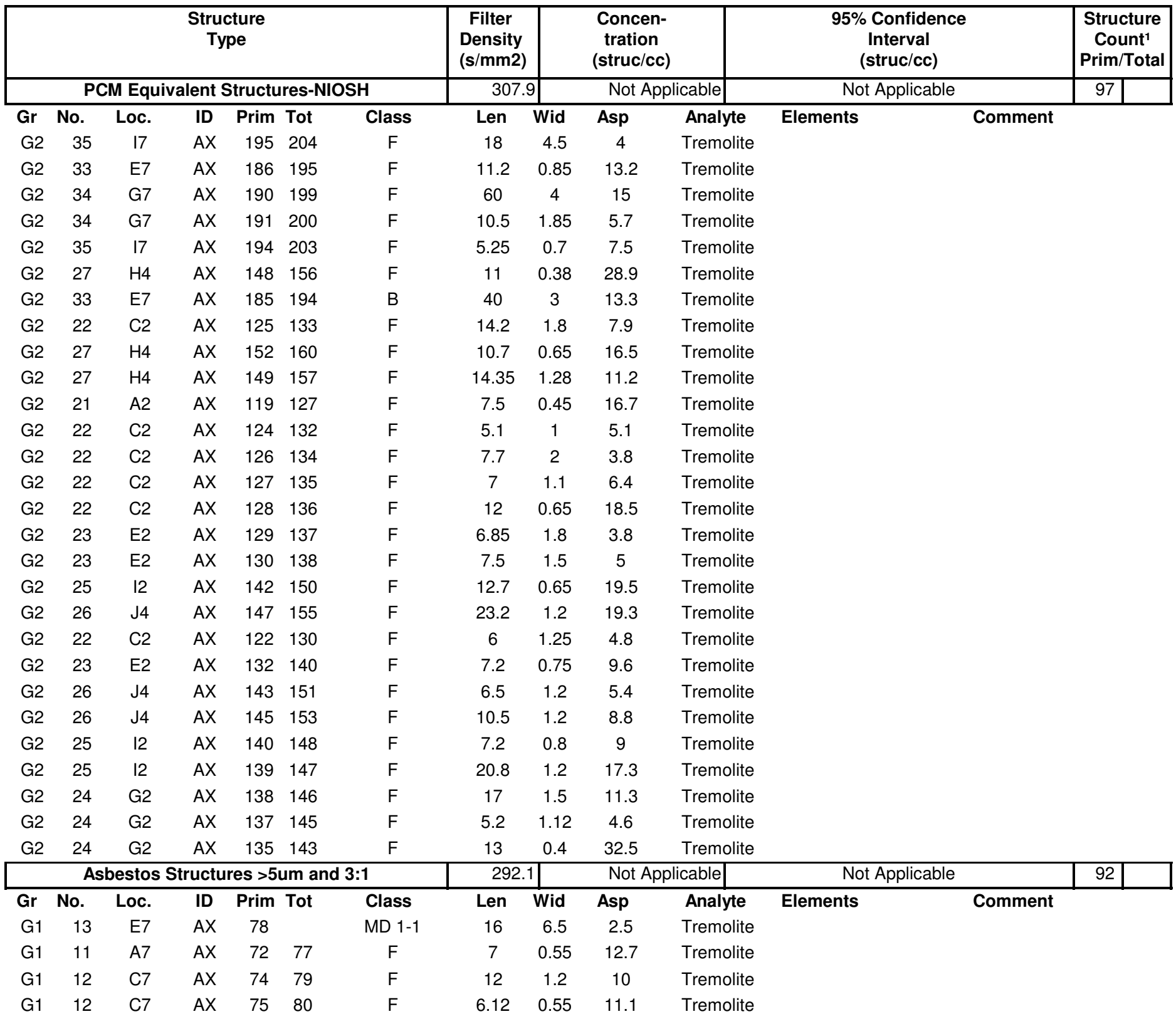


Job Number: 070434

SEA

ISO 10312, Direct Count Categories

Client: Idaho National Laboratory

Report Number: 070434R06

Date Received: 4/23/2007

Project Name: RARE

Lab/Cor Sample No.: S29

Client Sample No.: FB-2-R4

Description:

Filter Fraction: 1

Residual Ash Vol:
Aliquot Dilution: 0

Final Dilution: 0
Volume (L): 0

Lab Filter Area (mm2): 385

Grid Openings Analyzed: 35

Average Grid Opening Area: 0.009

Area Analyzed (mm2): 0.315

Analytical Sens. (struc/cc): 0

Dectection Limit. (struc/cc): 0

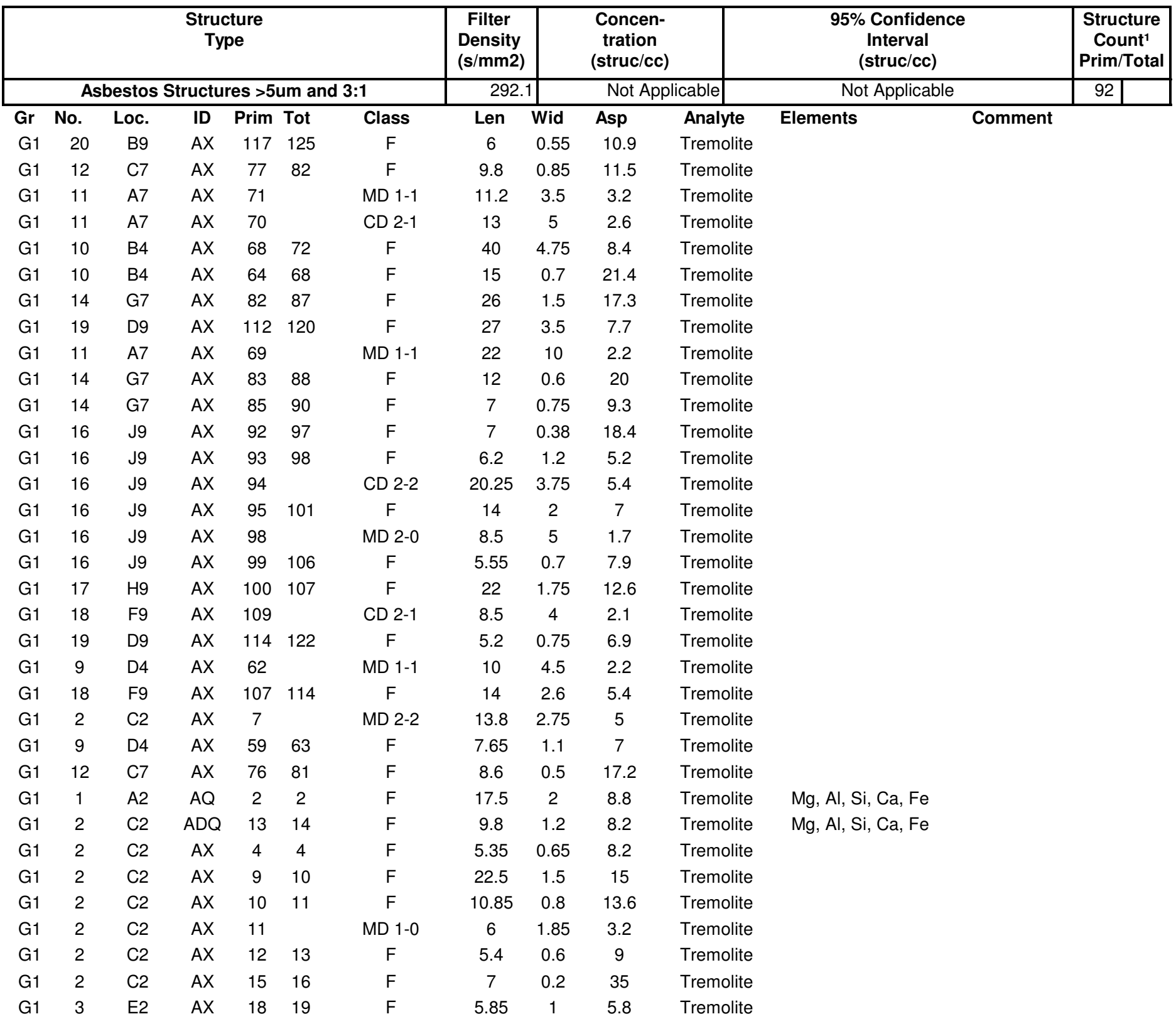


Job Number: 070434

SEA

ISO 10312, Direct Count Categories

Client: Idaho National Laboratory

Report Number: 070434R06

Date Received: 4/23/2007

Project Name: RARE

Lab/Cor Sample No.: S29

Client Sample No.: FB-2-R4

Description:

Filter Fraction: 1

Residual Ash Vol:
Aliquot Dilution: 0

Final Dilution: 0
Volume (L): 0

Lab Filter Area (mm2): 385

Grid Openings Analyzed: 35

Average Grid Opening Area: 0.009

Area Analyzed (mm2): 0.315

Analytical Sens. (struc/cc): 0

Dectection Limit. (struc/cc): 0

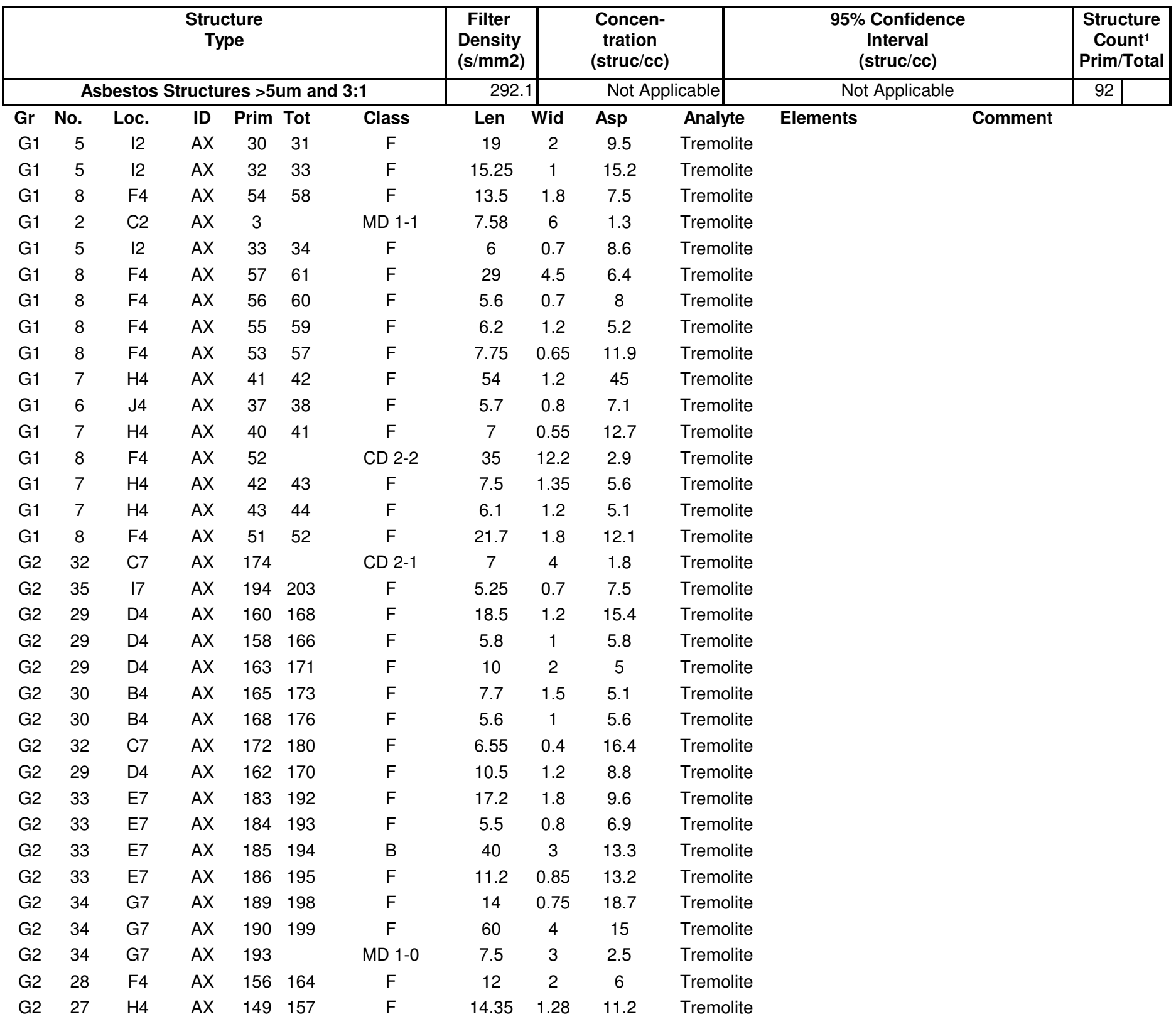


Job Number: 070434

SEA

ISO 10312, Direct Count Categories

Client: Idaho National Laboratory

Report Number: 070434R06

Date Received: 4/23/2007

Project Name: RARE

Lab/Cor Sample No.: S29

Client Sample No.: FB-2-R4

Description:

Filter Fraction: 1

Residual Ash Vol:
Aliquot Dilution: 0

Final Dilution: 0
Volume (L): 0

Lab Filter Area (mm2): 385

Grid Openings Analyzed: 35

Average Grid Opening Area: 0.009

Area Analyzed (mm2): 0.315

Analytical Sens. (struc/cc): 0

Dectection Limit. (struc/cc): 0

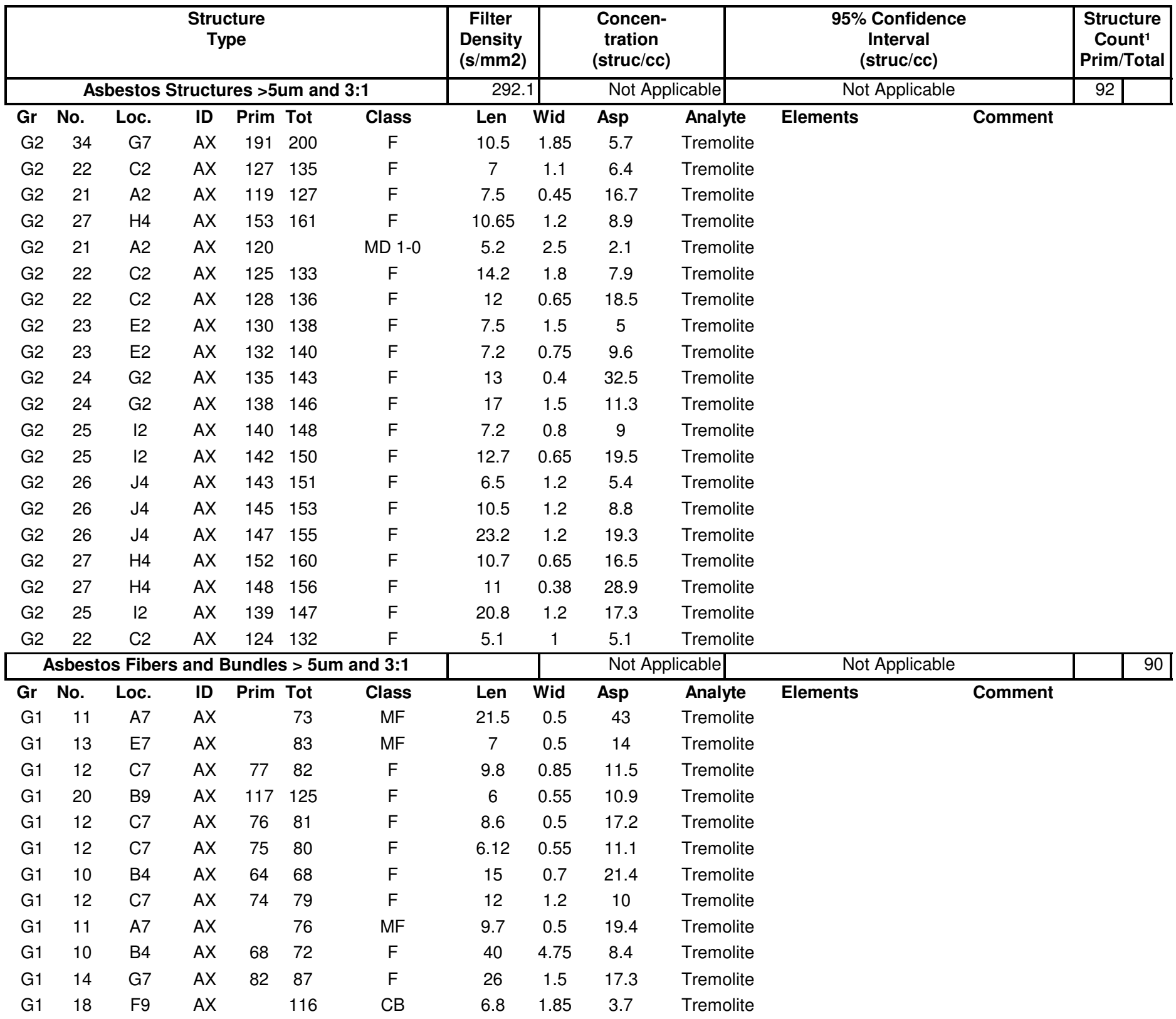


Job Number: 070434

SEA

ISO 10312, Direct Count Categories

Client: Idaho National Laboratory

Report Number: 070434R06

ct Name: RARE

Lab/Cor Sample No.: S29

Client Sample No.: FB-2-R4

Description:

Filter Fraction: 1

Residual Ash Vol:
Aliquot Dilution: 0

Final Dilution: 0
Date Received: 4/23/2007
Volume (L): 0

Lab Filter Area (mm2): 385

Grid Openings Analyzed: 35

Average Grid Opening Area: 0.009

Area Analyzed (mm2): 0.315

Analytical Sens. (struc/cc): 0

Dectection Limit. (struc/cc): 0

\begin{tabular}{|c|c|c|c|c|c|c|c|c|c|c|c|c|}
\hline & & & $\begin{array}{r}\text { Struc } \\
\text { Typ }\end{array}$ & $\begin{array}{l}\text { ture } \\
\text { oe }\end{array}$ & & & $\begin{array}{c}\text { Filter } \\
\text { Density } \\
(\mathrm{s} / \mathrm{mm} 2)\end{array}$ & & $\begin{array}{r}\text { Conce } \\
\text { tratio } \\
\text { (struc/ }\end{array}$ & & $\begin{array}{c}\text { 95\% Confidence } \\
\text { Interval } \\
\text { (struc/cc) }\end{array}$ & $\begin{array}{l}\text { Structure } \\
\text { Count }^{1} \\
\text { Prim/Total }\end{array}$ \\
\hline & Asbest & s Fibe & and $B$ & undles & $s>5 t$ & nd $3: 1$ & & & Not & licable & Not Applicable & 90 \\
\hline Gr & No. & Loc. & ID & Prim & Tot & Class & Len & Wid & Asp & Analyte & Elements & \\
\hline G1 & 11 & A7 & $A X$ & 72 & 77 & $\mathrm{~F}$ & 7 & 0.55 & 12.7 & Tremolite & & \\
\hline G1 & 14 & G7 & $A X$ & 83 & 88 & $F$ & 12 & 0.6 & 20 & Tremolite & & \\
\hline G1 & 14 & G7 & $A X$ & 85 & 90 & $F$ & 7 & 0.75 & 9.3 & Tremolite & & \\
\hline G1 & 16 & J9 & $A X$ & & 99 & $\mathrm{CF}$ & 16.85 & 1.5 & 11.2 & Tremolite & & \\
\hline G1 & 16 & J9 & $A X$ & & 100 & CF & 16.5 & 0.8 & 20.6 & Tremolite & & \\
\hline G1 & 16 & J9 & $A X$ & 92 & 97 & $\mathrm{~F}$ & 7 & 0.38 & 18.4 & Tremolite & & \\
\hline G1 & 16 & J9 & $A X$ & 93 & 98 & $\mathrm{~F}$ & 6.2 & 1.2 & 5.2 & Tremolite & & \\
\hline G1 & 16 & J9 & $A X$ & 95 & 101 & $F$ & 14 & 2 & 7 & Tremolite & & \\
\hline G1 & 17 & H9 & $A X$ & 100 & 107 & $\mathrm{~F}$ & 22 & 1.75 & 12.6 & Tremolite & & \\
\hline G1 & 9 & D4 & $A X$ & 59 & 63 & $\mathrm{~F}$ & 7.65 & 1.1 & 7 & Tremolite & & \\
\hline G1 & 18 & F9 & $A X$ & 107 & 114 & $F$ & 14 & 2.6 & 5.4 & Tremolite & & \\
\hline G1 & 19 & D9 & $A X$ & 112 & 120 & $\mathrm{~F}$ & 27 & 3.5 & 7.7 & Tremolite & & \\
\hline G1 & 19 & D9 & $A X$ & 114 & 122 & $F$ & 5.2 & 0.75 & 6.9 & Tremolite & & \\
\hline G1 & 7 & $\mathrm{H} 4$ & $A X$ & 41 & 42 & $\mathrm{~F}$ & 54 & 1.2 & 45 & Tremolite & & \\
\hline G1 & 16 & J9 & $A X$ & 99 & 106 & $\mathrm{~F}$ & 5.55 & 0.7 & 7.9 & Tremolite & & \\
\hline G1 & 2 & $\mathrm{C} 2$ & $A X$ & 4 & 4 & $\mathrm{~F}$ & 5.35 & 0.65 & 8.2 & Tremolite & & \\
\hline G1 & 7 & $\mathrm{H} 4$ & $A X$ & 43 & 44 & $\mathrm{~F}$ & 6.1 & 1.2 & 5.1 & Tremolite & & \\
\hline G1 & 9 & D4 & $A X$ & & 66 & MF & 6 & 0.8 & 7.5 & Tremolite & & \\
\hline G1 & 1 & $\mathrm{~A} 2$ & $A Q$ & 2 & 2 & $\mathrm{~F}$ & 17.5 & 2 & 8.8 & Tremolite & $\mathrm{Mg}, \mathrm{Al}, \mathrm{Si}, \mathrm{Ca}, \mathrm{Fe}$ & \\
\hline G1 & 2 & $\mathrm{C} 2$ & $\mathrm{ADQ}$ & 13 & 14 & $\mathrm{~F}$ & 9.8 & 1.2 & 8.2 & Tremolite & $\mathrm{Mg}, \mathrm{Al}, \mathrm{Si}, \mathrm{Ca}, \mathrm{Fe}$ & \\
\hline G1 & 2 & $\mathrm{C} 2$ & $A X$ & & 8 & MF & 5.1 & 0.25 & 20.4 & Tremolite & & \\
\hline G1 & 2 & $\mathrm{C} 2$ & $A X$ & 9 & 10 & $\mathrm{~F}$ & 22.5 & 1.5 & 15 & Tremolite & & \\
\hline G1 & 2 & $\mathrm{C} 2$ & $A X$ & 10 & 11 & $\mathrm{~F}$ & 10.85 & 0.8 & 13.6 & Tremolite & & \\
\hline G1 & 2 & $\mathrm{C} 2$ & $A X$ & 12 & 13 & $\mathrm{~F}$ & 5.4 & 0.6 & 9 & Tremolite & & \\
\hline G1 & 2 & $\mathrm{C} 2$ & $A X$ & 15 & 16 & $\mathrm{~F}$ & 7 & 0.2 & 35 & Tremolite & & \\
\hline G1 & 3 & E2 & $A X$ & 18 & 19 & $\mathrm{~F}$ & 5.85 & 1 & 5.8 & Tremolite & & \\
\hline G1 & 5 & 12 & $A X$ & 30 & 31 & $\mathrm{~F}$ & 19 & 2 & 9.5 & Tremolite & & \\
\hline G1 & 5 & 12 & $A X$ & 32 & 33 & $\mathrm{~F}$ & 15.25 & 1 & 15.2 & Tremolite & & \\
\hline G1 & 8 & $\mathrm{~F} 4$ & $A X$ & 54 & 58 & $F$ & 13.5 & 1.8 & 7.5 & Tremolite & & \\
\hline G1 & 8 & $\mathrm{~F} 4$ & $A X$ & 57 & 61 & $\mathrm{~F}$ & 29 & 4.5 & 6.4 & Tremolite & & \\
\hline G1 & 2 & $\mathrm{C} 2$ & $A X$ & & 7 & MF & 13.2 & 0.5 & 26.4 & Tremolite & & \\
\hline G1 & 5 & 12 & $A X$ & 33 & 34 & $\mathrm{~F}$ & 6 & 0.7 & 8.6 & Tremolite & & \\
\hline G1 & 8 & $\mathrm{~F} 4$ & $A X$ & 56 & 60 & $F$ & 5.6 & 0.7 & 8 & Tremolite & & \\
\hline G1 & 8 & $\mathrm{~F} 4$ & $A X$ & 55 & 59 & $\mathrm{~F}$ & 6.2 & 1.2 & 5.2 & Tremolite & & \\
\hline
\end{tabular}


Job Number: 070434

SEA

ISO 10312, Direct Count Categories

Client: Idaho National Laboratory

Report Number: 070434R06

Date Received: 4/23/2007

Project Name: RARE

Lab/Cor Sample No.: S29

Client Sample No.: FB-2-R4

Description:

Filter Fraction: 1

Residual Ash Vol:
Aliquot Dilution: 0

Final Dilution: 0
Volume (L): 0

Lab Filter Area (mm2): 385

Grid Openings Analyzed: 35

Average Grid Opening Area: 0.009

Area Analyzed (mm2): 0.315

Analytical Sens. (struc/cc): 0

Dectection Limit. (struc/cc): 0

\begin{tabular}{|c|c|c|c|c|c|c|c|c|c|c|c|c|}
\hline & & & Stru & $\begin{array}{l}\text { ture } \\
\text { pe }\end{array}$ & & & $\begin{array}{c}\text { Filter } \\
\text { Density } \\
(\mathrm{s} / \mathrm{mm} 2)\end{array}$ & & $\begin{array}{r}\text { Conc } \\
\text { tratic } \\
\text { (struc }\end{array}$ & & $\begin{array}{c}\text { 95\% Confidence } \\
\text { Interval } \\
\text { (struc/cc) }\end{array}$ & $\begin{array}{c}\text { Structure } \\
\text { Count }^{1} \\
\text { Prim/Total }\end{array}$ \\
\hline & Asbes & s Fibe & and & undle & $s>5 t$ & ind $3: 1$ & & & Not & licable & Not Applicable & 90 \\
\hline Gr & No. & Loc. & ID & Prim & Tot & Class & Len & Wid & Asp & Analyte & Elements & \\
\hline G1 & 8 & $\mathrm{~F} 4$ & $A X$ & 53 & 57 & $\mathrm{~F}$ & 7.75 & 0.65 & 11.9 & Tremolite & & \\
\hline G1 & 8 & $\mathrm{~F} 4$ & $A X$ & 51 & 52 & $\mathrm{~F}$ & 21.7 & 1.8 & 12.1 & Tremolite & & \\
\hline G1 & 8 & $\mathrm{~F} 4$ & $A X$ & & 55 & CF & 5.5 & 0.55 & 10 & Tremolite & & \\
\hline G1 & 8 & $\mathrm{~F} 4$ & $A X$ & & 54 & $\mathrm{CF}$ & 9 & 0.75 & 12 & Tremolite & & \\
\hline G1 & 8 & $\mathrm{~F} 4$ & $A X$ & & 53 & $\mathrm{CF}$ & 27.5 & 3.75 & 7.3 & Tremolite & & \\
\hline G1 & 7 & $\mathrm{H} 4$ & $A X$ & 42 & 43 & $\mathrm{~F}$ & 7.5 & 1.35 & 5.6 & Tremolite & & \\
\hline G1 & 7 & $\mathrm{H} 4$ & $A X$ & 40 & 41 & $\mathrm{~F}$ & 7 & 0.55 & 12.7 & Tremolite & & \\
\hline G1 & 6 & J4 & $A X$ & 37 & 38 & $\mathrm{~F}$ & 5.7 & 0.8 & 7.1 & Tremolite & & \\
\hline G2 & 29 & D4 & $A X$ & 158 & 166 & $\mathrm{~F}$ & 5.8 & 1 & 5.8 & Tremolite & & \\
\hline G2 & 34 & G7 & $A X$ & 190 & 199 & $\mathrm{~F}$ & 60 & 4 & 15 & Tremolite & & \\
\hline G2 & 29 & D4 & $A X$ & 162 & 170 & $\mathrm{~F}$ & 10.5 & 1.2 & 8.8 & Tremolite & & \\
\hline G2 & 29 & D4 & $A X$ & 163 & 171 & $\mathrm{~F}$ & 10 & 2 & 5 & Tremolite & & \\
\hline G2 & 30 & B4 & $A X$ & 165 & 173 & $\mathrm{~F}$ & 7.7 & 1.5 & 5.1 & Tremolite & & \\
\hline G2 & 30 & B4 & $A X$ & 168 & 176 & $\mathrm{~F}$ & 5.6 & 1 & 5.6 & Tremolite & & \\
\hline G2 & 32 & C7 & $A X$ & & 182 & CF & 5.75 & 1 & 5.8 & Tremolite & & \\
\hline G2 & 29 & D4 & $A X$ & 160 & 168 & $\mathrm{~F}$ & 18.5 & 1.2 & 15.4 & Tremolite & & \\
\hline G2 & 32 & C7 & $A X$ & 172 & 180 & $\mathrm{~F}$ & 6.55 & 0.4 & 16.4 & Tremolite & & \\
\hline G2 & 33 & E7 & $A X$ & 183 & 192 & $\mathrm{~F}$ & 17.2 & 1.8 & 9.6 & Tremolite & & \\
\hline G2 & 33 & E7 & $A X$ & 184 & 193 & $\mathrm{~F}$ & 5.5 & 0.8 & 6.9 & Tremolite & & \\
\hline G2 & 33 & E7 & $A X$ & 185 & 194 & B & 40 & 3 & 13.3 & Tremolite & & \\
\hline G2 & 34 & G7 & $A X$ & 189 & 198 & $\mathrm{~F}$ & 14 & 0.75 & 18.7 & Tremolite & & \\
\hline G2 & 34 & G7 & $A X$ & 191 & 200 & $\mathrm{~F}$ & 10.5 & 1.85 & 5.7 & Tremolite & & \\
\hline G2 & 35 & 17 & $A X$ & 194 & 203 & $\mathrm{~F}$ & 5.25 & 0.7 & 7.5 & Tremolite & & \\
\hline G2 & 28 & $\mathrm{~F} 4$ & $A X$ & 156 & 164 & $\mathrm{~F}$ & 12 & 2 & 6 & Tremolite & & \\
\hline G2 & 24 & $\mathrm{G} 2$ & $A X$ & 135 & 143 & $\mathrm{~F}$ & 13 & 0.4 & 32.5 & Tremolite & & \\
\hline G2 & 33 & E7 & $A X$ & 186 & 195 & $\mathrm{~F}$ & 11.2 & 0.85 & 13.2 & Tremolite & & \\
\hline G2 & 22 & $\mathrm{C} 2$ & $A X$ & 128 & 136 & $\mathrm{~F}$ & 12 & 0.65 & 18.5 & Tremolite & & \\
\hline G2 & 21 & A2 & $A X$ & 119 & 127 & $\mathrm{~F}$ & 7.5 & 0.45 & 16.7 & Tremolite & & \\
\hline G2 & 22 & $\mathrm{C} 2$ & $A X$ & 124 & 132 & $\mathrm{~F}$ & 5.1 & 1 & 5.1 & Tremolite & & \\
\hline G2 & 25 & 12 & $A X$ & 139 & 147 & $\mathrm{~F}$ & 20.8 & 1.2 & 17.3 & Tremolite & & \\
\hline G2 & 22 & $\mathrm{C} 2$ & $A X$ & 127 & 135 & $\mathrm{~F}$ & 7 & 1.1 & 6.4 & Tremolite & & \\
\hline G2 & 27 & $\mathrm{H} 4$ & $A X$ & 153 & 161 & $\mathrm{~F}$ & 10.65 & 1.2 & 8.9 & Tremolite & & \\
\hline G2 & 23 & E2 & $A X$ & 130 & 138 & $\mathrm{~F}$ & 7.5 & 1.5 & 5 & Tremolite & & \\
\hline G2 & 23 & E2 & $A X$ & 132 & 140 & $F$ & 7.2 & 0.75 & 9.6 & Tremolite & & \\
\hline
\end{tabular}


A Professional Service Corporation in the Northwest

ISO 10312, Direct Count Categories

Job Number: 070434

SEA

Report Number: 070434R06

Client: Idaho National Laboratory

Date Received: 4/23/2007

Project Name: RARE

Lab/Cor Sample No.: S29

Client Sample No.: FB-2-R4

Description:

Filter Fraction: 1

Residual Ash Vol:
Aliquot Dilution: 0

Final Dilution: 0
Volume (L): 0

Lab Filter Area (mm2): 385

Grid Openings Analyzed: 35

Average Grid Opening Area: 0.009

Area Analyzed (mm2): 0.315

Analytical Sens. (struc/cc): 0

Dectection Limit. (struc/cc): 0

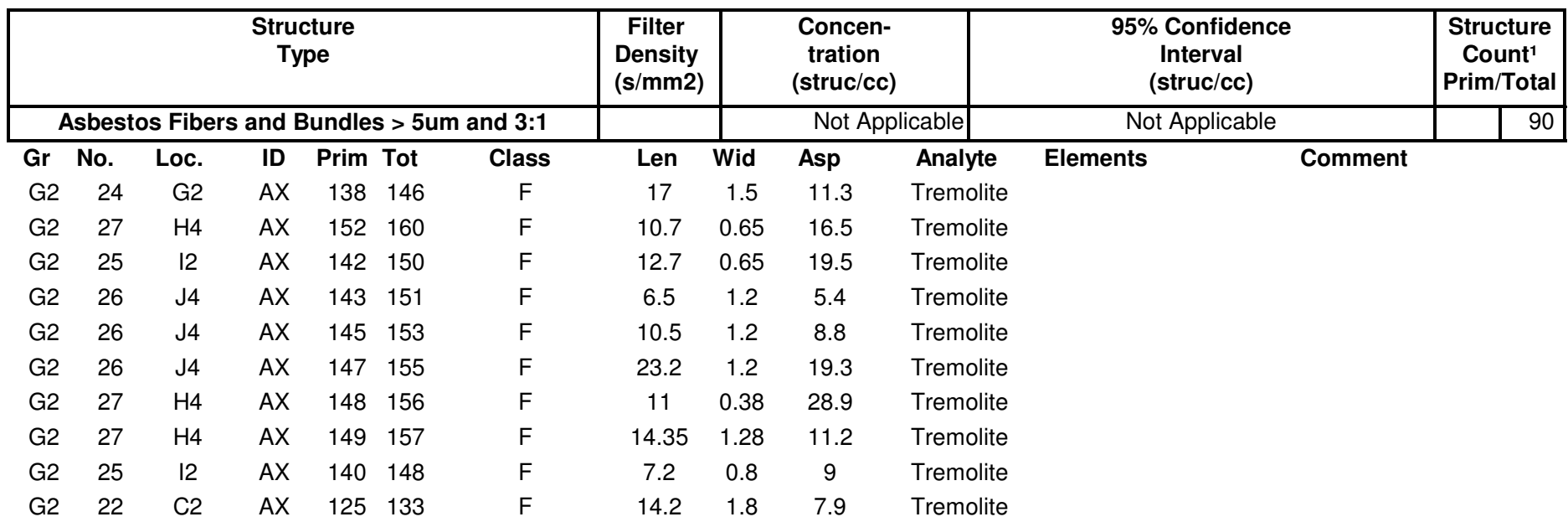


Job Number: 070434

SEA

ISO 10312, Direct Count Categories

Client: Idaho National Laboratory

Report Number: 070434R06

Date Received: 4/23/2007

Project Name: RARE

Lab/Cor Sample No.: S30

Client Sample No.: FB-2-R5

Description:

Filter Fraction: 1

Residual Ash Vol:
Aliquot Dilution: 0

Final Dilution: 0
Volume (L): 0

Lab Filter Area (mm2): 385

Grid Openings Analyzed: 35

Average Grid Opening Area: 0.009

Area Analyzed (mm2): 0.315

Analytical Sens. (struc/cc): 0

Dectection Limit. (struc/cc): 0

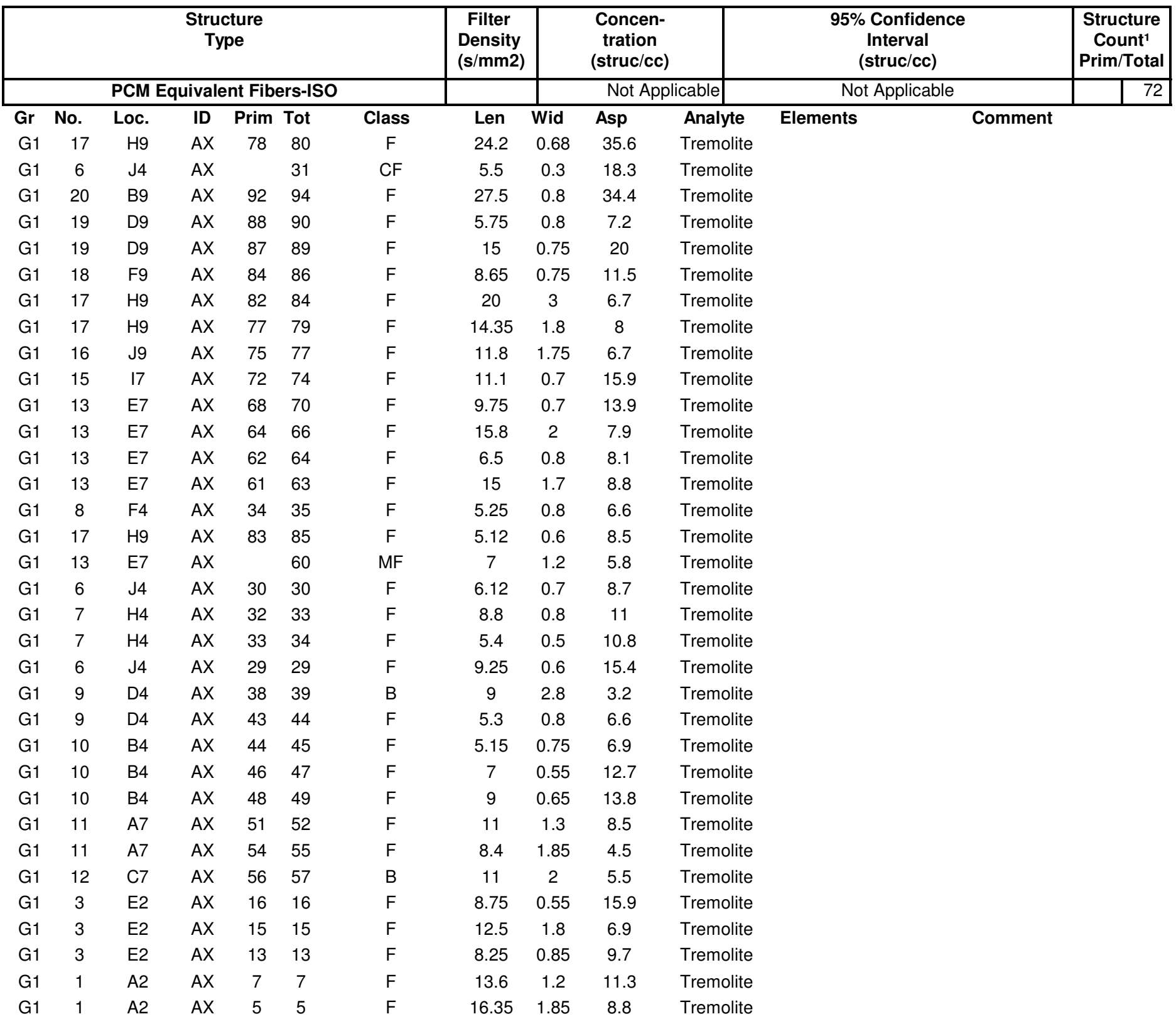


Job Number: 070434

SEA

ISO 10312, Direct Count Categories

Client: Idaho National Laboratory

Report Number: 070434R06

Date Received: 4/23/2007

Project Name: RARE

Lab/Cor Sample No.: S30

Client Sample No.: FB-2-R5

Description:

Filter Fraction: 1

Residual Ash Vol:
Aliquot Dilution: 0

Final Dilution: 0
Volume (L): 0

Lab Filter Area (mm2): 385

Grid Openings Analyzed: 35

Average Grid Opening Area: 0.009

Area Analyzed (mm2): 0.315

Analytical Sens. (struc/cc): 0

Dectection Limit. (struc/cc): 0

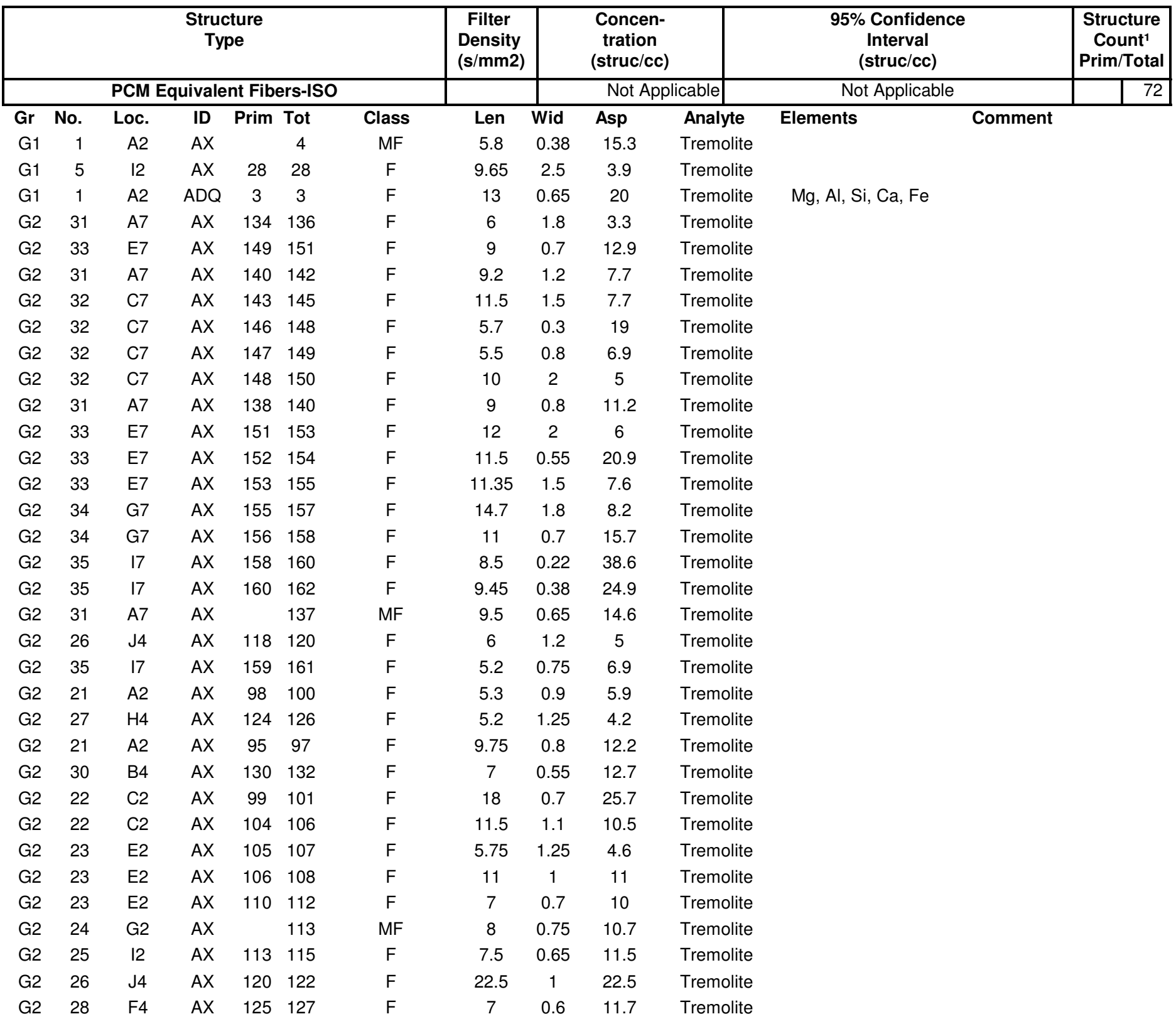


Job Number: 070434

SEA

ISO 10312, Direct Count Categories

Client: Idaho National Laboratory

Report Number: 070434R06

Date Received: 4/23/2007

Project Name: RARE

Lab/Cor Sample No.: S30

Client Sample No.: FB-2-R5

Description:

Filter Fraction: 1

Residual Ash Vol:
Aliquot Dilution: 0

Final Dilution: 0
Volume (L): 0

Lab Filter Area (mm2): 385

Grid Openings Analyzed: 35

Average Grid Opening Area: 0.009

Area Analyzed (mm2): 0.315

Analytical Sens. (struc/cc): 0

Dectection Limit. (struc/cc): 0

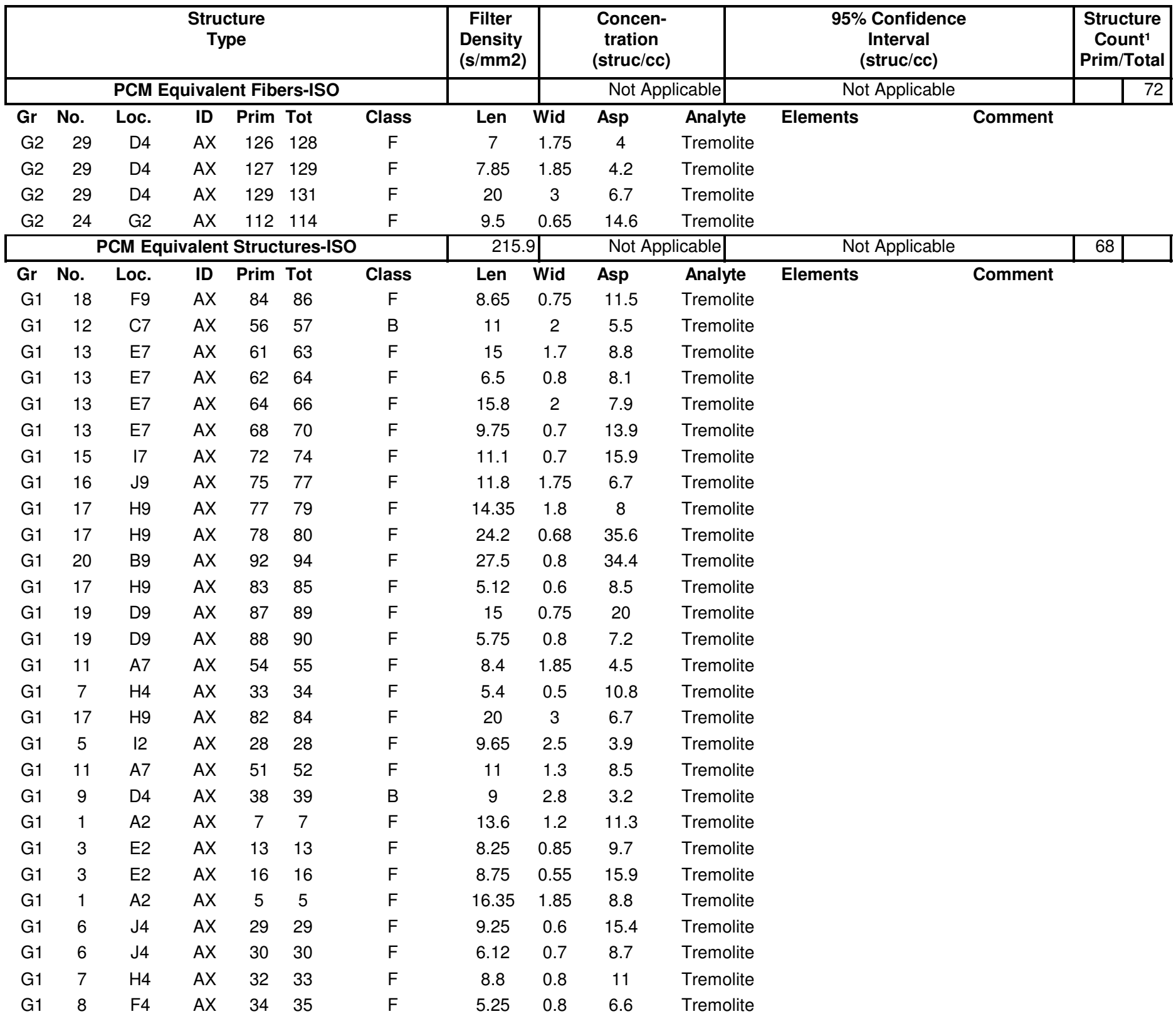


Job Number: 070434

SEA

ISO 10312, Direct Count Categories

Client: Idaho National Laboratory

Report Number: 070434R06

Date Received: 4/23/2007

Project Name: RARE

Lab/Cor Sample No.: S30

Client Sample No.: FB-2-R5

Description:

Filter Fraction: 1

Residual Ash Vol:
Aliquot Dilution: 0

Final Dilution: 0
Volume (L): 0

Lab Filter Area (mm2): 385

Grid Openings Analyzed: 35

Average Grid Opening Area: 0.009

Area Analyzed (mm2): 0.315

Analytical Sens. (struc/cc): 0

Dectection Limit. (struc/cc): 0

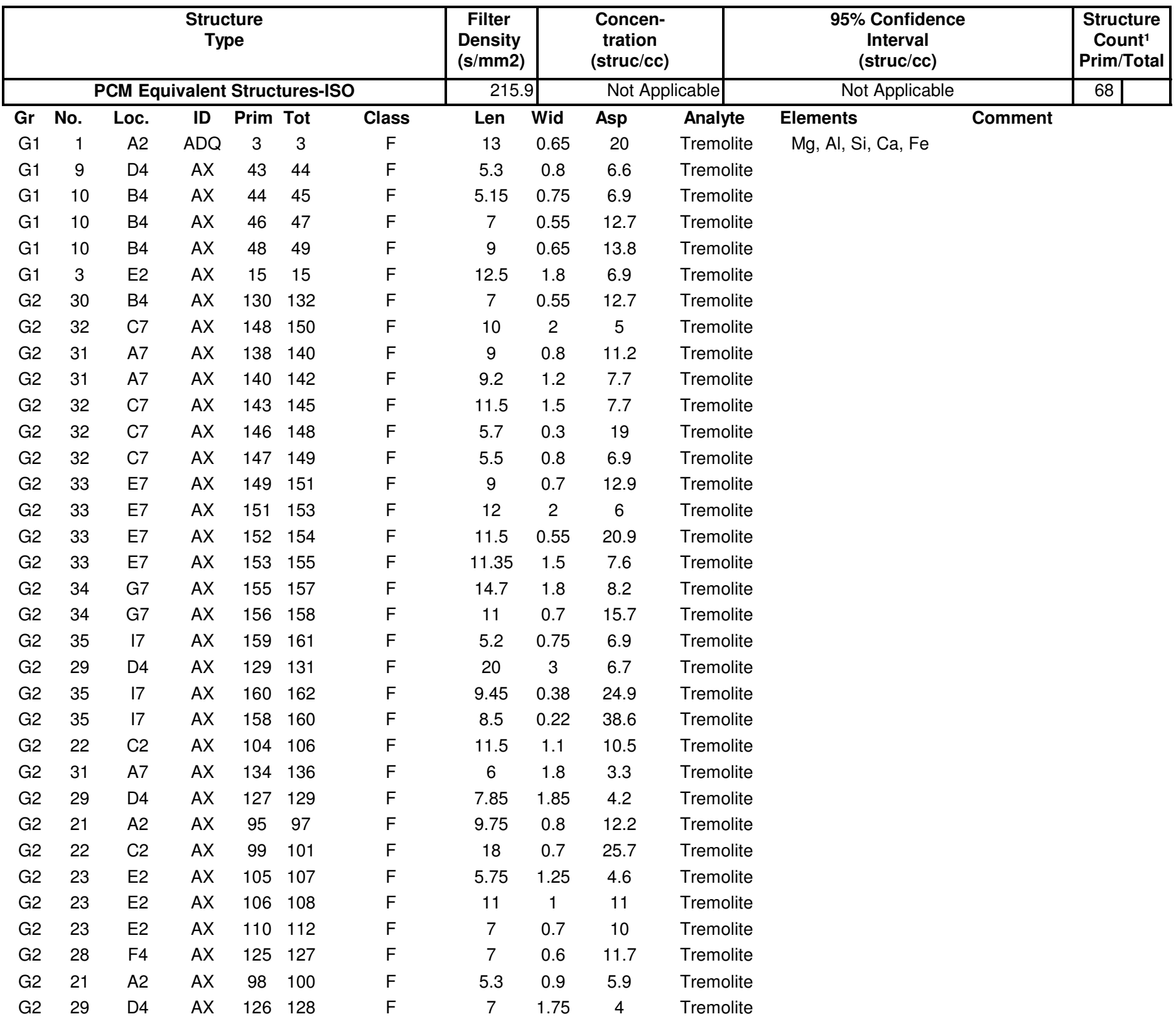


Job Number: 070434

SEA

ISO 10312, Direct Count Categories

Client: Idaho National Laboratory

Report Number: 070434R06

Date Received: 4/23/2007

Project Name: RARE

Lab/Cor Sample No.: S30

Client Sample No.: FB-2-R5

Description:

Filter Fraction: 1

Residual Ash Vol:
Aliquot Dilution: 0

Final Dilution: 0
Volume (L): 0

Lab Filter Area (mm2): 385

Grid Openings Analyzed: 35

Average Grid Opening Area: 0.009

Area Analyzed (mm2): 0.315

Analytical Sens. (struc/cc): 0

Dectection Limit. (struc/cc): 0

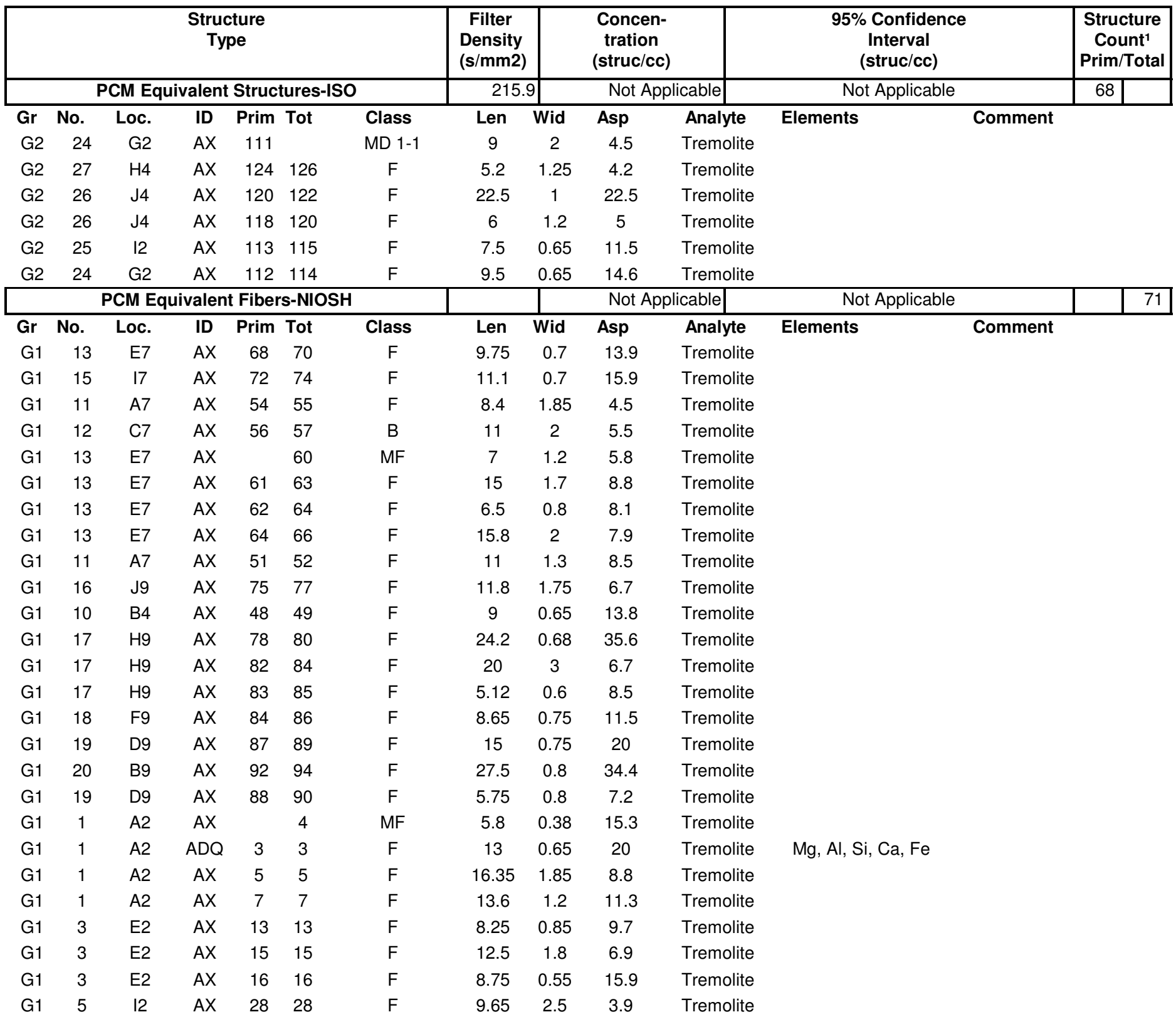


Job Number: 070434

SEA

ISO 10312, Direct Count Categories

Client: Idaho National Laboratory

Report Number: 070434R06

ct Name: RARE

Lab/Cor Sample No.: S30

Client Sample No.: FB-2-R5

Description:

Filter Fraction: 1

Residual Ash Vol:
Aliquot Dilution: 0

Final Dilution: 0
Date Received: 4/23/2007
Volume (L): 0

Lab Filter Area (mm2): 385

Grid Openings Analyzed: 35

Average Grid Opening Area: 0.009

Area Analyzed (mm2): 0.315

Analytical Sens. (struc/cc): 0

Dectection Limit. (struc/cc): 0

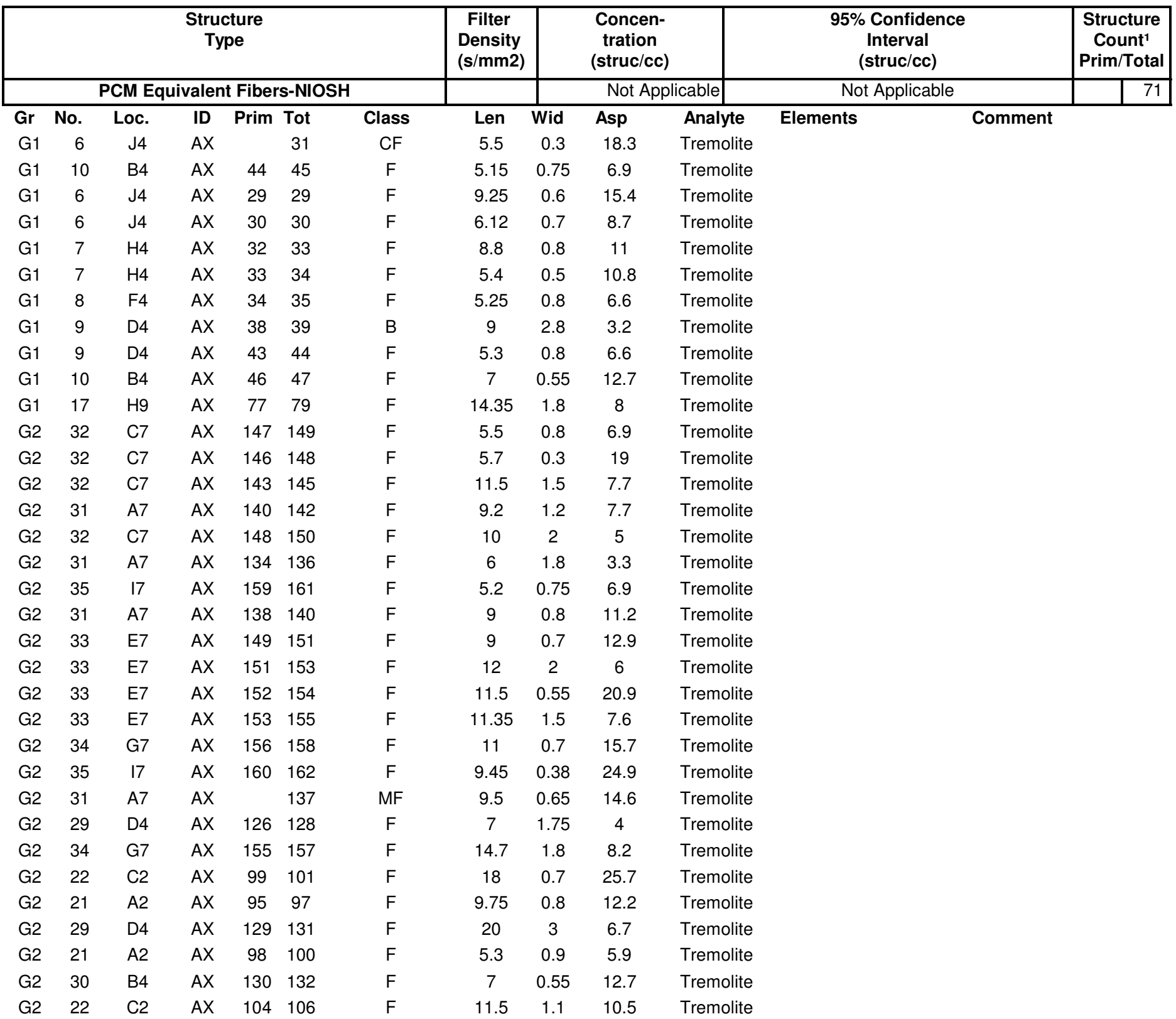


Job Number: 070434

SEA

ISO 10312, Direct Count Categories

Client: Idaho National Laboratory

Report Number: 070434R06

Date Received: 4/23/2007

Project Name: RARE

Lab/Cor Sample No.: S30

Client Sample No.: FB-2-R5

Description:

Filter Fraction: 1

Residual Ash Vol:
Aliquot Dilution: 0

Final Dilution: 0
Volume (L): 0

Lab Filter Area (mm2): 385

Grid Openings Analyzed: 35

Average Grid Opening Area: 0.009

Area Analyzed (mm2): 0.315

Analytical Sens. (struc/cc): 0

Dectection Limit. (struc/cc): 0

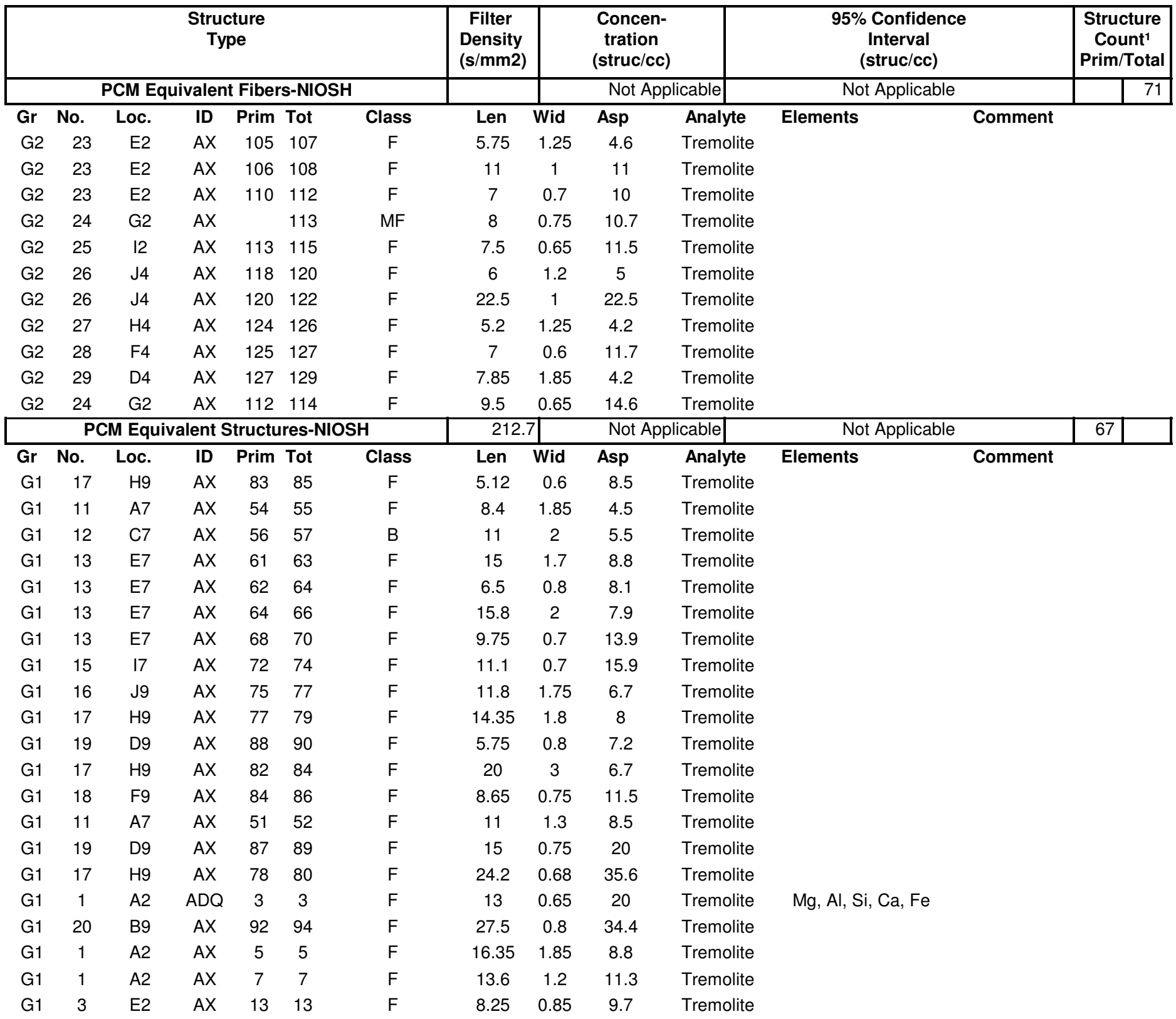


Job Number: 070434

SEA

ISO 10312, Direct Count Categories

Client: Idaho National Laboratory

Report Number: 070434R06

Date Received: 4/23/2007

Project Name: RARE

Lab/Cor Sample No.: S30

Client Sample No.: FB-2-R5

Description:

Filter Fraction: 1

Residual Ash Vol:
Aliquot Dilution: 0

Final Dilution: 0
Volume (L): 0

Lab Filter Area (mm2): 385

Grid Openings Analyzed: 35

Average Grid Opening Area: 0.009

Area Analyzed (mm2): 0.315

Analytical Sens. (struc/cc): 0

Dectection Limit. (struc/cc): 0

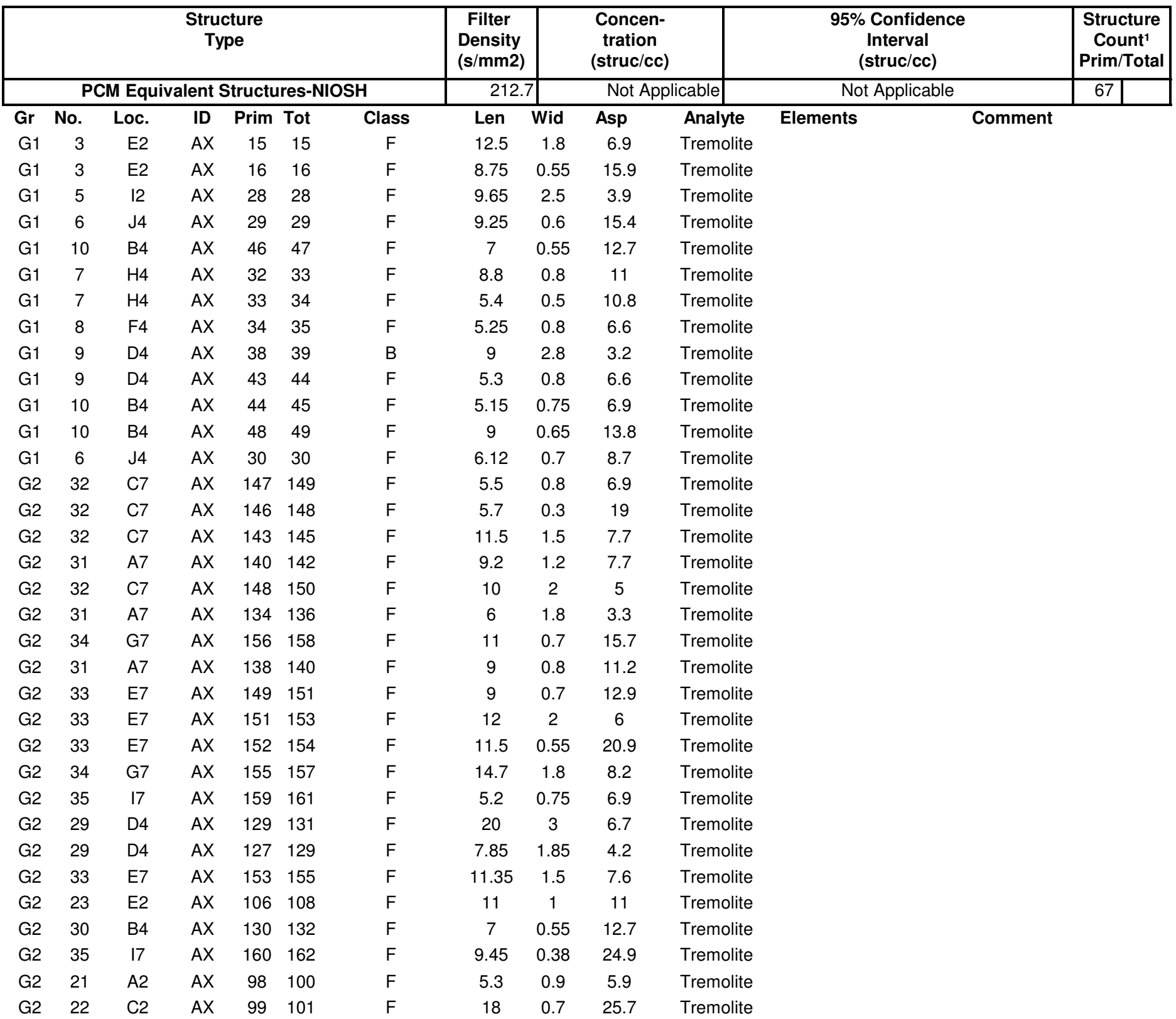


Job Number: 070434

SEA

ISO 10312, Direct Count Categories

Client: Idaho National Laboratory

Report Number: 070434R06

Date Received: 4/23/2007

Project Name: RARE

Lab/Cor Sample No.: S30

Client Sample No.: FB-2-R5

Description:

Filter Fraction: 1

Residual Ash Vol:
Aliquot Dilution: 0

Final Dilution: 0
Volume (L): 0

Lab Filter Area (mm2): 385

Grid Openings Analyzed: 35

Average Grid Opening Area: 0.009

Area Analyzed (mm2): 0.315

Analytical Sens. (struc/cc): 0

Dectection Limit. (struc/cc): 0

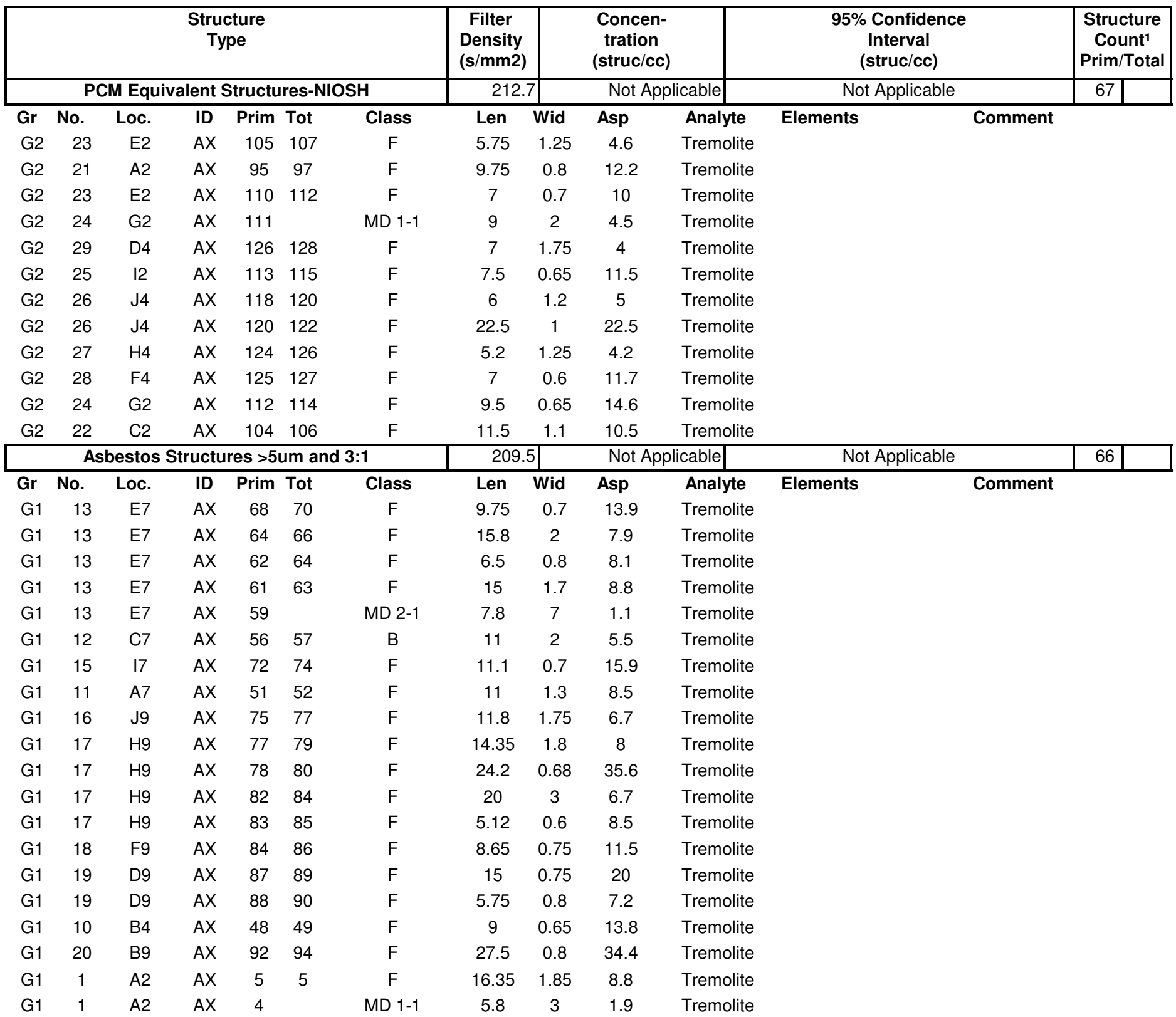


Job Number: 070434

SEA

ISO 10312, Direct Count Categories

Client: Idaho National Laboratory

Report Number: 070434R06

Date Received: 4/23/2007

Project Name: RARE

Lab/Cor Sample No.: S30

Client Sample No.: FB-2-R5

Description:

Filter Fraction: 1

Residual Ash Vol:
Aliquot Dilution: 0

Final Dilution: 0
Volume (L): 0

Lab Filter Area (mm2): 385

Grid Openings Analyzed: 35

Average Grid Opening Area: 0.009

Area Analyzed (mm2): 0.315

Analytical Sens. (struc/cc): 0

Dectection Limit. (struc/cc): 0

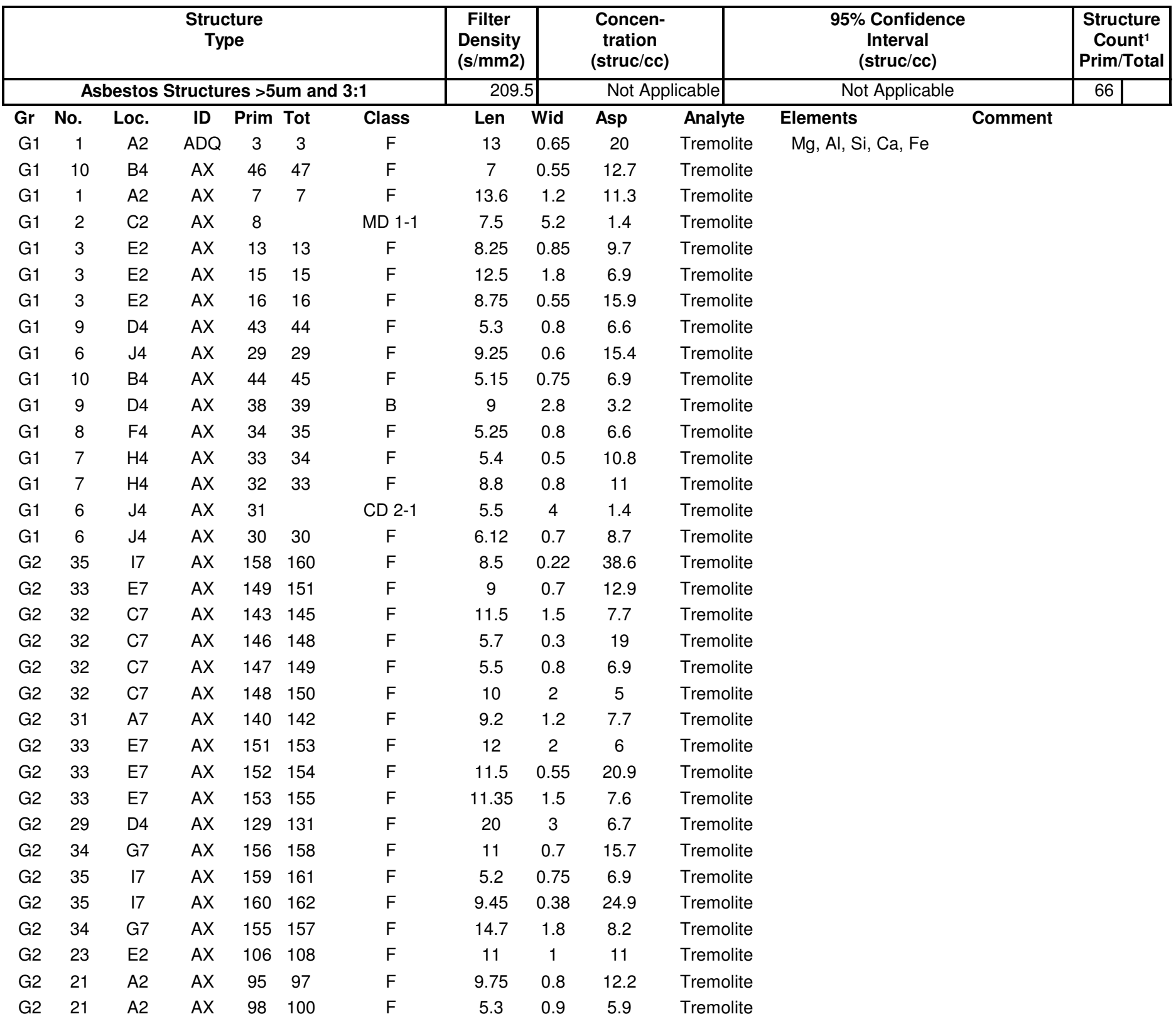


Job Number: 070434

SEA

ISO 10312, Direct Count Categories

Client: Idaho National Laboratory

Report Number: 070434R06

Date Received: 4/23/2007

Project Name: RARE

Lab/Cor Sample No.: S30

Client Sample No.: FB-2-R5

Description:

Filter Fraction: 1

Residual Ash Vol:
Aliquot Dilution: 0

Final Dilution: 0
Volume (L): 0

Lab Filter Area (mm2): 385

Grid Openings Analyzed: 35

Average Grid Opening Area: 0.009

Area Analyzed (mm2): 0.315

Analytical Sens. (struc/cc): 0

Dectection Limit. (struc/cc): 0

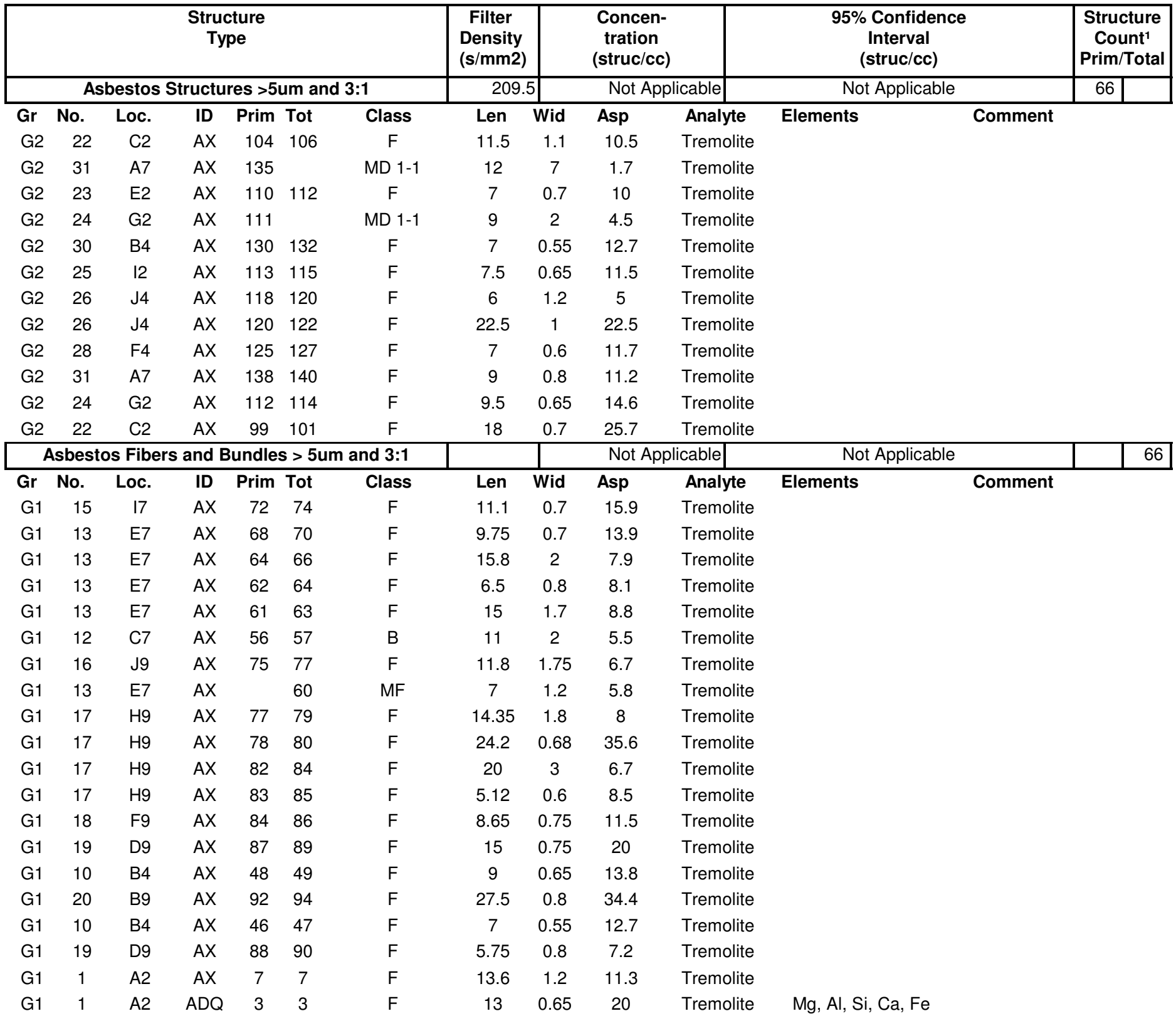


Job Number: 070434

SEA

ISO 10312, Direct Count Categories

Client: Idaho National Laboratory

Report Number: 070434R06

ct Name: RARE

Lab/Cor Sample No.: S30

Client Sample No.: FB-2-R5

Description:

Filter Fraction: 1

Residual Ash Vol:
Aliquot Dilution: 0

Final Dilution: 0
Date Received: 4/23/2007
Volume (L): 0

Lab Filter Area (mm2): 385

Grid Openings Analyzed: 35

Average Grid Opening Area: 0.009

Area Analyzed (mm2): 0.315

Analytical Sens. (struc/cc): 0

Dectection Limit. (struc/cc): 0

\begin{tabular}{|c|c|c|c|c|c|c|c|c|c|c|c|c|}
\hline & & & Stru & $\begin{array}{l}\text { ture } \\
\text { pe }\end{array}$ & & & $\begin{array}{c}\text { Filter } \\
\text { Density } \\
(\mathrm{s} / \mathrm{mm} 2)\end{array}$ & & $\begin{array}{r}\text { Conc } \\
\text { tratic } \\
\text { (struc }\end{array}$ & & $\begin{array}{c}\text { 95\% Confidence } \\
\text { Interval } \\
\text { (struc/cc) }\end{array}$ & $\begin{array}{c}\text { Structure } \\
\text { Count }^{1} \\
\text { Prim/Total }\end{array}$ \\
\hline & Asbes & s Fibe & and & undle & $s>5 t$ & ind $3: 1$ & & & Not & licable & Not Applicable & 66 \\
\hline Gr & No. & Loc. & ID & Prim & Tot & Class & Len & Wid & Asp & Analyte & Elements & \\
\hline G1 & 11 & A7 & $A X$ & 51 & 52 & $\mathrm{~F}$ & 11 & 1.3 & 8.5 & Tremolite & & \\
\hline G1 & 1 & $\mathrm{~A} 2$ & $A X$ & 5 & 5 & $\mathrm{~F}$ & 16.35 & 1.85 & 8.8 & Tremolite & & \\
\hline G1 & 2 & $\mathrm{C} 2$ & $A X$ & & 8 & MF & 5.5 & 0.18 & 30.6 & Tremolite & & \\
\hline G1 & 3 & E2 & $A X$ & 13 & 13 & $\mathrm{~F}$ & 8.25 & 0.85 & 9.7 & Tremolite & & \\
\hline G1 & 3 & E2 & $A X$ & 15 & 15 & $\mathrm{~F}$ & 12.5 & 1.8 & 6.9 & Tremolite & & \\
\hline G1 & 3 & E2 & $A X$ & 16 & 16 & $\mathrm{~F}$ & 8.75 & 0.55 & 15.9 & Tremolite & & \\
\hline G1 & 6 & $\mathrm{~J} 4$ & $A X$ & & 31 & $\mathrm{CF}$ & 5.5 & 0.3 & 18.3 & Tremolite & & \\
\hline G1 & 9 & D4 & $A X$ & 43 & 44 & $\mathrm{~F}$ & 5.3 & 0.8 & 6.6 & Tremolite & & \\
\hline G1 & 1 & $\mathrm{~A} 2$ & $A X$ & & 4 & MF & 5.8 & 0.38 & 15.3 & Tremolite & & \\
\hline G1 & 6 & $\mathrm{~J} 4$ & $A X$ & 29 & 29 & $\mathrm{~F}$ & 9.25 & 0.6 & 15.4 & Tremolite & & \\
\hline G1 & 10 & B4 & $A X$ & 44 & 45 & $F$ & 5.15 & 0.75 & 6.9 & Tremolite & & \\
\hline G1 & 9 & D4 & $A X$ & 38 & 39 & B & 9 & 2.8 & 3.2 & Tremolite & & \\
\hline G1 & 8 & $\mathrm{~F} 4$ & $A X$ & 34 & 35 & $\mathrm{~F}$ & 5.25 & 0.8 & 6.6 & Tremolite & & \\
\hline G1 & 7 & $\mathrm{H} 4$ & $A X$ & 33 & 34 & $\mathrm{~F}$ & 5.4 & 0.5 & 10.8 & Tremolite & & \\
\hline G1 & 7 & $\mathrm{H} 4$ & $A X$ & 32 & 33 & $\mathrm{~F}$ & 8.8 & 0.8 & 11 & Tremolite & & \\
\hline G1 & 6 & $J 4$ & $A X$ & 30 & 30 & $\mathrm{~F}$ & 6.12 & 0.7 & 8.7 & Tremolite & & \\
\hline G2 & 31 & A7 & $A X$ & 140 & 142 & $\mathrm{~F}$ & 9.2 & 1.2 & 7.7 & Tremolite & & \\
\hline G2 & 35 & 17 & $A X$ & 158 & 160 & $\mathrm{~F}$ & 8.5 & 0.22 & 38.6 & Tremolite & & \\
\hline G2 & 33 & E7 & $A X$ & 149 & 151 & $\mathrm{~F}$ & 9 & 0.7 & 12.9 & Tremolite & & \\
\hline G2 & 32 & C7 & $A X$ & 146 & 148 & $F$ & 5.7 & 0.3 & 19 & Tremolite & & \\
\hline G2 & 32 & C7 & $A X$ & 147 & 149 & $\mathrm{~F}$ & 5.5 & 0.8 & 6.9 & Tremolite & & \\
\hline G2 & 32 & C7 & $A X$ & 148 & 150 & $\mathrm{~F}$ & 10 & 2 & 5 & Tremolite & & \\
\hline G2 & 32 & C7 & $A X$ & 143 & 145 & $\mathrm{~F}$ & 11.5 & 1.5 & 7.7 & Tremolite & & \\
\hline G2 & 33 & E7 & $A X$ & 151 & 153 & $\mathrm{~F}$ & 12 & 2 & 6 & Tremolite & & \\
\hline G2 & 33 & E7 & $A X$ & 152 & 154 & $\mathrm{~F}$ & 11.5 & 0.55 & 20.9 & Tremolite & & \\
\hline G2 & 33 & E7 & $A X$ & 153 & 155 & $\mathrm{~F}$ & 11.35 & 1.5 & 7.6 & Tremolite & & \\
\hline G2 & 34 & G7 & $A X$ & 156 & 158 & $\mathrm{~F}$ & 11 & 0.7 & 15.7 & Tremolite & & \\
\hline G2 & 35 & 17 & $A X$ & 160 & 162 & $\mathrm{~F}$ & 9.45 & 0.38 & 24.9 & Tremolite & & \\
\hline G2 & 31 & A7 & $A X$ & 138 & 140 & $\mathrm{~F}$ & 9 & 0.8 & 11.2 & Tremolite & & \\
\hline G2 & 35 & 17 & $A X$ & 159 & 161 & $\mathrm{~F}$ & 5.2 & 0.75 & 6.9 & Tremolite & & \\
\hline G2 & 34 & G7 & $A X$ & 155 & 157 & $\mathrm{~F}$ & 14.7 & 1.8 & 8.2 & Tremolite & & \\
\hline G2 & 21 & $\mathrm{~A} 2$ & $A X$ & 98 & 100 & $\mathrm{~F}$ & 5.3 & 0.9 & 5.9 & Tremolite & & \\
\hline G2 & 31 & A7 & $A X$ & & 137 & MF & 9.5 & 0.65 & 14.6 & Tremolite & & \\
\hline G2 & 21 & $\mathrm{~A} 2$ & $A X$ & 95 & 97 & $F$ & 9.75 & 0.8 & 12.2 & Tremolite & & \\
\hline
\end{tabular}


A Professional Service Corporation in the Northwest

ISO 10312, Direct Count Categories

Job Number: 070434

SEA

Report Number: 070434R06

Client: Idaho National Laboratory

Date Received: 4/23/2007

Project Name: RARE

Lab/Cor Sample No.: S30

Client Sample No.: FB-2-R5

Description:

Filter Fraction: 1

Residual Ash Vol:
Aliquot Dilution: 0

Final Dilution: 0
Volume (L): 0

Lab Filter Area (mm2): 385

Grid Openings Analyzed: 35

Average Grid Opening Area: 0.009

Area Analyzed (mm2): 0.315

Analytical Sens. (struc/cc): 0

Dectection Limit. (struc/cc): 0

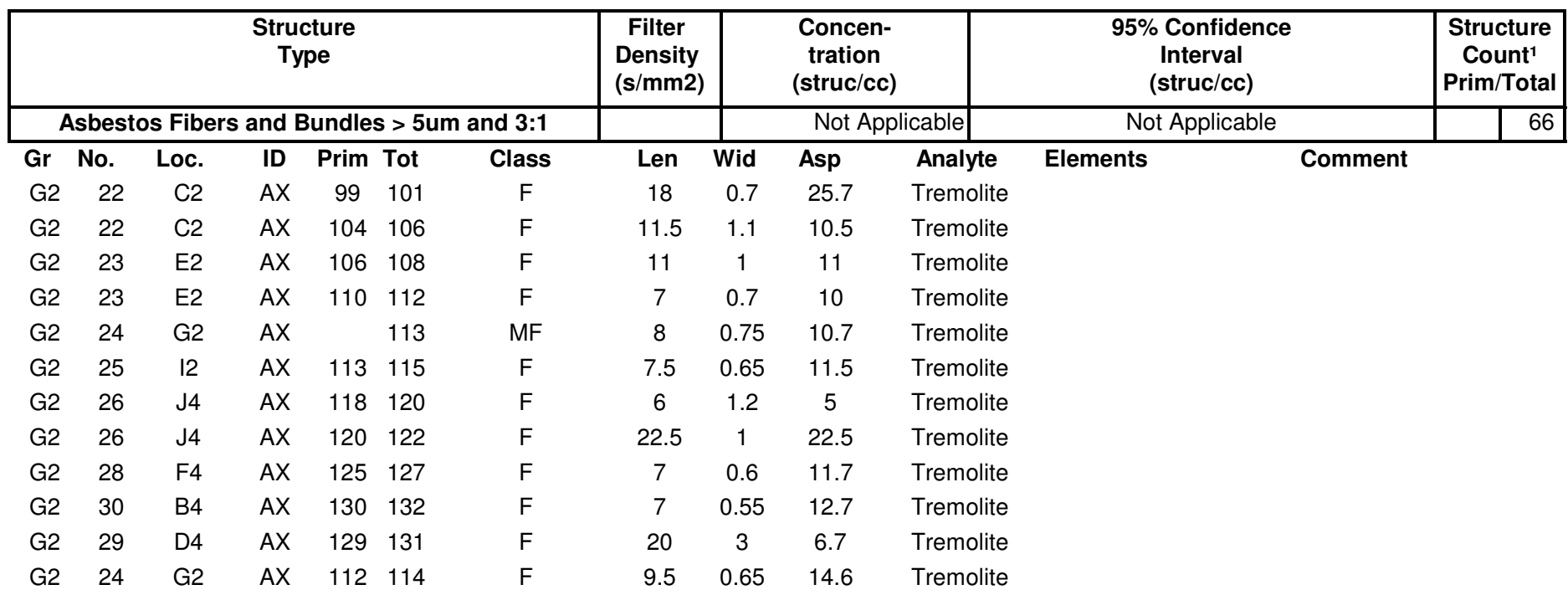


Job Number: 070434

SEA

ISO 10312, Direct Count Categories

Client: Idaho National Laboratory

Report Number: 070434R06

Date Received: 4/23/2007

Project Name: RARE

Lab/Cor Sample No.: S31

Client Sample No.: FB-2-R6

Description:

Filter Fraction: 1

Residual Ash Vol:
Aliquot Dilution: 0

Final Dilution: 0
Volume (L): 0

Lab Filter Area (mm2): 385

Grid Openings Analyzed: 10

Average Grid Opening Area: 0.009

Area Analyzed (mm2): 0.09

Analytical Sens. (struc/cc): 0

Dectection Limit. (struc/cc): 0

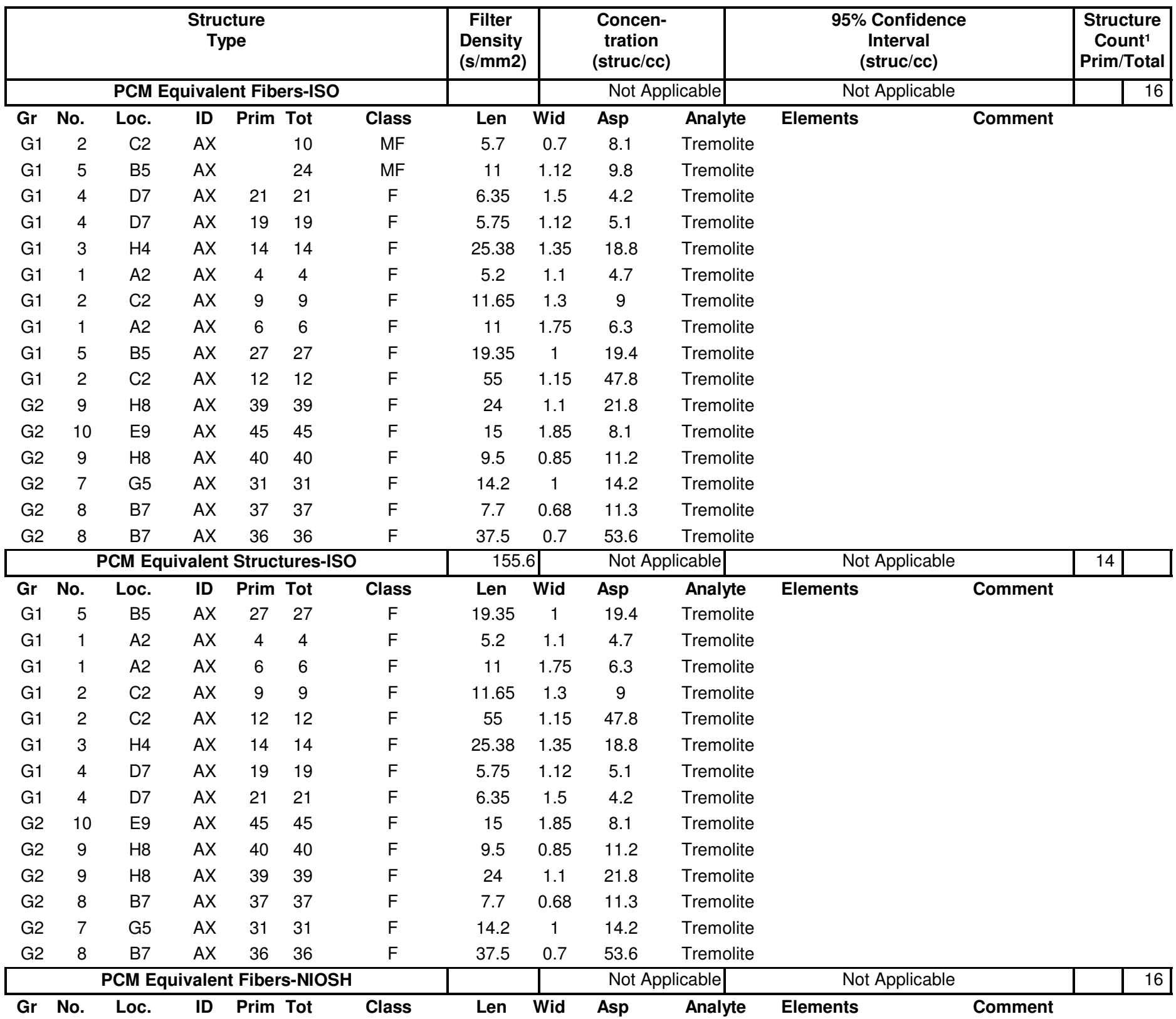


Job Number: 070434

SEA

ISO 10312, Direct Count Categories

Client: Idaho National Laboratory

Report Number: 070434R06

Date Received: 4/23/2007

Project Name: RARE

Lab/Cor Sample No.: S31

Client Sample No.: FB-2-R6

Description:

Filter Fraction: 1

Residual Ash Vol:
Aliquot Dilution: 0

Final Dilution: 0
Volume (L): 0

Lab Filter Area (mm2): 385

Grid Openings Analyzed: 10

Average Grid Opening Area: 0.009

Area Analyzed (mm2): 0.09

Analytical Sens. (struc/cc): 0

Dectection Limit. (struc/cc): 0

\begin{tabular}{|c|c|c|c|c|c|c|c|c|c|c|c|c|c|c|}
\hline & & & $\begin{array}{r}\text { Stru } \\
\mathrm{Ty}\end{array}$ & $\begin{array}{l}\text { ture } \\
\text { pe }\end{array}$ & & & $\begin{array}{c}\text { Filter } \\
\text { Density } \\
(\mathrm{s} / \mathrm{mm} 2)\end{array}$ & & $\begin{array}{r}\text { Conce } \\
\text { tratic } \\
\text { (struc/ }\end{array}$ & & $\begin{array}{c}95 \% \text { Confidence } \\
\text { Interval } \\
\text { (struc/cc) }\end{array}$ & & $\begin{array}{r}\text { Stru } \\
\text { Co } \\
\text { Prim }\end{array}$ & \\
\hline & & CM E & valen & Fiber & rs-NIC & & & & Not & licable & Not Applicable & & & 16 \\
\hline $\mathrm{Gr}$ & No. & Loc. & ID & Prim & Tot & Class & Len & Wid & Asp & Analyte & Elements & Comment & & \\
\hline G1 & 4 & D7 & $A X$ & 19 & 19 & $\mathrm{~F}$ & 5.75 & 1.12 & 5.1 & Tremolite & & & & \\
\hline G1 & 5 & B5 & $A X$ & & 24 & MF & 11 & 1.12 & 9.8 & Tremolite & & & & \\
\hline G1 & 1 & $\mathrm{~A} 2$ & $A X$ & 6 & 6 & $\mathrm{~F}$ & 11 & 1.75 & 6.3 & Tremolite & & & & \\
\hline G1 & 4 & D7 & $A X$ & 21 & 21 & $\mathrm{~F}$ & 6.35 & 1.5 & 4.2 & Tremolite & & & & \\
\hline G1 & 5 & B5 & $A X$ & 27 & 27 & $\mathrm{~F}$ & 19.35 & 1 & 19.4 & Tremolite & & & & \\
\hline G1 & 2 & $\mathrm{C} 2$ & $A X$ & 12 & 12 & $\mathrm{~F}$ & 55 & 1.15 & 47.8 & Tremolite & & & & \\
\hline G1 & 2 & $\mathrm{C} 2$ & $A X$ & & 10 & MF & 5.7 & 0.7 & 8.1 & Tremolite & & & & \\
\hline G1 & 1 & $\mathrm{~A} 2$ & $A X$ & 4 & 4 & $\mathrm{~F}$ & 5.2 & 1.1 & 4.7 & Tremolite & & & & \\
\hline G1 & 2 & $\mathrm{C} 2$ & $A X$ & 9 & 9 & $\mathrm{~F}$ & 11.65 & 1.3 & 9 & Tremolite & & & & \\
\hline $\mathrm{G} 1$ & 3 & $\mathrm{H} 4$ & $A X$ & 14 & 14 & $\mathrm{~F}$ & 25.38 & 1.35 & 18.8 & Tremolite & & & & \\
\hline G2 & 10 & E9 & $A X$ & 45 & 45 & $\mathrm{~F}$ & 15 & 1.85 & 8.1 & Tremolite & & & & \\
\hline G2 & 7 & G5 & $A X$ & 31 & 31 & $\mathrm{~F}$ & 14.2 & 1 & 14.2 & Tremolite & & & & \\
\hline G2 & 8 & B7 & $A X$ & 36 & 36 & $\mathrm{~F}$ & 37.5 & 0.7 & 53.6 & Tremolite & & & & \\
\hline G2 & 8 & B7 & $A X$ & 37 & 37 & $\mathrm{~F}$ & 7.7 & 0.68 & 11.3 & Tremolite & & & & \\
\hline G2 & 9 & $\mathrm{H} 8$ & $A X$ & 39 & 39 & $\mathrm{~F}$ & 24 & 1.1 & 21.8 & Tremolite & & & & \\
\hline $\mathrm{G} 2$ & 9 & $\mathrm{H} 8$ & $A X$ & 40 & 40 & $\mathrm{~F}$ & 9.5 & 0.85 & 11.2 & Tremolite & & & & \\
\hline & & M Equ & lent 5 & tructu & Ires-I & & 155.6 & & Not & licable & Not Applicable & & 14 & \\
\hline $\mathrm{Gr}$ & No. & Loc. & ID & Prim & Tot & Class & Len & Wid & Asp & Analyte & Elements & Comment & & \\
\hline G1 & 5 & B5 & $A X$ & 27 & 27 & $\mathrm{~F}$ & 19.35 & 1 & 19.4 & Tremolite & & & & \\
\hline G1 & 4 & D7 & $A X$ & 21 & 21 & $\mathrm{~F}$ & 6.35 & 1.5 & 4.2 & Tremolite & & & & \\
\hline G1 & 4 & D7 & $A X$ & 19 & 19 & $\mathrm{~F}$ & 5.75 & 1.12 & 5.1 & Tremolite & & & & \\
\hline G1 & 3 & $\mathrm{H} 4$ & $A X$ & 14 & 14 & $\mathrm{~F}$ & 25.38 & 1.35 & 18.8 & Tremolite & & & & \\
\hline $\mathrm{G} 1$ & 2 & $\mathrm{C} 2$ & $A X$ & 12 & 12 & $\mathrm{~F}$ & 55 & 1.15 & 47.8 & Tremolite & & & & \\
\hline G1 & 2 & $\mathrm{C} 2$ & $A X$ & 9 & 9 & $F$ & 11.65 & 1.3 & 9 & Tremolite & & & & \\
\hline G1 & 1 & $\mathrm{~A} 2$ & $A X$ & 4 & 4 & $\mathrm{~F}$ & 5.2 & 1.1 & 4.7 & Tremolite & & & & \\
\hline $\mathrm{G} 1$ & 1 & $\mathrm{~A} 2$ & $A X$ & 6 & 6 & $\mathrm{~F}$ & 11 & 1.75 & 6.3 & Tremolite & & & & \\
\hline G2 & 8 & B7 & $A X$ & 37 & 37 & $\mathrm{~F}$ & 7.7 & 0.68 & 11.3 & Tremolite & & & & \\
\hline G2 & 10 & E9 & $A X$ & 45 & 45 & $F$ & 15 & 1.85 & 8.1 & Tremolite & & & & \\
\hline G2 & 9 & $\mathrm{H} 8$ & $A X$ & 39 & 39 & $F$ & 24 & 1.1 & 21.8 & Tremolite & & & & \\
\hline G2 & 7 & G5 & $A X$ & 31 & 31 & $\mathrm{~F}$ & 14.2 & 1 & 14.2 & Tremolite & & & & \\
\hline G2 & 9 & $\mathrm{H} 8$ & $A X$ & 40 & 40 & $\mathrm{~F}$ & 9.5 & 0.85 & 11.2 & Tremolite & & & & \\
\hline G2 & 8 & B7 & $A X$ & 36 & 36 & $\mathrm{~F}$ & 37.5 & 0.7 & 53.6 & Tremolite & & & & \\
\hline & & estos & ructu & res $>5$ & um a & & 166.7 & & Not & licable & Not Applicable & & 15 & \\
\hline
\end{tabular}


Job Number: 070434

SEA

ISO 10312, Direct Count Categories

Client: Idaho National Laboratory

Report Number: 070434R06

Date Received: 4/23/2007

Project Name: RARE

Lab/Cor Sample No.: S31

Client Sample No.: FB-2-R6

Description:

Filter Fraction: 1

Residual Ash Vol:
Aliquot Dilution: 0

Final Dilution: 0
Volume (L): 0

Lab Filter Area (mm2): 385

Grid Openings Analyzed: 10

Average Grid Opening Area: 0.009

Area Analyzed (mm2): 0.09

Analytical Sens. (struc/cc): 0

Dectection Limit. (struc/cc): 0

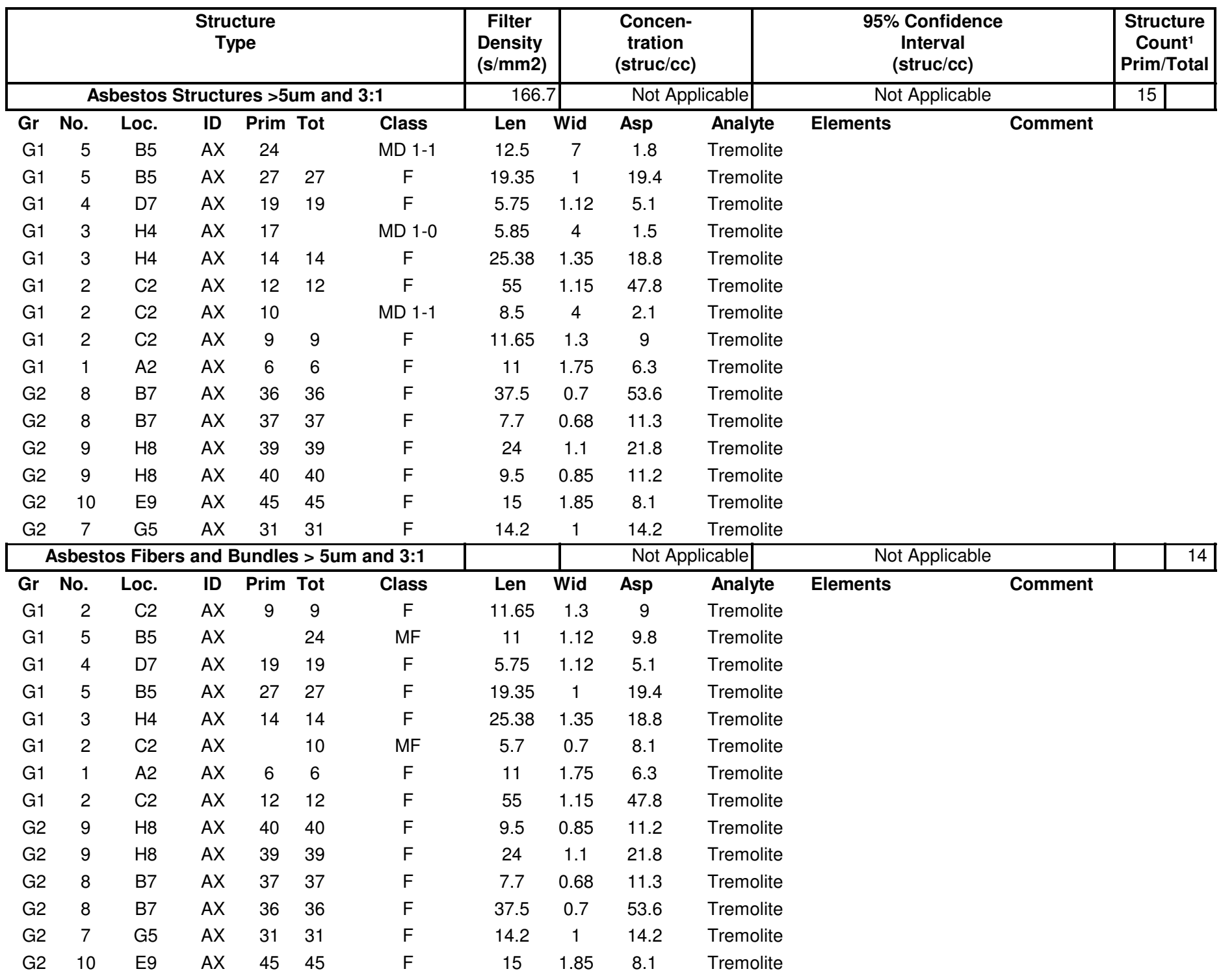


Job Number: 070434

SEA

ISO 10312, Direct Count Categories

Client: Idaho National Laboratory

Report Number: 070434R06

ct Name: RARE

Lab/Cor Sample No.: S32

Client Sample No.: FB-2-R7

Description:

Filter Fraction: 1

Residual Ash Vol:
Aliquot Dilution: 0

Final Dilution: 0
Date Received: 4/23/2007
Volume (L): 0

Lab Filter Area (mm2): 385

Grid Openings Analyzed: 10

Average Grid Opening Area: 0.009

Area Analyzed (mm2): 0.09

Analytical Sens. (struc/cc): 0

Dectection Limit. (struc/cc): 0

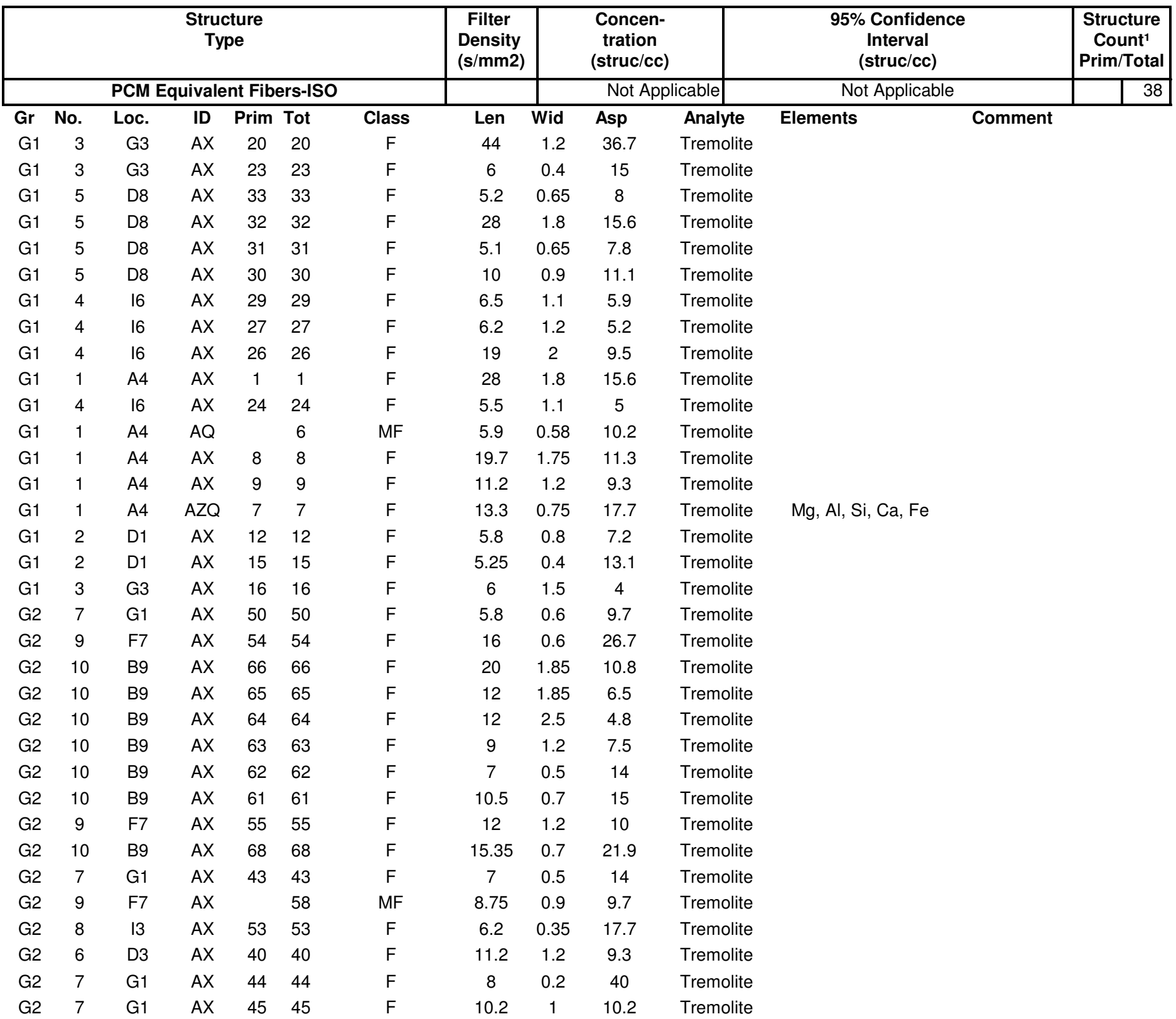


Job Number: 070434

SEA

ISO 10312, Direct Count Categories

Client: Idaho National Laboratory

Report Number: 070434R06

Date Received: 4/23/2007

Project Name: RARE

Lab/Cor Sample No.: S32

Client Sample No.: FB-2-R7

Description:

Filter Fraction: 1

Residual Ash Vol:
Aliquot Dilution: 0

Final Dilution: 0
Volume (L): 0

Lab Filter Area (mm2): 385

Grid Openings Analyzed: 10

Average Grid Opening Area: 0.009

Area Analyzed (mm2): 0.09

Analytical Sens. (struc/cc): 0

Dectection Limit. (struc/cc): 0

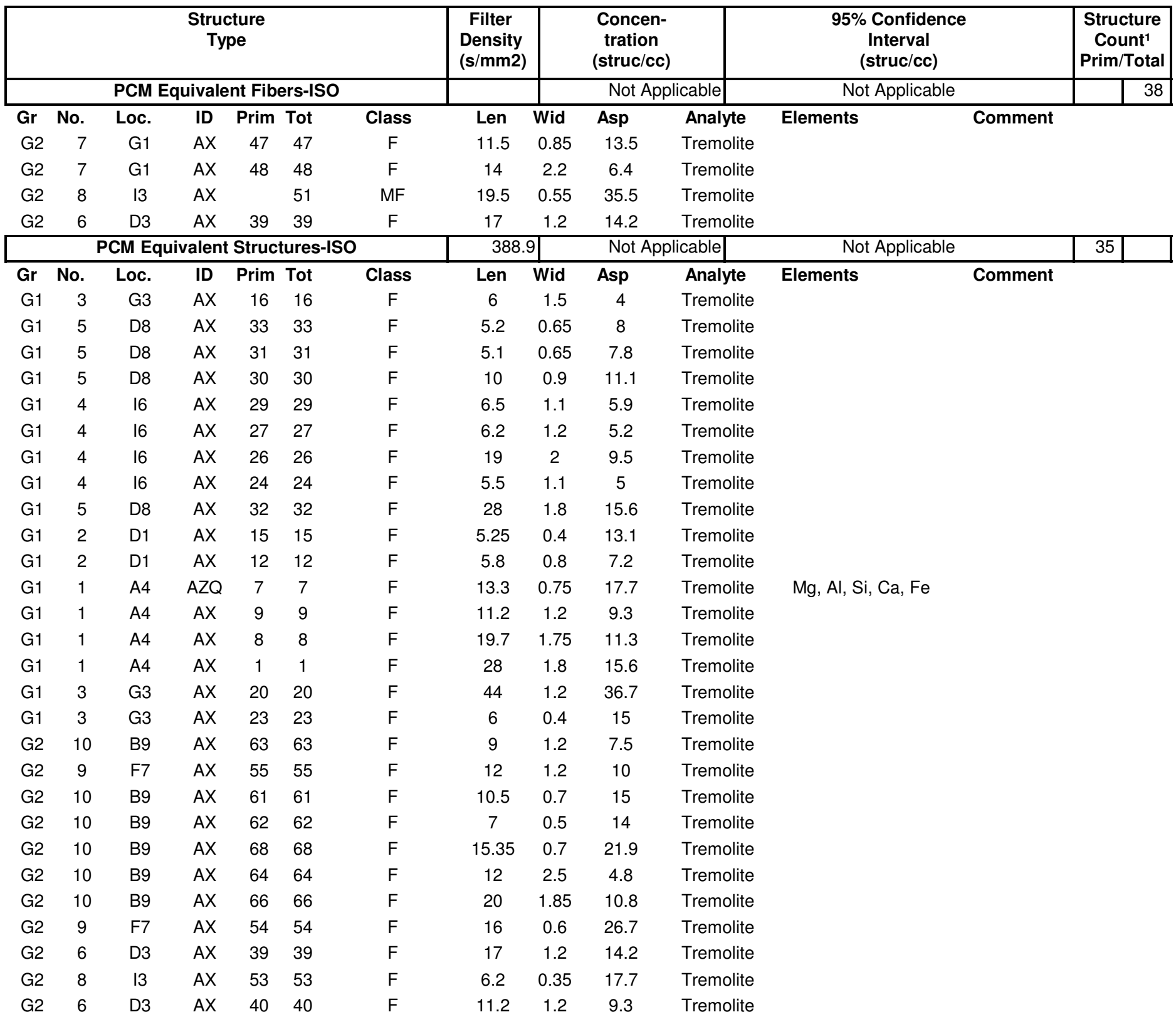


Job Number: 070434

SEA

ISO 10312, Direct Count Categories

Client: Idaho National Laboratory

Report Number: 070434R06

Date Received: 4/23/2007

Project Name: RARE

Lab/Cor Sample No.: S32

Client Sample No.: FB-2-R7

Description:

Filter Fraction: 1

Residual Ash Vol:
Aliquot Dilution: 0

Final Dilution: 0
Volume (L): 0

Lab Filter Area (mm2): 385

Grid Openings Analyzed: 10

Average Grid Opening Area: 0.009

Area Analyzed (mm2): 0.09

Analytical Sens. (struc/cc): 0

Dectection Limit. (struc/cc): 0

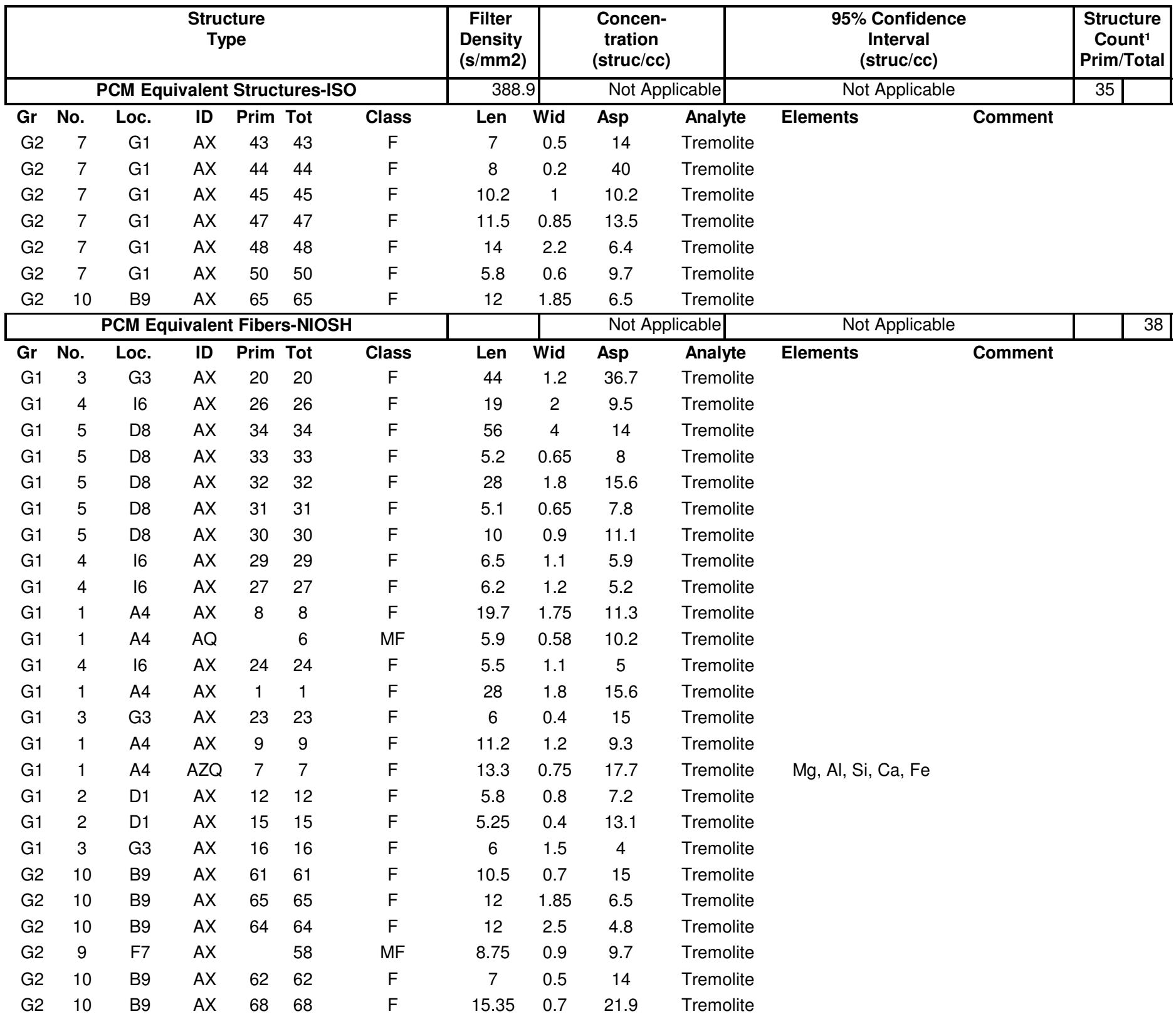


Job Number: 070434

SEA

ISO 10312, Direct Count Categories

Client: Idaho National Laboratory

Report Number: 070434R06

Date Received: 4/23/2007

Project Name: RARE

Lab/Cor Sample No.: S32

Client Sample No.: FB-2-R7

Description:

Filter Fraction: 1

Residual Ash Vol:
Aliquot Dilution: 0

Final Dilution: 0
Volume (L): 0

Lab Filter Area (mm2): 385

Grid Openings Analyzed: 10

Average Grid Opening Area: 0.009

Area Analyzed (mm2): 0.09

Analytical Sens. (struc/cc): 0

Dectection Limit. (struc/cc): 0

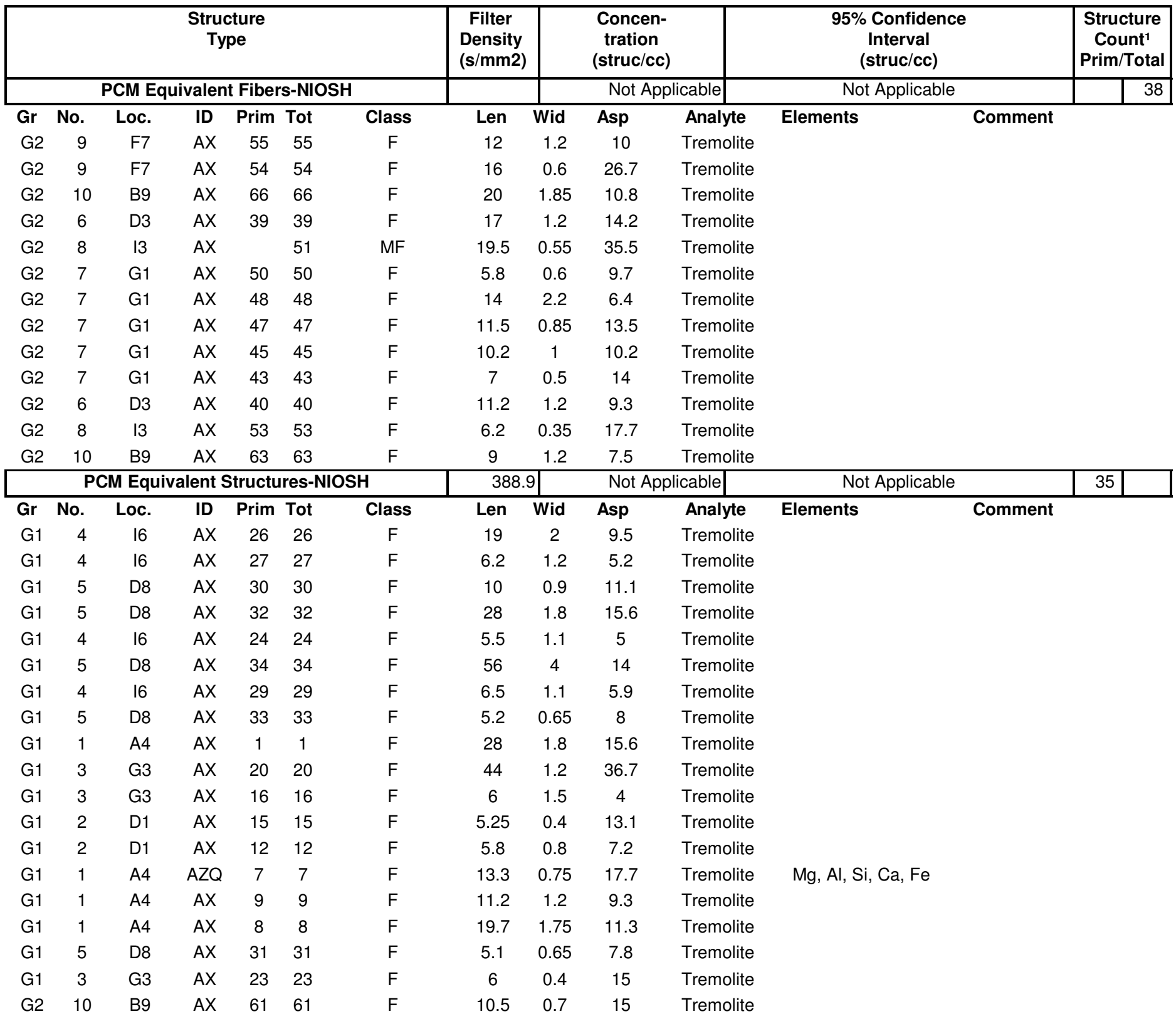


Job Number: 070434

SEA

ISO 10312, Direct Count Categories

Client: Idaho National Laboratory

Report Number: 070434R06

Date Received: 4/23/2007

Project Name: RARE

Lab/Cor Sample No.: S32

Client Sample No.: FB-2-R7

Description:

Filter Fraction: 1

Residual Ash Vol:
Aliquot Dilution: 0

Final Dilution: 0
Volume (L): 0

Lab Filter Area (mm2): 385

Grid Openings Analyzed: 10

Average Grid Opening Area: 0.009

Area Analyzed (mm2): 0.09

Analytical Sens. (struc/cc): 0

Dectection Limit. (struc/cc): 0

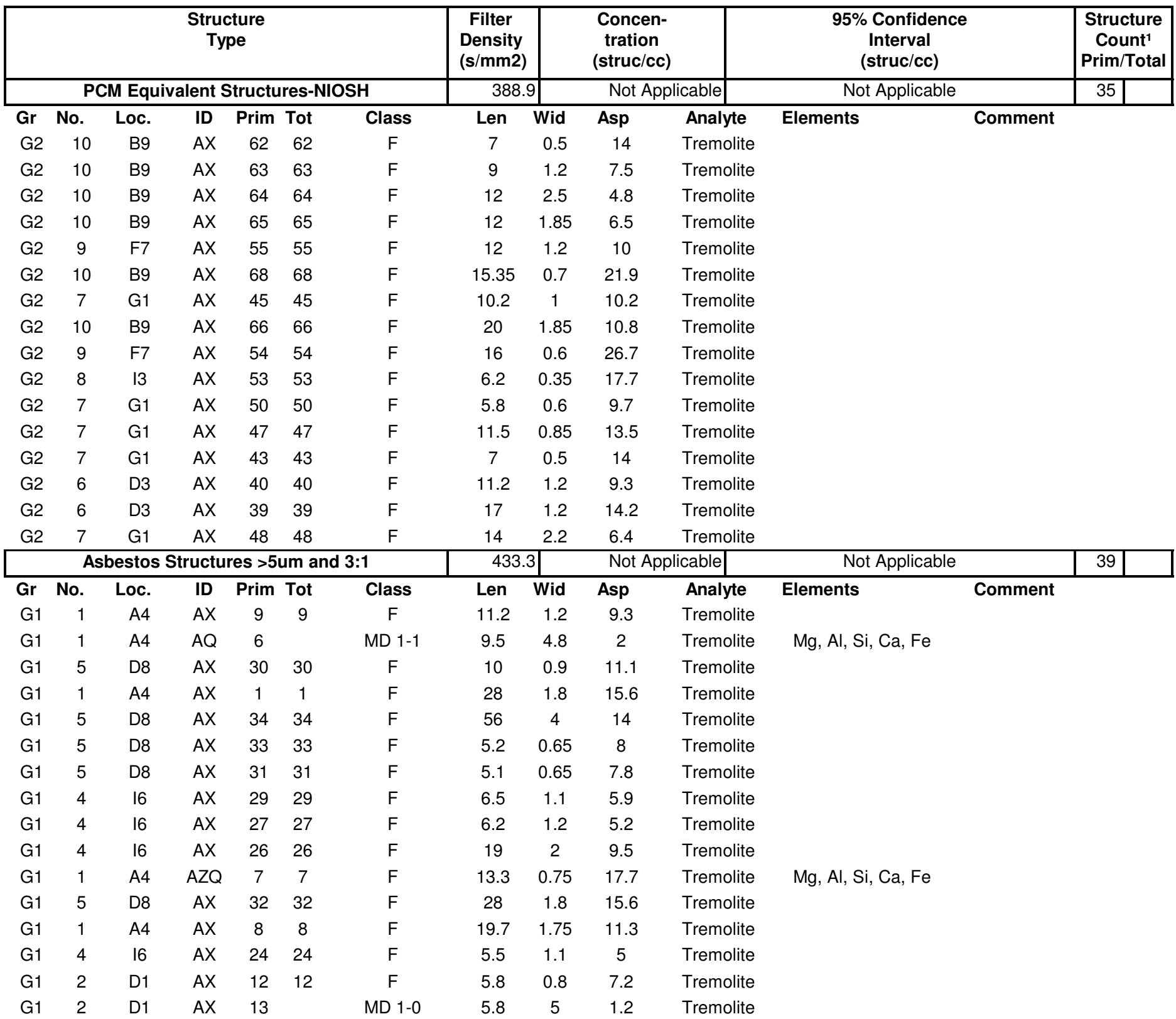


Job Number: 070434

SEA

ISO 10312, Direct Count Categories

Client: Idaho National Laboratory

Report Number: 070434R06

Date Received: 4/23/2007

Project Name: RARE

Lab/Cor Sample No.: S32

Client Sample No.: FB-2-R7

Description:

Filter Fraction: 1

Residual Ash Vol:
Aliquot Dilution: 0

Final Dilution: 0
Volume (L): 0

Lab Filter Area (mm2): 385

Grid Openings Analyzed: 10

Average Grid Opening Area: 0.009

Area Analyzed (mm2): 0.09

Analytical Sens. (struc/cc): 0

Dectection Limit. (struc/cc): 0

\begin{tabular}{|c|c|c|c|c|c|c|c|c|c|c|c|c|c|}
\hline \multicolumn{7}{|c|}{$\begin{array}{c}\text { Structure } \\
\text { Type }\end{array}$} & \multirow{2}{*}{\multicolumn{2}{|c|}{\begin{tabular}{|c|}
$\begin{array}{c}\text { Filter } \\
\text { Density } \\
\text { (s/mm2) }\end{array}$ \\
433.3 \\
\end{tabular}}} & \multirow{2}{*}{\multicolumn{2}{|c|}{\begin{tabular}{c|}
$\begin{array}{c}\text { Concen- } \\
\text { tration } \\
\text { (struc/cc) }\end{array}$ \\
Not Applicable
\end{tabular}}} & \multirow{2}{*}{$\begin{array}{c}\begin{array}{c}95 \% \text { Confidence } \\
\text { Interval } \\
\text { (struc/cc) }\end{array} \\
\text { Not Applicable }\end{array}$} & \multicolumn{2}{|c|}{$\begin{array}{c}\text { Structure } \\
\text { Count }^{1} \\
\text { Prim/Total }\end{array}$} \\
\hline \multicolumn{7}{|c|}{ Asbestos Structures >5um and 3:1 } & & & & & & 39 & \\
\hline Gr & No. & Loc. & ID & Prim & Tot & Class & Len & Wid & Asp & Analyte & Elements & & \\
\hline G1 & 2 & D1 & $A X$ & 15 & 15 & $\mathrm{~F}$ & 5.25 & 0.4 & 13.1 & Tremolite & & & \\
\hline G1 & 3 & G3 & $A X$ & 20 & 20 & $\mathrm{~F}$ & 44 & 1.2 & 36.7 & Tremolite & & & \\
\hline G1 & 3 & G3 & $A X$ & 23 & 23 & $\mathrm{~F}$ & 6 & 0.4 & 15 & Tremolite & & & \\
\hline G2 & 9 & F7 & $A X$ & 54 & 54 & $\mathrm{~F}$ & 16 & 0.6 & 26.7 & Tremolite & & & \\
\hline G2 & 7 & G1 & $A X$ & 45 & 45 & $\mathrm{~F}$ & 10.2 & 1 & 10.2 & Tremolite & & & \\
\hline G2 & 10 & B9 & $A X$ & 66 & 66 & $\mathrm{~F}$ & 20 & 1.85 & 10.8 & Tremolite & & & \\
\hline G2 & 10 & B9 & $A X$ & 65 & 65 & $\mathrm{~F}$ & 12 & 1.85 & 6.5 & Tremolite & & & \\
\hline G2 & 10 & B9 & $A X$ & 63 & 63 & $\mathrm{~F}$ & 9 & 1.2 & 7.5 & Tremolite & & & \\
\hline G2 & 10 & B9 & $A X$ & 62 & 62 & $\mathrm{~F}$ & 7 & 0.5 & 14 & Tremolite & & & \\
\hline G2 & 10 & B9 & $A X$ & 61 & 61 & $\mathrm{~F}$ & 10.5 & 0.7 & 15 & Tremolite & & & \\
\hline G2 & 9 & F7 & $A X$ & 58 & & MD 1-1 & 20 & 12 & 1.7 & Tremolite & & & \\
\hline G2 & 9 & F7 & $A X$ & 55 & 55 & $\mathrm{~F}$ & 12 & 1.2 & 10 & Tremolite & & & \\
\hline G2 & 10 & B9 & $A X$ & 68 & 68 & $\mathrm{~F}$ & 15.35 & 0.7 & 21.9 & Tremolite & & & \\
\hline G2 & 6 & D3 & $A X$ & 40 & 40 & $\mathrm{~F}$ & 11.2 & 1.2 & 9.3 & Tremolite & & & \\
\hline G2 & 7 & G1 & $A X$ & 48 & 48 & $\mathrm{~F}$ & 14 & 2.2 & 6.4 & Tremolite & & & \\
\hline G2 & 6 & D3 & $A X$ & 39 & 39 & $\mathrm{~F}$ & 17 & 1.2 & 14.2 & Tremolite & & & \\
\hline G2 & 8 & 13 & $A X$ & 53 & 53 & $F$ & 6.2 & 0.35 & 17.7 & Tremolite & & & \\
\hline G2 & 7 & G1 & $A X$ & 43 & 43 & $\mathrm{~F}$ & 7 & 0.5 & 14 & Tremolite & & & \\
\hline G2 & 7 & $\mathrm{G} 1$ & $A X$ & 44 & 44 & $\mathrm{~F}$ & 8 & 0.2 & 40 & Tremolite & & & \\
\hline G2 & 7 & G1 & $A X$ & 47 & 47 & $\mathrm{~F}$ & 11.5 & 0.85 & 13.5 & Tremolite & & & \\
\hline G2 & 7 & G1 & $A X$ & 50 & 50 & $F$ & 5.8 & 0.6 & 9.7 & Tremolite & & & \\
\hline G2 & 8 & 13 & $A X$ & 51 & & MD 1-1 & 23.8 & 10 & 2.4 & Tremolite & & & \\
\hline G2 & 6 & D3 & $A X$ & 37 & & MD 1-0 & 11 & 5 & 2.2 & Tremolite & & & \\
\hline \multicolumn{7}{|c|}{ Asbestos Fibers and Bundles > 5um and 3:1 } & & & \multicolumn{2}{|c|}{ Not Applicable } & Not Applicable & & 37 \\
\hline $\mathrm{Gr}$ & No. & Loc. & ID & Prim & Tot & Class & Len & Wid & Asp & Analyte & Elements & & \\
\hline G1 & 1 & A4 & $\mathrm{AX}$ & 1 & 1 & $\mathrm{~F}$ & 28 & 1.8 & 15.6 & Tremolite & & & \\
\hline G1 & 4 & 16 & $A X$ & 26 & 26 & $\mathrm{~F}$ & 19 & 2 & 9.5 & Tremolite & & & \\
\hline G1 & 5 & D8 & $A X$ & 34 & 34 & $\mathrm{~F}$ & 56 & 4 & 14 & Tremolite & & & \\
\hline G1 & 5 & D8 & $A X$ & 33 & 33 & $\mathrm{~F}$ & 5.2 & 0.65 & 8 & Tremolite & & & \\
\hline G1 & 5 & D8 & $A X$ & 32 & 32 & $\mathrm{~F}$ & 28 & 1.8 & 15.6 & Tremolite & & & \\
\hline G1 & 5 & D8 & $A X$ & 31 & 31 & $\mathrm{~F}$ & 5.1 & 0.65 & 7.8 & Tremolite & & & \\
\hline G1 & 5 & D8 & $A X$ & 30 & 30 & $\mathrm{~F}$ & 10 & 0.9 & 11.1 & Tremolite & & & \\
\hline G1 & 4 & 16 & $A X$ & 29 & 29 & $\mathrm{~F}$ & 6.5 & 1.1 & 5.9 & Tremolite & & & \\
\hline G1 & 4 & 16 & $A X$ & 27 & 27 & $\mathrm{~F}$ & 6.2 & 1.2 & 5.2 & Tremolite & & & \\
\hline
\end{tabular}


Job Number: 070434

SEA

ISO 10312, Direct Count Categories

Client: Idaho National Laboratory

Report Number: 070434R06

ct Name: RARE

Lab/Cor Sample No.: S32

Client Sample No.: FB-2-R7

Description:

Filter Fraction: 1

Residual Ash Vol:
Aliquot Dilution: 0

Final Dilution: 0
Date Received: 4/23/2007
Volume (L): 0

Lab Filter Area (mm2): 385

Grid Openings Analyzed: 10

Average Grid Opening Area: 0.009

Area Analyzed (mm2): 0.09

Analytical Sens. (struc/cc): 0

Dectection Limit. (struc/cc): 0

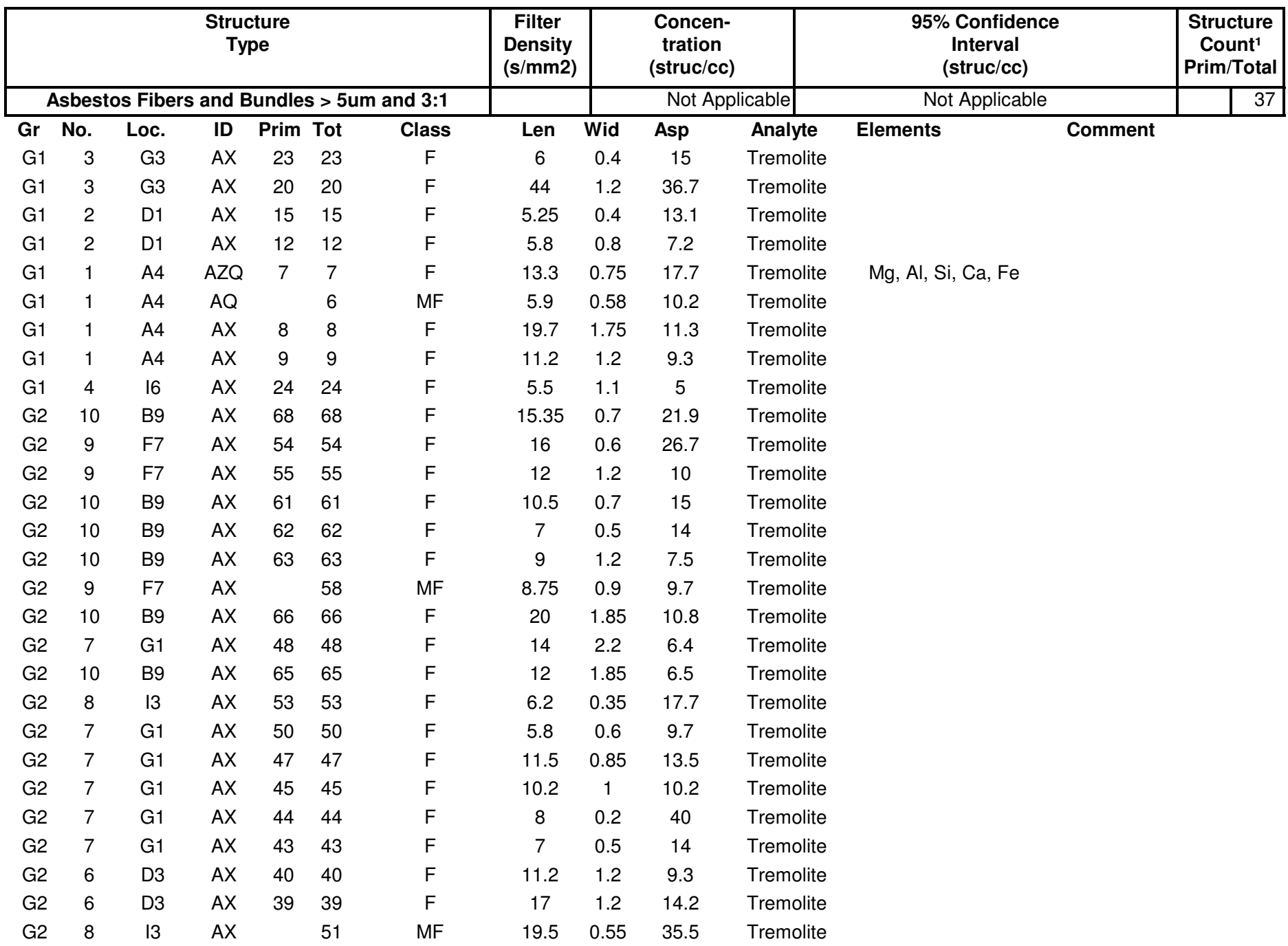


Job Number: 070434

SEA

ISO 10312, Direct Count Categories

Client: Idaho National Laboratory

Report Number: 070434R06

Date Received: 4/23/2007

Project Name: RARE

Lab/Cor Sample No.: S33

Client Sample No.: FB-1-R1

Description:

Filter Fraction: 1

Residual Ash Vol:
Aliquot Dilution: 0

Final Dilution: 0
Volume (L): 0

Lab Filter Area (mm2): 385

Grid Openings Analyzed: 10

Average Grid Opening Area: 0.009

Area Analyzed (mm2): 0.09

Analytical Sens. (struc/cc): 0

Dectection Limit. (struc/cc): 0

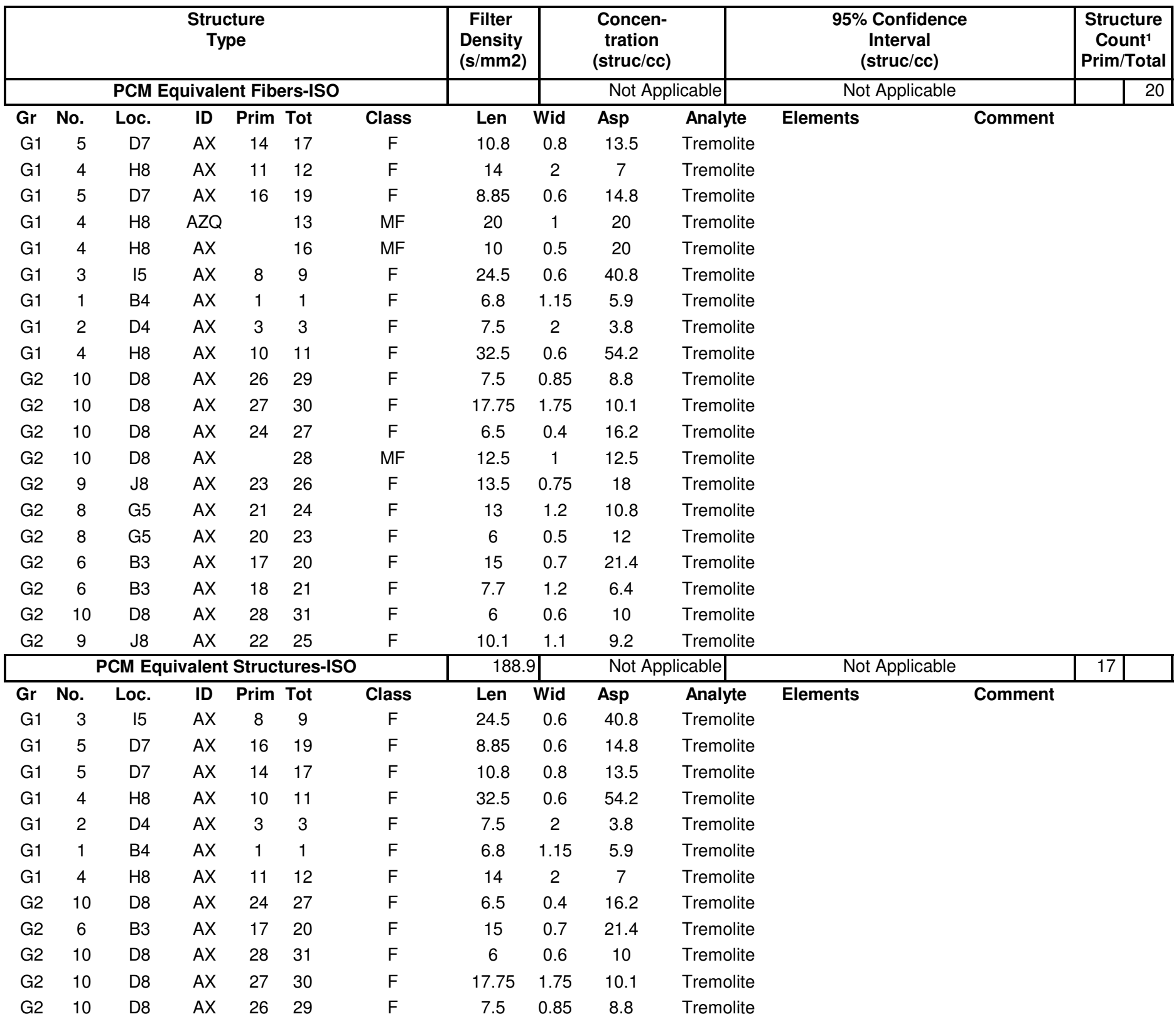


A Professional Service Corporation in the Northwest

Job Number: 070434

SEA

ISO 10312, Direct Count Categories

Client: Idaho National Laboratory

Report Number: 070434R06

Date Received: 4/23/2007

Project Name: RARE

Lab/Cor Sample No.: S33

Client Sample No.: FB-1-R1

Description:

Filter Fraction: 1

Residual Ash Vol:
Aliquot Dilution: 0

Final Dilution: 0
Volume (L): 0

Lab Filter Area (mm2): 385

Grid Openings Analyzed: 10

Average Grid Opening Area: 0.009

Area Analyzed (mm2): 0.09

Analytical Sens. (struc/cc): 0

Dectection Limit. (struc/cc): 0

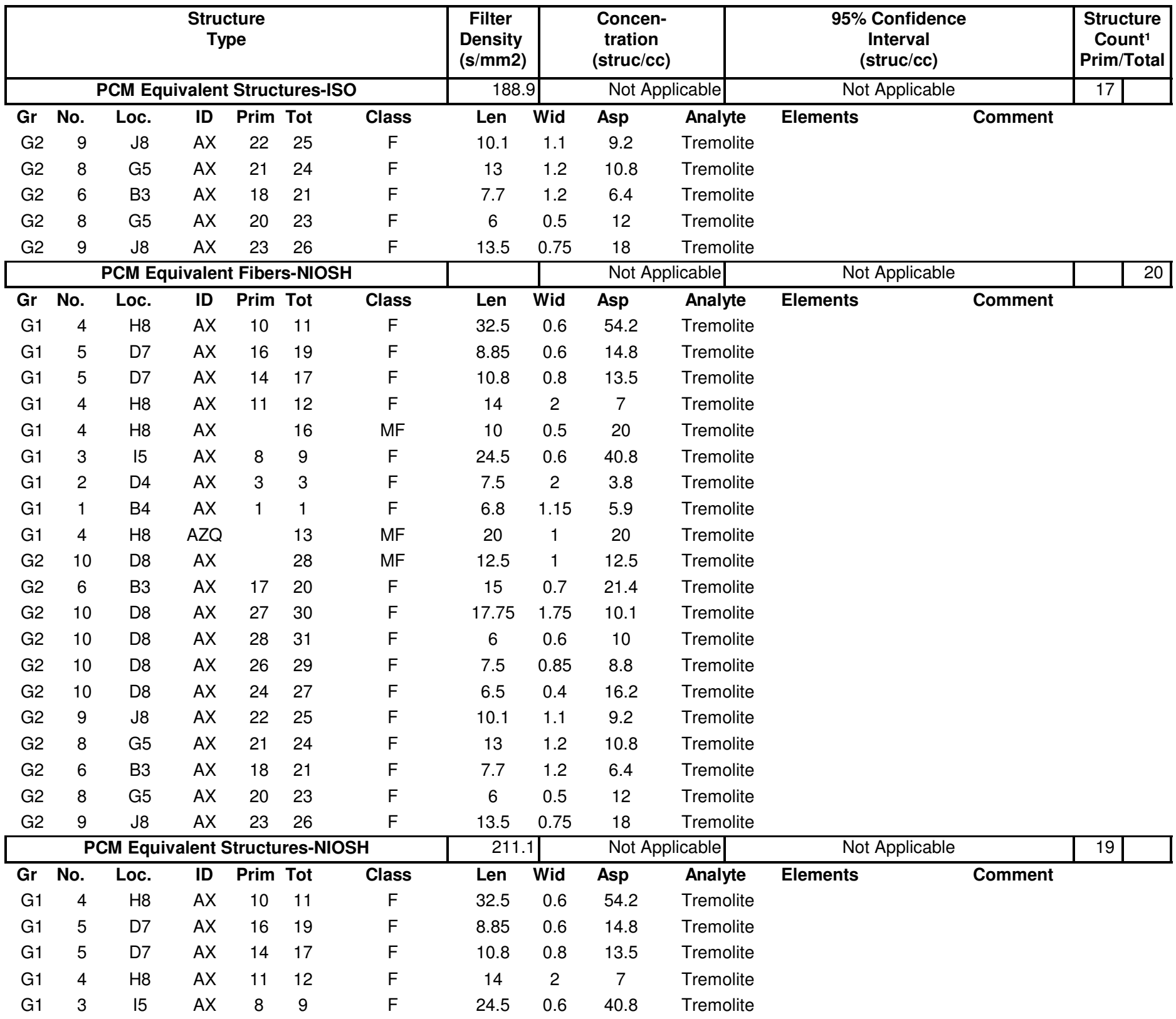


Job Number: 070434

SEA

ISO 10312, Direct Count Categories

Client: Idaho National Laboratory

Report Number: 070434R06

Date Received: 4/23/2007

Project Name: RARE

Lab/Cor Sample No.: S33

Client Sample No.: FB-1-R1

Description:

Filter Fraction: 1

Residual Ash Vol:
Aliquot Dilution: 0

Final Dilution: 0
Volume (L): 0

Lab Filter Area (mm2): 385

Grid Openings Analyzed: 10

Average Grid Opening Area: 0.009

Area Analyzed (mm2): 0.09

Analytical Sens. (struc/cc): 0

Dectection Limit. (struc/cc): 0

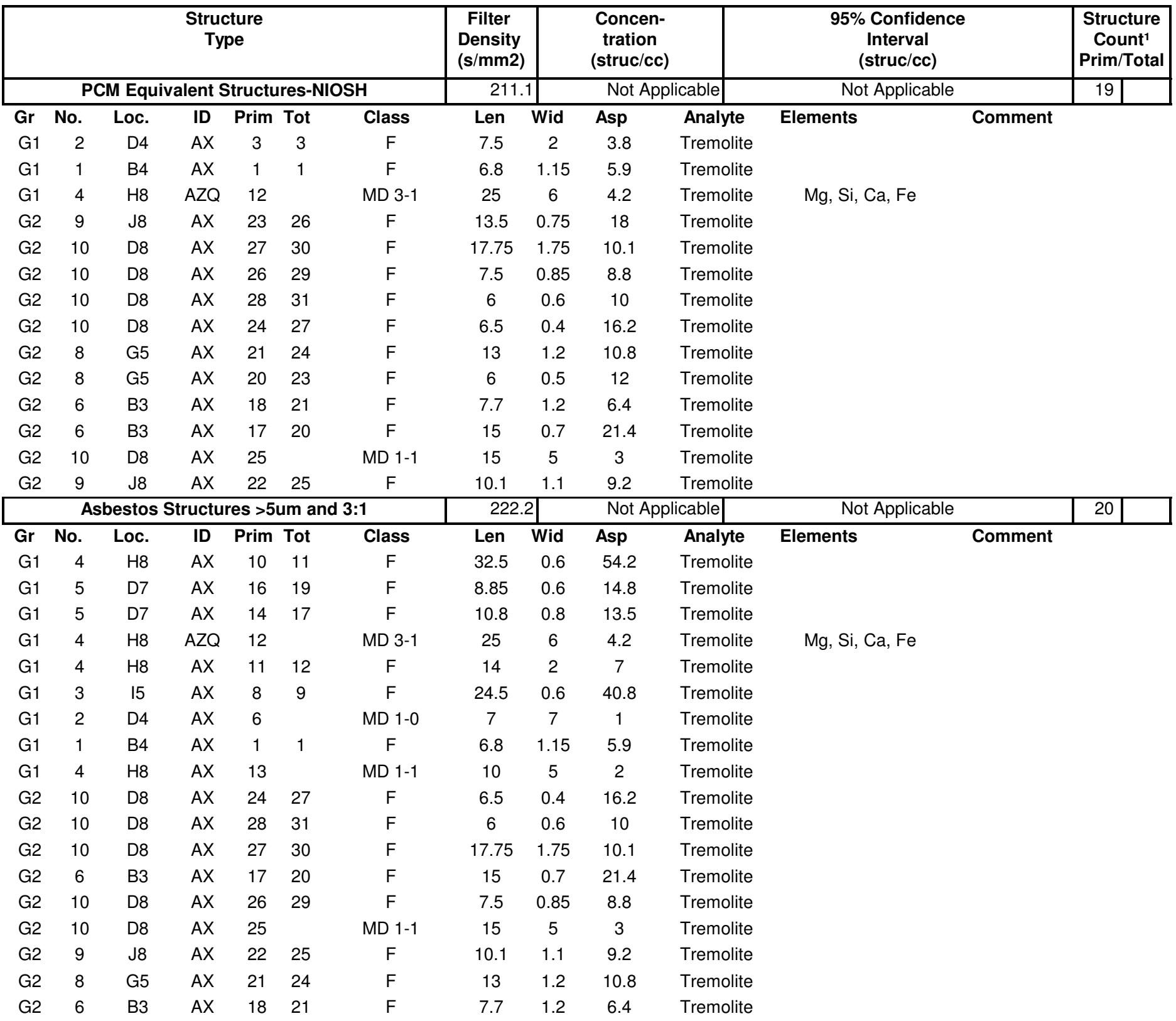


Job Number: 070434

SEA

ISO 10312, Direct Count Categories

Client: Idaho National Laboratory

Report Number: 070434R06

Date Received: 4/23/2007

Project Name: RARE

Lab/Cor Sample No.: S33

Client Sample No.: FB-1-R1

Description:

Filter Fraction: 1

Residual Ash Vol:
Aliquot Dilution: 0

Final Dilution: 0
Volume (L): 0

Lab Filter Area (mm2): 385

Grid Openings Analyzed: 10

Average Grid Opening Area: 0.009

Area Analyzed (mm2): 0.09

Analytical Sens. (struc/cc): 0

Dectection Limit. (struc/cc): 0

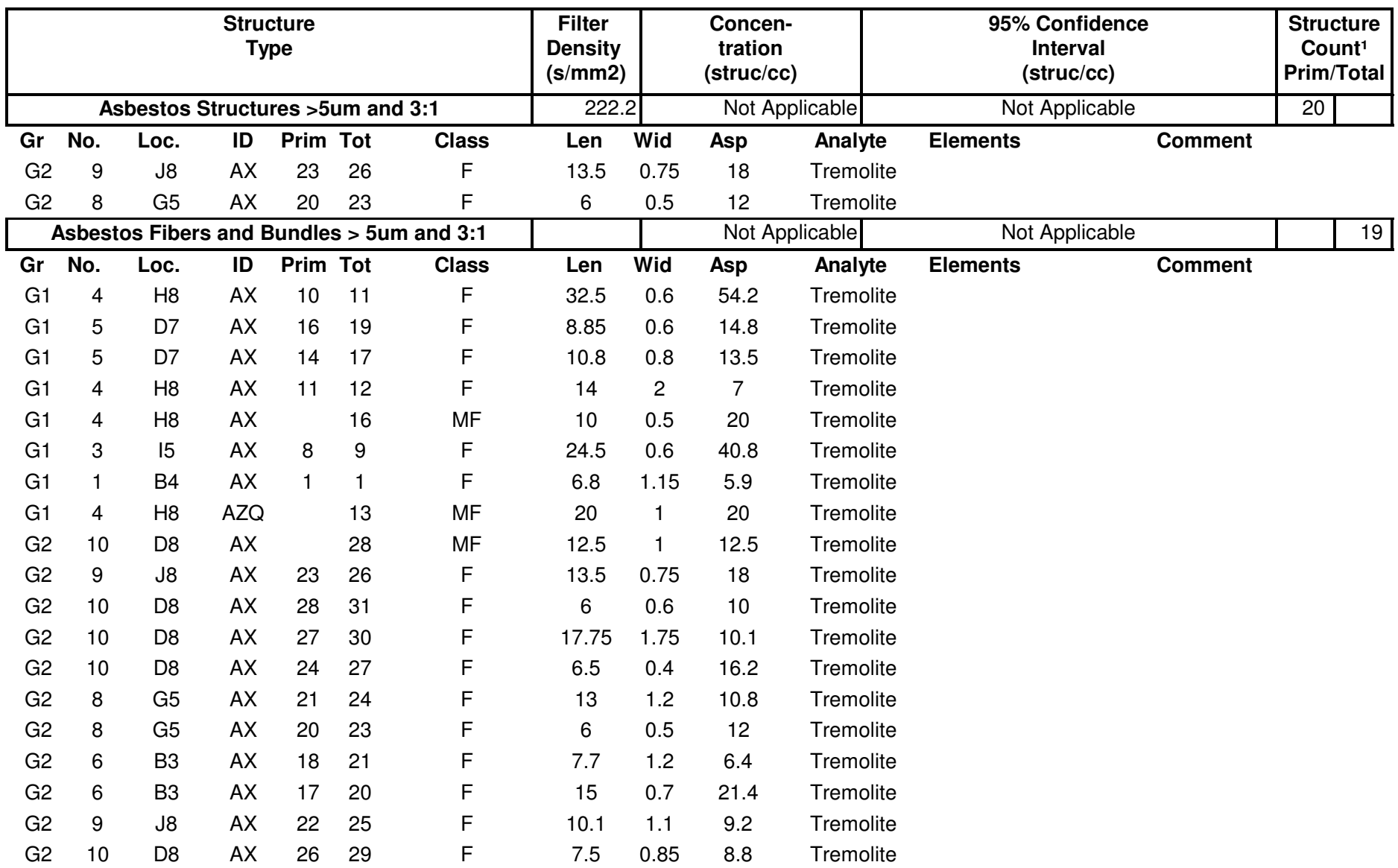


Job Number: 070434

SEA

ISO 10312, Direct Count Categories

Client: Idaho National Laboratory

Report Number: 070434R06

Date Received: 4/23/2007

Project Name: RARE

Lab/Cor Sample No.: S35

Client Sample No.: FB-1-R3

Description:

Filter Fraction: 1

Residual Ash Vol:
Aliquot Dilution: 0

Final Dilution: 0
Volume (L): 0

Lab Filter Area (mm2): 385

Grid Openings Analyzed: 10

Average Grid Opening Area: 0.009

Area Analyzed (mm2): 0.09

Analytical Sens. (struc/cc): 0

Dectection Limit. (struc/cc): 0

\begin{tabular}{|c|c|c|c|c|c|c|c|c|c|c|c|c|c|c|c|}
\hline & & & $\begin{array}{r}\text { Stru } \\
\text { Ty }\end{array}$ & $\begin{array}{l}\text { ture } \\
\text { pe }\end{array}$ & & & $\begin{array}{c}\text { Filter } \\
\text { Density } \\
(\mathrm{s} / \mathrm{mm} 2)\end{array}$ & & $\begin{array}{r}\text { Conce } \\
\text { tratic } \\
\text { (struc) }\end{array}$ & & & $\begin{array}{c}95 \% \text { Confidence } \\
\text { Interval } \\
\text { (struc/cc) }\end{array}$ & & $\begin{array}{r}\text { Stru } \\
\text { Co } \\
\text { Prim }\end{array}$ & \\
\hline & & PCM & uivale & nt Fib & ers-IS & & & & Not & licable & & Not Applicable & & & 19 \\
\hline $\mathrm{Gr}$ & No. & Loc. & ID & Prim & Tot & Class & Len & Wid & Asp & Anal & & Elements & Comment & & \\
\hline G1 & 2 & 13 & $A X$ & 9 & 9 & $\mathrm{~F}$ & 45 & 0.9 & 50 & Trem & lite & & & & \\
\hline G1 & 5 & E7 & $A X$ & 22 & 22 & $\mathrm{~F}$ & 6.2 & 0.4 & 15.5 & Trem & lite & & & & \\
\hline G1 & 5 & E7 & $A X$ & 21 & 21 & $\mathrm{~F}$ & 10.6 & 1.1 & 9.6 & Trem & lite & & & & \\
\hline G1 & 5 & E7 & $A X$ & 20 & 20 & $\mathrm{~F}$ & 6.2 & 0.55 & 11.3 & Trem & lite & & & & \\
\hline G1 & 5 & E7 & $A X$ & 19 & 19 & $\mathrm{~F}$ & 20.5 & 2 & 10.2 & Trem & lite & & & & \\
\hline G1 & 4 & J8 & $A X$ & 18 & 18 & $\mathrm{~F}$ & 5.8 & 0.85 & 6.8 & Trem & lite & & & & \\
\hline G1 & 4 & J8 & $A X$ & 17 & 17 & $\mathrm{~F}$ & 12.5 & 0.55 & 22.7 & Trem & lite & & & & \\
\hline G1 & 4 & J8 & $A X$ & 16 & 16 & $\mathrm{~F}$ & 12 & 1.75 & 6.9 & Trem & lite & & & & \\
\hline G1 & 3 & G6 & $A X$ & 12 & 12 & $\mathrm{~F}$ & 15.5 & 1.2 & 12.9 & Trem & lite & & & & \\
\hline $\mathrm{G} 1$ & 2 & І3 & $A X$ & 7 & 7 & $\mathrm{~F}$ & 7.25 & 0.4 & 18.1 & Trem & lite & & & & \\
\hline G1 & 1 & $\mathrm{E} 1$ & $A Z Q$ & 1 & 1 & $\mathrm{~F}$ & 9.8 & 0.6 & 16.3 & Trem & lite & $\mathrm{Mg}, \mathrm{Al}, \mathrm{Si}, \mathrm{Ca}, \mathrm{Fe}$ & & & \\
\hline G1 & 1 & E1 & $A X$ & 6 & 6 & $\mathrm{~F}$ & 5.5 & 1.25 & 4.4 & Trem & lite & & & & \\
\hline G1 & 1 & E1 & $A X$ & 4 & 4 & $\mathrm{~F}$ & 8.8 & 1.2 & 7.3 & Trem & lite & & & & \\
\hline G1 & 4 & J8 & $A X$ & 14 & 14 & $\mathrm{~F}$ & 48 & 1.85 & 25.9 & Trem & lite & & & & \\
\hline G2 & 7 & $\mathrm{~F} 2$ & $A X$ & 25 & 25 & $\mathrm{~F}$ & 35 & 2.25 & 15.6 & Trem & lite & & & & \\
\hline G2 & 10 & D7 & $A X$ & 31 & 31 & $\mathrm{~F}$ & 10 & 1.5 & 6.7 & Trem & lite & & & & \\
\hline G2 & 10 & D7 & $A X$ & 30 & 30 & $\mathrm{~F}$ & 8.85 & 0.8 & 11.1 & Trem & lite & & & & \\
\hline G2 & 9 & 18 & $A X$ & 29 & 29 & $\mathrm{~F}$ & 9 & 0.45 & 20 & Trem & lite & & & & \\
\hline G2 & 9 & 18 & $A X$ & 28 & 28 & $\mathrm{~F}$ & 10 & 1 & 10 & Trem & lite & & & & \\
\hline & & CM Ec & valent & Struc & tures & & 211. & & Not & licable & & Not Applicable & & 19 & \\
\hline $\mathrm{Gr}$ & No. & Loc. & ID & Prim & Tot & Class & Len & Wid & Asp & Anal & & Elements & Comment & & \\
\hline G1 & 4 & J8 & $A X$ & 14 & 14 & $\mathrm{~F}$ & 48 & 1.85 & 25.9 & Trem & lite & & & & \\
\hline G1 & 5 & E7 & $A X$ & 22 & 22 & $\mathrm{~F}$ & 6.2 & 0.4 & 15.5 & Trem & lite & & & & \\
\hline G1 & 5 & E7 & $A X$ & 21 & 21 & $\mathrm{~F}$ & 10.6 & 1.1 & 9.6 & Trem & lite & & & & \\
\hline G1 & 5 & E7 & $A X$ & 20 & 20 & $\mathrm{~F}$ & 6.2 & 0.55 & 11.3 & Trem & lite & & & & \\
\hline $\mathrm{G} 1$ & 5 & E7 & $A X$ & 19 & 19 & $\mathrm{~F}$ & 20.5 & 2 & 10.2 & Trem & lite & & & & \\
\hline G1 & 4 & J8 & $A X$ & 18 & 18 & $\mathrm{~F}$ & 5.8 & 0.85 & 6.8 & Trem & olite & & & & \\
\hline G1 & 4 & J8 & $A X$ & 16 & 16 & $\mathrm{~F}$ & 12 & 1.75 & 6.9 & Trem & lite & & & & \\
\hline G1 & 3 & G6 & $A X$ & 12 & 12 & $\mathrm{~F}$ & 15.5 & 1.2 & 12.9 & Trem & lite & & & & \\
\hline G1 & 2 & 13 & $A X$ & 9 & 9 & $\mathrm{~F}$ & 45 & 0.9 & 50 & Trem & lite & & & & \\
\hline $\mathrm{G} 1$ & 2 & І3 & $A X$ & 7 & 7 & $\mathrm{~F}$ & 7.25 & 0.4 & 18.1 & Trem & lite & & & & \\
\hline G1 & 1 & $\mathrm{E} 1$ & $A Z Q$ & 1 & 1 & $\mathrm{~F}$ & 9.8 & 0.6 & 16.3 & Trem & lite & $\mathrm{Mg}, \mathrm{Al}, \mathrm{Si}, \mathrm{Ca}, \mathrm{Fe}$ & & & \\
\hline G1 & 1 & E1 & $A X$ & 6 & 6 & $\mathrm{~F}$ & 5.5 & 1.25 & 4.4 & Trem & lite & & & & \\
\hline G1 & 1 & $\mathrm{E} 1$ & $A X$ & 4 & 4 & $\mathrm{~F}$ & 8.8 & 1.2 & 7.3 & Trem & lite & & & & \\
\hline
\end{tabular}


Job Number: 070434

SEA

ISO 10312, Direct Count Categories

Client: Idaho National Laboratory

Report Number: 070434R06

Date Received: 4/23/2007

Project Name: RARE

Lab/Cor Sample No.: S35

Client Sample No.: FB-1-R3

Description:

Filter Fraction: 1

Residual Ash Vol:
Aliquot Dilution: 0

Final Dilution: 0
Volume (L): 0

Lab Filter Area (mm2): 385

Grid Openings Analyzed: 10

Average Grid Opening Area: 0.009

Area Analyzed (mm2): 0.09

Analytical Sens. (struc/cc): 0

Dectection Limit. (struc/cc): 0

\begin{tabular}{|c|c|c|c|c|c|c|c|c|c|c|c|c|c|c|c|}
\hline \multicolumn{7}{|c|}{$\begin{array}{c}\text { Structure } \\
\text { Type }\end{array}$} & \multirow{2}{*}{\multicolumn{2}{|c|}{\begin{tabular}{|c|}
$\begin{array}{c}\text { Filter } \\
\text { Density } \\
\text { (s/mm2) }\end{array}$ \\
211.1 \\
\end{tabular}}} & \multirow{2}{*}{\multicolumn{2}{|c|}{\begin{tabular}{c|}
$\begin{array}{c}\text { Concen- } \\
\text { tration } \\
\text { (struc/cc) }\end{array}$ \\
Not Applicable
\end{tabular}}} & \multirow{2}{*}{\multicolumn{3}{|c|}{$\begin{array}{c}\begin{array}{c}\text { 95\% Confidence } \\
\text { Interval } \\
\text { (struc/cc) }\end{array} \\
\text { Not Applicable }\end{array}$}} & \multicolumn{2}{|c|}{$\begin{array}{l}\text { Structure } \\
\text { Count }^{1} \\
\text { Prim/Total }\end{array}$} \\
\hline \multicolumn{7}{|c|}{ PCM Equivalent Structures-ISO } & & & & & & & & 19 & \\
\hline $\mathrm{Gr}$ & No. & Loc. & ID & Prim & Tot & Class & Len & Wid & Asp & Anal & & Elements & Comment & & \\
\hline G1 & 4 & J8 & $A X$ & 17 & 17 & $\mathrm{~F}$ & 12.5 & 0.55 & 22.7 & Trem & olite & & & & \\
\hline G2 & 9 & 18 & $A X$ & 29 & 29 & $\mathrm{~F}$ & 9 & 0.45 & 20 & Trem & olite & & & & \\
\hline G2 & 10 & D7 & $A X$ & 30 & 30 & $\mathrm{~F}$ & 8.85 & 0.8 & 11.1 & Trem & olite & & & & \\
\hline G2 & 7 & $\mathrm{~F} 2$ & $A X$ & 25 & 25 & $\mathrm{~F}$ & 35 & 2.25 & 15.6 & Trem & olite & & & & \\
\hline G2 & 10 & D7 & $A X$ & 31 & 31 & $\mathrm{~F}$ & 10 & 1.5 & 6.7 & Tremc & olite & & & & \\
\hline G2 & 9 & 18 & $A X$ & 28 & 28 & $\mathrm{~F}$ & 10 & 1 & 10 & Tremc & olite & & & & \\
\hline \multicolumn{7}{|c|}{ PCM Equivalent Fibers-NIOSH } & & & \multicolumn{3}{|c|}{ Not Applicable } & \multicolumn{2}{|l|}{ Not Applicable } & & 20 \\
\hline Gr & No. & Loc. & ID & Prim & Tot & Class & Len & Wid & Asp & Analy & & Elements & Comment & & \\
\hline G1 & 4 & J8 & $A X$ & 14 & 14 & $\mathrm{~F}$ & 48 & 1.85 & 25.9 & \multicolumn{2}{|c|}{ Tremolite } & & & & \\
\hline G1 & 5 & E7 & $A X$ & 22 & 22 & $\mathrm{~F}$ & 6.2 & 0.4 & 15.5 & \multicolumn{2}{|c|}{ Tremolite } & & & & \\
\hline G1 & 5 & E7 & $A X$ & 21 & 21 & $\mathrm{~F}$ & 10.6 & 1.1 & 9.6 & \multicolumn{2}{|c|}{ Tremolite } & & & & \\
\hline G1 & 5 & E7 & $A X$ & 20 & 20 & $\mathrm{~F}$ & 6.2 & 0.55 & 11.3 & \multicolumn{2}{|c|}{ Tremolite } & & & & \\
\hline G1 & 5 & E7 & $A X$ & 19 & 19 & $\mathrm{~F}$ & 20.5 & 2 & 10.2 & \multicolumn{2}{|c|}{ Tremolite } & & & & \\
\hline G1 & 4 & J8 & $A X$ & 18 & 18 & $\mathrm{~F}$ & 5.8 & 0.85 & 6.8 & \multicolumn{2}{|c|}{ Tremolite } & & & & \\
\hline $\mathrm{G} 1$ & 4 & J8 & $A X$ & 16 & 16 & $F$ & 12 & 1.75 & 6.9 & \multicolumn{2}{|c|}{ Tremolite } & & & & \\
\hline G1 & 3 & G6 & $A X$ & 12 & 12 & $F$ & 15.5 & 1.2 & 12.9 & \multicolumn{2}{|c|}{ Tremolite } & & & & \\
\hline G1 & 2 & 13 & $A X$ & 9 & 9 & $F$ & 45 & 0.9 & 50 & \multicolumn{2}{|c|}{ Tremolite } & & & & \\
\hline G1 & 2 & 13 & $A X$ & 7 & 7 & $\mathrm{~F}$ & 7.25 & 0.4 & 18.1 & \multicolumn{2}{|c|}{ Tremolite } & & & & \\
\hline G1 & 1 & E1 & $A Z Q$ & 1 & 1 & $\mathrm{~F}$ & 9.8 & 0.6 & 16.3 & \multicolumn{2}{|c|}{ Tremolite } & $\mathrm{Mg}, \mathrm{Al}, \mathrm{Si}, \mathrm{Ca}, \mathrm{Fe}$ & & & \\
\hline G1 & 1 & E1 & $A X$ & 6 & 6 & $\mathrm{~F}$ & 5.5 & 1.25 & 4.4 & \multicolumn{2}{|c|}{ Tremolite } & & & & \\
\hline G1 & 1 & E1 & $A X$ & 4 & 4 & $\mathrm{~F}$ & 8.8 & 1.2 & 7.3 & \multicolumn{2}{|c|}{ Tremolite } & & & & \\
\hline G1 & 4 & J8 & $A X$ & 17 & 17 & $\mathrm{~F}$ & 12.5 & 0.55 & 22.7 & \multicolumn{2}{|c|}{ Tremolite } & & & & \\
\hline $\mathrm{G} 2$ & 9 & 18 & $A X$ & 28 & 28 & $\mathrm{~F}$ & 10 & 1 & 10 & Tremc & blite & & & & \\
\hline $\mathrm{G} 2$ & 10 & D7 & $A X$ & 31 & 31 & $\mathrm{~F}$ & 10 & 1.5 & 6.7 & Tremc & lite & & & & \\
\hline $\mathrm{G} 2$ & 10 & D7 & $A X$ & 32 & 32 & $\mathrm{~F}$ & 15.5 & 4.35 & 3.6 & Tremc & lite & & & & \\
\hline G2 & 7 & $\mathrm{~F} 2$ & $A X$ & 25 & 25 & $\mathrm{~F}$ & 35 & 2.25 & 15.6 & Tremc & lite & & & & \\
\hline G2 & 10 & D7 & $A X$ & 30 & 30 & $\mathrm{~F}$ & 8.85 & 0.8 & 11.1 & Tremc & lite & & & & \\
\hline G2 & 9 & 18 & $A X$ & 29 & 29 & $\mathrm{~F}$ & 9 & 0.45 & 20 & Tremc & olite & & & & \\
\hline & & M Equ & Ient S & tructu & Ires-I & & 222 & & Not & icable & & Not Applicable & & 20 & \\
\hline $\mathrm{Gr}$ & No. & Loc. & ID & Prim & Tot & Class & Len & Wid & Asp & Analy & & Elements & Comment & & \\
\hline G1 & 4 & J8 & $A X$ & 14 & 14 & $\mathrm{~F}$ & 48 & 1.85 & 25.9 & Tremc & lite & & & & \\
\hline G1 & 5 & E7 & $A X$ & 22 & 22 & $\mathrm{~F}$ & 6.2 & 0.4 & 15.5 & Tremc & lite & & & & \\
\hline G1 & 5 & E7 & $A X$ & 21 & 21 & $\mathrm{~F}$ & 10.6 & 1.1 & 9.6 & Tremc & lite & & & & \\
\hline G1 & 5 & E7 & $A X$ & 20 & 20 & $\mathrm{~F}$ & 6.2 & 0.55 & 11.3 & Tremc & lite & & & & \\
\hline
\end{tabular}


Job Number: 070434

SEA

ISO 10312, Direct Count Categories

Client: Idaho National Laboratory

Report Number: 070434R06

Date Received: 4/23/2007

Project Name: RARE

Lab/Cor Sample No.: S35

Client Sample No.: FB-1-R3

Description:

Filter Fraction: 1

Residual Ash Vol:
Aliquot Dilution: 0

Final Dilution: 0
Volume (L): 0

Lab Filter Area (mm2): 385

Grid Openings Analyzed: 10

Average Grid Opening Area: 0.009

Area Analyzed (mm2): 0.09

Analytical Sens. (struc/cc): 0

Dectection Limit. (struc/cc): 0

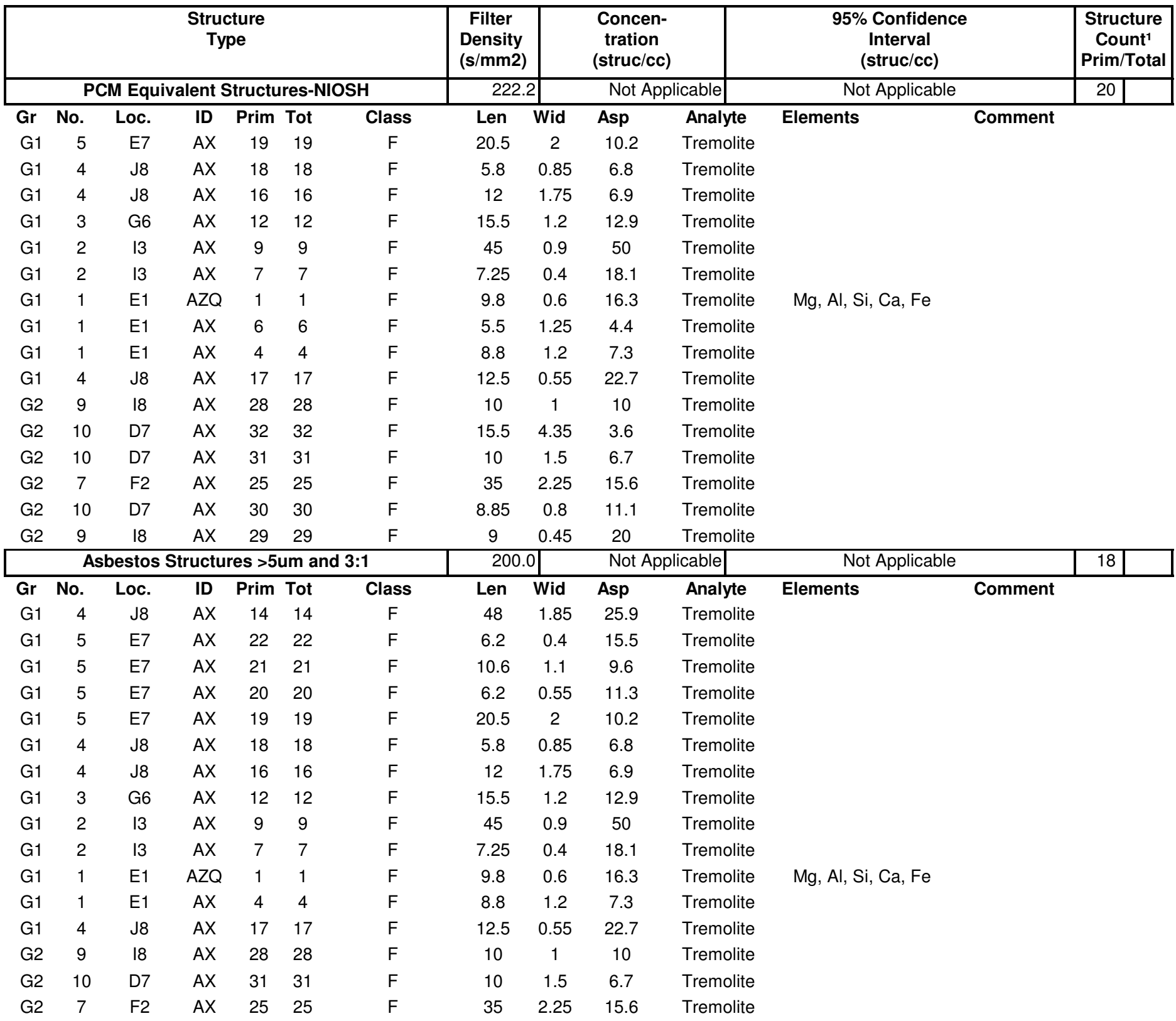


Job Number: 070434

SEA

ISO 10312, Direct Count Categories

Client: Idaho National Laboratory

Project Name: RARE

Lab/Cor Sample No.: S35

Client Sample No.: FB-1-R3

Description:

Filter Fraction: 1

Residual Ash Vol:
Aliquot Dilution: 0

Final Dilution: 0
Report Number: 070434R06

Date Received: 4/23/2007
Volume (L): 0

Lab Filter Area (mm2): 385

Grid Openings Analyzed: 10

Average Grid Opening Area: 0.009

Area Analyzed (mm2): 0.09

Analytical Sens. (struc/cc): 0

Dectection Limit. (struc/cc): 0

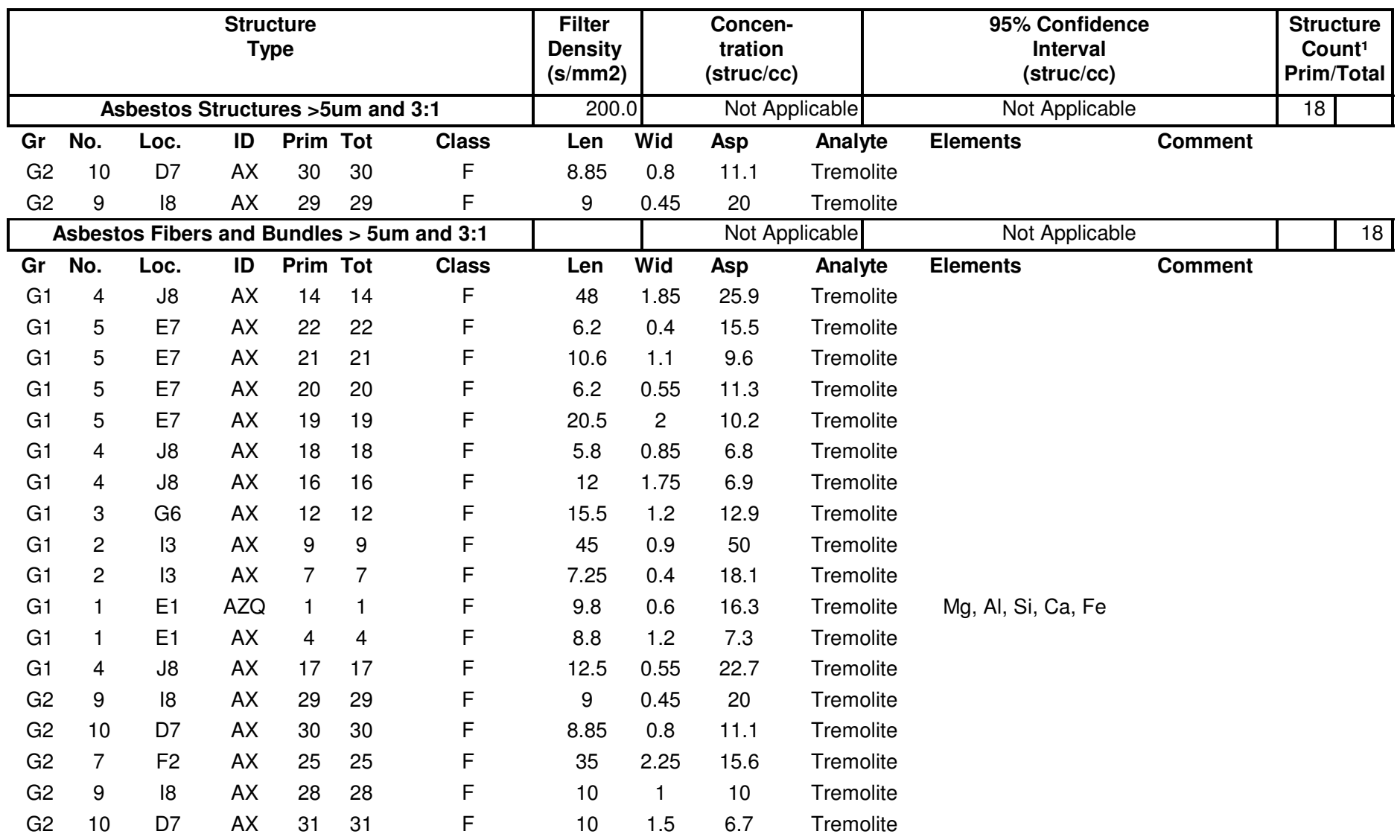


Job Number: 070434

SEA

ISO 10312, Direct Count Categories

Client: Idaho National Laboratory

Report Number: 070434R06

Date Received: 4/23/2007

Project Name: RARE

Lab/Cor Sample No.: S36

Client Sample No.: FB-1-R4

Description:

Filter Fraction: 1

Residual Ash Vol:
Aliquot Dilution: 0

Final Dilution: 0
Volume (L): 0

Lab Filter Area (mm2): 385

Grid Openings Analyzed: 10

Average Grid Opening Area: 0.009

Area Analyzed (mm2): 0.09

Analytical Sens. (struc/cc): 0

Dectection Limit. (struc/cc): 0

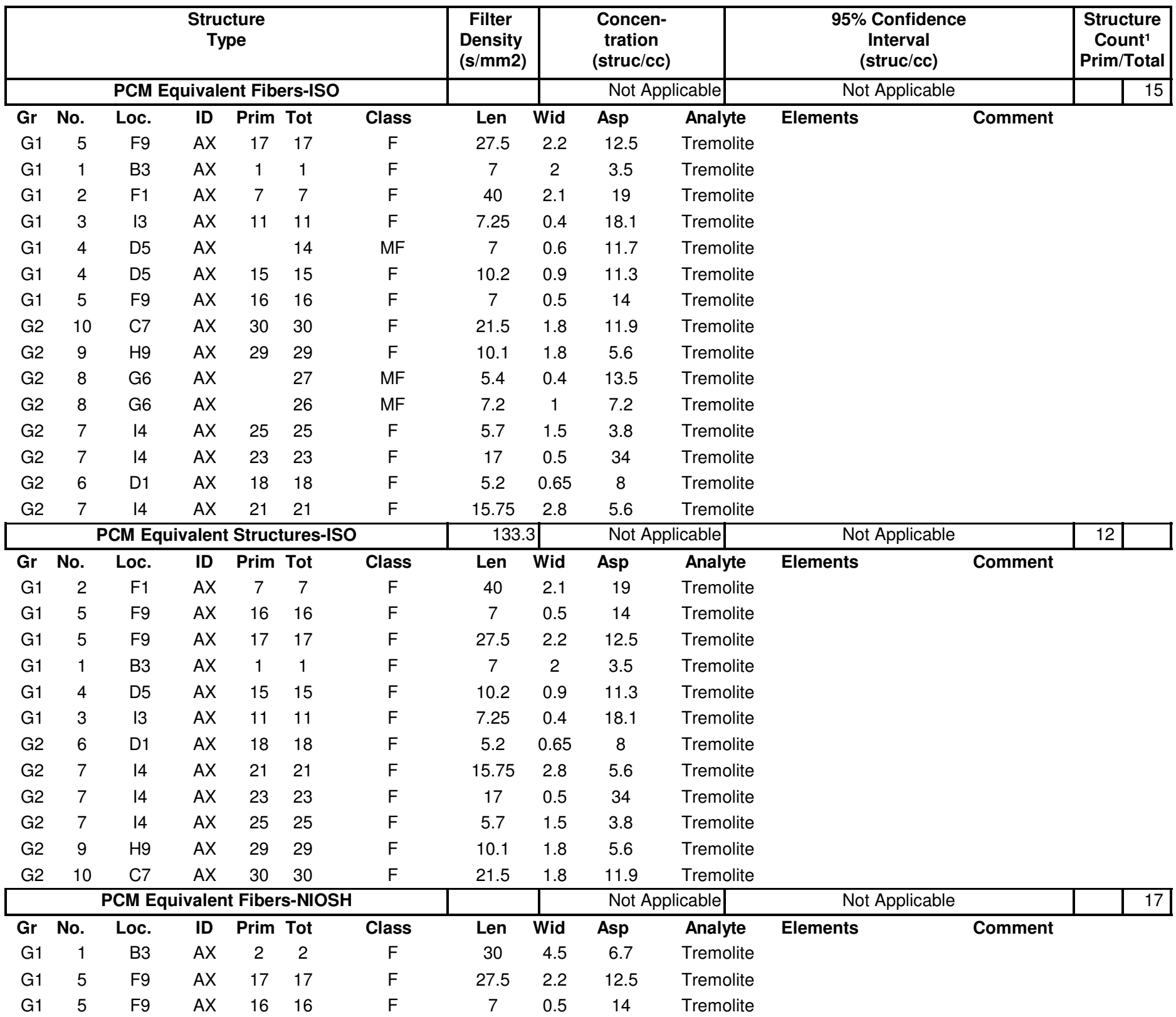


Job Number: 070434

SEA

ISO 10312, Direct Count Categories

Client: Idaho National Laboratory

Report Number: 070434R06

Date Received: 4/23/2007

Project Name: RARE

Lab/Cor Sample No.: S36

Client Sample No.: FB-1-R4

Description:

Filter Fraction: 1

Residual Ash Vol:
Aliquot Dilution: 0

Final Dilution: 0
Volume (L): 0

Lab Filter Area (mm2): 385

Grid Openings Analyzed: 10

Average Grid Opening Area: 0.009

Area Analyzed (mm2): 0.09

Analytical Sens. (struc/cc): 0

Dectection Limit. (struc/cc): 0

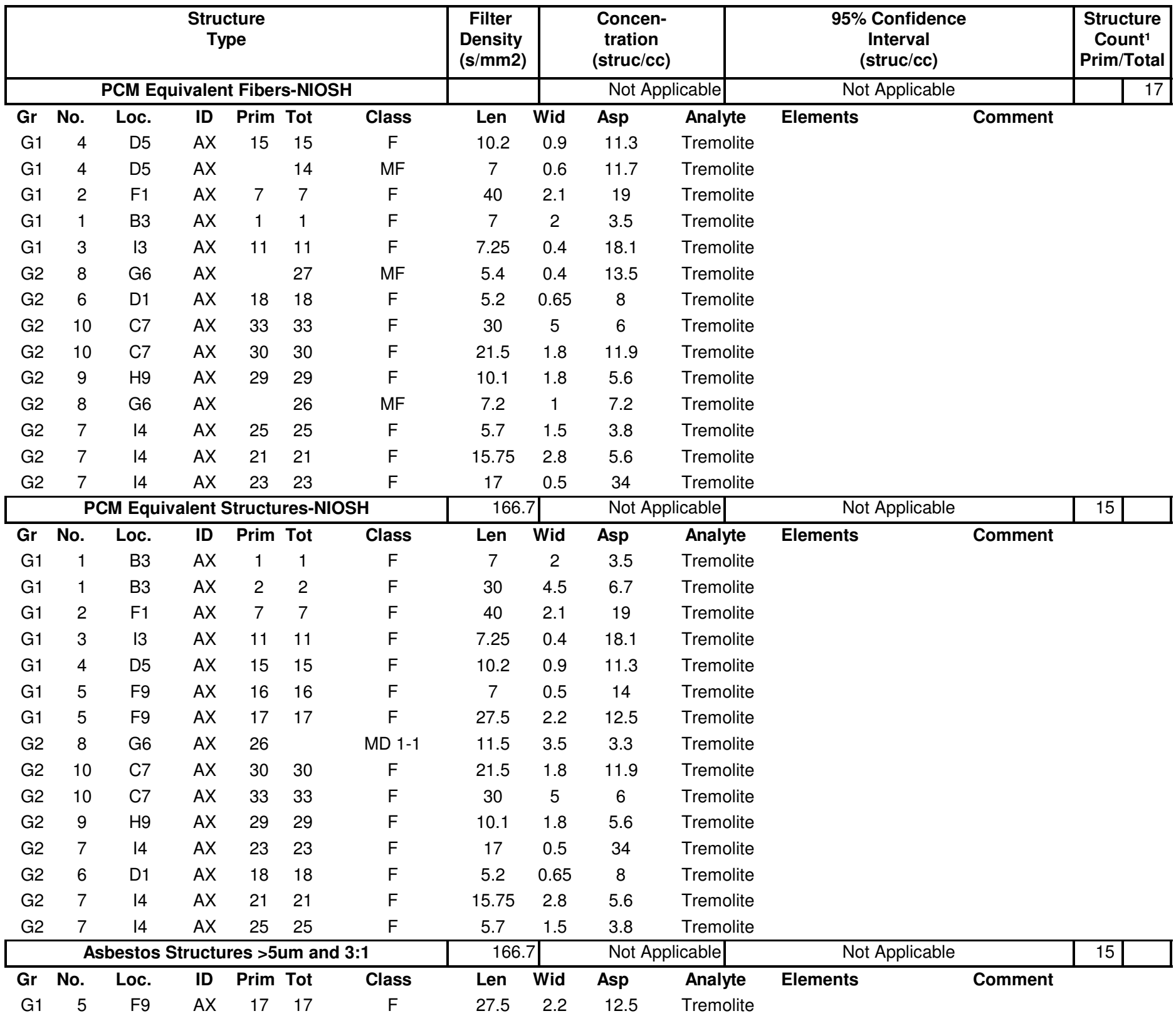


Job Number: 070434

SEA

ISO 10312, Direct Count Categories

Client: Idaho National Laboratory

Project Name: RARE

Lab/Cor Sample No.: S36

Client Sample No.: FB-1-R4

Description:

Filter Fraction: 1

Residual Ash Vol:
Aliquot Dilution: 0

Final Dilution: 0
Report Number: 070434R06

Date Received: 4/23/2007
Volume (L): 0

Lab Filter Area (mm2): 385

Grid Openings Analyzed: 10

Average Grid Opening Area: 0.009

Area Analyzed (mm2): 0.09

Analytical Sens. (struc/cc): 0

Dectection Limit. (struc/cc): 0

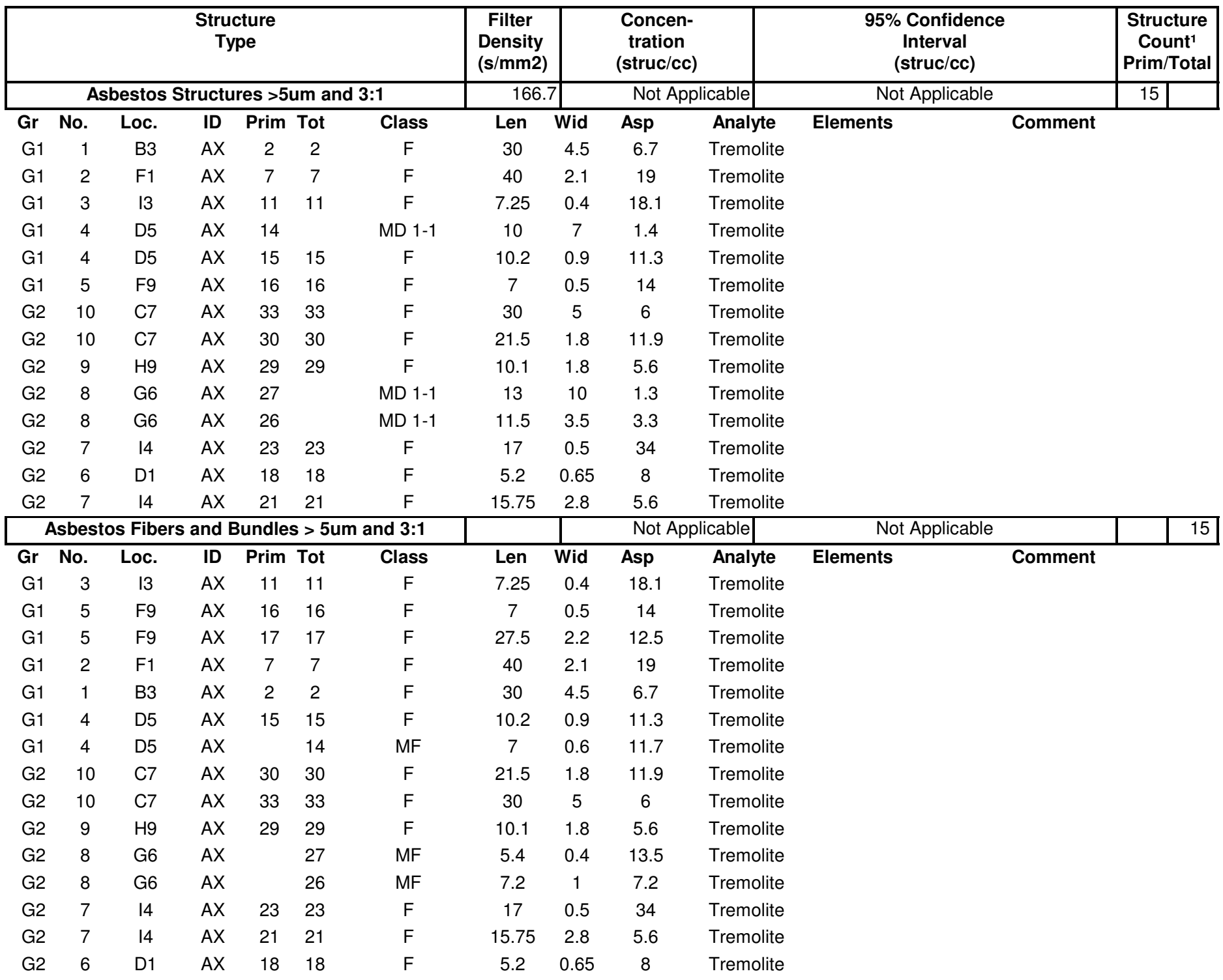


Job Number: 070434

SEA

ISO 10312, Direct Count Categories

Client: Idaho National Laboratory

Report Number: 070434R06

Date Received: 4/23/2007

Project Name: RARE

Lab/Cor Sample No.: S37

Client Sample No.: FB-1-R5

Description:

Filter Fraction: 1

Residual Ash Vol:
Aliquot Dilution: 0

Final Dilution: 0
Volume (L): 0

Lab Filter Area (mm2): 385

Grid Openings Analyzed: 10

Average Grid Opening Area: 0.009

Area Analyzed (mm2): 0.09

Analytical Sens. (struc/cc): 0

Dectection Limit. (struc/cc): 0

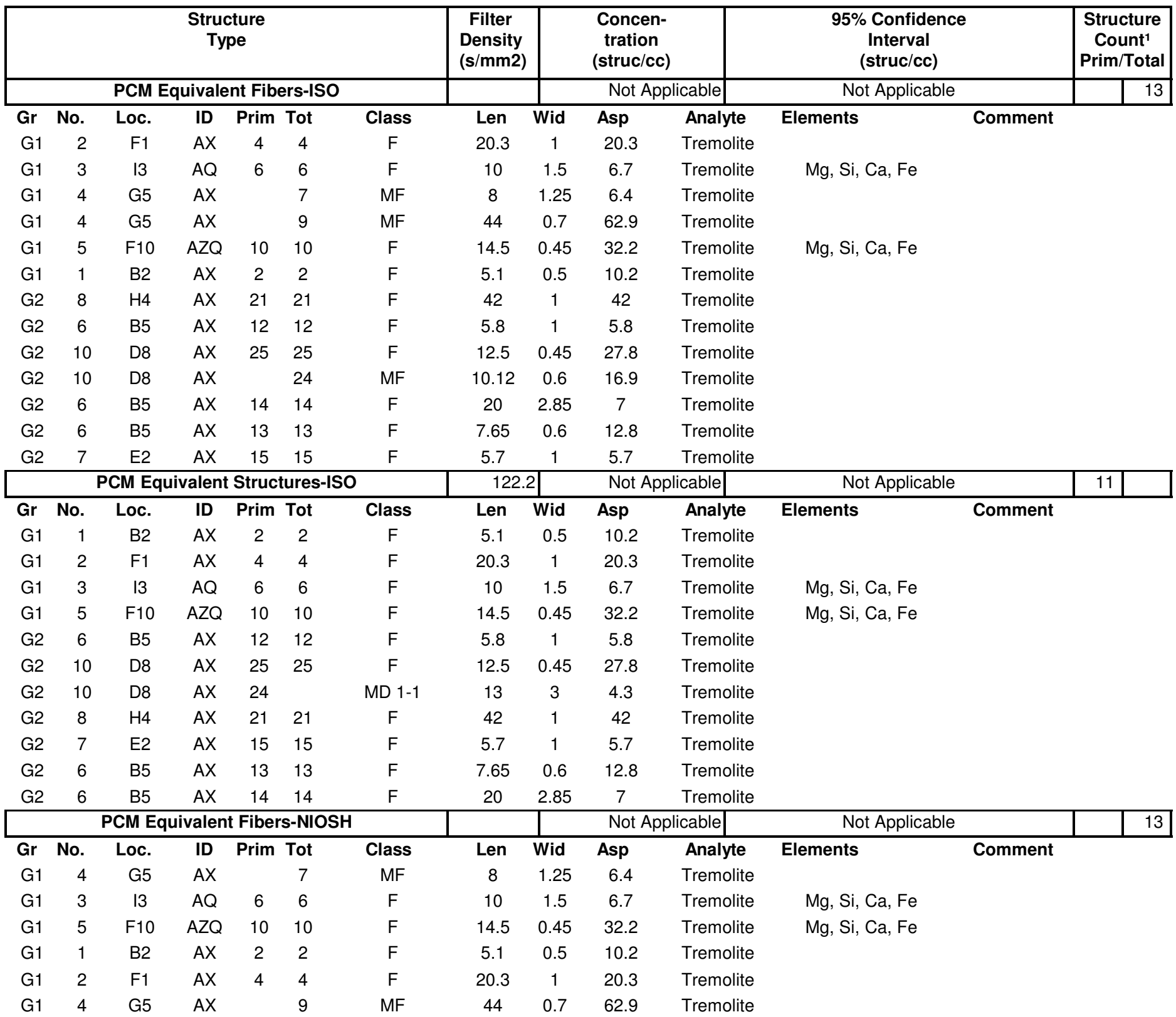


Job Number: 070434

SEA

ISO 10312, Direct Count Categories

Client: Idaho National Laboratory

Report Number: 070434R06

Date Received: 4/23/2007

Project Name: RARE

Lab/Cor Sample No.: S37

Client Sample No.: FB-1-R5

Description:

Filter Fraction: 1

Residual Ash Vol:
Aliquot Dilution: 0

Final Dilution: 0
Volume (L): 0

Lab Filter Area (mm2): 385

Grid Openings Analyzed: 10

Average Grid Opening Area: 0.009

Area Analyzed (mm2): 0.09

Analytical Sens. (struc/cc): 0

Dectection Limit. (struc/cc): 0

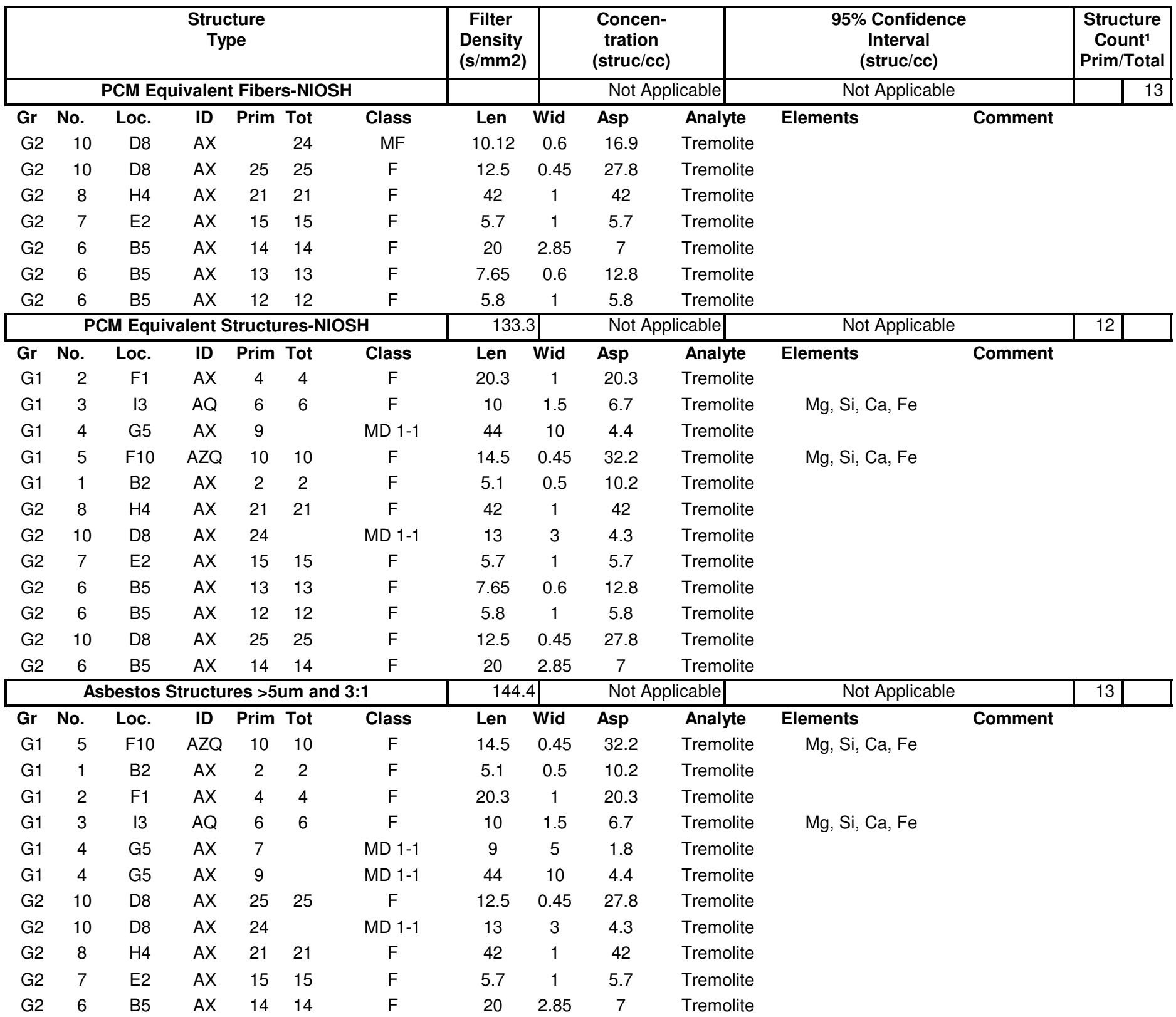


ISO 10312, Direct Count Categories

Job Number: 070434

SEA

Report Number: 070434R06

Client: Idaho National Laboratory

Date Received: 4/23/2007

Project Name: RARE

Lab/Cor Sample No.: S37

Client Sample No.: FB-1-R5

Description:

Filter Fraction: 1

Residual Ash Vol:
Aliquot Dilution: 0

Final Dilution: 0
Volume (L): 0

Lab Filter Area (mm2): 385

Grid Openings Analyzed: 10

Average Grid Opening Area: 0.009

Area Analyzed (mm2): 0.09

Analytical Sens. (struc/cc): 0

Dectection Limit. (struc/cc): 0

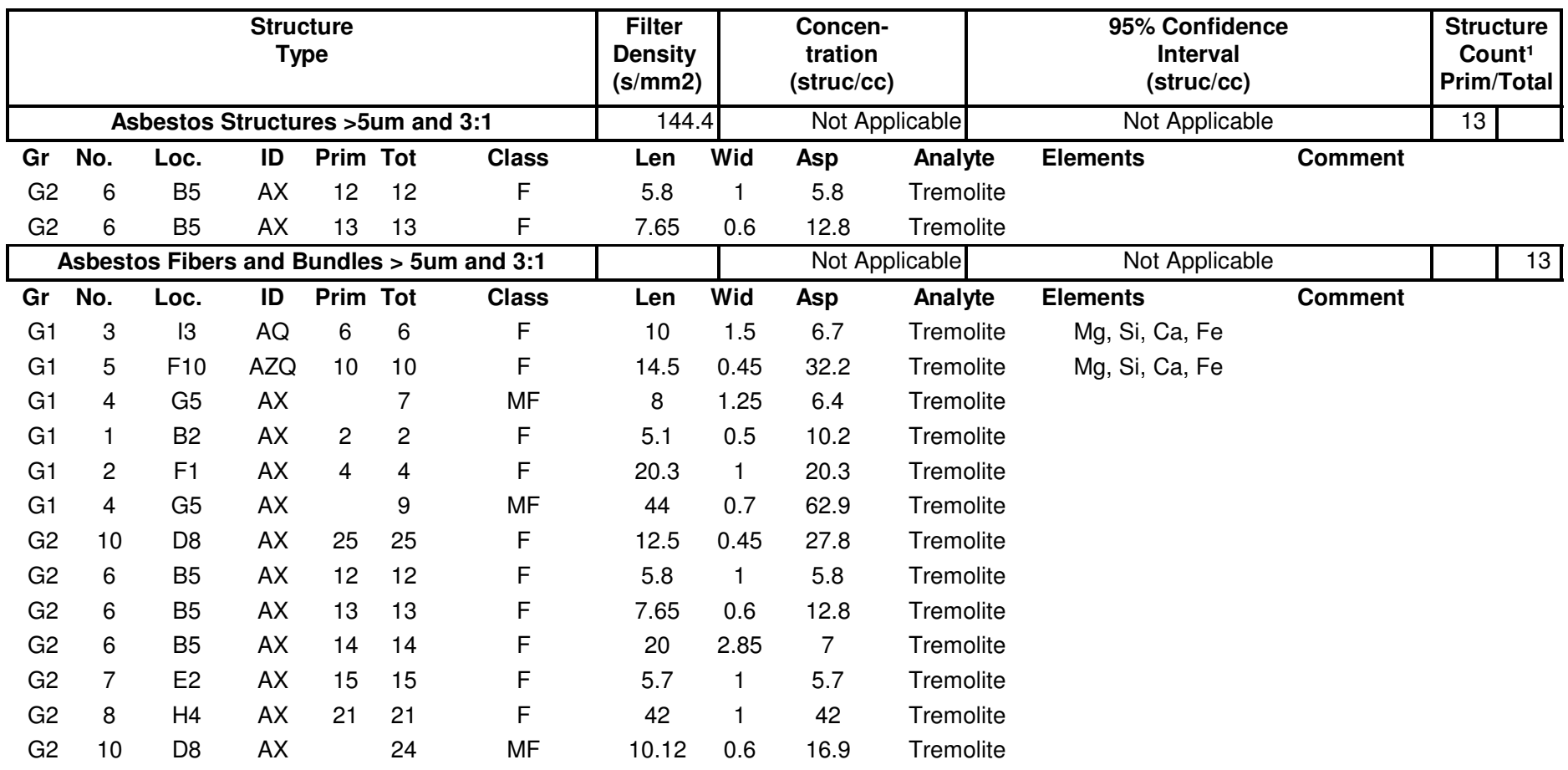


Job Number: 070434

SEA

ISO 10312, Direct Count Categories

Client: Idaho National Laboratory

Report Number: 070434R06

Date Received: 4/23/2007

Project Name: RARE

Lab/Cor Sample No.: S38

Client Sample No.: FB-1-R6

Description:

Filter Fraction: 1

Residual Ash Vol:
Aliquot Dilution: 0

Final Dilution: 0
Volume (L): 0

Lab Filter Area (mm2): 385

Grid Openings Analyzed: 10

Average Grid Opening Area: 0.009

Area Analyzed (mm2): 0.09

Analytical Sens. (struc/cc): 0

Dectection Limit. (struc/cc): 0

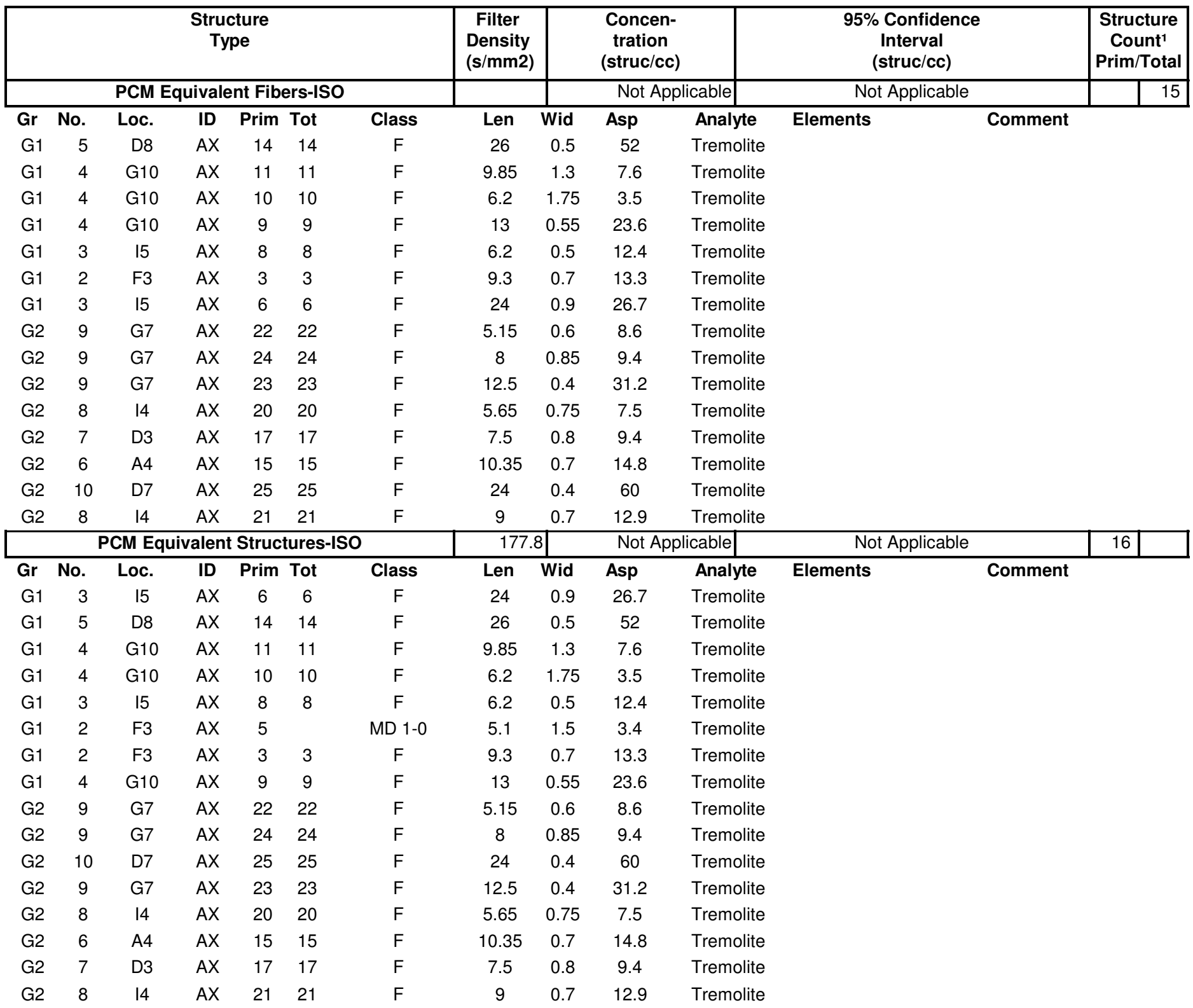


Job Number: 070434

SEA

ISO 10312, Direct Count Categories

Client: Idaho National Laboratory

Report Number: 070434R06

Date Received: 4/23/2007

Project Name: RARE

Lab/Cor Sample No.: S38

Client Sample No.: FB-1-R6

Description:

Filter Fraction: 1

Residual Ash Vol:
Aliquot Dilution: 0

Final Dilution: 0
Volume (L): 0

Lab Filter Area (mm2): 385

Grid Openings Analyzed: 10

Average Grid Opening Area: 0.009

Area Analyzed (mm2): 0.09

Analytical Sens. (struc/cc): 0

Dectection Limit. (struc/cc): 0

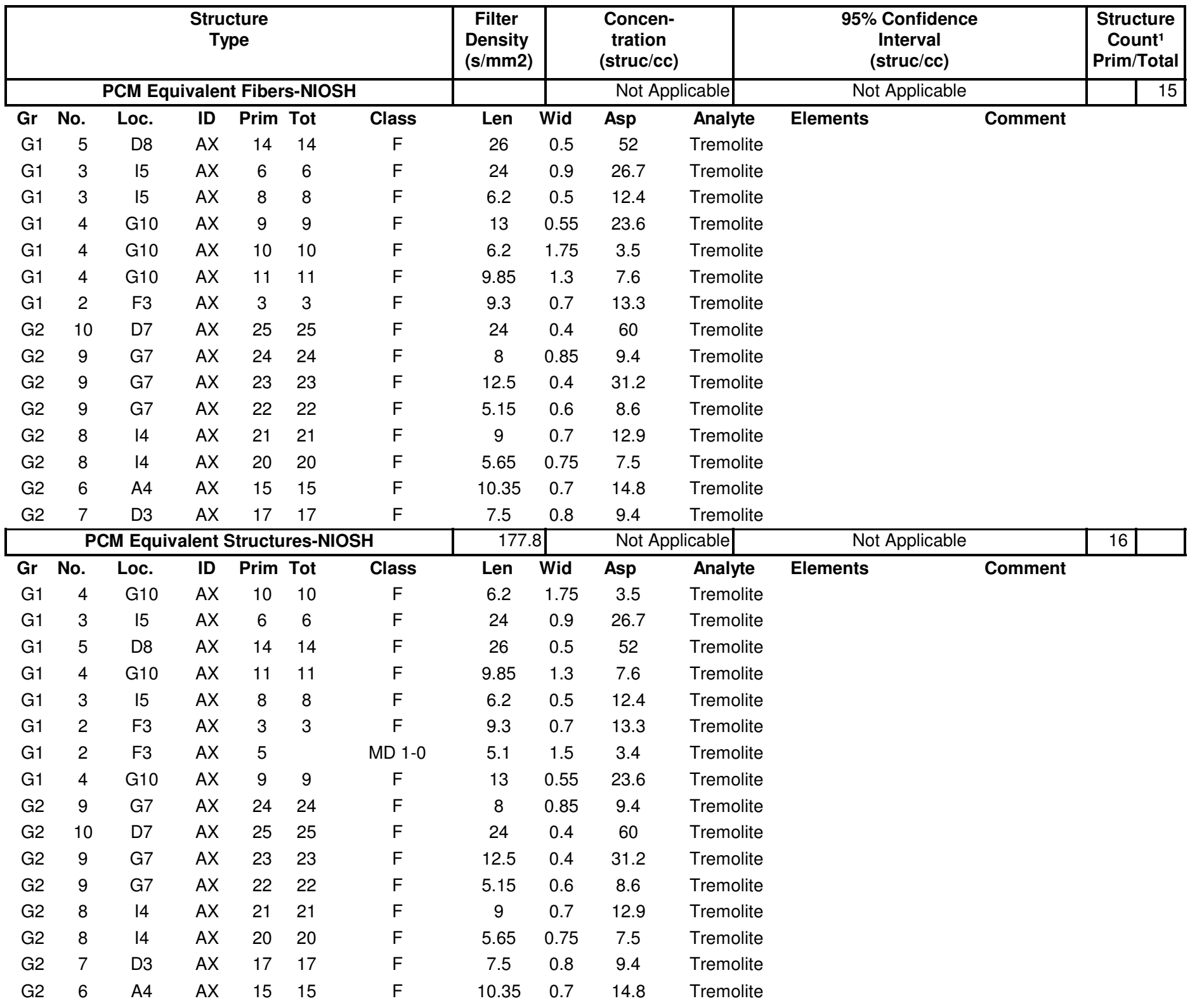


Job Number: 070434

SEA

ISO 10312, Direct Count Categories

Client: Idaho National Laboratory

Report Number: 070434R06

Date Received: 4/23/2007

Project Name: RARE

Lab/Cor Sample No.: S38

Client Sample No.: FB-1-R6

Description:

Filter Fraction: 1

Residual Ash Vol:
Aliquot Dilution: 0

Final Dilution: 0
Volume (L): 0

Lab Filter Area (mm2): 385

Grid Openings Analyzed: 10

Average Grid Opening Area: 0.009

Area Analyzed (mm2): 0.09

Analytical Sens. (struc/cc): 0

Dectection Limit. (struc/cc): 0

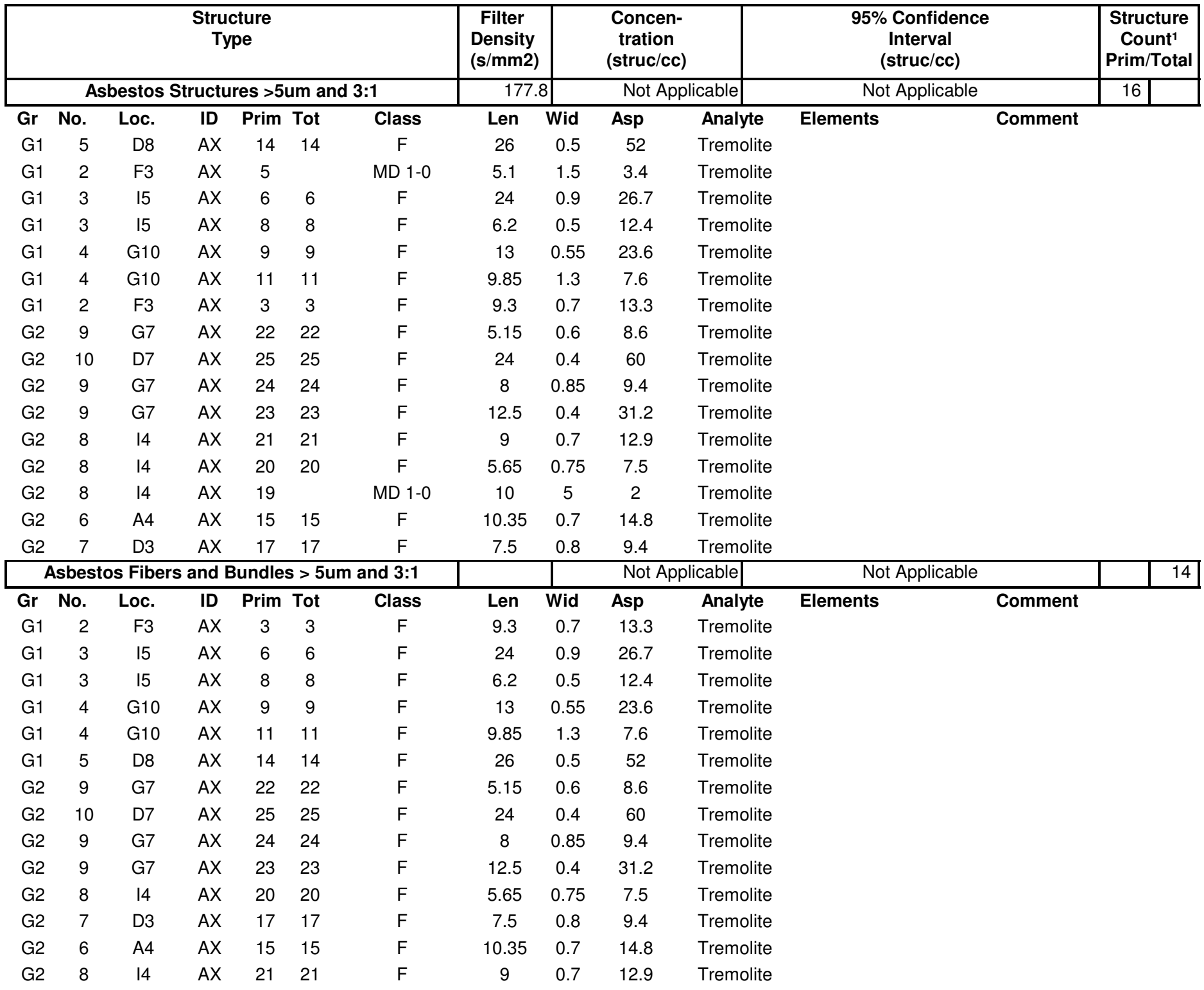


Job Number: 070434

SEA

ISO 10312, Direct Count Categories

Client: Idaho National Laboratory

Report Number: 070434R06

Date Received: 4/23/2007

Project Name: RARE

Lab/Cor Sample No.: S39

Client Sample No.: FB-1-R7

Description:

Filter Fraction: 1

Residual Ash Vol:
Aliquot Dilution: 0

Final Dilution: 0
Volume (L): 0

Lab Filter Area (mm2): 385

Grid Openings Analyzed: 10

Average Grid Opening Area: 0.009

Area Analyzed (mm2): 0.09

Analytical Sens. (struc/cc): 0

Dectection Limit. (struc/cc): 0

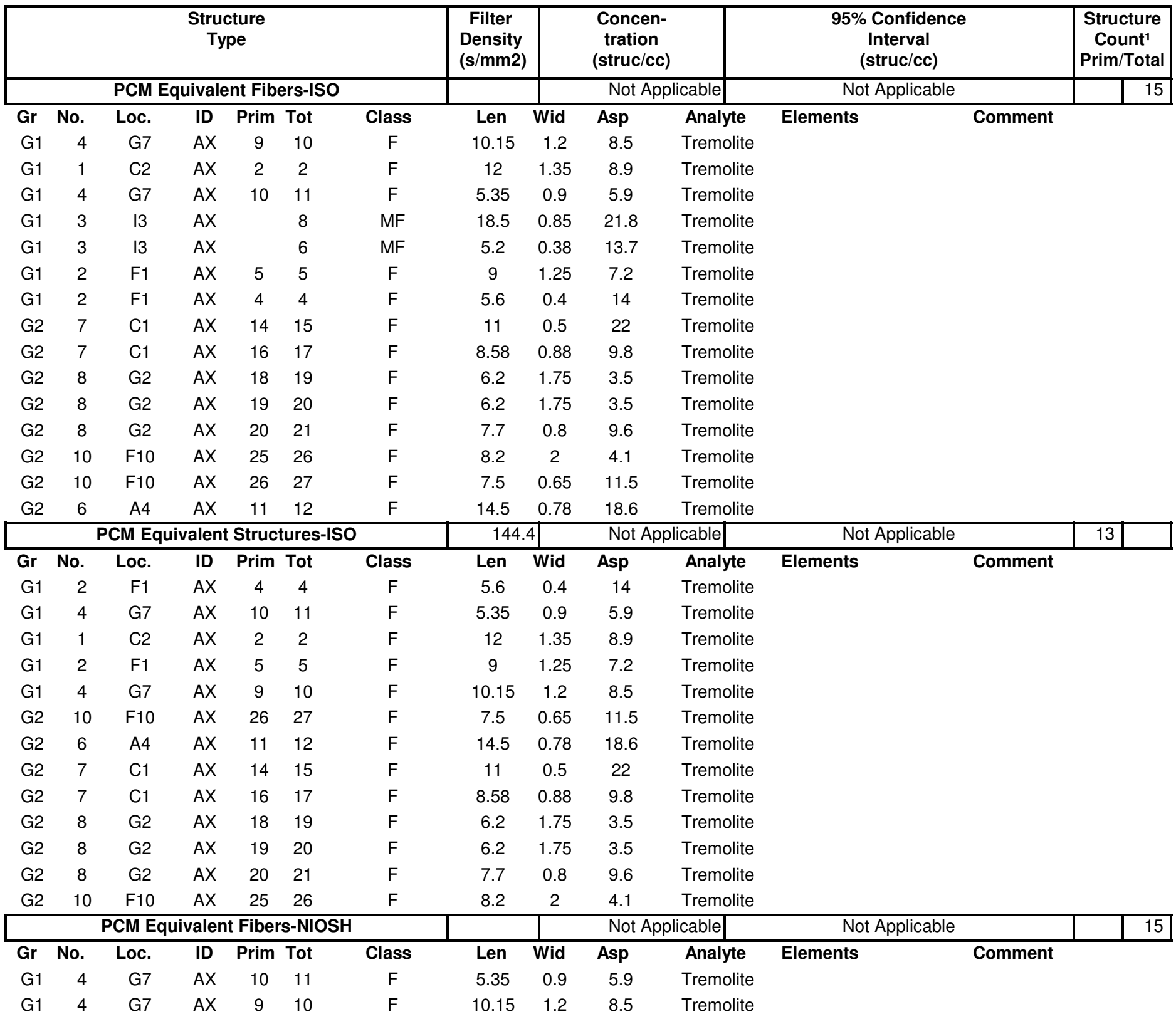


Job Number: 070434

SEA

ISO 10312, Direct Count Categories

Client: Idaho National Laboratory

Report Number: 070434R06

Date Received: 4/23/2007

Project Name: RARE

Lab/Cor Sample No.: S39

Client Sample No.: FB-1-R7

Description:

Filter Fraction: 1

Residual Ash Vol:
Aliquot Dilution: 0

Final Dilution: 0
Volume (L): 0

Lab Filter Area (mm2): 385

Grid Openings Analyzed: 10

Average Grid Opening Area: 0.009

Area Analyzed (mm2): 0.09

Analytical Sens. (struc/cc): 0

Dectection Limit. (struc/cc): 0

\begin{tabular}{|c|c|c|c|c|c|c|c|c|c|c|c|c|c|c|c|}
\hline & & & $\begin{array}{r}\text { Stru } \\
T_{y}\end{array}$ & $\begin{array}{l}\text { ture } \\
\text { pe }\end{array}$ & & & $\begin{array}{c}\text { Filter } \\
\text { Density } \\
(\mathrm{s} / \mathrm{mm} 2)\end{array}$ & & $\begin{array}{r}\text { Conc } \\
\text { trati } \\
\text { (struc }\end{array}$ & & & $\begin{array}{c}95 \% \text { Confidence } \\
\text { Interval } \\
\text { (struc/cc) }\end{array}$ & & $\begin{array}{r}\text { Struc } \\
\text { Col } \\
\text { Prim }\end{array}$ & \\
\hline & & $\mathrm{CM}$ EC & valer & Fiber & $s-\mathrm{NIC}$ & & & & Not & licable & & Not Applicable & & & 15 \\
\hline $\mathrm{Gr}$ & No. & Loc. & ID & Prim & Tot & Class & Len & Wid & Asp & Anal & & Elements & Comment & & \\
\hline G1 & 3 & 13 & $A X$ & & 8 & MF & 18.5 & 0.85 & 21.8 & Trem & lite & & & & \\
\hline G1 & 3 & 13 & $A X$ & & 6 & MF & 5.2 & 0.38 & 13.7 & Trem & lite & & & & \\
\hline G1 & 2 & $\mathrm{~F} 1$ & $A X$ & 5 & 5 & $\mathrm{~F}$ & 9 & 1.25 & 7.2 & Trem & lite & & & & \\
\hline G1 & 1 & $\mathrm{C} 2$ & $A X$ & 2 & 2 & $\mathrm{~F}$ & 12 & 1.35 & 8.9 & Trem & lite & & & & \\
\hline G1 & 2 & $\mathrm{~F} 1$ & $A X$ & 4 & 4 & $\mathrm{~F}$ & 5.6 & 0.4 & 14 & Trem & blite & & & & \\
\hline G2 & 7 & C1 & $A X$ & 16 & 17 & $\mathrm{~F}$ & 8.58 & 0.88 & 9.8 & Trem & lite & & & & \\
\hline G2 & 10 & F10 & $A X$ & 25 & 26 & $\mathrm{~F}$ & 8.2 & 2 & 4.1 & Trem & lite & & & & \\
\hline G2 & 8 & G2 & $A X$ & 20 & 21 & $\mathrm{~F}$ & 7.7 & 0.8 & 9.6 & Trem & lite & & & & \\
\hline G2 & 10 & F10 & $A X$ & 26 & 27 & $\mathrm{~F}$ & 7.5 & 0.65 & 11.5 & Trem & lite & & & & \\
\hline G2 & 7 & $\mathrm{C} 1$ & $A X$ & 14 & 15 & $\mathrm{~F}$ & 11 & 0.5 & 22 & Trem & lite & & & & \\
\hline G2 & 6 & A4 & $A X$ & 11 & 12 & $\mathrm{~F}$ & 14.5 & 0.78 & 18.6 & Trem & lite & & & & \\
\hline G2 & 8 & G2 & $A X$ & 19 & 20 & $\mathrm{~F}$ & 6.2 & 1.75 & 3.5 & Trem & lite & & & & \\
\hline G2 & 8 & G2 & $A X$ & 18 & 19 & $\mathrm{~F}$ & 6.2 & 1.75 & 3.5 & Trem & lite & & & & \\
\hline & & M Equ & lent & tructu & Ires-I & & 144. & & Not & licable & & Not Applicable & & 13 & \\
\hline $\mathrm{Gr}$ & No. & Loc. & ID & Prim & Tot & Class & Len & Wid & Asp & Anal & & Elements & Comment & & \\
\hline $\mathrm{G} 1$ & 1 & $\mathrm{C} 2$ & $A X$ & 2 & 2 & $\mathrm{~F}$ & 12 & 1.35 & 8.9 & Trem & lite & & & & \\
\hline G1 & 2 & $\mathrm{~F} 1$ & $A X$ & 4 & 4 & $\mathrm{~F}$ & 5.6 & 0.4 & 14 & Trem & lite & & & & \\
\hline G1 & 2 & $\mathrm{~F} 1$ & $A X$ & 5 & 5 & $\mathrm{~F}$ & 9 & 1.25 & 7.2 & Trem & lite & & & & \\
\hline G1 & 4 & G7 & $A X$ & 9 & 10 & $\mathrm{~F}$ & 10.15 & 1.2 & 8.5 & Trem & lite & & & & \\
\hline G1 & 4 & G7 & $A X$ & 10 & 11 & $\mathrm{~F}$ & 5.35 & 0.9 & 5.9 & Trem & lite & & & & \\
\hline G2 & 6 & A4 & $A X$ & 11 & 12 & $\mathrm{~F}$ & 14.5 & 0.78 & 18.6 & Trem & lite & & & & \\
\hline G2 & 10 & F10 & $A X$ & 26 & 27 & $\mathrm{~F}$ & 7.5 & 0.65 & 11.5 & Trem & lite & & & & \\
\hline G2 & 10 & F10 & $A X$ & 25 & 26 & $\mathrm{~F}$ & 8.2 & 2 & 4.1 & Trem & lite & & & & \\
\hline G2 & 8 & G2 & $A X$ & 20 & 21 & $\mathrm{~F}$ & 7.7 & 0.8 & 9.6 & Trem & lite & & & & \\
\hline $\mathrm{G} 2$ & 8 & G2 & $A X$ & 19 & 20 & $\mathrm{~F}$ & 6.2 & 1.75 & 3.5 & Trem & lite & & & & \\
\hline G2 & 8 & G2 & $A X$ & 18 & 19 & $\mathrm{~F}$ & 6.2 & 1.75 & 3.5 & Trem & lite & & & & \\
\hline G2 & 7 & $\mathrm{C} 1$ & $A X$ & 14 & 15 & $\mathrm{~F}$ & 11 & 0.5 & 22 & Trem & olite & & & & \\
\hline G2 & 7 & $\mathrm{C} 1$ & $A X$ & 16 & 17 & $\mathrm{~F}$ & 8.58 & 0.88 & 9.8 & Trem & lite & & & & \\
\hline & & estos & ructu & res $>5$ & $\mathrm{um} \mathrm{a}$ & & 144. & & Not & licable & & Not Applicable & & 13 & \\
\hline $\mathrm{Gr}$ & No. & Loc. & ID & Prim & Tot & Class & Len & Wid & Asp & Anal & & Elements & Comment & & \\
\hline $\mathrm{G} 1$ & 3 & 13 & $A X$ & 6 & & MD 2-1 & 15 & 12 & 1.2 & Trem & lite & & & & \\
\hline G1 & 4 & $\mathrm{G} 7$ & $A X$ & 10 & 11 & $F$ & 5.35 & 0.9 & 5.9 & Trem & lite & & & & \\
\hline G1 & 3 & 13 & $A X$ & 7 & & MD 1-1 & 20 & 10 & 2 & Trem & lite & & & & \\
\hline G1 & 2 & $\mathrm{~F} 1$ & $A X$ & 4 & 4 & $\mathrm{~F}$ & 5.6 & 0.4 & 14 & Trem & lite & & & & \\
\hline
\end{tabular}


Job Number: 070434

SEA

ISO 10312, Direct Count Categories

Client: Idaho National Laboratory

Report Number: 070434R06

Date Received: 4/23/2007

Project Name: RARE

Lab/Cor Sample No.: S39

Client Sample No.: FB-1-R7

Description:

Filter Fraction: 1

Residual Ash Vol:
Aliquot Dilution: 0

Final Dilution: 0
Volume (L): 0

Lab Filter Area (mm2): 385

Grid Openings Analyzed: 10

Average Grid Opening Area: 0.009

Area Analyzed (mm2): 0.09

Analytical Sens. (struc/cc): 0

Dectection Limit. (struc/cc): 0

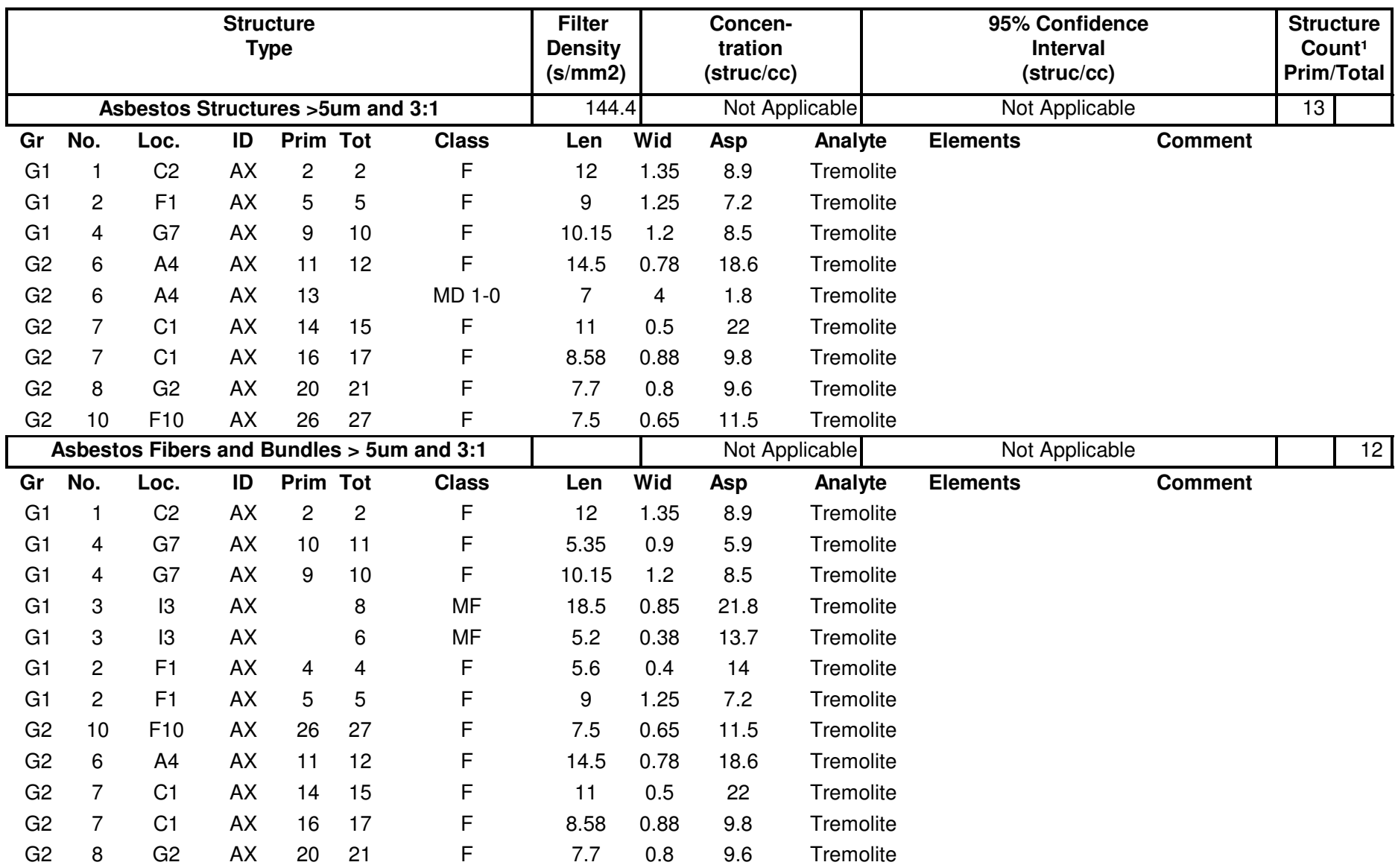


http://www.labcor.net

ISO 10312, Direct Count Categories

Job Number: 070434

SEA

Report Number: 070434R06

Client: Idaho National Laboratory

Date Received: 4/23/2007

Project Name: RARE

Reviewed by:

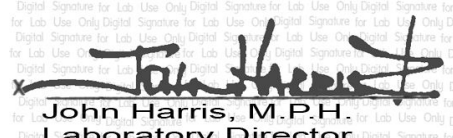

Laboratory Directo 


\section{Appendix F}

\section{TEM DATA for Berman Elutriator}


Analysis Report Cover Final Report
Phone: (206) 781-0155

Fax: (206) 789-8424

http://www.labcor.net
Job Number: 070451

Client: Idaho National Laboratory

Address: PO Box 1625 MS 2107

Idaho Falls, ID 83415-2107

Project Name: RARE

Project Num:

PO Number:

Sub Project:
Report Number: 070451R02

Report Date: 7/13/2007

Enclosed please find results for samples submitted to our laboratory. A list of samples and analyses follows:

\begin{tabular}{|c|c|c|c|}
\hline $\begin{array}{l}\text { Lab/Cor Sample \# } \\
070451 \text { - S1 }\end{array}$ & $\begin{array}{l}\text { Client Sample \# and Description } \\
\text { BR-0-R1 - }\end{array}$ & $\begin{array}{l}\text { Analysis } \\
\text { Soil - Elutriation Method }\end{array}$ & $\begin{array}{l}\text { Date Received: } \\
4 / 23 / 2007\end{array}$ \\
\hline $070451-\mathrm{S} 2$ & BR-1-R1 - & Soil - Elutriation Method & $4 / 23 / 2007$ \\
\hline $070451-\mathrm{S} 3$ & BR-2-R1 - & Soil - Elutriation Method & $4 / 23 / 2007$ \\
\hline $070451-\mathrm{S} 4$ & BR-3-R1 - & Soil - Elutriation Method & $4 / 23 / 2007$ \\
\hline $070451-\mathrm{S} 5$ & BR-4-R1 - & Soil - Elutriation Method & $4 / 23 / 2007$ \\
\hline
\end{tabular}

Soil - Elutriation Preparation and analysis of the above samples was conducted in accordance with the EPA Superfund method EPA $5402-90$ Method - EPA 540- 005a with modifications dated May 23, 2000 using ISO method 10312 (Direct) counting rules for the identification of asbestos. 2-90-005, Modified In an effort to create a sample with acceptable releasability, the samples submitted to the lab were combined with blank in-

May 23, 2000 house soil samples that were previously tested for asbestos using the elutriation method. The samples were conditioned overnight in a desiccator with $\mathrm{CaNO} 3-4 \mathrm{H} 2 \mathrm{O}$ including all filters used in the procedure.

Approximately 40-60 grams the combined sample are loaded in the tumbler and rotated at a speed of about $30 \mathrm{rpm}$ to initiate the run. Mixed cellulose ester (MCE) filters are placed at the sliding mechanism at the top of the vertical elutriator and moved in and out of the airstream at timed intervals. The filters are weighed after each timed interval. The weight values are used to monitor respirable dust release from the soil and to estimate optimal loading of particulate onto polycarbonate (PC) filters that are collected through an isokinetic port on top of the vertical elutriator. After collection of particulate during the timed intervals, the PC filters were weighed to 7 places, placed in a nonconductive box, then placed in a high vacuum carbon evaporator and carbon-coated to retain particulates in their original locations on the filter.

Carbon-coated filters were dissolved using 20\% 1-2-diaminoethane with 80\% 1-methyl-2-pyrrolidone (NMP) solution followed by a rinse in reagent alcohol. Samples were placed on 200 mesh copper or gold grids for examination by transmission electron microscopy (TEM).

TEM analysis was performed using a transmission electron microscope equipped with an appropriate $X$ ray analyzer. The air samples were analyzed at a screen magnification between 15,000 - 20,000x for asbestos structures greater than 0.5 micrometer lengths. An accelerating voltage of $100 \mathrm{KV}$ was applied.

Structures detected by this method are classified using the ISO fiber classification levels. The minimum acceptance level for Chrysotile is $\mathrm{CMQ}$, and the minimum acceptance level for Amphiboles is ADQ.

Disclaimer The results reported relate only to the samples tested or analyzed. Interpretation of these results is the sole responsibility of the client.

If further clarification of these results is needed, please call us. Thank you for allowing the staff at Lab/Cor, Inc. the opportunity to provide you with the analytical services.

Sincerely,

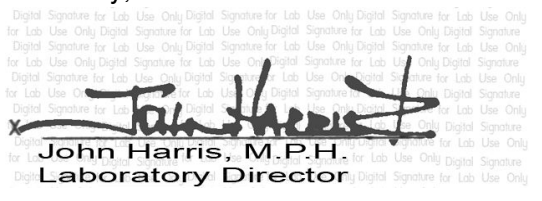


Job Number: 070451

\section{SEA}

\section{Soil - Elutriation Method Summary Data}

Client: Idaho National Laboratory

Report Number: 070451R02

Date Received: 4/23/2007

Project Name: RARE

Lab/Cor Sample No.: S1

Client Sample No.: BR-0-R1

Description:

$\begin{array}{cc}\text { Analyst(s) } & \text { Analysis Date } \\ \text { KM } & 6 / 20 / 2007 \\ \text { KM } & 6 / 21 / 2007\end{array}$

Mass (gPM10) : 0.0001012

Lab Filter Area (mm2) : 385

Grid Openings Analyzed : 89

Average Grid Opening Area (mm2) : 0.00945

Area Analyzed (mm2) : 0.84105

Analytical Sens. (struc/gPM10) : $4.52 \mathrm{E}+06$

Detection Limit. (struc/gPM10) : $1.35 \mathrm{E}+07$

\begin{tabular}{|c|c|c|c|c|c|}
\hline $\begin{array}{c}\text { Structure } \\
\text { Type }\end{array}$ & $\begin{array}{c}\text { Filter } \\
\text { Density } \\
(\mathbf{s} / \mathrm{mm} 2)\end{array}$ & $\begin{array}{c}\text { Concen- } \\
\text { tration* } \\
\text { (struc/gPM10) }\end{array}$ & $\begin{array}{c}\text { 95\% Confidence } \\
\text { Interval } \\
\text { (struc/gPM10) }\end{array}$ & \multicolumn{2}{|c|}{$\begin{array}{c}\text { Structure } \\
\text { Count }^{1} \\
\text { Prim/Total }\end{array}$} \\
\hline Primary Asbestos Structures & 0 & $<4.52 \mathrm{E}+06$ & $0.00 \mathrm{E}+00-3.69 \mathrm{E}+00-$ Poisson & 0 & \\
\hline Total Asbestos Structures & 0 & $<4.52 \mathrm{E}+06$ & $0.00 \mathrm{E}+00-3.69 \mathrm{E}+00-$ Poisson & & $\overline{0}$ \\
\hline PCM Equivalent Structures-ISO & 0 & $<4.52 \mathrm{E}+06$ & $0.00 \mathrm{E}+00-3.69 \mathrm{E}+00-$ Poisson & 0 & \\
\hline Total Chrysotile Structures, $>=0.5-<=5.0$, and $3: 1$ & 0 & $<4.52 \mathrm{E}+06$ & $0.00 \mathrm{E}+00-3.69 \mathrm{E}+00-$ Poisson & & 0 \\
\hline Total Chrysotile Structures, $>5-<=10$, and 3:1 & 0 & $<4.52 \mathrm{E}+06$ & $0.00 \mathrm{E}+00-3.69 \mathrm{E}+00-$ Poisson & & 0 \\
\hline Total Chrysotile Structures, > 10 and 3:1 & 0 & $<4.52 \mathrm{E}+06$ & $0.00 \mathrm{E}+00-3.69 \mathrm{E}+00-$ Poisson & & 0 \\
\hline Total Amphibole Structures, $>=0.5-<=5.0$, and $3: 1$ & 0 & $<4.52 \mathrm{E}+06$ & $0.00 \mathrm{E}+00-3.69 \mathrm{E}+00-$ Poisson & & 0 \\
\hline Total Amphibole Structures, $>5.0-<=10$, and 3:1 & 0 & $<4.52 \mathrm{E}+06$ & $0.00 \mathrm{E}+00-3.69 \mathrm{E}+00-$ Poisson & & 0 \\
\hline Total Amphibole Structures, $>10$, and 3:1 & 0 & $<4.52 \mathrm{E}+06$ & $0.00 \mathrm{E}+00-3.69 \mathrm{E}+00-$ Poisson & & 0 \\
\hline Primary Chrysotile Structures $>5.0 \mu \mathrm{m}$ & 0 & $<4.52 \mathrm{E}+06$ & $0.00 \mathrm{E}+00-3.69 \mathrm{E}+00-$ Poisson & 0 & \\
\hline Primary Chrysotile Structures $>=0.5-<=5.0 \mu \mathrm{m}$ & 0 & $<4.52 \mathrm{E}+06$ & $0.00 \mathrm{E}+00-3.69 \mathrm{E}+00-$ Poisson & 0 & \\
\hline Primary Amphibole Structures $>5.0 \mu \mathrm{m}$ & 0 & $<4.52 \mathrm{E}+06$ & $0.00 \mathrm{E}+00-3.69 \mathrm{E}+00-$ Poisson & 0 & \\
\hline Primary Amphibole Structures $>=0.5-<=5.0 \mu \mathrm{m}$ & 0 & $<4.52 \mathrm{E}+06$ & $0.00 \mathrm{E}+00-3.69 \mathrm{E}+00-$ Poisson & 0 & \\
\hline
\end{tabular}


Job Number: 070451

\section{SEA}

\section{Soil - Elutriation Method Summary Data}

Client: Idaho National Laboratory

Report Number: 070451R02

Date Received: 4/23/2007

Project Name: RARE

Lab/Cor Sample No.: S2

Client Sample No.: BR-1-R1

Description:

$\begin{array}{cc}\text { Analyst(s) } & \text { Analysis Date } \\ \text { KM } & 6 / 28 / 2007\end{array}$

Mass (gPM10) : 0.0001206

Lab Filter Area (mm2) : 385

Grid Openings Analyzed : 74

Average Grid Opening Area (mm2) : 0.00945

Area Analyzed (mm2) : 0.6993

Analytical Sens. (struc/gPM10) : $4.57 \mathrm{E}+06$

Detection Limit. (struc/gPM10) : $1.36 \mathrm{E}+07$

\begin{tabular}{|c|c|c|c|c|c|}
\hline $\begin{array}{c}\text { Structure } \\
\text { Type }\end{array}$ & $\begin{array}{c}\text { Filter } \\
\text { Density } \\
(\mathrm{s} / \mathrm{mm} 2)\end{array}$ & $\begin{array}{c}\text { Concen- } \\
\text { tration } \\
\text { (struc/gPM10) }\end{array}$ & $\begin{array}{c}\text { 95\% Confidence } \\
\text { Interval } \\
\text { (struc/gPM10) }\end{array}$ & \multicolumn{2}{|c|}{$\begin{array}{c}\text { Structure } \\
\text { Count }^{1} \\
\text { Prim/Total }\end{array}$} \\
\hline Primary Asbestos Structures & 8.6 & $2.74 \mathrm{E}+07$ & 1.01E+07 - 5.96E+07 - Poisson & 6 & \\
\hline Total Asbestos Structures & 8.6 & $2.74 \mathrm{E}+07$ & $1.01 \mathrm{E}+07-5.96 \mathrm{E}+07-$ Poisson & & 6 \\
\hline PCM Equivalent Structures-ISO & 4.3 & $1.37 \mathrm{E}+07$ & $2.83 \mathrm{E}+06-4.00 \mathrm{E}+07-$ Poisson & 3 & \\
\hline Total Chrysotile Structures, $>=0.5-<=5.0$, and $3: 1$ & 0 & $<4.57 \mathrm{E}+06$ & $0.00 \mathrm{E}+00-3.69 \mathrm{E}+00-$ Poisson & & 0 \\
\hline Total Chrysotile Structures, $>5-<=10$, and 3:1 & 0 & $<4.57 \mathrm{E}+06$ & $0.00 \mathrm{E}+00-3.69 \mathrm{E}+00-$ Poisson & & 0 \\
\hline Total Chrysotile Structures, $>10$ and 3:1 & 0 & $<4.57 \mathrm{E}+06$ & $0.00 \mathrm{E}+00-3.69 \mathrm{E}+00-$ Poisson & & 0 \\
\hline Total Amphibole Structures, $>=0.5-<=5.0$, and $3: 1$ & 2.9 & $9.13 \mathrm{E}+06$ & $1.10 \mathrm{E}+06-3.30 \mathrm{E}+07-$ Poisson & & 2 \\
\hline Total Amphibole Structures, $>5.0-<=10$, and 3:1 & 1.4 & $4.57 \mathrm{E}+06$ & 1.14E+05 - 2.54E+07 - Poisson & & 1 \\
\hline Total Amphibole Structures, > 10, and 3:1 & 4.3 & $1.37 \mathrm{E}+07$ & $2.83 \mathrm{E}+06-4.00 \mathrm{E}+07-$ Poisson & & 3 \\
\hline Primary Chrysotile Structures $>5.0 \mu \mathrm{m}$ & 0 & $<4.57 \mathrm{E}+06$ & $0.00 \mathrm{E}+00-3.69 \mathrm{E}+00-$ Poisson & 0 & \\
\hline Primary Chrysotile Structures $>=0.5-<=5.0 \mu \mathrm{m}$ & 0 & $<4.57 \mathrm{E}+06$ & $0.00 \mathrm{E}+00-3.69 \mathrm{E}+00-$ Poisson & 0 & \\
\hline Primary Amphibole Structures $>5.0 \mu m$ & 7.2 & $2.28 \mathrm{E}+07$ & $7.41 \mathrm{E}+06-5.33 \mathrm{E}+07-$ Poisson & 5 & \\
\hline Primary Amphibole Structures $>=0.5-<=5.0 \mu \mathrm{m}$ & 1.4 & $4.57 \mathrm{E}+06$ & $1.14 \mathrm{E}+05-2.54 \mathrm{E}+07-$ Poisson & 1 & \\
\hline
\end{tabular}


Job Number: 070451

\section{SEA}

\section{Soil - Elutriation Method Summary Data}

Client: Idaho National Laboratory

Report Number: 070451R02

Date Received: 4/23/2007

Project Name: RARE

Lab/Cor Sample No.: S3

Client Sample No.: BR-2-R1

Description:

$\begin{array}{cc}\text { Analyst(s) } & \text { Analysis Date } \\ \text { KM } & 6 / 28 / 2007\end{array}$

Mass (gPM10) : 0.0001270

Lab Filter Area (mm2) : 385

Grid Openings Analyzed : 70

Average Grid Opening Area (mm2) : 0.00945

Area Analyzed (mm2) : 0.6615

Analytical Sens. (struc/gPM10) : $4.58 \mathrm{E}+06$

Detection Limit. (struc/gPM10) : $1.37 \mathrm{E}+07$

\begin{tabular}{|c|c|c|c|c|c|}
\hline $\begin{array}{c}\text { Structure } \\
\text { Type }\end{array}$ & $\begin{array}{c}\text { Filter } \\
\text { Density } \\
(\mathrm{s} / \mathrm{mm} 2)\end{array}$ & $\begin{array}{c}\text { Concen- } \\
\text { tration } \\
\text { (struc/gPM10) }\end{array}$ & $\begin{array}{c}\text { 95\% Confidence } \\
\text { Interval } \\
\text { (struc/gPM10) }\end{array}$ & \multicolumn{2}{|c|}{$\begin{array}{c}\text { Structure } \\
\text { Count }^{1} \\
\text { Prim/Total }\end{array}$} \\
\hline Primary Asbestos Structures & 0 & $<4.58 \mathrm{E}+06$ & $0.00 \mathrm{E}+00-3.69 \mathrm{E}+00-$ Poisson & 0 & \\
\hline Total Asbestos Structures & 0 & $<4.58 \mathrm{E}+06$ & $0.00 \mathrm{E}+00-3.69 \mathrm{E}+00-$ Poisson & & $\overline{0}$ \\
\hline PCM Equivalent Structures-ISO & 0 & $<4.58 \mathrm{E}+06$ & $0.00 \mathrm{E}+00-3.69 \mathrm{E}+00$ - Poisson & 0 & \\
\hline Total Chrysotile Structures, >=0.5 - <=5.0, and 3:1 & 0 & $<4.58 \mathrm{E}+06$ & $0.00 \mathrm{E}+00-3.69 \mathrm{E}+00$ - Poisson & & 0 \\
\hline Total Chrysotile Structures, $>5-<=10$, and 3:1 & 0 & $<4.58 \mathrm{E}+06$ & $0.00 \mathrm{E}+00-3.69 \mathrm{E}+00-$ Poisson & & 0 \\
\hline Total Chrysotile Structures, $>10$ and 3:1 & 0 & $<4.58 \mathrm{E}+06$ & $0.00 \mathrm{E}+00-3.69 \mathrm{E}+00-$ Poisson & & 0 \\
\hline Total Amphibole Structures, $>=0.5-<=5.0$, and 3:1 & 0 & $<4.58 \mathrm{E}+06$ & $0.00 \mathrm{E}+00-3.69 \mathrm{E}+00-$ Poisson & & 0 \\
\hline Total Amphibole Structures, $>5.0-<=10$, and $3: 1$ & 0 & $<4.58 \mathrm{E}+06$ & $0.00 \mathrm{E}+00-3.69 \mathrm{E}+00-$ Poisson & & 0 \\
\hline Total Amphibole Structures, > 10, and 3:1 & 0 & $<4.58 \mathrm{E}+06$ & $0.00 \mathrm{E}+00-3.69 \mathrm{E}+00$ - Poisson & & 0 \\
\hline Primary Chrysotile Structures $>5.0 \mu \mathrm{m}$ & 0 & $<4.58 \mathrm{E}+06$ & $0.00 \mathrm{E}+00-3.69 \mathrm{E}+00$ - Poisson & 0 & \\
\hline Primary Chrysotile Structures $>=0.5-<=5.0 \mu \mathrm{m}$ & 0 & $<4.58 \mathrm{E}+06$ & $0.00 \mathrm{E}+00-3.69 \mathrm{E}+00-$ Poisson & 0 & \\
\hline Primary Amphibole Structures $>5.0 \mu \mathrm{m}$ & 0 & $<4.58 \mathrm{E}+06$ & $0.00 \mathrm{E}+00-3.69 \mathrm{E}+00$ - Poisson & 0 & \\
\hline Primary Amphibole Structures $>=0.5-<=5.0 \mu \mathrm{m}$ & 0 & $<4.58 \mathrm{E}+06$ & $0.00 \mathrm{E}+00-3.69 \mathrm{E}+00$ - Poisson & 0 & \\
\hline
\end{tabular}


Job Number: 070451

\section{SEA}

\section{Soil - Elutriation Method Summary Data}

Client: Idaho National Laboratory

Report Number: 070451R02

Date Received: 4/23/2007

Project Name: RARE

Lab/Cor Sample No.: S4

Client Sample No.: BR-3-R1

Description:

$\begin{array}{cc}\text { Analyst(s) } & \text { Analysis Date } \\ \text { KM } & 6 / 29 / 2007\end{array}$

Mass (gPM10) : 0.0001257

Lab Filter Area (mm2) : 385

Grid Openings Analyzed : 71

Average Grid Opening Area (mm2) : 0.00945

Area Analyzed (mm2) : 0.67095

Analytical Sens. (struc/gPM10) : 4.56E+06

Detection Limit. (struc/gPM10) : $1.36 \mathrm{E}+07$

\begin{tabular}{|c|c|c|c|c|c|}
\hline $\begin{array}{c}\text { Structure } \\
\text { Type }\end{array}$ & $\begin{array}{c}\text { Filter } \\
\text { Density } \\
(\mathrm{s} / \mathrm{mm} 2)\end{array}$ & $\begin{array}{c}\text { Concen- } \\
\text { tration } \\
\text { (struc/gPM10) }\end{array}$ & $\begin{array}{c}\text { 95\% Confidence } \\
\text { Interval } \\
\text { (struc/gPM10) }\end{array}$ & \multicolumn{2}{|c|}{$\begin{array}{c}\text { Structure } \\
\text { Count }^{1} \\
\text { Prim/Total }\end{array}$} \\
\hline Primary Asbestos Structures & 0 & $<4.56 \mathrm{E}+06$ & $0.00 \mathrm{E}+00-3.69 \mathrm{E}+00-$ Poisson & 0 & \\
\hline Total Asbestos Structures & 0 & $<4.56 \mathrm{E}+06$ & $0.00 \mathrm{E}+00-3.69 \mathrm{E}+00-$ Poisson & & $\overline{0}$ \\
\hline PCM Equivalent Structures-ISO & 0 & $<4.56 \mathrm{E}+06$ & $0.00 \mathrm{E}+00-3.69 \mathrm{E}+00$ - Poisson & 0 & \\
\hline Total Chrysotile Structures, >=0.5 - <=5.0, and 3:1 & 0 & $<4.56 \mathrm{E}+06$ & $0.00 \mathrm{E}+00-3.69 \mathrm{E}+00$ - Poisson & & 0 \\
\hline Total Chrysotile Structures, $>5-<=10$, and 3:1 & 0 & $<4.56 \mathrm{E}+06$ & $0.00 \mathrm{E}+00-3.69 \mathrm{E}+00-$ Poisson & & 0 \\
\hline Total Chrysotile Structures, $>10$ and 3:1 & 0 & $<4.56 \mathrm{E}+06$ & $0.00 \mathrm{E}+00-3.69 \mathrm{E}+00-$ Poisson & & 0 \\
\hline Total Amphibole Structures, $>=0.5-<=5.0$, and 3:1 & 0 & $<4.56 \mathrm{E}+06$ & $0.00 \mathrm{E}+00-3.69 \mathrm{E}+00-$ Poisson & & 0 \\
\hline Total Amphibole Structures, $>5.0-<=10$, and $3: 1$ & 0 & $<4.56 \mathrm{E}+06$ & $0.00 \mathrm{E}+00-3.69 \mathrm{E}+00-$ Poisson & & 0 \\
\hline Total Amphibole Structures, > 10, and 3:1 & 0 & $<4.56 \mathrm{E}+06$ & $0.00 \mathrm{E}+00-3.69 \mathrm{E}+00$ - Poisson & & 0 \\
\hline Primary Chrysotile Structures $>5.0 \mu \mathrm{m}$ & 0 & $<4.56 \mathrm{E}+06$ & $0.00 \mathrm{E}+00-3.69 \mathrm{E}+00$ - Poisson & 0 & \\
\hline Primary Chrysotile Structures $>=0.5-<=5.0 \mu \mathrm{m}$ & 0 & $<4.56 \mathrm{E}+06$ & $0.00 \mathrm{E}+00-3.69 \mathrm{E}+00-$ Poisson & 0 & \\
\hline Primary Amphibole Structures $>5.0 \mu \mathrm{m}$ & 0 & $<4.56 \mathrm{E}+06$ & $0.00 \mathrm{E}+00-3.69 \mathrm{E}+00$ - Poisson & 0 & \\
\hline Primary Amphibole Structures $>=0.5-<=5.0 \mu \mathrm{m}$ & 0 & $<4.56 \mathrm{E}+06$ & $0.00 \mathrm{E}+00-3.69 \mathrm{E}+00$ - Poisson & 0 & \\
\hline
\end{tabular}


Job Number: 070451

\section{SEA}

\section{Soil - Elutriation Method Summary Data}

Client: Idaho National Laboratory

Report Number: 070451R02

Date Received: 4/23/2007

Project Name: RARE

Lab/Cor Sample No.: S5

Client Sample No.: BR-4-R1

Description:

$\begin{array}{cc}\text { Analyst(s) } & \text { Analysis Date } \\ \text { KM } & 7 / 2 / 2007\end{array}$

Mass (gPM10) : 0.0001203

Lab Filter Area (mm2) : 385

Grid Openings Analyzed : 74

Average Grid Opening Area (mm2) : 0.00945

Area Analyzed (mm2) : 0.6993

Analytical Sens. (struc/gPM10) : $4.58 \mathrm{E}+06$

Detection Limit. (struc/gPM10) : $1.37 \mathrm{E}+07$

\begin{tabular}{|c|c|c|c|c|c|}
\hline $\begin{array}{c}\text { Structure } \\
\text { Type }\end{array}$ & $\begin{array}{c}\text { Filter } \\
\text { Density } \\
(\mathrm{s} / \mathrm{mm} 2)\end{array}$ & $\begin{array}{c}\text { Concen- } \\
\text { tration } \\
\text { (struc/gPM10) }\end{array}$ & $\begin{array}{c}\text { 95\% Confidence } \\
\text { Interval } \\
\text { (struc/gPM10) }\end{array}$ & \multicolumn{2}{|c|}{$\begin{array}{c}\text { Structure } \\
\text { Count }^{1} \\
\text { Prim/Total }\end{array}$} \\
\hline Primary Asbestos Structures & 0 & $<4.58 \mathrm{E}+06$ & $0.00 \mathrm{E}+00-3.69 \mathrm{E}+00-$ Poisson & 0 & \\
\hline Total Asbestos Structures & 0 & $<4.58 \mathrm{E}+06$ & $0.00 \mathrm{E}+00-3.69 \mathrm{E}+00-$ Poisson & & $\overline{0}$ \\
\hline PCM Equivalent Structures-ISO & 0 & $<4.58 \mathrm{E}+06$ & $0.00 \mathrm{E}+00-3.69 \mathrm{E}+00$ - Poisson & 0 & \\
\hline Total Chrysotile Structures, >=0.5 - <=5.0, and 3:1 & 0 & $<4.58 \mathrm{E}+06$ & $0.00 \mathrm{E}+00-3.69 \mathrm{E}+00$ - Poisson & & 0 \\
\hline Total Chrysotile Structures, $>5-<=10$, and 3:1 & 0 & $<4.58 \mathrm{E}+06$ & $0.00 \mathrm{E}+00-3.69 \mathrm{E}+00-$ Poisson & & 0 \\
\hline Total Chrysotile Structures, $>10$ and 3:1 & 0 & $<4.58 \mathrm{E}+06$ & $0.00 \mathrm{E}+00-3.69 \mathrm{E}+00-$ Poisson & & 0 \\
\hline Total Amphibole Structures, $>=0.5-<=5.0$, and 3:1 & 0 & $<4.58 \mathrm{E}+06$ & $0.00 \mathrm{E}+00-3.69 \mathrm{E}+00-$ Poisson & & 0 \\
\hline Total Amphibole Structures, $>5.0-<=10$, and $3: 1$ & 0 & $<4.58 \mathrm{E}+06$ & $0.00 \mathrm{E}+00-3.69 \mathrm{E}+00-$ Poisson & & 0 \\
\hline Total Amphibole Structures, > 10, and 3:1 & 0 & $<4.58 \mathrm{E}+06$ & $0.00 \mathrm{E}+00-3.69 \mathrm{E}+00$ - Poisson & & 0 \\
\hline Primary Chrysotile Structures $>5.0 \mu \mathrm{m}$ & 0 & $<4.58 \mathrm{E}+06$ & $0.00 \mathrm{E}+00-3.69 \mathrm{E}+00$ - Poisson & 0 & \\
\hline Primary Chrysotile Structures $>=0.5-<=5.0 \mu \mathrm{m}$ & 0 & $<4.58 \mathrm{E}+06$ & $0.00 \mathrm{E}+00-3.69 \mathrm{E}+00-$ Poisson & 0 & \\
\hline Primary Amphibole Structures $>5.0 \mu \mathrm{m}$ & 0 & $<4.58 \mathrm{E}+06$ & $0.00 \mathrm{E}+00-3.69 \mathrm{E}+00$ - Poisson & 0 & \\
\hline Primary Amphibole Structures $>=0.5-<=5.0 \mu \mathrm{m}$ & 0 & $<4.58 \mathrm{E}+06$ & $0.00 \mathrm{E}+00-3.69 \mathrm{E}+00$ - Poisson & 0 & \\
\hline
\end{tabular}


Phone: (206) 781-0155

7619 6th Ave Nw

http://www.labcor.net

Job Number: 070451

Client: Idaho National Laboratory

Soil - Elutriation Method Summary Data

Project Name: RARE

Reviewed by:

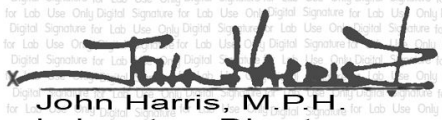

Harris, M.P.H.

SEA

Report Number: 070451R02

Date Received: 4/23/2007 
Phone: (206) 781-0155

7619 6th Ave Nw

A Professional Service Corporation in the Northwest

Fax: (206) 789-8424

http://www.labcor.net

oil - Elutriation Method Raw Data

Job Number: 070451

SEA

EPA 540-2-90-005, Modified May 23, 2000

Report Number: 070451R02

Client: Idaho National Laboratory

Date Received: 4/23/2007

Project Name: RARE

Client Sample No: BR-0-R1

Lab/Cor Sample No: S1

Client Description:

Date Sampled:

\begin{tabular}{|c|c|c|c|c|c|c|c|c|c|c|c|c|}
\hline Gr & No. & Loc. & ID & Prim Tot & Class & Len & Wid & Asp & Analyte & Elements & Comment & Count Categories \\
\hline G1 & 1 & A2 & & & NSD & & & & & & & \\
\hline G1 & 2 & $\mathrm{C} 2$ & & & NSD & & & & & & & \\
\hline G1 & 3 & E2 & & & NSD & & & & & & & \\
\hline G1 & 4 & $\mathrm{H} 2$ & & & NSD & & & & & & & \\
\hline G1 & 5 & 12 & & & NSD & & & & & & & \\
\hline G1 & 6 & J4 & & & NSD & & & & & & & \\
\hline G1 & 7 & $\mathrm{H} 4$ & & & NSD & & & & & & & \\
\hline G1 & 8 & $\mathrm{~F} 4$ & & & NSD & & & & & & & \\
\hline G1 & 9 & A4 & & & NSD & & & & & & & \\
\hline G1 & 10 & B5 & & & NSD & & & & & & & \\
\hline G1 & 11 & A7 & & & NSD & & & & & & & \\
\hline G1 & 12 & $\mathrm{C} 7$ & & & NSD & & & & & & & \\
\hline G1 & 13 & E7 & & & NSD & & & & & & & \\
\hline G1 & 14 & $\mathrm{H} 7$ & & & NSD & & & & & & & \\
\hline G1 & 15 & J7 & & & NSD & & & & & & & \\
\hline G1 & 16 & A8 & & & NSD & & & & & & & \\
\hline G1 & 17 & B8 & & & NSD & & & & & & & \\
\hline G1 & 18 & D8 & & & NSD & & & & & & & \\
\hline G1 & 19 & E8 & & & NSD & & & & & & & \\
\hline G1 & 20 & $\mathrm{H} 8$ & & & NSD & & & & & & & \\
\hline G1 & 21 & 18 & & & NSD & & & & & & & \\
\hline G1 & 22 & J8 & & & NSD & & & & & & & \\
\hline G1 & 23 & J9 & & & NSD & & & & & & & \\
\hline G1 & 24 & H9 & & & NSD & & & & & & & \\
\hline G1 & 25 & $\mathrm{Cg}$ & & & NSD & & & & & & & \\
\hline G1 & 26 & B9 & & & NSD & & & & & & & \\
\hline G1 & 27 & A9 & & & NSD & & & & & & & \\
\hline G1 & 28 & G5 & & & NSD & & & & & & & \\
\hline G1 & 29 & 15 & & & NSD & & & & & & & \\
\hline G1 & 30 & J6 & & & NSD & & & & & & & \\
\hline G2 & 31 & A2 & & & NSD & & & & & & & \\
\hline G2 & 32 & C2 & & & NSD & & & & & & & \\
\hline G2 & 33 & E2 & & & NSD & & & & & & & \\
\hline G2 & 34 & G2 & & & NSD & & & & & & & \\
\hline
\end{tabular}

Count Categories

PAmS_0.5-5

PAS

PCS_>5

TAmpSt, $>10$

TAmpSt,5-10
Primary Amphibole Structures $>=0.5-<=5.0 \mu \mathrm{m}$ Primary Asbestos Structures

Primary Chrysotile Structures $>5.0 \mu \mathrm{m}$

Total Amphibole Structures, > 10, and 3:1

Total Amphibole Structures, $>5.0-<=10$, and $3: 1$
PAmS_5 PCMES-ISO

PCS_0.5-5

TAmpSt,0.5-5

TAS
Primary Amphibole Structures $>5.0 \mu \mathrm{m}$ PCM Equivalent Structures-ISO

Primary Chrysotile Structures $>=0.5-<=5.0 \mu \mathrm{m}$

Total Amphibole Structures, $>=0.5-<=5.0$, and 3:1

Total Asbestos Structures 
Phone: (206) 781-0155

7619 6th Ave Nw

A Professional Service Corporation in the Northwest

Fax: (206) 789-8424

http://www.labcor.net

oil - Elutriation Method Raw Data

Job Number: 070451

SEA

EPA 540-2-90-005, Modified May 23, 2000

Report Number: 070451R02

Client: Idaho National Laboratory

Date Received: 4/23/2007

Project Name: RARE

Client Sample No: BR-0-R1

Lab/Cor Sample No: S1

Client Description:

Date Sampled:

\begin{tabular}{|c|c|c|c|c|c|c|c|c|c|c|c|c|}
\hline$\overline{\mathrm{Gr}}$ & No. & Loc. & 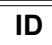 & Prim Tot & Class & Len & Wid & Asp & Analyte & Elements & Comment & "Count Categories \\
\hline G2 & 35 & 12 & & & NSD & & & & & & & \\
\hline G2 & 36 & J4 & & & NSD & & & & & & & \\
\hline G2 & 37 & $\mathrm{H} 4$ & & & NSD & & & & & & & \\
\hline G2 & 38 & $\mathrm{~F} 4$ & & & NSD & & & & & & & \\
\hline G2 & 39 & E4 & & & NSD & & & & & & & \\
\hline G2 & 40 & C4 & & & NSD & & & & & & & \\
\hline G2 & 41 & A4 & & & NSD & & & & & & & \\
\hline G2 & 42 & B3 & & & NSD & & & & & & & \\
\hline G2 & 43 & D3 & & & NSD & & & & & & & \\
\hline G2 & 44 & F3 & & & NSD & & & & & & & \\
\hline G2 & 45 & H3 & & & NSD & & & & & & & \\
\hline G2 & 46 & J3 & & & NSD & & & & & & & \\
\hline G2 & 47 & A7 & & & NSD & & & & & & & \\
\hline G2 & 48 & $\mathrm{C7}$ & & & NSD & & & & & & & \\
\hline G2 & 49 & E7 & & & NSD & & & & & & & \\
\hline $\mathrm{G} 2$ & 50 & G7 & & & NSD & & & & & & & \\
\hline G2 & 51 & 17 & & & NSD & & & & & & & \\
\hline G2 & 52 & J8 & & & NSD & & & & & & & \\
\hline G2 & 53 & $\mathrm{H} 8$ & & & NSD & & & & & & & \\
\hline G2 & 54 & F8 & & & NSD & & & & & & & \\
\hline G2 & 55 & D8 & & & NSD & & & & & & & \\
\hline G2 & 56 & B8 & & & NSD & & & & & & & \\
\hline G2 & 57 & A9 & & & NSD & & & & & & & \\
\hline G2 & 58 & $\mathrm{Cg}$ & & & NSD & & & & & & & \\
\hline G2 & 59 & E9 & & & NSD & & & & & & & \\
\hline G3 & 60 & $\mathrm{~A} 2$ & & & NSD & & & & & & & \\
\hline G3 & 61 & C2 & & & NSD & & & & & & & \\
\hline G3 & 62 & E2 & & & NSD & & & & & & & \\
\hline G3 & 63 & G2 & & & NSD & & & & & & & \\
\hline G3 & 64 & 12 & & & NSD & & & & & & & \\
\hline G3 & 65 & J3 & & & NSD & & & & & & & \\
\hline G3 & 66 & $\mathrm{H} 3$ & & & NSD & & & & & & & \\
\hline G3 & 67 & F3 & & & NSD & & & & & & & \\
\hline G3 & 68 & C3 & & & NSD & & & & & & & \\
\hline
\end{tabular}

Count Categories

Primary Amphibole Structures $>=0.5-<=5.0 \mu \mathrm{m}$

PAmS_5 PCMES-ISO

PCS_0.5-5

Primary Amphibole Structures $>5.0 \mu \mathrm{m}$

Page 9 of 23

PCS_>5

Primary Chrysotile Structures $>5.0 \mu \mathrm{m}$

TAmpSt, $>10$

Total Amphibole Structures, > 10, and 3:1

TAmpSt,0.5-5

PCM Equivalent Structures-ISO

Primary Chrysotile Structures $>=0.5-<=5.0 \mu \mathrm{m}$

TAmpSt,5-10

Total Amphibole Structures, $>5.0-<=10$, and

3:1

TAS 
A Professional Service Corporation in the Northwest

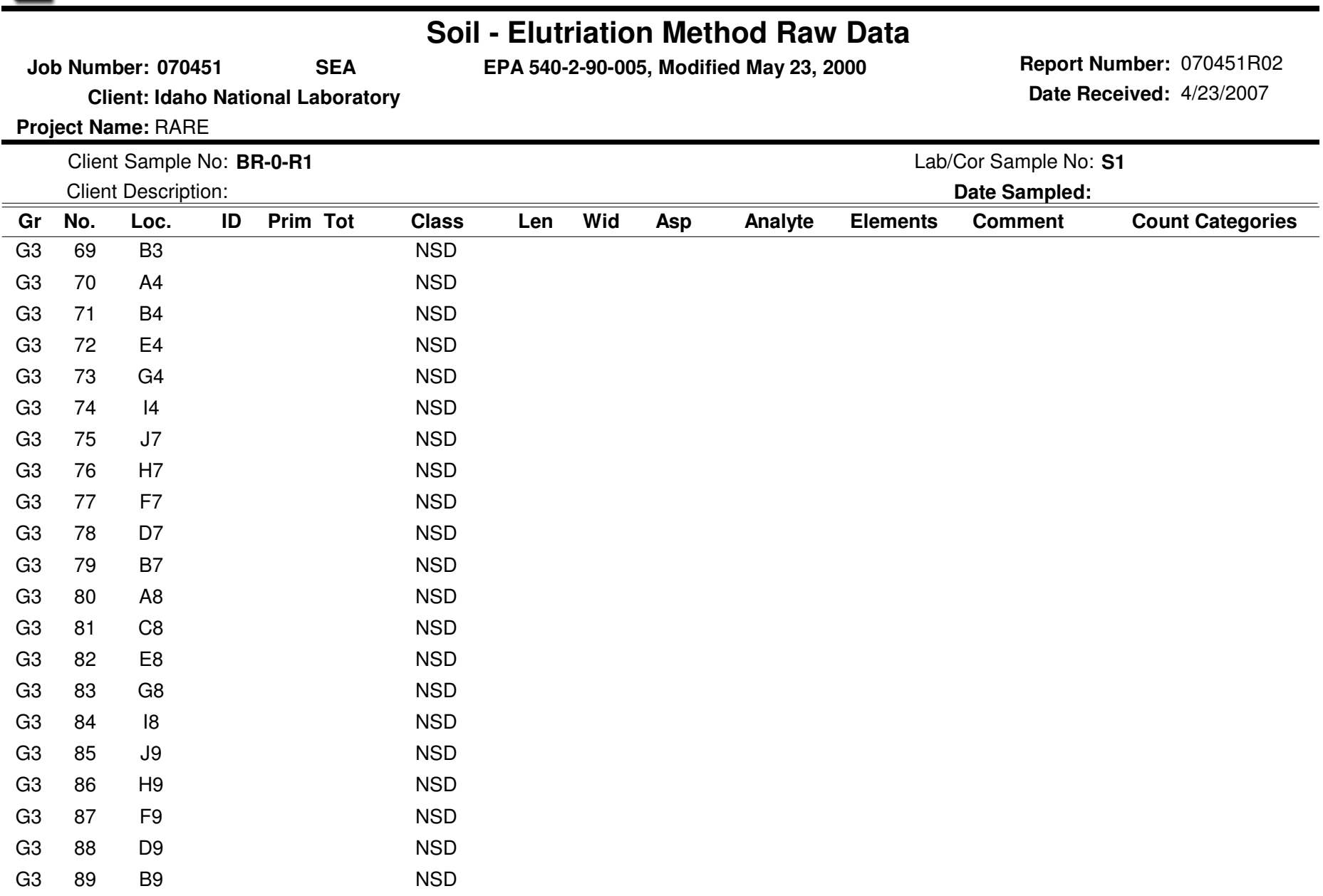

\section{Count Categories}

PAmS_0.5-5

PAS

PCS_>5

TAmpSt, $>10$

TAmpSt,5-10
Primary Amphibole Structures $>=0.5-<=5.0 \mu \mathrm{m}$ Primary Asbestos Structures

Primary Chrysotile Structures $>5.0 \mu \mathrm{m}$

Total Amphibole Structures, > 10, and 3:1

Total Amphibole Structures, $>5.0-<=10$, and

3:1
PAmS 5 PCMES-ISO PCS_0.5-5 TAmpSt,0.5-5

TAS
Primary Amphibole Structures $>5.0 \mu \mathrm{m}$ PCM Equivalent Structures-ISO

Primary Chrysotile Structures $>=0.5-<=5.0 \mu \mathrm{m}$

Total Amphibole Structures, $>=0.5-<=5.0$, and 3:1

Total Asbestos Structures 
Phone: (206) 781-0155

7619 6th Ave Nw

A Professional Service Corporation in the Northwest

Fax: (206) 789-8424

http://www.labcor.net

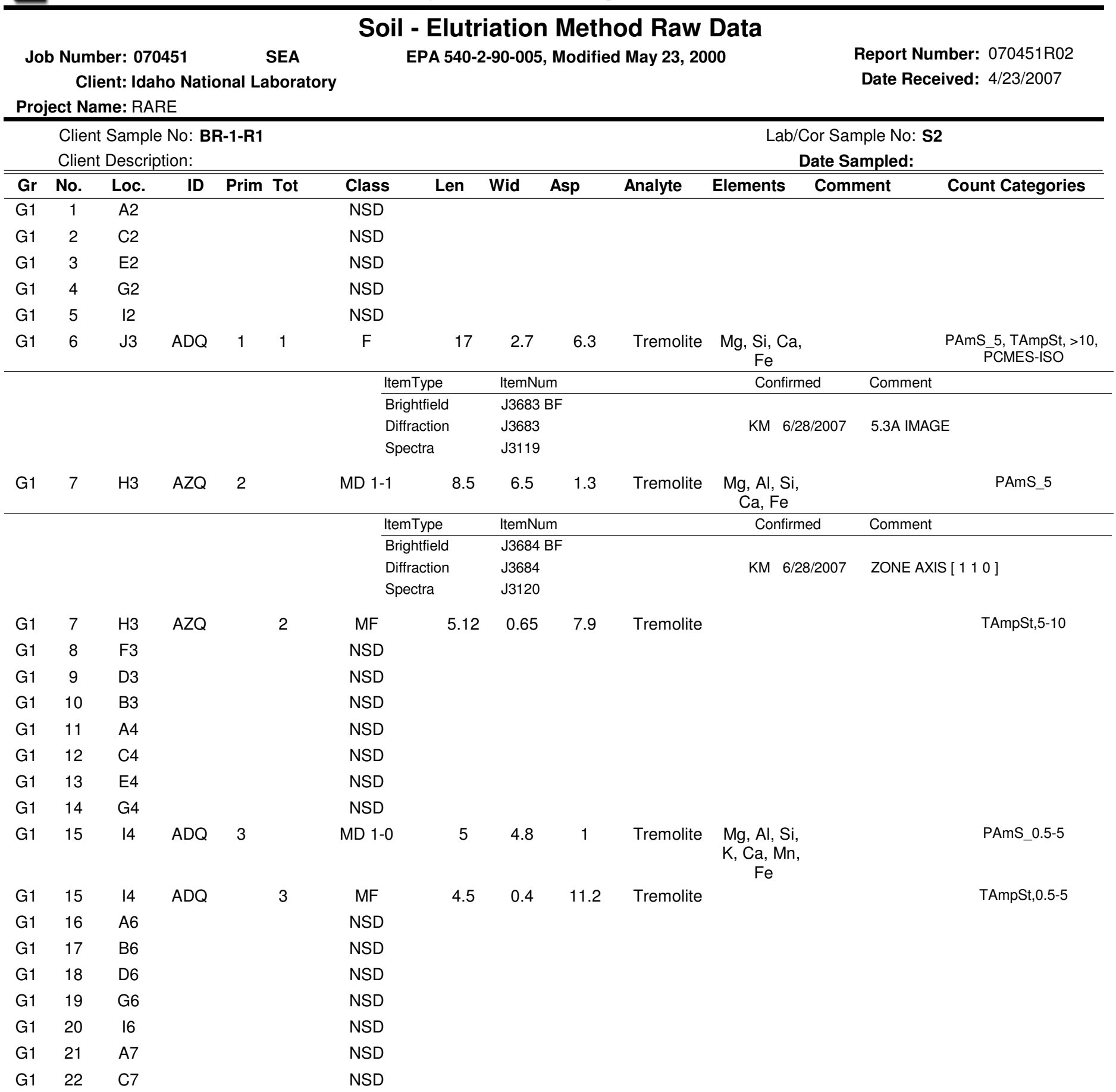

Count Categories

PAmS 0.5-5

PAS

PCS_>5

TAmpSt, $>10$

TAmpSt,5-10
Primary Amphibole Structures $>=0.5-<=5.0 \mu \mathrm{m}$ Primary Asbestos Structures

Primary Chrysotile Structures $>5.0 \mu \mathrm{m}$

Total Amphibole Structures, > 10, and 3:1

Total Amphibole Structures, $>5.0-<=10$, and 3:1
PAmS_5 PCMES-ISO PCS $0.5-5$

TAmpSt,0.5-5

TAS
Primary Amphibole Structures $>5.0 \mu \mathrm{m}$ PCM Equivalent Structures-ISO

Primary Chrysotile Structures $>=0.5-<=5.0 \mu \mathrm{m}$

Total Amphibole Structures, $>=0.5-<=5.0$, and 3:1

Total Asbestos Structures 
Phone: (206) 781-0155

7619 6th Ave Nw

A Professional Service Corporation in the Northwest

Fax: (206) 789-8424

http://www.labcor.net

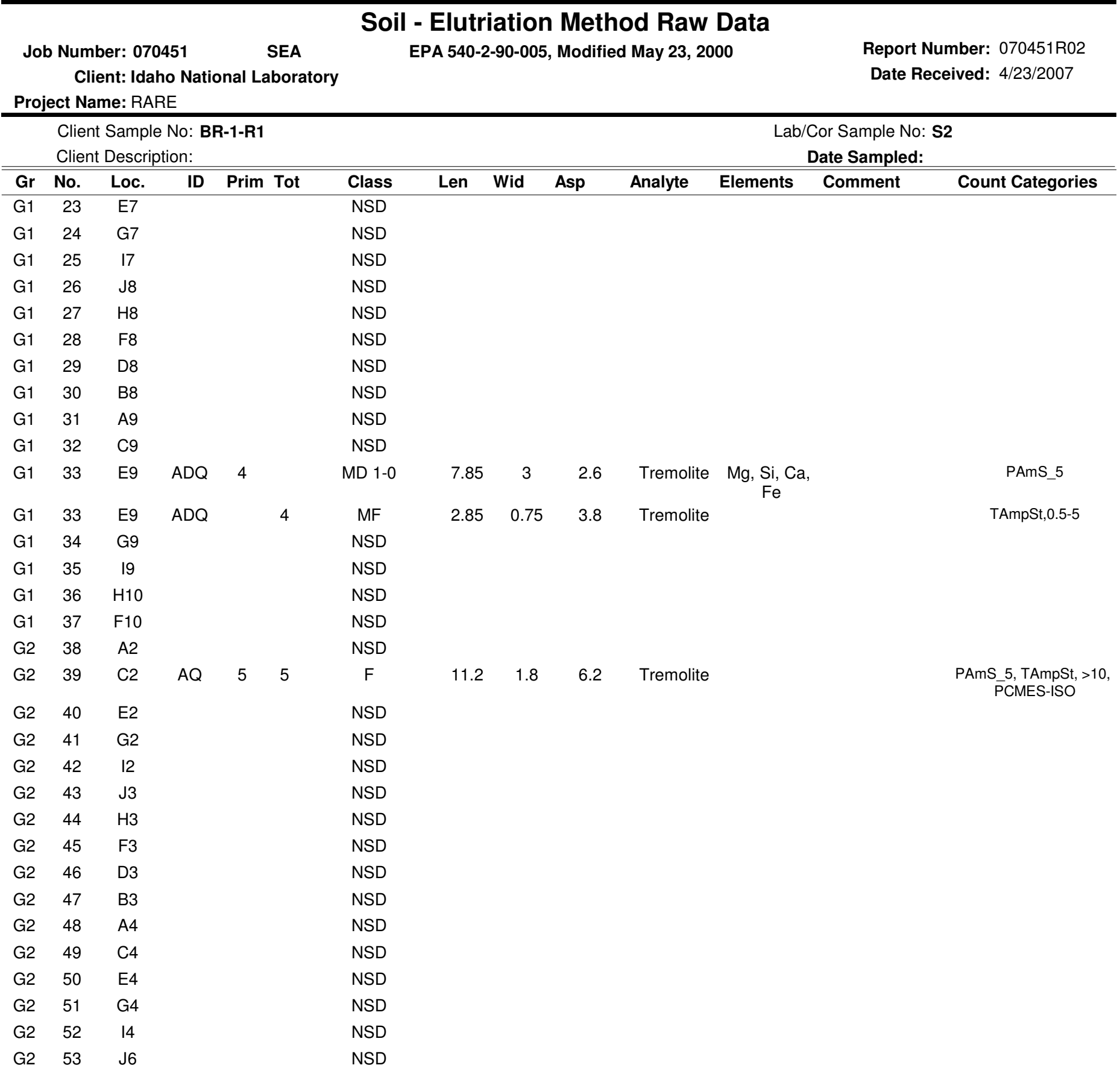

Count Categories

PAmS 0.5-5

PAS

PCS_>5

TAmpSt, $>10$

TAmpSt,5-10
Primary Amphibole Structures $>=0.5-<=5.0 \mu \mathrm{m}$

Primary Asbestos Structures

Primary Chrysotile Structures $>5.0 \mu \mathrm{m}$

Total Amphibole Structures, > 10, and 3:1

Total Amphibole Structures, $>5.0-<=10$, and

$3: 1$
PAmS_5 PCMES-ISO

PCS_0.5-5

TAmpSt,0.5-5

TAS
Primary Amphibole Structures $>5.0 \mu \mathrm{m}$ PCM Equivalent Structures-ISO

Primary Chrysotile Structures $>=0.5-<=5.0 \mu \mathrm{m}$

Total Amphibole Structures, $>=0.5-<=5.0$, and 3:1

Total Asbestos Structures 
Phone: (206) 781-0155

Fax: (206) 789-8424

7619 6th Ave Nw

A Professional Service Corporation in the Northwest

http://www.labcor.net

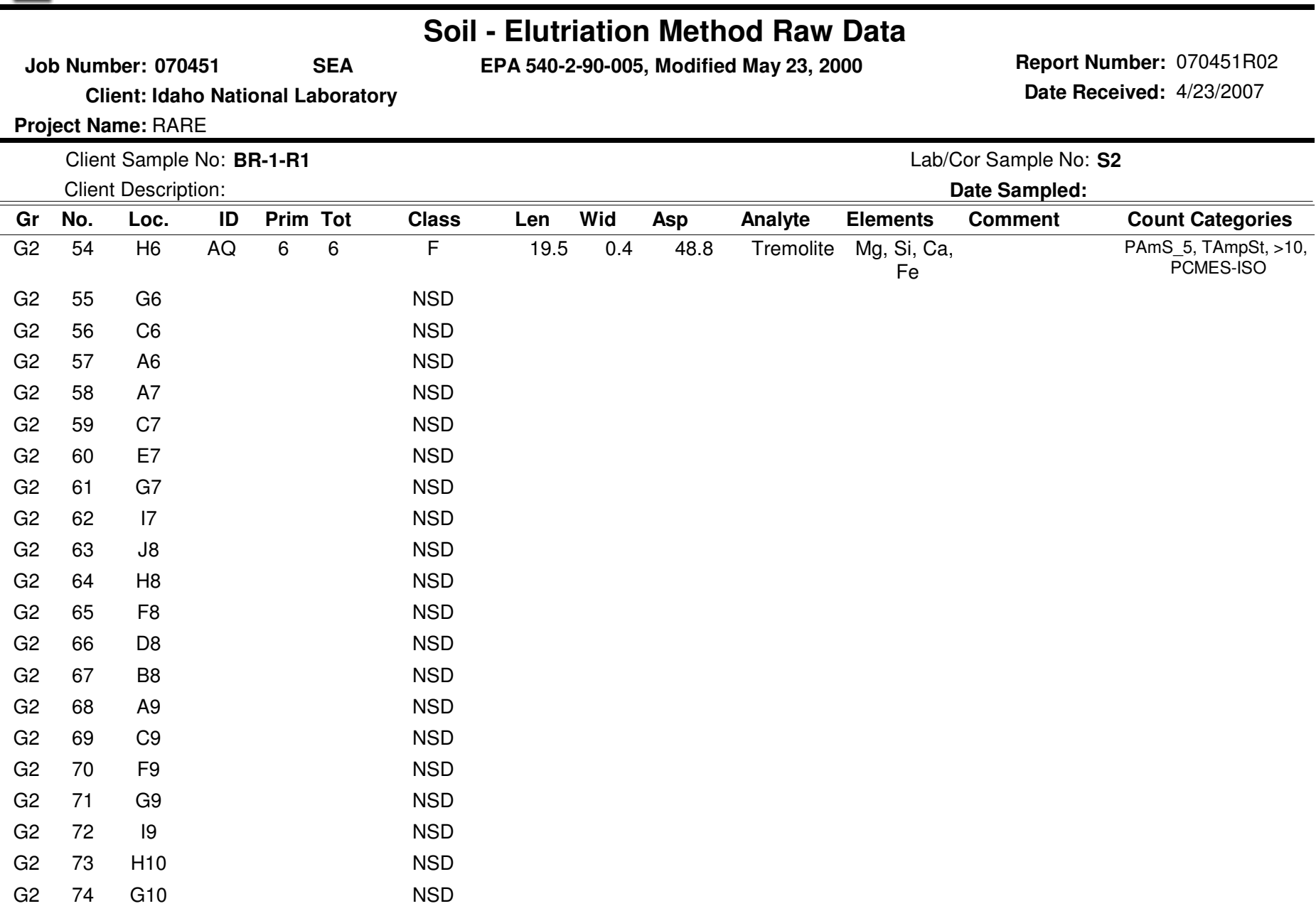

Count Categories

PAmS_0.5-5

PAS

PCS_>5

TAmpSt, $>10$

TAmpSt,5-10
Primary Amphibole Structures $>=0.5-<=5.0 \mu \mathrm{m}$ Primary Asbestos Structures

Primary Chrysotile Structures $>5.0 \mu \mathrm{m}$

Total Amphibole Structures, > 10, and 3:1

Total Amphibole Structures, $>5.0-<=10$, and $3: 1$
PAmS_5 PCMES-ISO

PCS_0.5-5

TAmpSt,0.5-5

TAS
Primary Amphibole Structures $>5.0 \mu \mathrm{m}$ PCM Equivalent Structures-ISO

Primary Chrysotile Structures $>=0.5-<=5.0 \mu \mathrm{m}$

Total Amphibole Structures, $>=0.5-<=5.0$, and 3:1

Total Asbestos Structures 
Phone: (206) 781-0155

7619 6th Ave Nw

A Professional Service Corporation in the Northwest

Fax: (206) 789-8424

http://www.labcor.net

oil - Elutriation Method Raw Data

Job Number: 070451

SEA

EPA 540-2-90-005, Modified May 23, 2000

Report Number: 070451R02

Client: Idaho National Laboratory

Date Received: 4/23/2007

Project Name: RARE

Client Sample No: BR-2-R1

Lab/Cor Sample No: S3

Client Description:

Date Sampled:

\begin{tabular}{|c|c|c|c|c|c|c|c|c|c|c|c|c|}
\hline $\mathrm{Gr}$ & No. & Loc. & ID & Prim Tot & Class & Len & Wid & Asp & Analyte & Elements & Comment & Count Categories \\
\hline G1 & 1 & $\mathrm{~A} 2$ & & & NSD & & & & & & & \\
\hline G1 & 2 & $\mathrm{C} 2$ & & & NSD & & & & & & & \\
\hline G1 & 3 & E2 & & & NSD & & & & & & & \\
\hline G1 & 4 & G2 & & & NSD & & & & & & & \\
\hline G1 & 5 & 12 & & & NSD & & & & & & & \\
\hline G1 & 6 & J3 & & & NSD & & & & & & & \\
\hline G1 & 7 & H3 & & & NSD & & & & & & & \\
\hline G1 & 8 & F3 & & & NSD & & & & & & & \\
\hline G1 & 9 & D3 & & & NSD & & & & & & & \\
\hline G1 & 10 & B3 & & & NSD & & & & & & & \\
\hline G1 & 11 & A4 & & & NSD & & & & & & & \\
\hline G1 & 12 & C4 & & & NSD & & & & & & & \\
\hline G1 & 13 & E4 & & & NSD & & & & & & & \\
\hline G1 & 14 & G4 & & & NSD & & & & & & & \\
\hline G1 & 15 & 14 & & & NSD & & & & & & & \\
\hline G1 & 16 & J6 & & & NSD & & & & & & & \\
\hline G1 & 17 & $\mathrm{H} 6$ & & & NSD & & & & & & & \\
\hline G1 & 18 & G6 & & & NSD & & & & & & & \\
\hline G1 & 19 & D6 & & & NSD & & & & & & & \\
\hline G1 & 20 & B6 & & & NSD & & & & & & & \\
\hline G1 & 21 & A7 & & & NSD & & & & & & & \\
\hline G1 & 22 & $\mathrm{C} 7$ & & & NSD & & & & & & & \\
\hline G1 & 23 & E7 & & & NSD & & & & & & & \\
\hline G1 & 24 & G7 & & & NSD & & & & & & & \\
\hline G1 & 25 & 17 & & & NSD & & & & & & & \\
\hline G1 & 26 & J8 & & & NSD & & & & & & & \\
\hline G1 & 27 & $\mathrm{H} 8$ & & & NSD & & & & & & & \\
\hline G1 & 28 & F8 & & & NSD & & & & & & & \\
\hline G1 & 29 & D8 & & & NSD & & & & & & & \\
\hline G1 & 30 & B8 & & & NSD & & & & & & & \\
\hline G1 & 31 & A9 & & & NSD & & & & & & & \\
\hline G1 & 32 & C9 & & & NSD & & & & & & & \\
\hline G1 & 33 & E9 & & & NSD & & & & & & & \\
\hline G1 & 34 & G9 & & & NSD & & & & & & & \\
\hline
\end{tabular}

Count Categories

Primary Amphibole Structures $>=0.5-<=5.0 \mu \mathrm{m}$

PAmS_5 PCMES-ISO

PCS_0.5-5

Primary Amphibole Structures $>5.0 \mu \mathrm{m}$

Page 14 of 23

PCS_>5

Primary Chrysotile Structures $>5.0 \mu \mathrm{m}$

TAmpSt, $>10$

Total Amphibole Structures, > 10, and 3:1

TAmpSt,0.5-5

PCM Equivalent Structures-ISO

Primary Chrysotile Structures $>=0.5-<=5.0 \mu \mathrm{m}$

TAmpSt,5-10

Total Amphibole Structures, $>5.0-<=10$, and

TAS 
Phone: (206) 781-0155

7619 6th Ave Nw

A Professional Service Corporation in the Northwest

Fax: (206) 789-8424

http://www.labcor.net

oil - Elutriation Method Raw Data

Job Number: 070451

SEA

EPA 540-2-90-005, Modified May 23, 2000

Report Number: 070451R02

Client: Idaho National Laboratory

Date Received: 4/23/2007

Project Name: RARE

Client Sample No: BR-2-R1

Lab/Cor Sample No: S3

Client Description:

Date Sampled:

\begin{tabular}{|c|c|c|c|c|c|c|c|c|c|c|c|c|}
\hline $\mathrm{Gr}$ & No. & Loc. & ID & Prim Tot & Class & Len & Wid & Asp & Analyte & Elements & Comment & Count Categories \\
\hline G1 & 35 & 19 & & & NSD & & & & & & & \\
\hline G2 & 36 & $\mathrm{~A} 2$ & & & NSD & & & & & & & \\
\hline G2 & 37 & $\mathrm{C} 2$ & & & NSD & & & & & & & \\
\hline G2 & 38 & E2 & & & NSD & & & & & & & \\
\hline $\mathrm{G} 2$ & 39 & G2 & & & NSD & & & & & & & \\
\hline G2 & 40 & 12 & & & NSD & & & & & & & \\
\hline G2 & 41 & J3 & & & NSD & & & & & & & \\
\hline G2 & 42 & H3 & & & NSD & & & & & & & \\
\hline G2 & 43 & F3 & & & NSD & & & & & & & \\
\hline G2 & 44 & D3 & & & NSD & & & & & & & \\
\hline G2 & 45 & B3 & & & NSD & & & & & & & \\
\hline $\mathrm{G} 2$ & 46 & A4 & & & NSD & & & & & & & \\
\hline G2 & 47 & C4 & & & NSD & & & & & & & \\
\hline G2 & 48 & E4 & & & NSD & & & & & & & \\
\hline G2 & 49 & G4 & & & NSD & & & & & & & \\
\hline G2 & 50 & 14 & & & NSD & & & & & & & \\
\hline G2 & 51 & J6 & & & NSD & & & & & & & \\
\hline G2 & 52 & $\mathrm{H} 6$ & & & NSD & & & & & & & \\
\hline G2 & 53 & G6 & & & NSD & & & & & & & \\
\hline G2 & 54 & D6 & & & NSD & & & & & & & \\
\hline G2 & 55 & B6 & & & NSD & & & & & & & \\
\hline G2 & 56 & A7 & & & NSD & & & & & & & \\
\hline G2 & 57 & $\mathrm{C7}$ & & & NSD & & & & & & & \\
\hline G2 & 58 & E7 & & & NSD & & & & & & & \\
\hline G2 & 59 & G7 & & & NSD & & & & & & & \\
\hline G2 & 60 & 17 & & & NSD & & & & & & & \\
\hline G2 & 61 & J8 & & & NSD & & & & & & & \\
\hline G2 & 62 & $\mathrm{H} 8$ & & & NSD & & & & & & & \\
\hline G2 & 63 & F8 & & & NSD & & & & & & & \\
\hline G2 & 64 & D8 & & & NSD & & & & & & & \\
\hline G2 & 65 & B8 & & & NSD & & & & & & & \\
\hline G2 & 66 & A9 & & & NSD & & & & & & & \\
\hline G2 & 67 & C9 & & & NSD & & & & & & & \\
\hline G2 & 68 & E9 & & & NSD & & & & & & & \\
\hline
\end{tabular}

Count Categories

Primary Amphibole Structures $>=0.5-<=5.0 \mu \mathrm{m}$

PAmS_5 PCMES-ISO

PCS_0.5-5

Primary Amphibole Structures $>5.0 \mu \mathrm{m}$

Page 15 of 23

PCS_>5

Primary Chrysotile Structures $>5.0 \mu \mathrm{m}$

TAmpSt, $>10$

Total Amphibole Structures, > 10, and 3:1

TAmpSt,0.5-5

PCM Equivalent Structures-ISO

Primary Chrysotile Structures $>=0.5-<=5.0 \mu \mathrm{m}$

TAmpSt,5-10

Total Amphibole Structures, $>5.0-<=10$, and

3:1

TAS 
Phone: (206) 781-0155

7619 6th Ave Nw

A Professional Service Corporation in the Northwest

Fax: (206) 789-8424

http://www.labcor.net

oil - Elutriation Method Raw Data

Job Number: 070451

SEA

EPA 540-2-90-005, Modified May 23, 2000

Report Number: 070451R02

Client: Idaho National Laboratory

Date Received: 4/23/2007

Project Name: RARE

Client Sample No: BR-2-R1

Lab/Cor Sample No: S3

Client Description:

\begin{tabular}{|c|c|c|c|c|c|c|c|c|c|c|c|c|}
\hline$\overline{\mathrm{Gr}}$ & No. & Loc. & ID & Prim Tot & Class & Len & "Wid & Asp & Analyte & Elements & Comment & "Count Categories \\
\hline G2 & 69 & G9 & & & NSD & & & & & & & \\
\hline $\mathrm{G} 2$ & 70 & 19 & & & NSD & & & & & & & \\
\hline
\end{tabular}

Count Categories

PAmS_0.5-5

PAS

PCS_>5

TAmpSt, $>10$

TAmpSt,5-10
Primary Amphibole Structures $>=0.5-<=5.0 \mu \mathrm{m}$ Primary Asbestos Structures

Primary Chrysotile Structures $>5.0 \mu \mathrm{m}$

Total Amphibole Structures, $>10$, and 3:1

Total Amphibole Structures, $>5.0-<=10$, and

3:1
PAmS_5 PCMES-ISO PCS 0.5-5

TAmpSt,0.5-5

TAS
Primary Amphibole Structures $>5.0 \mu \mathrm{m}$ PCM Equivalent Structures-ISO

Primary Chrysotile Structures $>=0.5-<=5.0 \mu \mathrm{m}$

Total Amphibole Structures, $>=0.5-<=5.0$, and 3:1

Total Asbestos Structures 
Phone: (206) 781-0155

7619 6th Ave Nw

A Professional Service Corporation in the Northwest

Fax: (206) 789-8424

http://www.labcor.net

oil - Elutriation Method Raw Data

Job Number: 070451

SEA

EPA 540-2-90-005, Modified May 23, 2000

Report Number: 070451R02

Client: Idaho National Laboratory

Date Received: 4/23/2007

Project Name: RARE

Client Sample No: BR-3-R1

Lab/Cor Sample No: S4

Client Description:

Date Sampled:

\begin{tabular}{|c|c|c|c|c|c|c|c|c|c|c|c|c|}
\hline $\mathrm{Gr}$ & No. & Loc. & ID & Prim Tot & Class & Len & Wid & Asp & Analyte & Elements & Comment & Count Categories \\
\hline G1 & 1 & $\mathrm{~A} 2$ & & & NSD & & & & & & & \\
\hline G1 & 2 & $\mathrm{C} 2$ & & & NSD & & & & & & & \\
\hline G1 & 3 & E2 & & & NSD & & & & & & & \\
\hline G1 & 4 & G2 & & & NSD & & & & & & & \\
\hline G1 & 5 & 12 & & & NSD & & & & & & & \\
\hline G1 & 6 & J3 & & & NSD & & & & & & & \\
\hline G1 & 7 & H3 & & & NSD & & & & & & & \\
\hline G1 & 8 & F3 & & & NSD & & & & & & & \\
\hline G1 & 9 & D3 & & & NSD & & & & & & & \\
\hline G1 & 10 & B3 & & & NSD & & & & & & & \\
\hline G1 & 11 & A4 & & & NSD & & & & & & & \\
\hline G1 & 12 & C4 & & & NSD & & & & & & & \\
\hline G1 & 13 & E4 & & & NSD & & & & & & & \\
\hline G1 & 14 & G4 & & & NSD & & & & & & & \\
\hline G1 & 15 & 14 & & & NSD & & & & & & & \\
\hline G1 & 16 & J5 & & & NSD & & & & & & & \\
\hline G1 & 17 & H5 & & & NSD & & & & & & & \\
\hline G1 & 18 & G5 & & & NSD & & & & & & & \\
\hline G1 & 19 & D5 & & & NSD & & & & & & & \\
\hline G1 & 20 & B5 & & & NSD & & & & & & & \\
\hline G1 & 21 & A6 & & & NSD & & & & & & & \\
\hline G1 & 22 & $\mathrm{C} 6$ & & & NSD & & & & & & & \\
\hline G1 & 23 & D6 & & & NSD & & & & & & & \\
\hline G1 & 24 & G6 & & & NSD & & & & & & & \\
\hline G1 & 25 & 16 & & & NSD & & & & & & & \\
\hline G1 & 26 & J7 & & & NSD & & & & & & & \\
\hline G1 & 27 & $\mathrm{H} 7$ & & & NSD & & & & & & & \\
\hline G1 & 28 & F7 & & & NSD & & & & & & & \\
\hline G1 & 29 & D7 & & & NSD & & & & & & & \\
\hline G1 & 30 & B7 & & & NSD & & & & & & & \\
\hline G1 & 31 & A8 & & & NSD & & & & & & & \\
\hline G1 & 32 & C8 & & & NSD & & & & & & & \\
\hline G1 & 33 & E8 & & & NSD & & & & & & & \\
\hline G1 & 34 & G8 & & & NSD & & & & & & & \\
\hline
\end{tabular}

Count Categories

Primary Amphibole Structures $>=0.5-<=5.0 \mu \mathrm{m}$

PAmS_5 PCMES-ISO

Primary Amphibole Structures $>5.0 \mu \mathrm{m}$

Page 17 of 23

PCS_>5

Primary Asbestos Structures

Primary Chrysotile Structures $>5.0 \mu \mathrm{m}$

TAmpSt, $>10$

Total Amphibole Structures, > 10, and 3:1

PCS_0.5-5

PCM Equivalent Structures-ISO

TAmpSt, 0.5-5

Primary Chrysotile Structures $>=0.5-<=5.0 \mu \mathrm{m}$

TAmpSt,5-10

Total Amphibole Structures, $>5.0-<=10$, and

TAS 
Phone: (206) 781-0155

7619 6th Ave Nw

A Professional Service Corporation in the Northwest

Fax: (206) 789-8424

http://www.labcor.net

oil - Elutriation Method Raw Data

Job Number: 070451

SEA

EPA 540-2-90-005, Modified May 23, 2000

Report Number: 070451R02

Client: Idaho National Laboratory

Date Received: 4/23/2007

Project Name: RARE

Client Sample No: BR-3-R1

Lab/Cor Sample No: S4

Client Description:

Date Sampled:

\begin{tabular}{|c|c|c|c|c|c|c|c|c|c|c|c|c|}
\hline $\mathrm{Gr}$ & No. & Loc. & ID & Prim Tot & Class & Len & Wid & Asp & Analyte & Elements & Comment & Count Categories \\
\hline G1 & 35 & 18 & & & NSD & & & & & & & \\
\hline G1 & 36 & J9 & & & NSD & & & & & & & \\
\hline G2 & 37 & $\mathrm{~A} 2$ & & & NSD & & & & & & & \\
\hline G2 & 38 & $\mathrm{C} 2$ & & & NSD & & & & & & & \\
\hline $\mathrm{G} 2$ & 39 & E2 & & & NSD & & & & & & & \\
\hline G2 & 40 & G2 & & & NSD & & & & & & & \\
\hline G2 & 41 & 12 & & & NSD & & & & & & & \\
\hline G2 & 42 & J3 & & & NSD & & & & & & & \\
\hline G2 & 43 & H3 & & & NSD & & & & & & & \\
\hline G2 & 44 & F3 & & & NSD & & & & & & & \\
\hline G2 & 45 & D3 & & & NSD & & & & & & & \\
\hline $\mathrm{G} 2$ & 46 & B3 & & & NSD & & & & & & & \\
\hline G2 & 47 & A4 & & & NSD & & & & & & & \\
\hline G2 & 48 & C4 & & & NSD & & & & & & & \\
\hline G2 & 49 & E4 & & & NSD & & & & & & & \\
\hline $\mathrm{G} 2$ & 50 & G4 & & & NSD & & & & & & & \\
\hline G2 & 51 & 14 & & & NSD & & & & & & & \\
\hline G2 & 52 & J5 & & & NSD & & & & & & & \\
\hline G2 & 53 & H5 & & & NSD & & & & & & & \\
\hline G2 & 54 & G5 & & & NSD & & & & & & & \\
\hline G2 & 55 & D5 & & & NSD & & & & & & & \\
\hline G2 & 56 & B5 & & & NSD & & & & & & & \\
\hline G2 & 57 & A6 & & & NSD & & & & & & & \\
\hline G2 & 58 & C6 & & & NSD & & & & & & & \\
\hline G2 & 59 & D6 & & & NSD & & & & & & & \\
\hline G2 & 60 & G6 & & & NSD & & & & & & & \\
\hline G2 & 61 & 16 & & & NSD & & & & & & & \\
\hline G2 & 62 & J7 & & & NSD & & & & & & & \\
\hline G2 & 63 & $\mathrm{H} 7$ & & & NSD & & & & & & & \\
\hline G2 & 64 & F7 & & & NSD & & & & & & & \\
\hline G2 & 65 & D7 & & & NSD & & & & & & & \\
\hline G2 & 66 & B7 & & & NSD & & & & & & & \\
\hline G2 & 67 & A8 & & & NSD & & & & & & & \\
\hline G2 & 68 & C8 & & & NSD & & & & & & & \\
\hline
\end{tabular}

Count Categories

Primary Amphibole Structures $>=0.5-<=5.0 \mu \mathrm{m}$

PAmS_5 PCMES-ISO

Primary Amphibole Structures $>5.0 \mu \mathrm{m}$

Page 18 of 23

PCS_>5

Primary Asbestos Structures

Primary Chrysotile Structures $>5.0 \mu \mathrm{m}$

TAmpSt, $>10$

Total Amphibole Structures, > 10, and 3:1

PCS_0.5-5

PCM Equivalent Structures-ISO

TAmpSt, 0.5-5

Primary Chrysotile Structures $>=0.5-<=5.0 \mu \mathrm{m}$

TAmpSt,5-10

Total Amphibole Structures, $>5.0-<=10$, and

TAS 
A Professional Service Corporation in the Northwest

Fax: (206) 789-8424

http://www.labcor.net

\section{Soil - Elutriation Method Raw Data}

EPA 540-2-90-005, Modified May 23, 2000

Report Number: 070451R02

Job Number: 070451

SEA

Client: Idaho National Laboratory

Project Name: RARE

Client Sample No: BR-3-R1

Lab/Cor Sample No: S4

Client Description:

\begin{tabular}{|c|c|c|c|c|c|c|c|c|c|c|c|c|}
\hline$\overline{G r}$ & No. & Loc. & ID & Prim Tot & Class & Len & Wid & Asp & Analyte & Elements & Comment & Count Categories \\
\hline G2 & 69 & E8 & & & NSD & & & & & & & \\
\hline G2 & 70 & G8 & & & NSD & & & & & & & \\
\hline G2 & 71 & 18 & & & NSD & & & & & & & \\
\hline
\end{tabular}

\section{Count Categories}

PAmS_0.5-5

PAS

PCS_>5

TAmpSt, $>10$

TAmpSt,5-10
Primary Amphibole Structures $>=0.5-<=5.0 \mu \mathrm{m}$ Primary Asbestos Structures

Primary Chrysotile Structures $>5.0 \mu \mathrm{m}$

Total Amphibole Structures, > 10, and 3:1

Total Amphibole Structures, $>5.0-<=10$, and 3:1
PAmS_5 PCMES-ISO PCS 0.5-5

TAmpSt,0.5-5

TAS
Primary Amphibole Structures $>5.0 \mu \mathrm{m}$ PCM Equivalent Structures-ISO

Primary Chrysotile Structures $>=0.5-<=5.0 \mu \mathrm{m}$

Total Amphibole Structures, $>=0.5-<=5.0$, and 3:1

Total Asbestos Structures 
Phone: (206) 781-0155

7619 6th Ave Nw

A Professional Service Corporation in the Northwest

Fax: (206) 789-8424

http://www.labcor.net

oil - Elutriation Method Raw Data

Job Number: 070451

SEA

EPA 540-2-90-005, Modified May 23, 2000

Report Number: 070451R02

Client: Idaho National Laboratory

Date Received: 4/23/2007

Project Name: RARE

Client Sample No: BR-4-R1

Lab/Cor Sample No: S5

Client Description:

Date Sampled:

\begin{tabular}{|c|c|c|c|c|c|c|c|c|c|c|c|c|}
\hline $\mathrm{Gr}$ & No. & Loc. & ID & Prim Tot & Class & Len & Wid & Asp & Analyte & Elements & Comment & Count Categories \\
\hline G1 & 1 & C1 & & & NSD & & & & & & & \\
\hline G1 & 2 & $\mathrm{E} 1$ & & & NSD & & & & & & & \\
\hline G1 & 3 & $\mathrm{~F} 1$ & & & NSD & & & & & & & \\
\hline G1 & 4 & G1 & & & NSD & & & & & & & \\
\hline G1 & 5 & $\mathrm{H} 1$ & & & NSD & & & & & & & \\
\hline G1 & 6 & J3 & & & NSD & & & & & & & \\
\hline G1 & 7 & H3 & & & NSD & & & & & & & \\
\hline G1 & 8 & F3 & & & NSD & & & & & & & \\
\hline G1 & 9 & D3 & & & NSD & & & & & & & \\
\hline G1 & 10 & B3 & & & NSD & & & & & & & \\
\hline G1 & 11 & A4 & & & NSD & & & & & & & \\
\hline G1 & 12 & C4 & & & NSD & & & & & & & \\
\hline G1 & 13 & E4 & & & NSD & & & & & & & \\
\hline G1 & 14 & G4 & & & NSD & & & & & & & \\
\hline G1 & 15 & 14 & & & NSD & & & & & & & \\
\hline G1 & 16 & J5 & & & NSD & & & & & & & \\
\hline G1 & 17 & H5 & & & NSD & & & & & & & \\
\hline G1 & 18 & G5 & & & NSD & & & & & & & \\
\hline G1 & 19 & D5 & & & NSD & & & & & & & \\
\hline G1 & 20 & B5 & & & NSD & & & & & & & \\
\hline G1 & 21 & A6 & & & NSD & & & & & & & \\
\hline G1 & 22 & C6 & & & NSD & & & & & & & \\
\hline G1 & 23 & D6 & & & NSD & & & & & & & \\
\hline G1 & 24 & G6 & & & NSD & & & & & & & \\
\hline G1 & 25 & 16 & & & NSD & & & & & & & \\
\hline G1 & 26 & J7 & & & NSD & & & & & & & \\
\hline G1 & 27 & $\mathrm{H} 7$ & & & NSD & & & & & & & \\
\hline G1 & 28 & F7 & & & NSD & & & & & & & \\
\hline G1 & 29 & D7 & & & NSD & & & & & & & \\
\hline G1 & 30 & B7 & & & NSD & & & & & & & \\
\hline G1 & 31 & A8 & & & NSD & & & & & & & \\
\hline G1 & 32 & C8 & & & NSD & & & & & & & \\
\hline G1 & 33 & E8 & & & NSD & & & & & & & \\
\hline G1 & 34 & G8 & & & NSD & & & & & & & \\
\hline
\end{tabular}

Count Categories

Primary Amphibole Structures $>=0.5-<=5.0 \mu \mathrm{m}$

PAmS_5 PCMES-ISO

Primary Amphibole Structures $>5.0 \mu \mathrm{m}$

Page 20 of 23

PCS_>5

Primary Asbestos Structures

Primary Chrysotile Structures $>5.0 \mu \mathrm{m}$

TAmpSt, $>10$

Total Amphibole Structures, > 10, and 3:1

PCS_0.5-5

PCM Equivalent Structures-ISO

TAmpSt, 0.5-5

Primary Chrysotile Structures $>=0.5-<=5.0 \mu \mathrm{m}$

TAmpSt,5-10

Total Amphibole Structures, $>5.0-<=10$, and

TAS 
Phone: (206) 781-0155

7619 6th Ave Nw

A Professional Service Corporation in the Northwest

Fax: (206) 789-8424

http://www.labcor.net

oil - Elutriation Method Raw Data

Job Number: 070451

SEA

EPA 540-2-90-005, Modified May 23, 2000

Report Number: 070451R02

Client: Idaho National Laboratory

Date Received: 4/23/2007

Project Name: RARE

Client Sample No: BR-4-R1

Lab/Cor Sample No: S5

Client Description:

Date Sampled:

\begin{tabular}{|c|c|c|c|c|c|c|c|c|c|c|c|c|}
\hline Gr & No. & Loc. & ID & Prim Tot & Class & Len & Wid & Asp & Analyte & Elements & Comment & Count Categories \\
\hline G1 & 35 & 18 & & & NSD & & & & & & & \\
\hline G1 & 36 & J9 & & & NSD & & & & & & & \\
\hline G1 & 37 & H9 & & & NSD & & & & & & & \\
\hline $\mathrm{G} 1$ & 38 & F9 & & & NSD & & & & & & & \\
\hline G1 & 39 & D9 & & & NSD & & & & & & & \\
\hline G1 & 40 & B9 & & & NSD & & & & & & & \\
\hline G2 & 41 & $\mathrm{~A} 2$ & & & NSD & & & & & & & \\
\hline G2 & 42 & $\mathrm{C} 2$ & & & NSD & & & & & & & \\
\hline G2 & 43 & E2 & & & NSD & & & & & & & \\
\hline G2 & 44 & G2 & & & NSD & & & & & & & \\
\hline G2 & 45 & 12 & & & NSD & & & & & & & \\
\hline G2 & 46 & J3 & & & NSD & & & & & & & \\
\hline G2 & 47 & H3 & & & NSD & & & & & & & \\
\hline G2 & 48 & F3 & & & NSD & & & & & & & \\
\hline G2 & 49 & D3 & & & NSD & & & & & & & \\
\hline G2 & 50 & B3 & & & NSD & & & & & & & \\
\hline G2 & 51 & A4 & & & NSD & & & & & & & \\
\hline G2 & 52 & C4 & & & NSD & & & & & & & \\
\hline G2 & 53 & E4 & & & NSD & & & & & & & \\
\hline G2 & 54 & G4 & & & NSD & & & & & & & \\
\hline G2 & 55 & 14 & & & NSD & & & & & & & \\
\hline G2 & 56 & J5 & & & NSD & & & & & & & \\
\hline G2 & 57 & $\mathrm{H} 5$ & & & NSD & & & & & & & \\
\hline G2 & 58 & G5 & & & NSD & & & & & & & \\
\hline G2 & 59 & D5 & & & NSD & & & & & & & \\
\hline G2 & 60 & B5 & & & NSD & & & & & & & \\
\hline G2 & 61 & A7 & & & NSD & & & & & & & \\
\hline G2 & 62 & $\mathrm{C7}$ & & & NSD & & & & & & & \\
\hline G2 & 63 & E7 & & & NSD & & & & & & & \\
\hline G2 & 64 & G7 & & & NSD & & & & & & & \\
\hline G2 & 65 & 17 & & & NSD & & & & & & & \\
\hline G2 & 66 & J8 & & & NSD & & & & & & & \\
\hline G2 & 67 & $\mathrm{H} 8$ & & & NSD & & & & & & & \\
\hline G2 & 68 & F8 & & & NSD & & & & & & & \\
\hline
\end{tabular}

Count Categories

Primary Amphibole Structures $>=0.5-<=5.0 \mu \mathrm{m}$

PAmS_5 PCMES-ISO

Primary Amphibole Structures $>5.0 \mu \mathrm{m}$

Page 21 of 23

PCS_>5

Primary Asbestos Structures

Primary Chrysotile Structures $>5.0 \mu \mathrm{m}$

TAmpSt, $>10$

Total Amphibole Structures, > 10, and 3:1

PCS_0.5-5

PCM Equivalent Structures-ISO

TAmpSt,0.5-5

Primary Chrysotile Structures $>=0.5-<=5.0 \mu \mathrm{m}$

TAmpSt,5-10

Total Amphibole Structures, $>5.0-<=10$, and

TAS 
A Professional Service Corporation in the Northwest

Fax: (206) 789-8424

http://www.labcor.net

\section{Soil - Elutriation Method Raw Data}

EPA 540-2-90-005, Modified May 23, 2000

Report Number: 070451R02

Job Number: 070451

SEA

Client: Idaho National Laboratory

Project Name: RARE

Client Sample No: BR-4-R1

Lab/Cor Sample No: S5

Client Description:

\begin{tabular}{|c|c|c|c|c|c|c|c|c|c|c|c|c|}
\hline $\mathrm{Gr}$ & No. & Loc. & ID & Prim Tot & Class & Len & Wid & Asp & Analyte & Elements & Comment & Count Categories \\
\hline G2 & 69 & D8 & & & NSD & & & & & & & \\
\hline G2 & 70 & B8 & & & NSD & & & & & & & \\
\hline $\mathrm{G} 2$ & 71 & A9 & & & NSD & & & & & & & \\
\hline $\mathrm{G} 2$ & 72 & $\mathrm{Cg}$ & & & NSD & & & & & & & \\
\hline $\mathrm{G} 2$ & 73 & E9 & & & NSD & & & & & & & \\
\hline G2 & 74 & 19 & & & NSD & & & & & & & \\
\hline
\end{tabular}

\section{Count Categories}

PAmS_0.5-5

PAS

PCS_>5

TAmpSt, $>10$

TAmpSt,5-10
Primary Amphibole Structures $>=0.5-<=5.0 \mu \mathrm{m}$ Primary Asbestos Structures

Primary Chrysotile Structures $>5.0 \mu \mathrm{m}$

Total Amphibole Structures, > 10, and 3:1

Total Amphibole Structures, $>5.0-<=10$, and

$3: 1$
PAmS_5 PCMES-ISO PCS_0.5-5 TAmpSt,0.5-5

TAS
Primary Amphibole Structures $>5.0 \mu \mathrm{m}$ PCM Equivalent Structures-ISO

Primary Chrysotile Structures $>=0.5-<=5.0 \mu \mathrm{m}$

Total Amphibole Structures, $>=0.5-<=5.0$, and 3:1

Total Asbestos Structures 
Phone: (206) 781-0155

7619 6th Ave Nw

A Professional Service Corporation in the Northwest

Fax: (206) 789-8424

http://www.labcor.net

Soil - Elutriation Method Raw Data

Job Number: 070451

SEA

EPA 540-2-90-005, Modified May 23, 2000

Report Number: 070451R02

Client: Idaho National Laboratory

Date Received: 4/23/2007

Project Name: RARE

Reviewed by:

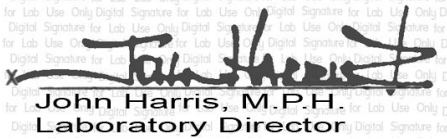

John Harris,

Count Categories

PAmS_0.5-5

PAS

PCS_>5

TAmpSt, $>10$

TAmpSt,5-10
Primary Amphibole Structures $>=0.5-<=5.0 \mu \mathrm{m}$ Primary Asbestos Structures

Primary Chrysotile Structures $>5.0 \mu \mathrm{m}$

Total Amphibole Structures, > 10, and 3:1

Total Amphibole Structures, $>5.0-<=10$, and $3: 1$
PAmS_5 PCMES-ISO

PCS_0.5-5

TAmpSt,0.5-5

TAS
Primary Amphibole Structures $>5.0 \mu \mathrm{m}$ PCM Equivalent Structures-ISO

Primary Chrysotile Structures $>=0.5-<=5.0 \mu \mathrm{m}$

Total Amphibole Structures, $>=0.5-<=5.0$, and 3:1

Total Asbestos Structures 